


\section{Fertile cities}

\section{Nutrient flows from new sanitation to urban agriculture}




\section{Thesis Committee}

\section{Promotor}

Prof. Dr G. Zeeman

Personal chair of New Public Sanitation

Wageningen University \& Research

\section{Promotor}

Prof. Dr O. Oenema

Special Professor of Nutrient Management and Soil Fertility

Wageningen University \& Research

\section{Co-promotor}

Dr J. Weijma

Assistant professor, Department of Environmental Technology

Wageningen University \& Research

\section{Other members}

Prof. Dr IJM de Boer - Wageningen University \& Research

Prof. A. van Timmeren - Delft University of Technology

Prof. Dr P.D. Jenssen - Norwegian University of Life Sciences (NMBU), Ås, Norway

Dr G. Metson - Linköping University, Sweden

This research was conducted under the auspices of the Graduate School for Socio-Economic and Natural Sciences of the Environment (SENSE) 


\title{
Fertile cities
}

\section{Nutrient flows from new sanitation to urban agriculture}

\author{
Rosanne Wielemaker
}

\section{Thesis}

submitted in fulfilment of the requirements for the degree of doctor at Wageningen University

by the authority of the Rector Magnificus

Prof. Dr A.P.J. Mol,

in the presence of the

Thesis Committee appointed by the Academic Board

to be defended in public

on Thursday 19 December 2019

at 11:00 a.m. in the Aula 
Rosanne Wielemaker

Fertile cities: Nutrient flows from new sanitation to urban agriculture, 318 pages

PhD thesis, Wageningen University, Wageningen, the Netherlands (2019)

With references, with summaries in English, Dutch and Spanish

ISBN: 978-94-6395-123-4

DOI: https://doi.org/10.18174/500300 
There is no guano comparable in fertility with the detritus of a capital. A great city is the most mighty of dung-makers.

Certain success would attend the experiment of employing the city to manure the plain. If our gold is manure, our manure, on the other hand, is gold.

What is done with this golden manure? It is swept into the abyss.

Victor Hugo, The Land Impoverished by the Sea, Les Miserables, 1862 



\section{TABLE OF CONTENTS}

Chapter $1+9$

Introduction

Chapter 2

Harvest to harvest: Recovering nutrients with new sanitation systems for reuse in urban agriculture

Chapter 3

Fertile cities: Nutrient management practices in urban agriculture

\section{Chapter 4}

Recycling nutrients contained in human excreta to agriculture: Pathways, processes, and products

\section{Chapter 5}

Identifying Amsterdam's nutrient hotspots: A new method to map human excreta at building and neighborhood scale

Chapter 6

Resource Dynamo: A GIS model to match urban nutrient supply with agricultural demand

Chapter 7

Human excreta management needs reframing

Chapter 8

Discussion

References

Supporting Information

Summary | Samenvatting | Resumen

Acknowledgements

Curriculum Vitae 


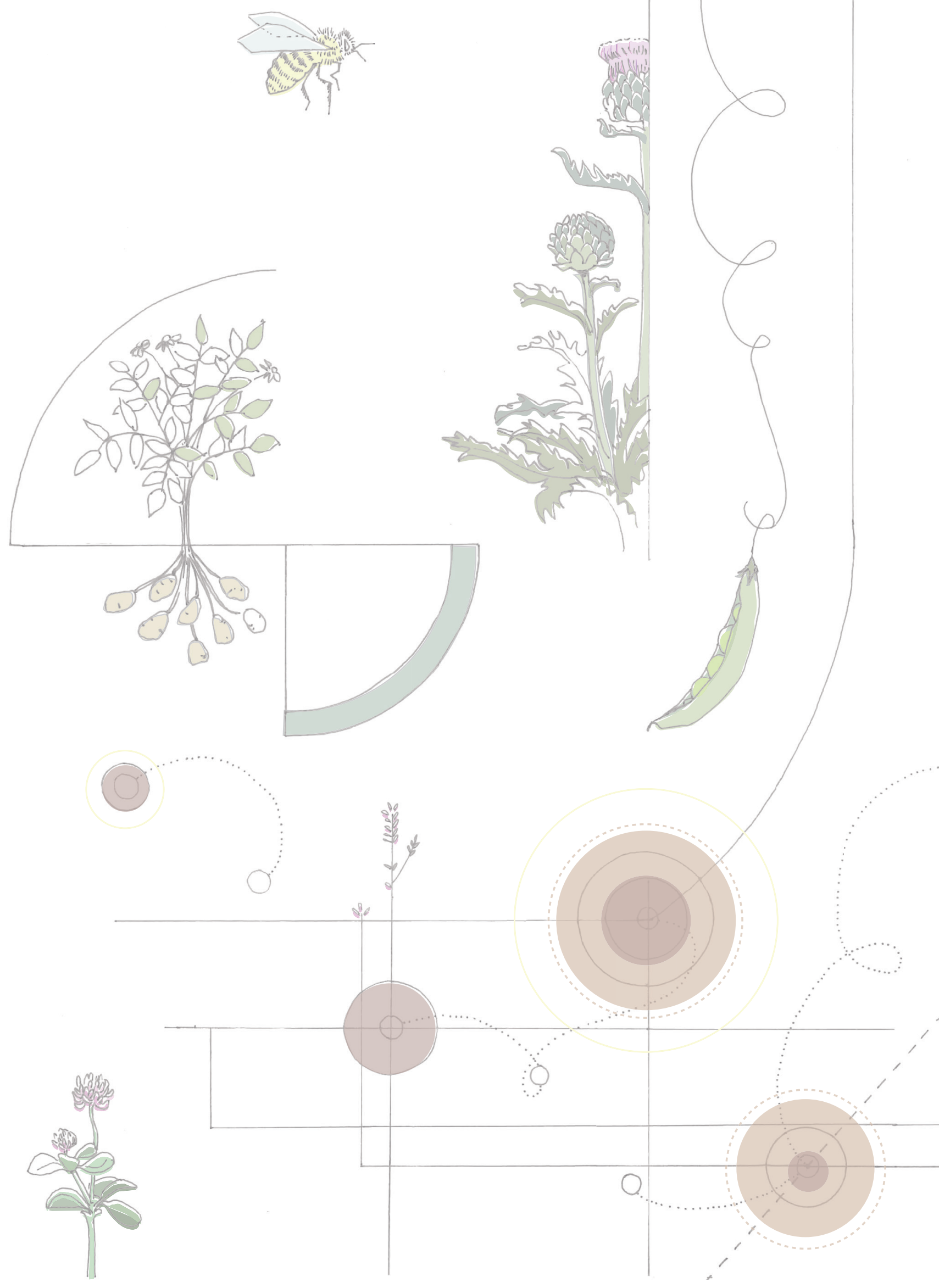




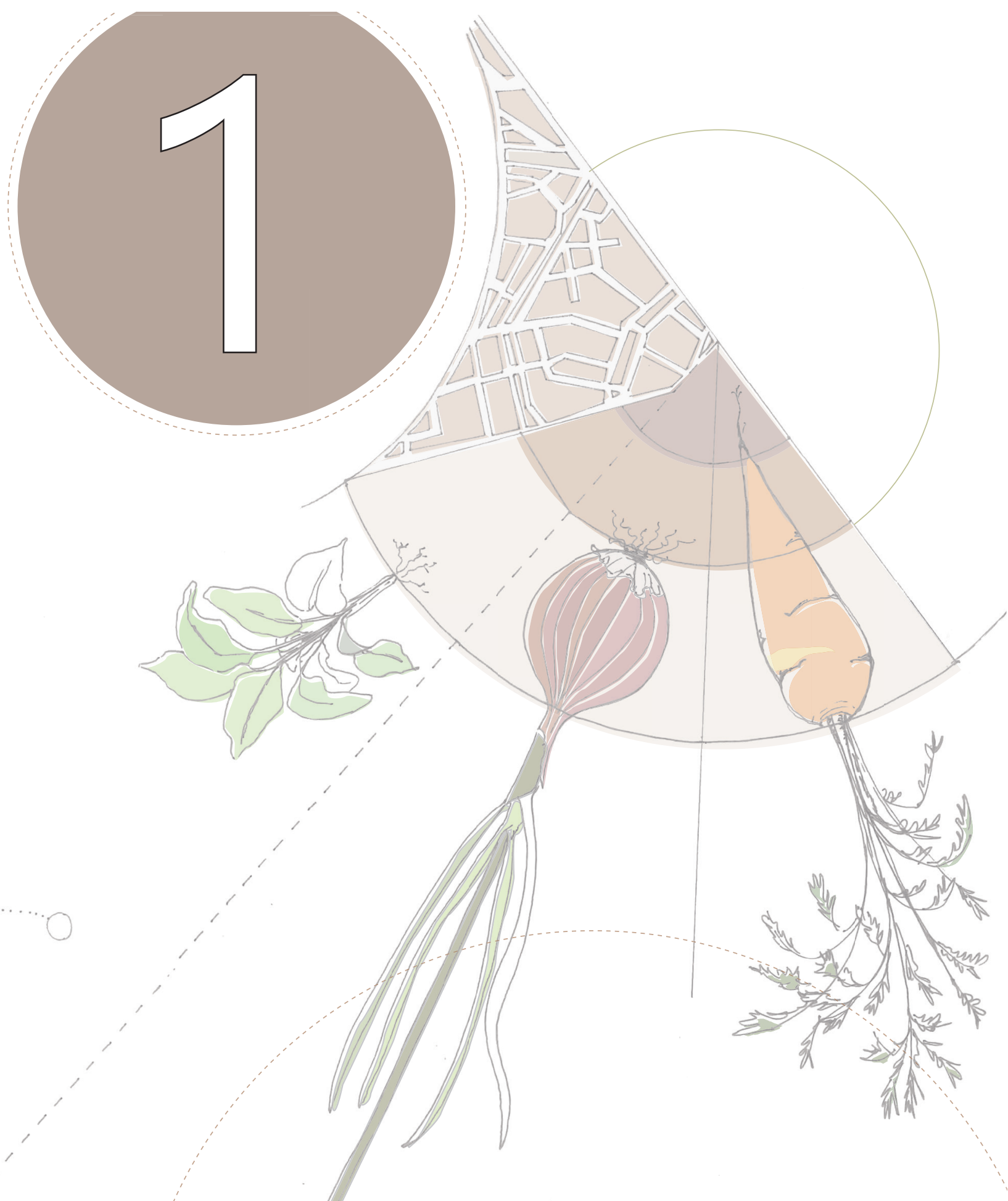


Minor parts of this chapter have been adapted from: Wielemaker, R. \& Weijma, J. in press. Redirecting nutrients in urban waste to urban agriculture. In: Wiskerke, J. S. (ed.) Acbieving Sustainable Urban Agriculture. Burleigh Dodds Science Publishing. 


\section{Background and problem outline}

\subsection{The nutrient cycle}

Nutrient elements are essential for all living organisms and thus are an important asset for soil fertility and crop growth. Plants need at least 14 different nutrient elements for growth and development (termed essential elements) (Maathuis, 2009, Marschner, 2011), notably nitrogen $(\mathrm{N})$, phosphorus $(\mathrm{P})$ and potassium $(\mathrm{K})$. Other elements taken up by plants are important for animal nutrition and for human health (e.g., cobalt and selenium); animals and humans need at least 22 different nutrient elements, including 15 micronutrient elements, for growth and development (Suttle, 2010). Nutrients move between the lithosphere, biosphere, hydrosphere and atmosphere, some in various organic and inorganic forms (Figure 1.1), to enable the provision, storage, transfer and regulation of these elements between biotic and abiotic components. Nutrient cycling occurs in ecosystems as a result of various natural driving sources (e.g., solar energy, tectonic energy, gravity), and interacts with the larger biogeochemical cycles through a system of inputs and outputs, which vary in space and time. Humans, like all organisms, assist in the flow of nutrients, most basically via the consumption of food and the excretion of urine and feces, and more fundamentally via respective human socio-economic activities including food and biomass production and processing, mining and processing of fertilizers, industrial activities, and waste management [Schroder et al., 2016]. These activities combined introduce more nitrogen $(\mathrm{N})$ and phosphorus $(\mathrm{P})$ into the nutrient cycles than would occur naturally (Steffen et al., 2015), leading to rising concerns about global efficiencies and sustainability of current nutrient management, further elaborated in Box 1.1 (Neset and Cordell, 2012, van Puijenbroek et al., 2019, Vitousek et al., 2009).

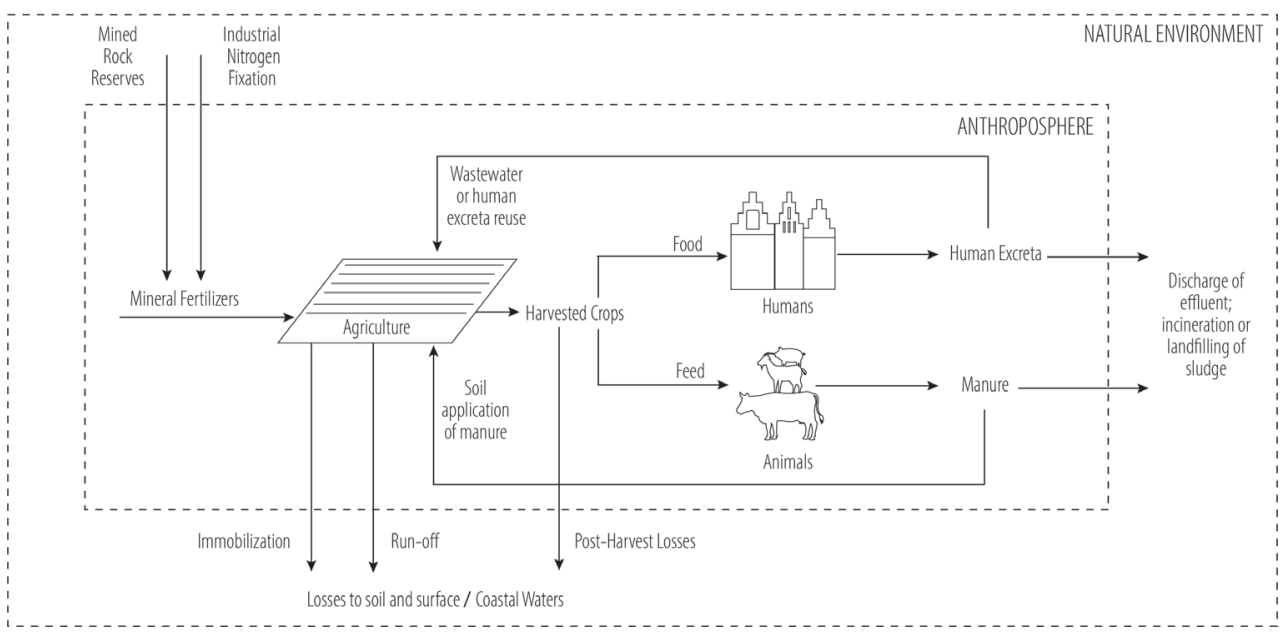

Figure 1.1 Basic scheme of key nutrient flows through global food production and consumption systems (adapted from Cordell et al., 2009a) 
BOX 1.1 Implications of current nutrient management for the nutrient cycle

Fertilizer inputs not only tackle the limited natural availability of nutrients in agroecosystems, but the use of synthetic fertilizers in particular has also increased global agricultural productivity immensely to sustain a growing global population (Schroder, 2014, Vitousek et al., 2009). Estimates suggest that approximately half of crop yield increase is due to mineral nitrogen fertilizer application (Erisman et al., 2008). Nonetheless, there are increasing concerns about the global efficiencies and sustainability of current nutrient use and management (Godfray et al., 2018, Steffen et al., 2015, Zhang et al., 2015).

First, the production of synthetic fertilizers exhausts fossil and mineral resources (Dawson and Hilton, 2011). While the production of nitrogen is energy intensive, currently sourcing energy from fossil fuels for the harvesting and conversion of atmospheric nitrogen $\left(\mathrm{N}_{2}\right)$ to ammonia $\left(\mathrm{NH}_{3}\right)$ (Maurer et al., 2003a), the sourcing of phosphorus and potassium, as well as several micronutrients, is dependent on finite, and spatially-concentrated, ore reserves (Cordell and White, 2011, van Dijk et al., 2016, Voortman, 2012a). The uneven spatial distribution of primary sources of nutrients could lead to geopolitical tensions, especially between countries with high nutrient dependency, such as countries in Europe, and countries rich in primary nutrient sources, e.g., China and Morocco (Rosemarin and Ekane, 2016, van Dijk et al., 2016, Withers et al., 2015).

Second, nutrient balances in agriculture are variable globally. In many developing economies, fertilizer inputs are inadequate to maintain soil fertility and thus contribute to soil nutrient deficiencies (e.g., $\mathrm{Zn}, \mathrm{Mg}$, $\mathrm{Cu}$ for crop yield) and 'nutrient stripping' (i.e., the disparity between food security and soil nutrient stocks) (Jones et al., 2013a, Nubé and Voortman, 2011, Vitousek et al., 2009). In developed and rapidly growing economies, excessive use of fertilizers has contributed to an accumulation of nutrients in soils and to increased leaching of nutrients to surface waters and groundwater (Glibert et al., 2014). Leaching of nitrogen and phosphorus to surface waters results in eutrophication, threatening water quality and freshwater ecosystem biodiversity and functioning (Cordell et al., 2009b, van Dijk et al., 2016, Vitousek et al., 1997).

High synthetic fertilizer inputs in agriculture are needed in part to account for nutrient losses from agriculture, but also for losses that occur further down the food chain in waste management systems. Nutrients contained in consumed food, which are subsequently excreted in the form of urine and feces, together contribute the largest fraction of nutrients to domestic wastewater (approximately $80 \%$ of nitrogen, $70 \%$ of phosphorus and $80 \%$ of potassium) (Kujawa-Roeleveld and Zeeman, 2006). The current management of human excreta leads to irretrievable losses of nutrients. In some countries, these losses occur through open defecation, pit latrine and septic systems or via direct discharge to surface waters. In developed countries, conventional wastewater treatment 
systems remove nutrients to meet discharge targets (e.g., as per the Water Framework Directive in European Union (EU) legislation), however, current systems ensue nutrient losses to the air or surface water (Chowdhury et al., 2014, Daigger, 2009, EC, 2016). The low level of recycling of nutrients in sewage sludge to agriculture follows from concerns regarding the presence of heavy metals and micro pollutants (Ott and Rechberger, 2012); sewage sludge is therefore often landfilled or incinerated (Kirchmann et al., 2016). Some semi-arid countries, in contrast, use untreated and/or treated wastewater for irrigation of agricultural land, though concerns regarding associated health risks persist, especially when the handling and application of the waste waters are not well managed (Jaramillo and Restrepo, 2017).

In agroecosystems, nutrient cycling and management refers to replacing nutrients, withdrawn during crop harvesting and soil cultivation, through biological processes such as nitrogen fixation or through the addition of organic material and/or mineral fertilizers to fields [Vitousek et al., 2009]. Historically, human excreta (also termed 'nightsoil') and organic residues from households, in addition to animal manure, were used to replenish (urban and peri-urban) agricultural land with nutrients and organic matter (Cooper, 2001a, Ferguson, 2014, van Zon, 1986, King, 2004). In many economically developing countries this is often still common practice, due to urgency for livelihood and food security (LeGrand et al., 2014, Mårald, 2006, Richardson, 2012, Ronteltap et al., 2007, Scheierling et al., 2011).

In economically developed countries, however, environmental and health concerns related to unsafe reuse and disposal of human excreta (e.g., eutrophication; cholera, E. coli) have led to the implementation of extensive sanitation infrastructures. The short preview of the Liernur system $(\sim 1870)$ with vacuum pressure pipes to export human excreta to agriculture, was quickly overtaken by waterborne sanitation, based on flush toilets and sewers, from the late 19th century onwards (Ferguson, 2014). Meanwhile, parallel developments in synthetic fertilizer production and use came to largely substitute the use of human excreta in agriculture, and enabled the expansion of agriculture on distant soils. The physical separation between urban settlements and agricultural land, no longer facilitated the reuse of waste in agriculture (Ferguson, 2014).

Population growth, urbanization and globalization have led to increased fluxes of nutrients from rural to urban areas, across borders and between continents. This has resulted in a distinct depletion of nutrients from agricultural soils in some places and the accumulation of nutrients in urban waste streams, soils and water systems in other places (Bouwman et al., 2009, van Dijk et al., 2016, Nesme et al., 2018). Sanitization, chemicalization (the use of chemical fertilizers) and industrialization have further contributed to critically altered nutrient flows, with corresponding consequences (Jones et al., 2013a, Kyllingsbæk and Hansen, 2007, Nesme et al., 2018, Cordell and White, 2011, Grimm et al., 2008, Ayala and Rao, 2002), illustrated in Figure 1.2a. 


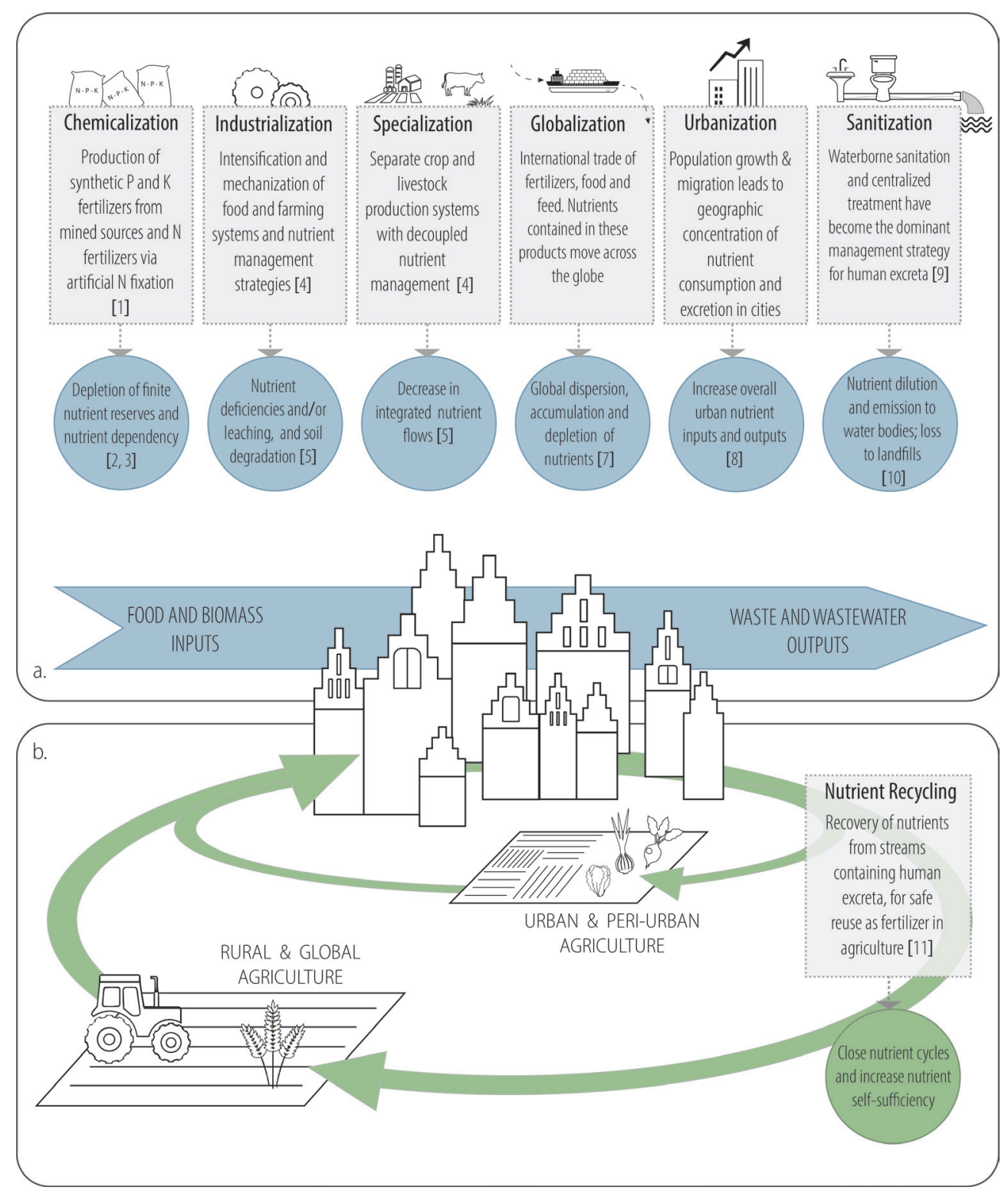

Figure 1.2 Nutrient flows through urban systems. (a) current nutrient flows from synthetic fertilizer to agriculture to food products to human excreta and other waste outputs, and associated consequences for the nutrient cycle. (b) strategies for nutrient recycling via nutrient recovery from streams containing human excreta to rural and global agricultural hinterlands, or alternatively, to urban and peri-urban agriculture. References: [1] (Dawson and Hilton, 2011) [2] (Cordell and White, 2011) [3] (van Dijk et al., 2016) [4] (Kyllingsbæk and Hansen, 2007) [5] (Jones et al., 2013a) [7] (Nesme et al., 2018) [8] (Grimm et al., 2008) [9] (Ferguson, 2014) [10] (Glibert et al., 2014) [11] (LeGrand et al., 2014) 


\subsection{From losses to loops}

Cognizant of the limitations of industrial fertilizer production and use, and of current sanitation infrastructures, it has become increasingly evident that present patterns of nutrient flows are unsustainable in the long term. This has urged industry, scientists and policy makers to rethink current nutrient management strategies and look towards solutions, to once again restore tight nutrient cycling, most notably for nitrogen and phosphorus (Chowdhury et al., 2017, Malila et al., 2019, Theregowda et al., 2019, Ulrich and Schnug, 2013, van Dijk et al., 2016, Withers et al., 2015). Interventions for improved nutrient management traverse multiple aspects of food and farming systems, as well as waste management, including a realignment of inputs to meet requirements in agriculture, a reduction of losses to water, and the reuse of agricultural byproducts as food, feed or fertilizer (de Boer and van Ittersum, 2018, Withers et al., 2015).

Among proposed interventions is the recycling of nutrients in human excreta and other waste streams to agricultural land (Figure 1.2b), following treatment and recovery processes, i.e., the production of safe fertilizer products from human excreta. The technological trend in recent decades in economically developed countries is towards facilitating resource recovery, and the extraction of nutrients from waste streams has gradually increased (Sartorius et al., 2012). Currently multiple technologies and combinations of technologies exist for the recovery of nutrients from human excreta, including precipitation, stripping, sorption, phototrophic biomass growth and leaching from sewage sludge incineration ashes (Tilley, 2014).

\subsection{Redirecting human excreta to urban and peri-urban agriculture}

The geographic concentration of food consumption and human excreta production in cities signifies that cities play a key role in new approaches for recycling nutrients to agriculture (Hodson et al., 2012b). Considering the global extent of current nutrient flows, one strategy for the recirculation of nutrients to agriculture could include the recovery of nutrients from (streams containing) human excreta and transporting these back to existing agricultural hinterlands. This would require concentrating recovered nutrients into transportable and exportable products for recycling on distant agricultural soils. Another strategy would be to recycle nutrients to closer locations, such as urban and peri-urban agricultural fields (within or along the perimeter of the city). Complemented with new sanitation solutions (systems for collection, transport, and treatment of, and recovery of resources from, streams containing human excreta), nutrient supply in human excreta and demand in urban agriculture can be linked to realize local solutions. From an environmental perspective, it is favorable to close resource cycles at local scales, rather than at regional and continental scales to avoid unnecessary transport and energy costs (Agudelo-Vera et al., 2012a, Tidåker et al., 2007, McConville et al., 2015).

Developments in both urban agriculture and new sanitation have occurred in parallel, yet autonomously. In the last few years, recognition of the mutual benefit for resource exchange between urban agriculture and new sanitation has increased (Goldstein et al., 2016, Grewal and 
Grewal, 2012, Chrispim et al., 2017b, Strauss, 2000). Even so, the quantification and assessment of recycling nutrients between the two remain mostly unexplored. The quantity and quality of the nutrient demand from urban agriculture systems needs to be matched by the quantity and quality of the nutrient flows produced by new sanitation systems - taking into account parameters for plant requirements, as well as human hygiene and environmental safety (e.g., pathogens, heavy metals). As nutrient supply and demand are variable in space and time, full considerations of spatio-temporal dynamics for optimized coupling of nutrient flows are also needed. This thesis focuses on uncovering the potential of redirecting nutrients in human excreta via new sanitation systems to urban agriculture, as further elucidated in the following sections.

\section{Key concepts}

This section provides background information and a general overview of urban agriculture and new sanitation individually and introduces their role in nutrient management.

\subsection{Urban agriculture}

Urban agriculture is widely practiced across the globe; an estimated 25-30\% of urban dwellers participate in urban farming, most prominently in emerging economies (Orsini et al., 2013). In these contexts urban agriculture is a means of income and increases local food security (Hamilton et al., 2014). In developed economies, urban agriculture is intensifying and formalizing as a means for creating a more resilient food system and increasing urban sustainability (fulfilling social, economic and environmental roles) (Cerón-Palma et al., 2012, Goldstein et al., 2016, Specht et al., 2013). Manifestations of urban agriculture include both low-tech and high-tech production systems, such as, community gardens, ground-based farms, rooftop farms, rooftop greenhouses, and (multi-story) indoor farms. These are either ground-based, contained or controlled systems (see examples in Figure 1.3). Especially controlled systems require higher energy and technology inputs such as climate control systems, artificial (LED) lighting and hydroponic growing systems. Various nomenclatures have been developed to differentiate forms of urban agriculture including: Continuous Productive Urban Landscape (CPUL) (Viljoen and Howe, 2012), Vertical Farming (Despommier, 2010), Building Integrated Agriculture (BIA) (Caplow, 2009), and ZeroAcreage Farming (Specht et al., 2013).

The diversity of activities, scales, locations and purposes attributable to urban agriculture, results in a broad range of definitions in literature (see Vejre, 2012). In this thesis the definition used is based on the one coined by (Mougeot, 2000): 'the production of food in (intra-urban) and around (peri-urban) a city, in which the practice of food production exchanges human and material resources, products and services with that city'. Inherent to this definition lies the notion of nutrient cycling; by exchanging resources, urban agriculture receives urban organic materials ('wastes') and supplies fresh produce ('food'). Smit and Nasr (1992) first alluded to the 
role that urban agriculture can play in recycling to improve urban sustainability; the topic has since received increasing, yet still meagre, attention from various academics (RUAF, 2006, Specht et al., 2013, Thomaier et al., 2015, McClintock, 2010).

Nutrient cycling in urban agriculture often includes the use of compost from garden waste of its premises, surrounding gardens and green public space, and/or kitchen waste from homes in the direct vicinity (Bergström et al., 2008). However, it can be assumed that the diversity in urban agricultural practices, demands equally diverse soil amendments and fertilizer inputs rather than solely compost. From a recycling perspective, recycled and recovered nutrient-rich products from streams containing human excreta could offer alternative solid (organic and inorganic) and liquid fertilizers to cover the demand for fertilizers and soil amendments from urban agriculture. In emerging economies the reuse of human excreta and raw wastewater is often researched with regards to health risks.

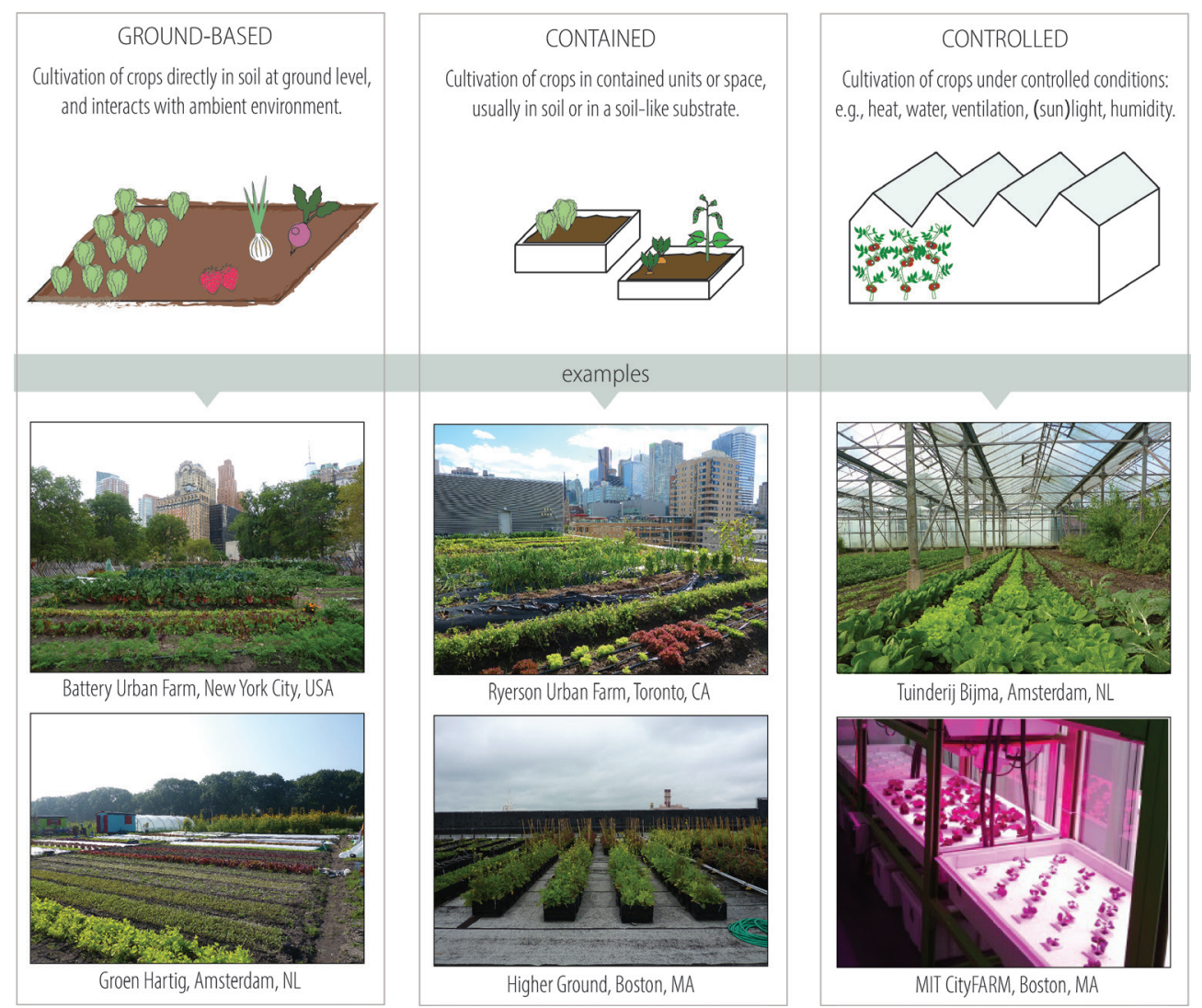

Figure 1.3 Three broad types of urban agriculture: ground-based (e.g., outdoor cultivation directly in soil), contained (e.g., rooftop; raised beds), controlled environment (e.g., greenhouse or indoor cultivation). Photographs by Rosanne Wielemaker. 


\subsection{New sanitation}

Human excreta contributes the majority of nutrients to domestic wastewater in a small fraction of the volume (see Figure 1.4a); urine contains the majority of the total nitrogen, phosphorus, and potassium in human excreta, while feces contains most of the organic matter, although exact values are variable in literature (Tervahauta et al., 2013, Simha and Ganesapillai, 2017). Human excreta, however, also contain pathogens, including enteric bacteria, and possibly viruses, protozoa and helminth eggs, pharmaceuticals and hormones, and heavy metals, albeit partitioned varyingly amongst urine and feces (Friedler et al., 2013b, Heinonen-Tanski and van Wijk-Sijbesma, 2005, Schönning et al., 2002). Thus, the challenge is to develop sanitation systems that effectively recover nutrients for direct reuse or as input for the fertilizer industry, comply with standards for public health, and prioritize environmental protection, while serving modern comfort preferences for toilet use (Lettinga et al., 2001, Shove, 2003).

The long-term sustainability and suitability of conventional sanitation systems, based on waterborne transport and aerobic sewage treatment, is increasingly called into question. The high infrastructure, maintenance and operation costs are prohibitive for widespread adoption in low-income countries (Larsen et al., 2016), while the high energy and water demand of the systems, and limited nutrient recycling are concerns in high-income countries (Brands, 2014). In recent decades, new sanitation systems (also referred to as 'ecological sanitation') have been developed for the collection, transport and treatment of and the recovery of nutrients from human excreta, that tend away from conventional urban wastewater infrastructures (Haddaway et al., 2019). One of the explicit objectives inherent to new sanitation is to facilitate nutrient recycling.

New sanitation systems are based on the premise that nutrient recovery is most cost-effective from streams with high nutrient and low contaminant concentrations (Larsen et al., 2009a, Zeeman and Kujawa-Roeleveld, 2011a). Source-separation prevents dilution and mutual contamination of streams via the implementation of toilets (e.g., low flush, vacuum and urine diversion) and sewer infrastructure (e.g., in house sewers and vacuum pipes). Human excreta (and possibly flush water), called blackwater, can be collected separately from grey water (used water from bath, shower, washing, etc.) industrial water and/or storm water; urine and feces (and possibly flush water) can be collected separately (see Figure 1.4b) to further segregate nutrient concentrations. Organic kitchen waste can be added to sewage or blackwater via disposal units (grinders). The recovery of nutrients from these separated streams occurs through the application of a (sequence of) treatment and recovery process(es), which result in output(s) of recovered products. Product composition depends on the primary input and the applied sequence of technologies, each with respective nutrient recovery efficiencies and potential to remove contaminants. The large number of possible combinations renders an equally diverse output of recovered products in terms of quantity and quality. Reusing recovered products in urban agriculture demands quality assurance across the treatment and reuse chain (Degaardt, 
2003a), not only for their effectiveness as fertilizers (e.g., plant availability of nutrients), but also to ensure human and environmental health by minimizing the risks associated with the introduction of human pathogens, hormones, pharmaceuticals, personal care products, heavy metals and other micro pollutants into the environment.
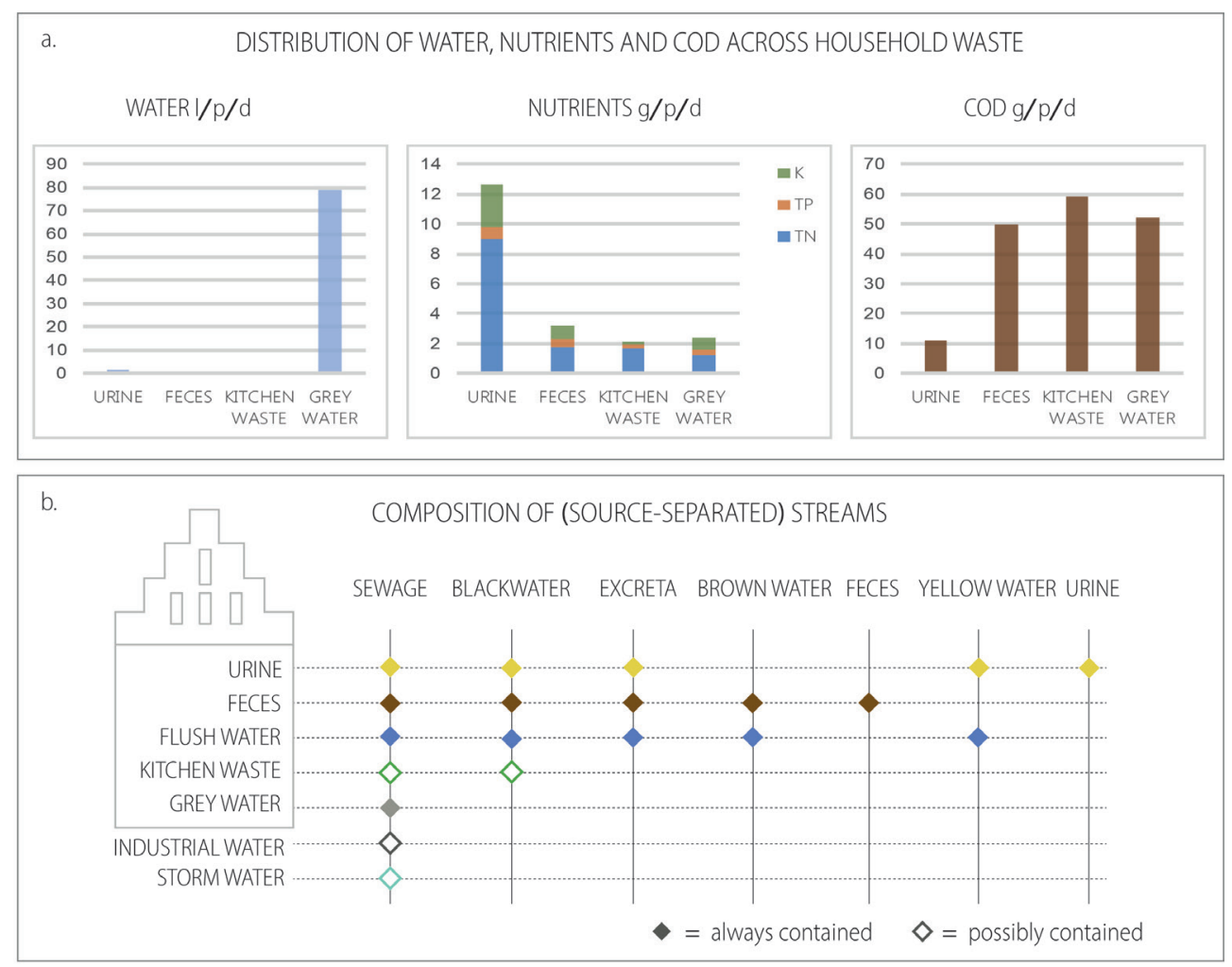

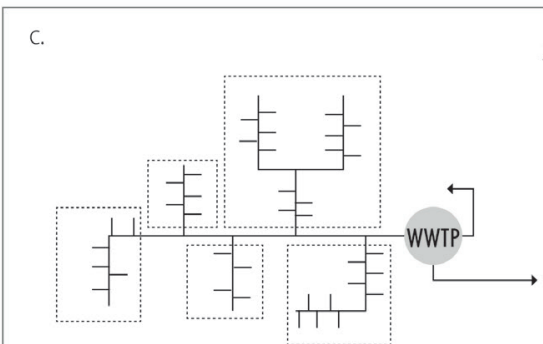

CENTRALIZED
SANITATION SCALES
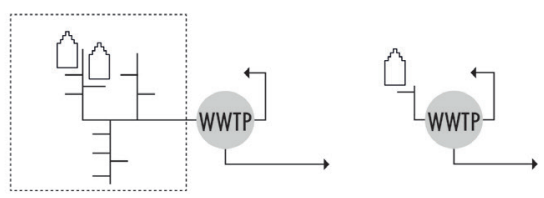

DECENTRALIZED

Figure 1.4 (a) Distribution of water, nutrients (nitrogen, phosphorus, potassium) and organics (indicated in chemical oxygen demand (COD)) across urine, feces, greywater and kitchen waste (data from Tervahauta et al. (2013)); (b) overview of combined inputs to form (source separated) streams for collection; (c) schematic of centralized (e.g., multiple-neighborhood or city-wide) and decentralized (e.g., neighborhood, block, street, or household) collection and treatment systems. 


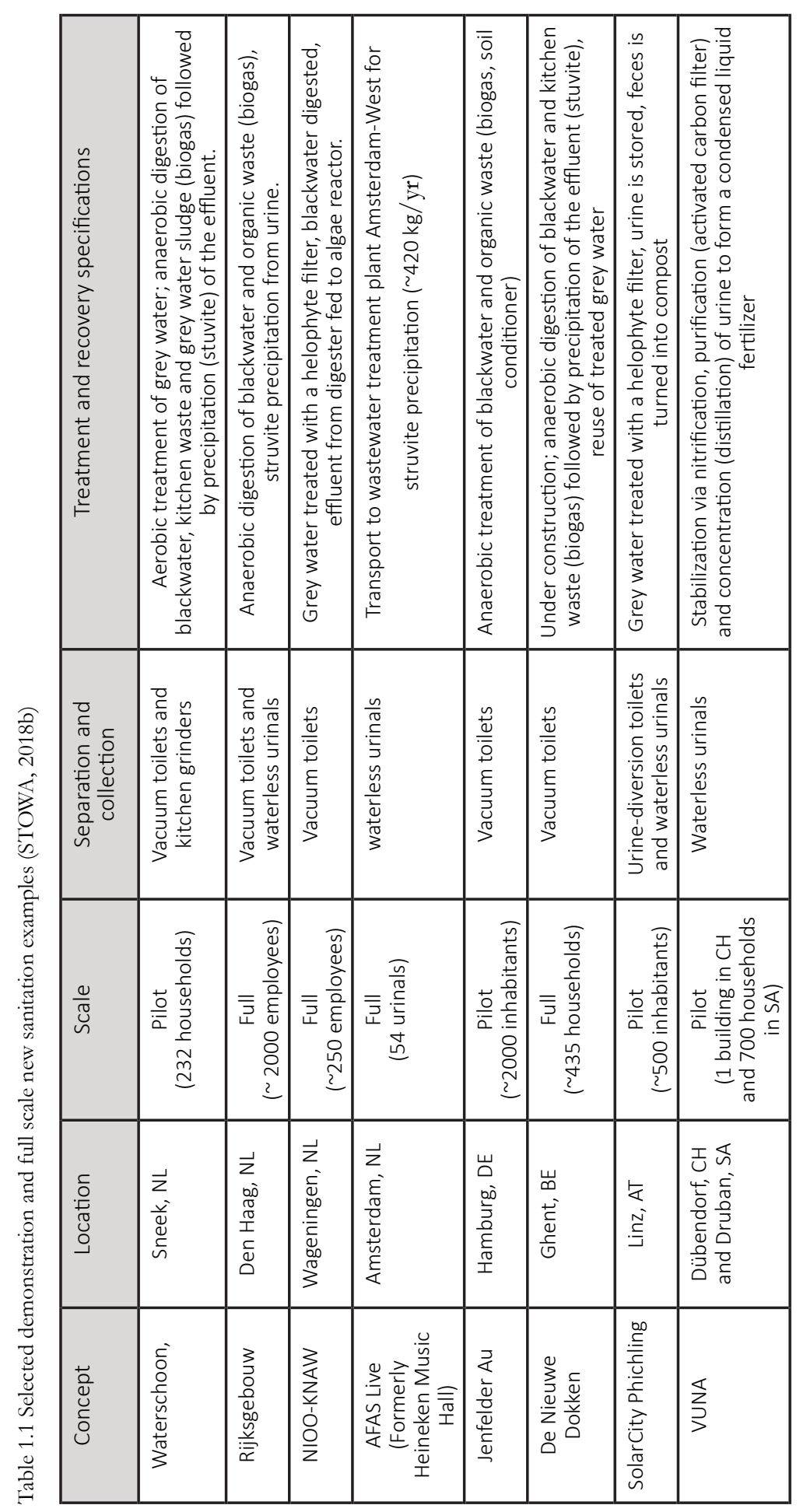


New sanitation systems are usually decentralized systems (see Figure 1.4c) since source-separation is currently easiest at this scale and due to the early development phase of many new sanitation technologies. The Waterschoon system implemented in Sneek, the Netherlands has shown to be economically competitive when scaled to approximately 3000 inhabitants, compared to conventional systems of 30,000 and 100,000 inhabitants (STOWA, 2018a). Nevertheless, there is no inherent limitation to apply such systems at a scale similar to that of centralized systems. State of the art demonstration and full scale examples of new sanitation for $>100$ inhabitants are listed in Table 1.1 (Winker et al., 2009b, Zeeman and Kujawa-Roeleveld, 2011a).

\section{Thesis Scope}

\subsection{Research objective}

The objective of this research is to contribute to uncovering the potential of integrating urban agriculture and new sanitation so as to establish nutrient recirculation between the two. In this regard, urban agriculture has a demand for nutrients and new sanitation a supply of nutrients, which if matched, can facilitate nutrient recycling and thereby minimize nutrient losses. Specific objectives include (1) an analysis of nutrient demand and supply, (2) an evaluation of spatial and temporal aspects of supply and demand matching, and (3) a reflection on trade-offs for improved nutrient recycling within the urban environment. This thesis primarily focuses on the three macronutrients, nitrogen $(\mathrm{N})$, phosphorus $(\mathrm{P})$ and potassium $(\mathrm{K})$, as well as organic matter $(\mathrm{OM})$, although, other macro- and micronutrients are tangentially discussed.

\subsection{Research questions}

The central question of this thesis research is: 'what is the potential to recycle nutrients present in human excreta as fertilizer to agriculture within the urban and peri-urban environment?' To address this question, four sub-questions are defined, as briefly explained in the succeeding text.

1. What is the demand for nutrients by urban agriculture?

Urban agriculture is diverse in practice and for the most part unregulated. Currently, there is little information in literature on how nutrients are managed on urban farms, which kinds and amounts of nutrient inputs are used, and their origin.

2. What quantity and quality of recovered nutrient-containing products can new sanitation systems render?

While research in and application of new sanitation systems has increased, a clear overview of all possible treatment and recovery configurations, recoverable products and their reuse potential in agriculture is still lacking.

3. How do spatial and temporal conditions influence the potential to match nutrient demand by urban agriculture with nutrient supply by recovered products? 
While mass balances between the two are an important first step, considerations of the spatial and temporal conditions for optimal nutrient recirculation are also needed. Such explorations can uncover, for instance, where to implement new sanitation systems and urban agriculture for increased mutual benefit or reduced transport between the two. It may also contribute to understanding the role of seasonality of nutrient demand and supply.

4. What trade-offs need to be considered when matching nutrient flows between urban agriculture and new sanitation systems?

Simultaneous consideration of urban agriculture and new sanitation may reveal new and unrealized potentials, and may also bring to the fore trade-offs between the two. For example, many recovery efforts have focused on energy and phosphorus only, which results in a neglect, and loss, of other nutrients and organic matter.

\subsection{Research context}

This research was carried out in the context of the Netherlands given the increased national interest in both the professionalization of urban agriculture (Green Deal Stadsgerichte Landbouw, 2013) and resource recovery (Government of the Netherlands, 2016). These interests stem from a general concern of the long-term sustainability of current resource management (Belevi and Baumgartner, 2003, Scheierling et al., 2011, van Der Schans, 2010). The results of this research are also expected to be relevant for other contexts because the transition towards closed nutrient cycles is of global interest.

\section{Thesis outline}

This thesis is presented in a publication-based format; the following six chapters have been published in or submitted to peer-reviewed scientific journals as stand-alone pieces, some in slightly modified versions. Figure 1.5 visualizes the connection of the chapters within the entirety of the thesis.

Chapter 2 presents a first exploration of closing cycles between urban agriculture and new sanitation using the Urban Harvest Approach, and shows the achievable nutrient self-sufficiency for phosphorus, nitrogen and organic matter in the city of Rotterdam. Chapter 3 presents a quantitative study of nutrient inputs at 25 urban farms across the Netherlands. Results are based on farm interviews and model calculations. This study serves as a quantitative benchmark for understanding nutrient management practices in urban agriculture, as well as fertilizer preferences and demands.

Research on nutrient supply from urban areas is presented in Chapter 4 and Chapter 5. Chapter 4 includes an extensive description of recovery pathways that exist to recover nutrients from streams containing human excreta. The chapter further identifies broad patterns and trends, and highlights the current focus on selective process technologies and on the targeted recovery of 
phosphorus. In addition, the review is meant to serve as a as basis for organizing and categorizing information on nutrient recovery pathways for more effective sharing and consolidation.

Chapter 5 moves the research towards including a spatial dimension to nutrient flows, recognizing the importance to understand where nutrient excretion takes place. The study used geographic information system (GIS) analysis to identify locations with high nutrient excretion (supply) at building and neighborhood scale (termed nutrient hotspots). Chapter 6 builds on the results presented in Chapter 5 and aims at matching the supply with nearby demand, while optimizing transportation distances between the two. Such understanding of spatially explicit data on the scale at which practitioners work, can increase capacity building for planning and decisionmaking with regards to intervention strategies for improved resource management.

Chapter 7 looks at the subject through a theoretical lens, and presents a plea for reframing human excreta management as part of food and farming systems.

Chapter 8 places the results of this thesis within the broader perspective on (urban) nutrient management. The chapter includes a synthesis of the results on the potential to redirect nutrients contained in human excreta to urban agriculture. It finishes by indicating several areas for future research.

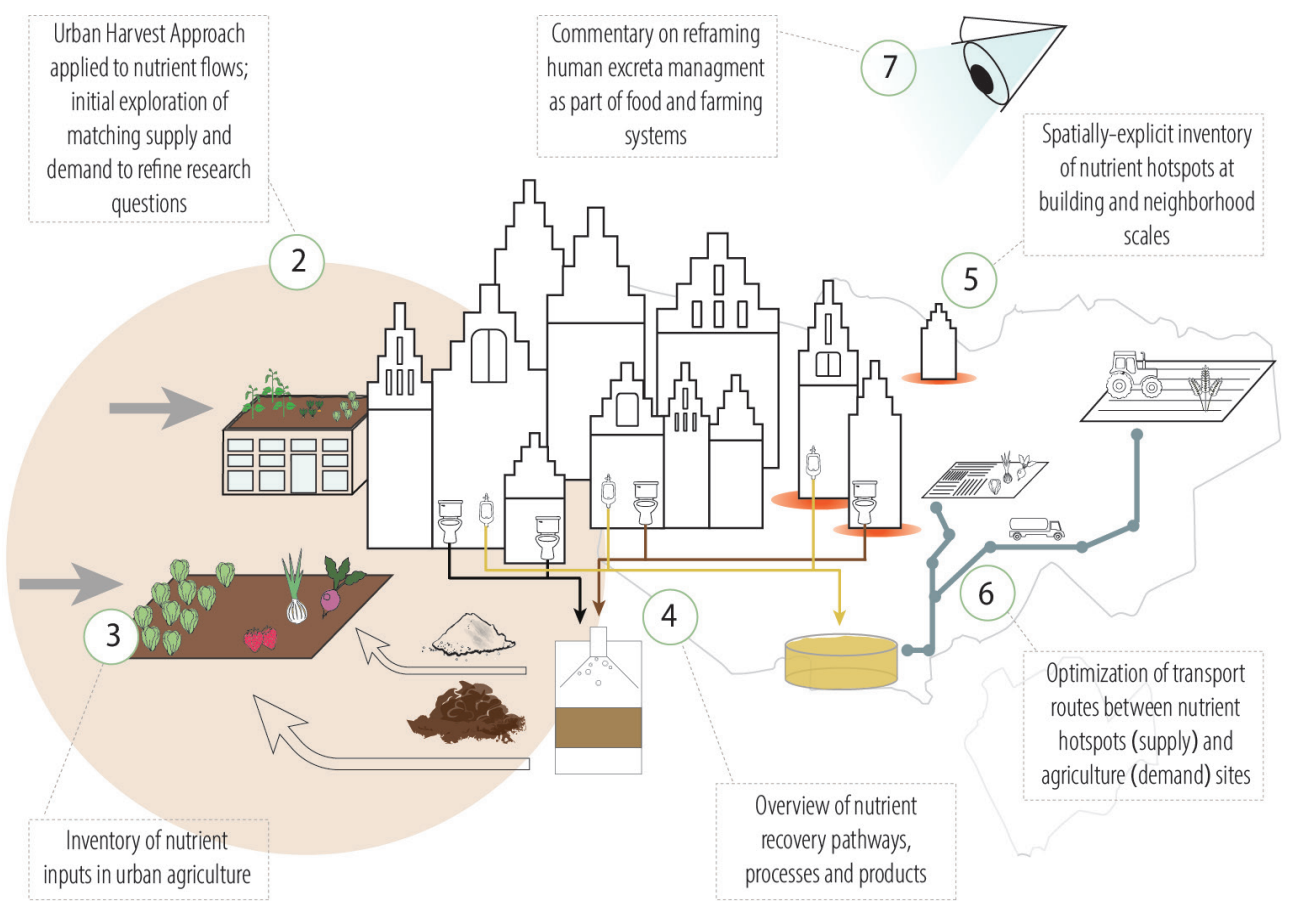

Figure 1.5 Scheme of presented chapters 


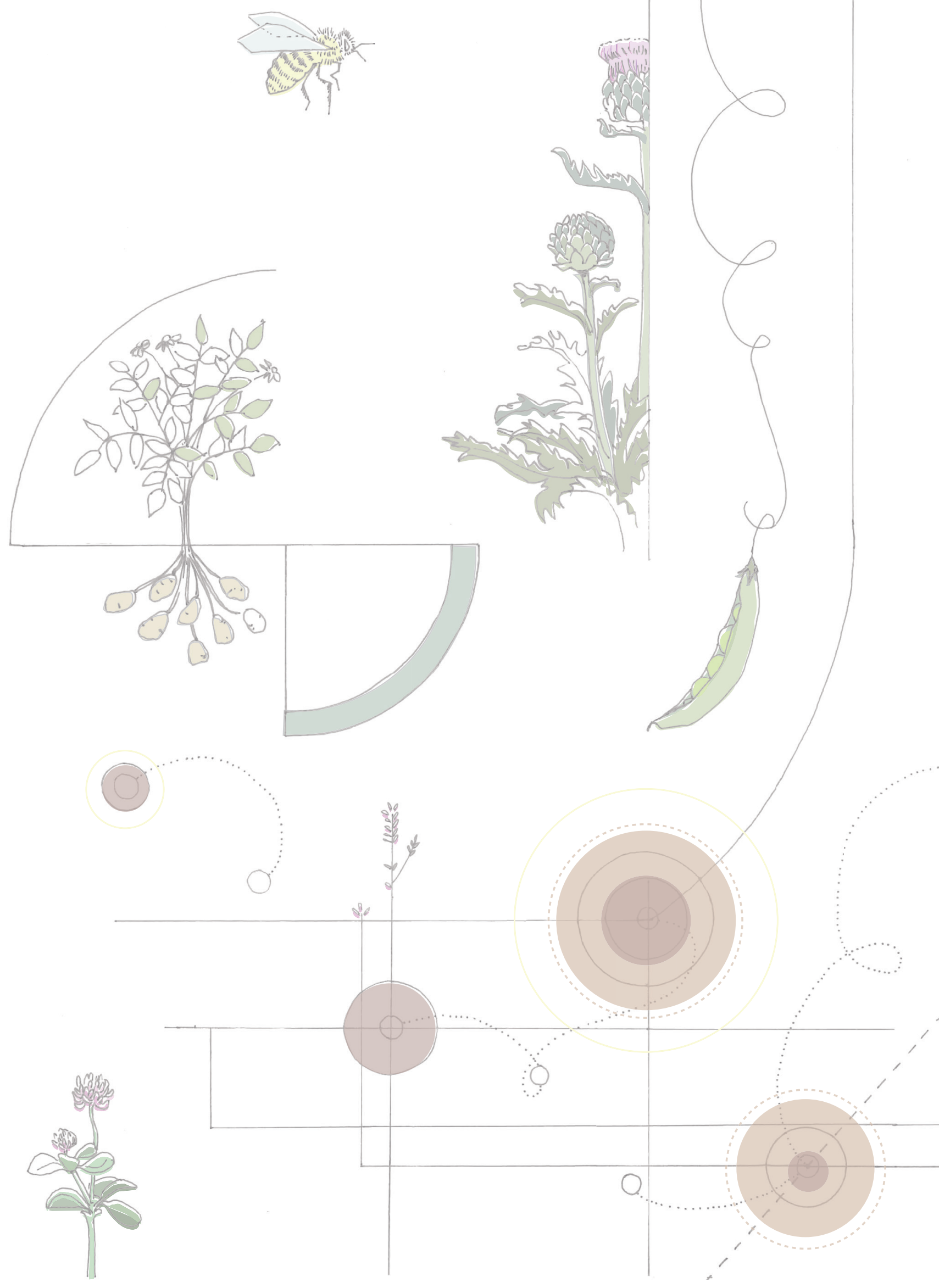




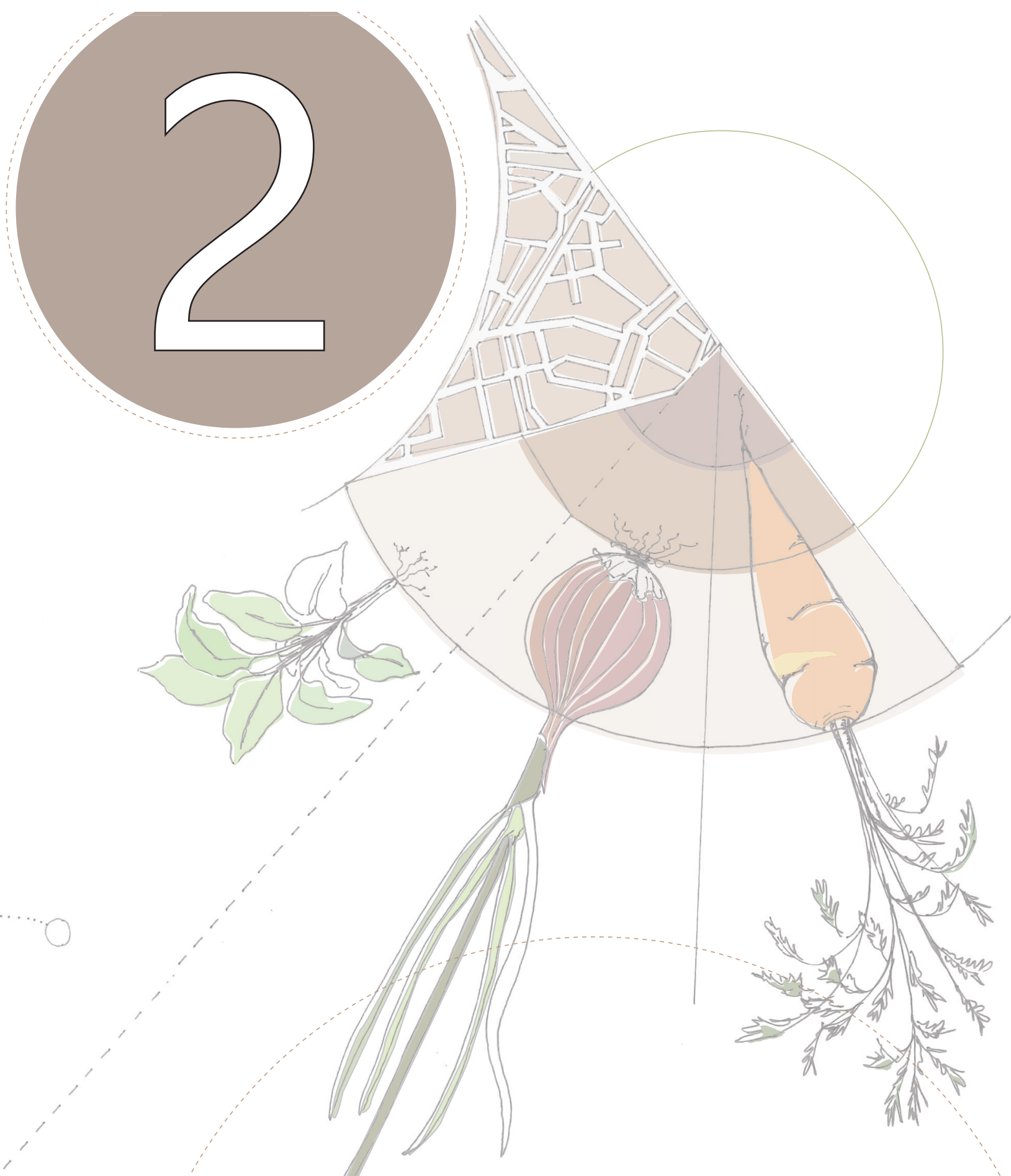

HARVEST TO HARVEST: RECOVERING NUTRIENTS WITH NEW SANITATION SYSTEMS FOR REUSE IN URBAN AGRICULTURE 


\begin{abstract}
To maintain the city as a viable concept for human dwelling in the long term, a circular metabolism needs to be adopted that relies on recovering, reusing and recycling resources, in which output ('waste') from one metabolic urban conversion equals input for another. Urban agriculture and source-separation-based new sanitation are gaining momentum as measures for improved urban resource management. Urban agriculture aims to localize food provisioning while new sanitation aims to reorganize wastewater and organic waste management to recover valuable and crucial resources. The objective of this paper is to assess the match between the supply by new sanitation systems and the demand from urban agriculture for nitrogen, phosphorus and organic matter in terms of quantity and quality, to foster a circular metabolism. The research is contextualized in the city of Rotterdam. The methodology used is based on the Urban Harvest Approach (UHA), developed previously for the urban water cycle. Novel to this research is adapting the UHA to nitrogen, phosphorus and organic matter loads for two practiced urban agriculture typologies (ground-based and rooftop) and four new sanitation concepts for the treatment of domestic urine, feces and organic kitchen waste. Results show that demand for nutrients and organic matter from urban agriculture can be minimized by $65-85 \%$ and a self-sufficiency of $100 \%$ for phosphorus can be achieved, while partial self-sufficiency for nitrogen and organic matter. This research reveals that integration of new sanitation and urban agriculture increases urban selfsufficiency.
\end{abstract}

Keywords: urban agriculture; new sanitation; urban metabolism; Urban Harvest Approach; nutrients; organic matter

This chapter is published as: Wielemaker, R. C., Weijma, J. \& Zeeman, G. 2018. Harvest to harvest: Recovering nutrients with New Sanitation systems for reuse in Urban Agriculture. Resources, Conservation and Recycling, 128, 426-437. 


\section{Introduction}

Cities depend on regional and global hinterlands for the supply of water, energy, nutrients and materials and for the disposal of wastes (Agudelo-Vera et al., 2012c, Brunner, 2007, Hodson et al., 2012a, Kennedy et al., 2007), deeming cities hotspots for resource conversion. This conversion presently follows a linear metabolism from high quality resource inputs and low quality waste outputs (Figure 2.1a). Few resources are currently recovered for reuse. This linear metabolism leads to two major challenges: first, cities' high rate of consumption puts stress on resource availability (e.g., phosphorus, fossil fuels), and second, the disposal of vast amounts of waste causes pollution (e.g., water and resource contamination, biodiversity loss, deforestation, and pollution in air, water and land). Cities currently import large quantities of food not only from their hinterlands, but also from locations across the globe. At the same time, they produce low or even negative value waste loads containing disposed and excreted nutrients. These are often mixed and collected via large-scale engineered infrastructures that endorse this linear tendency and make it difficult to effectively recover resources (Balkema et al., 2002, Hodson et al., 2012a). With more than half of the world's population currently residing in cities, this linear tendency is further intensified (United Nations, 2014).

As hotspots of resource conversion, however, cities also present an excellent opportunity to adopt a high-impact circular metabolism, in which output ('waste') from one process equals input ('resource') for another. As opposed to the current linear urban metabolism, a circular urban metabolism aims to recover and reuse (recycle) resources within or between urban functions to reduce both the external input of virgin resources and the output of waste (Agudelo-Vera et al., 2012c) (Figure 2.1b). To move towards a circular urban metabolism, resource input-output flows of urban functions need to be identified, described and matched in terms of quantity and quality. New sanitation and urban agriculture are currently gaining global interest individually as measures to improve urban resource management (Degaardt, 2003b, Metson and Bennett, 2015, Mougeot, 2006, Vernay et al., 2010). Linking these two urban functions could lead to mutual benefit in terms of resource cycling, especially for fertilizers.

a)

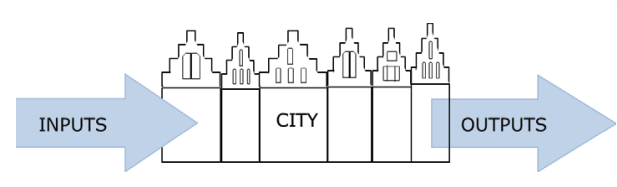

b)

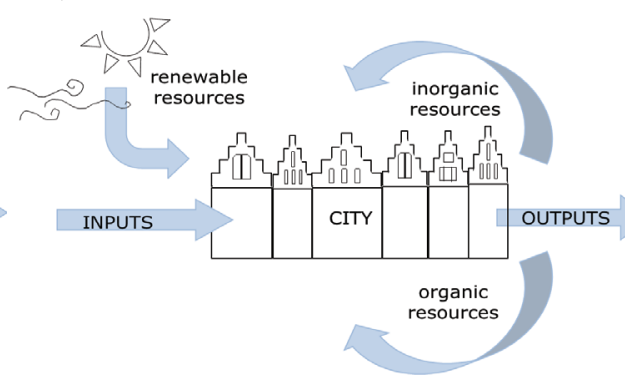

Figure 2.1 (a) A linear metabolism of inputs and outputs. (b) A circular metabolism reuses, recycles and recovers resources from urban waste streams, reducing resource inputs and outputs. 


\section{$1.1 \quad$ Urban agriculture}

Urban agriculture is the local production of food within (peri-)urban areas, which in addition fosters education, employment, community building and/or closing organic resource cycles (Mougeot, 2000, Smit et al., 2001). Urban agriculture involves intensive cultivation/breeding methods that yield a diverse selection of flora and fauna, and integrates it with the local urban economic, social and ecological systems; thus, urban agriculture assimilates a plurality of activities, locations, scales, purposes and engagement. Exemplary of this variety, urban agriculture can include low-tech and high-tech production systems, such as community gardens, rooftop farming, indoor controlled environment agriculture, and animal husbandry.

\subsection{New sanitation}

Sanitation is the promotion of hygiene via the management and treatment of wastes, and includes both the physical and organizational structure (Brikké and Bredero, 2003, Mihelcic et al., 2011). New sanitation is a new paradigm for the collection, transport, treatment, and recovery of solid waste and wastewater (e.g., urine deviated vacuum toilets, anaerobic digesters, struvite $\left(\mathrm{Mg}\left(\mathrm{NH}_{3}\right) \mathrm{PO}_{4}\right)$ precipitation) with the aim to recover resources (i.e., water, nutrients, organic matter), increase efficiency, reduce energy costs, and/or offer solutions to waste management (Kujawa-Roeleveld and Zeeman, 2006, Lens et al., 2001, Maurer et al., 2012, Zeeman, 2012). New sanitation systems minimize transport and are therefore locally oriented systems (source, recovery and reuse are in close proximity) and the technical design serves this aim. The design varies with the local context but often includes source separation of waste and wastewater streams, collecting organic kitchen waste, black water (urine and feces), grey water (shower/bath, sink, laundry, dish washer) and/or urine separately. Depending on the types of streams separated and the local context, new sanitation concepts can be configured for treatment and recovery to achieve reuse or discharge parameters. The respective recovery and removal efficiencies of the sanitation technologies determine the quantity of nutrients that can be harvested and the quality of the product for human and environmental hygiene.

\subsection{Linking urban agriculture and new sanitation}

Re-establishing a partnership between agriculture and sanitation is not a new phenomenon. Various studies have looked at the possible cycling between sanitation and crop production including: wastewater reuse/irrigation for crop production (Beuchler et al., 2006, Smit and Nasr, 1992, Strauss, 2001), treatment, recovery and reuse of fertilizers from wastewater (Jenkins, 2005, Lens et al., 2001, Mihelcic et al., 2011, Tervahauta et al., 2013, Tidåker et al., 2006), reuse of urine (Maurer et al., 2003b, Maurer et al., 2006a), bioavailability of recovered products to crops (Jönsson et al., 2004, Oenema et al., 2012), guidelines on urine and feces reuse in agriculture to ensure safe handling (Heinonen-Tanski and van Wijk-Sijbesma, 2005, Jönsson et al., 2004), risks of micro-pollutants, pathogens and heavy metals (Heinonen-Tanski and van Wijk-Sijbesma, 
2005, Tervahauta, 2014, Winker et al., 2009b), policymaking for resource recovery (van der Hoek et al., 2016) and the link between urban agriculture and sanitation systems as an economic and food security measure in developing countries (Cofie et al., 2013, Kone, 2010, Streiffeler, 2001).

The feasibility, however, to match input and output flows between urban agriculture and new sanitation systems at the urban scale is not known. To start, data on the quantity and quality of the input demands from urban agriculture systems is lacking, as urban agriculture is very diverse in practice and for the most part unregulated (Belevi and Baumgartner, 2003, Martellozzo et al., 2014). This diversity results in varied fertilization practices and therefore requires that urban agriculture typologies be clearly defined to identify respective input and output flows. Second, although data on the quantity and quality of the products produced by new sanitation systems has, and continues to be, researched, the extent of their reuse potential in urban agriculture is uncertain (e.g., plant availability, nutrient ratios, pathogen and micro-pollutant contamination) (Lens et al., 2001, Tervahauta et al., 2013, Zeeman and Kujawa-Roeleveld, 2011b).

\subsection{Scope of research and research objectives}

The scope of this research focuses on the recovery of nitrogen $(\mathrm{N})$, phosphorus $(\mathrm{P})$ and organic matter (OM) from domestic wastewater and organic kitchen waste to determine the extent to which these resources can cover the demand from urban agriculture, in Rotterdam, the Netherlands (population 620,000) (Gemeente Rotterdam, 2013). The reason for this focus is threefold. First is the global concern regarding resource depletion and environmental pollution due to current consumption and disposal trends of nutrients, N and P, and OM (Carter, 2002, Cordell and White, 2011, Galloway et al., 2004). Second is the increased regional interest in the Netherlands for the professionalization of urban agriculture and the recovery of resources from waste streams (Green Deal Stadsgerichte Landbouw, 2013). Third is Rotterdam's interest in improving local resource management and implementing urban agriculture (Cityportal Rotterdam, 2014, Gemeente Rotterdam, 2012). In fact, Rotterdam currently houses a few leading urban agriculture initiatives in the Netherlands, including: Uit Je Eigen Stad, Rotterdamse Munt, Rotterzwam, and De DakAkker.

The objective of this study is to model combined urban agriculture and new sanitation systems to evaluate the degree to which $\mathrm{N}, \mathrm{P}$ and OM input-output flows can be matched and quantify the degree of self-sufficiency. This will be done in three steps: a) select and characterize relevant urban agriculture typologies and quantify the demand of nutrients and OM for each selected typology, b) select the new sanitation technologies (proven at lab and pilot scale) most appropriate for the recovery nutrients from residual waste streams and quantify the harvested nutrients and $\mathrm{OM}, \mathrm{c})$ quantify the extent to which the demand for nutrients from urban agriculture can be met by recovered nutrients from the selected new sanitation systems. 


\section{Methodology}

\subsection{Methodological framework: urban harvest approach}

The methodology used in this research is an adaptation of the Urban Harvest Approach (UHA) developed at the Department of Environmental Technology (ETE) at Wageningen UR (Agudelo-Vera et al., 2012b, Leusbrock et al., 2015). It has been most extensively applied to the urban water cycle to improve urban resource management towards self-sufficiency starting with a baseline assessment and applying three management strategies: demand minimization, output minimization (by resource cascading, recycling and recovery), and multi-sourcing (harvesting local primary and secondary resources) (Agudelo-Vera et al., 2012c). Multi-sourcing will not be included in this research as there are few renewable sources of N, P and OM (e.g., N fixing cover crops). These strategies are shown in Figure 2.2 as applied in this research. The designed systems are evaluated using the two indices developed by Agudelo-Vera et al. (2012), including: Demand Minimization Index (DMI) and Self-Sufficiency Index (SSI).

\subsubsection{Strategy 0) baseline demand}

The baseline assessment describes the existing situation, including demand inventory and current technologies. Here the baseline identifies the quantity and type of nutrient input demand for urban agriculture, and the output of nutrient flows from domestic sanitation waste flows.

The baseline assessment was conducted for two selected urban agriculture typologies: groundbased urban agriculture (ground-based urban agriculture) and rooftop urban agriculture (rooftop urban agriculture). These were selected because both typologies can be found in Rotterdam, which served as reference case studies for this research. Ground-based urban agriculture grows edible plants at ground level in soil (e.g., commercial or community farms, permaculture farms and forest gardening). Rooftop urban agriculture involves cultivating crops on the rooftops of urban buildings, usually flat roofs that are most suited to carry additional weight (between 60-150 $\mathrm{kg} / \mathrm{m}^{2}$ ). This typology can cultivate plants in soil or in a soil-like substrate.

The nutrient baseline demand was calculated for each typology $(\mathrm{kg} / \mathrm{ha})$ from interviews with individual urban farmers and the respective records they had on the practiced fertilization regime. This demand was compared to fertilizer regulations for conventional agriculture in the Netherlands, and values for equilibrium fertilization (plant uptake). The conventional norms and the equilibrium fertilization values were averaged from 22 different types of horticultural crops ${ }^{1}$, to reflect the diversity of crops grown at the urban agriculture typologies (Fink et al., 1999, Rijksoverheid, 2014b, Rijksoverheid, 2014a). Equilibrium fertilization reflects the nutrients a

1 Dwarf bean, broccoli, Brussel sprouts, carrot, cauliflower, celery root, Chinese cabbage, cucumber, fennel, iceberg lettuce, kale, kohlrabi, leek, lettuce, onion, radicchio, radish, red beet, red cabbage, savoy cabbage, spinach, white cabbage 

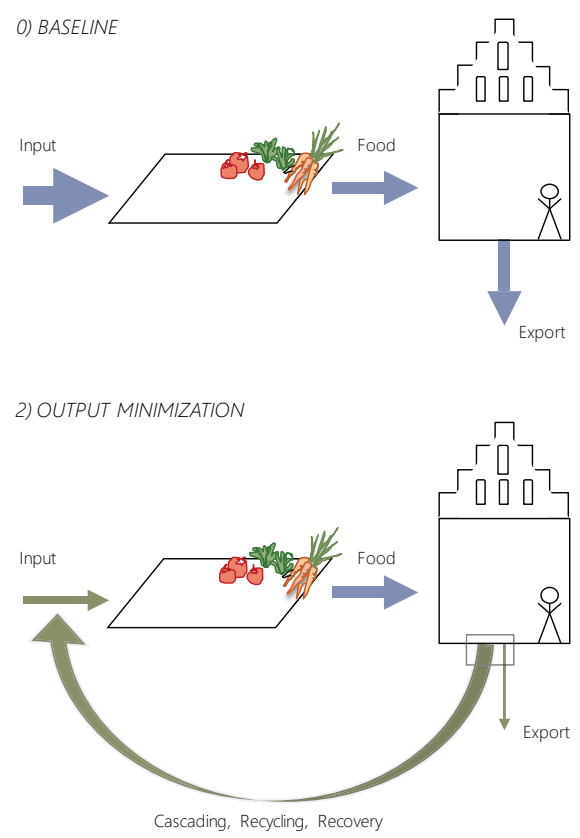

- Nutrient flows

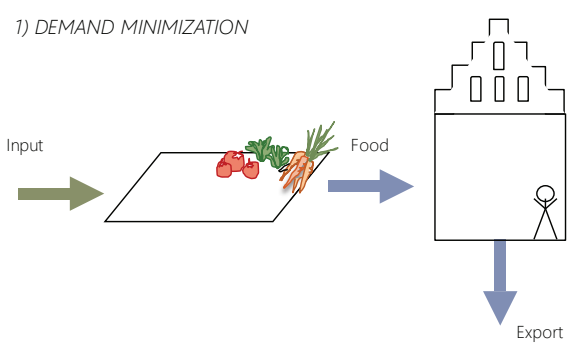

3) MULTI-SOURCING

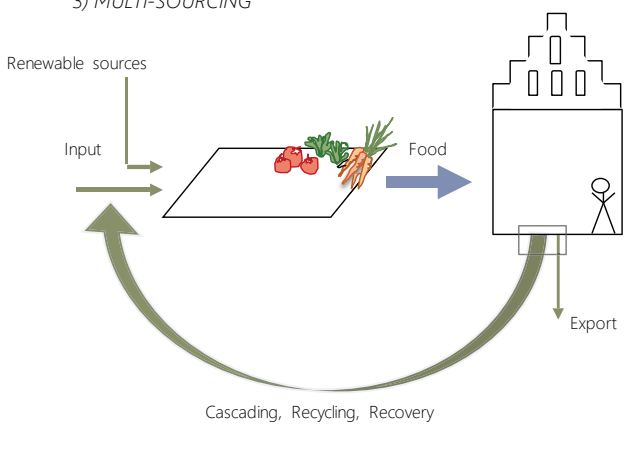

- Improved nutrient flows

Figure 2.2 Schematic of the management strategies of the UHA adapted to show nutrient flows between urban agriculture and new sanitation (nutrient losses are not shown)

plant takes up, or the nutrients contained in the total harvested fresh matter (harvest residues and marketable yield) assuming an optimal yield per hectare (Fink et al., 1999).

A further distinction was made between total $\mathrm{N}$ and $\mathrm{P}$ and available $\mathrm{N}$ and P. Available $\mathrm{N}$ and $P$ values take into account availability of organically-bound nutrients (slow release) as advised by Dutch fertilization regulations. According to set coefficients ('werkingscoëfficient') only a percentage of the $\mathrm{N}$ in organic fertilizers counts toward the regulatory norms. For instance, the $\mathrm{N}$ coefficient is $10 \%$ for compost and $30-60 \%$ for manure, depending on liquid or solid composition (Rijksoverheid, 2014b). Total P counts towards the norm with the exception of compost, for which only $50 \%$ counts.

The baseline assessment for the supply of nutrients first includes an overview of the current waste and wastewater treatment in Rotterdam. Second, the baseline supply from domestic sanitation was calculated per waste stream by using mean compositions (Table 2.1) of urine, feces, black water and organic kitchen waste generation per person as recorded in literature (Daigger, 2009, Friedler et al., 2013a, Kujawa-Roeleveld and Zeeman, 2006, Magid et al., 2006, Tervahauta et al., 2013). 
Table 2.1. Mean compositions of urine, feces, black water and organic kitchen waste calculated based on European data as reported in literature, including respective standard deviations (Daigger, 2009, Friedler et al., 2013a, Kujawa-Roeleveld and Zeeman, 2006, Magid et al., 2006, Tervahauta et al., 2013)

\begin{tabular}{|c|c|c|c|c|c|c|c|c|}
\hline Parameter & unit & Urine & s.d. & Feces & s.d. & $\begin{array}{c}\text { Kitchen } \\
\text { waste }\end{array}$ & s.d. & Total \\
\hline Volume & $\mathrm{L} / \mathrm{p} / \mathrm{d}$ & 1.3 & 0.12 & 0.13 & 0.06 & 0.2 & - & 1.63 \\
\hline $\mathrm{COD}$ & $\mathrm{g} / \mathrm{p} / \mathrm{d}$ & 12.5 & 1.91 & 47.9 & 12.23 & 59 & - & 119.4 \\
\hline $\mathrm{TN}$ & $\mathrm{g} / \mathrm{p} / \mathrm{d}$ & 10.2 & 1.10 & 1.4 & 0.38 & 1.4 & 0.52 & 13 \\
\hline $\mathrm{TP}$ & $\mathrm{g} / \mathrm{p} / \mathrm{d}$ & 1.1 & 0.34 & 0.5 & 0.05 & 0.2 & 0.06 & 1.8 \\
\hline COD= chemical oxygen demand, TN= total nitrogen, TP= total phosphorus \\
\hline
\end{tabular}

\subsubsection{Strategy 1) demand minimization}

The Demand Minimization Index (DMI) describes the change in demand in reference to the baseline demand. Baseline demand $\left(\mathrm{D}_{\mathrm{O}}\right)$ reflects the current resource demand (status quo) from urban agriculture and the minimized demand (D) describes the demand adjusted to reflect equilibrium fertilization values. A DMI of 0 indicates that no demand minimization has taken place. The DMI is calculated using Equation 2.1.

$$
\mathrm{DMI}=\left(\mathrm{D}_{\mathrm{O}}-\mathrm{D}\right) * \mathrm{D}_{\mathrm{O}}^{-1} * 100
$$

Equation 2.1

Demand minimization reduces the demand for nutrients via the implementation of new technologies or via changes in human behavior. For N, P and OM, a change in farming technologies or fertilizer regimes can reduce the initial demand. For this research, the minimized demand was based on equilibrium fertilization. The equilibrium values were used assuming an ideal scenario (zero waste) in which the fertilization regime reflects the amount of nutrients that crops take up, and not more. The baseline demand was used when these values were below the equilibrium values. The ratio of slow release vs. quick release fertilizer for the minimized demand was assumed to be the same as for the baseline demand. OM was minimized to reflect the suggested compost load per hectare in literature of $15,000 \mathrm{~kg}$ of compost, with a maximum of 3,000 kg OM/ha, or the baseline demand if below $3000 \mathrm{~kg} \mathrm{OM} / \mathrm{ha}$ (Goed boeren in kleinschalig landschap, 2011).

\subsubsection{Strategy 2) output minimization}

This strategy minimizes outputs via three strategies: cascading (direct use of outputs for a purpose with lower quality demand), recycling (the reuse of a resource flow after a quality upgrade, which generally costs energy) and/or recovery (the extraction of valuable resources from waste streams) from the outputs. Cascading will not be used because primary and/or secondary treatment of human excreta is needed to secure the removal of pathogens (Jönsson et al., 2004). 
For the recovery of nutrients, urine, feces and organic kitchen waste are the most promising streams since they have the highest loads of N, P, and OM (de Haan and van Geel, 2013b). Feces and organic kitchen waste contain most OM, suitable for making compost and soil conditioners, while urine contains the largest fraction of $\mathrm{N}$ and $\mathrm{P}$. Therefore, urine, feces, black water (BW) and organic kitchen waste (KW) were considered for recovery, whereas greywater (GW) was not considered.

Four new sanitation concepts (Figure 2.3) were selected based on systems demonstrated on lab and pilot scale. The sanitation system installed in Sneek, the Netherlands for source-separated BW, was used as a starting point for Concept 1 , and variations upon that system were configured for Concepts 2-4, further separating urine, feces, and/or organic kitchen waste with respective treatment systems (Tervahauta et al., 2013, Waterschoon, 2011). Concept 1 includes sourceseparation of BW combined with $\mathrm{KW}$ (via a grinder). The BW and $\mathrm{KW}$ are both treated anaerobically in an UASB (up-flow anaerobic sludge blanket) reactor, followed by an OLAND (oxygen limited anaerobic nitrification denitrification) reactor and a struvite precipitation reactor. Concept 2 includes the same treatment steps as Concept 1, although with separate collection of KW for composting (Dekker et al., 2010, Eklind and Kirchmann, 2000, Fricke and Vogtmann, 1994, Hargreaves et al., 2008). Concept 3 is similar to Concept 1 with the exception of urine, which is collected separately and stored (Jönsson et al., 1998, Jönsson et al., 2004, Maurer et al., 2006a). Concept 4 separates KW for composting and urine for either (a) storage or (b) struvite precipitation. Feces are not considered in Concept 4 for recovery of nutrients. Treatment systems for GW and for byproduct effluents from the technologies were not further quantified, and are therefore not shown in Figure 2.3. See Supporting Information I for substance flow analyses for each new sanitation concept for N, P and OM.

In Concepts 3 and 4, urine is separated at source via a urine-diverting toilet using 0.2L of water per flush. In Concept 3 and $4 \mathrm{a}$ urine is stored and in Concept $4 \mathrm{~b}$ urine undergoes struvite precipitation. In Concept 3, struvite precipitation was not considered for the separated urine because the treatment stream of the feces and KW already includes a struvite precipitation step.

\subsubsection{Sanitation technologies, removal efficiencies and harvested products}

The collection system for each concept depends on the separated waste streams. In Concepts 1 and 2 vacuum toilets are used with $1 \mathrm{~L}$ of flush water. In Concepts 3 and 4 urine-diverting vacuum toilets are used for collection with $0.2 \mathrm{~L}$ of water per flush. In urine-diverting toilets, it is assumed that the urine separation efficiency is $75 \%$, whereas $25 \%$ joins the feces stream (Larsen and Lienert, 2007, Tervahauta et al., 2013). With regards to KW, it is assumed that $100 \%$ of the $\mathrm{KW}$ per household is collected via a kitchen grinder in Concepts 1 and 3, where KW is digested together with feces streams. In Concepts 2 and $4 \mathrm{KW}$ is collected separately and composted.

De Graaff, et al., (2010) studied the fate of nutrients and OM in the anaerobic treatment of black water using a UASB reactor with a short HRT at $25^{\circ} \mathrm{C}$. Data for recovery and removal 
Concept 1

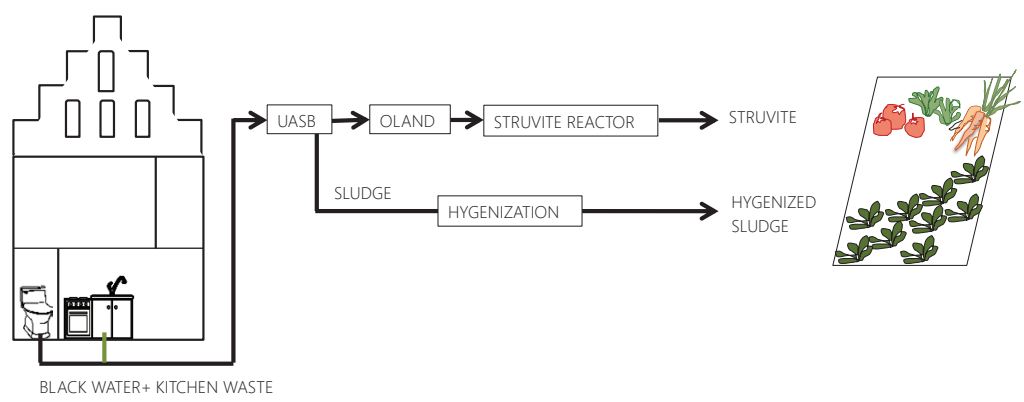

Concept 2
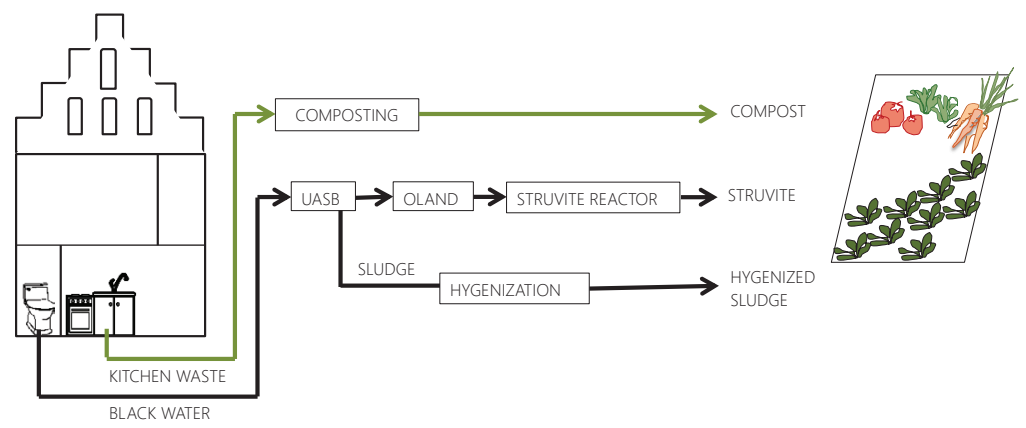

Concept 3
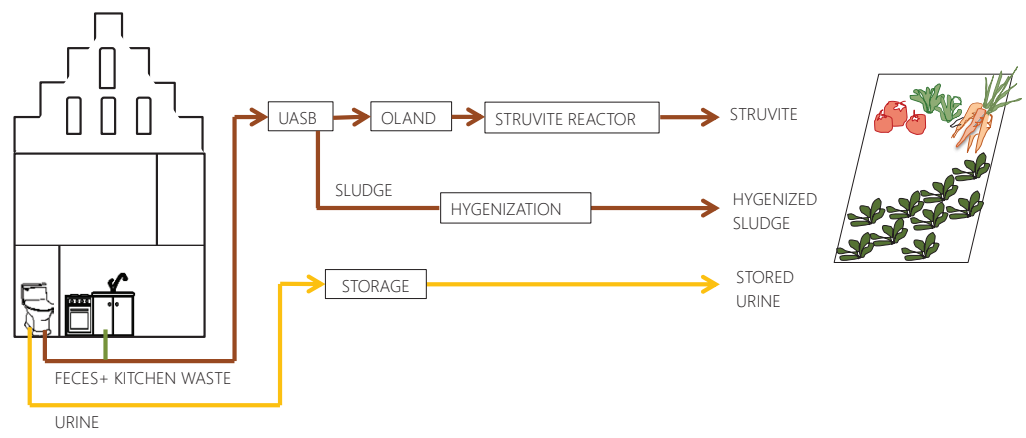

Concept 4a/4b
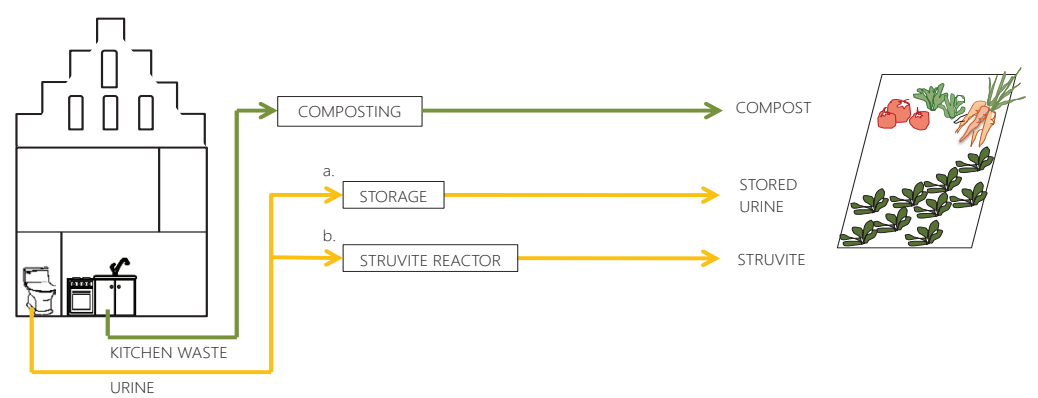

Figure 2.3 New sanitation concepts (adapted from Tervahauta et al., 2013). Arrows indicate nutrient flows. (For clarity of the figures, nutrient losses are not indicated, see section 2.1.4) 
efficiencies from de Graaf, et al. was used here for further calculations. The COD in the UASB reactor undergoes anaerobic biological decomposition reaching a methanization level of $54 \%$, $10 \mathrm{~L} \mathrm{CH}_{4} / \mathrm{p} / \mathrm{d}$ can be produced from black water. Of the remaining COD, $19 \%$ is found in the sludge and $27 \%$ remains in the effluent stream of the reactor (De Graaff et al., 2010a). The sludge from the UASB is thermally hygienized to deactivate pathogens (Capizzi-Banas et al., 2004). The OM of the sludge is calculated using a fixed COD to OM ratio of 1.4 (Zeeman and Gerbens, 2002). The available $\mathrm{N}$ from the UASB sludge is assumed to be the same percentage as what is available from sewage sludge identified by the Dutch fertilizer policies ('Mestbeleid: werkingscoefficient voor stikstof'). The available $\mathrm{P}$ is assumed to be $50 \%$, similar to compost, a comparable stabilized organic sludge. The removal efficiencies for the UASB, OLAND and Struvite reactors used in Concepts 1, 2, and 3 are provided in Table 2.2. Losses occur in the UASB (OM is methanized), in the OLAND reactor (release $\mathrm{NO}_{2}^{-}, \mathrm{NO}_{3}^{-}$and $\mathrm{N}_{2}$ ) and in the effluent of the struvite reactor ( $84 \%$ of $\mathrm{N}$ of the influent).

Table 2.2 Removal efficiencies for Concept 1, 2 and 3 (de Graaff, 2010, De Graaff et al., 2010a, Tervahauta et al., 2013)

\begin{tabular}{|c|c|c|c|}
\hline & \multicolumn{3}{|c|}{ Removal Efficiencies (\%) } \\
\hline Parameter & UASB & OLAND & Struvite \\
\hline COD & $73^{1}$ & $53^{2}$ & - \\
\hline $\mathrm{BOD}_{5}$ & $73^{1}$ & $53^{2}$ & - \\
\hline $\mathrm{TN}$ & $1^{2}$ & $73^{2}$ & $16^{3}$ \\
\hline TP & $33^{2}$ & - & $96^{2}$ \\
\hline \multicolumn{4}{|c|}{$\begin{array}{l}\left.{ }^{1} \text { (de Graaff, } 2010\right),{ }^{2} \text { (Tervahauta et al., } 2013 \text {, Wilsenach et al., 2007), }{ }^{3} \text { Calculated per concept based on the } \\
\text { molar ratio of N:P of } 1: 1 \\
\text { COD }=\text { Chemical Oxygen Demand, BOD5= Biological Oxygen Demand, TN= Total nitrogen } \text { TP= Total phosphorus }\end{array}$} \\
\hline
\end{tabular}

Struvite precipitation from UASB effluent (Concept 1, 2, and 3) and from urine (Concept 4) precipitates magnesium ammonium phosphate (MAP), conveying two nutrients, $\mathrm{N}$ and $\mathrm{P}$, in solid form at a molar ratio of 1:1 (Maurer et al., 2006a). However, urine contains ammonium and phosphate in a ratio of $20: 1$, meaning that only about $3 \%$ of the $\mathrm{N}$ can be recovered as struvite (Maurer et al., 2006a). The rest of the $\mathrm{N}$ remains in the effluent.

Urine is assumed to be collected via a well-sealed collection system and storage tank to prevent loss as gaseous $\mathrm{NH}_{3}$ (Jönsson et al., 2004, Maurer et al., 2003b). The $\mathrm{N}$ loss during collection is $0.02 \mathrm{~kg} \mathrm{NH} / \mathrm{yr}$ for 1000 inhabitants, which is considered negligible. Urine storage recovers the largest amount of $\mathrm{N}$ from wastewater compared to the other treatment steps. It is assumed that urine is stored for $>6$ months for hygienization and conserves $100 \%$ of the nutrients that are present in the fresh urine. During storage the urea hydrolyzes, increasing the $\mathrm{pH}$ and ammonium concentration, and precipitating struvite and calcium phosphate. The amount of struvite and 
calcium phosphate precipitated, both slow release fertilizers, is small and depends on the storage time. These are there not considered in further calculations and stored urine is assumed to be a quick release fertilizer. The stored urine is rich in $\mathrm{N}$ and $\mathrm{P}$, and also contains some OM. In this research, the $\mathrm{OM}$ found in stored urine is ineffective because it degrades quickly $(\sim 73 \%)$ in the first year (Kuntke, 2013), and therefore we do not take it into account in the OM balance.

Composting in Concept 2 and 4 is achieved in an open static pile composting system which allows for the regulation of temperature, humidity and $\mathrm{pH}$ by forcing air through the compost (Gomez, 1998). Source separated KW is N-rich (N ratio of 13:1) accounting for substantial gaseous $\mathrm{N}$ losses, (55\%) (Eklind and Kirchmann, 2000). The vegetable, fruit, and yard waste (VFY) produced is $0.338 \mathrm{~kg} / \mathrm{p} / \mathrm{d}$. The composition of the VFY can be calculated using the percentages of dry matter (DM) (40.6\%) and OM (65.3\% of DM) (van Haeff, 2012). The total available $\mathrm{N}$ and $\mathrm{P}$ from the compost is calculated using the 'werkingscoefficient' identified by the Dutch fertilizer policies (10\% of $\mathrm{N}$ is available and $50 \%$ of $\mathrm{P}$ ).

Increased self-sufficiency is achieved by reusing output as an input, (partially) covering the input demand. The Self-Sufficiency Index (SSI) was used as a measure for the extent to which the recovered nutrients from new sanitation systems fulfill the demand from urban agriculture. The SSI is defined by: the resources reused $(\mathrm{Rr})$ against the minimized demand (D). The SSI is calculated using Equation 2.2.

$$
\mathrm{SSI}=\mathrm{Rr}^{*} \mathrm{D}^{-1} * 100
$$

Equation 2.2

\section{Results}

\subsection{Baseline demand and demand minimization}

\subsubsection{Baseline demand}

The baseline demands for both ground-based urban agriculture and rooftop urban agriculture reflect the fertilizer regime followed by urban farms of respective typologies in Rotterdam. For ground-based urban agriculture, this fertilization regime included the use of both slow release $(15 \%)$ and quick release $(85 \%)$ fertilizers distributed in a compost mixture, chicken manure, and a liquid fertilizer. The baseline demand for rooftop urban agriculture was based on the fertilization regime of a rooftop farm that uses a growing substrate low in $\mathrm{OM}$, to decrease its weight, to adhere to the $180 \mathrm{~kg} / \mathrm{m}^{2}$ capacity of the roof. Therefore no compost is added for fertilization, but only slow release granulates $(100 \%$ slow release) and no quick release $(0 \%)$ fertilizers are used.

Figure 2.4 compares the baseline demand with the norms and regulations for $\mathrm{N}$ and $\mathrm{P}$ use in conventional agriculture in the Netherlands and with equilibrium fertilization values. This figure shows that the baseline demand for $\mathrm{N}$ for both ground-based urban agriculture and rooftop 
urban agriculture lies well below the equilibrium fertilization value. For both urban agriculture typologies, the baseline demand for $\mathrm{P}$, however, exceeds the conventional norms, meaning that over-fertilization of $\mathrm{P}$ is occurring. For ground-based urban agriculture, the baseline demand for

$\mathrm{N}$ available $(\mathrm{kg} / \mathrm{ha})$

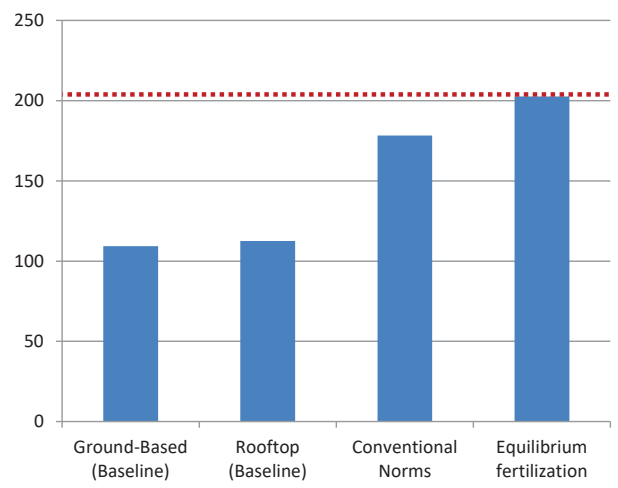

$\mathrm{P}_{2} \mathrm{O}_{5}$ available $(\mathrm{kg} / \mathrm{ha})$

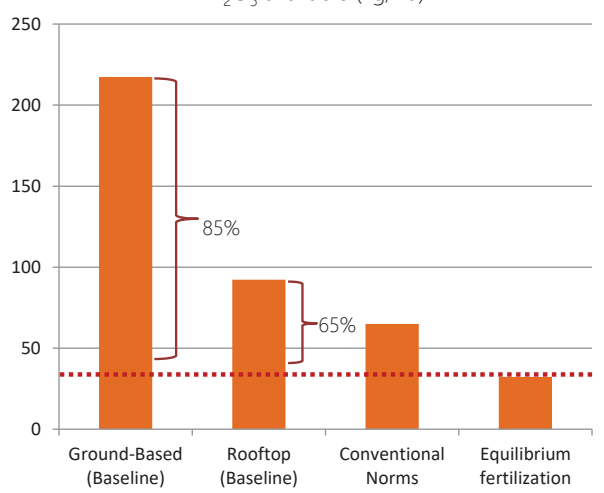

Figure 2.4 Comparison of nutrient demand from ground-based and rooftop urban agriculture to conventional norms and equilibrium fertilization. Where 'conventional norms' are the average $\mathrm{N}$ and $\mathrm{P}$ use norms and regulations (clay and sandy soils) in the Netherlands (Rijksoverheid, 2014a, Rijksoverheid, 2014b), and 'equilibrium fertilization' reflects the nutrients that crops take up, averaged for 22 vegetable crops (Fink et al., 1999)

Table 2.3 Annual baseline nutrient demand and minimized demand for ground-based and rooftop urban agriculture

\begin{tabular}{|c|c|c|c|c|}
\hline & units & Available $N^{*}$ & Available P* & $\mathrm{OM}^{3}$ \\
\hline \multicolumn{5}{|c|}{ Ground-based urban agriculture } \\
\hline Baseline Demand ${ }^{1}\left(D_{0}\right)$ & $\mathrm{kg} / \mathrm{ha}$ & 109 & 96 & 7861 \\
\hline Minimized Demand (D) & $\mathrm{kg} / \mathrm{ha}$ & 109 & 14 & 2685 \\
\hline DMI & $\%$ & 0 & 85 & 66 \\
\hline \multicolumn{5}{|c|}{ Rooftop urban agriculture } \\
\hline Baseline Demand $2\left(D_{0}\right)$ & $\mathrm{kg} / \mathrm{ha}$ & 113 & 41 & 1743 \\
\hline Minimized Demand (D) & $\mathrm{kg} / \mathrm{ha}$ & 113 & 14 & 1743 \\
\hline DMI & $\%$ & 0 & 65 & 0 \\
\hline \multicolumn{5}{|c|}{$\begin{array}{l}2 \text { Technische Fiche ECO-MIX } 1 \text { (DCM Nederland BV, 2014) and Organische Gedroogde Koemest (Humuforte, } \\
\text { 2014) } \\
3 \text { OM=32\% of dry matter, Samenstelling en werking van organische meststoffen (de Haan and van Geel, 2013b). } \\
\text { * Nutrient values for } \mathrm{N} \text { and } \mathrm{P} \text { are usually expressed by weight of } \mathrm{N} \text { and } \mathrm{P}_{2} \mathrm{O}_{5} . \mathrm{P} \text { is } 44 \% \text { of the } \mathrm{P}_{2} \mathrm{O}_{5} \text { value. } \mathrm{N} \text { is } \\
\text { expressed as elemental } \mathrm{N} \text {. Both } \mathrm{N} \text { and } \mathrm{P} \text { are calculated using the 'werkingscoefficient' for compost and animal } \\
\text { manure. Available } \mathrm{N} \text { is defined as } 10 \% \text { in compost and } 55 \% \text { from chicken manure. Available P is } 50 \% \text { in compost } \\
\text { with a maximum of } 3.5 \mathrm{~g} \mathrm{P} \mathrm{P}_{2} \mathrm{O}_{5} / \mathrm{kg} \text { dry matter of compost }\end{array}$} \\
\hline
\end{tabular}


P exceeds the conventional norms by a factor three and the equilibrium fertilization values by a factor seven. The amount of P over-fertilization that occurs in both typologies is wasteful and demands attention considering that $\mathrm{P}$ is a finite resource.

\subsubsection{Minimized demand}

The baseline demand was minimized (Table 2.3) to reflect a maximum value equivalent to that of equilibrium fertilization. For ground-based urban agriculture the $\mathrm{N}$ demand does not need to be minimized $(\mathrm{DMI}=0 \%$ ), while the demand for $\mathrm{P}$ and $\mathrm{OM}$ is minimized, with respective DMI values of $85 \%$ and $66 \%$. For rooftop urban agriculture the DMI for $\mathrm{N}$ and $\mathrm{OM}$ is $0 \%$, while the DMI for $\mathrm{P}$ is $65 \%$.

\subsubsection{Baseline supply from waste and wastewater}

Rotterdam, with an area of $319.35 \mathrm{~km}^{2}$, has a population of approximately 620,000 people (Gemeente Rotterdam, 2013). The city produces a total of 76,000 tons of household organic solid waste; however, most of this organic solid waste is collected together with municipal solid waste and incinerated for the generation of energy. A small fraction, 1\% of household VFY waste, is collected separately at source, composted and sold via a third party to the agricultural sector.
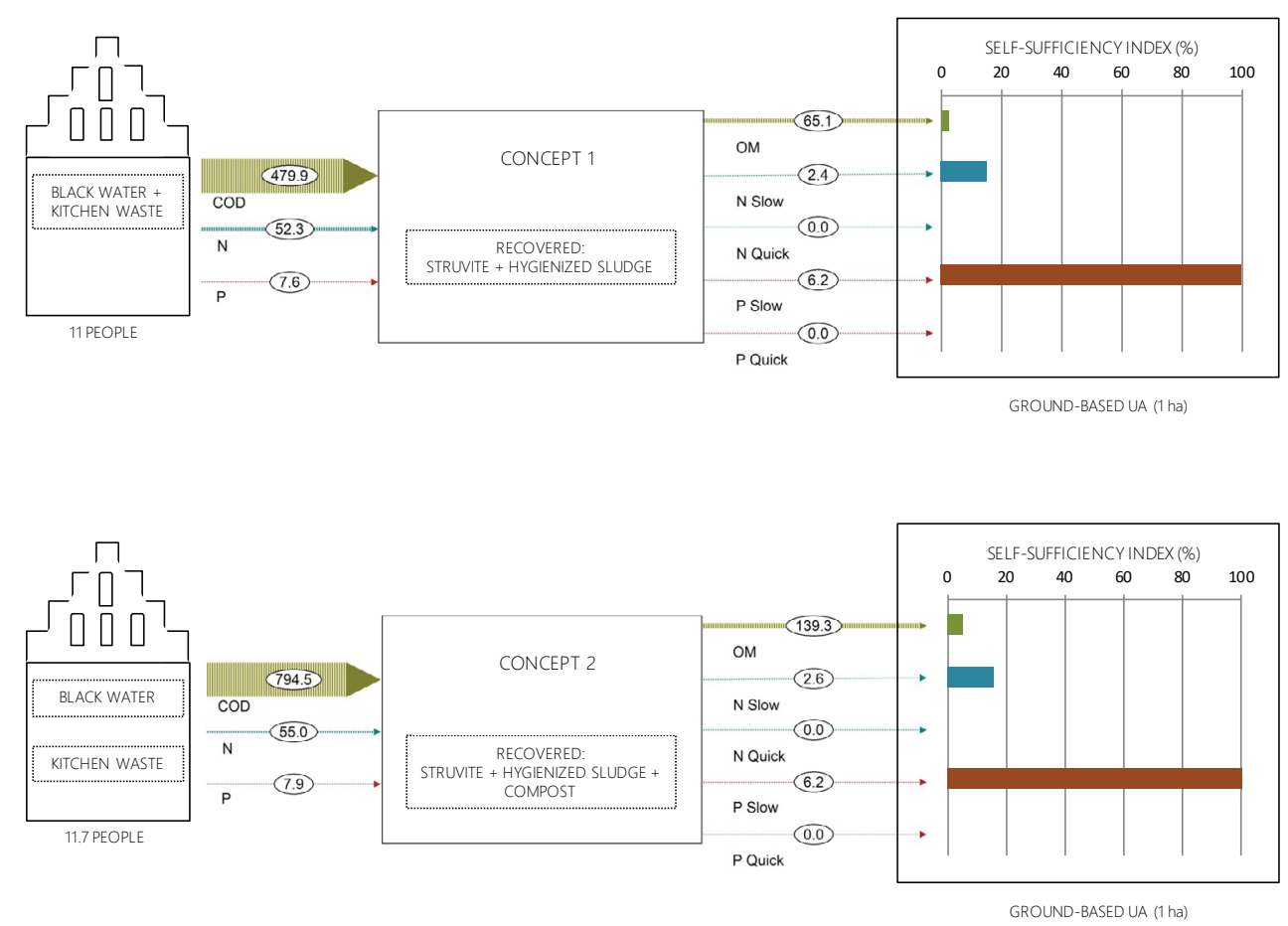

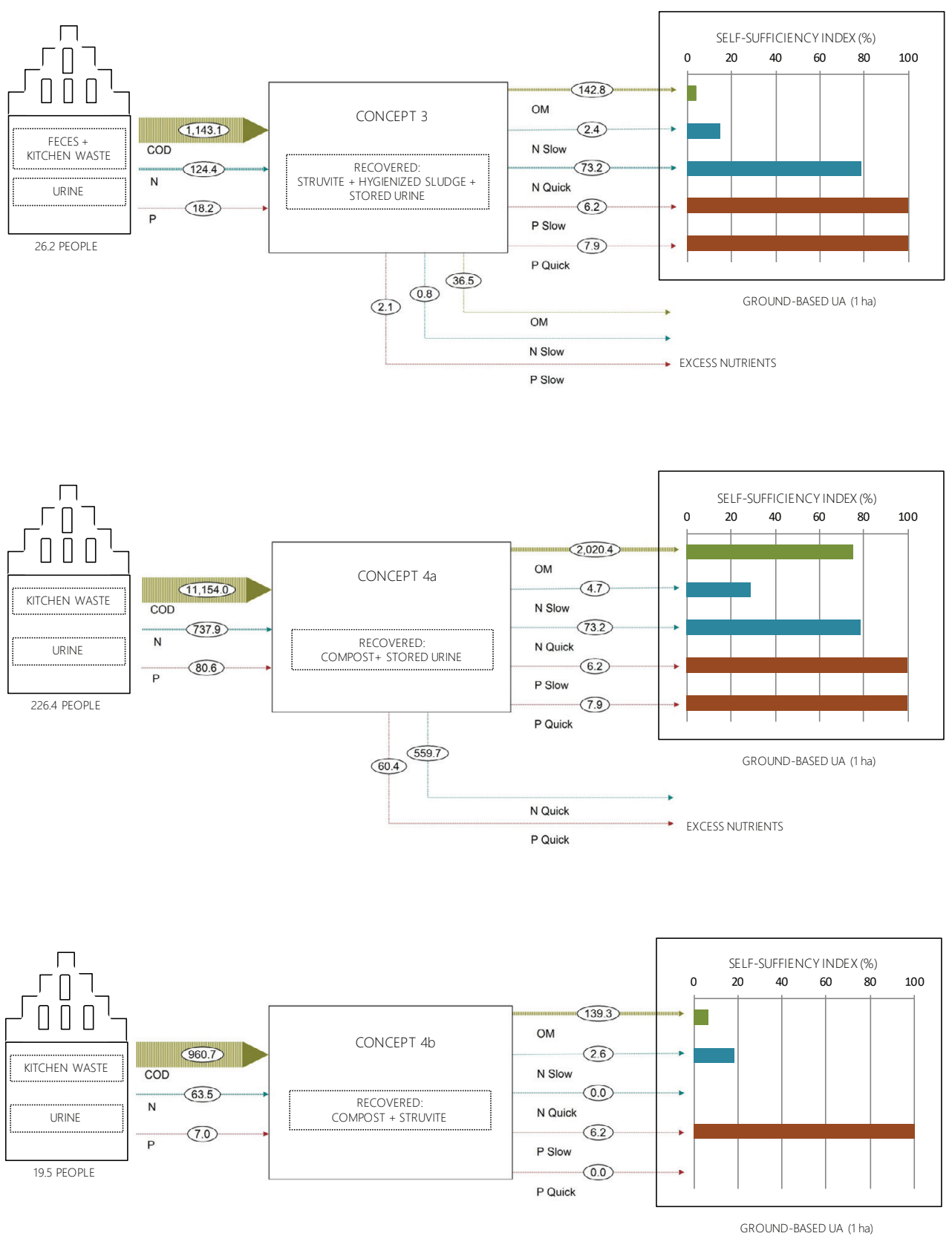

Figure 2.5 Nitrogen, phosphorus and organic matter mass flows $(\mathrm{kg} / \mathrm{ha})$ between new sanitation concepts and ground-based urban agriculture (1ha) with respective achieved self-sufficiency (\%) for organic matter, slow and quick release nitrogen, and slow and quick release phosphorus. The self-sufficiency for phosphorus is set to $100 \%$, determining the number of people needed per concept. 
The city's wastewater is treated at wastewater treatment plants by the Waterschap Hollandse Delta and Hoogheemraadschap Schielanden en Krimpenerwaard. Using Table 2.1, the loads of the nutrients can be calculated for the whole population of Rotterdam. Total household BW and KW generated daily represent a load of $1,356 \mathrm{~kg} P$ and 316,850 $\mathrm{kg} \mathrm{N}$ and 88,764 $\mathrm{kg} \mathrm{OM}$ per day.

\subsection{Output minimization}

The demand for N, P and OM from each urban agriculture typology was compared with the supply generated by each new sanitation concept. In total ten combinations were evaluated for the degree of self-sufficiency achieved using the self-sufficiency index (SSI) (Equation 2.2). The combinations aim at a SSI of $100 \%$ for P (as the most critical nutrient in terms of global scarcity and EU policies), both slow release and quick release; this determines the number of people (waste producers) needed per new sanitation concept per ha of urban agriculture to provide that self-sufficiency, as well as the respective reuse of the harvested N and OM. Figure 2.5 groundbased urban agriculture) and Figure 2.6 (rooftop urban agriculture) show the mass flows of the harvested N, P, and OM per concept and the respective self-sufficiency achieved for each for 1 ha of urban agriculture. The deficits of resources, which need to be imported into the urban agriculture system, and the excess nutrients harvested, resources which can be exported outside of the system, are also shown, as well as the number of people needed per concept to achieve the indicated SSI.
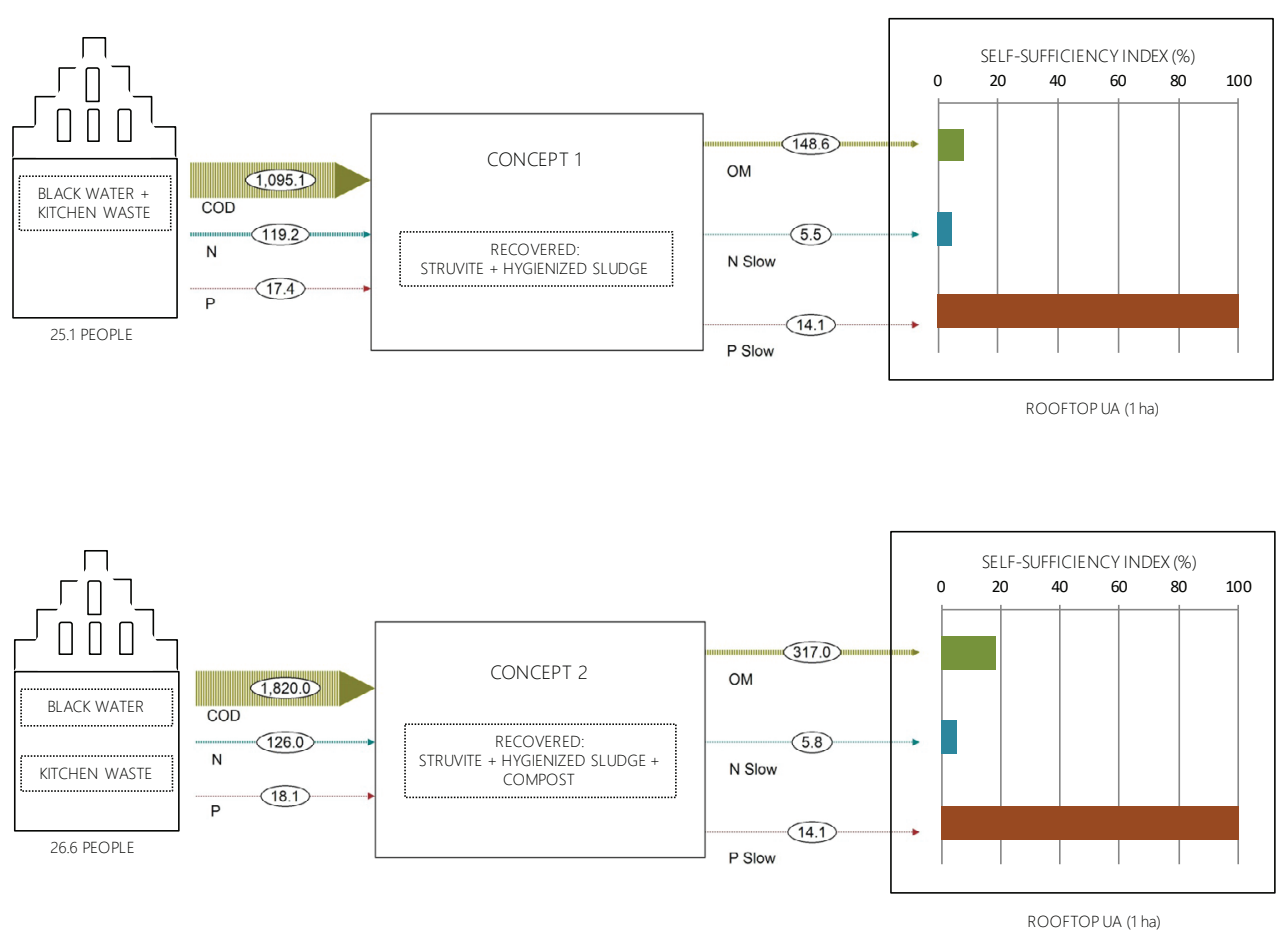

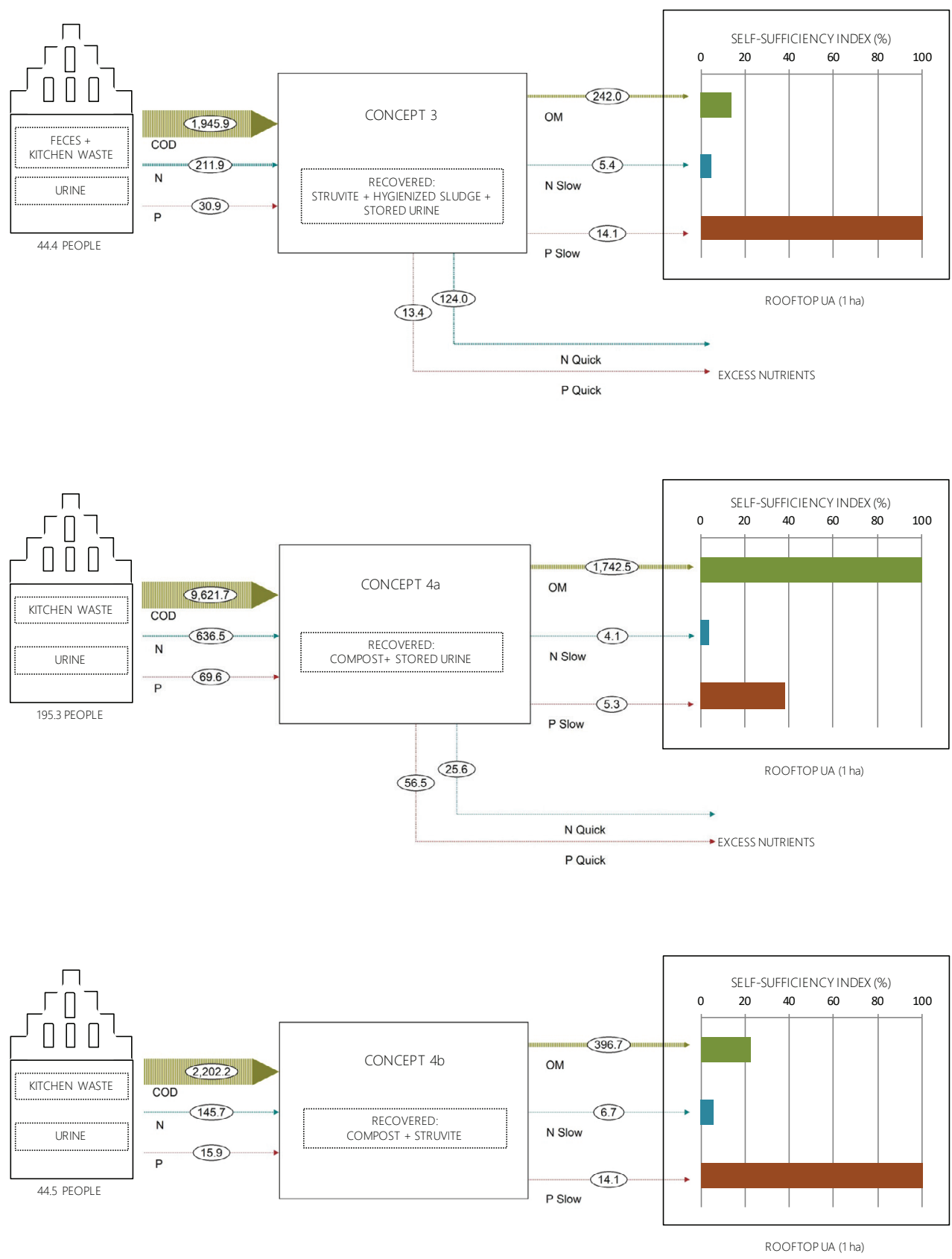

Figure 2.6 Nitrogen, phosphorus and organic matter mass flows $(\mathrm{kg} / \mathrm{ha})$ between new sanitation concepts and rooftop urban agriculture (1ha) with respective achieved self-sufficiency (\%) for organic matter, slow and quick release nitrogen, and slow and quick release phosphorus. The self-sufficiency for phosphorus is set to $100 \%$, determining the number of people needed per concept. 
Both the (SSI) and the number of persons needed to provide that SSI is relevant for the evaluation of the combined systems. While a high SSI is preferable for the sourcing of local resources, both the type of nutrient demand (slow vs quick release), and the removal and recovery efficiencies of the new sanitation technologies also determine the potential to implement the new sanitation concepts. The higher the recovery rate, the lower the number of people needed for each concept.

The combinations of ground-based urban agriculture with new sanitation Concepts 3 and $4 \mathrm{a}$ provide a SSI of 100\% for both slow and quick release P. Concept 4a, however, requires 10 times as many persons/ha to obtain this SSI, which is a possible barrier for the separate collection of VFY waste in densely (high rise) populated areas of Rotterdam. Concepts 1 and 2 fail to supply the demand for quick release $\mathrm{N}$ and $\mathrm{P}$ and seem less preferable. Rooftop urban agriculture does not have a demand for quick release fertilizer, and therefore the SSI for both quick release $\mathrm{N}$ and $\mathrm{P}$ is not applicable. The harvested quick release $\mathrm{N}$ and $\mathrm{P}$ in Concepts 3 and $4 \mathrm{a}$ are considered excess nutrient harvests. For all combinations, except with Concept $4 \mathrm{a}$, the SSI for slow release $\mathrm{P}$ was set to $100 \%$, resulting in low SSI values for both slow release $\mathrm{N}$ and organic matter. In Concept 4 a setting the SSI for P to $100 \%$ would result in a SSI for OM of $263 \%$. To prevent over-fertilization of OM, the SSI for OM was set to $100 \%$ instead. The combination of rooftop urban agriculture with Concept $4 \mathrm{~b}$ results in the highest combined SSI for N and P, followed by Concept 2.

\section{Discussion}

The UHA offers a step-by-step methodology to gain insight into the opportunities that lie in integrating urban agriculture and new sanitation, however, its application to N, P and OM inputoutput flows presented challenges at each step of the methodology.

\subsection{Baseline demand}

There are very few reliable empirical studies that quantify the demand from urban agriculture for nutrient inputs, as well as harvestable yield. In this study, the baseline N, P and OM demand from urban agriculture was based on two existing urban agriculture sites in Rotterdam. As these likely are not representative for fertilizer regimes of all urban agriculture initiatives within the studied typologies, more data is needed on nutrient demand to gain a broader view on the potential to couple urban agriculture to new sanitation. For example, whereas rooftop urban agriculture in this study did not have a demand for quick release $\mathrm{N}$ and $\mathrm{P}$, other rooftop urban agriculture initiatives might use quick release fertilizers.

In this research, both fertilization regimes showed strong over-fertilization of $\mathrm{P}$, a consequence of various factors including: lack of farmers' education and training on fertilization, the lack of regulations for fertilizer use in urban agriculture, the reuse of farm waste (i.e., chicken manure), and fertilizer use based on $\mathrm{N}$ limitation. Considering, however, that conventional agriculture in 
the Netherlands is heavily regulated in their $\mathrm{N}$ and $\mathrm{P}$ use to reduce pollution of water resources, and that $\mathrm{P}$ is a finite resource of increasing scarcity, urban agriculture fertilization regimes should also take measures to prevent over-fertilization. This study demonstrates the need for regulations for urban agriculture, especially when urban agriculture increases in scale, taking into account the wide range of urban agriculture typologies. The fertilization regime also has consequences on the nutrient loads discharged to the urban water cycle, such as the increase of nutrient loads to the sewer system via rooftop urban agriculture, especially after high rainfall. Therefore, expanding urban agriculture across cities has various implications for urban resource cycles and water treatment for which management systems need to developed

\subsection{Demand minimization}

Minimizing the demand for N, P and OM from urban agriculture is achieved by assuming equilibrium fertilization values, adjusting inputs to outputs and avoiding over-fertilization. This is a novel perspective for the application of nutrients in urban agriculture, although further research is needed to identify the optimal fertilization regime for each urban agriculture typology, considering that nutrients mineralize in the soil and runoff may occur. Especially the monitoring, collecting and sharing of data from urban agriculture (pilot) studies are needed in this respect. In addition, technological innovations (i.e., injection fertilization at the plant base as opposed to sprinkler systems) for the administration of fertilizers to minimize the demand were not considered in this research. Such measures, detailed by Schröder et al. (2011), could help farmers administer fertilizers where and when the plant needs them, reduce losses, and thereby minimize the demand.

\subsection{Output minimization}

The results of applying the output minimization strategies to N, P and OM flows between urban agriculture and new sanitation are determinedly context specific; these are dependent on the results of the baseline demand and the demand minimization, specific to the two reference initiatives in Rotterdam, and the specific new sanitation treatment systems selected, with their respective removal and recovery efficiencies. The main challenge in matching the input and output flows was accounting for the difference in N:P:OM ratios. While the demand from urban agriculture has one ratio of $\mathrm{N}: \mathrm{P}: \mathrm{OM}$ and a ratio of slow release to quick release fertilizer, the supply from the new sanitation concepts have different ratios of N:P:OM and of slow release to quick release fertilizers. This difference means that $100 \%$ self-sufficiency for all three resources, simultaneously, could never be achieved; there would always be a shortage or excess.

To address this challenge, and SSI of 100\% for P was assumed, which determined the respective SSI for N and OM achieved. Setting N or OM to 100\% self-sufficiency would mean overfertilizing in $\mathrm{P}$ per hectare. The ratio of slow release to quick release also influenced the matching of the demand and the supply, especially for ground-based urban agriculture. The 
characterization of the demand was context specific, based on the two reference initiatives in Rotterdam, and could very well be configured differently. This brings to question whether a difference between slow and quick release fertilizer should even be accounted for or that total available (effective in the first year after application) $\mathrm{N}, \mathrm{P}$ and $\mathrm{OM}$ would be a better approach. This again would change the ratios of N:P:OM, as well as the SSI for each.

The reuse of harvested products from wastewater in urban agriculture in this research prioritized the cycling of $\mathrm{P}$, a finite and scare resource, over $\mathrm{N}$ and OM. However, other criteria and indicators could also be considered for selecting the best combination of new sanitation concept and urban agriculture typology, and prioritizing the different harvested products. Criteria could include soil type and health, transport distance, storage requirements, availability of alternatives, costs, etc.

\subsection{Self-sufficiency}

Combining urban agriculture and new sanitation offers the possibility to increase urban selfsufficiency. The city of Rotterdam can fertilize the 2363 ha of available arable land and the 906 ha of rooftop area suitable for urban agriculture (calculated in a study carried out at the municipality of Rotterdam). With a population of 620,000 people (Gemeente Rotterdam, 2013) and assuming a marketable yield per hectare ${ }^{2}$ of $45,000 \mathrm{~kg} / \mathrm{ha}$, one hectare can supply the daily-recommended vegetable consumption $(200 \mathrm{~g} / \mathrm{p} / \mathrm{d})$ to circa 620 people, or $1010 \mathrm{ha}$ for the entire city. For the recommended consumption of $400 \mathrm{~g} / \mathrm{p} / \mathrm{d}$ of fruits and vegetables, one hectare can supply fruits and vegetables for circa 310 people, or 2020 ha for the entire city (Gezondheidsraad, 2015).

\section{Conclusion and outlook}

The UHA offers a methodology through which to reconsider urban resource flows through three management strategies: demand minimization, output minimization and multi-sourcing. Novel to this research is the application of the UHA on urban nutrient flows, showing preliminary results for future research in the domain of harvesting $\mathrm{P}, \mathrm{N}$ and $\mathrm{OM}$ from waste for reuse in urban food production. The application of this methodology in different contexts, including low-income countries, could offer new insight on opportunities for nutrient recovery and reuse. The results presented here are context specific and show that partial self-sufficiency can be reached. However, many uncertainties still remain when determining the extent to which urban agriculture and new sanitation can be integrated; future research needs to address remaining knowledge gaps of technical, operational and economic feasibility.

2 Equal to the national yield for conventional agriculture (based on conventional farming yields in the Netherlands for 'vegetables and melons' for 2013 as reported by FAOSTAT) with a reduction of $20 \%$ (organic yield gap) (FAOSTAT, 2013) 
Research on safety measures and technical feasibility studies for reuse of harvested products as fertilizers are needed to make sure that reuse does not impose risks to humans and the environment. This especially concerns the presence of heavy metals, micro-pollutants, pharmaceuticals and pathogens in the harvested products, which currently represent a barrier for reuse. In the Netherlands, the use of sewage sludge in agriculture is restricted because of the heavy metal content. Tervahauta et al. (2014), however, show that only $\mathrm{Cu}$ and $\mathrm{Zn}$ in black water sludge are high compared to Dutch standards and that these metals mainly originate from food intake. Therefore, Tervahauta et al. (2014) conclude that sludge from black water should be allowed as a fertilizer, to complete a circular metabolism of metals.

Micro-pollutants, pharmaceuticals, hormones and pathogens found in wastewater continue to be researched to determine the implications of the reuse of recovered products from human waste (Decrey et al., 2011, de Wilt et al., 2016, Escher et al., 2006, Ronteltap et al., 2007, Uysal et al., 2010). Either measures for removal of contaminants need to be developed, or risk reduction measures need to be implemented through handling and reuse protocols. Since January 2015, the Dutch fertilizer regulations have permitted the use of struvite, falling under the category of 'recovered phosphate', to be used as a fertilizer in the Netherlands, as long as the recovered struvite complies with heavy metal, pathogen and micro-pollutant guidelines (van der Grinten et al., 2015). Reuse of stored urine and sewage sludge as fertilizers are currently not permitted.

The operational feasibility of combined urban agriculture and new sanitation systems requires the evaluation of these systems in higher resolution, taking into account spatial and temporal conditions, including, seasonal patterns, storage, and infrastructure capacities. While humans produce waste and wastewater year-round, cultivation and the use of fertilizer only takes place during certain seasons. To account for this temporal disparity, appropriate storage tanks or the export of fertilizers (including to indoor farming and greenhouses) are needed, which also have respective spatial implications, let alone the logistics.

While the Netherlands is interested in closing resource cycles and moving towards a circular metabolism, the marketability of recovered products, especially $\mathrm{P}$, is limited due to the overabundance of animal manure in the country (van der Grinten et al., 2015). However, for urban agriculture, the reuse of struvite and other odorless products within cities could be a promising alternative to animal manure, as well as for synthetic fertilizers containing mined $\mathrm{P}$. Finally, the social perception of the reuse of human waste in urban agriculture is another barrier that needs to be relieved to secure a future for recovered products.

\section{Acknowledgements}

The authors gratefully acknowledge dr.ir. Ingo Leusbrock, for his critical review of the manuscript and valuable input, as well as, dr.ir. Frank de Ruijter for his advice on plant fertilizer requirements at the beginning stages of the research. 


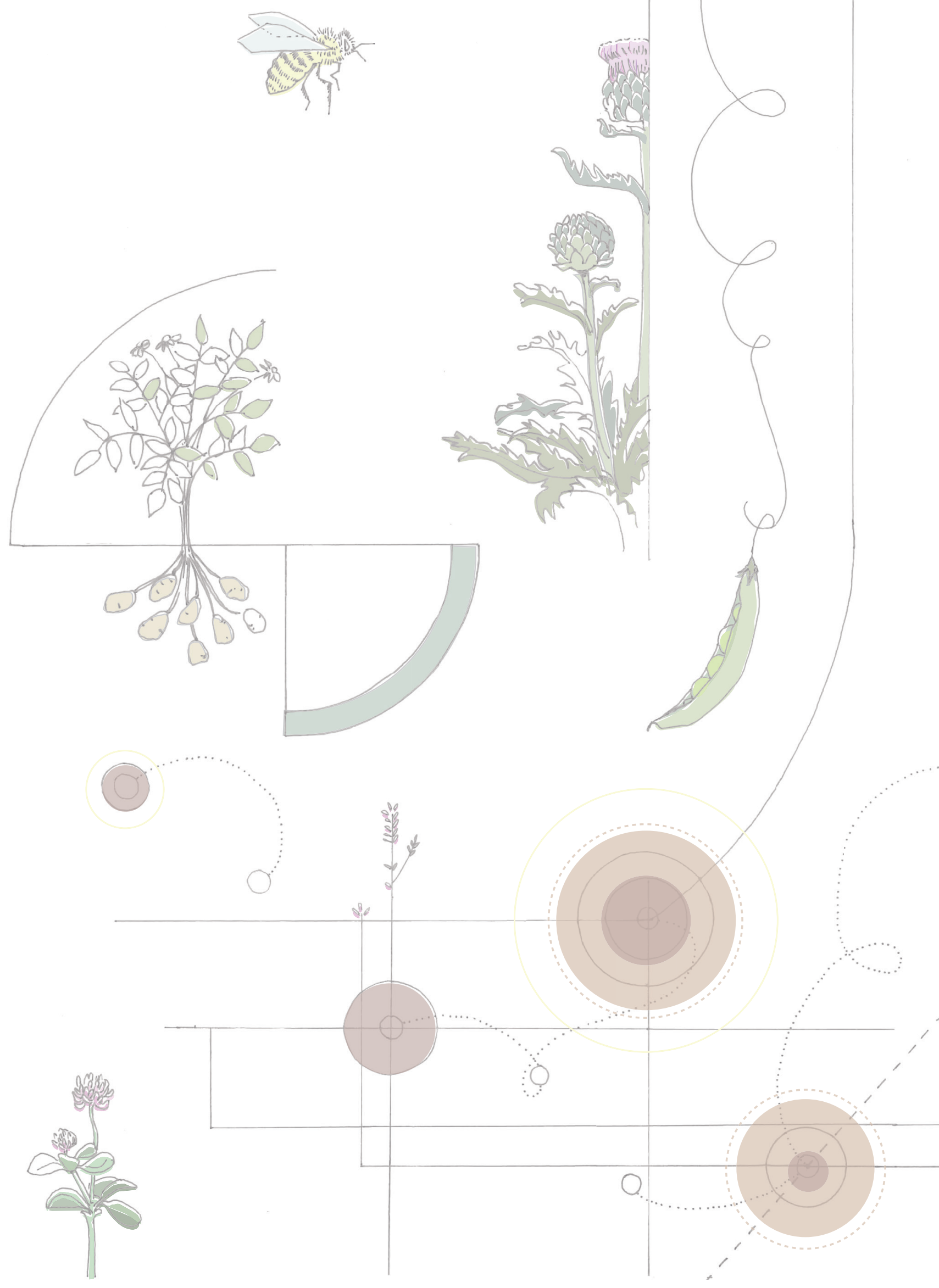




\begin{abstract}
Cities are increasingly targeted as centers for sustainable development and innovation of food systems. Urban agriculture is advocated by some as a multi-faceted approach to help achieve urban sustainability goals, as it provides possible social, economic and environmental benefits. The role of urban agriculture in restoring resource cycles receives increasing attention, especially with regard to assimilating urban waste. However, there is little information on how nutrients are managed in urban agriculture in industrialized countries. To examine nutrient management in urban agriculture, data was collected from a total of 25 ground-based urban agriculture initiatives in the Netherlands on i) preferences for types of fertilizers, and ii) quantity and quality of fertilizers used including nutrient composition and organic matter content. The main inputs at urban farms were compost and manure, high in organic matter content. The total nutrient inputs were compared to nutrient demand based on crop nutrient uptake in order to determine nutrient balances. Results show that mean nutrient inputs exceeded mean crop demand by roughly $450 \%$ for total nitrogen, $600 \%$ for phosphorus and $250 \%$ for potassium. Mean inputs for plant-available nitrogen were comparable to crop uptake values. The surpluses, particularly for phosphorus, are higher than fertilizer application limits used for conventional farming in The Netherlands. While nutrient input calculations were subject to several uncertainties, e.g., due to lack of accuracy of the data supplied by the farmers, results show a salient indication of overfertilization and thus a suboptimal nutrient use. If urban agriculture continues to expand across cities these observed nutrient surpluses may pose a risk for local surface waters and groundwater as well as soil quality. The need to improve nutrient management in urban agriculture is evident. Soil tests, harvest logging and book keeping of nutrient inputs would improve data quality and may help balance nutrient inputs with nutrient outputs.
\end{abstract}

Keywords: urban farming, nitrogen, phosphorus, potassium, organic matter, fertilizer use

This chapter is published as: Wielemaker, R., Oenema, O., Zeeman, G. \& Weijma, J. 2019. Fertile cities: Nutrient management practices in urban agriculture. Science of The Total Environment. 


\section{Introduction}

The long-term sustainability of urban areas has increasingly been called into question due to their dependency on non-renewable resources (McDonnell and MacGregor-Fors, 2016, Rees and Wackernagel, 1996). In response, the concern about the role and contribution of cities to sustainable development has prompted research agendas that regard cities as places that concentrate opportunities for change (Revi and Rosenzweig, 2013, Voytenko et al., 2016, Wolfram and Frantzeskaki, 2016). The production of food in or around urban areas, generally known as urban agriculture, has been proposed by many as an effective intervention to address various issues of urban sustainability (Ackerman et al., 2014, Deelstra and Girardet, 2000, Pearson et al., 2010, Smit et al., 1996, Specht et al., 2013). Especially the diversity in activities, scales, and purposes of urban agriculture offers ample opportunities to integrate urban agriculture in the built environment and to contribute to various sustainability goals. Urban agriculture has been advocated to increase local food provisioning, reduce supply chains and transportation distances, increase urban green, reduce the urban heat island effect, increase urban water retention and infiltration, increase bio-diversity in cities, provide opportunities for employment, education and recreation, and foster community cohesion (Lorenz, 2015, Mok et al., 2014, Artmann and Sartison, 2018b). Urban agriculture has also been identified as an auspicious component in repairing biological resource cycles within the built environment (Deelstra and Girardet, 2000, Drechsel and Kunze, 2001, Smit and Nasr, 1992). As such, water, energy, and materials can be recycled between urban agriculture and other urban functions.

Cities currently import nutrients contained in food and materials and discharge these as solid waste and wastewater streams with only meager nutrient and organic matter $(\mathrm{OM})$ recovery and reuse. By assimilating these nutrients as composts and bio-fertilizers, urban agriculture could reintroduce nutrients into the food system and contribute to restoring the nutrient cycle. The use of compost and animal manure is a widespread practice on urban farms (urban agriculture initiatives is used interchangeably hereafter) (Metson and Bennett, 2015). These soil amendments, high in OM, positively enhance soil quality. Increased soil OM and soil porosity facilitate water infiltration and water retention, serving as buffers during heavy rainfall (Taylor and Lovell, 2014) and retain water longer during episodes of drought. Soils with a history of urban uses such as housing, industrial production, and disposal activities, are often nutrient-poor, compacted and low in organic carbon. These urban soils may benefit from added soil amendments and farming practices (Lorenz, 2015). Moreover, using urban compost as well as other urban organic waste streams, allows urban farms to facilitate nutrient cycling on a local scale (Dewaelheyns et al., 2013). Metson and Bennett (2015) have shown that $73 \%$ of inputs used in urban farms in Montreal, Canada originated from local sources including green waste compost, vermicompost, and locally-produced manure. Grard et al. (2015) demonstrated the value of using urban organic waste as a growing substrate on rooftop farms in France; results indicated high crop yields and low levels of heavy metals in the harvested crops compared to European norms (No 1881/2006). The use of nutrients recovered from human excreta in urban agriculture can also contribute to 
closing urban nutrient cycles as shown by Chrispim et al. (2017a) and Wielemaker et al. (2018b). Increased technology developments in this field provide opportunities for nutrient recovery and reuse in urban agriculture in the form of fertilizers or soil amendments.

Despite the potential benefits for improved urban soil quality and increased nutrient availability, intensive urban agriculture production systems and fertilization practices can also result in negative ecological effects, both locally and regionally (Lorenz, 2015, Safi, 2011, Taylor and Lovell, 2014). The eager use of fertilizers and the lack of careful management of nutrients by urban farmers can lead to surpluses of macronutrients, nitrogen $(N)$, phosphorus $(P)$ and potassium (K), (Huang et al., 2006, Metson and Bennett, 2015, Wielemaker et al., 2018b, Witzling et al., 2011), as well as the accumulation of trace metals, such as cadmium and copper (Lorenz, 2015, Hargreaves et al., 2008).

Urban farms often follow organic farming practices (with or without certification), which means that they are limited to using the types of fertilizers that are permissible in organic agriculture. Commonly organic amendments such as manure and composts are the main fertilizer source (Bergström et al., 2009). When these amendments are applied at a rate to meet the $\mathrm{N}$ requirement of crops, the amounts of $\mathrm{P}$ and $\mathrm{K}$ applied often exceed crop requirements (Eghball, 2002, Maltais-Landry et al., 2015, Maltais-Landry et al., 2016, Mikkelsen and Hartz, 2008). Witzling et al. (2011) found high nutrient levels in community gardens in Chicago, with $\mathrm{P}$ and $\mathrm{K}$, and sometimes $\mathrm{N}$ levels exceeding soil fertility recommendations required for optimal crop growth. Similarly, Metson and Bennett (2015) found a surplus of $0.316 \mathrm{Gg} \mathrm{P} / \mathrm{yr}$ in surveyed urban farms in Montreal in 2012 (averaging to $1013 \mathrm{~kg} \mathrm{P} / \mathrm{ha} / \mathrm{yr}$ ). Another study on home gardens in Flanders, Belgium found $89 \%$ of vegetable gardens to fall under soil fertility class 'high' for soil P content (Dewaelheyns et al., 2013). The over-fertilization of P may result in the saturation of the soil $\mathrm{P}$ sorption capacity and increased P leaching and run-off, leading to environmental drawbacks such as eutrophication of surface waters (Schröder and Neeteson, 2008, van Grinsven and Bleeker, 2016, Maltais-Landry et al., 2016). The build-up of any nutrient in soils to beyond recommended levels is an inefficient use of resources; it decreases nutrient use efficiency and can result in soil-nutrient imbalances and/or unfavorable pH levels (Mikkelsen and Hartz, 2008, Tian and Niu, 2015).

In recent decades urban agriculture has been gaining ground in various forms such as allotment gardens, community gardens, harvest farms and commercial farms, across open spaces, rooftops, greenhouses, and as indoor farms (Lorenz, 2015, Thomaier et al., 2015). This trend is also visible in the Netherlands. There is, however, little information on how nutrients are managed on urban farms, which kinds and amounts of fertilizer inputs are used, their origin, and how current practices relate to the current regulatory frameworks for nutrient management in agriculture (i.e., Manure and Fertilizers Act). Research on nutrient budgeting has extensively been conducted in developing countries, such as Vietnam, China, Afghanistan, and several African countries (e.g., Abdulkadir et al., 2013, Huang et al., 2006, Khai et al., 2007, Safi, 2011, 
Wang et al., 2008). Previous studies on urban agriculture in industrialized countries primarily highlight the economic and social facets of urban agriculture (e.g., Duchemin et al., 2008, Smit et al., 1996, Specht et al., 2015, Thomaier et al., 2015, Zasada, 2011), and only few studies focus on nutrient management (e.g., Dewaelheyns et al., 2013, Grard et al., 2015, Metson and Bennett, 2015, Wielemaker et al., 2018b). If urban agriculture is to play a role in the closing of urban nutrient cycles, as is advocated by many (Goldstein et al., 2016, LeGrand et al., 2014, Grard et al., 2015), it is important to first quantify the current fertilizer use in urban agriculture and evaluate the contribution of urban agriculture to nutrient recycling.

The overall objective of this study was to evaluate nutrient use in urban agriculture farms in the Netherlands, a densely populated and industrialized country increasingly implementing urban agriculture. While stimulating urban agriculture has benefits across social, economic, and environmental facets, careful consideration of the cumulative effects of farm practices needs to be understood. We conducted interviews with ground-based urban agriculture farms (sizes between 0.1-1.7 ha) across cities to collect information on farmer preference for the type of nutrient inputs (fertilizers, manures, composts, soil amendments). We quantified N, P, and K (NPK) inputs, the three primary macronutrients, at farm level. In addition, OM inputs were also quantified, as soil OM is broadly recognized as an important aspect of soil quality and fertility (Hijbeek et al., 2017). The calculations allowed us to evaluate fertilization practices and identify over or under fertilization for NPK compared to crop nutrient uptake as well as compared to legal application limits for $\mathrm{N}$ and $\mathrm{P}$.

\section{Methods}

\subsection{Selection of urban agriculture initiatives, interviews and data collection}

The definition of urban agriculture used in this research is: the production of food in and around cities for the purpose of feeding local populations. Starting with an initial inventory (compiled via an internet search, word of mouth, and snowball sampling) of urban agriculture initiatives $(n=60)$ located across various cities, a final selection of 25 urban farms was included in this study, using the following criteria: (i) willingness to participate, and (ii) ability to provide quantitative data. Only ground-based urban farms were included in this study, which are the dominant type of urban farming in the Netherlands.

Interviews were conducted in person and onsite, or via telephone, with the head farmer or a farm volunteer between May 2017 - March 2018 using a semi-structured questionnaire (see Supporting Information II). Onsite surveying was preferred as it allowed for additional data collection through observation (e.g., cultivated area, management, maintenance of composting facilities, crop types) (Cohen and Crabtree, 2006). The semi-structured interviews were advantageous for both interviewer and interviewee as they intended to be conversational and allowed for flexibility to enquire for details when needed. Most interviews conducted in person were voice-recorded (with 
consent from the interviewees) and later transcribed to distill data for data processing; interviews conducted via telephone were not voice-recorded. To clarify or verify collected information, the interviews were followed-up with questions via email or telephone.

Data collected during the interviews, both qualitative data on farmer practices and preferences, and quantitative data on nutrient inputs, were processed per farm separately, as well as compounded into data spreadsheets to facilitate comparison. Nutrient contents of manure, compost, and organic fertilizers were derived from the labels of bags or from literature (see Supporting Information II). Occasionally farmers were able to present results of chemical analyses of the manures or composts applied on their farms; when available, we used these values. An overview of the data collected, data sources and assumptions (when input-specific information was not available), and of the specifications used to make nutrient input calculations is provided in the Supporting Information II.

\subsection{Data processing and analysis}

Total inputs for OM and NPK were calculated, and for comparison across farms, inputs were expressed as $\mathrm{kg} / \mathrm{ha} / \mathrm{yr}$. Inputs of NPK were compared with the uptake of NPK by crops commonly grown by urban agriculture initiatives. Reference values were used, and not an account of what was actually grown on each farm, because farmers could not supply detailed information on crops planted and respective harvests. Inputs were also compared to the legal application limits (2017) for N and P according to the Manure and Fertilizers Act (Meststoffenwet, 1986).

To assess the adequacy of soil OM inputs, these were expressed in terms of effective organic matter (EOM). EOM in manure, composts, and other organic fertilizers is defined as the fraction of OM that remains in the soil one year after its application to the soil (de Haan and van Geel, 2013a). The amount of EOM inputs ( $\mathrm{kg} / \mathrm{yr}$ ) at each farm was calculated using Equation 3.1, where HC stands for the humification coefficient (\%), as reported by de Haan and van Geel (2013a).

$$
\mathrm{EOM}=\mathrm{HC} * \mathrm{OM}
$$

Equation 3.1

Since plants acquire $\mathrm{N}$ from the soil only in plant-available forms, total $\mathrm{N}$ inputs $(\mathrm{kg} / \mathrm{yr})$ per farm were also expressed in terms of plant-available nitrogen (PAN) inputs ( $\mathrm{kg} / \mathrm{yr})$. PAN indicates the fraction of the total $\mathrm{N}$ (Ntot) input ( $\mathrm{kg} / \mathrm{yr}$ ) that is available to the plants during the first year after application. It also accounts for the $\mathrm{N}$ that is vulnerable to losses via leaching, denitrification, and (when in ammonium form) volatilization. The amounts of PAN were estimated for each fertilizer, using Equation 3.2 and fertilizer $\mathrm{N}$ equivalency (CNtot), expressed as percentage coefficients (\%). Fertilizer $\mathrm{N}$ equivalencies for composts, manures, and wastes are usually below $100 \%$, because the organically bound $\mathrm{N}$ has to be mineralized first to ammonium- $\mathrm{N}$ forms (van Dijk et al., 2005). We assumed that all P from composts, manures, and wastes becomes available at similar rates as synthetic $\mathrm{P}$ fertilizers on the longer term, and hence the fertilizer $\mathrm{P}$ equivalency 
for the $\mathrm{P}$ inputs was set at $100 \%$. Further, we assumed that all $\mathrm{K}$ is available and hence the fertilizer K equivalency was also set at 100\% for all fertilizers and soil amendments (de Haan and van Geel, 2013a).

$$
\text { Plant available } \mathrm{N}(\mathrm{PAN})=\mathrm{CN}_{\text {tot }} * \mathrm{~N}_{\text {tot }}
$$

Equation 3.2

The total nutrient inputs were then compared to nutrient demand based on crop nutrient uptake to determine nutrient balances. NPK uptake by crops, and thus withdrawal in harvested biomass, (kg/ha/yr) were retrieved from two literature sources: Bosch and De Jonge (1989) and Fink et al. (1999).

The Manure and Fertilizers Act of The Netherlands regulates $\mathrm{N}$ and $\mathrm{P}$ inputs to agricultural land via manure, and $\mathrm{N}$ and $\mathrm{P}$ application limits ('gebruiksnormen') (van Grinsven and Bleeker, 2016). Manure application limits are expressed in total $\mathrm{N}$ and are $170 \mathrm{~kg} \mathrm{~kg} / \mathrm{ha} / \mathrm{yr}$ for arable land, and 170 to $250 \mathrm{~kg} / \mathrm{ha} / \mathrm{yr}$ for grassland, depending on a farm-specific permit (derogation). Application limits for $\mathrm{N}$ (expressed in PAN) indicate the annual allowance of $\mathrm{N}$ per hectare per crop and soil type, and may vary with crop yield (RVO, 2017b). Legal fertilizer N equivalencies ('werkingscoëfficiënt') apply when calculating N application limits (RVO, 2014). Application limits for $\mathrm{P}$ indicate the annual allowance of $\mathrm{P}$ per hectare and varies relative to soil $\mathrm{P}$ content. In this study, the $\mathrm{N}$ and $\mathrm{P}$ application limits for 2017 were used for comparison. While commonly no fertilizer P equivalency (CP $(\%)$ ) is used for P inputs $(\mathrm{kg} / \mathrm{yr})$, the Manure and Fertilizers Act acknowledges that approximately half of the $\mathrm{P}$ in composts is soil-bound $\mathrm{P}$ and therefore uses a fertilizer P equivalency (CP) of 50\% for composts (RVO), calculated using Equation 3.3. All other fertilizers and soil amendments were counted with a $100 \%$ equivalent. There are no application limits for $\mathrm{K}$ in the Manure and Fertilizers Act.

$$
\text { Available } \mathrm{P}(\text { compost })=\mathrm{CP} * \mathrm{P}
$$

Equation 3.3

\section{Results and discussion}

\subsection{Characterization of the selected urban farms}

A total of 25 urban agriculture initiatives (Table 3.1) were interviewed in several cities across the Netherlands (Figure 3.1): Amsterdam (5), Apeldoorn (1), Culemborg (1), Dordrecht (3), Groningen (1), Haren (1), Leiden (1), Maastricht (1), Middelburg (1), Rotterdam (5), 's-Hertogenbosch (1), Utrecht (1), Veenhuizen (1), and Wageningen (3). The majority of the farms were established after 2005 with over half established after 2012. Only a handful of farms $(n=5)$ were over 1 ha in size, with $73 \%$ smaller than 0.5 ha. All farms showed ties to the local community and farmers emphasized the social benefits of their initiatives, including education, awareness raising, community building, and support for low-income and vulnerable groups. Meanwhile, over half of the farms had a commercial objective. The produce from these farms supplied own or local restaurants, were sold through membership shares (CSA), or were sold on-site or at local markets. 
With the exception of two farms, the initiatives can be classified as ground-based-non-conditioned (GB-NC). Goldstein et al. (2016) defines this typology as: occurring directly on the ground (as opposed to in or on a building) and connected to the ambient environment (in contrast to e.g., greenhouses). The other two farms can be classified as ground-based-conditioned, growing in raised beds within a frame (contained soil). A few of the farms also managed one or more smaller hoop-house(s) or greenhouse(s) for season extension. For a more detailed account of the farms, and an overview of the interview data can be found in the Supporting Information II.

In total $77 \%$ of the interviewees were satisfied with the crop yields. Almost a quarter were less content, which they related to poor yields, lack of labor force, lack of mechanization, or poor soil quality. All farmers listed measures to further improve harvests, including crop rotation and planning, pest, disease and weed management, and nutrient and soil $\mathrm{pH}$ management. The majority of the farmers considered their farming practices to be sustainable for a host of reasons. Many alluded to their organic or ecological farming practices referring to the use of organic manure, biological-control of pests, the absence of pesticide use and the use of a crop rotation plan. Onsite composting, waste management and recycling, rainwater harvest and reuse, green energy use and reducing transport and packaging of products were reasons given to support their sustainability claims. Many farmers also considered their role in raising awareness amongst the community as one of their main contributions to sustainability.

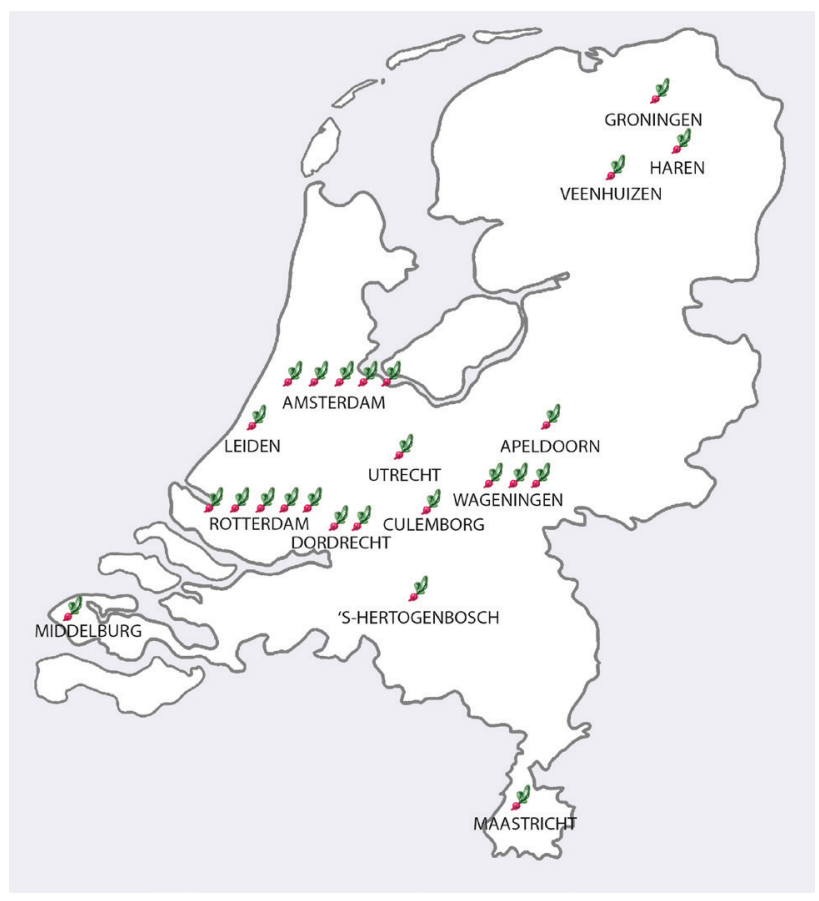

Figure 3.1 Map of the 25 interviewed urban agriculture initiatives across cities in the Netherlands 
All farmers also considered their resource management to be sustainable. The use of local, renewable, and/or organic sources of fertilizers as opposed to chemical fertilizers was the main reason provided. $35 \%$ of the farmers mentioned their onsite composting efforts as indication of their sustainable resource management. A meagre three of the farms considered their resource management to be less optimal because of the use of external inputs and uncertainty regarding the soil nutrient balance. To improve nutrient management many farmers indicated that a soil analysis would be appropriate, as well as (improved) onsite composting.

The preferences for fertilizers varied between farms. None of the farmers preferred synthetic fertilizers but rather preferred certified organic fertilizers derived from plant and animal residues, and sourced as locally as possible. However, in practice, farmers did not always/only use certified organic fertilizers, nor fertilizers sourced locally. Manure inputs at some farms were sourced onsite (i.e., from own farm animals), some were sourced directly from nearby (petting) farms, some from farms outside of the city, and others purchased packaged manure from retailers. Some farmers were adamantly against the use of fertilizers made from animal waste such as bone and blood meal, and sources of manure that could contain traces of antibiotics (administered to the animals). Most farmers conveyed that their fertilizer use practices were based on a mix of experience (62\%), feeling (42\%), knowledge (54\%), and trial-and-error (50\%); a minority indicated that advice found in literature (23\%), on the packaging (15), or from experts $(27 \%)$ and legislation (23\%) guided their fertilizer use.

\subsection{Fertilizer, organic matter and nutrient inputs}

Table 3.1 shows which types of fertilizer inputs were used. While some inputs were produced onsite (e.g., farm-made composts and manure from own farm animals), all farms used at least one fertilizer input from external sources (produced off-site). Compost (used at $80 \%$ of the farms), manure (60\%), and/or some other organic soil amendment (24\%) were the main inputs. Supplemental macronutrient (40\%), micronutrient $(20 \%)$ and potassium fertilizers $(16 \%)$ were also used at some farms. Calcium-rich soil amendments were also used (24\%), primarily to modify soil $\mathrm{pH}$. The variation in types and amounts of fertilizers used by farmers is reflected by thev variation in the total nutrient and organic matter inputs calculated for each farm (Table 3.1).

\subsubsection{Organic matter}

The calculations for farm inputs show a partiality for fertilizers rich in OM; particularly compost and manure contributed to OM loads with total OM inputs ranging between 700 and 138,100 $\mathrm{kg} \mathrm{OM} / \mathrm{ha} / \mathrm{yr}$ (Table 3.1 and Figure 3.2). Whether the application of organic inputs increases soil OM depends on the current amount of organic matter in the soil, the type of organic input applied, crop type, and crop residue management, as well as temperature, humidity, soil texture, and soil cultivation (Hijbeek et al., 2017). As found by Loveland and Webb (2003), it is difficult to establish a critical level for soil organic matter for temperate regions. A steady soil OM input- 


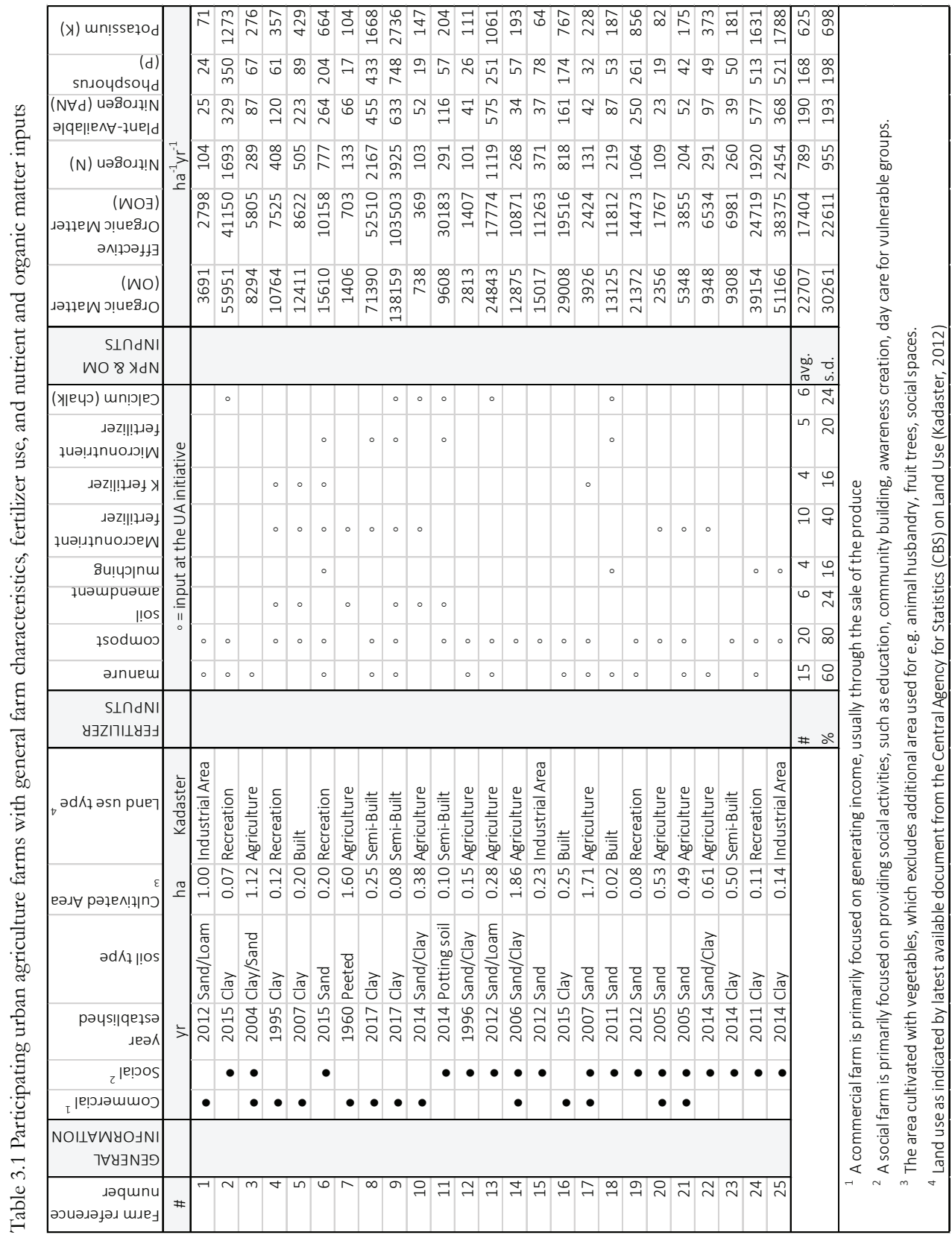


output balance requires a total annual supply of EOM that is equal to the amount of soil OM that is degraded annually. Degradation of soil organic matter depends on soil characteristics such as soil type, soil $\mathrm{pH}$, soil moisture, and temperature and $\mathrm{C} / \mathrm{N}$ ratio, as well as on the level of soil cultivation. The rate of degradation of soil organic matter may vary between $1-5 \%$ per year. For agricultural land in The Netherlands, it has been estimated at the average degradation is 2000 $\mathrm{kg} \mathrm{OM} / \mathrm{ha} / \mathrm{yr}$ (de Haan and van Geel, 2013a). Therefore, to replenish soil OM degradation, an average input of $2000 \mathrm{~kg} \mathrm{EOM} / \mathrm{ha} / \mathrm{yr}$ is recommended (de Haan and van Geel, 2013a). We observed that the average EOM input was much larger than the recommended EOM input; $84 \%$ of the farms $(n=22)$ applied more than $2000 \mathrm{~kg} E O M / \mathrm{ha} / \mathrm{yr}$ and $76 \%(\mathrm{n}=18)$ of the farms applied more than $5000 \mathrm{~kg} \mathrm{EOM} / \mathrm{ha} / \mathrm{yr}$. This indicates that most urban agriculture farms increase soil OM content.

\subsubsection{Nitrogen, phosphorus and potassium}

NPK inputs differed greatly between farms (Table 3.1 and Figure 3.2). Means \pm standard deviations indicate the wide range of inputs across the 25 urban agriculture initiatives. For NPK these values were $789 \pm 955 \mathrm{~kg} \mathrm{~N} / \mathrm{ha} / \mathrm{yr}, 168 \pm 198 \mathrm{~kg} \mathrm{P} / \mathrm{ha} / \mathrm{yr}$, and $625 \pm 698 \mathrm{~kg} \mathrm{~K} / \mathrm{ha} / \mathrm{yr}$. The variations in inputs for urban agriculture farms smaller than $0.3 \mathrm{ha}(\mathrm{n}=15)$ were especially large, and on average higher compared to the inputs for urban agriculture farms larger than 0.3 ha $(\mathrm{n}=11)$ (Figure 3.3a). The urban agriculture farms with the highest nutrient applications were also amongst the smaller farms. Another relationship was found between the year in which the urban farm was established and the nutrient input. Older farms ( $\mathrm{n}=9)$, established before 2010, displayed lower nutrient applications (average P application of $45 \mathrm{~kg} / \mathrm{ha} / \mathrm{yr}$ ) than younger farms $(\mathrm{n}=16)$ (average $\mathrm{P}$ application $237 \mathrm{~kg} / \mathrm{ha} / \mathrm{yr}$ ) (Figure 3.3b). The highest nutrient applications where on farms with clay soils (Figure 3.3c).

\subsubsection{Comparison of NPK inputs with NPK withdrawal in harvested crops}

Partial NPK input-output balances of urban agriculture farms provide an indication of the NPK surpluses or deficits, the potential NPK accumulation in the soil, as well as of the vulnerability of these urban agriculture farms for NPK losses to the wider environment. Mean total N inputs $(789 \pm 955 \mathrm{~kg} / \mathrm{ha} / \mathrm{yr})$ were 4 to 5 fold larger than the estimated $\mathrm{N}$ withdrawal via harvested crops (Figure 3.4a); N withdrawal averaged to $161 \pm 76 \mathrm{~kg} / \mathrm{ha} / \mathrm{yr}$ and $203 \pm 88 \mathrm{~kg} / \mathrm{ha} / \mathrm{yr}$, calculated from data extracted from two literature sources respectively (see Supplemental Information II). Independent-sample t-tests (confidence interval percentage 95\%) were conducted to compare the difference in means between $\mathrm{N}$ inputs and the withdrawal values. There was a significant difference in means (two tailed) for total $\mathrm{N}$ inputs and $\mathrm{N}$ withdrawal values found in Bosch and De Jonge (1989) ( $p=0.003$ ) and Fink et al. (1999) ( $p=0.005)$. Mean PAN inputs were only $191 \pm 192 \mathrm{~kg} / \mathrm{ha} / \mathrm{yr}$, which fall within the range for the $\mathrm{N}$ withdrawal estimates; the difference in means between PAN inputs and $\mathrm{N}$ withdrawal values found in Bosch and De Jonge (1989) 
$(p=0.480)$ and Fink et al. (1999) $(p=0.779)$ was not significantly different. The large difference between total $\mathrm{N}$ input and PAN input is due to the low fertilizer $\mathrm{N}$ equivalencies (CNtot) for composts, manures, and wastes. These fertilizers release only a small fraction of total $\mathrm{N}$ in plant-available forms in the year of application. However, the residual release of PAN during subsequent years is relatively high, and PAN may accumulate in the soil with continued use of these fertilizers at high rates. Urban agriculture farmers do seem to account for residual effects, as the total $\mathrm{N}$ input clearly decreased with the age of the farm (Figure 3.3).

Mean P inputs $(168 \pm 198 \mathrm{~kg} / \mathrm{ha} / \mathrm{yr})$ were much higher than, and significantly different from, estimated $\mathrm{P}$ withdrawal via harvested crops $(24 \pm 12 \mathrm{~kg} / \mathrm{ha} / \mathrm{yr}(\mathrm{p}=0.001)$ and $32 \pm 13 \mathrm{~kg} / \mathrm{ha} /$ $\mathrm{yr}(\mathrm{p}=0.002)$, for the two literature sources respectively) (Figure 3.4b). P inputs for $44 \%$ of the farms were below the maximum value of $\mathrm{P}$ withdrawal (brussel sprouts $\sim 60 \mathrm{~kg} \mathrm{P} / \mathrm{ha} / \mathrm{yr}$ ), the remaining $56 \%$ of $\mathrm{P}$ inputs surpassed this maximum value. Mean $\mathrm{K}$ inputs $(625 \pm 698 \mathrm{~kg} / \mathrm{ha}$ / yr) were also much higher than estimated $K$ withdrawal via harvested crops $(226 \pm 104 \mathrm{~kg} / \mathrm{ha} /$ $\mathrm{yr}(\mathrm{p}=0.009)$ and $372 \pm 102 \mathrm{~kg} / \mathrm{ha} / \mathrm{yr}(\mathrm{p}=0.020)$, for the two literature sources respectively). A total of $36 \%$ of the farms had $\mathrm{K}$ inputs that exceeded even the highest value for $\mathrm{K}$ output via harvested crops (red beet $\sim 460 \mathrm{~kg} / \mathrm{ha} / \mathrm{yr}$ ) (Figure 3.4c).

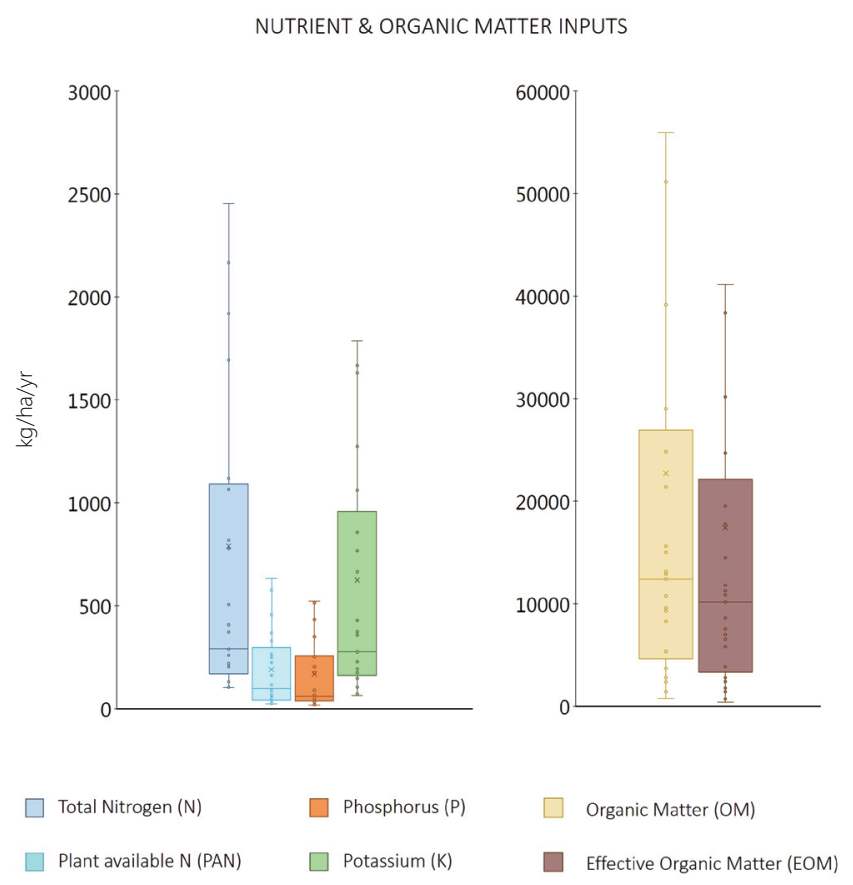

Figure 3.2 Box plots of organic matter $(\mathrm{OM})$ and effective organic matter (EOM) inputs (a) and nitrogen $(\mathrm{N})$, plant-available nitrogen (PAN), phosphorus $(\mathrm{P})$ and potassium $(\mathrm{K})$ inputs $(\mathrm{b})$ for all urban agriculture initiatives. Boxes show the interquartile range ( 25 to $75 \%$ values). The line in the boxes shows the median value and the ' $x$ ' shows the mean value. Whiskers indicate the total range of the values, outliers $(>1.5$ interquartile range) excluded. 

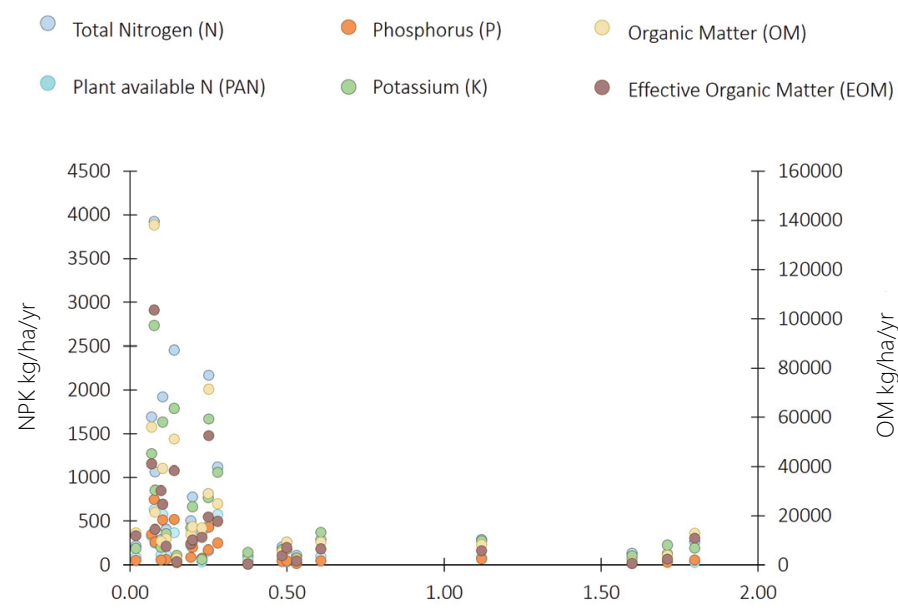

a.

Hectares

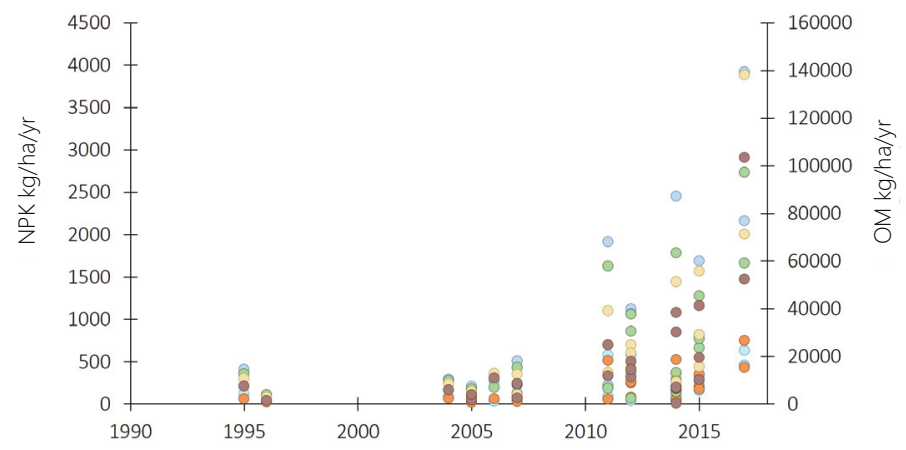

b.

Year Established

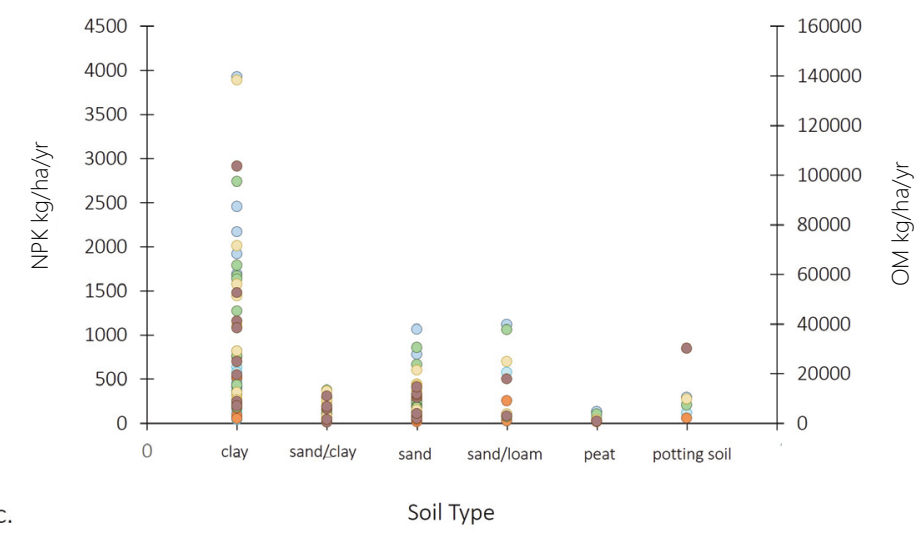

Figure 3.3 Nitrogen, phosphorus and potassium, and organic matter inputs plotted against (a) farms size, (b) year of farm establishment, and (c) per soil type 
The fertilization adequacy can further be evaluated on the basis of N:P:K mass ratios. Figure 3.5 shows the position of various common vegetable crops in the triangle based on their NPK contents and normalized to percentages (only selected crops are shown in Figure 3.5). The mean NPK inputs of the 25 urban agriculture farms are also plotted in the triangle. The mean NPK mass ratios of crops at harvest average 40:6:54 (calculated for both Bosch and De Jonge (1989) and Fink et al. (1999)). However, there is a significant variation between crops, for example for radish it is 53:4:43 and for endive 34:4:62). Ratios for the NPK inputs are also shown with PAN (PAN:P:K). The ratios of NPK inputs based on total $N$ (average $=49: 10: 41$ ) compare more closely to crop uptake ratios than NPK input ratios based on PAN (average= 21:16:63), indicating excess input of $\mathrm{P}$ and $\mathrm{K}$ relative to available N. Ratios of NPK inputs across farms are fairly similar with the exception of one farm, that is, urban farm 15 had only input of vermicompost, which has low N:P and N:K ratios.

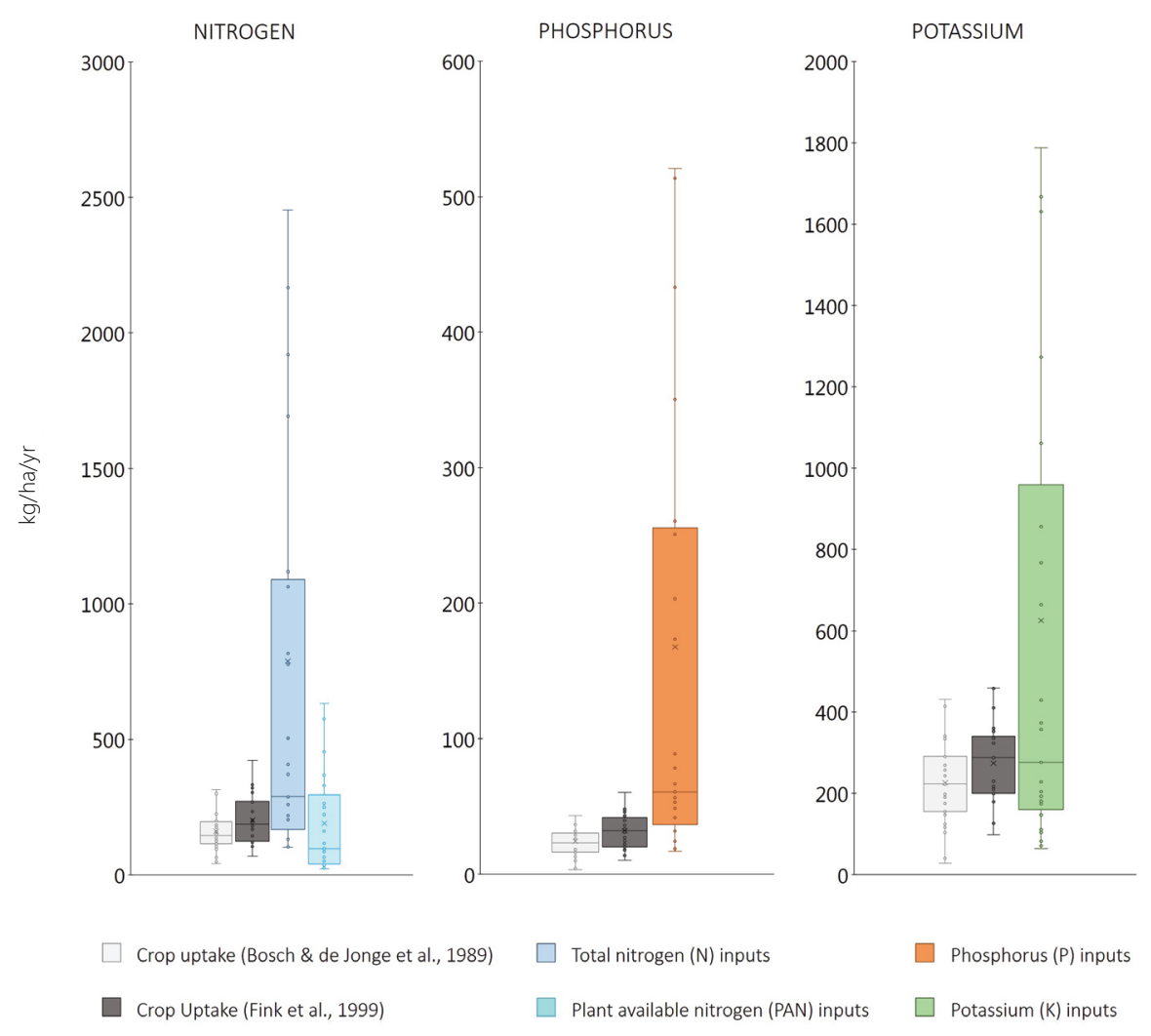

Figure 3.4 Nitrogen $(\mathrm{N})$, phosphorus $(\mathrm{P})$ and potassium $(\mathrm{K})$ inputs of the 25 interviewed farms compared to NPK crop uptake retrieved from two studies (Bosch and De Jonge, 1989, Fink et al., 1999). Total N and plantavailable N (PAN) inputs compared to crop uptake of N (a); P inputs compared to crop uptake of P (b); K inputs compared to crop uptake of $\mathrm{K}(\mathrm{c})$. Outliers ( $>1.5$ interquartile range) excluded. 


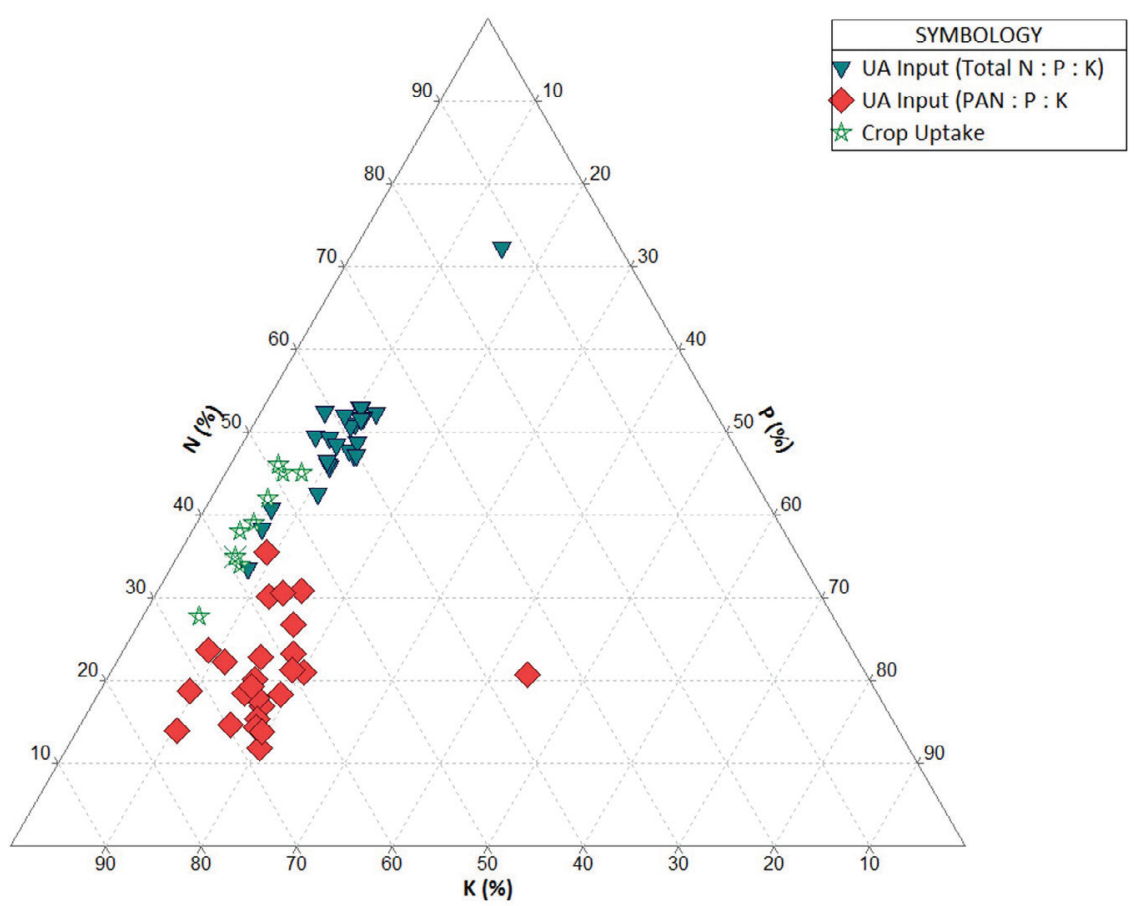

Figure 3.5 Comparison of ratios of nitrogen $(\mathrm{N})$, phosphorus $(\mathrm{P})$ and potassium $(\mathrm{K})$ inputs (shown separately for total $\mathrm{N}$ and plant-available nitrogen (PAN)) for 25 urban agriculture farms with ratios of $\mathrm{N}, \mathrm{P}$ and $\mathrm{K}$ uptake for 10 common crops grown on urban farms (beet root, broccoli, red cabbage, carrot, celery, green bean, lettuce, onion, radish and spinach). The ratios are normalized to percentages (e.g., $\mathrm{N} /(\mathrm{N}+\mathrm{P}+\mathrm{K})$ ). The $\mathrm{N}$ axis reads horizontally, the $\mathrm{P}$ axis reads diagonally from top right to bottom left and the $\mathrm{K}$ axis reads diagonally from bottom right to top left.

The 25 urban farms show relatively large mean NPK surpluses and a mismatch between inputs and demands in terms of NPK ratios, both indicating that current nutrient management practices on urban farms are not optimal. The preference among urban agriculture farms for manure and compost often leads to high P and K loads that exceed plant requirements (Maltais-Landry et al., 2016). While the nutrient surplus exhibited by some of the urban farms do not by default indicate total nutrient losses to the environment directly, they can be used as an integrated measure of nutrient loss potential (Öborn et al., 2003). The surplus may be stored within the system and may be lost to the environment; its dissipation into the environment depends on various factors including rainfall, soil type, and slope, soil tillage, crop type, and application method (Edwards and Withers, 1998, Lord et al., 1999). While some oversupply of P and K might be acceptable in single years, since they can be stored in the soil to a larger extent than $\mathrm{N}$, the long term balance would need to indicate ratios similar to those required by crops plus some unavoidable losses (Winker et al., 2009). Two options for improving the nutrient balance include: (1) increasing P export by cultivating plants with high plant $\mathrm{P}$ concentrations (e.g., grain, potato and cabbage 
crops), and (2) adjusting fertilizer inputs to better match crop uptake requirements (MaltaisLandry et al., 2016). Reducing over-fertilization of especially P, by simply reducing application rates of composts and manure would result in $\mathrm{N}$ under-fertilization, leading to lower crop yields and crop N deficiency (Maltais-Landry et al., 2016, Berry et al., 2002). Shifts in N:P:K ratios are needed. Instead of relying completely on composts and manures, with a constrained N:P stoichiometry, these organic amendments should be replaced by fertilizers with a high N:P ratio and/or decoupled nutrients (Maltais-Landry et al., 2016).

\subsubsection{Comparison of $\mathrm{N}$ and $\mathrm{P}$ inputs with legal $\mathrm{N}$ and $\mathrm{P}$ application limits}

Nutrient management practices in the Netherlands have long been discussed and criticized because of the high N and P surpluses in Dutch soils (Schröder and Neeteson, 2008, van Grinsven and Bleeker, 2016). The implementation of a series of governmental regulations in the late 1990's (especially in response to the 1991 Nitrate Directive (91/676/EEC)) have halved the mean $\mathrm{N}$ surplus from $250 \mathrm{~kg} / \mathrm{ha}$ in the mid-1990s, and have led to a strong decrease in mean P surpluses since the early 1990s, approaching zero kg/ha (van Grinsven and Bleeker, 2016). However, while conventional agriculture in the Netherlands has to comply with strict regulations for fertilization according to the Manure and Fertilizers Act, urban agriculture falls somewhere between existing categories. Due to their small size ( $<3 \mathrm{ha}$ ), and low number of animals (whose manure amounts to $<350 \mathrm{~kg} \mathrm{~N} / \mathrm{yr}$ ), these farms have an exemption from the compulsory $\mathrm{N}$ and $\mathrm{P}$ application limits and from nutrient management administration obligations, according to Article 43 of the Implementing Arrangement (Uitvoeringsregeling) of the Manure and Fertilizers Act (Meststoffenwet, 1986).

Further, of the included farms, 10 fall under the land use category for 'agricultural land'; the other farms fall under the following categories: 'recreation' (5), 'built' (4), 'semi-built' (3), and 'industrial' (3) (Kadaster, 2012). If classified as agricultural land, urban agriculture would have to adhere to the same regulations as conventional agriculture, with the maximum application rates for agricultural (grass land and arable land) of $35 \mathrm{~kg} \mathrm{P} / \mathrm{ha} / \mathrm{yr}$ and $170 \mathrm{~kg} \mathrm{~N} / \mathrm{ha} / \mathrm{yr}$ (article 2.4.b). However, if classified as 'other land' the maximum phosphorus application derived from animal manure, compost, recovered phosphorus fertilizers and organic fertilizers made form plant-derived materials (article 2.4.a, Dutch Manure and Fertilizer Act) amounts to $20 \mathrm{~kg} \mathrm{P}_{2} \mathrm{O}_{5} /$ ha/yr. For other land, a one-time dosage of vegetative compost of 200 ton dry matter/ha is also permitted (article 2a.1).

Total $\mathrm{N}$ and P inputs for all 25 farms were compared to the legal application limits (2017) for N and $\mathrm{P}$ (see Table 3.2). The $\mathrm{N}$ (as PAN) inputs were on average higher than the mean $\mathrm{N}$ application limits but lower than the highest $\mathrm{N}$ application limit (for white cabbage $=320 \mathrm{~kg} \mathrm{~N} / \mathrm{ha} / \mathrm{yr}$ ). Considering that the application standards for $\mathrm{N}$ are given in per hectare per year per crop, if two or more crops are planted in succession in one year, the application limit is increased to the sum of the individual limits per crop. Total $\mathrm{P}$ inputs are much higher than the $\mathrm{P}$ application 
limits for agricultural land. Total P inputs exceeded the lowest $\mathrm{P}$ application limit $(22 \mathrm{~kg} / \mathrm{ha} / \mathrm{yr})$ on $84 \%$ of the farms and $72 \%$ of the farms exceeded the highest $\mathrm{P}$ application limit $(33 / \mathrm{ha} / \mathrm{yr})$. The majority of farms that indicated to comply to legal application limits, largely over applied P. Using a fertilizer P equivalence for compost inputs shifted only one farm from a position of over-application of $\mathrm{P}$ to falling within the regulatory limits.

Table 3.2 Comparison of mean nutrient inputs at urban farms (this study) to legal nitrogen $(\mathrm{N})$ and phosphorus (P) application limits for conventional farming according to the Dutch Manure \& Fertilizers Act

\begin{tabular}{|c|c|c|c|c|c|c|c|c|c|c|c|c|}
\hline & \multicolumn{7}{|c|}{ Nitrogen } & \multicolumn{5}{|c|}{ Phosphorus } \\
\hline & \multicolumn{2}{|c|}{$\begin{array}{l}\text { N Input at } \\
\text { urban farms } \\
\text { kg/ha/yr }\end{array}$} & \multicolumn{5}{|c|}{$\begin{array}{l}\text { N Application Limits (Soil Type) } \\
\text { kg/ha/yr/crop }\end{array}$} & \multicolumn{2}{|c|}{$\begin{array}{l}\text { P Input at } \\
\text { urban farms } \\
\text { kg/ha/yr }\end{array}$} & \multicolumn{3}{|c|}{$\begin{array}{l}\text { P Application Limits } \\
\text { (Soil } \mathrm{P} \text { content) } \\
\text { kg } \mathrm{P}_{2} \mathrm{O}_{5} / \mathrm{ha} / \mathrm{yr}\end{array}$} \\
\hline & TN & PAN & Clay & $\begin{array}{l}\text { Sand- } \\
\text { NWC }\end{array}$ & $\begin{array}{c}\text { Sand- } \\
\text { S }\end{array}$ & Loam & Peat & $\mathrm{P}_{2} \mathrm{O}_{5}$ & $P$ & Low & Med. & High \\
\hline Mean & 789 & 197 & 209 & 187 & 152 & 152 & 194 & 267 & 116 & 75 & 60 & 50 \\
\hline s.d. & 955 & 189 & 66 & 59 & 45 & 45 & 63 & 272 & 119 & - & - & - \\
\hline \multicolumn{13}{|c|}{$\begin{array}{l}1 \text { The Manure and Fertilizers Act uses a fertilizer P equivalency (CP) of } 50 \% \text { for composts. For comparison to P } \\
\text { application limits, this equivalency was used when applicable to composts to calculate urban agriculture farm } \\
\text { inputs. }\end{array}$} \\
\hline
\end{tabular}

The Manure and Fertilizers Act was implemented to reduce $\mathrm{N}$ and $\mathrm{P}$ pollution of surface waters and groundwater by agricultural practices. However, small urban farms have an exemption from the compulsory $\mathrm{N}$ and $\mathrm{P}$ application limits and from nutrient management administration obligations because these farms have less than 3 ha of agricultural land and/or produce in total less than $350 \mathrm{~kg}$ of manure $\mathrm{N}$ per year on the farm. Because of this exemption, there is also no control and verification. Currently, the number of urban agriculture farms is relatively low, and they have a relatively small cultivated area. However, if current nutrient management practices on urban agriculture farms persist over long periods, and/or if the number of urban agriculture initiatives continues to grow and if new initiatives adopt similar practices, then these practices do raise concern from an environmental perspective. A further increase of the cultivated area will increase the environmental risks. Run-off from urban farms can either enter surface waters, leading to algal blooms, or, for cities with a combined sewer, wastewater treatment plants will have to manage increased nutrient loads from run-off. Equally of concern, the high compost and manure application rates may lead to accumulation of heavy metals in soils, and in vegetables. Heavy metal loads and organic micro pollutants through land application of fertilizers and soil amendments are also regulated by the Manure and Fertilizers Act; approved composts and soil amendments have to comply with heavy metal and micro pollutant concentration limits, and then may be increasingly applied until a maximum application per hectare of $100 \mathrm{~kg} \mathrm{~N}$, 
$35 \mathrm{~kg} \mathrm{P}, 150 \mathrm{~kg} \mathrm{~K}$, or $3000 \mathrm{~kg}$ OM is reached (RVO, 2017a, RVO, 2017c). The lack of data on heavy metals and micro pollutant concentrations of the inputs used on urban agriculture farms makes it difficult to assess whether concentration limits are exceeded. Regardless, considering that many farms exceed the indicated application limits, most interviewed farms may breach the heavy metal and micro pollutant legislation.

\subsection{Data uncertainties}

Several uncertainties affected the accuracy to calculate farm-level nutrient inputs, especially given the high-demand for data for this research, these included: (1) the (lack of accuracy and comprehensiveness of the data supplied by the farmers, (2) the estimates on the nutrient composition of manures and composts, (3) the lack of information on past fertilization regimes and soil nutrient stocks, and (4) the lack of information of farm management (e.g., tillage practices, fertilizer placement and timing). Despite these limitations, we consider the quality of the partial nutrient balances sufficiently robust to assess the nutrient management practices of urban agriculture farms, which was our main goal. For instance, assuming a magnitude of possible error for $\mathrm{N}$ content in manure of 30\% (as used by Mulier et al. (2003) in a similar study), to account for variability in nutrient composition estimates, does not change the main findings of this study (mean PAN inputs only change by $\pm 3 \%$ ). Likewise, the inclusion of past fertilization regimes and the mineralization of organic $\mathrm{N}$ from previous fertilizer applications, would only magnify the surpluses already observed. While $\mathrm{P}$ from previous applications accumulates in the soil, organic nitrogen is further released as PAN; a yearly application of chicken, pig or cow manure, for example, increases the fertilizer $\mathrm{N}$ equivalence by 20 and $35 \%$ respectively (de Haan and van Geel, 2013a).

The initial intention to conduct full farm-gate balances was discarded early on as collecting data on fertilizers used and the respective amounts applied was challenging enough and farmers could not supply detailed information on crop harvests $(\mathrm{kg} / \mathrm{yr})$ and succession planting. Planting multiple crops in succession throughout a year changes the amount of crop nutrient withdrawal, which could not be accounted for in this study. However a quick analysis considering two crop plantings in a year for comparison returned the following conclusions, which echo the conclusions already presented: (1) the majority of the farms risk under fertilization of PAN, and (2) the mean $\mathrm{P}$ and $\mathrm{K}$ inputs for the urban agriculture farms would still exceed $\mathrm{P}$ and $\mathrm{K}$ crop withdrawal. Especially for $\mathrm{P}$, the difference in means between inputs and crop withdrawal, considering two plantings, remains significantly different compared to the two literature sources: Bosch and De Jonge (1989) ( $p=0.006)$ and Fink et al. (1999) ( $p=0.016)$.

Furthermore, most farmers had not recorded which fertilizers they had used that year and many could provide only rough estimates of the amounts applied, let alone the exact placement and timing of the fertilizer application, and crop residue, mulching, and soil cultivation practices. Detailed farm nutrient balances would however benefit farmers in targeting and improving their 
nutrient management practices. Periodic soil testing and book keeping of all fertilizer inputs as well as yield and harvest logging, would make it possible to calculate input-output balances at farm resolution. Farm specific data on yield would have allowed for further analyses between fertilizer inputs and respective yield success. To achieve even more complete farm nutrient balances, $\mathrm{N}$ deposition, $\mathrm{N}$ fixation, and nutrient sedimentation could be included.

\section{Conclusions and outlook}

NPK inputs varied greatly among the 25 urban agriculture farms studied. Mean total NPK inputs were much larger than NPK output via harvested crops. There was a considerable range in fertilizer types, with a clear preference for composts and animal manures rich in OM. However, most OM-rich inputs are also the culprit of the excess fertilization due to the small faction of total $\mathrm{N}$ inputs that is plant-available and their constrained $\mathrm{N}: \mathrm{P}: \mathrm{K}$ stoichiometry. While, mean input of PAN was roughly similar to the mean $\mathrm{N}$ withdrawal via crop uptake, the $\mathrm{P}$ and $\mathrm{K}$ inputs largely exceeded their withdrawal via harvested crops. The persistence of current nutrient management practices on urban farms over long periods, and/or the adoption of similar practices at new urban farms increases the risks of inefficient nutrient management and excessive nutrient losses. While this research is unable to determine whether the excess NPK inputs have accumulated in the soil or are lost to the environment, the excesses do call to attention the need for increasing nutrient use efficiency and merits further examination. Just as conventional agriculture in the Netherlands has to comply with $\mathrm{N}$ and $\mathrm{P}$ application limits, urban agriculture initiatives might require similar attention. Longer term monitoring of nutrient inputs, outputs and soil nutrient pools will better help determine which targeted measures and tools could assist farmers in improving nutrient use efficiency and better inform whether measures are needed to regulate fertilizer use in urban agriculture.

The broader perspective of this study was to examine whether the nutrient demand of urban agriculture could be used to assess how much NPK from urban waste streams could be absorbed by urban agriculture, so as to achieve effective nutrient recycling within urban boundaries. Currently it is difficult to quantify how much NPK can be assimilated by urban agriculture, based on current urban agriculture practices, due to the huge diversity in urban agriculture practices and limited amounts of quantitative data. Only with a stark decrease in nutrient inputs could actual urban agriculture fertilization practices be taken as a point of departure to determine the extent to which urban agriculture may assimilate nutrients from urban waste streams to repair nutrient cycles within the built environment. However, in cities saturated with nutrients in solid waste and wastewater, it seems unsuited to perpetuate the current nutrient management practices of urban agriculture farms, including importing manure from rural areas to urban agriculture. Because urban agriculture is inherently urban and thus is in proximity to nutrient sources in waste, urban agriculture lends itself for establishing local nutrient cycles, especially for nutrients in forms too costly to export back to other agricultural areas (i.e., voluminous and heavy). 


\section{Acknowledgements}

The authors thank MSc Mathieu Chaix-Bar for conducting interviews with farmers and collecting data. We appreciate the participation of the urban farmers surveyed at each urban agriculture initiative, and thank them for taking the time to answer our questions and share data with us. We also thank Inge de Weerd, former employee at the Nutrient Platform NL, for her explanation of the Dutch Manure and Fertilizers Act as it applies to urban agriculture, and Willem van Geel and Janjo de Haan, from Wageningen Plant Research, for their input on crop yield and crop nutrient uptake. 


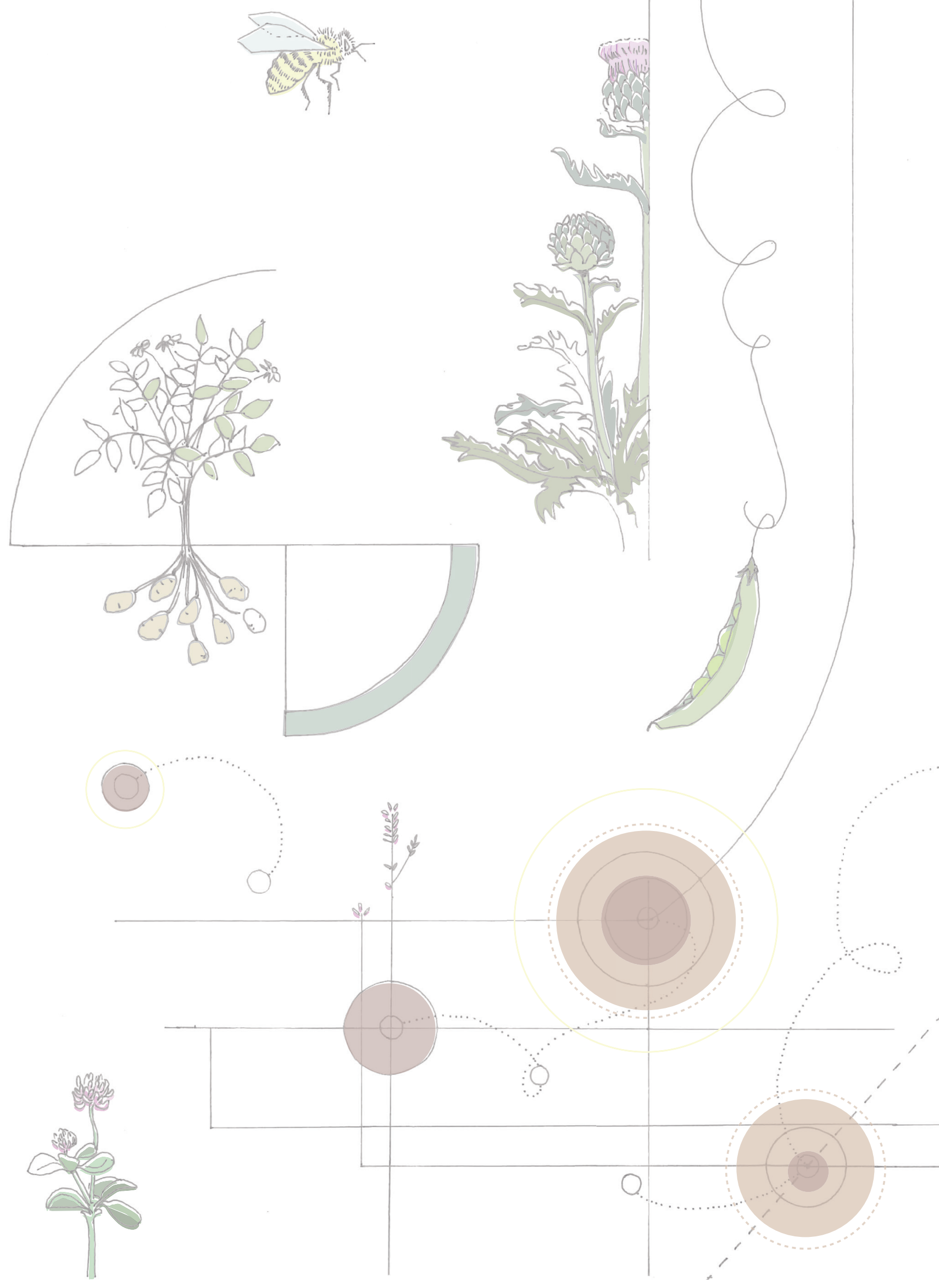




\section{Abstract}

The need for better nutrient management has spurred efforts towards more comprehensive recycling of nutrients contained in human excreta to agriculture. Research in this direction has intensified throughout the past years, continuously unfolding new knowledge and technologies. The present review aspires to provide a systematic synthesis of the field by providing an accessible overview of terminology, recovery pathways and treatment options, and products rendered by treatment. Our synthesis suggests that, rather than focusing on a specific recovery pathway or product and on a limited set of nutrients, there is scope for exploring how to maximize nutrient recovery by combining individual pathways and products and including a broader range of nutrients. To this end, finding ways to more effectively share and consolidate knowledge and information on recovery pathways and products would be beneficial. The present review aims to provide a template that aims to facilitate designing human excreta management for maximum nutrient recovery, and that can serve as foundation for organizing and categorizing information for more effective sharing and consolidation.

Keywords: phosphorus, nitrogen, potassium, carbon, organic matter, recovery, sewage, wastewater, urine, feces, black water, source-separation, fertilizer, soil amendment, resourceoriented sanitation

This chapter is published as: Harder, R., Wielemaker, R., Larsen, T. A., Zeeman, G. \& Öberg, G. 2019. Recycling nutrients contained in human excreta to agriculture: Pathways, processes, and products. Critical Reviews in Environmental Science and Technology, 1-49. 


\section{Introduction}

Growing concern about future fertilizer availability has re-emphasized the need for better nutrient management, including comprehensive recycling of nutrients contained in human excreta to agriculture (Elser and Bennett, 2009, Dawson and Hilton, 2011, McConville et al., 2015). Human excreta have a long history of being used as fertilizer and organic soil amendment but urbanization, the introduction of water closets and sewer networks, and the growing and nowadays widespread use of synthetic fertilizers has contributed to a significant departure from this practice (Rockefeller, 1998, Ferguson, 2014).

In urban areas in industrialized countries, water is used to convey human excreta through extensive sewer networks to municipal sewage treatment plants (STPs). Treatment renders a treated effluent, gaseous emissions, and a solid residual referred to as sewage sludge (in European regulations) or biosolids (in North American regulations). Land application of sewage sludge is a common practice in many countries and allows for partial recycling of nutrients to agriculture. The practice has been heavily debated for a long time, however, due to concerns about contaminants such as pathogens, organic pollutants, and heavy metals in the sludge (Petrik, 1954, Renner, 2000, McBride, 2003, Bengtsson and Tillman, 2004, Singh et al., 2017, Öberg and Mason-Renton, 2018). As a result, there is a trend towards incineration of a larger portion of the sludge (Kelessidis and Stasinakis, 2012, Kirchmann et al., 2017).

The adequacy and long-term sustainability of conventional urban water and sanitation systems has increasingly been called into question. For low-income countries, the high infrastructure costs are prohibitive for widespread adoption (Larsen et al., 2016). In the context of highincome countries, issues of concern include high energy and water demand, sludge disposal problems, and limited nutrient recycling (Brands, 2014). Some scholars hold on to the idea of municipal sewers and call for more comprehensive resource recovery at municipal STPs (Peccia and Westerhoff, 2015, Puyol et al., 2017). Other scholars hold that source separation and control provide greater opportunities for resource recovery, as it minimizes dilution and contamination of human excreta (Larsen and Gujer, 1997, Otterpohl et al., 1997, Wilsenach et al., 2003, Larsen et al., 2009a). Approaches based on source separation and control are commonly referred to as new, ecological, resource-oriented, source-separating, or decentralized sanitation or wastewater management.

Overall, significant research and development has taken place in recent decades to enable more comprehensive recovery of nutrients contained in human excreta. New knowledge and technologies are continuously unfolding, as evidenced by the number and scope of recent reviews published in the scientific literature, see Table 4.1. These reviews provide detailed insights into certain aspects of nutrient recovery. It is, however, challenging to identify broad patterns and opportunities in the field as a whole, when technical details or certain technologies are studied in isolation. 
Table 4.1 Examples of previous reviews on the recovery of nutrients found in human excreta and streams containing human excreta. These reviews have generally focused on a specific nutrient (notably phosphorus), recovery technology (e.g., membrane separation), input stream (e.g., urine), or a combination thereof. Note that this list is not exhaustive.

\begin{tabular}{|c|c|}
\hline Technology & Reference(s) \\
\hline Phosphorus recovery & $\begin{array}{l}\text { (Balmér, 2004, Cornel and Schaum, 2009, Petzet and Cornel, 2011, } \\
\text { Rittmann et al., 2011, Sartorius et al., 2012, Desmidt et al., 2015, } \\
\text { Egle et al., 2015, Karunanithi et al., 2015, Cieslik et al., 2015, Melia } \\
\text { et al., 2017) }\end{array}$ \\
\hline Struvite crystallisation & $\begin{array}{l}\text { (Doyle and Parsons, 2002, Le Corre et al., 2009, Rahman et al., } \\
\text { 2014, Kumar and Pal, 2015, Darwish et al., 2016) }\end{array}$ \\
\hline Membrane separation & (Lutchmiah et al., 2014, Xie et al., 2016, Ansari et al., 2017) \\
\hline Sorption & (Wang and Peng, 2010, Loganathan et al., 2014) \\
\hline Ecological sanitation & (Winker et al., 2009a, Haq and Cambridge, 2012, Roy, 2017) \\
\hline Biological systems & (Nancharaiah et al., 2016, Puyol et al., 2017) \\
\hline Phototrophic biomass growth & $\begin{array}{l}\text { (Hülsen et al., 2014, Sukačová and Červený, 2017, Abinandan et } \\
\text { al., 2018, Santos and Pires, 2018) }\end{array}$ \\
\hline Bioelectrochemical systems (BES) & $\begin{array}{l}\text { (Kelly and He, 2014, Rodríguez Arredondo et al., 2015, } \\
\text { Nancharaiah et al., 2016) }\end{array}$ \\
\hline BES applied to urine & (Ledezma et al., 2015) \\
\hline Nutrient recovery from urine & (Maurer et al., 2006b, Pronk and Koné, 2009) \\
\hline Nutrient recovery from digestate & (Monfet et al., 2017, Vaneeckhaute et al., 2013) \\
\hline Nutrient recovery from waste water & (Batstone et al., 2015, Mehta et al., 2015) \\
\hline $\begin{array}{l}\text { Nutrient recovery from sewage } \\
\text { sludge incineration ash (SSA) }\end{array}$ & (Donatello and Cheeseman, 2013) \\
\hline
\end{tabular}

The present review aims to provide a rigorously informed and systematic synthesis of available and proposed recovery pathways designed to facilitate recycling of nutrients contained in human excreta to agriculture, covering treatment processes as well as products rendered by treatment. Our aspiration is to present the material in a way that is accessible across diverse yet relevant fields of expertise. The focus is on highlighting broad patterns and opportunities in the field as a whole, and to point to literature that specifically describes certain selected aspects, technologies, or products in more detail.

Most importantly, we hope to facilitate communication and cross-fertilization not only among the various engineering groups that work on the recovery of nutrients found in human excreta, but also between these groups and research communities active in the fields of soil sciences and food and farming systems, as well as other related fields such as industrial ecology, urban metabolism, circular economy, and environmental systems analysis. 


\section{Human excreta}

As our intention is to write for a diverse audience, we start by providing a short description of human excreta and how they may get mixed with other streams prior to treatment. To clarify what it is that treatment aims at recovering or removing, we also describe factors that impact the composition of different streams that consist of, or contain human excreta, and can form the starting point for the recovery of resources contained in human excreta.

\subsection{Carbon and nutrient content of human urine and feces}

Human urine consists of more than $90 \%$ water $\left(\mathrm{H}_{2} \mathrm{O}\right)$ by weight, the remainder being inorganic salts and organic compounds (Rose et al., 2015b). The dried solids contain about 13\% carbon $(\mathrm{C}), 14-18 \%$ nitrogen $(\mathrm{N}), 3.7 \%$ phosphorus $(\mathrm{P})$, and 3.7\% potassium $(\mathrm{K})$ (Rose et al., 2015b). Urea $\left(\mathrm{CH}_{4} \mathrm{~N}_{2} \mathrm{O}\right)$ is the dominant solute in fresh urine, making up over $50 \%$ of the organic compounds (Rose et al., 2015b). About $85 \%$ of $\mathrm{N}$ is fixed in urea and about $5 \%$ as total ammonia $\left(\mathrm{NH}_{3}\right.$ and $\mathrm{NH}_{4}+$ ) (Udert et al., 2003b, Udert et al., 2006b). Shortly after urination, the nonvolatile urea is broken down into bicarbonate $\left(\mathrm{HCO}_{3}^{-}\right)$and carbonate $\left(\mathrm{CO}_{3}^{2-}\right)$ as well as nonvolatile ammonium $\left(\mathrm{NH}_{4}{ }^{+}\right)$and volatile ammonia $\left(\mathrm{NH}_{3}\right)$ (Udert et al., 2003b). Urea hydrolysis is a spontaneous process because the bacteria that produce the urea hydrolyzing enzyme urease are ubiquitous (Udert et al., 2003a). After urea hydrolysis, about $90 \%$ of total $\mathrm{N}$ in urine is present as ammonia or ammonium (Udert et al., 2006b). Urea hydrolysis implies the potential for ammonia volatilization during collection, storage, transport and application of urine, especially because the $\mathrm{pH}$ can increase up to 9 during the process, shifting the equilibrium from nonvolatile ammonium to volatile ammonia (Hellström et al., 1999, Chang et al., 2015). Human feces consist of about $75 \% \mathrm{H}_{2} \mathrm{O}$ by weight and $25 \%$ solid material, mainly organic matter (Rose et al., 2015b). C is a major constituent of the dried solids as approximately half of organic matter generally is C (Vassilev et al., 2010) and this is also true for feces (Rose et al., 2015b). N, P, and K make up 5.0-7.0\%, 3.0-5.4\%, and 1.0-2.5\% of the dried solids respectively (Rose et al., 2015b). Both urine and feces also contain a range of micronutrients such as magnesium $(\mathrm{Mg})$ and selenium $(\mathrm{Se})$. The amount of excreted nutrients depends on dietary intake, while the digestibility of the diet determines the partitioning of nutrients between urine (digested) and feces (undigested) (Jönsson et al., 2004). Generally, urine contains the majority of $\mathrm{N}$ and about half of $\mathrm{P}$ and $\mathrm{K}$ contained in human excreta, while feces are rich in $\mathrm{P}$ and $\mathrm{K}$ and contain the majority of C (Heinonen-Tanski and van Wijk-Sijbesma, 2005).

\subsection{Contaminants of concern in human urine and feces}

Human excreta commonly contain pathogens. Feces always contain high numbers of enteric bacteria (e.g., Campylobacter, Salmonella) and may also contain high numbers of viruses (e.g., Norovirus, Rotavirus), protozoa (e.g., Cryptosporidium, Giardia), and parasitic worm eggs (e.g., Ascaris) (Heinonen-Tanski and van Wijk-Sijbesma, 2005). Fresh urine, especially from healthy 
persons, contains few pathogens (Heinonen-Tanski and van Wijk-Sijbesma, 2005, Udert et al., 2006b). Fecal cross-contamination of urine during and after excretion, however, can increase the number of pathogens in urine (Jönsson et al., 1997, Schönning et al., 2002). Human excreta can also contain heavy metals and organic pollutants, notably pharmaceutically active substances such as pharmaceuticals, pharmaceutical residues, and (synthetic) hormones. Heavy metal concentrations in urine are generally very low in relation to the nutrients; feces constitute a much higher heavy metal load compared to urine (Jönsson et al., 1997, Tervahauta et al., 2014b). Some of the organic pollutants are mainly excreted with urine, while others are excreted mostly with feces (Lienert et al., 2007).

\begin{tabular}{|c|c|c|c|c|c|c|c|c|}
\hline \multirow{7}{*}{$\begin{array}{c}\text { HUMAN EXCRETA } \\
\text { STREAMS } \\
\text { CONSISTING OF } \\
\text { OR CONTAINING } \\
\text { HUMAN EXCRETA }\end{array}$} & $\begin{array}{l}\text { Faeces } \\
\text { Urine }\end{array}$ & \multirow{7}{*}{\multicolumn{2}{|c|}{ 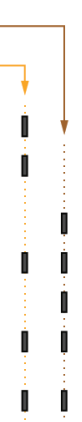 }} & \multirow{3}{*}{\multicolumn{4}{|c|}{$\begin{array}{l}\text { Flush and Anal Cleansing Water } \\
{\left[\begin{array}{c}\text { Domestic Used Water } \\
- \text { Industrial Used Water } \\
\quad \text { Stormwater }\end{array}\right.}\end{array}$}} & \multirow{4}{*}{$\begin{array}{c}\text { OTHER } \\
\text { STREAMS }\end{array}$} \\
\hline & $\begin{array}{l}\text { Urine } \\
\text { Yellowwater }\end{array}$ & & & & & & & \\
\hline & Faeces & & & & & & & \\
\hline & Excreta & & & & & & & \\
\hline & Brownwater & & & 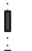 & & & Given stream is: & \\
\hline & Blackwater & & & i & & & I always - & part of given \\
\hline & Sewage & & & 【 & i & 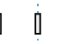 & 『 possibly - & primary input \\
\hline CONSTITUENTS OF & $\begin{array}{l}\text { Nutrients } \\
\text { Organic Matter }\end{array}$ & $\begin{array}{l}\oplus \Theta \\
+\epsilon\end{array}$ & & & & & $\begin{array}{l}\text { Given stream: } \\
+ \text { always } \\
\text { - possibly }\end{array}$ & $\begin{array}{l}\text { contains given } \\
\text { constituent }\end{array}$ \\
\hline $\begin{array}{l}\text { HUMIAN EXCREIA } \\
\text { AND OTHER STREAMS }\end{array}$ & $\begin{array}{l}\text { Pathogens } \\
\text { Heavy Metals } \\
\text { Organic Pollutants }\end{array}$ & 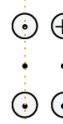 & & & & $\odot$ & $\begin{array}{l}\text { Constituent in giv } \\
\text { of main inter } \\
\text { in treatment }\end{array}$ & $\begin{array}{l}\text { n stream is: } \\
\text { t or concern } \\
\text { f primary input }\end{array}$ \\
\hline
\end{tabular}

Figure 4.1 Overview of: (1) how human excreta and other streams are combined into a range of primary inputs that form the starting point for recovery pathways reported in peer-reviewed studies dealing with recovery of resources from human excreta; and (2) constituents of interest of concern in human excreta and other streams. Note that used diapers are not considered in the present review, even though they also represent a stream containing human excreta.

\subsection{Mixing of human excreta with other streams}

Collection of human excreta often involves mixing with other streams (see Figure 4.1). Separate collection of urine, depending on the type of toilet or urinal, may involve mixing with flush water and the respective stream is commonly referred to as source-separated urine or yellow water. Separate collection of human feces, depending on the type of toilet, may involve mixing with urine, flush water, anal cleansing water, toilet paper, and additives such as ash, lime, or dried soil. The respective stream is commonly referred to as source-separated feces, brown 
water, excreta, or black water. In conventional urban water and sanitation infrastructures, human excreta generally become mixed with flush water, anal cleansing water, toilet paper, domestic used water, industrial used water, and possibly even storm water. The stream resulting from this form of collecting human excreta is commonly referred to as domestic or municipal sewage or wastewater.

\subsection{Sources of carbon, nutrients, and contaminants in mixed streams}

Human excreta normally are the major contributor of nutrients and organic matter in any of the streams containing human excreta, although the amount of nutrients and organic matter will increase if organic kitchen refuse is collected through the same collection system or added to treatment as supplemental feedstock (Kujawa-Roeleveld and Zeeman, 2006, Friedler et al., 2013b). Flush water can add heavy metals and organic pollutants originating from the water supply system, as for example copper $(\mathrm{Cu})$ and lead $(\mathrm{Pb})$ can be released from metal pipes (Renner, 2008, Schock et al., 2008) or organic compounds from polymeric pipes (Zhang and Liu, 2014). Contamination levels are further increased following mixing with used water from households, hospitals, industry, and the commercial sector, and with storm water where it is also discharged to the same sewer. Pathogens mainly originate from human excreta (Dumontet et al., 2001), but can also originate from meat preparation in domestic kitchens (e.g., Salmonella and Campylobacter during the preparation of chicken) (Cogan et al., 1999) or commercial processing of animal products (e.g., in tanneries, meat markets, abattoirs) (Dumontet et al., 2001). Organic pollutants include substances such as pharmaceutically active compounds and hormones, personal care products, and detergents (Kümmerer, 2013). Pharmaceutically active compounds and hormones mainly originate from human excreta whereas other organic pollutants mainly originate from other sources. Heavy metals originate from different sources (Sörme and Lagerkvist, 2002). Compared with other sources of heavy metals, those contained in human excreta typically account for less than a tenth of total load in sewage (Tervahauta et al., 2014b).

\section{Recovery pathways}

Efforts to recover resources from human excreta or streams containing human excreta have typically targeted water, energy, carbon, nutrients, metals, or a combination of these resources. Here, we compile and describe recovery pathways that facilitate nutrient recovery. Given the iterative nature of the literature search and analysis, information about what we found is presented along with how we found it.

\subsection{Conceptual model and terminology}

A simple input-output model (Figure 4.2) guided our literature analysis and is useful to explain central terms. We define as primary input any stream that contains human urine and/or feces 
and that forms the starting point for resource recovery. Treatment aims to facilitate recovery and recycling of resources found in the primary input. Where treatment comprises more than one treatment process, the output from one process can become the input to another. Products are defined as outputs that do not become the input to another treatment process. We refer to a specific sequence of treatment processes as a treatment train. A treatment train either transforms a primary input into one single product, or into a number of different products. The combination of a certain primary input, a certain treatment train, and a certain product we refer to as a recovery pathway. Where multiple products are obtained from the same primary input and treatment train, each product comprises a separate recovery pathway.

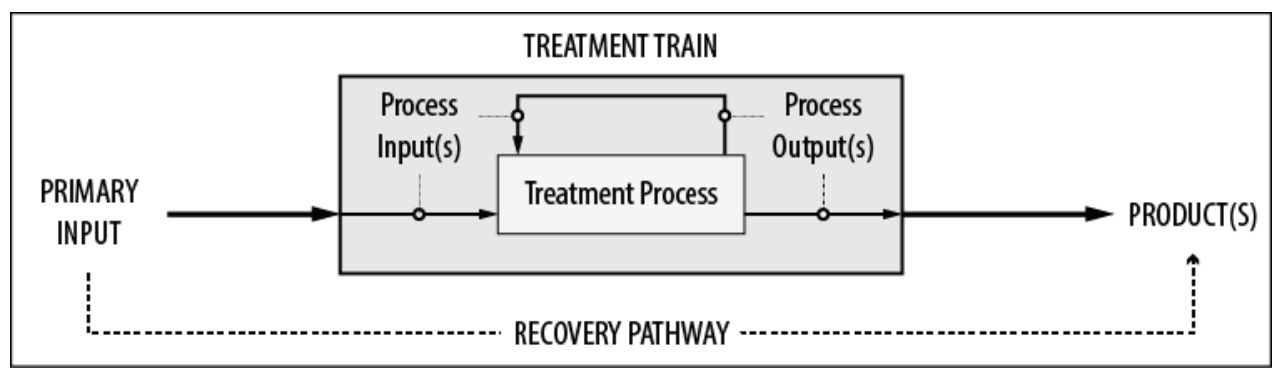

Figure 4.2 Simple input-output model that guided our analysis of peer-reviewed scientific studies dealing with resource recovery from human excreta and streams containing human excreta.

\subsection{Recovery pathways facilitating nutrient recovery}

We searched the scientific literature for documents describing recovery pathways specifically targeted towards nutrient recovery through the search strategies described in Supporting Information III. In doing so, we iteratively identified and developed categories for treatment processes and products rendered by treatment. Recovery pathways were arranged into clusters of pathways that start from similar primary inputs, feature similar treatment processes, and/or render similar products. These clusters as well as variations within clusters and a list of documents constituting each cluster are detailed in Supporting Information III. A simplified representation of common recovery pathways is shown in Figure 4.3 and explained in the remainder of this section.

Treatment trains starting from urine or yellow water represent two broad strategies. The first strategy applies treatment processes that aim at prevention of ammonia volatilization, separation of water from nutrients, and/or contaminant reduction (through separation of contaminants from nutrients and/or the pathogen inactivation and/or organic pollutant degradation). The second strategy is characterized by selective nutrient extraction. Treatment processes applied to this end often also imply volume reduction through separation of nutrients from water) and contaminant reduction (through separation of nutrients from contaminants) (Maurer et al., 2006b). 


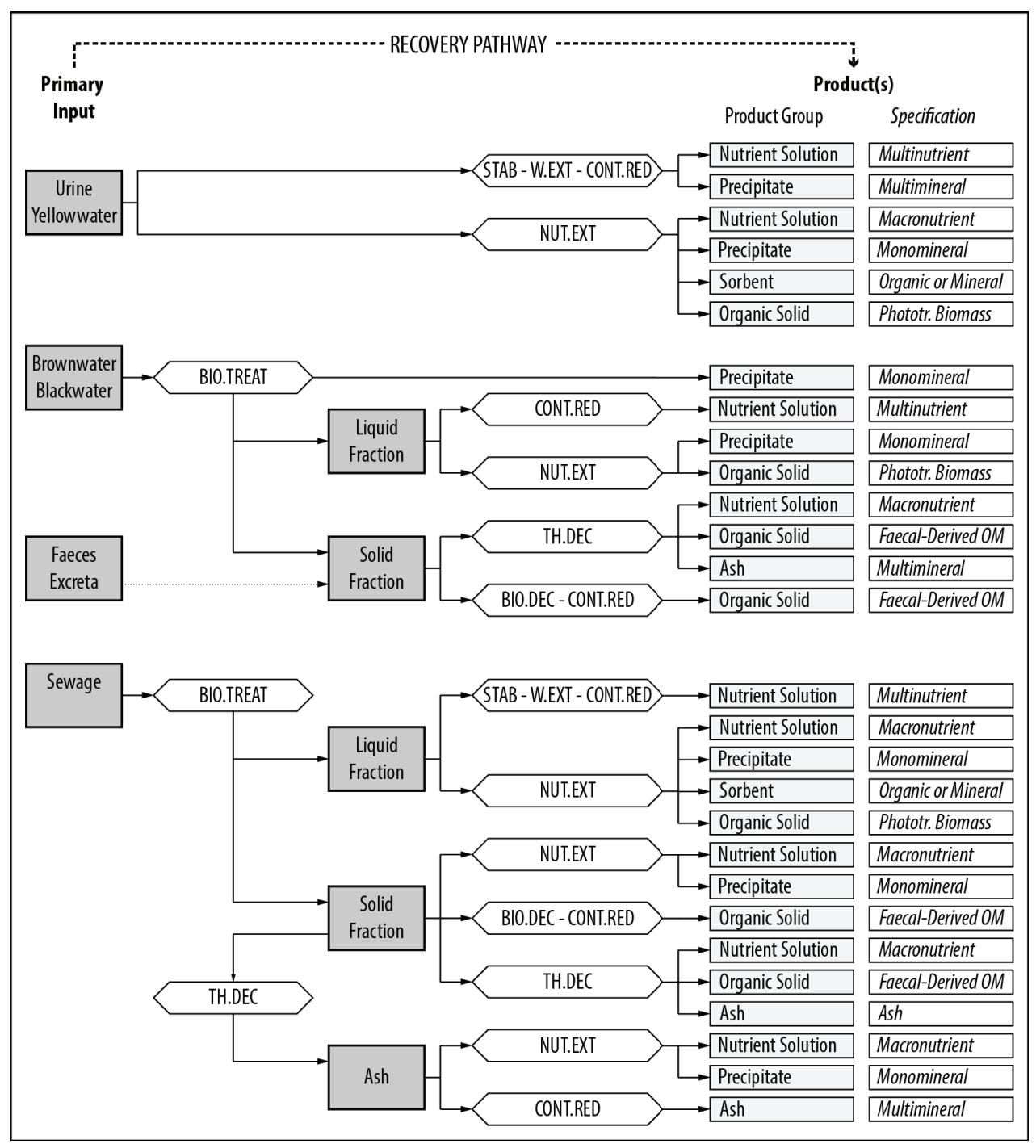

Figure 4.3 Simplified representation of recovery pathways targeted towards nutrient recovery from human excreta and streams containing human excreta, as reported in the peer-reviewed scientific studies included in the present review. Abbreviations: BIO-TREAT = biological treatment; BIO.DEC = biological decomposition; TH.DEC $=$ thermal decomposition; STAB = stabilization; W.EXT $=$ water extraction; CONT.RED $=$ contaminant reduction; NUT.EXT $=$ nutrient extraction. A more comprehensive representation can be found in Figure S3.1 in Supporting Information III. For further explanation of product groups and specifications the reader is referred to section 5 .

Treatment trains starting from brown water or blackwater commonly begin with (anaerobic) biological treatment followed by liquid-solid separation. Biological treatment can be designed such as to enable simultaneous nutrient extraction, for instance through precipitation or granulation. The liquid fraction can be the input to processes aimed at depollution (notably 
Figure 4.4 Option space for nutrient recovery. Note that the option space presented here can easily be extended to accommodate additional primary inputs, products, and treatment processes, in order to also map novel recovery pathways.

RECOVERY PATHWAY

\begin{tabular}{lll} 
& & TREATMENT TRAIN \\
\hline $\begin{array}{c}\text { Process } \\
\text { Input }\end{array}$ & Process Group & Process(es)
\end{tabular}

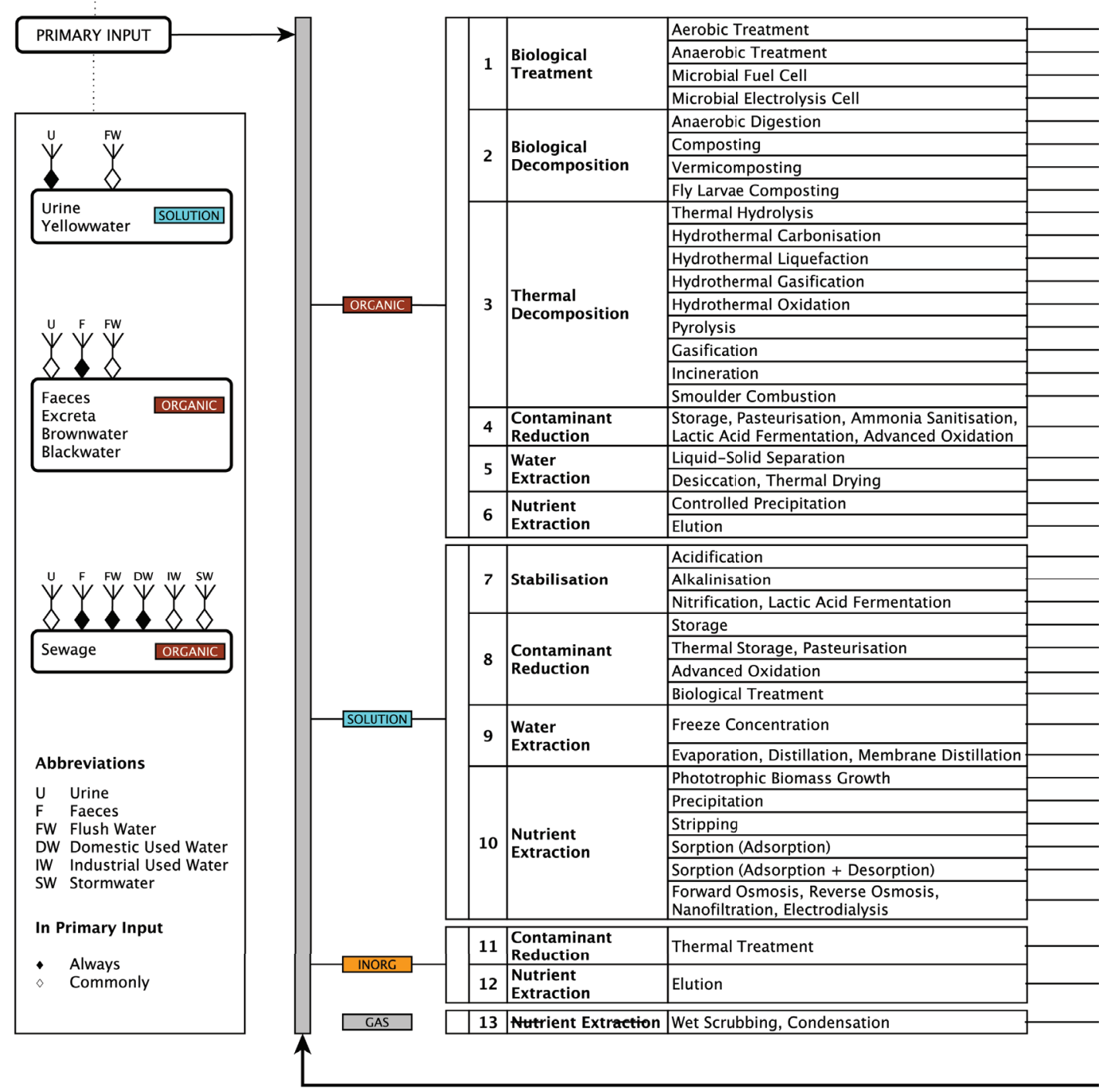




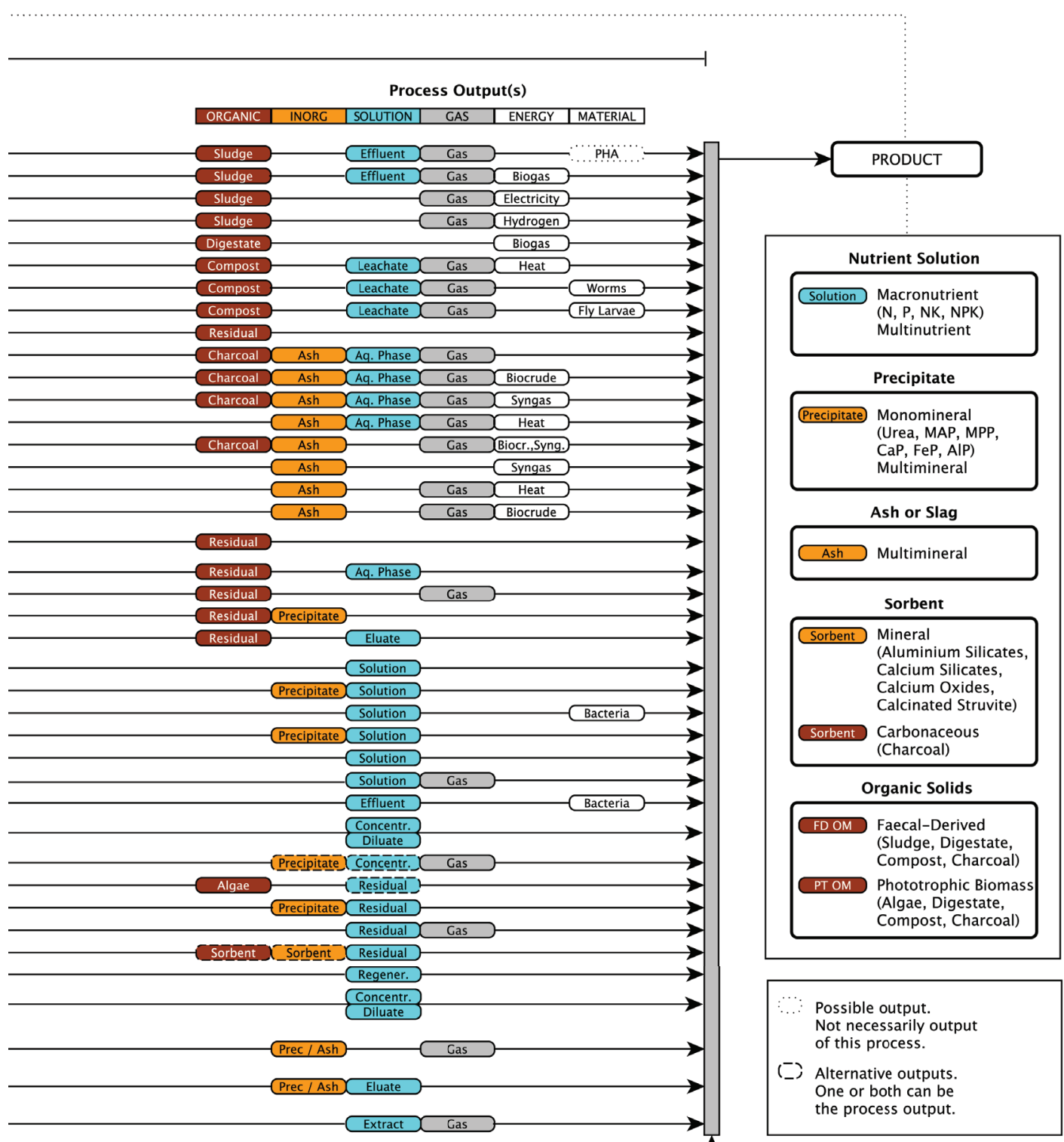

ITERATION: Process output becomes process input for additional process. SHORTCUT: Input directly becomes product without treatment. 
pathogen inactivation) or nutrient extraction. The solid fraction, or the dryer primary inputs feces and excreta, can be the input to depollution (notably pathogen inactivation), or to biological decomposition (possibly enhanced by additional pathogen inactivation) or thermal decomposition of organic matter.

Treatment trains starting from sewage commonly involve liquid-solid separation, usually preceded by or as a part of (aerobic) biological treatment. The liquid fraction or effluent can be the input to processes aimed at contaminant reduction or nutrient extraction. The solid fraction (sewage sludge) can be the input to processes aimed at contaminant reduction (pathogen inactivation), nutrient extraction, and/or biological or thermal decomposition of organic matter. Ash, the inorganic residual rendered by some thermal decomposition processes, can be the input to processes aimed at nutrient extraction or contaminant reduction (heavy metal removal).

Note that there are certain thermal decomposition processes that have generally been targeted towards recovering carbon in the form of energy carriers rather than nutrient recovery. Processes of this kind include hydrothermal carbonization (HTC) (Danso-Boateng et al., 2015a, DansoBoateng et al., 2013, Danso-Boateng et al., 2015b), hydrothermal gasification (HTG) (Afif et al., 2011, He et al., 2014), and gasification (Rong et al., 2015) with feces or sewage sludge as feedstock. We have included these processes because they can facilitate nutrient recovery, in principle.

\subsection{Developing the option space for nutrient recovery}

Mapping recovery pathways quickly becomes subject to redundancy, even in a simplified representation like Figure 4.3, because different primary inputs can be subjected to similar treatment trains, and different treatment trains can feature similar treatment processes and render similar products. Our aspiration was to produce a map of primary inputs, treatment processes, products rendered by treatment, and their relationships. This map we refer to as the 'option space' for nutrient recovery. To avoid redundancy, we identified four broad categories of similar process inputs and outputs (including primary inputs and products). We mapped these four categories of process inputs and outputs, indicating how treatment processes can convert an input belonging to one category to an output belonging to the same or a different category. This resulted in a refined input-output model that forms the backbone of the option space for nutrient recovery. The step-wise process leading to the option space is illustrated in Figure S4.1 in Supporting Information IV. The actual option space is shown in Figure 4.4. Note that the option space allows for the output from one process to become the input to a following process. The option space is a generic representation that can map any recovery pathway that builds on the primary inputs, treatment processes, and products featured as building blocks of the option space. Treatment processes are described in more detail in Section 4, products in Section 5. 


\section{Treatment processes}

A brief description of each treatment process featured in the option space, a simple input-output diagram, and details regarding the fate of constituents (nutrients, organic matter, pathogens, organic pollutants, and heavy metals) during treatment are provided in Supporting Information IV. Here, we summarize treatment processes and the fate of constituents during treatment.

\subsection{Decomposition of organic matter}

Decomposition refers to the breakdown of organic matter into smaller and more stable molecules, which can be achieved via biotic (biological) or abiotic (mechanical, thermal, chemical, or thermo-chemical) processes (Atay and Akbal, 2016). As for biotic processes, we here distinguish biological treatment and biological decomposition. Biological treatment refers to processes wh $\neg$ ere the solid retention time (SRT) is larger than the hydraulic retention time (HRT), that is, the solid fraction of the input stays in the system longer than the liquid fraction. Biological decomposition refers to processes where solid and liquid fraction stay in the system for the same period of time (SRT equals HRT).

\subsubsection{Biological treatment}

The activated sludge process, invented roughly a century ago, is still at the core of many contemporary municipal STPs and was originally designed to remove organic matter from municipal sewage or industrial wastewaters (Orhon, 2015). Over the years, biological $\mathrm{N}$ and biological or chemical $\mathrm{P}$ removal processes have been incorporated into overall process design to meet ever stricter effluent standards aimed at minimizing the release of $\mathrm{N}$ and $\mathrm{P}$ to the aquatic environment (Cooper, 2001b). In tropical climates, anaerobic treatment of sewage is a frequently applied alternative to the activated sludge process (Seghezzo et al., 1998). The upflow anaerobic sludge blanket (UASB) reactor, developed in the 1980s (Lettinga et al., 1980), is the most applied anaerobic system for treatment of sewage and industrial wastewaters. Also blackwater is succesfully treated applying UASB technology (de Graaff et al., 2010b, Hernández Leal et al., 2017). Bioelectrochemical systems such as microbial fuel cells (MFCs) and microbial electrolysis cells (MECs) represent an emerging technology for treating wastewater under anaerobic conditions and can be applied to urine, low-strength wastewater such as municipal sewage, as well as high-strength industrial wastewaters (Gude, 2016). While initially designed for efficient wastewater treatment, nutrient recovery has become an integral part of process design (Kelly and He, 2014, Nancharaiah et al., 2016, Goglio et al., 2019). More recently, treatment configurations that enable the formation of polyhydroxyalkanoates (PHA) or other precursors for high-value products have received increased attention (Morgan-Sagastume et al., 2014, Modin et al., 2016, Pittmann and Steinmetz, 2017, Puyol et al., 2017). The decomposition of organic matter releases nutrients from the organic matter to the liquid phase, adding to those nutrients already present in their dissolved form. Process designs based on the activated sludge process can 
achieve much lower concentrations of $\mathrm{N}$ and $\mathrm{P}$ in the effluent compared to anaerobic treatment (Seghezzo et al., 1998). Retaining nutrients in the effluent is beneficial if the effluent is used for fertigation in agriculture. Treatment under anaerobic conditions avoids consuming energy for aeration. Instead, energy is recovered in the form of biogas (in anaerobic treatment such as UASB) (Seghezzo et al., 1998), electricity (in microbial fuel cells) or biofuels such as ethanol, methane, or hydrogen (in microbial electrolysis cells). Pathogens tend to only partly accumulate in the sludge (Wen et al., 2009, Agudelo-Vera et al., 2012b, Li et al., 2015, Huang et al., 2018). In aerobic treatment, heavy metals partition fairly equally between effluent and sludge (Karvelas et al., 2003). In anaerobic treatment, heavy metal precipitation to the sludge is generally higher due to sulphide precipitation (Cowling et al., 1992, De la Varga et al., 2013). The partitioning behavior of organic pollutants depends on the compound, with a tendency towards sorbing to the sludge (Katsoyiannis and Samara, 2005). In addition, both aerobic and anaerobic treatment schemes have the potential to inactivate some pathogens and degrade some organic pollutants (Butkovskyi et al., 2016b). Bioelectrochemical systems in particular have been shown to have the potential for high removal of recalcitrant pollutants (Huang et al., 2011).

\subsubsection{Biological decomposition}

Anaerobic digestion enables recovery of energy in the form of biogas and nutrients in the form of digestate. Composting renders a soil amendment while vermicomposting and fly larvae composting render a soil amendment as well as worms or fly larvae for potential use as animal feed. These processes are commonly applied to more concentrated streams such as feces or excreta, or fecal, blackwater, or sewage sludge.

As in biological treatment, nutrients are released from organic matter upon its decomposition. If biological treatment takes place in an open system, as often the case for composting and usually the case for vermicomposting and fly larvae composting, volatile forms of $\mathrm{N}$ can be lost to the atmosphere, and soluble nutrients to a liquid leachate (Ulén, 1997, Jönsson et al., 2004, Lalander et al., 2014, Nigussie et al., 2016). Biological decomposition can inactivate some pathogens (especially when temperatures above $60^{\circ} \mathrm{C}$ are achieved) (Gajurel et al., 2007, Lalander et al., 2013), decompose some organic pollutants (Butkovskyi et al., 2016a), and influence heavy metal speciation (He et al., 2016).

\subsubsection{Thermal decomposition}

Thermal decomposition processes can be geared towards facilitating further treatment or safe disposal of an organic feedstock, but can also be designed to facilitate the recovery of resources such as energy, carbon, nutrients, and/or metals. Thermal hydrolysis and advanced oxidation processes (AOPs) such as ozonation aim to make wet organic matter, usually sewage sludge, more biodegradable and are commonly applied as pre-treatment to anaerobic digestion (Barber, 2016). Hydrothermal carbonization (HTC) (Danso-Boateng et al., 2015a, Danso-Boateng et al., 
2013, Danso-Boateng et al., 2015b), liquefaction (HTL) (Aida et al., 2016, Lu et al., 2017), and gasification (HTG) (Afif et al., 2011, He et al., 2014) aim to convert wet organic matter into charcoal, biocrude, or syngas, respectively. Hydrothermal oxidation (HTO) processes such as low pressure wet oxidation (LOPROX) (Blöcher et al., 2012) and supercritical water oxidation (SCWO) (Stendahl and Jäfverström, 2004) aim at complete destruction and conversion of wet organic matter to carbon dioxide. Pyrolysis (Bridle and Pritchard, 2004, Shepherd et al., 2016, Bai et al., 2017) aims to convert dry organic matter into charcoal and/or biocrude. Gasification (Rong et al., 2015) aims to convert dry organic matter into syngas. Incineration (Li et al., 2017) usually involves complete decomposition of organic matter by means of oxidation to carbon dioxide. Smoulder combustion (Yermán et al., 2015, Fabris et al., 2017) also aims at complete decomposition of organic matter but can be designed to yield pyrolysis products such as biocrude. Pyrolysis and HTL have been investigated with recovery of both nutrients and energy in mind, while the other processes have typically been targeted primarily towards recovering carbon in the form of charcoal or energy carriers.

Hydrothermal processes (i.e., HTC, HTL, HTG, and HTO) generally yield an inorganic residual in addition to the target product(s). This residual generally consists of a liquid fraction and a solid fraction, which can be separated from one another by means of liquid-solid separation. Nutrients are partitioned to the carbonaceous target product as well as the inorganic (liquid or solid) residual (Stendahl and Jäfverström, 2004, Blöcher et al., 2012, Kruse et al., 2016, Yao et al., 2016, Lu et al., 2017). Monovalent ions (e.g., $\mathrm{NH}_{4}{ }^{+}$and $\mathrm{K}^{+}$) tend to partition to the liquid fraction of the inorganic residual, multivalent ions (e.g., $\mathrm{PO}_{4}{ }^{3-}$ and most metal ions) to the solid fraction (Toufiq Reza et al., 2016). Pyrolysis, gasification, and incineration are subject to $\mathrm{N}$ volatilization, while $\mathrm{P}$ and $\mathrm{K}$ as well as most metals are retained in the char or ashes, respectively (Bridle and Pritchard, 2004, Hossain et al., 2011, Gorazda et al., 2017). Pathogens are generally fully inactivated while organic pollutants are partly or fully decomposed depending on process conditions and type of compound (Libra et al., 2011).

\subsection{Stabilisation processes}

Stabilization of urine and other liquid streams such as treated effluent is specifically directed to prevent volatilization of ammonia as this can help avoid $\mathrm{N}$ losses and negative impacts associated with released ammonia gas such as odour nuisance (Hellström et al., 1999) and acidification of soils and water bodies (Hunter et al., 2011). Stabilization of urea-rich solutions (e.g., fresh urine) aims at preventing urea hydrolysis and hence preserving $\mathrm{N}$ in the form of non-volatile urea. Stabilization of ammonia-rich solutions (e.g., urine after urea hydrolysis) aims at converting volatile ammonia to ammonium and other non-volatile forms of $\mathrm{N}$. While stabilization can be applied as standalone process, it is typically applied in combination with other processes such as storage (e.g., Hellström et al., 1999), or as pre-treatment to other processes, notably evaporation (e.g., Senecal and Vinnerås, 2017), distillation (e.g., Fumasoli et al., 2016), membrane distillation 
(e.g., Tun et al., 2016) and phototrophic biomass growth (e.g., Coppens et al., 2016). Processes geared towards stabilization of liquid streams that have gained most traction include chemical processes such as acidification (e.g., Hellström et al., 1999) and alkalinization (e.g., Randall et al., 2016) as well as biological processes such as partial nitrification (e.g., Sun et al., 2012) and lactic acid fermentation (e.g., Andreev et al., 2017). Stabilization processes generally have some potential to inactivate some pathogens (Hellström et al., 1999, Bischel et al., 2015, Randall et al., 2016). Biological processes in addition also have the potential to degrade some organic pollutants (Fumasoli et al., 2016, Andreev et al., 2017).

\subsection{Separation processes}

The main purpose of separation processes is to separate various constituents in the process input from one another. Several treatment processes have been investigated for liquid streams such as urine or treated effluent, aiming at separating water and/or contaminants from nutrients, or nutrients from water and/or contaminants.

\subsubsection{Freeze concentration}

Processes geared towards water extraction from liquid streams include freeze concentration, that is, the concentration of a solution through freezing and melting. Freeze concentration has the potential to retain most nutrients in the concentrate (Lind et al., 2000, Gulyas et al., 2004).

\subsubsection{Vaporization and membrane separation}

Processes based on vaporization and/or membrane separation include passive evaporation (e.g., Pahore et al., 2010; Bethune et al., 2014, 2016; Dutta and Vinnerås, 2016), thermal (e.g., Ek et al., 2006; Senecal and Vinnerås, 2017) or solar thermal evaporation (e.g., Antonini et al., 2012), high temperature (Jiang et al., 2017a) or low pressure distillation (e.g., Udert and Wächter, 2012; Fumasoli et al., 2016), membrane distillation (MD) (e.g., Tun et al., 2016), forward osmosis (FO) (e.g., Ek et al., 2006; Zhang et al., 2014; Liu et al., 2016), reverse osmosis (RO) (e.g., Ek et al., 2006), and nanofiltration (NF) (e.g., Pronk et al., 2006b; Lazarova and Spendlingwimmer, 2008). These processes can enable the separation of water from nutrients in liquids as well as liquidsolid separation in slurries that also contain particulate organic matter or minerals. In thermal drying of sewage sludge (e.g., Horttanainen et al., 2017), for example, water is extracted from a slurry through evaporation. Separation of the liquid and solid fractions can also be achieved through membrane separation processes such as FO, RO and NF, as well as a number of other processes such as centrifugation or sedimentation.

$\mathrm{N}$ losses can occur due to ammonia volatilization during vaporization (Bethune et al., 2016; Dutta and Vinnerås, 2016; Tun et al., 2016; Jiang et al., 2017b), and due to low rejection of urea, ammonia, nitrite and nitrate in membrane separation (Ek et al., 2006; Pronk et al., 2006b; Zhang et al., 2014). Pathogens and heavy metals are generally retained in the concentrate, both in case 
of vaporization and membrane separation (Pronk et al., 2006b; Liu et al., 2016). The fate of organic pollutants depends on their volatility during vaporization (Wijekoon et al., 2014), and upon membrane and pollutant properties during membrane separation (Alturki et al., 2013). Electrodialysis (ED) is another membrane separation process that has been investigated and enables the transfer of nutrients from a liquid stream (such as urine) or the liquid fraction of a slurry (such as sewage sludge) to another liquid stream (Pronk et al., 2006a, 2007; Tice and Kim, 2014). Desalination degrees of up to $99 \%$ have been achieved (Pronk, Biebow, et al., 2006). Retention is high for pathogens while organic pollutants and heavy metals permeate through the membrane to some extent (Pronk et al., 2006a, 2007).

\subsubsection{Phototrophic biomass growth}

Through phototrophic biomass growth in aquatic or terrestrial systems, nutrients can be extracted from liquid streams, notably urine and treated effluent, and incorporated into phototrophic biomass. Algal systems have been found to have the potential to simultaneously extract $\mathrm{N}$ and P (as well as K and micronutrients) (Shilton et al., 2012; Vasconcelos Fernandes et al., 2015; Sukačová and Červený, 2017), but have also been found to extract organic pollutants (de Wilt et al., 2016) or heavy metals (Zeraatkar et al., 2016; Demey et al., 2018) through sorption. Moreover, algae were found to have the potential to degrade some organic pollutants (de Wilt et al., 2016; Wang et al., 2017).

\subsubsection{Sorption}

Sorption processes have been investigated to transfer nutrients from liquid streams (notably urine and treated effluent) to a range of carbonaceous or mineral sorbents. The sorbent itself can be the target product, or it can be an intermediary nutrient carrier from which nutrients can be transferred back to a desorption solution or regenerant. In the latter case, also synthetic sorbents/resins have been investigated.

Charcoal has been shown to have the potential to adsorb urea (Kameda et al., 2017), $\mathrm{NH}_{4}{ }^{+}$(Cai, Qi, Liu, and He, 2016), and $\mathrm{PO}_{4}{ }^{3-}$ (Takaya et al., 2016; Trazzi et al., 2016). Mineral sorbents generally have good cation exchange properties and good affinity for $\mathrm{NH}_{4}{ }^{+}$and $\mathrm{K}^{+}$(Hedström, 2006; Jaskūnas, 2015), and have also been shown to act as precipitation nuclei for the surface precipitation of phosphates, for instance as calcium phosphate, notably if $\mathrm{Ca}_{2}{ }^{+}$is released in exchange for $\mathrm{NH}_{4}{ }^{+}$and $\mathrm{K}^{+}$(Hedström, 2006; Gustafsson et al., 2008; Karapinar, 2009; Köse and Kivanç, 2011; Guaya et al., 2016; Wan et al., 2017). Charcoal and mineral sorbents do not only remove nutrients from aqueous solutions. Charcoal, notably in the form of activated carbon, has the potential to remove some waterborne pathogens (Busscher et al., 2006), organic pollutants (Nam et al., 2014; Tong et al., 2016), and heavy metals (Kołodyńska et al., 2012) from solutions. Mineral sorbents have the potential to remove organic pollutants (Tsai et al., 2008; De Ridder et al., 2012; Chraibi et al., 2016) and heavy metals (Zorpas et al., 2000; Babel, 2003; 
Shaheen et al., 2012; Choi and Lee, 2015) from solutions. There are indications, however, that sorbents (e.g., some types of activated carbon) can be designed to remove either nutrients or pollutants but not both.

\subsubsection{Controlled precipitation}

Through precipitation, crystallization, or granulation, nutrients can be transferred from a liquid stream or the liquid fraction of a slurry to a mineral in amorphous or crystalline form. Precipitation in the $\mathrm{Mg}-\mathrm{Ca}-\mathrm{NH}_{3}-\mathrm{PO}_{4}$ system has been explored thoroughly (Ronteltap et al., 2007a, 2010; Marti et al., 2008; Triger et al., 2012; Liu et al., 2013; Muster et al., 2013; Vasenko and $\mathrm{Qu}, 2017)$. In the absence of ammonium, it is possible to precipitate the struvite analogue magnesium potassium phosphate (MPP, also referred to as potassium struvite) (Wilsenach et al., 2007; Xu et al., 2012b, 2015; Nakao et al., 2017). Other studies have targeted calcium phosphate (Cunha et al., 2018) aluminium phosphate (Huang et al., 2015) ferric phosphate (Lin et al., 2017), or magnesium and sodium phosphates (Huang et al., 2015). Key mineral precipitates are described in Table S4.1 in Supporting Information IV. Pathogens may accumulate in the precipitate (Udert et al., 2006; Decrey et al., 2011; Lahr et al., 2016). Pharmaceuticals have been found to attach to the surface of precipitates rather than being incorporated in the crystal structure (Escher et al., 2006; Ronteltap et al., 2007b) and can be removed by washing (Schürmann et al., 2012).

\subsubsection{Ammonia volatilization and capture}

The transfer of volatile components from a liquid to a gas stream is referred to as stripping and has long been known to be useful to remove ammonia from concentrated streams such as urine (e.g., Liu et al., 2015, Maurer et al., 2003c) or ammonia-rich wastewater (e.g., Siegrist, 1996, Yuan et al., 2016). Process variations include stripping columns (e.g., Antonini et al., 2011, Huang et al., 2015, Katehis et al., 1998, Morales et al., 2013) as well as a range of setups where ammonia release is facilitated by (bio)electrochemical systems such as microbial fuel cells (e.g., Kuntke et al., 2012, Zhou et al., 2015), microbial electrolysis cells (e.g., Kuntke et al., 2014, Wu and Modin, 2013), or electrochemical cells (e.g., Desloover et al., 2012, Luther et al., 2015). Ammonia release from a liquid to a gas stream can also occur as side-effect of water extraction processes and in these cases often turns into a nitrogen loss, although the released ammonia can be captured, in principle (e.g., Horttanainen et al., 2017). Ammonia is commonly captured by wet scrubbing (i.e., absorbtion in an acid such as sulphuric acid) which renders an ammonia-rich solution (for example ammonium sulphate). Pathogens, organic pollutants, and heavy metals can be expected to largely remain in the stream from which ammonia has been released. 


\subsubsection{Mobilization - separation}

Prior to liquid-solid separation of slurry-like organics, release of $\mathrm{P}$ from the solid to the liquid fraction can be facilitated by processes such as bioelectrochemical systems (e.g., Fischer et al., 2011, Happe et al., 2016), ozonation (e.g., Suzuki et al., 2006), additional anaerobic tanks or zones in enhanced biological phosphorus removal (EBPR) schemes (e.g., Heinzmann, 2005), or acid elution (e.g., Antakyali et al., 2013, Güney et al., 2008, Niewersch et al., 2008). Elution has also been investigated for the extraction of $\mathrm{P}$ from ashes. Alkaline elution dissolves phosphorus and aluminium to some extent but not iron and heavy metals; acid elution dissolves phosphorus as well as metals. Elution is often followed by processes such as membrane separation, sorption, or solvent extraction in order to separate P from heavy metals, and possibly by processes aiming at the precipitation of phosphates (Egle et al., 2015).

\subsubsection{Thermal ash treatment}

Separation of $\mathrm{P}$ and heavy metals found in ashes can be achieved through thermal ash treatment designed to release heavy metals or P from the ash, or both (sequentially) (e.g., Adam et al., 2009, Nowak et al., 2012, Schönberg et al., 2014).

\subsection{Pathogen inactivation and degradation of organic pollutants}

Pathogen inactivation and removal aim to ensure hygienic safety of the fertilizer product while organic pollutant degradation and removal target pharmaceuticals, pharmaceutical residues, and (synthetic) hormones. Some of the separation processes above have aimed to separate desired products (i.e., nutrients, organic matter) from those not desired (i.e., pathogens, organic pollutants, heavy metals). Treatment can also be geared towards the inactivation of pathogens or degradation of organic pollutants. Storage (e.g., Tilley et al., 2008), thermal storage (e.g., Zhou et al., 2017), and pasteurization (e.g., Lahr et al., 2016) are processes that focus on pathogen inactivation in liquid streams. These processses need concentrated streams to limit storage capacity and/or energy use. Advanced oxidation processes (Lazarova and Spendlingwimmer, 2008, Pronk et al., 2007) have the potential to achieve inactivation of pathogens (Deng and Zhao, 2015, Giannakis et al., 2017) as well as degradation of organic pollutants (Deng and Zhao, 2015). Biological treatment of liquid streams such as urine has been targeted mainly towards the degradation of organic pollutants (e.g., Abdel-Shafy and Mansour, 2016). Pathogen inactivation in slurry-like organic matter has been investigated by means of storage (e.g.Fidjeland et al., 2013), pasteurization (e.g., Forbis-Stokes et al., 2016), ammonia sanitization (e.g., Fidjeland et al., 2015)(e.g., Fidjeland et al., 2015), lime stabilization (e.g., Anderson et al., 2015), and desiccation (e.g., Magri et al., 2015). 


\section{Products}

Products rendered by treatment of human excreta and streams containing human excreta can be in the form of for example energy carriers, fertilizers, or feed, while in other cases the products can be utilized for the production of biopolymers, biofuels or other high-value chemicals (Chen et al., 2018, Puyol et al., 2017). Here, we focus on products that are useful as fertilizers or for the production of synthetic fertilizers. The composition and quality of these products vary widely within the same product category. This is in part because the quality of the primary input varies between locations as a result of for example the type of industries or habits of the population, in part because different treatment trains can yield similar products (see Figure 4.3 and Figure S4.2 in Supporting Information IV). Below we describe different products with respect to composition and usability (Figure 4.5).

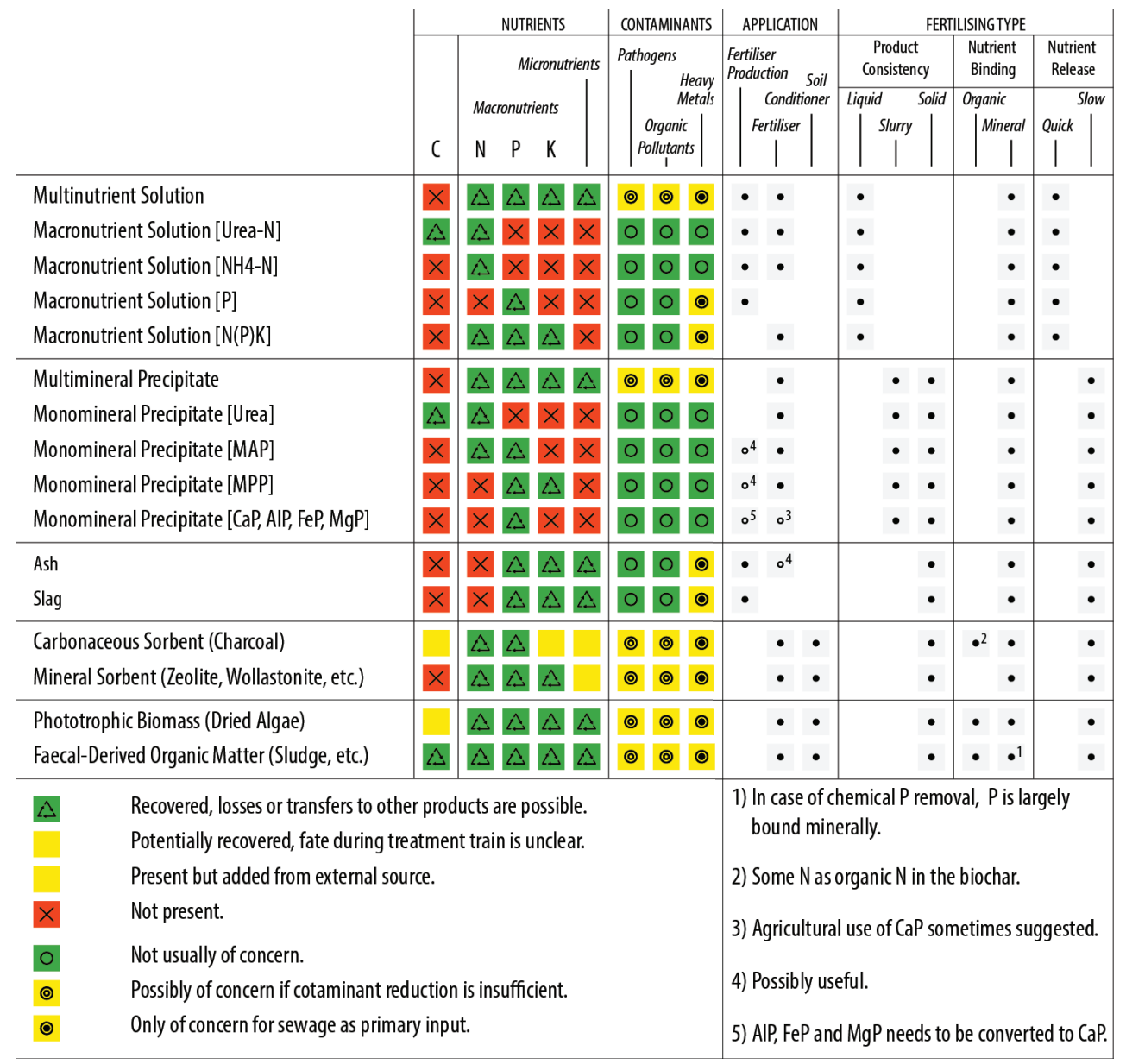

Figure 4.5: Characteristics, application potential, and fertilizing type of different product subcategories. 


\subsection{Nutrient solutions}

Excreta-derived nutrient solutions contain nutrients derived from human excreta but are devoid of suspended organic matter. Given the wide variety of combinations of primary inputs and treatment trains that render nutrient solutions, there is considerable variation within this product category. If used as fertilizers, nutrient solutions generally are considered quick-release fertilizers as the nutrients are present as dissolved ionic species and thus directly available for plant uptake. Alternatively, some of the nutrient solutions can be used as input for the production of fertilizers. Broadly, nutrient solutions fall into two subgroups we refer to as multinutrient and macronutrient solutions. The term multinutrient solution here is used to refer to nutrient solutions that generally contain both macro- and micronutrients. The term macronutrient solution here is used to refer to nutrient solutions that contain one or several of the macronutrients NPK but no or only traces of micronutrients.

\subsubsection{Multinutrient solutions}

One set of recovery pathways that has received considerable attention is based on treatment trains starting from urine or yellow water and featuring (a combination of) stabilization, contaminant reduction, and water extraction processes, or nutrient extraction processes; but the same (combinations of) processes have also been applied to other liquid streams, notably treated effluent and liquid process side streams rendered during treatment of primary inputs that contain feces. Human urine has long been known for its usefulness as fertilizer particularly rich in $\mathrm{N}$ (and that also contains $\mathrm{P}, \mathrm{K}$ and micronutrients) (Heinonen-Tanski and van Wijk-Sijbesma, 2005). Urine-based liquid fertilizers can be expected to be similarly useful (e.g., Bonvin et al., 2015). Aurin is an example of a marketable urine-based liquid fertilizer that is obtained through nitrification-distillation of hydrolyzed urine (Eawag, 2018a). Human urine has also been shown to be useful as liquid fertilizer for aquaculture (e.g., Jana et al., 2012, Rana et al., 2017), and has been investigated as input for the production of methylene urea, a slow-release synthetic nitrogen fertilizer (e.g., Ito et al., 2013). Treated effluent is useful for fertigation.

\subsubsection{Macronutrient solutions (urea-N)}

Solutions rich in urea-N have been obtained, starting from unhydrolyzed urine, through sorption to and desorption from activated carbon (e.g., Ganesapillai et al., 2016, Simha et al., 2016), and through membrane separation processes, notably nanofiltration (e.g., Lazarova and Spendlingwimmer, 2008, Pronk et al., 2006). In membrane separation, pathogens and organic pollutants are retained by the membrane (Pronk et al., 2006) and heavy metals are not of concern for urine as primary input. For sorption, the fate of contaminants is less reported. Also solutions rich in urea-N can be expected to be useful as fertilizer rich in nitrogen (Pronk et al., 2006) and possibly as feedstock for the production of synthetic fertilizers such as methylene urea. 


\subsubsection{Macronutrient solutions (ammonia-N)}

Solutions rich in ammonia- $\mathrm{N}$ have been obtained, starting from hydrolyzed urine, through membrane separation, notably nanofiltration e.g.(e.g., Lazarova and Spendlingwimmer, 2008, Pronk et al., 2006). A more widely researched approach to obtaining a solution rich in ammonia- $\mathrm{N}$ is the release of ammonia from liquid streams (e.g., through air stripping from urine or treated effluent) (e.g., Desloover et al., 2012, Luther et al., 2015) or organics (e.g., during thermal drying of sewage sludge) (e.g., Horttanainen et al., 2017) followed by absorption in an acid trap. Depending on the acid trap used, the respective product is ammonium sulfate $\left(\left(\mathrm{NH}_{4}\right)_{2} \mathrm{SO}_{4}\right)$ (e.g., Desloover et al., 2012), ammonium borate $\left(\left(\mathrm{NH}_{4}\right)_{3} \mathrm{BO}_{3}\right)$ (e.g., Kuntke et al., 2014), ammonium chloride $\left(\mathrm{NH}_{4} \mathrm{Cl}\right)$ (e.g., Wu and Modin, 2013), ammonium nitrate $\left(\mathrm{NH}_{4} \mathrm{NO}_{3}\right)$ (e.g., Horttanainen et al., 2017), or diammonium phosphate $\left(\left(\mathrm{NH}_{4}\right)_{2} \mathrm{HPO}_{4}\right)$ (e.g., Licon Bernal et al., 2016). These products are generally free of pathogens, organic pollutants, and heavy metals. Yet other studies have used sorption followed by desorption to render ammonia water, starting from urine (e.g., Tarpeh et al., 2017) or treated effluent (e.g., Sancho et al., 2017, You et al., 2017). The fate of contaminants is less reported. Ammonium nitrate and ammonium sulfate are common fertilizer products applied for instance in combination with CULTAN (controlled uptake long-term ammonia nutrition) fertilization (Deppe et al., 2016). Ammonium nitrate is also a common ingredient in the production of synthetic fertilizers.

\subsubsection{Macronutrient solutions (NK or NPK)}

Sorption followed by desorption has also been investigated for the simultaneous recovery of $\mathrm{NH}_{4}{ }^{+}$and $\mathrm{K}^{+}$from urine and their separation from $\mathrm{Na}^{+}$(e.g., Casadellà et al., 2016). If two or more sorbent materials are combined, sorption can also render a solution rich in in $\mathrm{N}, \mathrm{P}$ and $\mathrm{K}$. A less explored pathway to render a solution rich in N, P an K is HTL of wet organic matter such as feces (e.g., Lu et al., 2017). HTL transfers N, P, and K to a liquid residue while most metals (e.g., Ca, Mg, Zn, Al, Fe) are transferred to a solid residue (Lu et al., 2017). While the respective studies do not point towards a specific end use, it seems likely the macronutrient solutions would be useful as liquid fertilizers.

\subsubsection{Macronutrient solutions $(P)$}

Solutions rich in $\mathrm{P}$ have been obtained through a broad range of treatment trains designed to extract P from organics (e.g., sewage sludge) or inorganics (e.g., sewage sludge ash) where sewage is the primary input, as reviewed extensively in (Egle et al., 2015). These treatment trains generally yield a phosphoric acid, ranging from rather diluted to very pure and concentrated. Pathogens and organic pollutants are not usually of concern. Depending on the treatment train, heavy metals can be of concern, but several efforts are under way to reduce heavy metal contamination by subsequent processes such as membrane separation (e.g., Parés Viader et al., 2017, Schaum et al., 2007), sorption (e.g., Xu et al., 2012), or solvent extraction (e.g., Hong et al., 2005). Another 
way to obtain a solution rich in $\mathrm{P}$ is through adsorption from treated effluent or other liquid process side-streams followed by desorption (e.g., Ohura et al., 2011). Phosphoric acid is rarely used for direct agricultural application but is instead commonly used in the production of synthetic fertilizers. P-rich desorption solutions seem to be most useful as starting point for the precipitation of phosphate minerals such as struvite (e.g., O'Neal and Boyer, 2013).

\subsection{Precipitates}

Precipitates are rendered by a wide range of processes, either as non-target (e.g., during storage) or target product (e.g., in crystallization reactors). Common precipitates include struvite (MAP) and potassium struvite (MPP) as well as calcium, aluminium, and iron phosphates. Precipitates range from slurries consisting of individual precipitated nuclei, which can be filtered and dried to obtain a powder, to larger crystals or granules. Broadly, precipitates fall into two subgroups we refer to as multimineral and monomineral precipitates. The term multimineral precipitate here refers to precipitates that contain a range of different minerals. The term monomineral precipitate here refers to precipitates that only contain one mineral, or at least where only one mineral is the target mineral.

\subsubsection{Multimineral precipitates}

Treatment trains involving dehydration of urine, for instance, usually yield an inhomogeneous slurry or powder containing most of the nutrients found in the original solution, incorporated into a broad range of minerals (Antonini et al., 2012, Bethune et al., 2016, Jiang et al., 2017); where applicable also minerals originating from drying agents such as ash or lime (Dutta and Vinnerås, 2016, Senecal and Vinnerås, 2017). Multimineral precipitates have also been obtained through dehydration of anaerobic digester liquor (Ek et al., 2006). These multimineral precipitates are commonly held to be directly useful as fertilizers (Lemming et al., 2017), although their usefulness can be hampered by high salt contents (Jiang et al., 2017).

\subsubsection{Monomineral precipitates}

Spontaneous precipitation of MAP or calcium phosphate $(\mathrm{CaP})$ is a common phenomenon in urine collection systems (Tilley et al., 2008, Udert et al., 2003a) and pipes returning anaerobic digester supernatant to the STP inflow. Treatment trains that induce precipitation through $\mathrm{pH}$ adjustment and/or the addition of metal ions most commonly target MAP or CaP (notably hydroxylapatite) (Melia et al., 2017), but co-precipitation of a wide variety of non-target minerals may occur (Muster et al., 2013). As amorphous precipitates may easily be overlooked, what is believed to be mostly struvite may in fact contain more other precipitates than thought, particularly in higher $\mathrm{pH}$ ranges (Hao et al., 2008). Other precipitates that have been targeted include MPP, $\mathrm{AlP}, \mathrm{FeP}$, and MgP. Slurries and powders tend to be less homogeneous and more prone to contain non-target minerals as well as pathogens, organic pollutants, and heavy metals. Crystals 
and granules can have a very high purity and homogeneity, and their quality is rather independent of the primary input and treatment train (Antonini et al., 2012); even if sewage is the primary input (and e.g., anaerobic digester supernatant the process input), heavy metal concentrations are generally lower than in commercial fertilizers (Krüger et al., 2016). The usefulness of struvite as slow-release fertilizer has long been known (Bridger et al., 1962, Degryse et al., 2017, Johnston and Richards, 2003, Talboys et al., 2016). Marketed struvite pellets include Ostara Crystal Green (Ostara, 2018) and Berliner Pflanze (Wasserbetriebe, 2018). Calcium phosphate in the form of hydroxylapatite, and to a lesser extent also aluminium and ferric phosphate are held to be more useful to produce synthetic fertilizers (Melia et al., 2017).

\subsection{Ashes and slags}

Ashes and slags are rendered by thermal decomposition of organic matter. Ashes and slags contain non-volatile nutrients and heavy metals incorporated in a range of minerals. These minerals are not normally further specified in the respective studies. Ashes and slags are free of pathogens and organic pollutants but heavy metals can be of concern, notably for primary inputs with higher heavy metal loads, such as sewage. Several treatment processes are under development that aim to separate P from heavy metals contained in ashes or slags, and render a decontaminated ash or slag, as described in detail in (Egle et al., 2015). Ashes and slags generally are not considered a product of direct use for agriculture unless subjected to additional treatment (Lemming et al., 2017, Melia et al., 2017). Thermo-chemical ash treatment, for example, has been shown to increase the bioavailability of $\mathrm{P}$ in the ash, making the product (calcined ash) potentially useful for direct agricultural application (Adam et al., 2008, Herzel et al., 2016). More commonly, however, ashes and slags are the starting point for the recovery of fertilizer products such as struvite or the production of synthetic fertilizers (Cabeza et al., 2011).

\subsection{Sorbents}

A wide range of sorbents has been investigated to extract one or several of the macronutrients NPK from liquid streams. Sorbents can be broadly divided into two subgroups: carbonaceous and mineral sorbents. The main carbonaceous sorbent is charcoal. Key mineral sorbents include calcinated struvite as well as aluminium silicates, calcium silicates, or calcium oxides. These sorbents are described in Table S3.3 in Supporting Information III. Sorbents can be applied as a combined soil amendment and fertilizer (Bai et al., 2017, Nakhli et al., 2017, Zhang et al., 2015). They are generally considered slow-release fertilizers, as nutrients are released from the sorbent to soil pore water over time. Salinity potentially present in the feed solution can be reduced as sorbents have a higher affinity for desired nutrient cations (i.e., $\mathrm{NH}_{4}{ }^{+}$and $\mathrm{K}^{+}$) than for undesired salts cations (e.g., $\mathrm{Na}^{+}$) (Beler-Baykal et al., 2011). Sorbents, however, are also commonly applied to remove organic micropollutants and heavy metals from aqueous solutions (Babel, 2003, Choi and Lee, 2015, Kołodyńska et al., 2012, Shaheen et al., 2012, Zorpas et al., 2000). The respective 
bodies of literature are largely separate and studies using sorbents for selective nutrient extraction remain largely silent about potential sorption of micropollutants and heavy metals along with nutrients, as well as desorption characteristics of these contaminants. Some sorbents may also contain heavy metals to start with, for example charcoal where the feedstock is sewage sludge or sewage-derived algal biomass.

\subsection{Organic solids}

Organic solids include a wide variety of products that contain organic matter originating from human excreta or biomass produced during treatment of human excreta or streams containing human excreta. We here distinguish between phototrophic biomass and excreta-derived organic matter.

\subsubsection{Phototrophic biomass}

Phototrophic algae and cyanobacteria have received much attention in recent years and have been grown in urine and yellowater but also in liquid streams rendered by treatment of primary inputs containing feces, such as treated effluent, anaerobic digester supernatant, or the aqueous phase after HTL. High removal of $\mathrm{N}$ and $\mathrm{P}$ from the substrate have generally been achieved (Shilton et al., 2012, Sukačová and Červený, 2017). Also heterotrophic or mixotrophic growth of microalgae has received some attention, but mostly in combination with algal biofuel production (Perez-Garcia et al., 2011). Contaminants may be of concern as algae have been shown to extract not only nutrients but also micropollutants and heavy metals from liquid streams through uptake or sorption (Demey et al., 2018, de Wilt et al., 2016, Zeraatkar et al., 2016). Algal biomass is a promising product potentially useful as plant fertilizer or animal feed (Cole et al., 2017, Wells et al., 2017). The nutrient-rich biomass is usually dried before application as a soil conditioner and fertilizer (Mulbry et al., 2005). Alternatively, it can be used as feedstock for biological decomposition (e.g., composting) or thermal decomposition (e.g., HTL).

\subsubsection{Faecal-derived organic matter}

Fecal-derived organic matter includes a wide variety of products that contain organic matter originating from feces or biomass produced during treatment of fecal-derived organic matter. This product type includes products that closely resemble the primary input (e.g., hygienized feces), products rendered after collection and treatment of the primary input (e.g., blackwater sludge or sewage sludge), as well as products rendered after further decomposition of aforementioned fecal-based feedstocks. These feedstocks can possibly be supplemented by other organic feedstocks (e.g., organic kitchen, yard, or wood waste) and additives (e.g., charcoal, lime, or ash) prior to (biological or thermal) decomposition. Biological decomposition renders digestate or compost (including vermicompost and fly larvae compost), whereas charcoal is the result of thermal decomposition. 
These products are useful as combined soil amendments and fertilizers (Grigatti et al., 2014, Horta, 2017, Kathijotes et al., 2016, Liu et al., 2018, Sangare et al., 2015). When treatment takes place in a closed system, the product can contain $\mathrm{N}$ both in the form of inorganic and organic $\mathrm{N}$. Treatment in open systems, however, is prone to $\mathrm{N}$ losses through volatilization and/or leaching. Similarly, if liquid-solid separation is applied, inorganic $\mathrm{N}$ can be transferred to the liquid fraction. Most $\mathrm{N}$ in sewage sludge and in compost in fact is organically bound and not immediately available to plants (Cogger et al., 2006, Horttanainen et al., 2017). The majority of $\mathrm{P}$ in fecal-derived organics is bound in mineral form. In feces, for instance, $\mathrm{P}$ is mainly present as calcium and iron phosphate (Rose et al., 2015b). In sewage sludge, P can be present as polyphosphate incorporated in microbial biomass (biological $\mathrm{P}$ removal), or as aluminium or iron phosphate (chemical P removal). P availability is variable and strongly depends on the treatment train. Sludge from biological P removal was found to be superior to precipitation with high Fe:P ratios regarding P availability and recycling (Kahiluoto et al., 2015, Lemming et al., 2017, Römer, 2006). There are indications, however, that phosphorus recovery from iron phosphate can be substantially improved by a better understanding of iron-phosphorus chemistry (Wilfert et al., 2015).

It is commonly held that satisfactory pathogen inactivation can be achieved in processes that involve exposure to elevated temperatures (Jönsson et al., 2004). Decomposition processes have the potential to fully or partially decompose organic pollutants. Heavy metals are generally of concern for (derivatives of) sewage (Tervahauta et al., 2014b). As heavy metals present in human excreta were found to primarily originate from dietary sources, agricultural use of human excreta would not increase the amount of heavy metals in the food cycle (Tervahauta et al., 2014b). Therefore, the importance of distinguishing black water sludge from municipal sewage sludge in sludge reuse regulations has been emphasized (Tervahauta et al., 2014b). Heavy metals present in sewage sludge are mainly adsorbed to the cell surfaces of the microorganisms in the sludge (Yoshizaki and Tomida, 2000). Several studies have shown at the laboratory scale the possibility of extracting heavy metals from sewage sludge by means of acid leaching (e.g., Naoum et al., 2001, Stylianou et al., 2007, Usharani and Vasudevan, 2016, Yoshizaki and Tomida, 2000). Acid leaching, however, also dissolves phosphorus (Guilayn et al., 2017) and thus would leave a product in the form of fecal-derived organic matter depleted of heavy metals as well as phosphorus and other nutrients.

\section{Patterns and trends}

The synthesis presented in this review was informed by a rigorous process of organizing and and extracting information from the pertinent literature. There is clearly no shortage of proposed recovery pathways, treatment processes, and products rendered by treatment. Here we outline a number of broader trends and patterns regarding efforts to facilitate recycling of nutrients contained in human excreta to agriculture. 


\subsection{Trends in process technology}

For a long time, agricultural use of human excreta and streams containing human excreta was the dominant way to recycle nutrients and organic matter found in human excreta and wastewater back to agriculture (Ferguson, 2014, Petrik, 1954, Rockefeller, 1998). Beginning in the 1970s, the extraction of nutrients started to complement recycling of the streams themselves. Early efforts include: extraction of nutrients from liquid streams through precipitation (e.g., Salutsky et al., 1972), algae growth (e.g., Mcgarry et al., 1971), or sorption (e.g., Liberti et al., 1981); and extraction of phosphorus from sewage sludge ash (e.g., Hino et al., 1998). Roughly since the mid 2000s, efforts towards nutrient extraction have intensified. Approaches that have been investigated include: extraction of nutrients from liquid streams and wet organic matter through precipitation, sorption, membrane processes, or phototrophic biomass growth; extraction of $\mathrm{P}$ from sewage sludge or ash; and extraction of $\mathrm{N}$ through various forms of ammonia release and capture (see Supporting Information III). Also, bioelectrochemical systems have gained currency, among others to support the extraction of nutrients through electrodialysis (e.g., Zhang et al., 2013), ammonia release (e.g., Desloover et al., 2012, Wu and Modin, 2013), or precipitation (e.g., Hug and Udert, 2013). Continued research and development is taking place. For source-separated primary inputs, recent developments range from simple (e.g., struvite precipitation from urine in a simple sedimentation reactor) to more complicated approaches (e.g., bioelectrochemical systems) and include pathways that decontaminate and concentrate nutrients (to a liquid or solid product) as well as pathways based on selective nutrient extraction (notably of NPK), or a combination thereof. For sewage as primary input, recent developments are predominantly technology-intensive approaches based on selective nutrient extraction, notably of $\mathrm{P}$ (e.g., $\mathrm{P}$ leaching from sewage sludge incineration ashes). These approaches are reviewed extensively in Egle et al. (2015). The great variety of recovery pathways that involve extraction of nutrients (notably P) from sewage sludge or sewage sludge ash aligns with the trend towards incineration of a larger portion of the sludge (Kelessidis and Stasinakis, 2012, Kirchmann et al., 2017) and the anticipation of more stringent future regulation for pathogens, heavy metals, organic pollutants, and other emerging contaminants in sludge intended for land application (Mininni et al., 2015, Peccia and Westerhoff, 2015).

\subsection{Focus on macronutrients NPK}

The trend towards nutrient extraction coincides with a focus on the macronutrients NPK. There is no single recovery pathway that captures all nutrients and carbon in human excreta in a single product free of contamination. We see a clear divide between recovery pathways that target the recovery of (some of the) macronutrients NPK and those that more broadly target a wider selection of nutrients, and possibly also organic matter. Products obtained from source-separated primary inputs such as urine, feces, or blackwater in general are less polluted, notably regarding heavy metals. When sewage is the primary input, products containing a 
broader spectrum of nutrients as well as organic matter generally are prone to also contain higher levels of contamination. Recovery pathways that render a product of high purity and homogeneity (e.g., macronutrient solutions, monomineral precipitates) achieve this through selective extraction of only some of the nutrients, notably macronutrients $\mathrm{N}$ and P. Many studies in fact do not even investigate or report the fate of $\mathrm{K}$ and micronutrients. For conventional urban water management and sanitation systems, and often also for new sanitation systems, the discourse generally focuses even more narrowly on P extraction and recovery. P extraction and recovery in fact is expected to become an established process in the coming decades in industrialized countries (Sartorius et al., 2012).

\subsection{Multiple uses for carbon}

Human excreta, notably feces, contain carbon that can be valuable to improve soil quality. In conventional sewage treatment, the more readily biodegradable fraction of this carbon is usually converted into biogas and carbon dioxide through microbial metabolism. Unless sewage sludge is incinerated, the less readily biodegradable fraction of the carbon is preserved in the organic residual and potentially available to improve soil quality. But carbon may increasingly be appropriated for other purposes. Human feces and streams containing human feces can potentially serve as feedstock for the production of biocrude, bioethanol, biodiesel, biohydrogen, and syngas (Gomaa and Abed, 2017, Manyuchi et al., 2018, Puyol et al., 2017).

Likewise, feces and streams containing feces can serve as feedstock for the production of higher-value industrial chemicals, for example precursors for biopolymer synthesis and bioplastic production (Pittmann and Steinmetz, 2017, Puyol et al., 2017). These different uses of carbon do not necessarily exclude one another. But appropriation of a larger fraction of the carbon for the production of energy carriers or higher-value chemicals means that less carbon is available for the improvement of soils. The appropriation of organic matter for the production of energy or chemicals thus may to some extent compete with the recovery of organic matter to improve soil quality.

\subsection{Synergies and opportunities for combining recovery pathways}

While many of the studies covered in the present review target a single product with agricultural value, some studies report on a combination of recovery pathways leading to multiple products, or at least point to the possibility for a combination of recovery pathways. For example, NF of unhydrolyzed urine followed by precipitation yields a solution rich in urea and a precipitate containing N and P (e.g., Pronk et al., 2006). Likewise, ammonia stripping from urine can be complemented by struvite precipitation, yielding a solution rich in ammonium and a precipitate containing N and P (e.g., Antonini et al., 2011, Wei et al., 2018). Evaporation in a vertical evaporation pipe preceded by alkalinization yields one precipitate rich in $\mathrm{P}$ and a one precipitate containing the other nutrients (e.g., Eawag, 2018b). The combination of ammonia stripping, 
struvite precipitation, and biomass growth in a hydroponic system to treat source-separated urine even yields three products useful for agriculture: a solution rich in ammonia, a precipitate containing $\mathrm{N}$ and $\mathrm{P}$, and a residual solution used as input to the hydroponic system (e.g., Yang et al., 2015). Other possible combinations are: pyrolysis of for instance sewage sludge and use of the char thus obtained as sorbent (e.g., Shepherd et al., 2016), or urea extraction from unhydrolyzed urine through sorption in order to facilitate MPP (magnesium potassium phosphate) precipitation in the absence of N (e.g., Simha et al., 2016). Similarly, CaP granulation during anaerobic digestion of blackwater would yield three products from one reactor system: CaP, digestate, and a concentrated liquid with $\mathrm{N}, \mathrm{K}$ and micronutrients.

The combination of recovery pathways can enhance overall nutrient recovery and recycling. What might be nutrient losses in a single pathway might well be captured in another product if several pathways are combined to target more than one product. This means that individual recovery pathways or products should not be judged in isolation. For example, one could argue that recovery pathways based on urine separation fail to capture about half of the $\mathrm{P}$ and most of the $\mathrm{C}$ in human excreta. Urine separation, however, does not prevent recovery of nutrients and organic matter from the stream containing the feces. On the contrary, the fact that most of the $\mathrm{N}$ is in the urine means that any recovery pathway starting from the stream containing the feces will be subject to lesser $\mathrm{N}$ losses than would be the case if urine were in this stream. Similarly, one could argue that struvite precipitation usually only captures a fraction of the $\mathrm{P}$ if sewage is the primary input (and anaerobic digester reject water the process input). Struvite precipitation during sewage treatment, however, does not prevent the subsequent recovery of additional $\mathrm{P}$ from sewage sludge or sewage sludge ashes; though systems become more complicated. Finally, individual products can also be applied in combination, for example mineral sorbents and precipitates (Lind et al., 2000, Xu et al., 2001), or compost and precipitates (Karak et al., 2015).

\section{Discussion and outlook}

As outlined in this review and elsewhere in the literature, a broad range of recovery technologies and pathways to facilitate recovery of nutrients and organic matter contained in human excreta is available or under development. The two currently most mature recovery pathways are struvite crystallization from anaerobic digester supernatant and incineration of sewage sludge with subsequent $\mathrm{P}$ recovery from incineration ashes. While further development and refinement of these and other recovery technologies and pathways is valuable in its own right, we believe that there is scope to ask questions that go beyond individual recovery technologies and pathways, and that better integrate end-user needs and the bigger picture.

The call for further development of technologies that recover $\mathrm{N}$ and $\mathrm{K}$ in addition to $\mathrm{P}$ (Mehta et al., 2015) is a step in the right direction. But in light of soil nutrient stripping (Jones et al., 2013b) and soil carbon losses (Amundson et al., 2015), we think the scope of nutrient recovery should be even broader and also include micronutrients and organic matter. This will ultimately 
require a shift away from thinking in terms of individual recovery pathways, towards thinking in terms of sensible combinations of recovery pathways that maximize recovery of nutrients and organic matter while minimizing risks associated with contaminants.

Recognizing that comprehensive nutrient recovery will again have to become a key function of human excreta management in order to help reinvigorate soil and food security, it becomes evident that a conceptual change towards framing human excreta management as part of the food cycle rather than the urban water cycle might be productive. We believe that broadening the discourse along these lines would strongly benefit from the integration of perspectives and considerations from food and farming systems with those from managing human excreta.

In other words, we argue that it is not sufficient to ask: how to recover (some of the) nutrients from (streams containing) human excreta? It is also necessary to ask: which kind of production system is envisioned as recipient of the nutrients and organic matter? How can the products best support a given production system and the achievement of specific goals such as food and soil security? Are there specific functions that need to be fulfilled by recycling from human excreta and that cannot be fulfilled by other ways of (re)cycling nutrients and organic matter? Still, there is only little research into how various recovered products fit the needs of soils and farmers (e.g., Wielemaker et al., 2018a). While the present review briefly touches upon general product characteristics, further research on the effects of nutrients and contaminants from products recovered from human excreta on soil health, plant growth and human well-being would be helpful.

We recognize that recycling nutrients (and organic matter) from human excreta and streams containing human excreta to food production is only one dimension of and establishing a circular nutrient metabolism where nutrients from food are recycled back to the production of food. Establishing such a circular nutrient metabolism requires action along the entire food chain from agriculture and food processing to consumers and waste management; this includes proper management of harvest residues, animal manure, food processing residuals and waste, and human excreta (McConville et al., 2015). But nutrient recycling is currently constrained by spatial disconnects between livestock intensive areas and areas where feed is produced, and between rural areas where food is produced and urban areas where food is consumed and human excreta produced (Jones et al., 2013b, Nesme et al., 2018).

Other factors that are critical for a smooth and effective transition to the widespread use of recovered fertilizer products but were not considered in the present review include legislation (Hukari et al., 2016) and social acceptance (Dahlin et al., 2016), as well as technological maturity, environmental performance, and costs (Egle et al., 2016).

We agree with Trimmer et al. (2017) that sanitation systems could become an inspirational component of societal infrastructure and an amplifying force for sustainable development. We hope that the present review can make valuable contributions to this end, by providing inspiration 
to look upon the recovery of nutrients and organic matter from a broader perspective, and to better integrate perspectives from food and farming systems, the recipients of the recovered fertilizer products. The organization and classification of recovery pathways that underpins the present review could also serve as a foundation to more effectively share and consolidate what we already know about various aspects of human excreta management, and to keep track of further technological advancements.

\section{Acknowledgements}

Robin Harder contributed to this review in relation to the project 'Recycling organic matter and nutrients from sanitation to farming systems to regenerate soil and land: identifying approaches that are feasible and preferable', which is funded by a mobility grant from the Swedish Research Council for Environment, Agricultural Sciences and Spatial Planning (Formas) under grant agreement 2016-00859. 


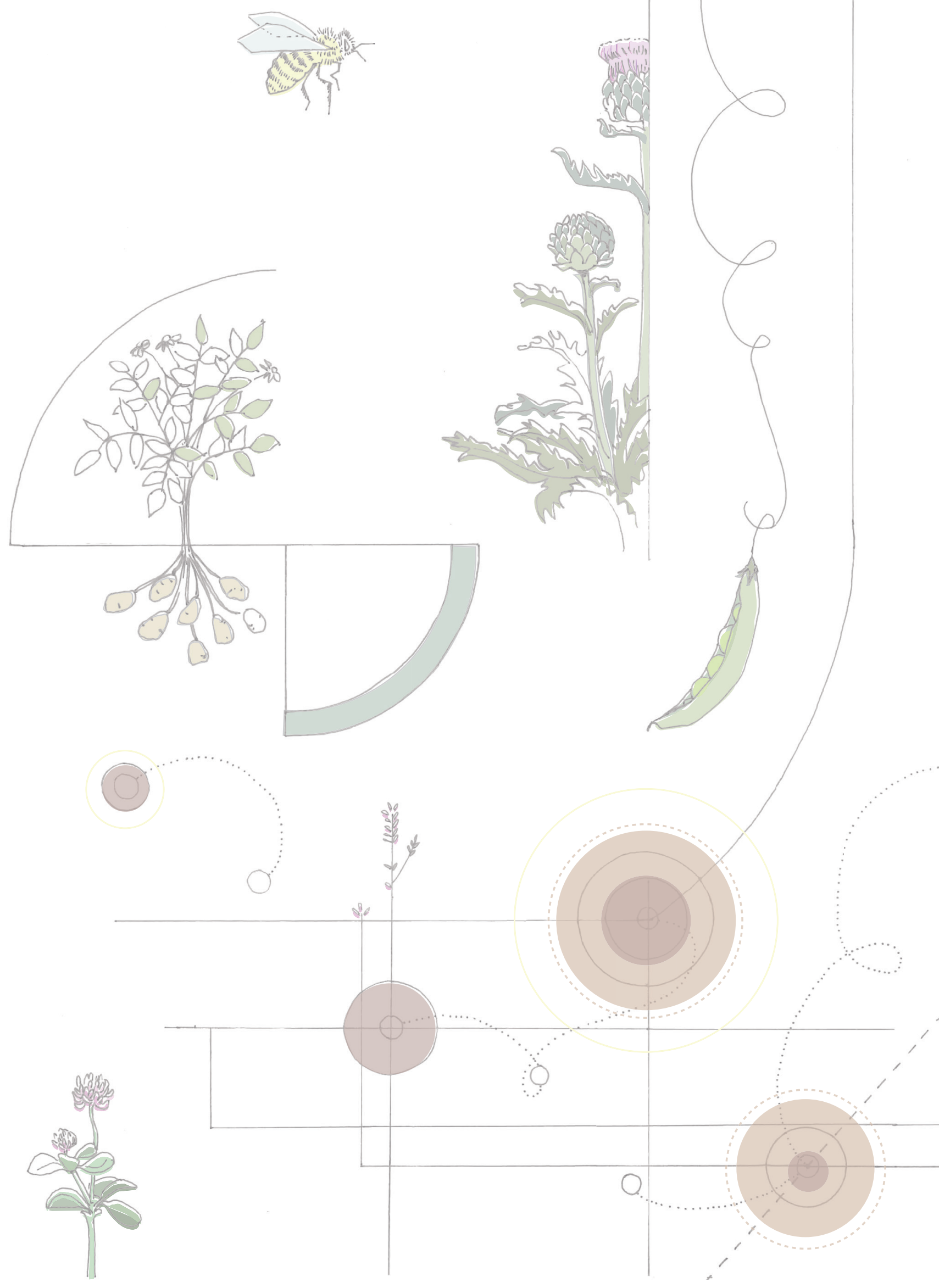




\begin{abstract}
Recovering nutrients from human excreta and wastewater has been receiving increasing attention as a means to supplement or replace synthetic fertilizer production. Apart from technologies for nutrient recovery at centralized wastewater treatment plants, numerous decentralized, sourceseparated sanitation systems, also known as new sanitation systems, have been developed to facilitate recovery. Decision making for the planning and implementation of new sanitation systems would benefit from a spatially explicit inventory of nutrient hotspots in urban areas. To provide visual representations of nutrient loads, we developed a methodology that combines spatial-temporal modelling with geographic information systems (GIS) analysis, and used it for the city of Amsterdam. The methodology is new in the field of nutrient mapping, especially at the smallest geographical scale: building. Nitrogen, phosphorus and potassium loads and hotspots are mapped at both building and neighborhood scale, drawing attention to the need for multiple scale analyses in decision making. This study concludes with a discussion on the potential to further develop the method proposed to include more detailed and verified data and to identify nutrient hotspots that are promising as nutrient recovery sites with new sanitation systems.
\end{abstract}

Keywords: wastewater; nutrient recycling; geographic information systems (GIS); urban metabolism; resource recovery

This chapter is published as: Wielemaker, R., Stuiver, J., Zeeman, G. \& Weijma, J., 2019. Identifying Amsterdam's nutrient hotspots: A new method to map human excreta at building and neighborhood scale. Journal of Industrial Ecology. 


\section{Introduction}

The need for improved nutrient management, including increased recycling of nutrients from wastes back to agriculture, is increasingly emphasized in research to minimize ecosystem damage, and ensure food security and access to sufficient fertilizers (Cooper et al., 2011, Dawson and Hilton, 2011, Elser and Bennett, 2011, Harder et al., 2019, Malila et al., 2019, Trimmer and Guest, 2018, Wielemaker et al., 2018a). Numerous technologies have been developed for centralized wastewater treatment plants (WWTP) to facilitate the recovery of nutrients at the end of the pipe, as outlined by Egle et al. (2015). In recent decades, localized, source-separated sanitation systems, also known as new sanitation, have been developed not only to treat wastewater, but also to recover resources from wastewater (Tervahauta et al., 2013, Zeeman and Kujawa-Roeleveld, 2011a). New sanitation systems keep streams separate and concentrated (e.g., low flush toilet, separation of black and grey water) to minimize mutual contamination and dilution of streams, which facilitates nutrient recovery (Larsen et al., 2009b). These systems can include low-tech and high-tech recovery technologies, as reviewed by (Harder et al., 2019), which are suitable for decentralized scales. New sanitation systems are especially interesting for neighborhoods, particularly for new developments or neighborhoods undergoing renovation, and larger commercial or public buildings (STOWA, 2014).

As interest in new sanitation increases, decision-making for the implementation of new sanitation systems would benefit from a spatially explicit inventory of promising locations for nutrient 'harvesting' from human excreta. However, people are transient in space, moving between home, work, commercial and public domains, and their toilet-use patterns are equally dispersed. It can be expected that there is a spatial variance in composition and volume of wastewater across urban areas, and that therefore certain locations, particularly locations with high nutrient loads ('hotspots'), might be more interesting for recovery via new sanitation systems than others. Yet, hardly any data is available on this variability in toilet-use patterns and wastewater generation across geographical locations.

The need for spatial representation of urban nutrient loads has been underlined in research (Chowdhury et al., 2014, Metson et al., 2012, Metson et al., 2018, Li and Kwan, 2018); visualization can play an important role in comprehensibility of information and provide clarity of results (Li and Kwan, 2018). The benefit of a geospatial inventory, as opposed to presenting substance flow box and arrow diagrams, is that the visualization of nutrient availability aids the subsequent planning capacity of interventions, such as technologies, policies and behavioral changes, to facilitate the recycling of nutrients (Metson et al., 2018). Previous studies have mapped phosphorus (P) fluxes for the city of Phoenix in Arizona, USA (Metson et al., 2012), and for Sydney, Australia (Metson et al., 2018), however the spatial resolution of the datasets for potentially recyclable $\mathrm{P}$ was rasterized. A rasterized dataset is advantageous for calculating net nutrient balances for a given area (raster cell), but it does not identify exact locations for intervention. Agricultural P losses have been mapped by Scherer and Pfister (2015) for global 
P emissions, and by Wang et al. (2018) for nitrogen $(\mathrm{N})$ and P losses from food production in China at county scale. Urban nutrient load profiles and hotspots have not been mapped and reported before this time for nutrients originating from human excreta, and more specifically at the high resolution that we present here.

The objective of this study is to provide a method that can produce a spatial-temporal representation of nutrient excretion estimates. The method maps urban N, P and K (potassium) loads per building and neighborhood, and pinpoints those that display comparatively high nutrient loads as 'nutrient hotspots'. We applied the method to the city of Amsterdam, the Netherlands, though the method can be adjusted to and applied in other contexts. The method uses accessible geographic and population data, together with data on nutrient composition of urine and feces and general toilet-use patterns, in this case for the Netherlands. Geographical Information Systems (GIS) analysis was used to create maps of the nutrient loads across space that help visualize buildings and neighborhoods in Amsterdam that can be identified as nutrient hotspots. The results can provide valuable input to determine the viability of new sanitation interventions at these locations.

\section{Methods}

This study spatially maps nutrient load profiles based on geographic and governmental population data. The developed method combines spatial-temporal modelling with geographic information systems (GIS) analysis to develop 2D (ArcMap 10.5) maps that show nutrient peaks across space. Nutrient loads are mapped at one or multiple spatial and temporal scale combinations. The spatial scale can include: city, city district, neighborhood, or building, while the temporal scale can include: year, month, week, day, or hour. The resolution of the results depends on the detail of the available data. Data that is not available or is non-existing is supplemented with estimates based on scientific literature.

The method includes the following steps (see Figure 5.1 for a visual depiction of the outlined steps):

1. Delineation of geographical scales: Area of interest is delineated (e.g., city, district, neighborhood or building). An appropriate spatial extent can be selected accordingly for a diversity of research objectives.

2. Description of distribution of people in space (as a function of time): Identifying the locations where people engage in activities, as well as the number of hours that they spend at each location.

3. Definition of nutrient excretion and frequency of excretion: Nutrient content of excreta and toilet use patterns, as well as frequency of excretion (how often a person uses the toilet over a period of time). 

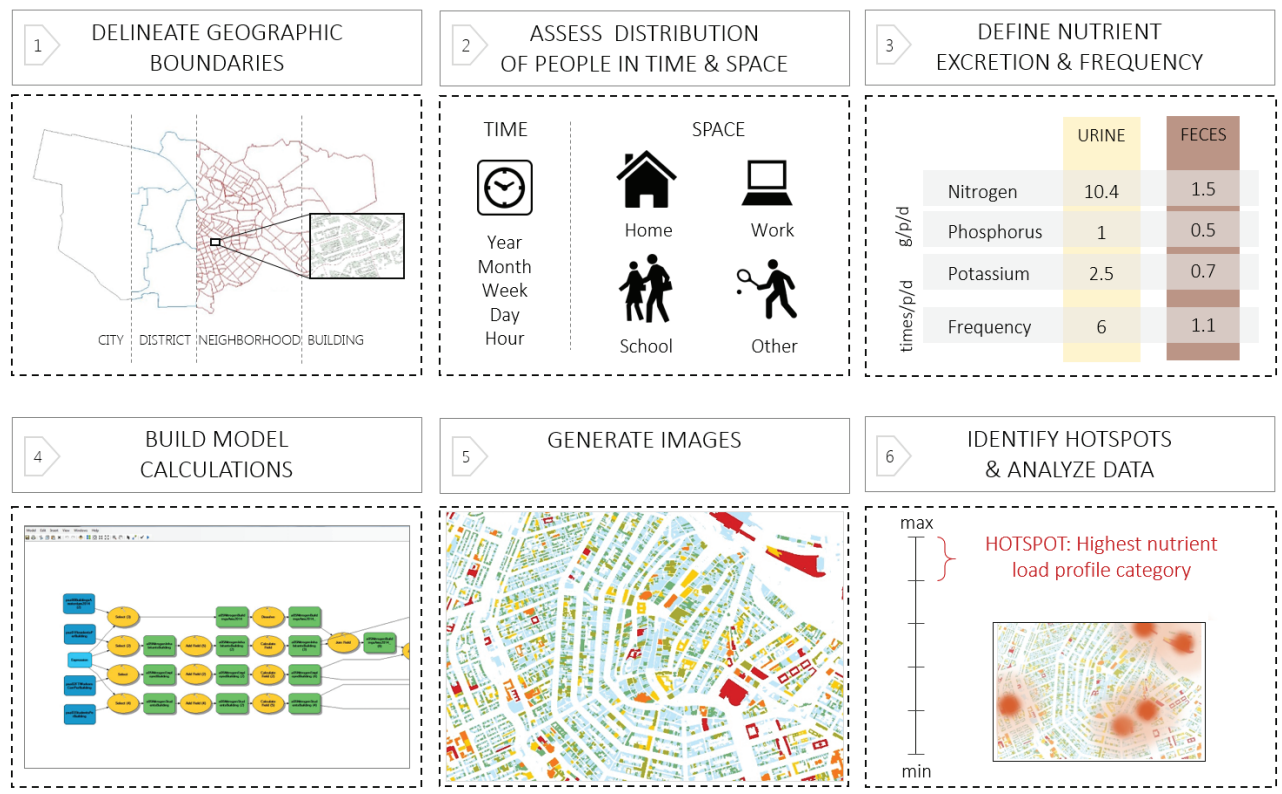

Figure 5.1. Graphic representation of the developed method.

4. Calculation of nutrient loads: Using GIS model builder and input data to calculate the values for nutrient excretion in space and time for each delineated boundary (shown in equations below). The nutrient load $\left(\mathrm{N}_{\mathrm{x}}\right)$, for a select nutrient, within spatial boundary $\mathrm{X}$ is calculated using Equation 5.1. Where $\mathrm{I}_{\mathrm{X}}$ is the sum of the number of individuals within boundary $\mathrm{X}, \mathrm{P}_{\mathrm{UX}}$ is the percentage of urine excreted within boundary $\mathrm{X}$, and $\mathrm{N}_{\mathrm{U}}$ is the total nutrient load in urine, $\mathrm{P}_{\mathrm{Fx}}$ is the percentage of feces excreted within boundary $\mathrm{X}$ and $\mathrm{N}_{\mathrm{F}}$ is the nutrient load in feces. $\mathrm{P}_{\mathrm{Ux}}$ and $\mathrm{P}_{\mathrm{Fx}}$ can be calculated using Equation 5.2 and Equation 5.3, where $\mathrm{T}_{\mathrm{x}}$ is the average time (e.g., hours/week) per individual spent within boundary $\mathrm{X}, f_{\mathrm{U}}$ and $f_{\mathrm{F}}$ are the frequency of urination and defecation (toilet visits per individual per hour) and $\mathrm{V}_{\mathrm{U}}$ and $\mathrm{V}_{\mathrm{F}}$ are the total number of urination or defecation visits (e.g., per individual per week). The units for $\mathrm{N}_{\mathrm{x}}$ directly reflect the units defined for the total nutrient load in urine and feces.

$$
\begin{aligned}
& \mathrm{N}_{\mathrm{x}}=\mathrm{I}_{\mathrm{x}}\left(\mathrm{PU}_{\mathrm{x}} * \mathrm{~N}_{\mathrm{U}}+\mathrm{P}_{\mathrm{Fx}} * \mathrm{~N}_{\mathrm{F}}\right) \\
& \mathrm{P}_{\mathrm{Ux}_{\mathrm{x}}}=\mathrm{T}_{\mathrm{x}} * f_{\mathrm{U}} * \mathrm{~V}_{\mathrm{U}^{-1}} \\
& \mathrm{P}_{\mathrm{Fx}}=\mathrm{T}_{\mathrm{x}} * f_{\mathrm{F}} * \mathrm{~V}_{\mathrm{F}^{-1}}
\end{aligned}
$$

Equation 5.1

Equation 5.2

Equation 5.3

5. Generate Images: The use of GIS analysis allows for the visualization of the nutrient loads across space. Several symbology and classification tools can enhance the visual representation and communication of results. 
6. Identification of nutrient hotspots: Nutrient hotspots are defined as the location where the nutrient loads are highest, either relative to the other locations or over an identified threshold. Locations with higher loads than others are deemed 'hotter' than others.

\subsection{Input data for the Amsterdam context}

The developed method requires several input data to complete the calculations outlined previously. An overview of all the collected input data can be found in the Supporting Information VI (Table S6.1).

\subsubsection{Geographic boundaries, population data and time-use data}

We selected two geographical scales, namely building and neighborhood, for this study based on data available from the Dutch Land Registry and Mapping Agency (Kadaster) and the Municipality of Amsterdam. Mapping nutrient loads at the building scale was a deliberate choice as normally each building is connected to the main sewer through one outlet pipe. The outlet pipe at the building is therefore the potential intervention point source for nutrient recovery, especially when nutrient content in the stream could be expected to be relatively high, e.g., office buildings without showers. Neighborhoods are of course an administrative boundary and do not necessarily imply that sewage from this area is collected together. In fact, it might be more conducive to draw boundaries that delineate specific catchment areas that drain into the sewage system. However, the choice to map nutrient loads at the neighborhood scale was to show profiles based on aggregated data. The temporal resolution selected for this study is one year. This means that the total annual P excretion across the city of Amsterdam is represented per delineated geographic boundary.

Population data for numbers of inhabitants, numbers of people employed and numbers of students per building was kindly provided by the Municipality of Amsterdam, department of Planning and Sustainability. It is emphasized that all data was anonymized. The population data for numbers of people employed was corrected to better estimate their temporal presence in the buildings, as some jobs do not take place at the location where they are registered (i.e., cleaners, consultants, electricians, etc.). The correction was calculated using coefficients provided by the municipality that are commonly used for traffic estimations. To determine how people move in time and space, time use data ('het tijdsbestedingsonderzoek' (TBO)), collected via population surveying every 5 years for the Netherlands, was used. Time use data records several activities, including: hours spent on work, personal care, sleeping, eating, household chores, etc. (Harvey, 1993, Cloïn et al., 2013). The last TBO (2011-2012) was led under collaboration between the Netherlands Institute for Social Research (SCP) and the Central Agency for Statistics (CBS) (CBS, 2013) and includes 1806 respondents (sample size) of 12 years and older (Cloïn et al., 2013). For this study, the complete time use distribution across home, work, school and other can be found in the Supporting Information VI (Table S6.2), summarized as follows: 
Home: Dutch citizens (>12 years old) spend 76\% (127.3 h) of their week at home (calculated from (CBS, 2014)). The hours spent working from home are not included in this percentage.

Work: Dutch citizens (>12 years old) spend 12\% (19.6 h) of the week engaged in paid work (CBS, 2014).

School: Students between 12 and 18 years of age spend on average 14.8\% (24.8 hours) of the week at school (CBS, 2014). Elementary students in the Netherlands spend a minimum of 7520 hours in class during their complete elementary education (ages 4-12). This averages to $940 \mathrm{~h} / \mathrm{yr}$ (Rijksoverheid). Weekly data is not available for educational facilities.

Out of house activities: The remaining 13\% (20.9 h) of the week (ages >12 years old) is spent on other out of house activities (CBS, 2014). This data was not included because the locations at which these activities took place was unknown.

Additionally, private and public institutions such as museums, theaters, and concert halls were also included. The number of visitors per location is recorded by the Department of Research, Information and Statistics (OIS) and the Consultative Association of the Museums of Amsterdam (OAM). The amount of time visitors spent at each location could not be deduced from the TBO study, therefore the reported time people typically spent at the respective institutions according to Google (https:// wnw.google.com/ business/) were used.

\subsubsection{Nutrient excretion, toilet-use patterns, stool frequency and frequency of urination}

It is not practicable to calculate exact nutrient excretion for each person in an entire urban population as toilet-use patterns and nutrient concentrations in excreta are based on individual behavioral patterns and diet. In addition, it may be unethical, in terms of privacy, to obtain such exact information about individual whereabouts and their toilet-use patterns. Therefore, average parameters or design values are used to estimate nutrient excretion for larger groups. General parameters from Meinzinger and Oldenburg (2009) and Kujawa-Roeleveld and Zeeman (2006) are used in this study. See Table 5.1 for composition values. Frequency of urination and defecation over a 24 hour period varies with a persons' fluid intake, their food intake, as well as other health and environmental factors; digestibility of the diet, determines the partitioning of nutrients between urine and feces (Jönsson et al., 2004). A report on household water use in the Netherlands concludes an average toilet use at 5.9 flushes per day in 2013 (van Thiel, 2014). The frequency of urination is assumed to be 6 times per day with $60 \%$ of total urine volume excreted between 9:00-21:00 and the remaining 40\% during 21:00- 9:00, and a stool frequency of 1.1 times/day (Rose et al., 2015a). According to the STOWA report from 1998, the average Dutch person prefers to use the toilet at their home; For urine, $85 \%$ is excreted at home and $15 \%$ is excreted away from home, while $96 \%$ of feces is excreted at home while $4 \%$ is excreted 
away from home (Wijst and Groot-Marcus, 1998). Based on the time use data, we calculate that $72 \%$ of urine is excreted at home, and $14 \%$ at work. The remaining fraction is excreted during the time spent on activities such as hobbies, sports, and social activities which are difficult to attribute to a specific location. These might be excreted at home before or after these activities, or elsewhere.

Table 4.1 Composition of urine and feces and frequency of excretion

\begin{tabular}{|c|c|c|c|c|}
\hline Parameter & Unit & Urine & Feces & Reference \\
\hline \multicolumn{5}{|l|}{ Composition } \\
\hline Volume & $\mathrm{L} / \mathrm{p} / \mathrm{d}$ & 1.37 & 0.14 & (Meinzinger \& Oldenburg, 2009) \\
\hline COD & $\mathrm{g} / \mathrm{p} / \mathrm{d}$ & 10 & 60 & (Meinzinger \& Oldenburg, 2009) \\
\hline TSS & $\mathrm{g} / \mathrm{p} / \mathrm{d}$ & 57 & 38 & (Meinzinger \& Oldenburg, 2009) \\
\hline $\mathrm{TN}$ & $\mathrm{g} / \mathrm{p} / \mathrm{d}$ & 10.4 & 1.5 & (Meinzinger \& Oldenburg, 2009) \\
\hline TP & $\mathrm{g} / \mathrm{p} / \mathrm{d}$ & 1 & 0.5 & (Meinzinger \& Oldenburg, 2009) \\
\hline TK & $\mathrm{g} / \mathrm{p} / \mathrm{d}$ & 2.5 & 0.7 & (Meinzinger \& Oldenburg, 2009) \\
\hline \multicolumn{5}{|l|}{ Frequency } \\
\hline Per day & times/d & 6 & 1.1 & (Rose et al., 2015) \\
\hline Per hour (9:00-21:00) & times/h & 0.3 & & (Rose et al., 2015) \\
\hline Per hour (21:00-9:00) & times/h & 0.2 & & (Rose et al., 2015) \\
\hline
\end{tabular}

\section{Results}

The compiled input data allowed us to create maps to depict nutrient loads across space, at both building and neighborhood scales. The maps for $\mathrm{P}$ are presented in the following section and are compared with the results for $\mathrm{N}$ and $\mathrm{K}$. The figures for $\mathrm{N}$ and $\mathrm{K}$ individually can be found in the Supporting Information VI.

\subsection{Nutrient load profiles at building scale}

Using the method, $\mathrm{P}$ load profiles were calculated for each registered building $(\mathrm{n}=188,483)$ in the city of Amsterdam, shown in Figure 5.2. The load profile value range (0-544 kg P/yr/building) was classified into five equal interval load classes, dividing the data in $20 \%$ increments: $0-108 \mathrm{~kg} /$ yr (Group I), 109-218 kg/yr (Group II), 219-327 kg/yr (Group III), 328-435 kg/yr (Group IV), and 436-544 kg/yr (Group V). We retrieved a data set that was unevenly distributed; the large majority of buildings had low P loads placing them in Group I. More than $98 \%$ of the buildings had P loads under $15 \mathrm{~kg} / \mathrm{yr}$ and the mean value was $1.75 \mathrm{~kg} \mathrm{P} / \mathrm{yr} /$ building. Only 193 buildings 


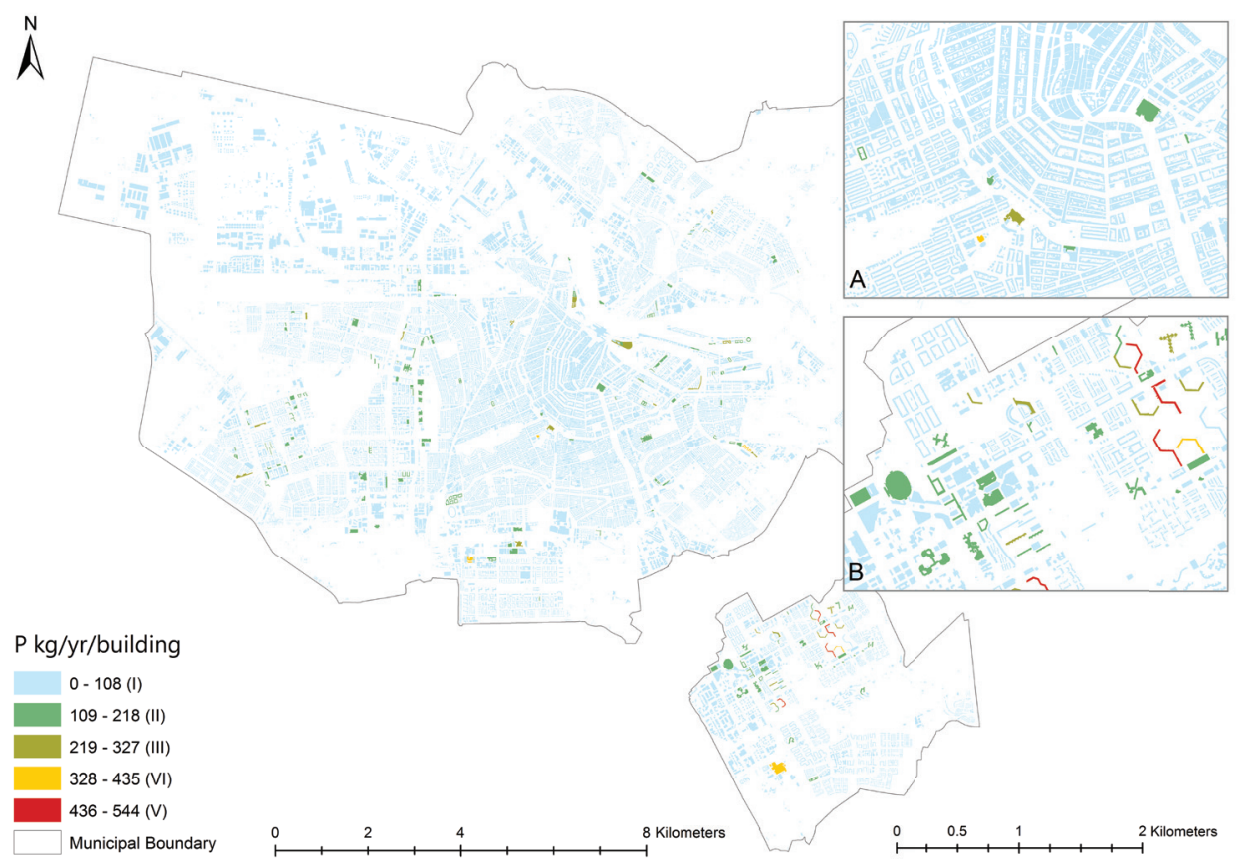

Figure 5.2 Phosphorus load profile for the city of Amsterdam at building scale. The distribution of equal interval places the vast majority of the buildings in the lowest load class (Group I). Inset map A and B show the difference in spatial distribution of phosphorus loads between areas in the inner city (A) and outer city (B). Inset map B also includes the buildings with the highest phosphorus loads (Group V)

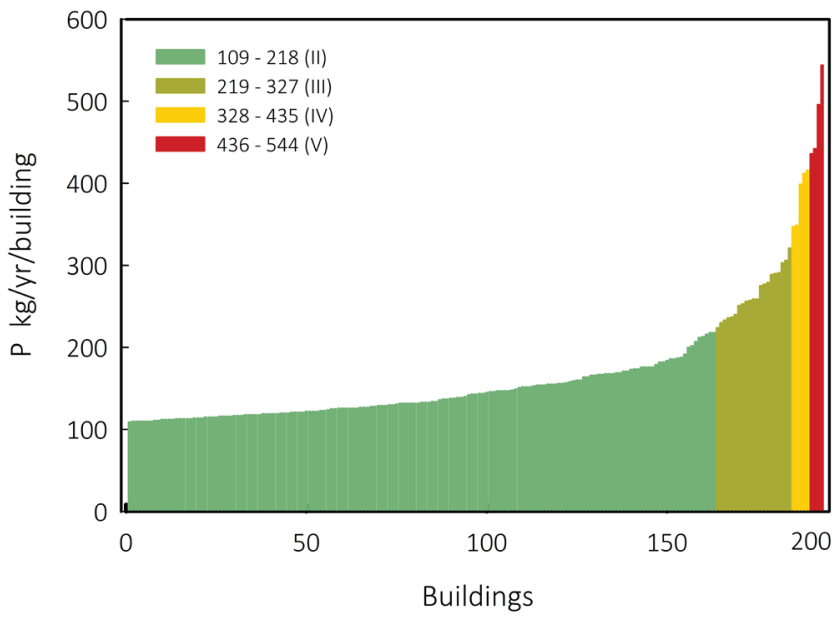

Figure 5.3 Phosphorus loads for buildings in Group II ( $n=163)$, Group III ( $n=21)$, Group IV (n=5) and Group $\mathrm{V}(\mathrm{n}=4)$. The slope increases after $160 \mathrm{~kg} \mathrm{P} / \mathrm{yr} /$ building with smaller numbers of buildings in higher load classes. The vast majority of the buildings are in the lowest load classes Group I (not shown) and Group II. 


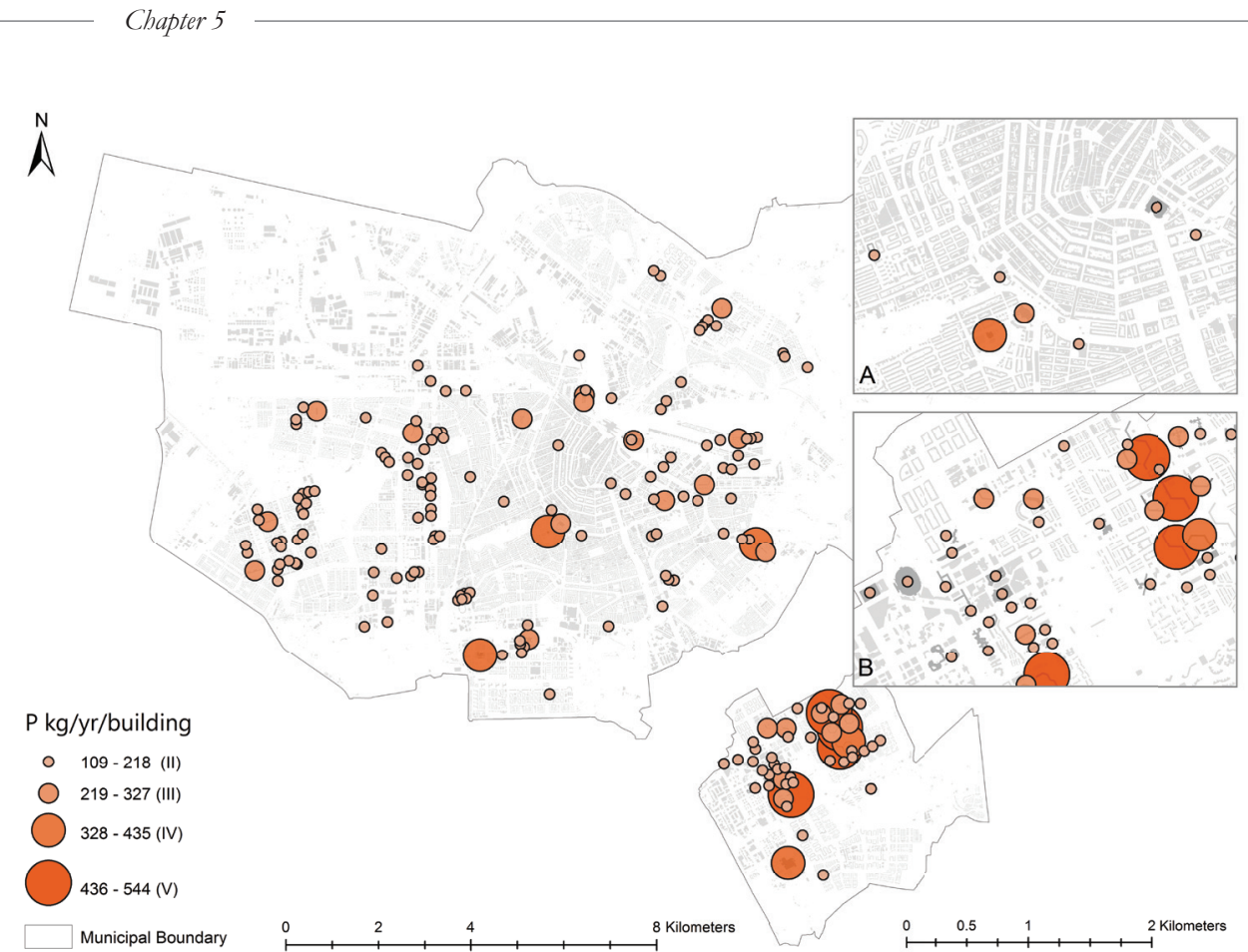

Figure 5.4. Phosphorus hotspots (Groups II-V) for the city of Amsterdam at building scale. Inset map A shows the low hotspot density in the inner city of Amsterdam. Inset map B improves visual clarity of densely populated hotspots and includes all hotspots in Group V.

are represented by the top four interval load classes, Group II-Group V, with a mean P load of $168 \mathrm{~kg} / \mathrm{yr} /$ building and median value of $143 \mathrm{~kg} \mathrm{P} / \mathrm{yr} /$ building. Given the comparatively high loads of the buildings in these four classes, we considered these buildings $\mathrm{P}$ hotspots. Most hotspots are located beyond the city center of Amsterdam (Figure 5.2, inset map A) and the majority of the hotspots in Group IV and Group V were located in Amsterdam Zuid-Oost (Figure 5.2, inset map B). Figure 5.3 further shows the distribution of the hotspots across the load values, displaying a steep increase after $160 \mathrm{~kg} \mathrm{P} / \mathrm{yr} /$ building.

Figure 5.4 represents the same data, this time using circles of various sizes to reflect the magnitude of the P loads. To better focus on the buildings with relatively higher loads, we omitted the data points in the bottom $20 \%$ of the data values, which includes all buildings in Group I. The representation of the data in this form improves the visualization of the data. While the circles overlap with a zoomed out view, zooming in (Figure 5.4, inset maps A and B) improves clarity of their placement, with the center of the circle coinciding with the center of the respective building. The majority of the building hotspots receive their largest $\mathrm{P}$ load from inhabitants residing in those buildings (Figure 5.5). A few however, are company headquarters, museums, universities, and hospitals that receive their largest loads from employees or visitors. Many hotspots attribute their total load to a sum of loads from different functions. 


\section{Largest contributing phosphorus load by building function}

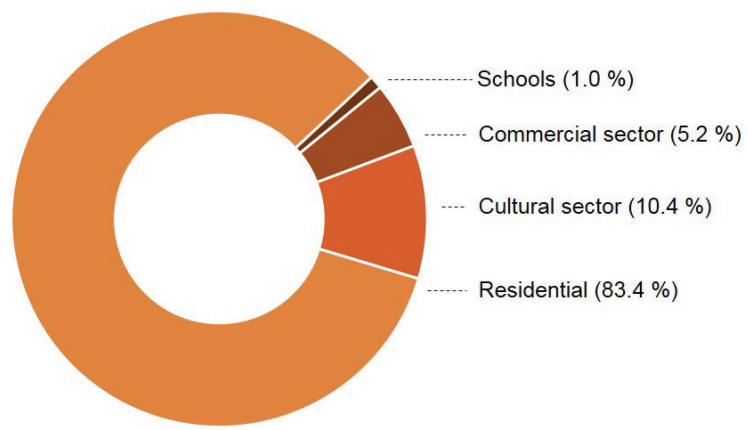

Figure 5.5. Distribution across building functions responsible for the largest contributing phosphorus load for the identified phosphorus hotspots at building scale.

\subsection{Phosphorus load profiles at neighborhood scale}

The choice to map P loads at the neighborhood scale (Figure 5.6) was to show profiles based on aggregated data. The data was defined in the same manner as at building scale, in five equal interval load classes. P loads per neighborhood have a mean value of $812 \mathrm{~kg} \mathrm{P} / \mathrm{yr} /$ neighborhood and are attributed primarily to the number of inhabitants. The predominance of the $\mathrm{P}$ loads originating from residential functions is not surprising, however, as more urine and feces is expelled at home. Notable is the distribution of neighborhoods among the five interval classes relative to their surface area, that is, neighborhoods in the highest load class are some of the smaller neighborhoods, while the larger neighborhoods have lower P loads. In Figure 5.6, the P loads at building scale have been superimposed on the P loads at neighborhood scale to indicate the importance of spatial resolution for the generation and interpretation of results. Noteworthy is that buildings in the highest load class are not located in neighborhoods in the highest load class per se (inset map, label A). Likewise, neighborhoods in the highest load class do not necessarily accommodate any buildings that are in the highest load class (inset map, label B). Figure S6.1 (in the Supporting Information VI) shows the distribution of the P loads per neighborhood across the load values, again indicating that a few neighborhoods are clearly 'hotter' than the majority.

\subsection{Nitrogen, phosphorus and potassium load profiles and hotspots, building and neighborhood scale}

Mapping hotspots for $\mathrm{N}$ and $\mathrm{K}$ (Supporting Information VI, Figure S6.2, and Figure S6.3) separately returned almost the same number of hotspots, 202 and 197 respectively, and also at the same locations, as for $\mathrm{P}(\mathrm{n}=193)$. The majority of the hotspots across the 3 nutrients also fell in the 


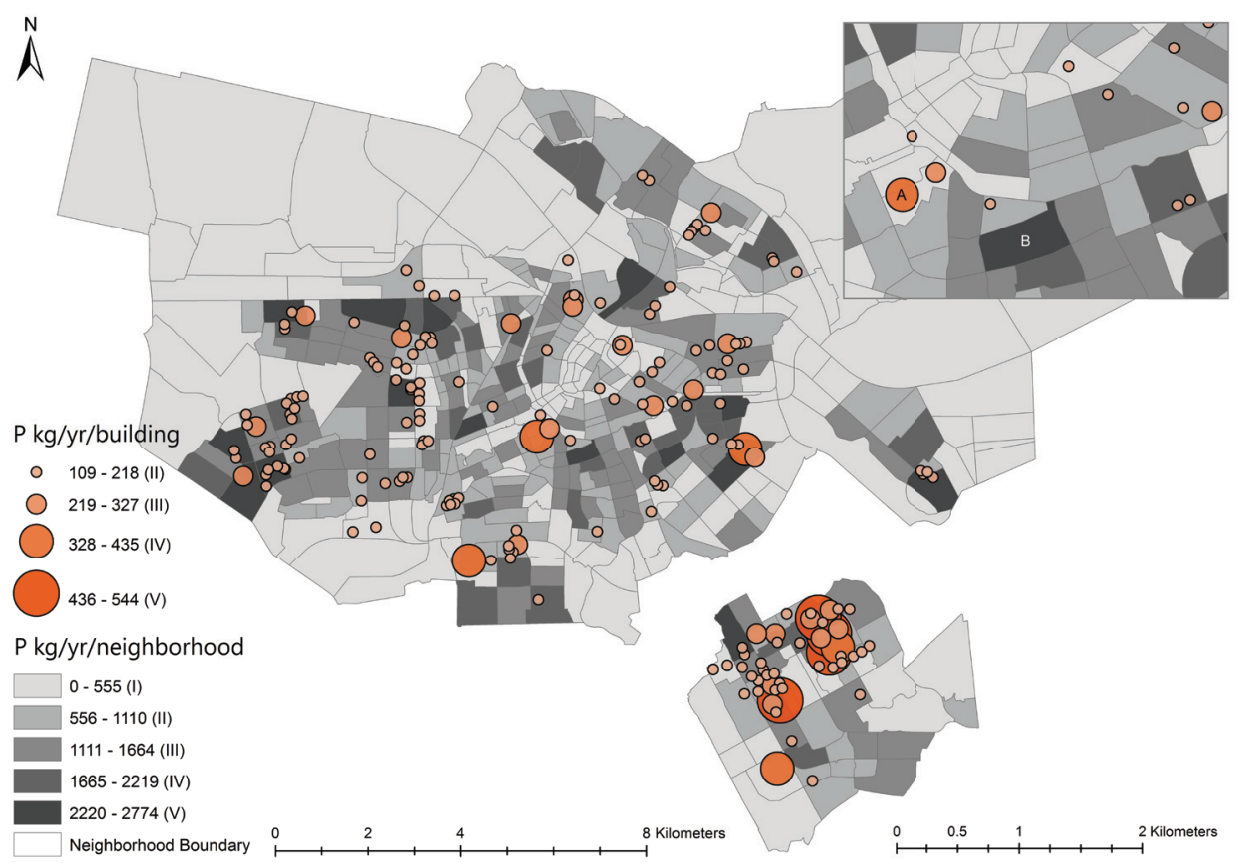

Figure 5.6. Building and neighborhood phosphorus loads. The inset map clearly shows the overlay of building and neighborhood loads: Buildings with high phosphorus loads don't necessarily fall in neighborhoods with high phosphorus loads (A) and vice versa, neighborhoods with high phosphorus loads don't necessarily contain buildings with high phosphorus loads (B).

same interval load classes with the exception of a few. These exceptions can be attributed to: (1) the manner of demarcation of the interval load classes which resulted in some loads being pushed into the next interval class, and (2) the ratio of urine to feces excreted at each location and the resulting nutrient ratios. Meanwhile, the value range within each interval class is large and therefore the variation in the proportion of $\mathrm{N}$ to $\mathrm{P}$ to $\mathrm{K}$ loads for each location is not easily deducible by comparing Figures 5.6, and Figure S6.2 and Figure S6.3 (Supporting Information VI); two locations identified in the same $\mathrm{N}$ interval class and same $\mathrm{P}$ interval class can have different $\mathrm{N}$ to $\mathrm{P}(\mathrm{N}: \mathrm{P})$ ratios. A calculation of nutrient ratios increases clarity of proportional loads of nutrients. Figure 5.7 indicates the N:P ratio for the identified hotspots. The combination of the N:P ratios with the $\mathrm{P}$ load profile in the figure reveals the relevance of depicting nutrient ratios. For example, building 1 and 2 have P loads that classify in the same interval (Group I), however, building 1 has a high N:P ratio whereas building 2 has a smaller N:P ratio. High N:P ratios also occur in other interval classes, e.g., building 3 shows a high ratio with $\mathrm{P}$ load in Group III. Building 4 has a large P load (Group V), although a small N:P ratio, indicating its primarily residential function. Another combination of N:P ratio and P load is shown by building 5 . 
Especially office buildings, schools and public institutions have higher N:P ratios. N:P ratios serve as an indication of whether relatively more urine (N:P ratio 10.4) versus feces (3) are excreted. While overall human excreta has an N:P ratio of 7.9 (refer to Table 4.1), the ratios at the building hotspots varied between 7.5 to 10.1 .

The nutrient load profiles across neighborhoods were mostly similar, that is, neighborhoods fell into the same interval load classes for N, P and K. A few neighborhoods were pushed into the next interval category because of the demarcation of the interval load classes and not because the nutrient ratios at those locations were significantly higher or lower. The similarity in interval categorization shows that certain neighborhoods have consistently higher nutrient loads than others. While total $\mathrm{N}$ to $\mathrm{P}$ load ratios for most neighborhoods is equal to or lower than that of human excreta (7.9:1), for neighborhoods that are predominantly residential, $25 \%$ of the neighborhoods had ratios higher than that of human excreta.

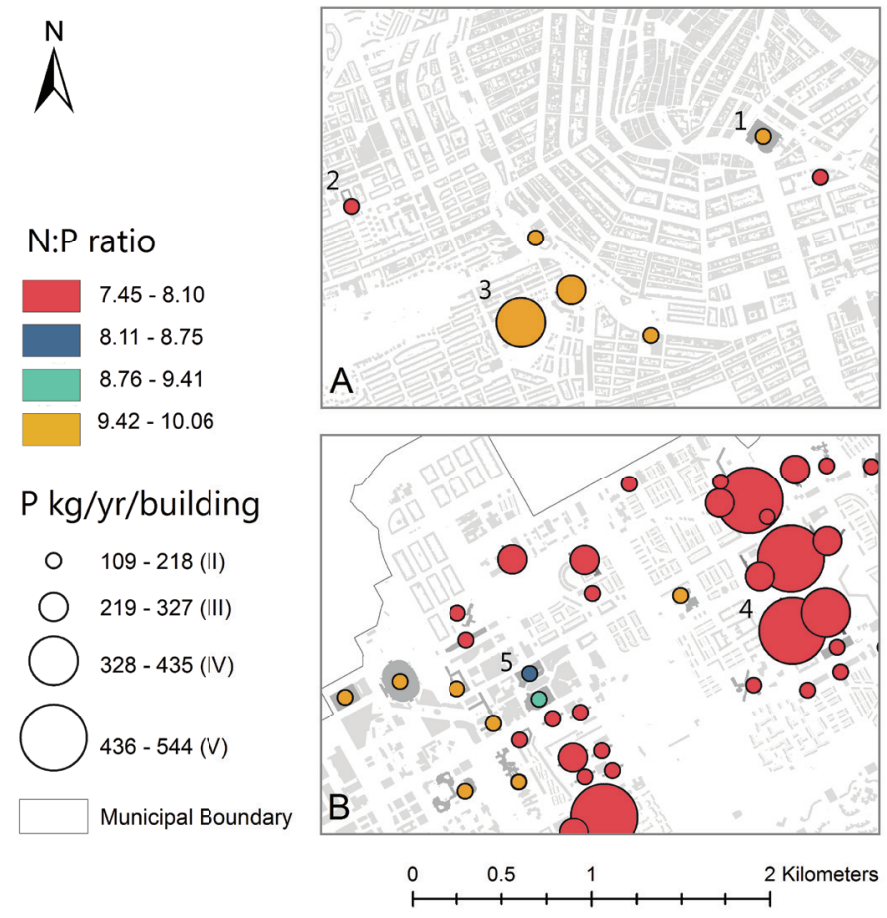

Figure 5.7. Combination of nitrogen to phosphorus (N:P) ratios for the identified building hotspots (color scheme) and the nitrogen loads (circle size). Building 1 and 2 (map A) have phosphorus loads that classify in the same interval class (Group I), however, building 1 has a high N:P ratio whereas building 2 has a small N:P ratio. High N:P ratios also occur in other interval classes, e.g., building 3 (map A) shows a high ratio with phosphorus load in Group III. Building 4 (map B) has a large phosphorus load (Group V), although a small N:P ratio. Another combination of N:P ratio and phosphorus load is shown by building 5 (map B). 


\section{Discussion}

\subsection{Spatially explicit inventories of nutrient loads}

The method developed allows for a spatially explicit visualization of nutrient loads, applied here to the city of Amsterdam. By decreasing the level of spatial abstraction inherent to nutrient flow diagrams, the method developed here can better inform decision makers in the planning of next steps. With straightforward data processing to remove buildings in the lowest interval class(es), locations with higher loads are easily visible on the maps, locating low hanging fruit for the implementation of nutrient recovery and recycling strategies. Particularly the representation of nutrient hotspots, as opposed to population density maps or rasterized data per area, has the advantage of accounting for and differentiating between nutrient compositions in urine versus feces, and also accounts for excretion away from home. The differences in N:P ratios attest to the value of considering nutrients individually and differentiating between urine and feces excretion.

The nutrient hotspots identified in our study indicate that there is potential to improve $\mathrm{P}$ management in Amsterdam by targeting low hanging fruit. The 193 buildings identified as nutrient hotspots, $0.1 \%$ of the buildings included in this study, together produce 32.5 tons of $\mathrm{P}$ annually, $10 \%$ of the city's annual load of 330.5 tons. The sum for $\mathrm{N}$ hotspots, and $\mathrm{K}$ hotspots, indicate similar percentages, meaning that the implementation of new sanitation systems at these locations would already contribute considerably to the nutrient recovery in the city of Amsterdam, while the economics of scale are more favorable. This demonstrates the added value of looking at the building scale. However, the rate of nutrient load increase accelerated at higher interval classes and the wide variation affects cost effectiveness of recovery as well as technology choice per location.

The function of the building is particularly important for understanding the composition of the wastewater and an appropriate sanitation system for the location. While feces are mostly excreted at home, at office buildings and institutions urine is predominantly excreted, according to the partition of excreta between home and away from home (Wijst and Groot-Marcus, 1998). This distinction in function allows for some assumptions about the characterization of the streams from which nutrients can be harvested. Most of the identified hotspots were residential buildings. These locations will have a mixed composition of urine and feces and grey water, requiring sanitation systems for the treatment of and recovery of nutrients from mixed and diluted household water. Large renovations of residential buildings provide an opportunity to implement vacuum collection and transport of separated, concentrated black water. For the case of office buildings or museums, collecting urine separately via (waterless) urinals for men (and even urine deviating toilets for women) would allow for the implementation of nutrient recovery technologies from urine. Urinals are more feasible at locations such as office buildings and museums because of the higher percentage of urine excreted compared to feces and available 
space; in homes, a urinal would be less appropriate. The review by Harder et al. (2019) provides an overview of the possible technologies and recovery pathways that are possible starting from either blackwater or urine.

The mapping of nutrient loads and hotspots at two geographical scales showed noteworthy value, as has been emphasized previously by others (Chowdhury et al 2014.) The assumption that buildings have one outlet pipe that connects them to the main sewer lines was the motivation behind mapping nutrients at this resolution; the one outlet pipe becomes the point for intervention for new sanitation systems, especially at buildings with high nutrient loads. Buildings with low nutrient loads might require grouping to then consider the implementation of new sanitation systems for building conglomerations. The maps at neighborhood scale show results by aggregating the building data, in this case defined by an administrative boundary. In fact, the aggregation of data for some neighborhoods, including neighborhoods that did not contain a building hotspot, caused them to classify in the highest load class. The maps for nutrient loads at neighborhood scale are useful for situations wherein the municipality of Amsterdam would, for example, need to renovate or replace sewer infrastructure in a neighborhood and decide whether to employ alternative sanitation systems in the area. Luckily, these are among some of the smaller neighborhoods in terms of area, most likely requiring fewer kilometers of piping. Surprising was that some neighborhoods that contained building hotspots fell into lower load classes. Here the overlap of building and neighborhood hotspot maps attest the value of presenting the data at each scale when selecting appropriate locations for nutrient recovery systems and infrastructure renovations.

\subsection{Data limitations and method refinement}

The maps were deliberately mapped using vector data as opposed to raster data because of the benefit of calculating nutrient loads per urban boundary, building or neighborhood, as opposed to a raster grid, for which the quality of the results are subject to the resolution of the raster grid (Spiller and Agudelo, 2011). Many previous studies have chosen a raster grid to map resource flows because it allows for the combination of input data at varying spatial scales which are either aggregated or disaggregated per raster cell ((Batty et al., 2004, Metson et al., 2012, Metson et al., 2018). Using vector data, however, keeps results together per structural unit, aiding the identification of actual intervention points.

The new method is still subject to various assumptions and the resolution of the results is as high as the lowest resolution of the input data. For example, increased accuracy on nutrient excretion and time-use data would change nutrient load values calculated per building and neighborhood. The question is how much and would the identified nutrient hotspots shift to other locations. Sensitivity analyses were not within the scope of the current paper, but we discuss the items that are relevant for such analyses. For the city of Amsterdam we were able to collect data on number of people registered as an inhabitant or employee in each building, however, to increase 
the accuracy of nutrient excretion at each location, population demographics would have made it possible to account for variance in nutrient excretion and frequency as is known for different age groups, for example. While age demographics are known at neighborhood scale, it was not available to us at building scale, and there may be ethical limitations to obtaining such data as well. Again increased data heterogeneity to account for age demographics would allow for differentiation across the time use data to determine how much time people spend engaged in different activities, and thus at different locations, per age group. For instance, a younger person spends more time away from home (at school, at work or engaged in leisure activities) than an elderly person. If age demographics are known, age specific value for time use can be used instead of average values.

Toilet use patterns, excretion frequency and nutrient concentrations were assumed to be constant per person and over time, with the exception of frequency of excretion during hours of sleep. However, toilet use is not only dependent on the amount of time spent at a location, as we assume, but also depends on consumption of food and water, personal preference (comfort of own home, bathroom hygiene), age (as discussed), access to a toilet, etc. Moreover, toilet use is a discrete event, and while we assume a frequency of 0.3 times per hour, in a period of three hours a person might either go 0 times (they went to the bathroom elsewhere before and will go elsewhere afterwards), 1 time, or 2 times (at the beginning and at the end of the three hours). An increase in the number of consecutive hours people spend at one place will most likely also increase the probability that people use the bathroom at that place.

Last, certain input data was not attainable. For example, the number of patients that visit or are admitted to hospitals, and the length of their stay, hotel guests and their length of stay and time spent at the location, as well as restaurant goers, sport center visitors, and those engaged in other leisure activities were not included in the dataset. However, the dataset could be easily updated to include this information, provided that these data remain anonymized. In addition, large events and national holidays, which include large numbers of people were also not accounted for in the calculations. Since these events are not necessarily associated with specific buildings, it would be difficult to attribute a location and time stamp to the toilet use of the many party-goers. The use of mobile toilet units at such events, however, already provides source-separated collection of urine, or black water.

\section{Outlook}

The benefit of the method developed here is its flexibility for further refinement depending on the resolution of available input data as well as its ability to integrate new input data appropriate per context. The context for which this method is used and motivation of a study determines the hotspot definition selected, along with the generation of the respective maps. We chose to divide the data values into five equal intervals, each interval including $20 \%$ of the data values. The uneven distribution of our data quickly led us to focus on the data excluding the lowest load 
class. However other possibilities to define a hotspot exist. A quantile classification would allow for the selection of a percentage of buildings with the highest nutrient loads, for example, by classifying the top $1 \%$ of buildings with the highest nutrient loads as nutrient hotspots. Another option would be to define a threshold load value, hotspots would include buildings with loads higher than the threshold. If a threshold becomes known above which recovery becomes costeffective, then these can be easily identified.

The chosen neighborhood scale was an arbitrary boundary selection to show the possibility of determining hotspots through the aggregation of data. However, other manners of aggregating data exist. Using GIS, data can be aggregated within radial distances or by rasterized grid. In this way conglomerations of buildings, within a defined proximity, can be identified which together have a high nutrient load. Another possibility would be to draw boundaries around 'sewage catchment areas' or areas with connected smaller sewage infrastructures whose waste then join the larger sewage network at one outlet. At these pipe outlets wastewater could be diverted to new sanitation systems.

The value of a hotspot analysis is to visualize large nutrient loads relative to others. This is considered the first step to be able to determine the viability of sanitation interventions at certain locations. Maps such as the ones produced in this study can inform management decisions, aiding decision makers in determining next steps that need to be undertaken and in creating planning capacity (Metson et al., 2018). After all, the actual suitability to recover nutrients and select appropriate recovery technologies depends on various criteria. Follow up studies can, for instance, include modeling toilet flush water (and greywater) to determine dilution and respective nutrient concentrations. Recovery of nutrients is more effective at higher concentration (Zeeman and Kujawa-Roeleveld, 2011a). While most nutrient hotspots are residential, greywater from bathing, laundry and cooking also dilutes the wastewater stream. Office buildings, with no or few showering facilities or kitchens, can be expected to have higher nutrient concentrations, though generally applied flush toilets cause considerable dilution of feces and urine. With data from showering intensity and per capita water consumption, similar mapping can be done for concentrations as for loads. Likewise, expanding input data to include contaminants such as pharmaceuticals, heavy metals and hormones helps to assess quality parameters. Keeping locations with higher contaminant loads such as elderly homes and hospitals separate, could improve the quality of collected wastewater, and the respective recovered products.

The spatial representation of the hotspots can be used to further model other spatial aspects such as available area to house new sanitation systems and distance between nutrient supply and demand. Using the GIS analysis, the available space at the locations suitable for the installation of treatment and recovery technologies can be mapped. Vacant land, parking spaces, available basements or sturdy rooftops could all be considered for such an assessment. Lastly, the results from our study can be paired with a reverse logistics analysis to assess the distance between the nutrient hotspots (supply) and locations where harvested nutrients can be reused (demand), 
such as at urban, peri-urban or rural farms. Depending on the demand for fertilizer types from farms, the appropriate new sanitation systems can be determined, followed by calculations of the distance from the points of recovery to land application and the respective transport costs.

The spatially explicit inventory of nutrient loads and hotspots presented in this study at varying spatial scales is the first step in quantifying the recycling potential of nutrients in human excreta. With this we have identified low hanging fruit for increased recovery in the city of Amsterdam. Method refinement and expansion can further increase its usefulness for informing decision management and development plans for the recycling of nutrients to agricultural fields.

\section{Acknowledgements}

The authors thank Tony Dashorst, at the Municipality of Amsterdam for providing valuable input data for the developed method. 


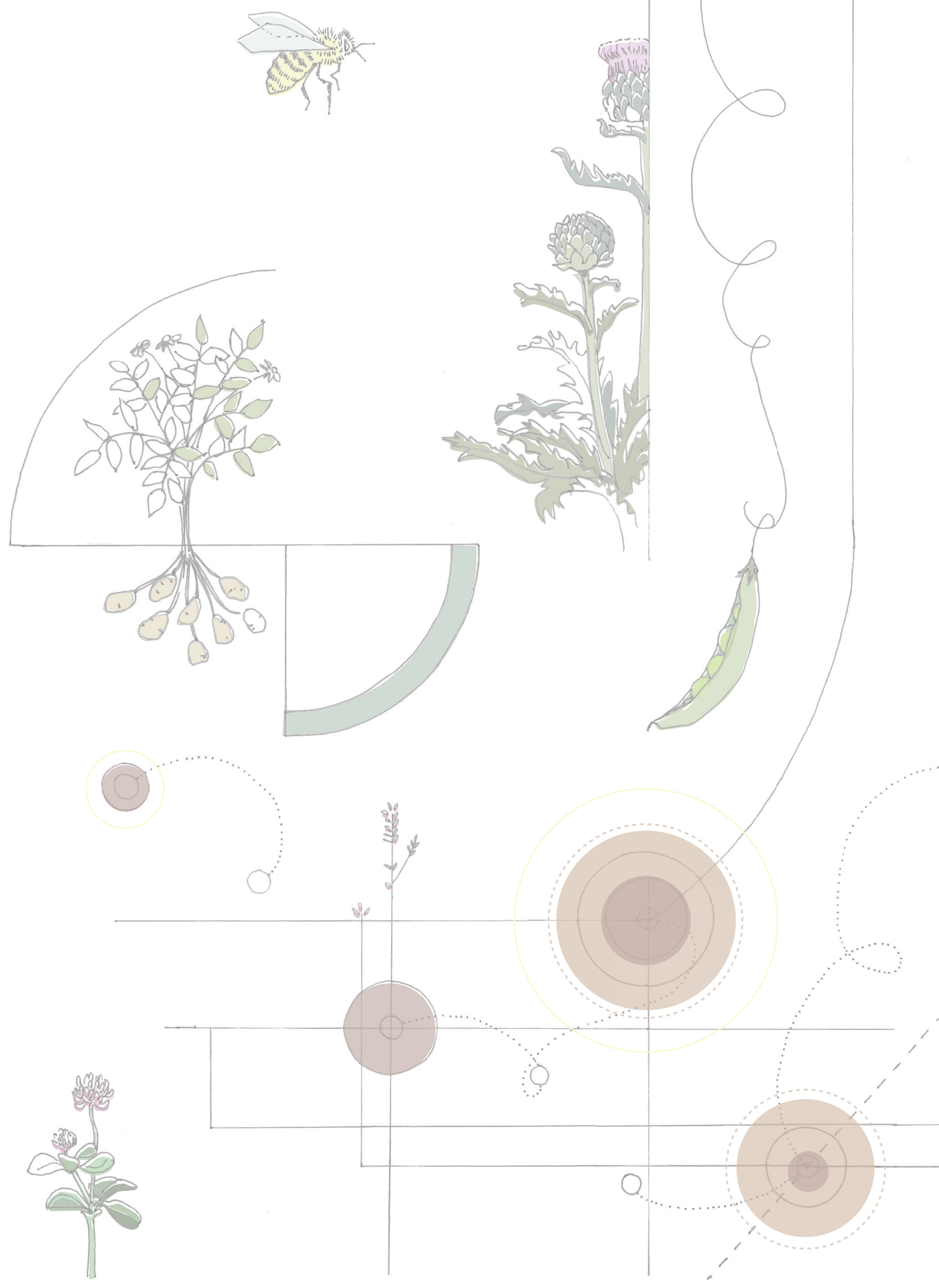




\section{Abstract}

Buildings in cities represent potential point sources of human excreta-derived nutrients, while agricultural land in (peri)urban areas demand nutrients. Here we present a new geographic information system (GIS)-based modelling tool, Resource Dynamo, that optimizes the allocation of excreta-derived nutrients from buildings in cities to nearby agricultural fields. The model minimizes the number of supply sites needed to match the demand by prioritizing sites with the highest nutrient supply, i.e., the hotspots. Furthermore the model delineates exact transport routes between the discrete supply and demand sites. The transport routes are minimized for transport costs as transport costs are a key factor for economic viability of nutrient management. The high resolution of the model allows it to function as a decision-support tool for bringing cyclic nutrient management into practice. To disclose the potential of the model, we used it to match phosphorus supply in human-derived urine with phosphorus demands from agricultural fields within the municipality of Amsterdam on a temporal scale of 1 year.

Keywords: nutrient recycling; geographic information systems (GIS); optimization; resource recovery

This chapter has been submitted for publication as: Wielemaker, R., Wilken, C., Chen, W.S., Oenema, O., \& Weijma, J., Resource Dynamo: A GIS model to match urban nutrient supply with agricultural demand 


\section{Introduction}

The transition to a circular nutrient economy, including the recirculation of human excretaderived (hereafter referred to as excreta-derived) nutrients to agriculture, will require a shift in wastewater management (Guest et al., 2009, Kennedy et al., 2012, Wilsenach et al., 2003). Human excreta comprises the majority of the nutrients present in domestic wastewater, and most of these are currently irretrievably lost (Egle et al., 2016, Kujawa-Roeleveld and Zeeman, 2006). Innovative sanitation and wastewater management solutions offer opportunities to recycle excreta-derived nutrients in the form of recovered fertilizer products (Haddaway et al., 2019, Harder et al., 2019). Recycling these products back to agriculture requires first and foremost a matching of product quantity and quality with agricultural demand; however, consideration of spatial and temporal dimensions of nutrient recirculation is equally important (Nicholson et al., 2012, Wielemaker et al., 2018a). A commonly noted barrier for the recirculation of excreta-derived nutrients to agriculture is the relatively high cost of transportation, given that the production of human excreta is predominantly urban (cities concentrate 55\% (2018) of the world's population) while agriculture is largely rural (United Nations, 2018). The (long-distance) transport of bulky products between urban supply and rural demand sites may limit the economic feasibility of nutrient recycling (Keplinger and Hauck, 2006). Several studies have sought to reconcile spatial dimensions of nutrient flows including: Akram et al. (2019a), Akram et al. (2019b), Chowdhury et al. (2016), Chowdhury et al. (2018), Cordell et al. (2012), Metson et al. (2018), Nicholson et al. (2012), Parchomenko and Borsky (2018), Trimmer and Guest (2018), Wadsworth et al. (2018). However, the applied geographic resolution is coarse in these studies, i.e., on city or regional level (Chowdhury et al., 2018), or resolution is increased by disaggregating aggregated data over a grid (Akram et al., 2019b). While these studies are valuable to indicate broad trends at system level, the use of aggregated data over entire areas is insufficient for scenario development and decision-making processes at executive level: where to collect nutrients and where to use these for crop growth. Instead of connecting 'areas' of supply and demand, as in previous studies, a tool that identifies economically promising points (buildings) of nutrient supply and minimizes transport distances between these discrete points of supply and nearby agricultural fields is useful for practitioners.

We developed a geographic information system (GIS)-based model (Resource Dynamo), that determines minimum transport distances between discrete locations of urban nutrient supply and locations of nutrient demand. Unique to this model is that it uses the highest possible resolution of the spatial dimension of nutrient supply and demand: nutrient supply per building is matched with the nutrient demand of individual agriculture fields in and around that city. Identifying the supply per building (e.g., house, apartment block, office, hospital) was a deliberate choice as these normally have one piping system to collect and transport the produced wastewater, and this piping system may serve as a point of intervention for nutrient recovery. 
The model provides calculations and visualizations of transport distances between available supply and actual demand along the shortest route on existing roads. This required an iterative approach to negotiate three objectives: (1) match nutrient supply and demand quantities, (2) minimize the distance between supply and demand sites, and (3) minimize the number of supply sites needed to meet the demand. This latter objective holds that the recovery of nutrients from human excreta should optimally first occur at supply sites with large nutrient loads, in order to reduce costs for the collection, storage and treatment of human excreta and the recovery of nutrients (Wielemaker et al., in press). Supply sites with large loads, nutrient hotspots, are given a high priority as points of intervention.

Here we present first results of Resource Dynamo for allocating phosphorus from human urine collected in buildings to agricultural fields according to their phosphorus demands, within the municipal boundary of Amsterdam. We made the deliberate choice to focus on urine since it contains by far the largest fraction of nutrients expelled with domestic wastewater in only a fraction $(1 \%)$ of the total flow, and it can be easily separated at source via urine-diverting toilets or urinals (Karak and Bhattacharyya, 2011, Kujawa-Roeleveld and Zeeman, 2006, Maurer et al., 2003d, Spångberg et al., 2014, Udert et al., 2006a). Moreover, urine is touted as a viable alternative for fertilizers because the nitrogen and phosphorus is plant-available in the form of ammonium and orthophosphate (Spångberg et al., 2014). The demand site included three types of agricultural typologies: urban agriculture (2016), cropland, and grassland (2018), using respective fertilizer recommendations for each. The inclusion of urban and peri-urban agriculture is unique, as previous studies have focused on rural agriculture (e.g., Akram et al. (2019b), Nicholson et al. (2012), Trimmer and Guest (2018), Wadsworth et al. (2018)). The focus on phosphorus is because current phosphorus fertilizer production depends on non-renewable, regionally concentrated supplies of phosphate rock, and phosphorus management is important with regards to food security and environmental protection (Cordell and White, 2011).

\section{Methods}

\subsection{Define nutrient demand}

The nutrient demand in the municipality of Amsterdam was calculated and modelled with the use of the geographic information system ArcMap (Version 10.4.1). The nutrient demand is the product of the cultivated agriculture area and the fertilizer recommendations per area. Three types of agriculture were included: urban agriculture, cropland, and grassland. The area of urban agriculture in the city of Amsterdam was derived from municipal data (Gemeente Amsterdam and Ruimte en Duurzaamheid, 2016) and corrected to account for non-farmed area (e.g., buildings and paths). Based on exact parcel area information found on 27 out of 44 urban farm websites, an average of $45 \%$ of the total area is cultivated. The area of cropland and grassland was derived from the shapefile 'Basisregistratie Gewaspercelen (BRP)' from the 'Publieke Dienstverlening 
Op de Kaart' (PDOK, 2018). In total, there are 141.0 ha of urban farms, 94.6 ha of cropland, and 1907.1 ha of grassland within the municipal boundary of Amsterdam.

To assign appropriate demand to urban agriculture farms, cropland and grassland, we used soil phosphorus concentrations ( $\mathrm{Pw} / \mathrm{P}-\mathrm{AL}$ ) and assumed respective legal phosphorus allowances. These allowances are based on guidelines for allowed phosphate application rates (valid in the Netherlands from 2020 onwards) reported in the 'Zesde Nederlandse actieprogramma betreffende de Nitraatrichtlijn (2018 - 2021)' (Ministerie van Landbouw and Waterstaat, 2017). In the context of the Netherlands, with a long history of phosphorus-saturated soils (CBS et al., 2019), phosphorus allowances based on soil phosphorus content was considered most appropriate. Soil phosphate concentrations (expressed as either $\mathrm{Pw}\left(\mathrm{mg} \mathrm{P}_{2} \mathrm{O}_{5} / \mathrm{L}\right)$ concentration for farmland or P-AL(mg $\left.\mathrm{P}_{2} \mathrm{O}_{5} / \mathrm{L}\right)$ concentration for grassland) and are divided into 5 classes: these range from 'poor/phosphate fixing' $\left(<25 \mathrm{Pw}\left(\mathrm{mg} \mathrm{P}_{2} \mathrm{O}_{5} / \mathrm{L}\right)\right.$ or $\left.<16 \mathrm{P}-\mathrm{AL}\left(\mathrm{mg} \mathrm{P}_{2} \mathrm{O}_{5} / \mathrm{L}\right)\right)$ to 'high' (> $55 \mathrm{Pw}\left(\mathrm{mg} \mathrm{P}_{2} \mathrm{O}_{5} / \mathrm{L}\right)$ or $\left.>50 \mathrm{P}-\mathrm{AL}\left(\mathrm{mg} \mathrm{P}_{2} \mathrm{O}_{5} / \mathrm{L}\right)\right)$. The corresponding allowances for phosphorus application based on these concentrations range from $120 \mathrm{~kg} \mathrm{P}_{2} \mathrm{O}_{5} / \mathrm{ha}$ (cropland and grassland) for 'poor/phosphate fixing' soils to $40 \mathrm{~kg} \mathrm{P}_{2} \mathrm{O}_{5} / \mathrm{ha}$ (cropland) and $75 \mathrm{~kg} \mathrm{P}_{2} \mathrm{O}_{5} /$ ha (grassland) 'high' phosphorus-rich soils (Ministerie van Landbouw and Waterstaat, 2017). A complete overview of the guidelines can be found in (Table S7.1 and Table S7.2, Supporting Information VII). Soil phosphate concentrations per postal codes (measured between 2015 and 2018)(Kros et al., 2019) were attributed to cropland and grassland sites located in the respective postal codes. The $\mathrm{Pw}\left(\mathrm{mg} \mathrm{P}_{2} \mathrm{O}_{5} / \mathrm{L}\right)$ concentration of cropland ranged between 13-61 $\mathrm{Pw}(\mathrm{mg}$ $\left.\mathrm{P}_{2} \mathrm{O}_{5} / \mathrm{L}\right)$ and the concentrations of grassland soil between 25-91 P-AL $\left(\mathrm{mg} \mathrm{P}_{2} \mathrm{O}_{5} / \mathrm{L}\right)$. Since not all cropland or grassland areas in Amsterdam had a measured phosphate concentration, these agriculture sites without $\mathrm{Pw}$ or PAL values were assigned the highest class $\left(55 \mathrm{Pw}\left(\mathrm{mg} \mathrm{P}_{2} \mathrm{O}_{5} / \mathrm{L}\right)\right.$ and $50 \mathrm{P}-\mathrm{AL}\left(\mathrm{mg} \mathrm{P}_{2} \mathrm{O}_{5} / \mathrm{L}\right)$ ), as per the normal procedure in the Netherlands. Following these recommendations for phosphate application, the nutrient demand per parcel was calculated according to respective parcel areas.

Urban farms in the Netherlands have been found to generally over fertilize soils with phosphorus, as nutrient management remains unmanaged (Wielemaker et al., 2019). Since no information was available about the actual phosphate concentration of the soil at urban farms, the same approach of assigning the highest $\mathrm{P}$ saturation for the soil class was used. Since urban farms are more similar to cropland, the value of the highest class for cropland ( $>55 \mathrm{Pw}\left(\mathrm{mg} \mathrm{P} \mathrm{P}_{2} \mathrm{O}_{5} / \mathrm{L}\right)$ was assigned to the urban farm plots. To identify demand points in the transport-distance analysis, the polygon fields are transformed to points (at the centre of each polygon).

A description of assumptions and specifications, as well as the input data used for the model can be found in Table S7.3 (in Supporting Information VII). 


\subsection{Define nutrient supply}

All buildings in which people reside, work, study, or visit are taken as potential supply sources of nutrients in the city of Amsterdam. (Wielemaker et al., in press) quantified nutrient loads per building in Amsterdam over one year based on the number of individuals working, residing, studying, and visiting per building (Gemeente Amsterdam, 2016, OIS, 2016, OAM, 2016), the number of hours individuals spend within buildings (CBS, 2014, Cloïn et al., 2013), based on time use data, urine nutrient composition (Meinzinger and Oldenburg, 2009) and the frequency of urination per hour and number of urination events per day (Rose et al., 2015a) (See Table S7.3, Supporting Information VII). Based on this previous study we retrieved data on urine production per building and respective phosphorus loads. Supply points with the largest loads, termed hotspots, were selected first to match a certain demand in descending order, to later match with the demand points. The reasoning here is that infrastructural adjustments for the collection, treatment and recovery of nutrients should be kept to a minimum.

\subsection{Levels of demand coverage}

To begin, a fixed demand coverage (viz. 10-20-40-100\%) was matched with an equal supply to identify the number of supply points needed (largest hotspots in descending order) to cover the demand. We executed this in phases (scenarios) to show the gradual increase in necessary supply points with an increasing demand from agriculture. We first selected a demand coverage of (1) $10 \%$, (2) $20 \%$, (3) $40 \%$ and finally (4) $100 \%$. The selection of incremental stages of demand coverage was intentional to parallel a stepwise approach to the transition to closing phosphorus cycles and support decision-making. It is further assumed that each supply point has storage capacity for the total volume of urine produced. It is also assumed that the storage facility contains proper measures to store and sterilize the urine for a six month period before its application as a fertilizer, as is general practice when working with urine in agriculture.

\subsection{Transport distance between demand and supply}

To match demand and supply, the closest demand to a supply point was determined with the use of the 'Network Analysist' toolbox of ArcMap, with the function 'OD Closest Facility'. For the objective of this study, the existing road network (Geofabrik, 04-2019) was first filtered to exclude roads unsuitable for truck transport (e.g., bike paths). The tool uses the road network to calculate the most optimal route (thus taking main roads preferably) between all supply and all demand points. The results are stored in a table with one row per connection between a supply and demand point and a column that indicates the total travel distance between the two points. This table was exported to an Excel file for data analysis using the python interface IDLE (Python Version 2.7.10). A python script then calculated the amount of urine transported from each supply point to any demand point, based on the shortest distance. It is assumed that the application of urine to the fields occurs only once a year in the spring, by being transported 
directly from each hotspot to the demand sites.

The python scrips first sorted the Point-Distance table based on minimum distance, so that demand points closer to a supply point will be favoured in the calculation. Then the script checked whether the supply and demand at each match was larger than 0 . If both were not 0 , the script executed the calculation row by row (top to bottom), based on 3 situations:

1. Supply at point $X$ is smaller than demand at point $Y$ : In this case it is assumed that the total amount of urine supplied at point $\mathrm{X}$ is transported to point $\mathrm{Y}$. Afterwards, the values in the whole table for point $\mathrm{X}$ and $\mathrm{Y}$ are updated for the rows thereafter with the use of a lookup dictionary. In this scenario, the supply for point $\mathrm{X}$ is 0 afterwards and the demand of point $\mathrm{Y}$ is reduced by the amount of point $\mathrm{X}$. The advantage of updating the table using a lookup table is that double calculations are prevented. Even so, the table contains as many rows per supply point as existing demand points (in our case every supply point has 193 records stored in that table, summing up to $282,000+$ rows), that supply point can only supply as many demand points until its supply volume is 0 . Therefore; it is crucial to update the values for each point with the new/reduced volumes present at that point.

2. Supply at point $X$ is larger than demand at point $Y$ : In this case it is assumed that the amount of urine transported from point $\mathrm{X}$ equals the amount of the demand at point $\mathrm{Y}$. Afterwards, the values in the whole table for point $\mathrm{X}$ and $\mathrm{Y}$ are updated, so that the supply for point $\mathrm{X}$ is reduced by the demand of point $\mathrm{Y}$ and the demand at point $\mathrm{Y}$ is 0 .

3. Supply at point $X$ is equal to demand at point $Y$ : In this case all the urine from point $X$ is transported to point $\mathrm{Y}$. Afterwards, both values for point $\mathrm{X}$ and $\mathrm{Y}$ are updated to 0 .

The result is another Excel table, that includes a new column ('Transfer') that stored the units of urine that is transferred between the supply ('Transfer_from') and demand points ('Transfer_ to'). For connections/rows where nothing has been transferred, the columns are left blank.

To show how such a re-use system of urine could look in practice, the number of trucks that could potentially deliver the urine from the supply to the demand points is calculated. It was chosen to include two types of trucks in this calculation: (1) with a volume of $35 \mathrm{~m}^{3}$ and (2) with $10 \mathrm{~m}^{3}$ for smaller or residual volumes. For that, the urine that is transferred between point $\mathrm{X}$ and point $\mathrm{Y}$ was transformed to volumetric values by taking average nutrient load of $\mathrm{P}$ in urine (0.8 $\mathrm{kgP} / \mathrm{m}^{3}$ (Meinzinger and Oldenburg, 2009). The script then checked how many times the resulting volume could be divided by 35 , to derive the number of trucks with a volume of 35 $\mathrm{m} 3$ needed (rounded down to whole numbers). If the remaining volume after this calculation lays between 10 and $35 \mathrm{~m}^{3}$, another truck of $35 \mathrm{~m}^{3}$ was added to the column of $35 \mathrm{~m} 3$ trucks needed. If the remaining amount was between $5-10 \mathrm{~m}^{3}$, or if the amount was between $5-10 \mathrm{~m}^{3}$ to begin with, a number of 1 was added to the column for the number of $10 \mathrm{~m}^{3}$ trucks needed. If the (remaining) volume was below $5 \mathrm{~m}^{3}$, no truck was calculated to pick up the urine. This threshold was decided to prevent an uneconomical transportation system with almost empty 
trucks. Based on that threshold the amount of urine that is actually transferred or left at the supply point could be calculated. The total number of all trucks was then used to determine the total transport distance needed to be covered between supply and demand points. The results of this calculation are afterwards imported back to ArcMap, to visualized the actual roads that are actually needed to supply the urine, by selecting only the connections in which urine has been transferred between point $\mathrm{X}$ and point $\mathrm{Y}$. In addition to that, the results are used to derive the actual demand points that are supplied in each scenario.

\subsection{Environmental impact and cumulative energy requirements}

Standard values for environmental impact $\left(\mathrm{CO}_{2}\right.$ equivalent) and cumulative energy requirements (MJ) were collected from the ReCiPe LCA Methodology database. For transportation, values obtained from the databased included impact and energy per kilometer travelled by a freight lorry of 16-32 metric tons and for a freight lorry of $>32$ metric tons. For fertilizer production, values obtained from the database included impact and energy requirements for the production per kilogram of phosphate fertilizer $\left(\mathrm{P}_{2} \mathrm{O}_{5}\right)$ including ammonium nitrate phosphate, monoammonium phosphate, single superphosphate, and triple superphosphate. Using the conversion of $\mathrm{P}_{2} \mathrm{O}_{5}$ to phosphorus, values were corrected for use in subsequent calculations. The obtained values were multiplied by the respective distances travelled by freight truck of $35 \mathrm{~m}^{3}$ and $10 \mathrm{~m}^{3}$ to measure total $\mathrm{CO}_{2}$ equivalencies and energy requirements per demand coverage; the obtained values for the production of phosphorus fertilizers was multiplied by the total agricultural demand for each level of demand coverage.

\section{Results}

\subsection{Demand and supply sites}

Most demand sites were located at the periphery of the municipality of Amsterdam. Urban agriculture included 43 community farms and plot gardens covering a total cultivated area of 142 ha. Cropland, for the cultivation of primarily winter barley, sugar beets, corn, wheat, potatoes, included 51 parcels spanning 95 ha. Grassland comprised by far the largest agricultural area (2025 ha) within the municipality, with 1420 parcels. The total demand for phosphorus for the three typologies was 75,465 $\mathrm{kg} \mathrm{P} / \mathrm{yr}$ with 2,470 $\mathrm{kg} \mathrm{P} / \mathrm{yr}$ for urban agriculture, $1,851 \mathrm{~kg} \mathrm{P} / \mathrm{yr}$ for cropland, and 71,144 kg P/yr for grassland.

The total phosphorus supply derived from human urine was 196,890 $\mathrm{kg} \mathrm{P}$ in 2015, distributed across 189,551 cadastral buildings in the municipality of Amsterdam. The supply per building was based on building population data (number of individuals that reside, work, study, or visit in each building) and nutrient excretion and toilet frequency estimations (Wielemaker et al., 2019). The phosphorus supply per building derived from urine ranged between 0-389 $\mathrm{kg} \mathrm{P} / \mathrm{yr}$ with the highest supply located at public institutions, office buildings and large apartment complexes. 
a

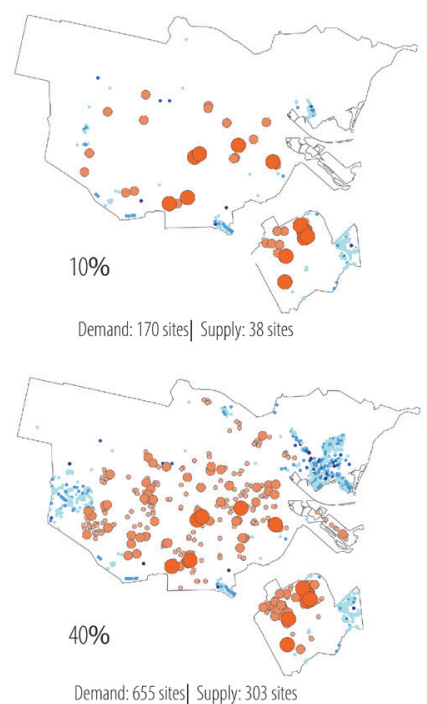

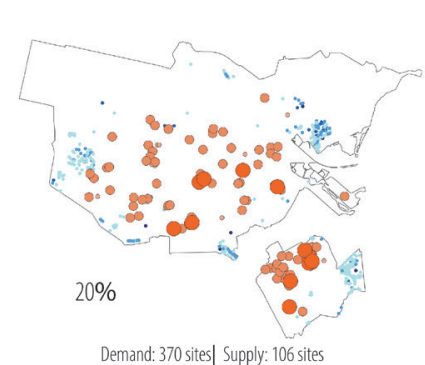

b Number of supply points needed to cover demand

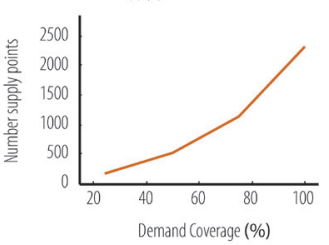

Phosphorus Supply (kg P/yr)

○ 1-100

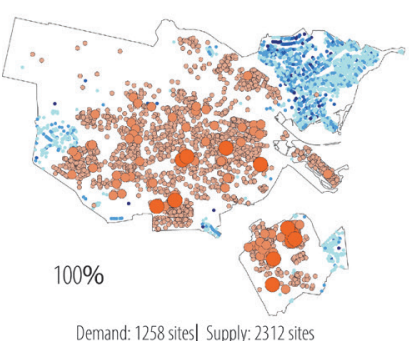

101-200

$200+$

Phosphorus Demand (kg P/yr/ha)

○ $0-20$

- 20-40

- $40+$

Municipal Boundary

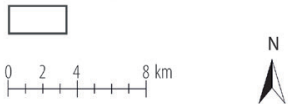

Figure 6.1 (a) Number and location of discrete urine phosphorus supply sites needed to fulfil 10\%, 20\%, 40\%, $100 \%$ of the phosphorus demand of agricultural land in the municipality of Amsterdam, (b) non-linear regression of the number of discrete supply sites needed to meet higher demand coverage.

The total demand $(100 \%)$ within the municipality of Amsterdam can be met with a mere 38\% of the available supply of phosphorus in urine. However, the minimum number of buildings, i.e., the ones with the highest phosphorus-supply, needed to meet the demand amounts to a staggering 2,312 (Figure 6.1 a). We suspect that the feasibility of collecting urine from such a large number of buildings, in the near future, is extremely low (Borsuk et al., 2008). To demonstrate a stepwise transition to a circular nutrient economy we show the results for matching $10 \%, 20 \%$, and $40 \%$ of the calculated phosphorus demand with supply from urine. Figure 6.1 $a$ indicates the number and location of the supply sites and the closest demand sites for each of these demand coverages. The non-linear regression of the number of supply sites needed to meet increasing demand coverage is shown in Figure 6.1b, with 38, 106 and 303 buildings needed to meet the $10 \%, 20 \%$ and $40 \%$ demand coverage.

\subsection{Transport distances and routes between supply and demand}

Resource Dynamo calculates and selects the shortest connections to cover the demand with the available supply. Figure 6.2 depicts all transport routes for the $10 \%$ scenario, linking the buildings, prioritizing sites with the highest nutrient supply (i.e., hotspots), with their respective, closest agricultural field. Routes are concentrated along main roads because the model prioritizes main roads over secondary and tertiary roads. Transport routes for the $20 \%$ and $40 \%$ scenarios are shown in Figure 6.3. 


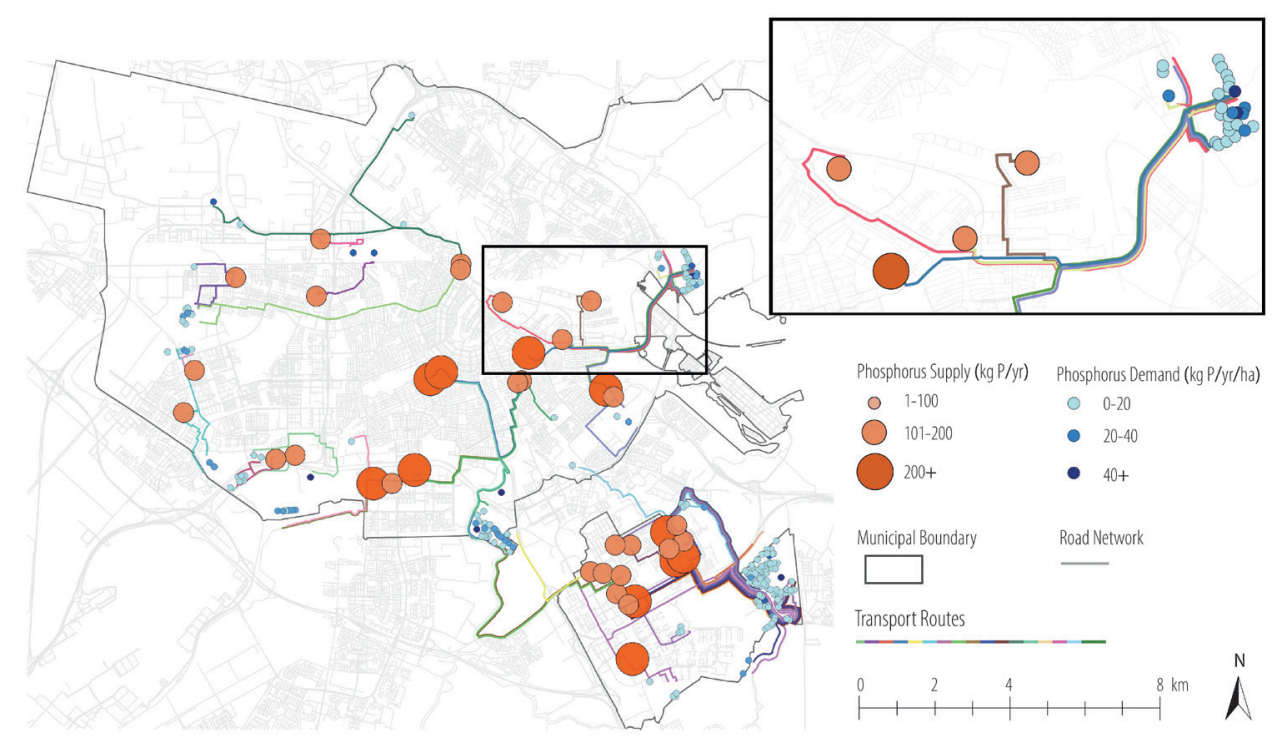

Figure 6.2 Supply and demand sites connected by respective transport routes for $10 \%$ demand coverage. The inset map shows the detail of the indicated area.
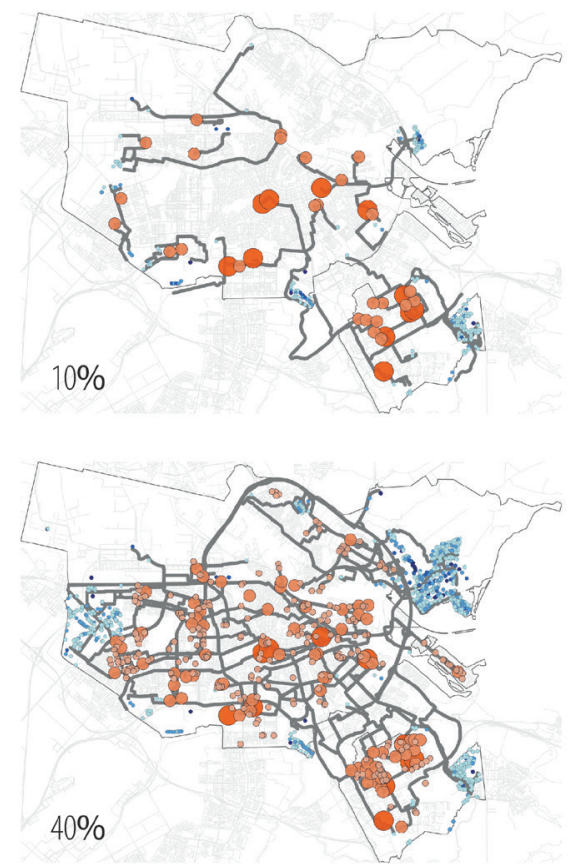

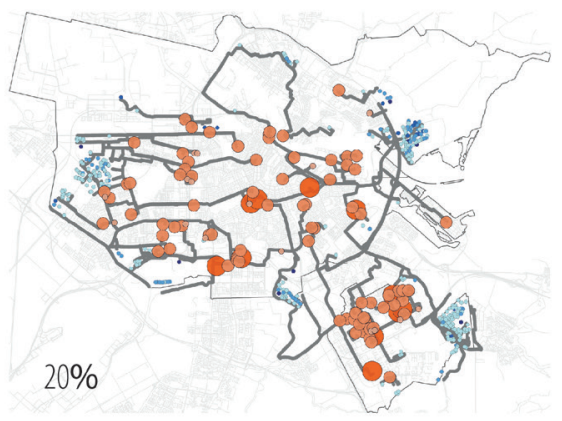

Phosphorus Supply (kg P/yr) Phosphorus Demand (kg P/yr/ha)
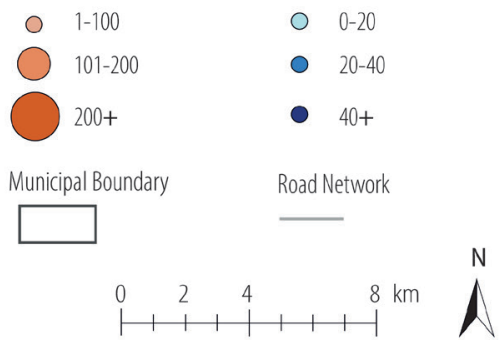

Figure 6.3 Transport routes between supply sites (buildings) and demand sites (agricultural fields) for 10\%, 20\% and $40 \%$ demand coverage. 

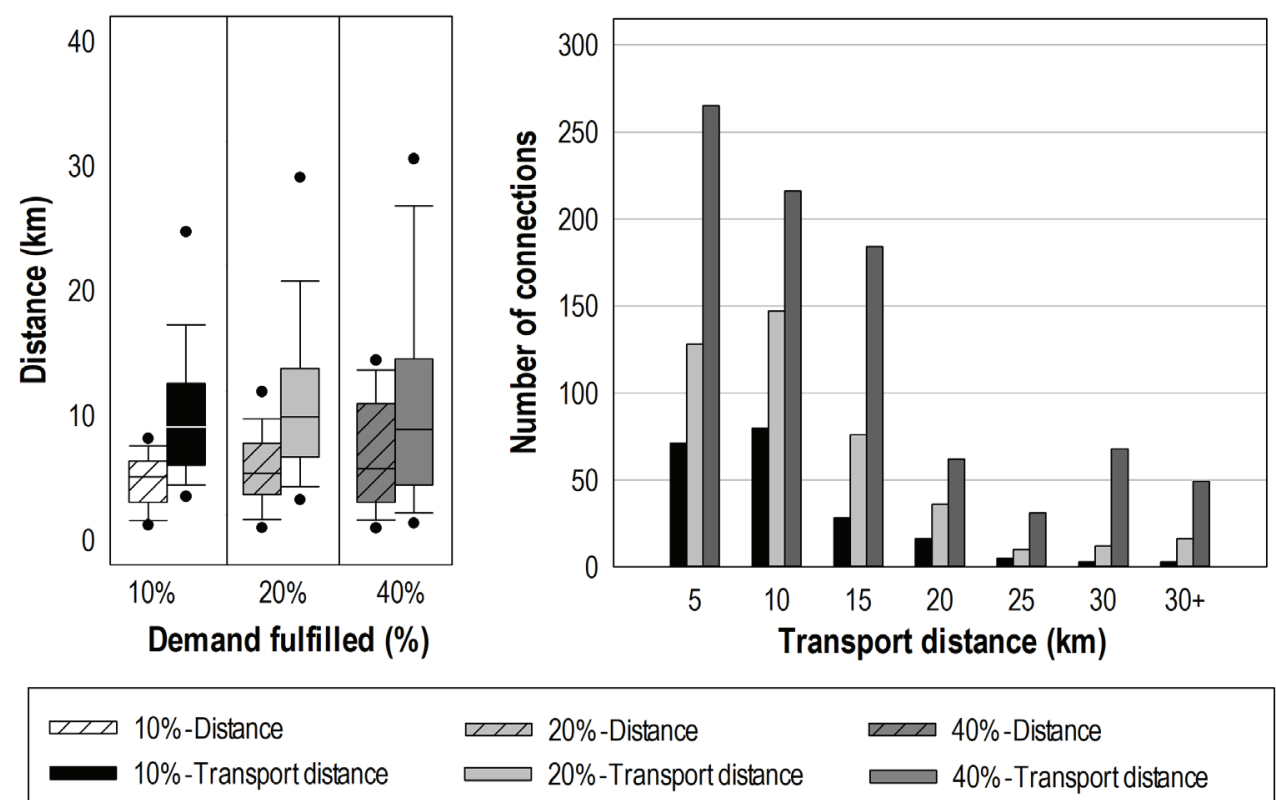

Figure 6.4 (a) Comparison of average distances and transport distances and (b) distribution of transport distances between discrete supply and demand sites for the scenarios with 10\%,20\% and 40\% demand coverage.

Table 6.1 Transport distance per unit phosphorus $(\mathrm{km} / \mathrm{kg})$

\begin{tabular}{|c|c|c|c|}
\hline & Scenario 10\% & Scenario 20\% & Scenario 40\% \\
\hline Urban Agriculture & 0.16 & 0.18 & 0.18 \\
\hline Cropland + Grassland & 0.24 & 0.30 & 0.37 \\
\hline All agriculture & 0.23 & 0.28 & 0.36 \\
\hline
\end{tabular}

Average distances between buildings and fields were $4.75 \mathrm{~km}$ (s.d. 2.18) for the $10 \%$ scenario, $5.75 \mathrm{~km}$ (s.d. 3.18) for the $20 \%$ scenario and $6.88 \mathrm{~km}$ (s.d. 4.47) for the $40 \%$ scenario. Actual transport distances, however, also depend on the volume of collected urine and the truck size. Assuming truck volume capacities of $35 \mathrm{~m}^{3}$ for relatively large volumes and $10 \mathrm{~m}^{3}$ for small volumes, we estimated that the average transport distances were $7.38 \mathrm{~km}$ (s.d. 6.54) $(10 \%)$, $9.05 \mathrm{~km}$ (s.d. 8.43) (20\%), and $10.37 \mathrm{~km}$ (s.d. 10.75) (40\%). The difference between distance and transport distance values (Figure 6.4a) was significant $(\mathrm{P}<0.001)$. The increased transport distances and respective standard deviations, show the relevance of including truck size and volume transported in distance calculations. The distribution of the transport distances is shown in Figure 6.4b. Transport distances between supply and demand points sites under $15 \mathrm{~km}$ were most frequent; $87 \%(10 \%), 83 \%(20 \%), 76 \%(40 \%)$ of transport distances were under $15 \mathrm{~km}$. 
Total transport distances ranged between $1,690 \mathrm{~km}(10 \%)$ to $4,156 \mathrm{~km}(20 \%)$ to $10,288 \mathrm{~km}$ $(40 \%)$, reflecting the nonlinear increase in supply sites needed to meet an increasing demand coverage (Figure 6.1b), in addition to the increasing inclusion of demand sites located on average further away from supply sites. For further standardized comparison, we normalized the transport distances per unit of phosphorus (shown in Table 6.1). Clear is that an increase in demand coverage led to an increase in transport distance per unit of phosphorus. As expected, transport distances to urban agriculture per unit of phosphorus were smaller than to cropland and grassland.

\subsection{Environmental impact and cumulative energy requirement}

For further evaluation, we compared the environmental impact $\left(\mathrm{CO}_{2}\right.$ Equivalent $)$ and cumulative energy requirement $(\mathrm{MJ})$ of the total transport distance between matched supply and demand sites for the transported urine with the impact and energy requirement avoided from the production of chemical phosphorus fertilizers (such as ammonium nitrate phosphate and single superphosphate) to meet the demand per increasing coverage. Table 6.2 indicates that the impact of the production of chemical phosphorus fertilizers alone (not even considering the transport of the product to agriculture) is larger than the transport of urine between the identified supply and demand sites.

Table 6.2 Environmental impact and energy demand of urine transport versus conventional phosphorus (P) fertilizer production

\begin{tabular}{|c|c|c|c|c|c|c|c|c|}
\hline & \multirow[t]{2}{*}{$\begin{array}{l}\text { Transport } \\
\text { distance } \\
(\mathrm{km})\end{array}$} & \multirow[t]{2}{*}{$\begin{array}{l}\text { Demand } \\
(\mathrm{kg})\end{array}$} & \multicolumn{3}{|c|}{$\begin{array}{l}\text { Produced (+) or avoided (-) impact } \\
\left(\mathrm{kg} \mathrm{CO}_{2} \text { Equivalent per demand }\right. \\
\text { coverage scenario) }\end{array}$} & \multicolumn{3}{|c|}{$\begin{array}{l}\text { Consumed (+) or avoided (-) } \\
\text { energy (MJ per demand coverage } \\
\text { scenario) }\end{array}$} \\
\hline & & & $\begin{array}{c}\text { Transport } \\
\text { of } \\
\text { Urine* }^{*}\end{array}$ & $\begin{array}{l}\text { P fertilizer } \\
\text { production } \\
\text { (ANP) }\end{array}$ & $\begin{array}{l}\text { P fertilizer } \\
\text { production } \\
\text { (SSP) }\end{array}$ & $\begin{array}{l}\text { Transport } \\
\text { of Urine* }\end{array}$ & $\begin{array}{l}\text { P fertilizer } \\
\text { production } \\
\text { (ANP) }\end{array}$ & $\begin{array}{l}\text { P fertilizer } \\
\text { production } \\
\text { (SSP) }\end{array}$ \\
\hline $10 \%$ & 1690 & 7547 & 164 & -3357 & -5840 & 2777 & -51077 & -96741 \\
\hline $20 \%$ & 4156 & 15094 & 402 & -6713 & -11679 & 6799 & -102154 & -193487 \\
\hline $40 \%$ & 10288 & 30188 & 1031 & -13426 & -23358 & 17344 & -204309 & -386963 \\
\hline \multicolumn{9}{|c|}{ * calculations account for freight trucks with volume capacity of $35 \mathrm{~m}^{3}$ and $10 \mathrm{~m}^{3}$} \\
\hline
\end{tabular}

\section{Discussion}

The recirculation of phosphorus from human excreta to agriculture aims to replace conventional fertilizers currently mined from nonrenewable reserves. The mining of phosphate rock and its use results in ecosystem damage and other environmental issues (e.g., Cordell et al., 2009b). The 
model presented here identifies the agricultural demand sites within a boundary and matches this with the available supply and the respective locations of the supply. For the case of Amsterdam there is enough phosphorus in excreted urine to fulfill the total agricultural demand from urban agriculture, cropland and grassland in the municipality, and more (62\% of the total urine left). In this regard, Amsterdam could become completely self-sufficient in fulfilling current local phosphorus demand. This finding also indicates that local recycling of urine, let alone human excreta, is not enough to achieve a circular nutrient economy, as noted in similar studies (Akram et al., 2019a, Trimmer and Guest, 2018). Either an increase in cultivated area in and around the city is needed, or the excess of nutrients in human excreta will need to find their way to rural hinterlands. Despite the abundance of nutrients in the municipality of Amsterdam, the number of supply sites needed to reach full demand coverage for phosphorus is large and an unlikely target on the short term. The stepwise increased coverage (Figure 6.1) indicates the possible incremental transition towards phosphorus recirculation, matching the hottest supply sites with the closest demand sites.

Resource Dynamo selects minimum distances between the supply and demand sites and maps routes along existing road networks; such high resolution is novel in this field. Previous studies often used aggregated data, allocated across grid cells. Such rasterized data effect the quality of the results. For example, for the $10 \%$ coverage sketched here, the aggregation of data to a grid $(100 \mathrm{x}$ $100 \mathrm{~m}$ cell resolution) was tested and showed differences of $10.8 \%$ less in total distance and $1.7 \%$ less in total transport distance measurements. Grid rasterization measures distances between cell centroids, and thus fails to account for transport routes to exact supply and demand sites. For coarser cell resolutions we expect these differences to increase. Previous studies have shown that the use of high resolution and local datasets results in more exact results and often presents realities that are otherwise overlooked (Sharpley et al., 2016, Akram et al., 2019b, Wielemaker et al., in press). Previous studies also measured distances without considering the road network, assuming connections between two points as straight lines (as the crow flies) (e.g., (Akram et al., 2019b, Trimmer and Guest, 2018)). Nor do these account for truck volume capacities. Trimmer and Guest (2018) indicate that broad trends in average distances per studied city did not change when using estimated distances versus actual road network distances, however we argue that the difference they observed of $7-21 \%$ is relevant for planning capacity at municipal level, and that the inclusion of truck capacity better indicates expected transport distances. The identification and visualization of transport routes can inform decision-makers the extent of the transport infrastructure that will be needed to support recirculation of nutrients.

The delineation and measurement of routes along existing roads also allows for a more representative environmental impact assessment associated transport emissions ( $\mathrm{CO}_{2}$ equivalent). With this we are able to show that the critiques concerning the added impacts of transporting bulky products (Keplinger and Hauck, 2006), such as urine, is unmerited when weighed against the impact of conventional fertilizer production. The production of phosphorus fertilizers from finite rock supplies, such as ammonium nitrate phosphate and single superphosphate, has 
a much larger impact still, even without considering transport from locations of mining and processing of phosphate rock to agriculture (Table 6.2). The replacement of mined fertilizer with urine is a substantial improvement to the status quo. While in this regard further optimization of transport distances has little effect (as it is a matter of better versus best), it may be an important parameter in a future where phosphorus recycling becomes more commonplace, and conventional fertilizer production is no longer the baseline measure.

\section{Outlook and model expansion}

The case of matching nutrient supply and demand in the municipality of Amsterdam was selected to (1) demonstrate the potential of the model, and (2) present first results, in this case applied to phosphorus loads in urine. The model can be used for other spatial contexts and is flexible to accommodate context-specific conditions or preferences, and study objectives. For the context of Amsterdam a handful of input datasets were publicly available. We expect similar datasets to be available in many countries, although Resource Dynamo is flexible to be used with aggregated datasets or datasets that span different boundaries (e.g., provincial, regional). It is important, however, to consider the effect of aggregated data on results as discussed previously.

The model identifies potential promising connections between specific buildings as supply sites of nutrients and specific agricultural fields as nearest demand sites. This allows a further step, i.e., evaluating the viability to bring such specific connections into reality by considering for example options for nutrient collection in the building and options for truck transport to and from that building. To this end, the model could serve as a decision-making tool, that when used by practitioners can help identify (based on e.g., available investment potential) first points of intervention for the recovery of nutrients (in this case, urine).

Further refinement of the model would benefit from more accurate data on fertilizer use at the specific farm locations such as the inclusion of crop-specific nutrient requirements in demand calculations, and the frequency of fertilizer applications. We expect a higher resolution in temporal variations, i.e., more than one application of fertilizer per year, would have an effect on transport requirements due to more frequent emptying of storage tanks (although reduces needed storage capacity on site). Further supply site selection can be improved based on parameters such as feasibility to collect urine via urinals from buildings, or sites located within a predefined radius from demand sites. Particularly study boundary demarcations can affect the results for identifying optimal matches between supply and demand, for instance when optimal matches lie across boundaries, as is most likely the case for Amsterdam, with its jagged and partitioned municipal boundary.

Moreover, it is important to consider that the recirculation of nutrients to agriculture in urine competes with other fertilizer inputs on the market. This study assumes complete substitution of agricultural phosphorus demand with urine-derived phosphorus. However, in a country such as 
the Netherlands, with abundant manure, complete substitution is not realistic on the short term. In future applications of the model, demand should reflect nutrient percentages substitutable by urine-derived nutrients, taking into consideration farmer preferences and the fraction of nutrients in urine available to crops. Manure allocation, as well as other available nutrient supply streams, could also be included in the model to combine nutrient supply and demand. For the case of Amsterdam, further model expansion could generate alternative scenarios for the collection, transport and storage of urine. For instance, considering centralized storage units or the implementation of recovery technologies that target volume and mass reduction (e.g., struvite precipitation, nitrogen stripping, nitrification-distillation) before transport. 


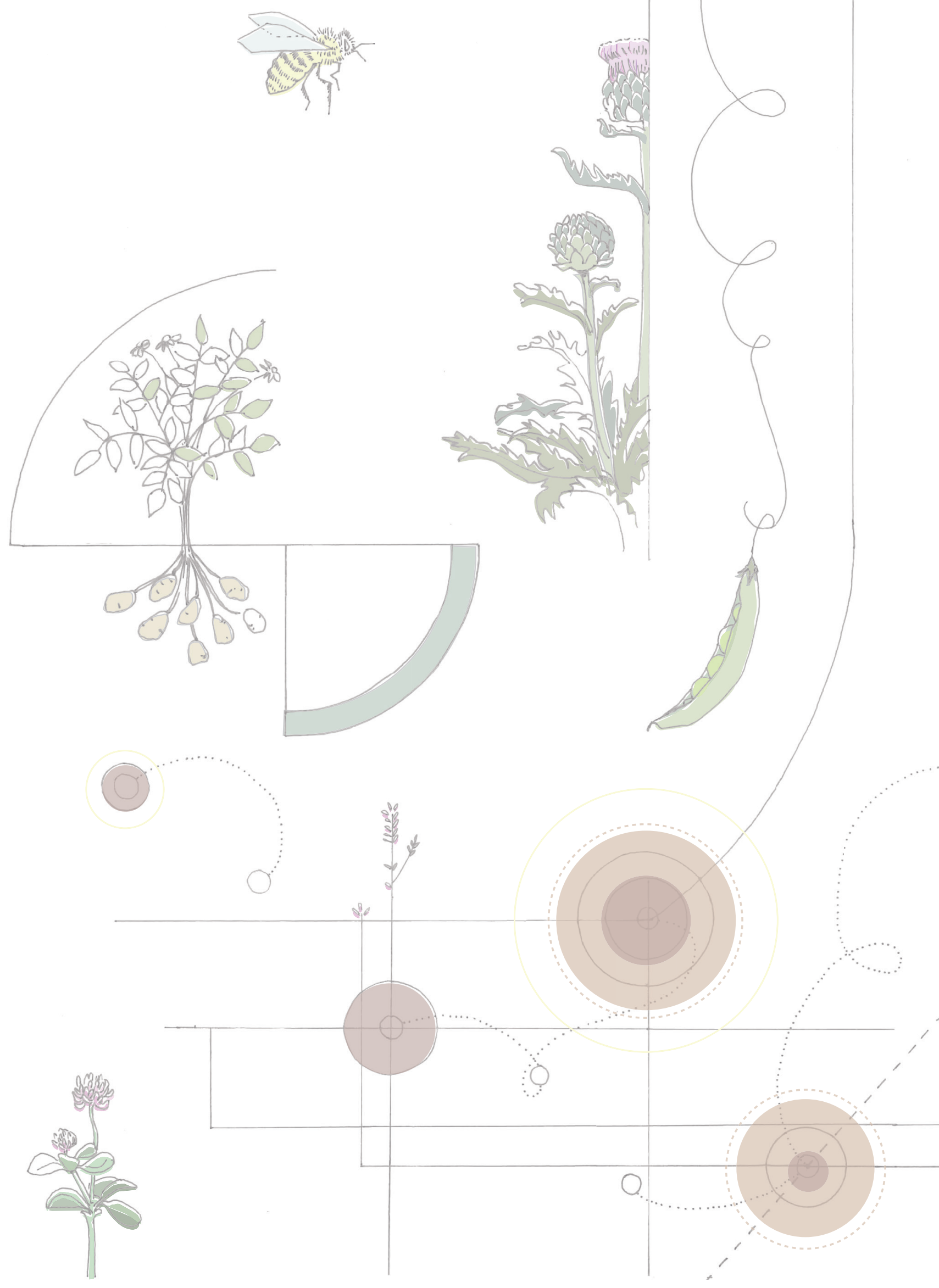




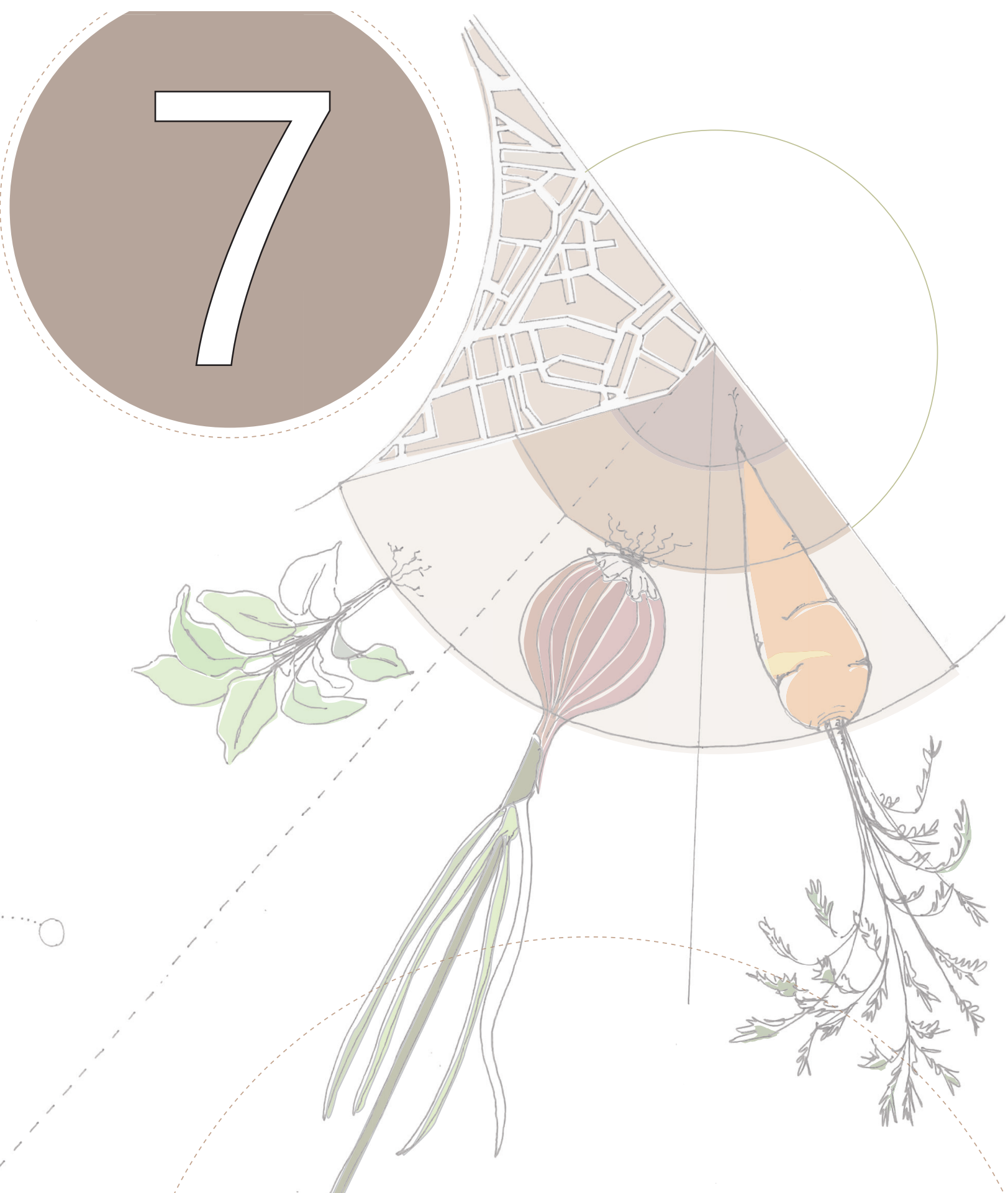




\section{Abstract}

Recognition of human excreta as a resource, rather than just as waste, has led to the emergence of a range of new and innovative nutrient recovery solutions. Nevertheless, the management of human excreta remains largely rooted in current sanitation and wastewater management approaches, which often makes nutrient recovery an add-on to existing infrastructures. In this paper, we argue that framing human excreta management as a resource recovery challenge within waste management obscures important trade-offs. We call for human excreta management to be framed as part of food and farming systems and show that such reframing will bring to the fore (at least) six aspects of critical importance that are currently largely overlooked. We conclude that increased consideration of these aspects has the potential to better guide human excreta management towards global food, soil, and nutrient security without compromising other priorities related to human and environmental health.

Keywords: human excreta management; nutrient management; nutrient recovery; wastewater management; sanitation; resource recovery

This chapter has been submitted for publication as: Harder, R*., Wielemaker, R.*, Molander, S., and Öberg, G., Human excreta management needs reframing

\footnotetext{
* Shared first authorship
} 


\section{Introduction}

Finding ways to feed a growing and increasingly urbanized population while reducing environmental and social impacts is a major global challenge (Foley et al., 2011, Willett et al., 2019). One of the key prerequisites to achieving and maintaining global food security is improved nutrient management along the entire food chain, which includes farming practices, food processing, consumer behavior, and waste management (McConville et al., 2015). Better nutrient management also requires the recirculation of nutrients from human excreta to food production (Drangert et al., 2018, Trimmer and Guest, 2018). In most cultures, human excreta has historically been used for fertilization and soil improvement (Ferguson, 2014). However, the introduction of the water closet and sewer networks (that is, waterborne sanitation) has led to a decoupling from food production (Ferguson, 2014). Other contributing factors have been the rise of synthetic fertilizers, increased urbanization, as well as specialization, globalization, chemicalization, and industrialization of farming systems (Jones et al., 2013b). Taken together, these factors have profoundly altered nutrient flows at the local, regional, and global scales, leading to a linearization and globalization of nutrient flows as illustrated and explained in Figure 7.1 .

It has become increasingly evident over the past few decades that the patterns of nutrient flows associated with current approaches to farming and human excreta management are unsustainable. Global estimates of current recirculation rates are highly variable, but suggest that, at most, 15 percent of nitrogen and 55 percent of phosphorus in human excreta are recirculated to cropland (Trimmer et al., 2017). Also, emissions of nutrients from human excreta to water bodies are projected to increase even further in the future due to increased population and urbanization, as well as the widespread perception of waterborne sanitation as the 'gold standard' (Van Drecht et al., 2009, del Carmen Morales et al., 2014). Concerns about nutrient pollution in freshwater and marine environments (Glibert et al., 2014), combined with the anticipation of insecurities related to future phosphorus supplies (Cordell et al., 2009b), have fuelled the development of new and innovative human excreta management solutions that facilitate the recovery of nutrients (and organic matter) from human excreta for reuse in agriculture (Haddaway et al., 2019, Harder et al., 2019). The development of nutrient recovery and reuse solutions reflects an ongoing shift from perceiving human excreta as waste towards recognizing its value as resource, and is part of a broader trend towards more comprehensive resource recovery in the sanitation and wastewater management sectors (Larsen and Gujer, 1997, Otterpohl et al., 1997, Wilsenach et al., 2003, Larsen et al., 2009a, Verstraete et al., 2009, Peccia and Westerhoff, 2015). While several scholars have highlighted that new and innovative sanitation and wastewater management solutions that embrace resource recovery have the potential to achieve multiple sustainable development goals (SDGs) (Andersson et al., 2016, Andersson et al., 2018, Anderson et al., 2015, Trimmer et al., 2017, Orner and Mihelcic, 2018), trade-offs are rarely considered. For instance, resource recovery efforts have long focused on energy and phosphorus, which generally results in loss of other nutrients and organic matter. Consequently, resource recovery can lead to sub-optimal solutions 
$$
\text { 音 }
$$$$
\frac{\pi}{ \pm}
$$$$
\text { 考 }
$$$$
\text { ป }
$$$$
\text { 先 }
$$$$
\text { 苗 }
$$$$
\text { 氠 }
$$$$
\pi
$$$$
\text { केष्ठ }
$$$$
\text { त्व }
$$$$
\text { तั }
$$$$
\text { 离 }
$$$$
0
$$$$
\text { 믕 }
$$$$
\text { 产完 }
$$

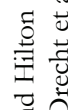

롬

हี त्ञ

赵

छঙ

$\ddot{\ddot{d}}$

苞

崩

完

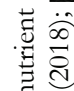

茨

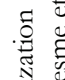

골

$\frac{0}{60}$

चี

.ี ปิ

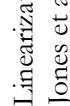

듬

(n)
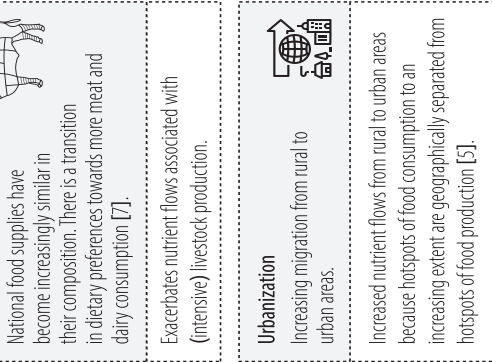

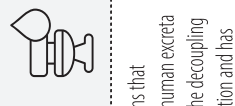

高害

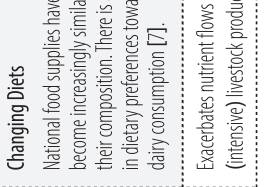

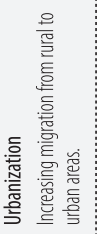

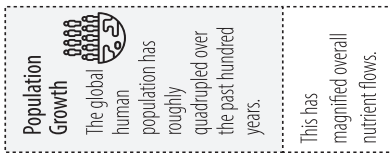
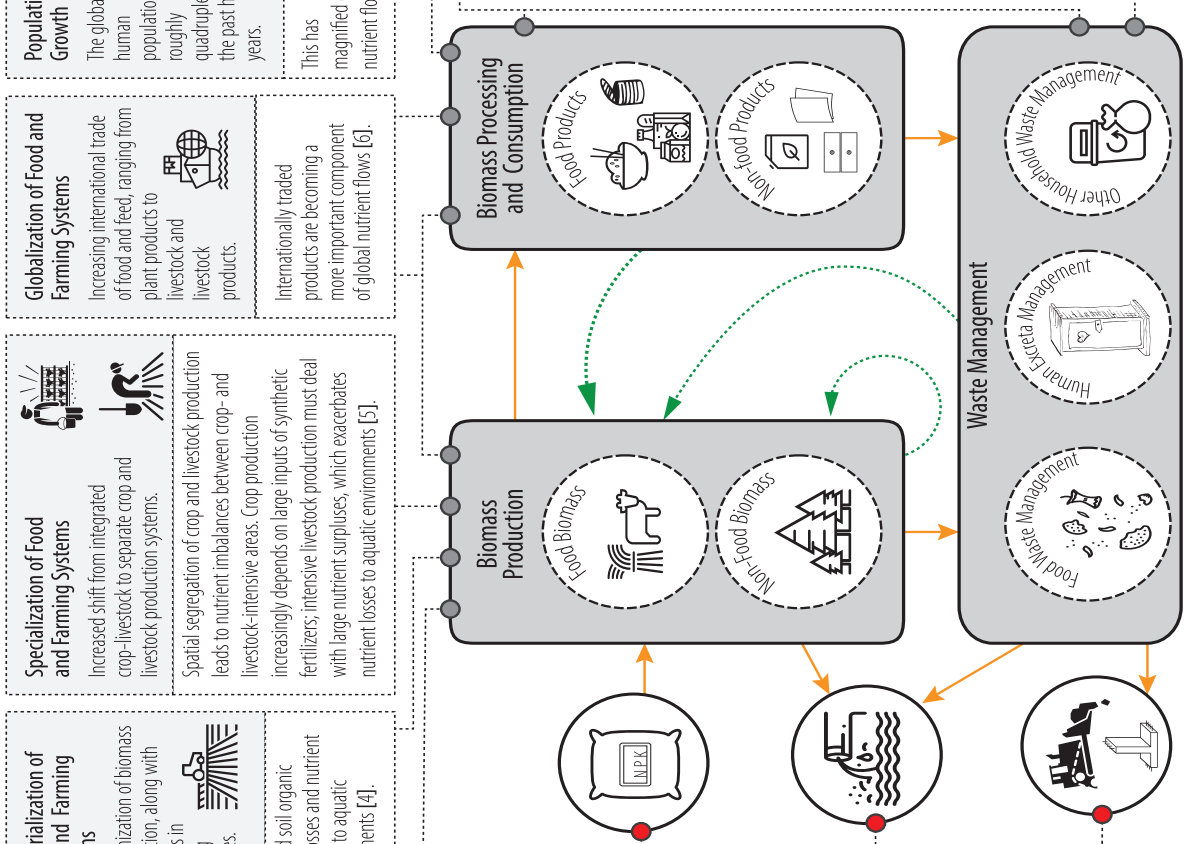

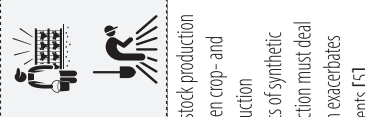
흠

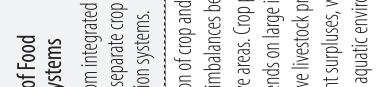

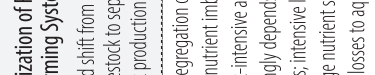

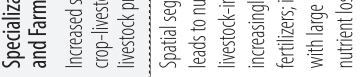
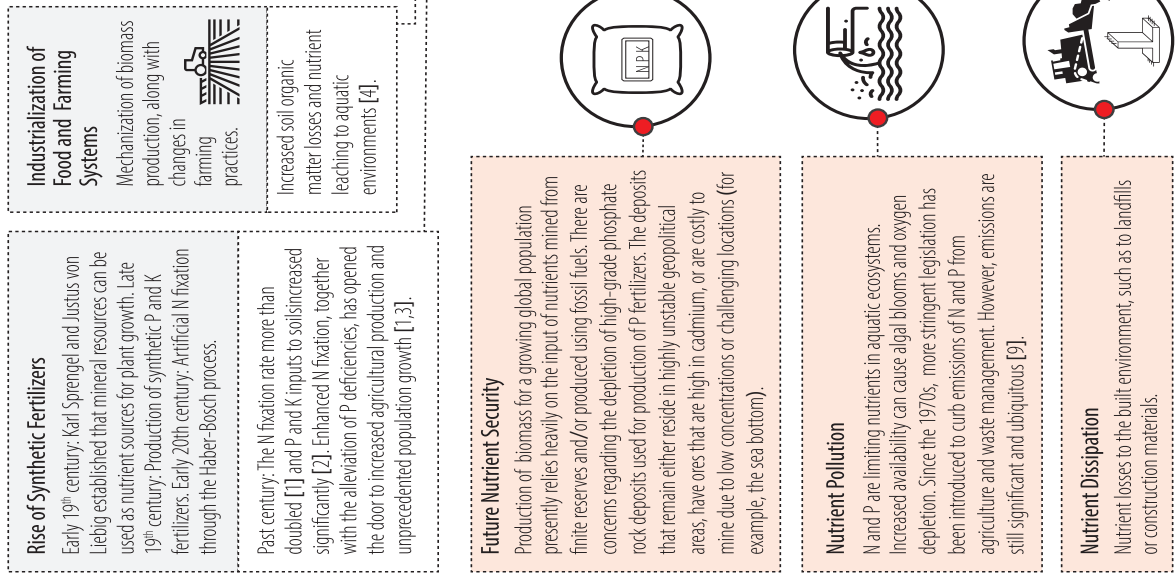

易 
if the development of nutrient recovery technologies is driven primarily by what can easily be recovered (for example, phosphorus from side-streams at existing wastewater treatment plants) rather than asking which contributions human excreta management ought to make to food and nutrient security. It is likely that such neglect of trade-offs related to nutrient recovery and reuse stem from the currently dominant framing to human excreta management. Although human excreta is increasingly recognized as a resource, its management is still largely rooted in current sanitation and wastewater management approaches (Simha and Ganesapillai, 2017). Under these premises, nutrient recovery often becomes an add-on to existing infrastructures.

It is well documented that the way in which an issue is framed has a major impact on the perception of what the problem is and how it might be handled (Beck, 1992, Vliegenthart and van Zoonen, 2011, Giampietro, 2018, Ahlborg et al., 2019). We argue that reframing human excreta management as part of food and farming systems has the potential to shift the perception of opportunities and challenges and can reveal central trade-offs that are currently underrated. Since vocabulary guides our thinking (Schön, 2012), we believe that a shift in thought patterns and framing also requires a shift in terminology. Much of the current vocabulary related to human excreta management is rooted in the perception of human excreta as waste and contributes to the technological, institutional, and mental lock-in to conventional solutions. For example, the terms 'human waste' and 'wastewater' directly allude to the notion of 'waste'. Similarly, the term 'sewage' requires sewers and, like 'wastewater', it implies the use of water as means of transportation. Therefore, we have chosen to avoid these terms and consistently speak of human excreta, streams that contain human excreta, and human excreta management.

\section{Underrated aspects of human excreta management}

In this paper, we argue that reframing human excreta management as a part of food and farming systems, would give prominence to (at least) six aspects that are currently largely overlooked. We propose that better consideration of these aspects has the potential to contribute to global food, soil, and nutrient security in the long term. The six underrated aspects are illustrated in Figure 7.2 and elaborated upon below. While presented separately, they are connected and there are potential synergies among them.

\subsection{Prioritizing nutrient recovery and reuse}

Energy recovery from human excreta has received more attention than nutrient recovery (Grant et al., 2012, Van Loosdrecht and Brdjanovic, 2014). From a food and farming systems perspective, however, nutrient recovery is a higher priority than the recovery of energy. This is partly because the potential contribution of nutrient recovery to meet the global fertilizer demand far surpasses the potential contribution of energy recovery to meet the global energy demand (Trimmer et al., 2017). Nevertheless, recent research and the development of novel energy recovery technologies 
CURRENTLY DOMINANT FRAMING

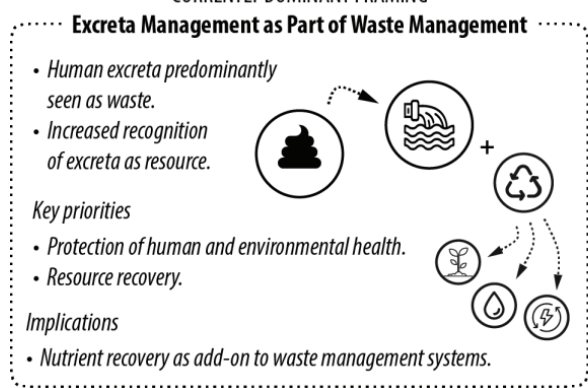

REFRAMING

SUGGESTED NEW FRAMING

$\ldots \ldots$....... Excreta Management as Part of Food and Farming Systems ...

- Wide recognition of excreta as important nutrient source for the production of food and other biomass.

Key priorities

- Protection of human and environmental health.

- Food, soil, and nutrient security.

Implications

- Nutrient recovery as integrated part of food and farming systems.

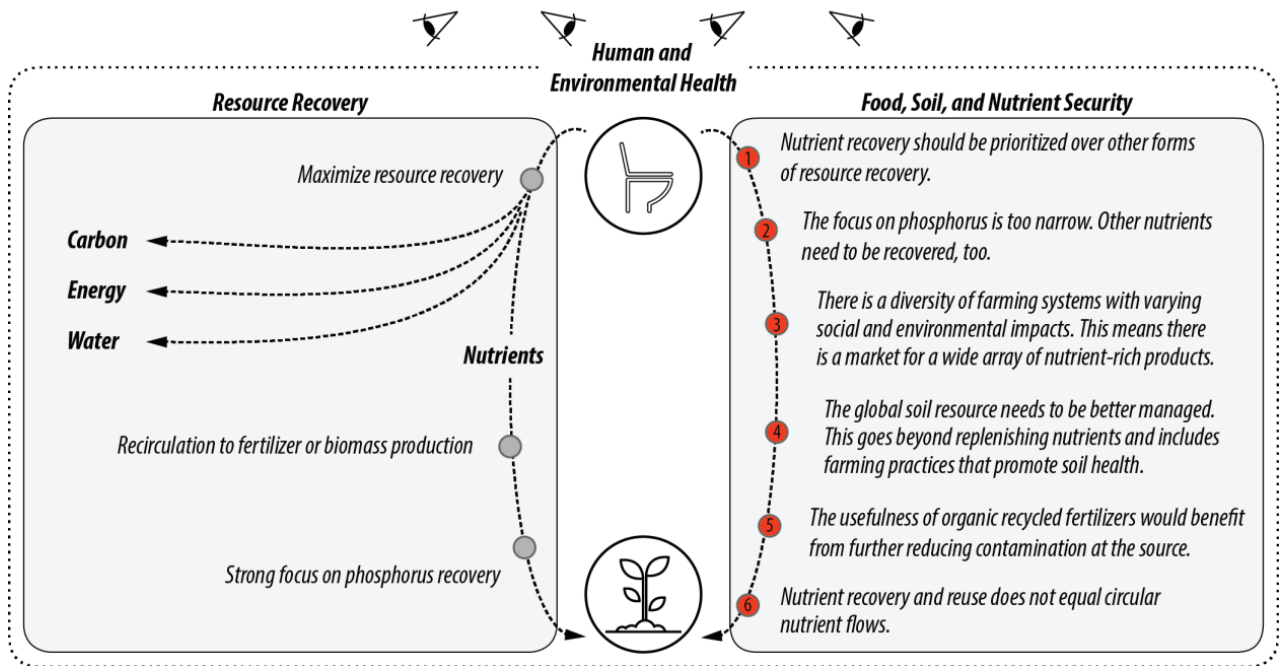

Figure 7.2 Current and proposed framing of human excreta management, as well as, underrated aspects of human excreta management that emerge upon reframing.

that use human excreta and streams containing human excreta as feedstock for the production of biocrude, bioethanol, biodiesel, biohydrogen, and syngas (Gomaa and Abed, 2017, Puyol et al., 2017, Manyuchi et al., 2018) has rarely indicated what fraction of nutrients, if any, is recovered in parallel and in what types of residual products. Given the potential role of human excretaderived nutrients in supplying nutrients to food and farming systems, we argue that treatment of human excreta should be optimized for nutrient recovery rather than energy recovery. While we acknowledge the importance of soil organic matter (Crews and Rumsey, 2017), we do not take a stance here as to whether nutrients should be recirculated with or without organic matter.

\subsection{Broadening the scope of nutrient recovery}

Plants need at least 17 essential elements to grow (Hänsch and Mendel, 2009, Maathuis, 2009). Certain other elements, even if they are not essential for plant growth, can be essential for animal nutrition (such as cobalt) (Voortman, 2012b) or human health (such as selenium) (Jones et 
al., 2017). Insufficient and imbalanced fertilization has led to a systematic stripping of nutrients from soil at the global level (Jones et al., 2013b). For instance, potassium limitation is common in terrestrial ecosystems globally (Sardans and Peñuelas, 2015) and it has been estimated that only about half of the potassium removed from soil as offtake is replenished through fertilizers and soil amendments (Sheldrick et al., 2002, Manning, 2018). Concerns have also been raised regarding micronutrient stripping (Voortman, 2012b, Jones et al., 2013b) and micronutrient deficiencies, notably regarding copper, zinc, and selenium (Udo de Haes, 2012, Jones et al., 2017).

The depletion of high-grade phosphate rock deposits has led to increasing attention being given to alternative phosphorus sources. Several jurisdictions have implemented legislation for comprehensive phosphorus recovery (such as Switzerland, Germany, and Austria), or are in the process of developing such legislation (Sweden, for example). However, potassium and micronutrients are also currently mined from finite deposits. Some scholars have argued that potassium is not anticipated to be in limited supply, nor is there a significant energy requirement for the production of potassium fertilizers (Dawson and Hilton, 2011). Others have highlighted that high-grade potassium ore is also limited and concentrated in a small number of countries, and have advised the exploration and use of novel sources of potassium (Ciceri et al., 2015, Manning, 2015, Manning, 2018). Likewise, micronutrients might become increasingly scarce (Voortman, 2012b). In contrast, nitrogen is not in short supply as it is abundant in the atmosphere where key challenges are to develop less energy-intensive ways of nitrogen fixation from the atmosphere (Razon, 2018).

There is little doubt that phosphorus recovery plays a critical role in slowing down the depletion of high-grade phosphate rock deposits. From a food and farming systems perspective, however, a narrow focus on phosphorus recovery falls short of addressing the broader issues of soil nutrient stripping, increased micronutrient deficiencies, and long-term food security. Many recovery technologies that have focused on phosphorus recovery have actually resulted in high losses of other nutrients (such as nitrogen to the atmosphere and potassium to the effluent). Therefore, there is a risk that an overly narrow focus on phosphorus may lead to sub-optimal solutions. In the long term, recirculation of nutrients contained in human excreta (and other organic residuals) to farming systems will need to include potassium and micronutrients (and possibly nitrogen), as is already the case for some of the recovery and reuse technologies and approaches that are under development (Harder et al., 2019).

\subsection{Catering to diversity in food and farming systems}

One of the largest challenges, globally, is to find ways to feed a growing and increasingly urbanized population while reducing environmental and social impacts (Foley et al., 2011, Willett et al., 2019). Not surprisingly, there is fierce debate on what the future of food should look like (Garnett, 2014, Willett et al., 2019, Fraser et al., 2016). Because it is highly unlikely that one single solution will work in every context, we assume that a combination of different types of 
production systems will be required (Cunningham et al., 2013). The future food system will likely include 'soil-based' production systems (where soil is the growth medium) as well as 'soilless' production systems (where substances other than soil are the growth medium), varying from low-tech to high-tech, and located in rural as well as urban settings. Among the soil-based production systems, there will most likely be scope for a portfolio of systems that are adapted to the local ecological conditions and resource base (Struik and Kuyper, 2017). Among the soilless systems, there are opportunities for hydroponics and aquaculture in various configurations, including vertical farms (Muller et al., 2017), as well as, reactor-based production of microbial protein (Pikaar et al., 2017, Linder, 2019). The variety in production systems for food (and other biomass) will require a variety of nutrient inputs. Hydroponic production systems, for instance, require a carefully crafted combination of mineral salts to produce a nutrient solution, possibly in combination with granular fertilizers such as struvite. Aquaculture systems require fish feed, which could contain protein rendered by treatment of human excreta or streams containing human excreta. In principle, soil-based production systems can handle a wide variety of nutrient inputs, ranging from nutrient-rich liquids and organic matter to granular and powdery inorganic matter, although different soil-based production systems and different farmers have different preferences. In other words, there will most likely be a need for a wide array of nutrient-rich products rendered by treatment of, among other things, human excreta and streams containing human excreta.

Until very recently, however, most research on developing and assessing nutrient recovery and reuse solutions was not designed to meet the needs of specific production systems. This is probably because nutrient recovery and reuse is seen primarily as a way to replace conventional mineral fertilizers and curb the demand for mined nutrients. However, different food and farming systems differ widely regarding environmental and social impacts (Hilborn et al., 2018, Poore and Nemecek, 2018, Rasmussen et al., 2018). When the overarching question becomes how human excreta management can best support future food and farming systems, as opposed to how to best replace conventional mineral fertilizers, there is a need to distinguish between different human excreta-derived recycled fertilizers and clarify their usefulness for different farming systems. Viewing recycled fertilizers as an integral part of food and nutrient security highlights that better understanding of the compatibility of different recycled fertilizers and production systems is key to ensure that nutrient recirculation can cater to a diversity of farming systems.

\subsection{Invigorating soil and ecosystem health}

One of the major challenges facing food and farming systems is the global degradation of arable soil (Montgomery, 2007, Montanarella et al., 2016, Amundson et al., 2015). It has been proposed that the concept of soil security can better translate soil science into policy for sustainable development (Koch et al., 2013) and facilitate a shift of focus away from the prevention of 
negative soil changes such as soil degradation and pollution and towards actively striving for soil changes in a positive direction through management practices that mitigate soil degradation (Baumhardt et al., 2015, Lal, 2015) and regenerate soil health (Sherwood and Uphoff, 2000, Cardoso et al., 2013). In this regard, the role of soil biodiversity for sustaining or improving food supply and human health has been emphasized (Wall et al., 2015), with several scholars calling for soil and land management practices that promote soil biodiversity (Wall et al., 2015, Bender et al., 2016). Ultimately, to help protect the global soil resource, recycled fertilizers (including those that contain human excreta-derived nutrients and organic matter) will need to be compatible with farming practices and production systems that maintain or improve soil and ecosystem health. This goes beyond just replenishing inorganic plant nutrients and relates more broadly to farming practices and production systems.

Agronomic evaluation of (recycled) fertilizers has long focused primarily on nutrient availability, nutrient offtake, and crop yield. Testing often takes place in pot experiments, which means it does not allow for a differentiated evaluation of fertilizers in the broader context of soil and ecosystem health at the field and landscape scales. Therefore, a better understanding of the compatibility of different recycled fertilizers and biomass production systems is crucial to guide the development and assessment of recovery and reuse of human excreta-derived nutrients towards invigorating soil and ecosystem health. Recent research into the effects of (recycled) fertilizers on microbial communities and biodiversity (van der Bom et al., 2018, Ibekwe et al., 2018, Staley et al., 2018), and into aligning recycled fertilizer chemistry and soil context (Trimmer et al. 2019), are important steps in this direction. We acknowledge that the recirculation of organic matter from human excreta to soil is one way to build and maintain soil organic matter (Crews and Rumsey, 2017).

\subsection{Further reducing contamination at the source}

Contaminants that are found in human excreta or added from other sources to streams containing human excreta represent a major challenge when it comes to recirculating human excreta-derived recycled fertilizers to agricultural production, since the presence of contaminants restricts the use of these products (Mininni et al., 2015, Röös et al., 2018). Contaminants of concern include pathogens, heavy metals, and organic pollutants. There is a lack of knowledge about the occurrence of emerging contaminants such as microplastics and nanomaterials in human excreta and their fate during treatment. Source separation and control measures can help to significantly reduce contamination, as illustrated by the successful reduction of heavy metal concentrations in sewage sludge in Sweden from the 1970s onwards (Kirchmann et al., 2017). Separate collection of human excreta, without mixing with domestic and industrial used water or stormwater, can further reduce heavy metal contamination. As human excreta only contain heavy metals that originate from dietary sources, the use of human excreta-derived fertilizers does not increase the amount of heavy metals in the food cycle (Tervahauta et al., 2014a). However, 
separate collection of human excreta does not prevent contamination with pharmaceuticals and hormones. While several treatment processes exist that can partially or fully remove or break down some pharmaceuticals and hormones, complete decomposition of all such substances can currently only be achieved through thermal processes, simultaneously resulting in loss of volatile elements like carbon, nitrogen and sulfur. There is presently little to no evidence that suggest that the presence of pharmaceuticals and hormones poses a threat to human or environmental health (Corcoran et al., 2010). Even so, as such substances are designed to impact biological systems, concerns have been raised that potential effects are yet to be discovered. Therefore, more efforts are needed to avoid further contamination at the source, as well as to develop and use less recalcitrant organic chemicals, such as pharmaceuticals and synthetic hormones that are easily (bio)degradable once they leave the human body (Daughton and Ruhoy, 2011, Leder et al., 2015).

\subsection{Nutrient recovery and reuse does not equal circular nutrient flows}

Once recovered, human excreta-derived nutrients are now reused in both food and non-food production. From the food and farming systems framing we suggest, nutrient recirculation goes beyond the recovery and reuse of nutrients. Reuse of human-excreta-derived nutrients in the production of non-food biomass - for example, in forestry (Marron, 2015) and biofuel production (Canter et al., 2015) or on green roofs and sports fields - represents a nutrient loss from food production, at least at shorter time scales. In other words, in such a scenario there is a risk that nutrient recovery and reuse simply substitutes two linear pathways ( fertilizer to food production to waste and fertilizer to non-food production to waste) with one longer one (fertilizer to food production to non-food production to waste). This means that human excretaderived nutrients may still become dispersed into the environment despite initial recovery and reused in non-food biomass production. From a food and farming systems perspective, it is important that nutrients originating from food production eventually find their way back to food production, at least in longer time scales. In this regard, reframing highlights the importance of considering whether nutrient recirculation from human excreta via the production of nonfood products to food production increases or reduces the contaminant load when potentially entering food production with recycled fertilizers.

\section{Conclusions}

With the present paper, we corroborate calls for major transdisciplinary efforts in research, policy, and practice to develop, assess, and implement alternative human excreta management practices. Along with others, we argue that the recirculation of human excreta-derived nutrients to food production will need to (again) become an important tenet of food systems (Drangert et al., 2018, McConville et al., 2015). The following presently underrated aspects require more attention if we are to guide human excreta management towards solutions that truly contribute to soil, food, and nutrient security in the long term: 
1. Optimized treatment for nutrient recovery.

2. A broader perspective on nutrient recirculation than the present focus on phosphorus and nitrogen.

3. Differentiation of recovery and reuse solutions that re-circulate nutrients to food production and those that do not.

4. Acknowledgement of diverse farming systems that are potential recipients of nutrientrich products.

5. Ensuring that recovered products are compatible with farming systems that are conducive to long-term soil and ecosystem health.

6. The development and use of less recalcitrant organic chemicals to benefit recirculation solutions that render products that retain organic matter.

A constructive dialogue on how human excreta management can best support future food and farming systems would also benefit from consolidation of existing evidence on the performance of various excreta management and farming systems, as this would help identify knowledge and synthesis gaps under different pre-analytical framing choices.

\section{Acknowledgements}

Robin Harder contributed to this manuscript in relation to the project 'Recycling organic matter and nutrients from sanitation to farming systems to regenerate soil and land: identifying approaches that are feasible and preferable', which is funded by a mobility grant from the Swedish Research Council for Environment, Agricultural Sciences and Spatial Planning (Formas) under grant agreement 2016-00859. 


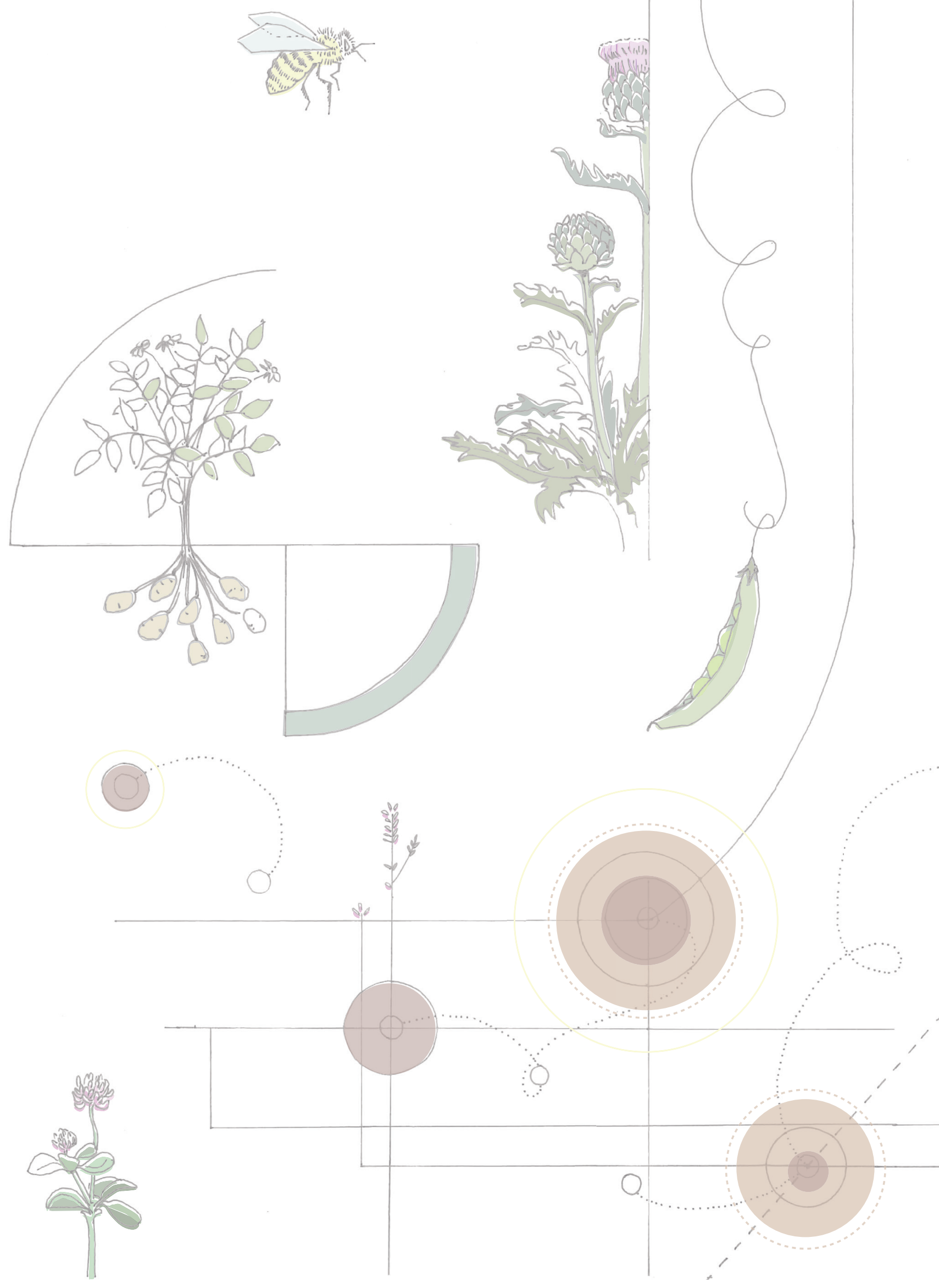


Table 8.1 Summary of main findings

\begin{tabular}{|c|c|c|}
\hline Chapter & Research Objective & Method \\
\hline $\begin{array}{c}\text { Chapter } 2 \\
\text { Harvest to Harvest } \\
\text { Resources, Conservation } \\
\text { and Recycling }\end{array}$ & $\begin{array}{c}\text { Apply the Urban Harvest Approach } \\
\text { to the nutrient cycle by comparing } \\
\text { supply from new sanitation with } \\
\text { demand from urban agriculture and } \\
\text { assess the potential self-sufficiency } \\
\text { for nitrogen, phosphorus and organic } \\
\text { matter }\end{array}$ & $\begin{array}{c}\text { Urban Harvest Approach (see } \\
\text { Agudelo-Vera et al., 2012a) }\end{array}$ \\
\hline $\begin{array}{c}\text { Chapter } 3 \\
\text { Fertile Cities } \\
\text { Science of the Total } \\
\text { Environment }\end{array}$ & $\begin{array}{l}\text { Evaluate current nutrient use on } \\
\text { Dutch urban agriculture farms } \\
\text { including i) type of nutrient inputs } \\
\text { ii) Input quantities for nitrogen, } \\
\text { phosphorus, potassium and organic } \\
\text { matter }\end{array}$ & Interviews; Nutrient budgeting \\
\hline $\begin{array}{c}\text { Chapter } 4 \\
\text { Pathways, Processes and } \\
\text { Products } \\
\text { Critical Reviews in } \\
\text { Environmental Science }\end{array}$ & $\begin{array}{l}\text { Provide a systematic synthesis of } \\
\text { the sanitation and nutrient recovery } \\
\text { fields by providing an overview of } \\
\text { terminology, recovery pathways and } \\
\text { treatment options, and products } \\
\text { rendered by treatment }\end{array}$ & $\begin{array}{l}\text { Literature review and desk } \\
\text { research }\end{array}$ \\
\hline $\begin{array}{c}\text { Chapter } 5 \\
\text { Nutrient Hotspots } \\
\text { Journal of Industrial Ecology }\end{array}$ & $\begin{array}{c}\text { Identify and quantitatively assess } \\
\text { hotspots of nutrient excretion in } \\
\text { buildings and neighborhoods in } \\
\text { Amsterdam }\end{array}$ & $\begin{array}{l}\text { Modelling using Geographic } \\
\text { Information Systems (GIS) }\end{array}$ \\
\hline $\begin{array}{l}\text { Chapter } 6 \\
\text { Resource Dynamo }\end{array}$ & $\begin{array}{l}\text { Match phosphorus supply in } \\
\text { human-derived urine with demand } \\
\text { by agricultural fields within the } \\
\text { municipality of Amsterdam and } \\
\text { delineate minimum transport routes } \\
\text { between the two }\end{array}$ & $\begin{array}{l}\text { Modelling using Geographic } \\
\text { Information Systems (GIS) }\end{array}$ \\
\hline $\begin{array}{c}\text { Chapter } 7 \\
\text { Reframing Human Excreta } \\
\text { Management }\end{array}$ & $\begin{array}{l}\text { Call for the reframing of human } \\
\text { excreta management as part of food } \\
\text { and farming systems. }\end{array}$ & NA: Commentary \\
\hline
\end{tabular}




\begin{tabular}{|c|c|}
\hline Main findings & Conclusions \\
\hline $\begin{array}{l}\text { - Demand for nutrients and organic matter in urban } \\
\text { agriculture can be minimized by } 65-85 \% \\
\text { - } 100 \% \text { self-sufficiency for phosphorus, partial } \\
\text { self-sufficiency achievable for nitrogen and organic } \\
\text { matter demand in urban agriculture with recovered } \\
\text { products from new sanitation }\end{array}$ & $\begin{array}{l}\text { UHA is applicable to urban nutrient flows and the } \\
\text { integration of new sanitation and urban agriculture } \\
\text { substantially increases urban self-sufficiency }\end{array}$ \\
\hline $\begin{array}{l}\text { - Main fertilizer inputs at urban farms were } \\
\text { compost and manure } \\
\text { - Mean nutrient inputs exceeded mean crop } \\
\text { demand by roughly } 450 \% \text { for total nitrogen, } 600 \% \\
\text { for phosphorus and } 250 \% \text { for potassium }\end{array}$ & $\begin{array}{l}\text { Over-fertilization in urban farms is a salient } \\
\text { indication for the need to improve nutrient } \\
\text { management in urban agriculture }\end{array}$ \\
\hline $\begin{array}{l}\text { - Identification and categorization of recovery } \\
\text { pathways, technological processes and recovered } \\
\text { products from (streams containing) human excreta } \\
\text { - Development of an 'option space', i.e., a map of } \\
\text { primary inputs, treatment processes, products } \\
\text { rendered by treatment, and their relationships }\end{array}$ & $\begin{array}{l}\text { Trends in nutrient recovery include: } 1 \text { ) Increased } \\
\text { developments in selective nutrient extraction, 2) } \\
\text { Focus on the recovery of macronutrients (notable } \\
\text { NPK) 3) a trade-off in carbon harvesting as } \\
\text { energy carriers, chemicals or as soil amendment, } \\
\text { 4) potential to combine recovery pathways for } \\
\text { increased nutrient recovery }\end{array}$ \\
\hline $\begin{array}{l}\text { - } 10 \% \text { of phosphorus excreted in the city of } \\
\text { Amsterdam recoverable in } 0.1 \% \text { of the city's } \\
\text { buildings }\end{array}$ & $\begin{array}{l}\text { A novel tool to identify and quantify nutrient } \\
\text { hotspots. Results at building versus neighborhood } \\
\text { scale draw attention to the need for multiple scale } \\
\text { analyses in decision-making }\end{array}$ \\
\hline $\begin{array}{l}\text { - Total phosphorus demand can be met with } 38 \% \text { of } \\
\text { the available supply of phosphorus in urine }\end{array}$ & $\begin{array}{c}\text { A novel tool for scenario development and } \\
\text { assessment of nutrient cycling from human excreta } \\
\text { at the highest resolution. }\end{array}$ \\
\hline $\begin{array}{l}\text { - Reframing bring to the fore six overlooked } \\
\text { aspects: 1) prioritize nutrient recovery over } \\
\text { recovery of other resources; } 2 \text { ) broaden the focus } \\
\text { on the recovery of nutrients beyond phosphorus; } \\
\text { 3) cater recovered products to a diversity of } \\
\text { farming systems; 4) promote soil health; 5) reduce } \\
\text { contamination at source; 6) nutrient recovery and } \\
\text { reuse does not equal circular nutrient flows }\end{array}$ & $\begin{array}{l}\text { Reframing human excreta management as part } \\
\text { of food and farming systems can better guide } \\
\text { global food, soil, and nutrient security, without } \\
\text { compromising priorities related to human and } \\
\text { environmental health }\end{array}$ \\
\hline
\end{tabular}




\section{Introduction}

Anthropogenic activities have drastically altered nutrient flows and cycling, calling into question the long-term sustainability of current nutrient management. The extensive use of synthetic fertilizer in agriculture and the widespread implementation of waterborne sanitation systems have especially contributed to irretrievable losses of nutrients to water bodies and landfills, contributing to a host of environmental issues (see Figure 1.1, Chapter 1) (Erisman et al., 2008, Dawson and Hilton, 2011, van Puijenbroek et al., 2019, Vitousek et al., 2009). Improved nutrient management to facilitate a transition from nutrient losses to nutrient loops extends the entire food chain. Efforts across fertilizer sourcing and production, nutrient use and post-harvest waste recycling in agriculture, and nutrient recycling from organic refuse and human excreta to agriculture can help tighten flows in the nutrient cycle, and contribute to achieving several of the Sustainable Development Goals (SDGs) of the United Nations 2030 Agenda for Sustainable Development (e.g., SDG 2 (food security), SDG 6 (clean water and sanitation), and SDG 11 (urban sustainability)).

The increase in urban agriculture and new sanitation in recent decades brings about new narratives to the status quo of both food production and human excreta management. The case for urban agriculture and new sanitation individually is clear. Cities need innovative solutions to safeguard the food security of growing urban populations, meanwhile innovations in source-separated sanitation facilitate targeted and efficient recovery of valuable resources, echoing tenets of a circular economy. Taken together, an integration of urban agriculture and new sanitation reopens the opportunity to recycle nutrients at urban and peri-urban scales; a previously common practice until the rise of artificial fertilizers and water-borne sanitation. To this end, urban agriculture can source nutrients from available urban organic refuse and human excreta; new sanitation can supply nutrient-rich products recovered from source-separated streams containing human excreta to nearby agriculture (Ackerman, 2012, Goldstein et al., 2016, Specht et al., 2013). The extent to which nutrient flows between the two can be matched in terms of quantity and quality, and in time and space, however, remains largely uninvestigated.

Main findings (summarized in Table 8.1) of this thesis contribute to uncovering the potential to establish nutrient cycling between urban agriculture and new sanitation. The presented studies in preceding Chapters 2-6 focus primarily on descriptive, quantitative and spatial assessments of urban nutrient demand and supply. Results indicate that urban agriculture's embeddedness in and/or proximity to the urban environment can be harnessed to establish opportune and context-appropriate matching of nutrient supply and demand in space, though nutrient recycling at regional and global scales will also be necessary. Chapter 7 looks at the subject through a theoretical lens, and suggests that reframing human excreta management as part of food and farming systems has the potential to better guide human excreta management towards global food, soil, and nutrient security. 
This chapter places the results of this thesis within the broader perspective on nutrient management. The following sections first introduce a framework which aligns future spatial arrangements of agricultural systems and the potential reuse of recovered products at various proximities from the urban center, adapted from Von Thünen's model for agricultural land use first proposed in 1826 (Sinclair, 1967). Next, a taxonomy of nutrient profiles for urban agriculture in presented, hinging on the work by Goldstein et al. (2016), to discuss the role of urban agriculture in nutrient management. A second taxonomy is proposed for organizing new sanitation systems based on similar attributes of the recovered nutrient-rich products. We conclude that the diversity in urban agriculture and new sanitation typologies increases the potential to match nutrient demand and supply, though further compatibility assessments are needed. A discussion on spatial modelling of nutrient flows between sanitation and agriculture follows, and pleas in support of increased resolution in future modelling work follows. Finally, this chapter discusses opportunities for increased nutrient recycling and indicates pending and newly identified knowledge gaps in nutrient management research, especially concerning integrated urban agriculture and new sanitation systems.

\section{Radial levels of nutrient cycling and self-sufficiency}

Nutrient recycling between farms is increasingly stimulated as part of circular agriculture strategies, notably in the Netherlands (de Boer and van Ittersum, 2018). The recycling of farm wastes and animal manure on and between farms can considerably reduce nutrient losses in the food chain. Nevertheless, dominant current nutrient flows in harvested crops follow from farms to cities, where nutrients contained in food are consumed and excreted. Returning nutrients in excreta to agriculture begs the question: how much can be returned, to where, and how? On the one hand, this depends on the nutrient demand from agriculture and the location of these with respect to the city, currently and in the future. On the other hand, it depends on the economic feasibility of the (long-distance) transport of (often bulky) products from human excreta to agriculture (Akram et al., 2019b, Nicholson et al., 2012, Sharpley et al., 2016, Trimmer and Guest, 2018).

To this end, there is scope to carefully consider a hierarchy for both the type of agriculture located in and close to the city and the types of nutrient-rich products recovered from human excreta that can be reused in these different types of agriculture, much like the model of agricultural land use conceived by Von Thünen in 1826. Von Thünen noted that crops that were highly perishable, of large volume or heavy, and thus difficult to transport, should be grown and produced within or close to the city, while less intensive agriculture and grazing should be located farther away from the city (Sinclair, 1967). The onset of industrialization and developments to preserve crops and transport them more efficiently left Von Thünen's model outdated to describe current agricultural land use, as observed by Sinclair (1967). Recent developments in urban agriculture are advocating a model similar to Von Thünen's: intensive growing facilities in 


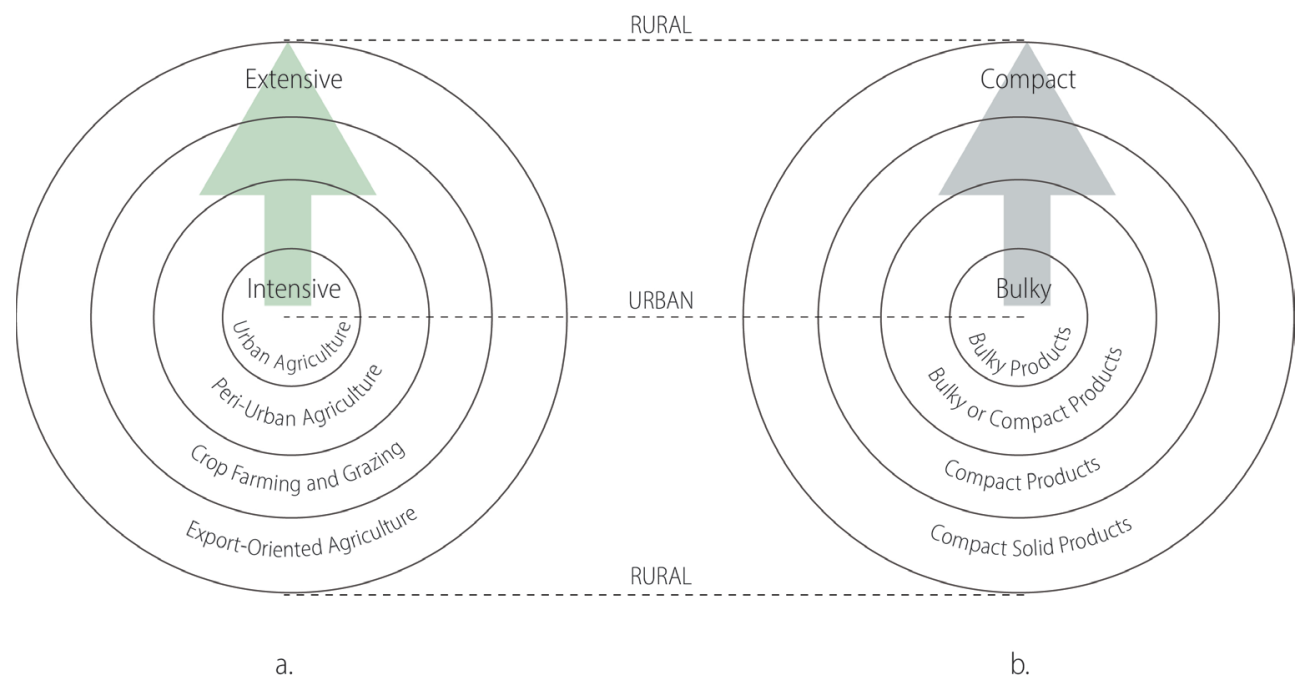

Figure 8.1 Radial hierarchies for (a) agricultural land use and (b) reuse of recovered products in relation to the urban center. For agricultural land use, the hierarchy spans intensive urban farms producing high-value, highquality, perishable fruits and vegetables to extensive crop farming (e.g., staple foods) and animal grazing and export-oriented agriculture. For the reuse of recovered products, products that are bulky (i.e., voluminous and/or heavy) should be used closer to the city, whereas concentrated and compact products can be transported further away. The hierarchy for recovered products is not meant to indicate where sanitation systems should be located.

and around the city for the cultivation of perishable leafy greens and high-quality, high-value vegetables and fruit (representing the most profitable crops for urban agriculture) (van der Schans and Wiskerke, 2012). Located farther away from the urban center, agricultural practices would include more extensive crop farming, grazing and export oriented agriculture (Figure 8.1 a).

A similar hierarchy for the transport and use of recovered products is conceivable. Transport is both costly and increases environmental externalities (e.g., greenhouse gas emissions). In this regard, heavy and voluminous products (e.g., treated effluent, stored urine, sludge, compost) should be reused closer to the urban center (e.g., in urban and peri-urban agriculture) and products with high nutrient concentrations (e.g., precipitates, concentrated urine) should be transported to agricultural land in rural areas or exported to international markets (directly or via fertilizer processing plants)(Figure 8.1b). Important to consider is that reductions in volume and mass often also call for increased technological investments, and that the use of technologies for the concentration or targeted recovery of nutrients often also accounts for additional nutrient losses between processing steps, and further energy and chemicals consumption. Therefore, with regard to maximizing nutrient cycling and minimizing transport, there is an obvious advantage in recycling nutrients at smaller scales first before moving outward (e.g., from city, region, continent, world). As such, urban agriculture is the first target as assimilator of nutrients derived from human excreta. 
While urban agriculture opens the opportunity to recycle nutrients locally, rough mass balances quickly reveal that the magnitude of this potential is limited. Results presented in Chapter 6 show that for the case of Amsterdam, current urban agriculture only requires 1\% of the phosphorus supply in urine to cover its phosphorus demand, with an even smaller percentage of the total available phosphorus in total human excreta and other organic waste streams. Further, periurban cropland and grassland located within the municipality of Amsterdam requires 38\% of the phosphorus supply in urine to cover its phosphorus demand. Metson and Bennett (2015) similarly conclude that the area of urban agricultural land in the city of Montreal is too small to accommodate the supply of phosphorus in the city's urban waste. Martellozzo et al. (2014) show that approximately one third of the total global urban area is required to meet the global urban vegetable demand $(300 \mathrm{~g} / \mathrm{cap} / \mathrm{d})$, though this is highly variable across cities. In Chapter 2 we indicate that the city of Rotterdam has enough available land acreage to meet the cities fruit and vegetable demand $(400 \mathrm{~g} / \mathrm{cap} / \mathrm{d})$, and that the supply of phosphorus recovered via new sanitation was sufficient to cover crop phosphorus demand at such scales. Nonetheless, the restricted acreage within cities limits the production of all food products, and thus impedes the recycling of all nutrients in human excreta within cities. Especially the cultivation of cereals and production of proteins is expected to remain beyond urban areas since, for example, global cereal production currently requires a land area ten times that occupied by cities worldwide (Hamilton et al., 2014). In short, nutrient recycling to rural land will be required next to nutrient recycling to urban farms.

In theory, nutrient recycling would be balanced across the hierarchies, meaning that equal and opposite nutrient loads flow between locations of agricultural production and locations of consumption and excretion, a self-sufficiency if it were across the radial hierarchies. The debate persists: what level of self-sufficiency can cities pursue in both food provisioning and nutrient cycling, and what level of self-sufficiency should a city sustain at the subsequent hierarchies. The two inform one another, and thus requires a rather iterative analysis. Where the availability of vacant land, rooftops and buildings might determine the extent and type of urban agriculture suitable in a city, population density and diet trends ${ }^{1}$ will determine the amount of nutrients produced in a city. Further, building and neighborhood function, planning and demography might determine the recovery pathways suitable per context, let alone compatible with the type of urban agriculture selected. Meanwhile, the requirement for storage of bulky products also deserves consideration, especially in space-constrained cities. The level of potential selfsufficiency is thus expected to be variable across different cities and municipalities (e.g., based on land use patterns and population density) (Trimmer and Guest, 2018, Akram et al., 2019a), and is also expected to be variable across time.

1 van Kernebeek et al. (2018) and van Zanten et al. (2019) suggest an optimum for meat consumption (23 g of protein per person per day) for an improved circular food system and particularly increased phosphorus circularity, not to mention the benefit of reduced meat consumption on climate change and land use change. 


\section{The role of urban agriculture in nutrient management}

While the impact of urban agriculture in assimilating nutrients is currently slim, several studies show that there is scope to scale up urban agriculture to increase its contribution to urban resource management and nutrient cycling (Goldstein et al., 2016, Haberman et al., 2014, Martellozzo et al., 2014, Smit and Nasr, 1992, Taylor and Lovell, 2012). The extent to which urban agriculture can be scaled up depends on several factors, including land and real-estate prices, market demand for urban agricultural products and services, dietary choices and competition for space with other services (e.g., solar panels on rooftops) (Artmann and Sartison, 2018a, Thomaier et al., 2015). Though the physical scalability of urban agriculture is, first and foremost, dependent on available area for cultivation, whether in soil, on rooftops or in buildings (Clinton et al., 2018), which is variable across cities.

The potential contribution to the absorption and recycling of nutrients from human excreta, however, depends not only on the scaled-up acreage, but also on the type of urban agriculture to be scaled up. Different types of urban agriculture have different resource use profiles as respective nutrient demands differ depending on context-specific factors such as: type of production (e.g., soil-based or soil-less production), growing medium (e.g., soil type and structure), crop type (e.g., nutrient offtake and crop rotation), climate (e.g., precipitation, solar radiation) and farm management (e.g., use of amendments, tillage practices, farming styles) (Sarkar and Baishya 2017). The potential of recycling nutrients to urban agriculture therefore varies across urban agriculture types.

Goldstein et al. (2016) proposed a taxonomy for urban agriculture typologies as an organizing framework for research that connects urban agriculture to urban resource fluxes, including

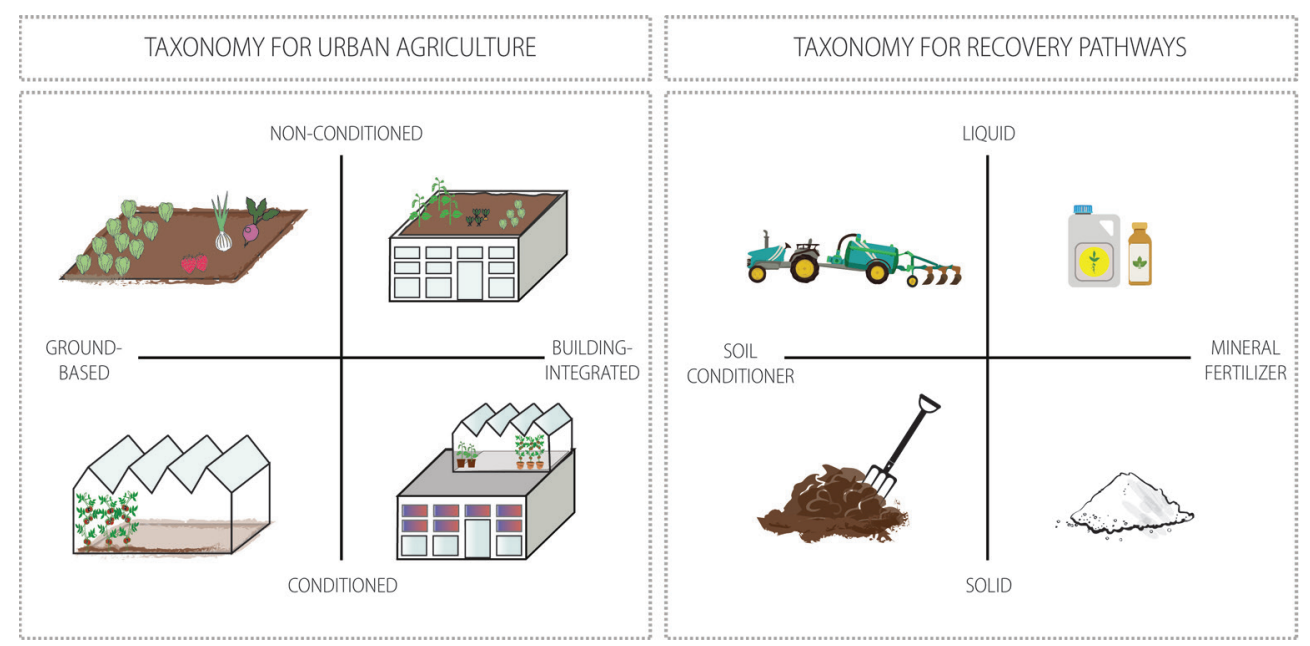

Figure 8.2. Taxonomies urban agriculture and new sanitation 
nutrient flows. The taxonomy is based on two organizing principles: (1) level of integration with buildings (ground-based vs building integrated), and (2) conditioning of growing space (conditioned vs non-conditioned), describing four main urban agriculture typologies and their respective resource profiles, as shown in Figure 8.2. Building integration is a measure for how physically embedded a growing system is within the built environment and the opportunities that exist to exchange resource flows with (adjoining) buildings (e.g., rainwater harvesting). The conditioning of growing spaces describes the level of control over physical and material flows, including light, temperature, nutrient dosing, pest management (e.g., in outdoor, greenhouse, or indoor farming systems).

The research presented in this thesis focused primarily ${ }^{2}$ on ground-based, non-conditioned systems, that is, the cultivation of crops directly in soil that interact with the ambient environment. Goldstein et al. (2016) note that these systems often show low nutrient use efficiency due to a diminished potential to minimize nutrient losses. However, these systems can act as a significant assimilator of urban organic refuse such as compost. To better understand the role of this type of urban agriculture in closing nutrient cycles and its prospective capacity to assimilate human excreta to cover nutrient demands, a baseline assessment of current nutrient management practices was conducted.

\subsection{Nutrient use efficiency in ground-based urban agriculture}

The inventory of nutrient management practices and the quantification of nutrient inputs at ground-based urban farms in the Netherlands showed that current nutrient management practices could not be used as a point of departure to define nutrient demand in urban agriculture (Chapter 3). Nutrient use across the interviewed ground-based urban farms included primarily compost and animal manure, and varied greatly in amounts applied per hectare. Results provide a salient indication of over-fertilization compared to crop uptake values, as well as compared to application limits for nitrogen and phosphorus in the Netherlands for conventional agriculture ${ }^{3}$. Such surpluses follow from constrained N:P:K stoichiometry of the preferred organic soil amendments used on farms. The low nitrogen ratio in compost and manure often leads to high phosphorus and potassium loads that exceed crop requirements when applications aim at meeting plant nitrogen demand (Maltais-Landry et al., 2016, Eghball, 2002). Studies on nutrient budgeting in both emerging and post-industrial economies show similar tendencies of overfertilization in urban and peri-urban agriculture (Abdulkadir et al., 2013, Dewaelheyns et al., 2013, Huang et al., 2006, Metson and Bennett, 2015, Wang et al., 2008). On the short term, such

2 Equal to the national yield for conventional agriculture (based on conventional farming yields in the Netherlands for 'vegetables and melons' for 2013 as reported by FAOSTAT) with a reduction of 20\% (organic yield gap) (FAOSTAT, 2013)

3 Application limits set by the Manure and Fertilizers Act are applicable for conventional cropland and grassland. Urban agriculture is often exempt from these application limits due to their small size $(<3 \mathrm{ha})$, and low number of animals (whose manure amounts to $<350 \mathrm{~kg} \mathrm{~N} / \mathrm{yr}$ ), according to Article 43 of the Implementing Arrangement (Uitvoeringsregeling) of the Manure and Fertilizers Act (Meststoffenwet, 1986). 
high nutrient surpluses increase the risk of nutrient losses and eutrophication of downstream water bodies (Schröder and Neeteson, 2008, van Grinsven and Bleeker, 2016). At the same time, high inputs of phosphorus can hamper crop uptake of micronutrients, like zinc (Dewaelheyns et al., 2013). On the long term, continual nutrient surpluses are a critical sustainability issue (Cordell et al., 2009a, Maltais-Landry et al., 2015). Before practices can be taken as a point of departure to determine the extent to which (future) urban agriculture may assimilate nutrients from (streams containing) human excreta, a more balanced nutrient input is required.

The extent to which nutrient inputs need to be reduced and through which measures, however is yet unclear. Merely cutting back on current inputs in urban agriculture may not provide crops with all essential nutrients they need to grow well. A reduction of compost and manure inputs may result in nitrogen shortage and crop nitrogen deficiencies (Berry et al., 2002, Maltais-Landry et al., 2016). Nutrient inputs need to be adjusted and perhaps diversified to better match crop uptake requirements to achieve balanced fertilization (Maltais-Landry et al., 2016). Instead of relying on compost and manures with fixed nutrient stoichiometry, the use of other nutrient sources with relatively high nitrogen ratios, or decoupled nutrients, could help increase flexibility in nutrient application (Winker et al., 2009b). A reduced input in compost could then be complemented with single nutrient mineral fertilizers.

Longer-term monitoring of nutrient inputs, outputs and soil nutrient stocks (e.g., logbook of fertilizers and harvests, and soil sampling and analysis) may help determine which targeted measures and tools could assist farmers in improving nutrient use efficiency and better inform whether measures are needed to regulate nutrient inputs in urban agriculture. Regulatory measures need to accommodate the unique cropping systems of urban farms, taking into consideration, for example, the often higher nutrient demands by intensive cropping systems (Beniston and Lal, 2012) and the effect of different growing substrates or growing space conditioning (e.g., greenhouse, indoor farming) has on nutrient flows in production systems (Goldstein et al., 2016).

\subsection{Beyond ground-based urban agriculture}

Ground-based urban agriculture is the most common type of urban agriculture practiced globally. More recent developments in urban agriculture, however, include rooftop farming, container farming, urban greenhouses and indoor farming systems (Specht et al., 2013, Thomaier et al., 2015). Drawing on the organizing framework (see Figure 8.1) of urban agriculture taxonomies proposed by Goldstein et al. (2016), these systems have different resource use profiles, which have different implications for nutrient recycling. For instance, rooftop systems often demand lightweight substrates in line with the building's rooftop carrying capacity. Meanwhile, high nutrient loads in rooftop farming systems, such as the rooftop farm presented in Chapter 2, may contribute to environmental pollution and nutrient losses, as additional nutrient inputs join storm water runoff (Emilsson et al., 2007, Kong et al., 2015). Alternatively, soil-less (e.g., hydroponic or aeroponic) systems, often located in greenhouses or in indoor (vertical) farms, 
have yet other implications for nutrient flows and recycling. These systems require liquid/ soluble nutrient inputs, and not soil amendments like compost, as preferred by the interviewed ground-based urban agriculture initiatives interviewed in Chapter 3. Closed systems also increase the capacity to recirculate nutrients (e.g., in captured irrigation water) and minimize losses to the ambient environment (Benke and Tomkins, 2017). Evident is that the diversity in urban agriculture typologies presents an array of nutrient profiles. It would therefore be conducive to understand these typology-specific nutrient profiles to better take advantage of nutrient cycling opportunities, including the potential for targeted recovery of nutrients from human excreta and allocation of recovered products amongst the typologies. In this regard, the demand should direct supply. After all, recovered products need to be useful for agriculture, i.e., these need to be recyclable.

\section{Towards a heuristic for recovery pathways}

The recovery of nutrients from wastewater has predominantly been supply-driven. This has materialized in the implementation of add-on technologies to current wastewater treatment plants (WWTPs) with a focus on phosphorus recovery. The new German Sewage Sludge ordinance is a representative example of such developments: recovery of phosphorus from sewage sludge (if sludge contains more than $2 \%$ phosphorus per kilogram of dry solids) is obligatory by 2029 at WWTPs servicing more than 100,000 person equivalents, and by 2032 at WWTPs servicing more than 50,000 person equivalents. While recovery of phosphorus should be stimulated, and even enforced, the myopic focus on phosphorus of current regulations and the corresponding ignoring of other nutrients, results in a loss of these other nutrients and organic matter, and surrenders recovery potential to fit current conventional infrastructures.

The recovery of nutrients from streams containing human excreta towards closing nutrient cycles should in principle maximize recovery of all nutrients into products useful for direct application or for the production of synthetic fertilizers. Chapter 4 presents a comprehensive overview of recovery pathways that exist to recover nutrients from (streams containing) human excreta (see Figure 4.4) and discusses the fate of nutrients and product quality in a range of products including: organic solids, mineral precipitates, nutrient solutions, ashes or slags, and sorbents. Notable, however, are the trade-offs that arise between the various recovery pathways; there is no single recovery pathway that captures all nutrients and carbon present in human excreta into a single product free of contaminants (e.g., pathogens, heavy metals and organic pollutants). For instance, fecal-derived feed stocks that are rich in carbon and usually contain a broad spectrum of nutrients, have varying nutrient quantity and product quality depending on the primary input and the treatment system. Conversely, some products are of high purity and homogeneity (Antonini et al., 2012), but with a limited nutrient spectrum (notably of NPK) and little organic carbon. Fortunately, the combination of several recovery pathways to target more than one product can increase overall nutrient recovery and can prevent losses in byproducts and effluent streams. For example, nanofiltration and ammonia stripping of urine, 
complimented with struvite precipitation (Pronk et al., 2006, Wei et al., 2018, Antonini et al., 2011), yield two products useful for agriculture. Additional fecal treatment can render another complementary product, for instance through biological decomposition, to maximize recovery.

\subsection{Taxonomy of recovery pathways and recovered products}

To better understand the compatibility between the typologies for urban agriculture and the range in products rendered by recovery pathways, there is scope to conceive a heuristic for recovery pathways in the form of a taxonomic scheme based on the characterization of the recovered products, similar to the taxonomy proposed by Goldstein et al. (2016) for urban agriculture typologies. The taxonomy groups recovery pathways based on the similarity of the attributes of their rendered products (e.g., nutrient spectrum, organic matter content, form). The two organizing principles proposed here for this taxonomy include: state of matter (liquid vs. solid) and product composition (organic soil conditioner vs. inorganic nutrient source) (see Figure 8.2).

\subsubsection{State of matter}

The state of matter refers to the physical phase of the product rendered per recovery pathway, classified as either liquid (or soluble) or solid. More specifically, it refers to nutrient solutions and slurries versus organic and inorganic solids (e.g., compost, sludge, granular and powdery inorganic fertilizers, ashes and sorbents, etc.). The state of matter influences the usability of a product in agriculture because certain agricultural systems have preferences or requirements regarding product form. Liquid fertilizers (e.g urine-based nutrient solutions) and soluble fertilizers are appropriate for use in hydroponic growing systems or for administering through irrigation (fertigation), although preventive measures may have to be taken to prevent blockages of the systems due to precipitation of salts (Andreev et al., 2017, Jönsson et al., 2004). Sludge has a similar fluidity as cattle and pig slurries, and can therefore be applied to land via similar (injection) equipment, as is currently obligatory for animal manure in The Netherlands. Conversely, solid products (e.g., compost, precipitates, sorbents, algae biomass) may be applied via conventional spreaders, followed by ploughing or rototilling. Soluble products can be broadcasted or dissolved into irrigation systems.

\subsubsection{Product composition}

This organizing principle refers to recovery pathways that render a soil conditioner with ample organic matter versus an inorganic mineral fertilizer. Soil conditioners, as preferred by the farmers interviewed in the study presented Chapter 3, support soil structure, water infiltration capacity, nutrient retention and aeration. Soil conditioners (e.g., composts, sludge, algal biomass) also serve as a source of nutrients for plant growth, albeit that the nutrients often only become available after mineralization processes. Mineral fertilizers on the other hand target nutrient 
demand by crops directly, provided as either slow-release fertilizer or quick-release fertilizer. Recovery pathways render concentrated nutrient-rich products that are either rich in (mostly) one nutrient (e.g., ammonium nitrogen through air stripping or phosphorus-rich struvite via precipitation) or multiple nutrients (e.g., concentrated urine, mineral sorbents, dehydrated urine). Such extracted nutrient-rich products can offer farmers higher flexibility in nutrient dosing, especially when decoupled nutrients are dosed individually to match crop uptake demands (Maltais-Landry et al., 2016).

\subsubsection{Recovery pathway typologies}

The four recovery pathway typologies can then be defined as: solid-mineral, solid-soil conditioner, liquid-mineral and liquid-soil conditioner (Figure 8.2). A new sanitation system which renders two products, such as a mineral nutrient solution and an inorganic mineral precipitate from urine stripping and precipitation, includes two recovery pathways categorized in separate typologies. The taxonomy can be used as a tool to roughly organize recovery pathways and their respective products. Taken together with the taxonomy for urban agriculture, broad matches can be identified between the two, for example, between building integrated-conditioned urban agriculture and recovery pathways that render liquid-mineral products. In theory, the indicated diversity in cropping systems and recovered products across the taxonomies presents ample opportunities for establishing compatibilities between the two. Yet, product-specific quality in terms of exact nutrient content, nutrient ratios and contaminants is not described by the taxonomy. Understanding of the compatibility between the taxonomies requires further assessment of fertilizer preferences, compatibility of recovered products with current growing systems and application techniques, and safety of product use for different crops and human health. Important too is that the development of compatible systems occur at complementary rates.

\section{Harnessing diversity in nutrient cycling strategies}

The inherent complexity of nutrient cycling relayed in the presented hierarchies and taxonomies of the previous sections, points towards the conclusion: there is no one-size-fits-all solution to nutrient cycling.

\subsection{A Modernized Mixtures Approach}

The deliberate and reflexive reconstruction of systems to deal with contemporary social, economic and environmental challenges is identified as a modernized mixture. The Modernized Mixtures Approach, first proposed by Hegger (2007) for sanitation systems, argues that multiple systems may coexist in the optimum, the applicability of which depends on context-specific spatial and temporal situations. As such, sanitation infrastructures consist of a mixture of scales, 
technologies, payment systems and cultural and institutional structures (Hegger and van Vliet, 2010). A modernized mixtures approach here understands the coexistence of infrastructures that range between the 'large is robust and efficient' type (often centralized) and the 'small is beautiful' type (often decentralized) in both agriculture and sanitation (Hegger and van Vliet, 2010). Closing cycles between human excreta and agriculture deserves a similar approach.

In some contexts the implementation of add-on technologies at existing conventional wastewater treatment plants are appropriate for nutrient recovery. Consider locations where the asset lifetime of a conventional systems has not yet been reached (i.e., younger systems). Here the recovery of phosphorus from bottom ash after the incineration of sludge (e.g., EcoPhos, BE) and struvite recovery from rejection water (Fosvaatje, NL) are developments that demand few changes to existing infrastructure and management practices. The recovered phosphorus can be used by fertilizer industries for the production of synthetic fertilizers. However, coming back to the hierarchy for recovered products, compact, solid products are better suited for transport out of cities. Alternative technologies and strategies, often decentralized in scale, lend themselves for other contexts, for instance in buildings and neighborhoods undergoing renovations, or un-serviced or new-build areas where no existing infrastructure sets preliminary restraints. Newbuild areas can also include agriculture into planning designs (e.g., adjacent to buildings or on rooftops) to facilitate reuse locally.

Such novel and niche solutions help facilitate the transition towards societal embedding and adoption of these new technological concepts and strategies (Schot and Geels, 2008). Knowledge development and capacity building for novel and niche solutions, such as coupling urban agriculture and new sanitation, is useful to realize larger scale implementation. In the Netherlands, for example, developments in new sanitation have multiplied in the last decades and have led to implementation of several full scale systems (STOWA, 2018b) (see Table 1.1). The coexistence of systems may also establish synergies. One example is the separate collection of urine via waterless urinals at the AFAS Live concert hall (formerly the Heineken Music Hall) in Amsterdam. The collected urine is then transported to the WWTP as additional feed to the struvite reactor already installed for phosphorus precipitation from sludge digestate (STOWA, 2018b).

Most importantly, the modernized mixtures approach illustrates that new and conventional sanitation systems, and a range of combinations in-between can coexist, or perhaps even must coexist, to attend to the diversity across contexts. As such, local and global food production, as well as new and conventional systems can coexist and work in synergy. Specifically for urban agriculture and new sanitation, the taxonomies alone illustrate the mixture of systems that might coexist even just within the urban center, while the hierarchies framework again calls a different mixture of strategic variables to bridge spatial distance. 


\subsection{Scaled solutions}

A consideration of multiple systems, however, demands careful selection in terms of economies of scale. In such regard, current conventional systems have been shown to outperform other systems and their robustness proves to cope with future population growth and variability in weather events (Roefs et al., 2017). The certainty they offer, however, only holds when human excreta is framed as waste and the objective is to efficiently get rid of it (Öberg et al., 2014). When human excreta is framed as a resource (Chapter 7), and economies of scale take into account social and environmental benefits across the sanitation chain (i.e., from capture to reuse/ disposal), then alternative systems may be more lucrative. For example, the latest report on the Waterschoon new sanitation system in Sneek, NL (based on source separation of black water and grey water) shows that the system scores slightly higher overall in an life cycle assessment (LCA) analysis than conventional WWTPs for 100,000 person equivalents (p.e) and 30,000 p.e., assuming correct and full capacity operation of the new sanitation system for 1500 p.e. (STOWA, 2018a). Especially the positive energy balance of the system was determining in its high score. Similar reasoning can be applied to agricultural systems, where the value of urban agriculture extends social, economic and environmental co-benefits (Artmann and Sartison, 2018a). While multiple systems increases operational complexity and implementation costs, there is scope to exploit various systems at appropriate economies of scale that take into account their value across the sanitation chain.

\section{The value of high-resolution, spatially-explicit data in nutrient flow assessments}

Optimizing nutrient management demands a coordinated strategy for nutrient conservation and recovery (Scholz et al., 2015). Several studies have assessed nutrient flows, stocks and stores using accessible datasets to draw attention to the pressing issues of the abundance-scarcity paradox, as well as strategies to restore nutrient cycles (Sharpley et al., 2016). Data resolution, however, is often coarse. While meta-analyses are able to indicate broad trends in nutrient transfers and imbalances, closing nutrient cycles demands understanding at higher resolution to determine subsequent interventions (Sharpley et al., 2016).

The GIS analyses presented in Chapter 5 and Chapter 6 bring together several aspects concerning quantity, quality, space and time. Chapter 5 first presents a novel tool to identify locations (buildings and neighborhoods) where nutrient excretion is highest (termed nutrient hotspots), where the implementation of new sanitation systems was assumed to be most promising. Chapter 6 builds on this first spatial study by matching the supply with nearby demand and optimizing transportation distances between the two. Both chapters impart the benefit of modelling nutrient flows at high resolution. As discussed in other studies, such spatial analyses better equip decision-makers to evaluate the viability of site-specific interventions and specific supply and demand connections for improved resource management (Agudelo-Vera, 2012, Metson et al., 
2018). Especially the provision of spatially explicit data on the scale at which practitioners work is useful for determining intervention strategies (Voskamp et al., 2018). The relevance of scale is illustrated in Chapter 5 as building hotspots were not located in hotspot neighborhoods per se, likewise, hotspot neighborhoods did not necessarily accommodate any building hotspots. The presentation of results at two spatial scales attests to the value of spatial resolution for the generation and interpretation of results, as well as for selecting appropriate locations for new sanitation and other interventions. We expect results to further emphasize the relevance of resolution of spatially explicit data if applied to cities that exhibit sprawl (e.g., Phoenix, AZ, USA; Melbourne, Australia) versus high density development (e.g., Shanghai, China; New York City, NY, USA).

The increased momentum in big data collection at high resolution signifies a potential increase in understanding nutrient flows and identifying possibilities for closing cycles. Such data in addition to other model refinements would help expand upon current results, such as data on nutrient inputs and harvest yields per crop type per agricultural parcel, location and size of available arable land and/or rooftop acreage per city, and data on expected dates for needed sewage renovations in buildings and neighborhoods. Especially the inclusion of temporal data on nutrient demand and supply would help optimize transport versus storage requirements of collected streams of recovered products.

\section{A reflection on nutrient cycling in the Netherlands}

The focus on the Dutch context in this thesis is convoluted. To start, agricultural land area is in relatively close proximity to cities already. Many farms could mistakenly be classified as urban agriculture. While many of the grown vegetables and potatoes, and produced dairy and meat products do end up on urban tables, most Dutch agriculture is oriented towards the international market (van Der Schans, 2010). In terms of nutrient management, such proximity only increases opportunities to convey nutrients from cities back to agriculture.

Further convolution exists in that agricultural soils across the Netherlands are often already saturated with nutrients, particularly phosphorus (CBS et al., 2019, Smit et al., 2015). Moreover the large livestock farming industry offers an abundance of nutrient-rich manure, which equally contributes to closing nutrient cycles. Movements in circular agriculture are increasingly advocating improved nutrient cycling of both manure and other farm wastes back to agriculture (de Boer and van Ittersum, 2018). Recovered products from human excreta thus compete with existing and future fertilizer inputs on the market. Complete substitution of current fertilizer inputs with recovered products, as assumed in Chapter 6 with human urine, is not realistic. In future applications of the model, partial substitution of fertilizer demand with recovered products is suggested. For example the fraction of fertilizer demand currently satisfied with synthetic fertilizer might be substitutable by recovered products, while other products, such as manure and compost should also be accounted for. 
Regardless of the abundance of nutrients in the Netherlands, there is still sufficient argumentation in favor of their recovery from streams containing human excreta, as shown in Figure 1.1, in Chapter 1. In addition to reducing losses, the Netherlands can reduce their dependency on finite foreign sources of phosphorus, and to cope with the excess, establish an export market for recovered nutrients from human excreta and/or animal manure to regions where it is profitable and useful (Smit et al., 2015).

\section{Inputs for future research agendas}

This thesis focused primarily on quantitative and spatial assessments of nutrient demand and supply between urban agriculture and new sanitation. The outcomes of this research give inspiration to sharpen and expand on these first results, not only concerning nutrient quantities and nutrient flows in space, but also by including qualitative aspects and temporal dynamics of nutrient recycling in a systems approach. Selected pending and newly identified knowledge gaps are elaborated in the following sub-sections. Other areas for future research not explicitly elucidated here, though also require attention, include social acceptance, market value, and regulatory frameworks for appropriate and safe reuse of recovered products.

\subsection{Quantity \& Quality}

This research looked to maximize matching of nutrient quantities between supply and demand, with only minor tangents to the importance of quality. In Chapter 2 a distinction between slow versus quick release fertilizer is made, and in Chapter 4 contaminants in the recovered products are roughly indicated as found in literature. However in-depth understanding of quality are left aside in this thesis. As a result the importance of fertilizer quality for plant (e.g., nutrient availability), environmental (e.g., soil health and water quality) and human (i.e., risk) health is undermined. Understanding the fate and risks of pathogens, pharmaceuticals, hormones, heavy metals and other persistent contaminants in the soil and receiving water bodies, during plant uptake, and post consumption of harvested crops is critical, and demands better knowledge on sorption capacities and biodegradability of the substances, and formation of intermediates. Risk assessments can help establish threshold values for quality assurance. With established threshold values for the reuse of recovered products in agriculture follows research on (1) the development of new, or selection of existing technologies, that can comply with these values and (2) the formation of appropriate legislation to facilitate product reuse $e^{4}$.

In light of quality thresholds and preferences, future research can refine and expand upon the quantitative assessments to match supply and demand conducted in this thesis. First, this requires defining nutrient demand per fertilizer type per urban agriculture typology, so as to describe their

\footnotetext{
4 For example, in the Netherlands the reuse of all human excreta-derived sludge is prohibited, however as shown by Tervahauta et al. (2014), heavy metals in human excreta are exclusively food-derived and closing cycles implies returning these to the land. As such, legislation on the reuse of sludge in agriculture on basis of heavy metals should differentiate between sludge derived from human excreta and black water, versus sewage sludge.
} 
respective nutrient profiles quantitatively and qualitatively. The assessment of nutrient inputs in ground-based urban agriculture in Chapter 3 indicate the need for improved understanding of crop yields and nutrient uptake in urban agriculture to better assign demand values in groundbased agriculture. Similar values should be obtained for the other typologies.

Second, the recovery pathways presented in Chapter 4 require further assessment of potential nutrient recovery quantities and respective quality per pathway. Detailed process descriptions of new sanitation systems are currently fragmented across literature, per primary input (e.g., Maurer et al. (2003a)), per targeted nutrient (e.g., De Graaff et al. (2011), (Egle et al., 2015), per technology (e.g., Zeeman et al. (2008)). A comparison of product composition per applied sequence of technologies, each with respective nutrient recovery efficiencies and potential to destroy contaminants, is useful. After, further cross-checking of compatibilities between the demand and supply are needed, and the identification of opportunities in practice or technology to secure better matching, for example, attuning products to current application methods or introducing new application methods and technologies, manipulating product form for improved nutrient release when and where needed, or developing recovery technologies for new recovered products).

\subsection{Space $\&$ time}

Demand and supply vary in space and time, prompting questions such as: how often does agriculture apply fertilizer and what are the necessary retention times for treatment and recovery of nutrients from new sanitation? Where is agriculture located with respect to where nutrients are collected and recovered? Fluctuations in nutrient demand based on seasonality in crop production (e.g., winter dormancy) or, on the contrary, the continuous demand for nutrients in hydroponic systems, influences not only the product type, but also the requirements for product storage and housing of treatment technologies. The distance between demand and supply influences the feasibility of transportation between the two, albeit by truck or piped systems. To evaluate an exchange of nutrients between urban agriculture and new sanitation, these systems also have to be described in a dynamic way considering spatial and temporal patterns.

\subsection{Model expansion}

Literature shows an increase in the use of GIS to understand both urban agriculture (e.g., Haberman et al., 2014, Martellozzo et al., 2014, Taylor and Lovell, 2012) and nutrient flows and stocks (e.g., Akram et al., 2019a, Akram et al., 2019b, Metson et al., 2018, Trimmer and Guest, 2018). The GIS analyses presented in Chapter 5 and Chapter 6 are first results in bringing together several dimensions of demand and supply using spatially explicit data. Several model refinements and expansion opportunities span both the identification of nutrient hotspots and the optimization of transport distances from hotspots to agriculture, which have been elaborated in the chapter discussions. In short, these include: (1) calibration on nutrient excretion input 
data (2) refined fertilizer use input data (amount, form and quality, frequency of application), (3) inclusion of storage capacity to determine frequency of collection and total transport requirements, (4) inclusion of other recovered products and nutrient sources to better represent fertilizer market share and match demand and supply, (5) assessment of economic factors (e.g., transport distance, economies of scale, infrastructure, operation and maintenance costs), and (6) scenario development to illustrate nutrient cycling opportunities and application of model in other contexts, including an evaluation of environmental impact (e.g., life cycle assessment) for comparison of scenarios.

\section{Conclusions}

Increased nutrient use efficiency in agriculture and increased recycling of nutrients from domestic wastes back to agriculture should be prioritized to both minimize ecosystem damage, and ensure food security and access to sufficient fertilizers. In cities saturated with nutrients in organic waste and human excreta, it seems unsuited to perpetuate current nutrient management practices in urban agriculture, including the import of manure from rural areas and the excess of nutrient inputs observed in general. The proximity of urban agriculture to nutrient sources from human excreta, lends itself for establishing local nutrient cycles, especially for nutrients in forms too costly (i.e., bulky) to export back to rural and global agricultural hinterlands.

Various studies consider the potential to scale up urban agriculture to secure food provisioning but few look at the extent to which urban agriculture should be scaled up to recycle nutrients locally. To determine the scalability of urban agriculture it may be useful to strike a balance between food that is viable (e.g., perishable or profitable crops) for urban production and a supply of human excreta-derived fertilizer products that meet the predefined demand in terms of type and form. To this end, there is scope to consider a hierarchy of agricultural land use in proximity to the urban center, paired with recovered products suited for transport to each respective type of agriculture.

The diversity in urban agriculture typologies as well as the diversity in recovery pathways and recovered products signifies an increased potential to match nutrient demand and supply between one another. More research is needed, however, to better define demand and supply across typologies. The study of nutrient flows, in terms of quantity and quality, is best complemented with an understanding of point sources, direction and connection of nutrient flows to other spatial characteristics. Spatial models of nutrient sources and sinks can aid capacity building for planning and decision-making with regards to intervention strategies for improved nutrient management. Such models can be further optimized and expanded to further examine the potential of closing local nutrient cycles, taking into consideration context-specific factors and parameters. Although rough mass balances reveal that the contribution of integrated urban agriculture and new sanitation systems towards a circular nutrient economy will remain partial, the necessity for systems changes and reframing in both food provisioning and human excreta management strategies welcomes further exploration of their integration. 


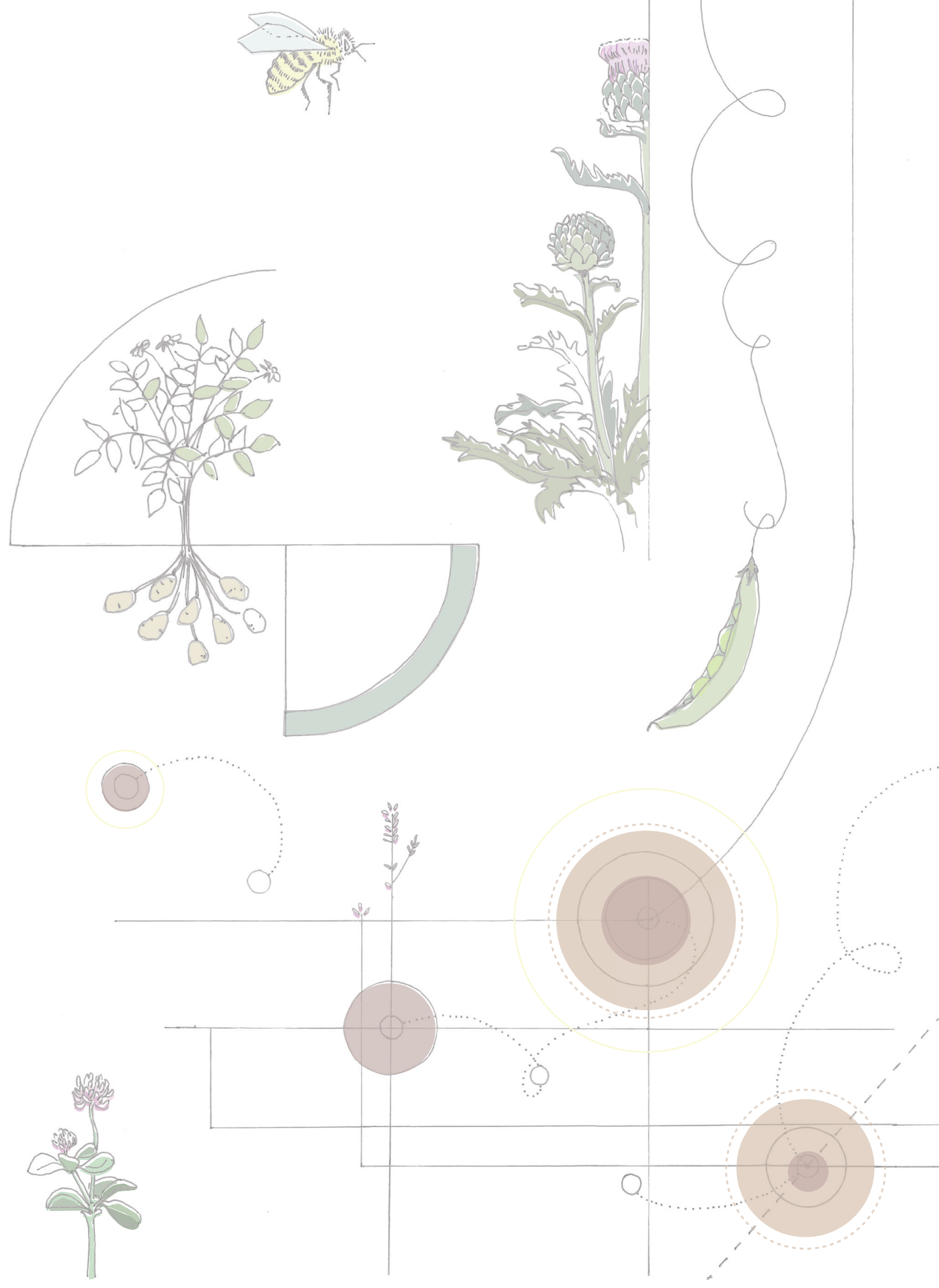


Abdel-Shafy, H. I. \& Mansour, M. S. M. 2016. Integration of effective microorganisms and membrane bioreactor for the elimination of pharmaceutical active compounds from urine for safe reuse. Journal of Water Reuse and Desalination, 6, 495-504.

Abdulkadir, A., Leffelaar, P. A., Agbenin, J. O. \& Giller, K. E. 2013. Nutrient flows and balances in urban and peri-urban agroecosystems of Kano, Nigeria. Nutrient cycling in agroecosystems, 95, 231-254.

Abinandan, S., Subashchandrabose, S. R., Venkateswarlu, K. \& Megharaj, M. 2018. Nutrient removal and biomass production: advances in microalgal biotechnology for wastewater treatment. Critical Reviews in Biotechnology, $38,1-17$.

Ackerman, K. 2012. Urban agriculture: Opportunities and constraints. Metropolitan Sustainability: Understanding and Improving the Urban Environment. Elsevier Ltd.

Ackerman, K., Conard, M., Culligan, P., Plunz, R., Sutto, M.-P. \& Whittinghill, L. 2014. Sustainable food systems for future cities: The potential of urban agriculture. The economic and social review, 45, 189-206.

Adam, C., Peplinski, B., Kley, G., Kratz, S., Schick, J. \& Schnug, E. 2008. Phosphorrückgewinnung aus Klärschlammaschen - Ergebnisse aus dem EU-Projekt SUSAN. Osterreichische Wasser- und Abfallwirtschaft, 60, 55-64.

Adam, C., Peplinski, B., Michaelis, M., Kley, G. \& Simon, F. G. 2009. Thermochemical treatment of sewage sludge ashes for phosphorus recovery. Waste Management, 29, 1122-1128.

Afif, E., Azadi, P. \& Farnood, R. 2011. Catalytic hydrothermal gasification of activated sludge. Applied Catalysis B: Environmental, 105, 136-143.

Agudelo-Vera, C. M. 2012. Dynamic water resource management for achieving self-sufficiency of cities of tomorrow.

Agudelo-Vera, C. M., Leduc, W. R., Mels, A. R. \& Rijnaarts, H. H. 2012a. Harvesting urban resources towards more resilient cities. Resources, conservation and recycling, 64, 3-12.

Agudelo-Vera, C. M., Leduc, W. R. W. A., Mels, A. R. \& Rijnaarts, H. H. M. 2012b. Harvesting urban resources towards more resilient cities. Resources Conservation and Recycling, 64, 3-12.

Agudelo-Vera, C. M., Mels, A., Keesman, K. \& Rijnaarts, H. 2012c. The Urban Harvest Approach as an Aid for Sustainable Urban Resource Planning. Journal of Industrial Ecology, 16, 839-850.

Ahlborg, H., Ruiz-Mercado, I., Molander, S. \& Masera, O. 2019. Bringing technology into social-ecological systems research-Motivations for a socio-technical-ecological systems approach. Sustainability (Switzerland).

Aida, T. M., Nonaka, T., Fukuda, S., Kujiraoka, H., Kumagai, Y., Maruta, R., Ota, M., Suzuki, I., Watanabe, M. M., Inomata, H. \& Smith, R. L. 2016. Nutrient recovery from municipal sludge for microalgae cultivation with two-step hydrothermal liquefaction. Algal Research, 18, 61-68.

Akram, U., Quttineh, N.-H., Wennergren, U., Tonderski, K. \& Metson, G. S. 2019a. Enhancing nutrient recycling from excreta to meet crop nutrient needs in Sweden - a spatial analysis. Scientific Reports, 9, 10264.

Akram, U., Tonderski, K., Quttineh, N.-H., Wennergren, U. \& Metson, G. S. 2019b. Optimizing nutrient recycling from excreta in Sweden and Pakistan: Higher spatial resolution makes transportation more attractive. Frontiers in Sustainable Food Systems, 3, 50.

Amundson, R., Berhe, A. A., Hopmans, J. W., Olson, C., Sztein, A. E. \& Sparks, D. L. 2015. Soil and human security in the 21 st century. Science, 348.

Anderson, C., Malambo, D. H., Perez, M. E. G., Nobela, H. N., de Pooter, L., Spit, J., Hooijmans, C. M., van de Vossenberg, J., Greya, W., Thole, B., van Lier, J. B. \& Brdjanovic, D. 2015. Lactic acid fermentation, urea and lime addition: Promising faecal sludge sanitizing methods for emergency sanitation. International Journal of Environmental Research and Public Health, 12, 13871-13885.

Andersson, K., Dickin, S. \& Rosemarin, A. 2016. Towards "sustainable" sanitation: Challenges and opportunities in urban areas. Sustainability (Switzerland), 8.

Andersson, K., Otoo, M. \& Nolasco, M. 2018. Innovative sanitation approaches could address multiple development challenges. Water Science and Technology.

Andreev, N., Ronteltap, M., Boincean, B., Wernli, M., Zubcov, E., Bagrin, N., Borodin, N. \& Lens, P. 2017. Lactic acid fermentation of human urine to improve its fertilizing value and reduce odour emissions. Journal of environmental management, 198, 63-69.

Ansari, A. J., Hai, F. I., Price, W. E., Drewes, J. E. \& Nghiem, L. D. 2017. Forward osmosis as a platform for resource recovery from municipal wastewater - A critical assessment of the literature. Journal of Membrane Science, 529, 195-206.

Antakyali, D., Meyer, C., Preyl, V., Maier, W. \& Steinmetz, H. 2013. Large-scale application of nutrient recovery from digested sludge as struvite. Water Practice and Technology, 8, 256-262.

Antonini, S., Nguyen, P. T., Arnold, U., Eichert, T. \& Clemens, J. 2012. Solar thermal evaporation of human urine for nitrogen and phosphorus recovery in Vietnam. Science of the Total Environment, 414, 592-599. 
Antonini, S., Paris, S., Eichert, T. \& Clemens, J. 2011. Nitrogen and phosphorus recovery from human urine by struvite precipitation and air stripping in Vietnam. CLEAN-Soil, Air, Water, 39, 1099-1104.

Artmann, M. \& Sartison, K. 2018a. The role of urban agriculture as a nature-based solution: a review for developing a systemic assessment framework. Sustainability, 10, 1937.

Artmann, M. \& Sartison, K. 2018b. The Role of Urban Agriculture as a Nature-Based Solution: A Review for Developing a Systemic Assessment Framework. Sustainability, 10, 1-32.

Atay, Ş. \& Akbal, F. 2016. Classification and Effects of Sludge Disintegration Technologies Integrated Into Sludge Handling Units: An Overview. Clean - Soil, Air, Water, 44, 1198-1213.

Ayala, S. \& Rao, E. 2002. Perspectives of soil fertility management with a focus on fertilizer use for crop productivity. CURRENT SCIENCE-BANGALORE-, 82, 797-807.

B

Babel, S. 2003. Low-cost adsorbents for heavy metals uptake from contaminated water: a review. Journal of Hazardous Materials, 97, 219-243.

Bai, X., Li, Z., Zhang, Y., Ni, J., Wang, X. \& Zhou, X. 2017. Recovery of Ammonium in Urine by Biochar Derived from Faecal Sludge and its Application as Soil Conditioner. Waste and Biomass Valorization, 0, 1-10.

Balkema, A. J., Preisig, H. A., Otterpohl, R. \& Lambert, F. J. 2002. Indicators for the sustainability assessment of wastewater treatment systems. Urban water, 4, 153-161.

Balmér, P. 2004. Phosphorus recovery - An overview of potentials and possibilities. Water Science and Technology, 49, 185-190.

Barber, W. P. F. 2016. Thermal hydrolysis for sewage treatment: A critical review. Water Research, 104, 53-71.

Batstone, D. J., Hülsen, T., Mehta, C. M. \& Keller, J. 2015. Platforms for energy and nutrient recovery from domestic wastewater: A review. Chemosphere, 140, 2-11.

Batty, M., Besussi, E., Maat, K. \& Harts, J. J. 2004. Representing multifunctional cities: density and diversity in space and time. Built Environment, 30, 324-337.

Baumhardt, R. L., Stewart, B. A. \& Sainju, U. M. 2015. North American soil degradation: Processes, practices, and mitigating strategies. Sustainability (Switzerland), 7, 2936-2960.

Beck, U. 1992. Risk Society. Towards a New Modernity.

Beler-Baykal, B., Allar, A. D. \& Bayram, S. 2011. Nitrogen recovery from source-separated human urine using clinoptilolite and preliminary results of its use as fertilizer. Water Science and Technology, 63, 811-817.

Belevi, H. \& Baumgartner, B. 2003. A systematic overview of urban agriculture in developing countries from an environmental point of view. International Journal of Environmental Technology and Management, 3, 193-211.

Bender, S. F., Wagg, C. \& van der Heijden, M. G. A. 2016. An Underground Revolution: Biodiversity and Soil Ecological Engineering for Agricultural Sustainability. Trends in Ecology and Evolution, 31, 440-452.

Bengtsson, M. \& Tillman, A. M. 2004. Actors and interpretations in an environmental controversy: The Swedish debate on sewage sludge use in agriculture. Resources, Conservation and Recycling, 42, 65-82.

Beniston, J. \& Lal, R. 2012. Improving Soil Quality for Urban Agriculture in the North Central U.S. In: LAL, R. \& AUGUSTIN, B. (eds.) Carbon Sequestration in Urban Ecosystems. Dordrecht: Springer Netherlands.

Benke, K. \& Tomkins, B. 2017. Future food-production systems: vertical farming and controlled-environment agriculture. Sustainability: Science, Practice and Policy, 13, 13-26.

Bergström, L., Kirchmann, H., Aronsson, H., Torstensson, G. \& Mattsson, L. 2008. Use Efficiency and Leaching of Nutrients in Organic and Conventional Cropping Systems in Sweden. In: KIRCHMANN, H. \& BERGSTRÖM, L. (eds.) Organic Crop Production - Ambitions and Limitations. Dordrecht: Springer Netherlands.

Bergström, L., Kirchmann, H., Aronsson, H., Torstensson, G. \& Mattsson, L. 2009. Use efficiency and leaching of nutrients in organic and conventional cropping systems in Sweden. Organic Crop Production-Ambitions and Limitations. Springer.

Berry, P., Sylvester-Bradley, R., Philipps, L., Hatch, D., Cuttle, S., Rayns, F. \& Gosling, P. 2002. Is the productivity of organic farms restricted by the supply of available nitrogen? Soil Use and Management, 18, 248-255.

Bethune, D. N., Chu, A. \& Ryan, M. C. 2016. Passive evaporation of source-separated urine from dry toilets: UES optimization and dry product accumulation over time. Journal of Water, Sanitation and Hygiene for Development, 6, 96-103.

Beuchler, S., Mekala, G. D. \& Keraita, B. 2006. Wastewater Use for Urban and Peri-urban Agriculture. In: VAN VEENHUIZEN, R. (ed.) Cities Farming for the Future: Urban Agriculture for Green and Productive Cities. the Philippines: RUAF Foundation.

Bischel, H. N., Schertenleib, A., Fumasoli, A., Udert, K. M. \& Kohn, T. 2015. Inactivation kinetics and mechanisms of viral and bacterial pathogen surrogates during urine nitrification. Environ. Sci.: Water Res. Technol., 1, 65-76. 
Blöcher, C., Niewersch, C. \& Melin, T. 2012. Phosphorus recovery from sewage sludge with a hybrid process of low pressure wet oxidation and nanofiltration. Water Research, 46, 2009-2019.

Bonvin, C., Etter, B., Udert, K. M., Frossard, E., Nanzer, S., Tamburini, F. \& Oberson, A. 2015. Plant uptake of phosphorus and nitrogen recycled from synthetic source-separated urine. Ambio, 44, 217-227.

Borsuk, M. E., Maurer, M., Lienert, J. \& Larsen, T. A. 2008. Charting a Path for Innovative Toilet Technology Using Multicriteria Decision Analysis. Environmental Science \& Technology, 42, 1855-1862.

Bosch, H. \& De Jonge, P. 1989. Handboek voor de Akkerbouw en de Groenteteelt in de Vollegrond 1989. PAGV.

Bouwman, A., Beusen, A. \& Billen, G. 2009. Human alteration of the global nitrogen and phosphorus soil balances for the period 1970-2050. Global Biogeochemical Cycles, 23.

Brands, E. 2014. Prospects and challenges for sustainable sanitation in developed nations: a critical review. Environmental Reviews, 22, 346-363.

Bridger, G. L., Salutsky, M. L. \& Starostka, R. W. 1962. Micronutrient Sources, Metal Ammonium Phosphates as Fertilizers. Journal of Agricultural and Food Chemistry, 10, 181-188.

Bridle, T. \& Pritchard, D. 2004. Energy and nutrient recovery from sewage sludge via pyrolysis. Water Science Technololgy, 50, 169-175.

Brikké, F. \& Bredero, M. 2003. Linking technology choice with operation and maintenance in the context of community water supply and sanitation: A reference document for planners and project staff. In: ORGANIZATION, W. H. (ed.). Geneva: World Health Organization, IRC Water, Sanitation Centre.

Brunner, P. H. 2007. Reshaping urban metabolism, na.

Butkovskyi, A., Ni, G., Hernandez Leal, L., Rijnaarts, H. H. M. \& Zeeman, G. 2016a. Mitigation of micropollutants for black water application in agriculture via composting of anaerobic sludge. Journal of Hazardous Materials, $303,41-47$.

Butkovskyi, A., Rijnaarts, H. H. M., Zeeman, G. \& Hernandez Leal, L. 2016b. Fate of personal care and household products in source separated sanitation. Journal of Hazardous Materials, 320, 427-434.

Cabeza, R., Steingrobe, B., Römer, W. \& Claassen, N. 2011. Effectiveness of recycled P products as P fertilizers, as evaluated in pot experiments. Nutrient Cycling in Agroecosystems, 91, 173-184.

Canter, C. E., Blowers, P., Handler, R. M. \& Shonnard, D. R. 2015. Implications of widespread algal biofuels production on macronutrient fertilizer supplies: Nutrient demand and evaluation of potential alternate nutrient sources. Applied Energy, 143, 71-80.

Capizzi-Banas, S., Deloge, M., Remy, M. \& Schwartzbrod, J. 2004. Liming as an advanced treatment for sludge sanitisation: helminth eggs elimination--Ascaris eggs as model. Water Res, 38, 3251-8.

Caplow, T. 2009. Building integrated agriculture: Philosophy and practice. Urban futures, 2030, 54-58.

Cardoso, E. J. B. N., Vasconcellos, R. L. F., Bini, D., Miyauchi, M. Y. H., Santos, C. A. d., Alves, P. R. L., Paula, A. M. d., Nakatani, A. S., Pereira, J. d. M. \& Nogueira, M. A. 2013. Soil health: looking for suitable indicators. What should be considered to assess the effects of use and management on soil health? Scientia Agricola, 70, 274 289.

Carter, M. R. 2002. Soil quality for sustainable land management: Organic matter and aggregation interactions that maintain soil functions. Agronomy Journal, 94, 38-47.

Casadellà, A., Kuntke, P., Schaetzle, O. \& Loos, K. 2016. Clinoptilolite-based mixed matrix membranes for the selective recovery of potassium and ammonium. Water Research, 90, 62-70.

CBS 2013. Bijlage B TBO 2011/2012 Onderzoeksdocumentatie. Centraal Bureau voor de Statistiek. Available: https:// www.scp.nl/Publicaties/Alle_publicaties/Publicaties_2013/Met_het_oog_op_ de_tijd/Bijlage_B_TBO_2011_2012_Onderzoeksdocumentatie

CBS 2014. Tijdsbesteding in uren per week; persoonskenmerken. 29-07-2014 ed. Den Haag: Centraal Bureau voor de Statistiek

CBS, PLB, RIVM \& WUR 2019. Stikstof- en fosfaatbalans voor landbouwgrond, 1990-2017. Centraal Bureau voor de Statistiek (CBS), Den Haag; PBL Planbureau voor de Leefomgeving, Den Haag; RIVM Rijksinstituut voor Volksgezondheid en Milieu, Bilthoven; Wageningen University and Research, Wageningen.

Cerón-Palma, I., Sanyé-Mengual, E., Oliver-Solà, J., Montero, J. I. \& Rieradevall, J. 2012. Barriers and Opportunities Regarding the Implementation of Rooftop Eco.Greenhouses (RTEG) in Mediterranean Cities of Europe. Journal of Urban Technology, 19, 87-103.

Chang, Y., Deng, C., Dore, A. J. \& Zhuang, G. 2015. Human excreta as a stable and important source of atmospheric ammonia in the megacity of Shanghai. PLoS ONE, 10, 1-13. 
Chen, P., Anderson, E., Addy, M., Zhang, R., Cheng, Y., Peng, P., Ma, Y., Fan, L., Zhang, Y., Lu, Q., Liu, S., Zhou, N., Deng, X., Zhou, W., Omar, M., Griffith, R., Kabir, F., Lei, H., Wang, Y., Liu, Y. \& Ruan, R. 2018. Breakthrough Technologies for the Biorefining of Organic Solid and Liquid Wastes. Engineering, 4, 574-580.

Choi, H. J. \& Lee, S. M. 2015. Heavy metal removal from acid mine drainage by calcined eggshell and microalgae hybrid system. Environmental Science and Pollution Research, 22, 13404-13411.

Chowdhury, R. B., Moore, G. A. \& Weatherley, A. J. 2018. A multi-year phosphorus flow analysis of a key agricultural region in Australia to identify options for sustainable management. Agricultural systems, 161, 42-60.

Chowdhury, R. B., Moore, G. A., Weatherley, A. J. \& Arora, M. 2014. A review of recent substance flow analyses of phosphorus to identify priority management areas at different geographical scales. Resources, Conservation and Recycling, 83, 213-228.

Chowdhury, R. B., Moore, G. A., Weatherley, A. J. \& Arora, M. 2016. A novel substance flow analysis model for analysing multi-year phosphorus flow at the regional scale. Science of the Total Environment, 572, 1269-1280.

Chowdhury, R. B., Moore, G. A., Weatherley, A. J. \& Arora, M. 2017. Key sustainability challenges for the global phosphorus resource, their implications for global food security, and options for mitigation. Journal of Cleaner Production, 140, 945-963.

Chrispim, M. C., Tarpeh, W. A., Salinas, D. T. \& Nolasco, M. A. 2017a. The sanitation and urban agriculture nexus: urine collection and application as fertilizer in São Paulo, Brazil. Journal of Water Sanitation and Hygiene for Development, 7, 455-465.

Chrispim, M. C., Tarpeh, W. A., Salinas, D. T. \& Nolasco, M. A. 2017b. The sanitation and urban agriculture nexus: urine collection and application as fertilizer in São Paulo, Brazil. Journal of Water, Sanitation and Hygiene for Development, 7, 455-465.

Ciceri, D., Manning, D. A. C. \& Allanore, A. 2015. Historical and technical developments of potassium resources. Science of the Total Environment, 502, 590-601.

Cieslik, B. M., Namiesnik, J. \& Konieczka, P. 2015. Review of sewage sludge management: Standards, regulations and analytical methods. Journal of Cleaner Production, 90, 1-15.

Cityportal Rotterdam. 2014. Food Council [Online]. Cityportal Rotterdam. Available: http://www.rotterdam.nl/foodcouncil [Accessed January 29, 2014 2014].

Clinton, N., Stuhlmacher, M., Miles, A., Uludere Aragon, N., Wagner, M., Georgescu, M., Herwig, C. \& Gong, P. 2018. A Global Geospatial Ecosystem Services Estimate of Urban Agriculture. Earth's Future, 6, 40-60.

Cloïn, M., van den Broek, A., van den Dool, R., de Haan, J., de Hart, J., van Houwelingen, P., Tiessen-Raaphorst, A., Sonck, N. \& Spit, J. 2013. Met oog op de tijd: Een blik op de tijdsbesteding van Nederlanders. In: CLOÏN, M. (ed.). Den Haag: Sociaal en Cultureel Planbureau. Available: https://www.scp.nl/Publicaties/Alle_publicaties/Publicaties_2013/Met_het_oog_op_de_tijd

Cofie, O., Jackson, L. \& van Veenhuizen, R. 2013. Thematic paper 1: Innovative experiences with the reuse of organic wastes and wastewater in (peri-) urban agriculture in the global south. RUAF Foundation; SUPURBFOOD.

Cogan, T. A., Bloomfield, S. F. \& Humphrey, T. J. 1999. The effectiveness of hygiene procedures for prevention of cross-contamination from chicken carcases in the domestic kitchen. Letters in Applied Microbiology, 29, 354-358.

Cogger, C. G., Forge, T. A. \& Neilsen, G. H. 2006. Biosolids recycling: Nitrogen management and soil ecology. Canadian Journal of Soil Science, 86, 613-620.

Cohen, D. \& Crabtree, B. 2006. Qualitative Research Guidelines Project.

Cole, A. J., Paul, N. A., de Nys, R. \& Roberts, D. A. 2017. Good for sewage treatment and good for agriculture: Algal based compost and biochar. Journal of Environmental Management, 200, 105-113.

Cooper, J., Lombardi, R., Boardman, D. \& Carliell-Marquet, C. 2011. The future distribution and production of global phosphate rock reserves. Resources, Conservation and Recycling, 57, 78-86.

Cooper, P. 2001a. Historical aspects of wastewater treatment. In: LENS, P., ZEEMAN, G. \& LETTINGA, G. (eds.) Decentralised Sanitation and Reuse. Padstow, Cornwall, UK: IWA Publishing.

Cooper, P. F. 2001b. Historical aspects of wastewater treatment. In: LENS, P. N. L., ZEEMAN, G. \& LETTINGA, G. (eds.) Decentralised sanitation and reuse: concepts, systems and implementation. IWA.

Corcoran, J., Winter, M. J. \& Tyler, C. R. 2010. Pharmaceuticals in the aquatic environment: A critical review of the evidence for health effects in fish. Critical Reviews in Toxicology.

Cordell, D., Drangert, J.-O. \& White, S. 2009a. The story of phosphorus: Global food security and food for thought. Global environmental change, 19, 292-305.

Cordell, D., Drangert, J. O. \& White, S. 2009b. The story of phosphorus: Global food security and food for thought. Global Environmental Change, 19, 292-305.

Cordell, D., Neset, T.-S. S. \& Prior, T. 2012. The phosphorus mass balance: identifying 'hotspots' in the food system as a roadmap to phosphorus security. Current Opinion in Biotechnology, 23, 839-845. 
Cordell, D. \& White, S. 2011. Peak Phosphorus: Clarifying the Key Issues of a Vigorous Debate about Long-Term Phosphorus Security. Sustainability, 3, 2027-2049.

Cornel, P. \& Schaum, C. 2009. Phosphorus recovery from wastewater: Needs, technologies and costs. Water Science and Technology, 59, 1069-1076.

Cowling, S. J., Gardner, M. J. \& Hunt, D. T. E. 1992. Removal of heavy metals from sewage by sulphide precipitation: Thermodynamic calculations and tests on a pilot-scale anaerobic reactor. Environmental Technology (United Kingdom), 13, 281-291.

Crews, T. \& Rumsey, B. 2017. What agriculture can learn from native ecosystems in building soil organic matter: A review. Sustainability, 9, 578.

Cunningham, S. A., Attwood, S. J., Bawa, K. S., Benton, T. G., Broadhurst, L. M., Didham, R. K., McIntyre, S., Perfecto, I., Samways, M. J., Tscharntke, T., Vandermeer, J., Villard, M. A., Young, A. G. \& Lindenmayer, D. B. 2013. To close the yield-gap while saving biodiversity will require multiple locally relevant strategies. Agriculture, Ecosystems and Environment, 173, 20-27.

\section{$\mathrm{D}$}

Dahlin, J., Halbherr, V., Kurz, P., Nelles, M. \& Herbes, C. 2016. Marketing green fertilizers: Insights into consumer preferences. Sustainability (Switzerland), 8, 1-15.

Daigger, G. T. 2009. Evolving Urban Water and Residuals Management Paradigms: Water Reclamation and Reuse, Decentralization, and Resource Recovery. Water Environment Research, 81, 809-823.

Danso-Boateng, E., Holdich, R. G., Martin, S. J., Shama, G. \& Wheatley, A. D. 2015a. Process energetics for the hydrothermal carbonisation of human faecal wastes. Energy Conversion and Management, 105, 1115-1124.

Danso-Boateng, E., Holdich, R. G., Shama, G., Wheatley, A. D., Sohail, M. \& Martin, S. J. 2013. Kinetics of faecal biomass hydrothermal carbonisation for hydrochar production. Applied Energy, 111, 351-357.

Danso-Boateng, E., Shama, G., Wheatley, A. D., Martin, S. J. \& Holdich, R. G. 2015b. Hydrothermal carbonisation of sewage sludge: Effect of process conditions on product characteristics and methane production. Bioresource Technology, 177, 318-327.

Darwish, M., Aris, A., Puteh, M. H., Abideen, M. Z. \& Othman, M. N. 2016. Ammonium-nitrogen recovery from wastewater by struvite crystallization technology. Separation and Purification Reviews, 45, 261-274.

Daughton, C. G. \& Ruhoy, I. S. 2011. Green pharmacy and pharmEcovigilance: Prescribing and the planet. Expert Review of Clinical Pharmacology.

Dawson, C. J. \& Hilton, J. 2011. Fertiliser availability in a resource-limited world: Production and recycling of nitrogen and phosphorus. Food Policy, 36, S14-S22.

DCM Nederland BV 2014. DCM ECO-MIX 1 Technische Fiche. In: DCM (ed.).

de Boer, I. J. \& van Ittersum, M. K. 2018. Circularity in agricultural production. Animal production systems and Plant production systems. Wageningen University and Research.

de Graaff, M. 2010. Resource recovery from black water, Wageningen Universiteit (Wageningen University).

de Graaff, M., Temmink, H., Zeeman, G. \& Buisman, C. 2011. Energy and phosphorus recovery from black water. Water Science and Technology, 63, 2759-2765.

de Graaff, M. S., Temmink, H., Zeeman, G. \& Buisman, C. J. 2010a. Anaerobic treatment of concentrated black water in a UASB reactor at a short HRT. Water, 2, 101-119.

de Graaff, M. S., Temmink, H., Zeeman, G. \& Buisman, C. J. N. 2010b. Anaerobic Treatment of Concentrated Black Water in a UASB Reactor at a Short HRT. Water, 2, 101-119.

de Haan, J. J. \& van Geel, W. 2013a. Advies voor de bemesting van akkerbouw- en vollegrondsgroentengewassen. Praktijkonderzoek Plant \& Omgeving (PPO).

de Haan, J. J. \& van Geel, W. 2013b. Adviesbasis voor de bemesting van akkerbouwgewassen - Samenstelling en werking van organische meststoffen [Online]. Available: http://www.kennisakker.nl/kenniscentrum/handleidingen/ adviesbasis-voor-de-bemesting-van-akkerbouwgewassen-samenstelling-en-wer.

de la Varga, D., Díaz, M. A., Ruiz, I. \& Soto, M. 2013. Heavy metal removal in an UASB-CW system treating municipal wastewater. Chemosphere, 93, 1317-1323.

de Wilt, A., Butkovskyi, A., Tuantet, K., Leal, L. H., Fernandes, T. V., Langenhoff, A. \& Zeeman, G. 2016. Micropollutant removal in an algal treatment system fed with source separated wastewater streams. Journal of Hazardous Materials, 304, 84-92.

Decrey, L., Udert, K. M., Tilley, E., Pecson, B. M. \& Kohn, T. 2011. Fate of the pathogen indicators phage $Ф X 174$ and Ascaris suum eggs during the production of struvite fertilizer from source-separated urine. Water research, 45, 4960-4972. 
Deelstra, T. \& Girardet, H. 2000. Urban agriculture and sustainable cities. Bakker N., Dubbeling M., Gündel S., Sabel-Koshella U., de Zeeuw H. Growing cities, growing food. Urban agriculture on the policy agenda. Feldafing, Germany: Zentralstelle für Ernährung und Landwirtschaft (ZEL), 43-66.

Degaardt, S. 2003a. Human urine from city to field-towards sustainable co-operation.pdf $>$. 2nd international symposium on ecological sanitation.

Degaardt, S. 2003b. Human urine from city to field-towards sustainable co-operation.pdf $>$.

Degryse, F., Baird, R., da Silva, R. C. \& McLaughlin, M. J. 2017. Dissolution rate and agronomic effectiveness of struvite fertilizers - effect of soil pH, granulation and base excess. Plant and Soil, 410, 139-152.

Dekker, P., van Zeeland, M. \& Paauw, J. 2010. Levenscyclusanalyse groencompost: grootschalig en zelf composteren.

del Carmen Morales, M., Harris, L. \& Öberg, G. 2014. Citizenshit: The right to flush and the urban sanitation imaginary. Environment and Planning A, 46, 2816-2833.

Demey, H., Vincent, T. \& Guibal, E. 2018. A novel algal-based sorbent for heavy metal removal. Chemical Engineering Journal, 332, 582-595.

Deng, Y. \& Zhao, R. 2015. Advanced Oxidation Processes (AOPs) in Wastewater Treatment. Current Pollution Reports, $1,167-176$

Deppe, M., Well, R., Kücke, M., Fuß, R., Giesemann, A. \& Flessa, H. 2016. Impact of CULTAN fertilization with ammonium sulfate on field emissions of nitrous oxide. Agriculture, Ecosystems and Environment, 219, 138-151.

Desloover, J., Abate Woldeyohannis, A., Verstraete, W., Boon, N. \& Rabaey, K. 2012. Electrochemical resource recovery from digestate to prevent ammonia toxicity during anaerobic digestion. Environmental Science and Technology, 46, 12209-12216.

Desmidt, E., Ghyselbrecht, K., Zhang, Y., Pinoy, L., Van Der Bruggen, B., Verstraete, W., Rabaey, K. \& Meesschaert, B. 2015. Global phosphorus scarcity and full-scale P-recovery techniques: A review. Critical Reviews in Environmental Science and Technology, 45, 336-384.

Despommier, D. 2010. The vertical farm: feeding the world in the 21st century, Macmillan.

Dewaelheyns, V., Elsen, A., Vandendriessche, H. \& Gulinck, H. 2013. Garden management and soil fertility in Flemish domestic gardens. Landscape and Urban Planning, 116, 25-35.

Donatello, S. \& Cheeseman, C. R. 2013. Recycling and recovery routes for incinerated sewage sludge ash (ISSA): A review. Waste Management.

Doyle, J. D. \& Parsons, S. A. 2002. Struvite formation, control and recovery. Water Research, 36, 3925-3940.

Drangert, J.-O., Tonderski, K. \& McConville, J. 2018. Extending the European Union Waste Hierarchy to Guide Nutrient-Effective Urban Sanitation toward Global Food Security-Opportunities for Phosphorus Recovery. Frontiers in Sustainable Food Systems, 2, 1-13.

Drechsel, P. \& Kunze, D. 2001. Waste composting for urban and peri-urban agriculture: Closing the rural-urban nutrient cycle in sub-Saharan Africa, CABI.

Duchemin, E., Wegmuller, F. \& Legault, A.-M. 2008. Urban agriculture: multi-dimensional tools for social development in poor neighbourhoods. Field Actions Science Reports. The journal of field actions, 1.

Dumontet, S., Scopa, A., Kerje, S. \& Krovacek, K. 2001. The importance of pathogenic organisms in sewage and sewage sludge. Journal of the Air and Waste Management Association, 51, 848-860.

Dutta, S. \& Vinnerås, B. 2016. Fertilizer from dried human urine added to ash and lime -A potential product from eco-sanitation system. Water Science and Technology, 74, 1436-1445.

\section{$E$}

Eawag 2018a. Aurin - Fertilisers from urine.

Eawag 2018b. Blue Diversion AUTARKY Wastewater Treatment off the Grid.

EC. 2016. Introduction to the EU Water Framework Directive [Online]. Available: http://ec.europa.eu/environment/ water/water-framework/info/intro_en.htm.

Edwards, A. \& Withers, P. 1998. Soil phosphorus management and water quality: a UK perspective. Soil use and Management, 14, 124-130.

Eghball, B. 2002. Soil Properties as Influenced by Phosphorus- and Nitrogen-Based Manure and Compost Applications Joint contribution of USDA-ARS and Univ. of Nebraska Agric. Res. Div., Lincoln, NE, as paper no. 12996. Agronomy Journal, 94, 128-135.

Egle, L., Rechberger, H., Krampe, J. \& Zessner, M. 2016. Phosphorus recovery from municipal wastewater: An integrated comparative technological, environmental and economic assessment of P recovery technologies. Science of the Total Environment, 571, 522-542. 
Egle, L., Rechberger, H. \& Zessner, M. 2015. Overview and description of technologies for recovering phosphorus from municipal wastewater. Resources, Conservation and Recycling, 105, 325-346.

Ek, M., Bergström, R., Bjurhem, J. E., Björlenius, B. \& Hellström, D. 2006. Concentration of nutrients from urine and reject water from anaerobically digested sludge. Water Science and Technology, 54, 437-444.

Eklind, Y. \& Kirchmann, H. 2000. Composting and storage of organic household waste with different litter amendments. II: nitrogen turnover and losses. Bioresource technology, 74, 125-133.

Elser, J. \& Bennett, E. 2011. Phosphorus cycle: a broken biogeochemical cycle. Nature, 478, 29.

Elser, J. \& Bennett, E. M. 2009. A broken geochemical cycle. Nature, 2009-2011.

Emilsson, T., Berndtsson, J. C., Mattsson, J. E. \& Rolf, K. 2007. Effect of using conventional and controlled release fertiliser on nutrient runoff from various vegetated roof systems. Ecological engineering, 29, 260-271.

Erisman, J. W., Sutton, M. A., Galloway, J., Klimont, Z. \& Winiwarter, W. 2008. How a century of ammonia synthesis changed the world. Nature Geoscience, 1, 636.

Escher, B. I., Pronk, W., Suter, M. J.-F. \& Maurer, M. 2006. Monitoring the removal efficiency of pharmaceuticals and hormones in different treatment processes of source-separated urine with bioassays. Environmental science \& technology, 40, 5095-5101.

\section{F}

Fabris, I., Cormier, D., Gerhard, J. I., Bartczak, T., Kortschot, M., Torero, J. L. \& Cheng, Y. L. 2017. Continuous, self-sustaining smouldering destruction of simulated faeces. Fuel, 190, 58-66.

Ferguson, D. T. 2014. Nightsoil and the 'Great Divergence': human waste, the urban economy, and economic productivity, 1500-1900. Journal of Global History, 9, 379-402.

Fidjeland, J., Magri, M. E., Jönsson, H., Albihn, A. \& Vinnerås, B. 2013. The potential for self-sanitisation of faecal sludge by intrinsic ammonia. Water Research, 47, 6014-6023.

Fidjeland, J., Svensson, S. E. \& Vinnerås, B. 2015. Ammonia sanitization of blackwater for safe use as fertilizer. Water Science and Technology, 71, 795-800.

Fink, M., Feller, C., Scharpf, H. C., Weier, U., Maync, A., Ziegler, J., Paschold, P. J. \& Strohmeyer, K. 1999. Nitrogen, phosphorus, potassium and magnesium contents of field vegetables-Recent data for fertiliser recommendations and nutrient balances. Journal of Plant Nutrition and Soil Science, 162, 71-73.

Fischer, F., Bastian, C., Happe, M., Mabillard, E. \& Schmidt, N. 2011. Microbial fuel cell enables phosphate recovery from digested sewage sludge as struvite. Bioresource Technology, 102, 5824-5830.

Foley, J. A., Ramankutty, N., Brauman, K. A., Cassidy, E. S., Gerber, J. S., Johnston, M., Mueller, N. D., O'Connell, C., Ray, D. K., West, P. C., Balzer, C., Bennett, E. M., Carpenter, S. R., Hill, J., Monfreda, C., Polasky, S., Rockström, J., Sheehan, J., Siebert, S., Tilman, D. \& Zaks, D. P. M. 2011. Solutions for a cultivated planet. Nature, 478, 337-342.

Forbis-Stokes, A. A., O'Meara, P. F., Mugo, W., Simiyu, G. M. \& Deshusses, M. A. 2016. On-Site Fecal Sludge Treatment with the Anaerobic Digestion Pasteurization Latrine. Environmental Engineering Science, 33, 898-906.

Fraser, E., Legwegoh, A., KC, K., CoDyre, M., Dias, G., Hazen, S., Johnson, R., MartiKCn, R., Ohberg, L., Sethuratnam, S., Sneyd, L., Smithers, J., Van Acker, R., Vansteenkiste, J., Wittman, H. \& Yada, R. 2016. Biotechnology or organic? Extensive or intensive? Global or local? A critical review of potential pathways to resolve the global food crisis. Trends in Food Science and Technology, 48, 78-87.

Fricke, K. \& Vogtmann, H. 1994. Compost quality: physical characteristics, nutrient content, heavy metals and organic chemicals. Toxicological \& Environmental Chemistry, 43, 95-114.

Friedler, E., Butler, D. \& Alfiya, Y. 2013a. Wastewater composition. In: LARSEN, T. A., UDERT, K. M. \& LIENERT, J. (eds.) Source Separation and Decentralization for Wastewater Management. London, UK: IWA Publishing.

Friedler, E., Butler, D. \& Alfiya, Y. 2013b. Wastewater composition. Source separation and decentralization for wastewater management, 241-257.

Fumasoli, A., Etter, B., Sterkele, B., Morgenroth, E. \& Udert, K. M. 2016. Operating a pilot-scale nitrification/distillation plant for complete nutrient recovery from urine. Water Science and Technology, 73, 215-222.

\section{G}

Gajurel, D., Deegener, S., Shalabi, M. \& Otterpohl, R. 2007. Potential of filter-vermicomposter for household wastewater pre-treatment and sludge sanitisation on site. Water Science and Technology.

Galloway, J. N., Dentener, F. J., Capone, D. G., Boyer, E. W., Howarth, R. W., Seitzinger, S. P., Asner, G. P., Cleveland, C. C., Green, P. A., Holland, E. A., Karl, D. M., Michaels, A. F., Porter, J. H., Townsend, A. R. \& Vorosmarty, C. J. 2004. Nitrogen cycles: past, present, and future. Biogeochemistry, 70, 153-226. 
Ganesapillai, M., Simha, P., Gupta, K. \& Jayan, M. 2016. Nutrient Recovery and Recycling from Human Urine: A Circular Perspective on Sanitation and Food Security. Procedia Engineering, 148, 346-353.

Garnett, T. 2014. Three perspectives on sustainable food security: Efficiency, demand restraint, food system transformation. What role for life cycle assessment? Journal of Cleaner Production, 73, 10-18.

Gemeente Amsterdam 2016. AMSInwoners.

Gemeente Amsterdam \& Ruimte en Duurzaamheid 2016. Stadslandbouw. 07-12-2016 ed.

Gemeente Rotterdam 2012. Stimuleren van stadslandbouw in en om Rotterdam. Food \& the City.

Gemeente Rotterdam 2013. Rotterdam in Cijfers. http://www.rotterdamincijfers.nl/.

Geofabrik 04-2019. Open StreetMap-Netherlands-latest.osm.pbf.

Gezondheidsraad 2015. Richtlijnen goede voeding 2015. Den Haag: Gezondheidsraad.

Giampietro, M. 2018. Perception and representation of the resource nexus at the interface between society and the natural environment. Sustainability (Switzerland).

Giannakis, S., Rtimi, S. \& Pulgarin, C. 2017. Light-assisted advanced oxidation processes for the elimination of chemical and microbiological pollution of wastewaters in developed and developing countries. Molecules, 22.

Glibert, P. M., Maranger, R., Sobota, D. J. \& Bouwman, L. 2014. The Haber Bosch-harmful algal bloom (HB-HAB) link. Environmental Research Letters, 9, 105001.

Godfray, H. C. J., Aveyard, P., Garnett, T., Hall, J. W., Key, T. J., Lorimer, J., Pierrehumbert, R. T., Scarborough, P., Springmann, M. \& Jebb, S. A. 2018. Meat consumption, health, and the environment. Science, 361, eaam5324.

Goed boeren in kleinschalig landschap. 2011. Hoeveel compost mag ik als agrarische ondernemer aanwenden? [Online]. Available: http://www.goedboereninkleinschaliglandschap.nl/hoeveel-compost-mag-ik-als-agrarische-ondernemer-aanwenden.

Goglio, A., Tucci, M., Rizzi, B., Colombo, A., Cristiani, P. \& Schievano, A. 2019. Microbial recycling cells (MRCs): A new platform of microbial electrochemical technologies based on biocompatible materials, aimed at cycling carbon and nutrients in agro-food systems. Science of The Total Environment, 649, 1349-1361.

Goldstein, B., Hauschild, M., Fernández, J. \& Birkved, M. 2016. Urban versus conventional agriculture, taxonomy of resource profiles: a review. Agronomy for Sustainable Development, 36, 1-19.

Gomaa, M. A. \& Abed, R. M. 2017. Potential of fecal waste for the production of biomethane, bioethanol and biodiesel. Journal of biotechnology, 253, 14-22.

Gomez, A. 1998. The evaluation of compost quality. TrAC Trends in Analytical Chemistry, 17, 310-314.

Gorazda, K., Tarko, B., Werle, S. \& Wzorek, Z. 2017. Sewage sludge as a fuel and raw material for phosphorus recovery: Combined process of gasification and P extraction. Waste Management, 73, 404-415.

Government of the Netherlands 2016. A Circular Economy in the Netherlands by 2050: Government-wide Programme for a Circular Economy. Ministry of Infrastructure and the Environment, Ministry of Economic Aff airs,

Grant, S. B., Saphores, J.-D., Feldman, D. L., Hamilton, A. J., Fletcher, T. D., Cook, P. L. M., Stewardson, M., Sanders, B. F., Levin, L. A., Ambrose, R. F., Deletic, A., Brown, R., Jiang, S. C., Rosso, D., Cooper, W. J. \& Marusic, I. 2012. Taking the \&quot;waste\&quot; out of \&quot;wastewater\&quot; for human water security and ecosystem sustainability. Science (New York, N.Y.), 337, 681-6.

Grard, B. J.-P., Bel, N., Marchal, N., Madre, F., Castell, J.-F., Cambier, P., Houot, S., Manouchehri, N., Besancon, S. \& Michel, J.-C. 2015. Recycling urban waste as possible use for rooftop vegetable garden. Future of Food: Journal on Food, Agriculture and Society, 3, 21-34.

Green Deal Stadsgerichte Landbouw 2013. Stadsboeren in Nederland: Professionalisering van de stadsgerichte landbouw. Rotterdam: Van Bergen Kopla Architecten, Wageningen UR/LEI, de Volharding Breda, Priva, Ministerie van Economische Zaken, Ministerie van Infrastructuur en Milieu.

Grewal, S. S. \& Grewal, P. S. 2012. Can cities become self-reliant in food? Cities, 29, 1-11.

Grigatti, M., Cavani, L., Marzadori, C. \& Ciavatta, C. 2014. Recycling of dry-batch digestate as amendment: Soil C and N dynamics and ryegrass nitrogen utilization efficiency. Waste and Biomass Valorization, 5, 823-833.

Grimm, N. B., Faeth, S. H., Golubiewski, N. E., Redman, C. L., Wu, J., Bai, X. \& Briggs, J. M. 2008. Global change and the ecology of cities. science, 319, 756-760.

Gude, V. G. 2016. Wastewater treatment in microbial fuel cells - An overview. Journal of Cleaner Production, 122, 287307.

Guest, J. S., Skerlos, S. J., Barnard, J. L., Beck, M. B., Daigger, G. T., Hilger, H., Jackson, S. J., Karvazy, K., Kelly, L. \& Macpherson, L. 2009. A new planning and design paradigm to achieve sustainable resource recovery from wastewater. ACS Publications.

Guilayn, F., Braak, E., Piveteau, S. \& Daumer, M. L. 2017. Sequencing biological acidification of waste-activated sludge aiming to optimize phosphorus dissolution and recovery. Environmental Technology (United Kingdom), 38 , 1399-1407. 
Gulyas, H., Bruhn, P., Furmanska, M., Hartrampf, K., Kot, K., Lüttenberg, B., Mahmood, Z., Stelmaszewska, K. \& Otterpohl, R. 2004. Freeze concentration for enrichment of nutrients in yellow water from no-mix toilets. Water Science and Technology, 50, 61-68.

Güney, K., Weidelener, A. \& Krampe, J. 2008. Phosphorus recovery from digested sewage sludge as MAP by the help of metal ion separation. Water Research, 42, 4692-4698.

\section{$\mathrm{H}$}

Haberman, D., Gillies, L., Canter, A., Rinner, V., Pancrazi, L. \& Martellozzo, F. 2014. The potential of urban agriculture in Montréal: A quantitative assessment. ISPRS International Journal of Geo-Information, 3, 1101-1117.

Haddaway, N. R., Johannesdottir, S. L., Piniewski, M. \& Macura, B. 2019. What ecotechnologies exist for recycling carbon and nutrients from domestic wastewater? A systematic map protocol. Environmental Evidence, 8, 1.

Hamilton, A. J., Burry, K., Mok, H. F., Barker, S. F., Grove, J. R. \& Williamson, V. G. 2014. Give peas a chance? Urban agriculture in developing countries. A review. Agronomy for Sustainable Development, 34, 45-73.

Hänsch, R. \& Mendel, R. R. 2009. Physiological functions of mineral micronutrients (Cu, Zn, Mn, Fe, Ni, Mo, B, Cl). Current Opinion in Plant Biology, 12, 259-266.

Hao, X. D., Wang, C. C., Lan, L. \& Van Loosdrecht, M. C. M. 2008. Struvite formation, analytical methods and effects of $\mathrm{pH}$ and $\mathrm{Ca} 2+$. Water Science and Technology, 58, 1687-1692.

Happe, M., Sugnaux, M., Cachelin, C. P., Stauffer, M., Zufferey, G., Kahoun, T., Salamin, P. A., Egli, T., Comninellis, C., Grogg, A. F. \& Fischer, F. 2016. Scale-up of phosphate remobilization from sewage sludge in a microbial fuel cell. Bioresource Technology, 200, 435-443.

Haq, G. \& Cambridge, H. 2012. Exploiting the co-benefits of ecological sanitation. Current Opinion in Environmental Sustainability, 4, 431-435.

Harder, R., Wielemaker, R., Larsen, T. A., Zeeman, G. \& Öberg, G. 2019. Recycling nutrients contained in human excreta to agriculture: Pathways, processes, and products. Critical Reviews in Environmental Science and Technology, $1-49$.

Hargreaves, J. C., Adl, M. S. \& Warman, P. R. 2008. A review of the use of composted municipal solid waste in agriculture. Agriculture, Ecosystems \& Environment, 123, 1-14.

Harvey, A.S., 1993. Guidelines for time use data collection. Social Indicators Research, 30(2-3), pp.197-228.

He, C., Chen, C. L., Giannis, A., Yang, Y. \& Wang, J. Y. 2014. Hydrothermal gasification of sewage sludge and model compounds for renewable hydrogen production: A review. Renewable and Sustainable Energy Reviews, 39, $1127-1142$.

He, X., Zhang, Y., Shen, M., Zeng, G., Zhou, M. \& Li, M. 2016. Effect of vermicomposting on concentration and speciation of heavy metals in sewage sludge with additive materials. Bioresource Technology, 218, 867-873.

Hegger, D. \& van Vliet, B. 2010. End user perspectives on the transformation of sanitary systems. Social Perspectives on the Sanitation Challenge. Springer.

Hegger, D. L. 2007. Greening sanitary systems: and end-user perspectives.

Heinonen-Tanski, H. \& van Wijk-Sijbesma, C. 2005. Human excreta for plant production. Bioresource technology, 96, 403-411.

Heinzmann, B. 2005. Phosphorus recycling in sewage treatment plants with biological phosphorus removal. Water science and technology : a journal of the International Association on Water Pollution Research, 52, 543-8.

Hellström, D., Johansson, E. \& Grennberg, K. 1999. Storage of human urine: Acidification as a method to inhibit decomposition of urea. Ecological Engineering, 12, 253-269.

Hernández Leal, L., Tervahauta, T. \& Zeeman, G. 2017. Resource recovery from source separated domestic wastewater; energy, water, nutrients, and organics. Innovative Wastewater Treatment \& Resource Recovery Technology: Impacts on Energy, Economy and Environment.

Herzel, H., Krüger, O., Hermann, L. \& Adam, C. 2016. Sewage sludge ash - A promising secondary phosphorus source for fertilizer production. Science of the Total Environment, 542, 1136-1143.

Hijbeek, R., van Ittersum, M. K., ten Berge, H. F. M., Gort, G., Spiegel, H. \& Whitmore, A. P. 2017. Do organic inputs matter - a meta-analysis of additional yield effects for arable crops in Europe. Plant and Soil, 411, 293-303.

Hilborn, R., Banobi, J., Hall, S., Pucylowski, T. \& Walsworth, T. 2018. The environmental cost of producing animal source foods. Frontiers in Ecology and the Environment, 16, 329-335.

Hino, A., Hirai, T. \& Komasawa, I. 1998. Te recovery of phosphorus value from incineration ashes of sewage sludge using solvent extraction. Kagaku Kogaku Ronbunshu, 24, 277-278.

Hodson, M., Marvin, S., Robinson, B. \& Swilling, M. 2012a. Reshaping Urban Infrastructure. Journal of Industrial Ecology, 16, 789-800. 
Hodson, M., Marvin, S., Robinson, B. \& Swilling, M. 2012b. Reshaping urban infrastructure: material flow analysis and transitions analysis in an urban context. Journal of Industrial Ecology, 16, 789-800.

Hong, K. J., Tarutani, N., Shinya, Y. \& Kajiuchi, T. 2005. Study on the recovery of phosphorus from waste-activated sludge incinerator ash. Journal of Environmental Science and Health - Part A Toxic/Hazardous Substances and Environmental Engineering, 40, 617-631.

Horta, C. 2017. Bioavailability of phosphorus from composts and struvite in acid soils. Revista Brasileira de Engenharia Agrícola e Ambiental, 21, 459-464.

Horttanainen, M., Deviatkin, I. \& Havukainen, J. 2017. Nitrogen release from mechanically dewatered sewage sludge during thermal drying and potential for recovery. Journal of Cleaner Production, 142, 1819-1826.

Hossain, M. K., Strezov Vladimir, V., Chan, K. Y., Ziolkowski, A. \& Nelson, P. F. 2011. Influence of pyrolysis temperature on production and nutrient properties of wastewater sludge biochar. Journal of Environmental Management, 92, 223-228.

Huang, B., Shi, X., Yu, D., Öborn, I., Blombäck, K., Pagella, T. F., Wang, H., Sun, W. \& Sinclair, F. L. 2006. Environmental assessment of small-scale vegetable farming systems in peri-urban areas of the Yangtze River Delta Region, China. Agriculture, Ecosystems \& Environment, 112, 391-402.

Huang, H., Liu, J. \& Ding, L. 2015. Recovery of phosphate and ammonia nitrogen from the anaerobic digestion supernatant of activated sludge by chemical precipitation. Journal of Cleaner Production, 102, 437-446.

Huang, K., Mao, Y., Zhao, F., Zhang, X.-X., Ju, F., Ye, L., Wang, Y., Li, B., Ren, H. \& Zhang, T. 2018. Free-living bacteria and potential bacterial pathogens in sewage treatment plants. Applied Microbiology and Biotechnology, 102, 2455-2464.

Huang, L., Cheng, S. \& Chen, G. 2011. Bioelectrochemical systems for efficient recalcitrant wastes treatment. Journal of Chemical Technology and Biotechnology, 86, 481-491.

Hug, A. \& Udert, K. M. 2013. Struvite precipitation from urine with electrochemical magnesium dosage. Water Research, 47, 289-299.

Hülsen, T., Batstone, D. J. \& Keller, J. 2014. Phototrophic bacteria for nutrient recovery from domestic wastewater. Water Research, 50, 18-26.

Humuforte 2014. Humuforte Technische Fiche: Gedroogde Koemest.

Hunter, K. A., Liss, P. S., Surapipith, V., Dentener, F., Duce, R., Kanakidou, M., Kubilay, N., Mahowald, N., Okin, G., Sarin, M., Uematsu, M. \& Zhu, T. 2011. Impacts of anthropogenic $\mathrm{SO}<\operatorname{sub}>_{\mathrm{x}}</$ sub $>, \mathrm{NO}<\operatorname{sub}>_{\mathrm{x}}<$ / sub $>$ and $\mathrm{NH}<\mathrm{sub}>3</$ sub $>$ on acidification of coastal waters and shipping lanes. Geophysical Research Letters, 38, n/a-n/a.

\section{I}

Ibekwe, A. M., Gonzalez-rubio, A. \& Suarez, D. L. 2018. Science of the Total Environment Impact of treated wastewater for irrigation on soil microbial communities. Science of the Total Environment, 622-623, 1603-1610.

Ito, R., Takahashi, E. \& Funamizu, N. 2013. Production of slow-released nitrogen fertilizer from urine. Environmental Technology (United Kingdom), 34, 2813-2819.

\section{$J$}

Jana, B., Bag, S. K. \& Rana, S. 2012. urine, cow manure and their mix for the production. Aquaculture International, 20, 735-749.

Jaramillo, M. F. \& Restrepo, I. 2017. Wastewater Reuse in Agriculture: A Review about Its Limitations and Benefits. Sustainability, 9, 1734.

Jenkins, J. C. 2005. The humanure handbook: A guide to composting human manure, Joseph Jenkins, Incorporated.

Jiang, S., Wang, X., Yang, S. \& Shi, H. 2017. Effect of initial pH and pH-adjusted acid on nutrient recovery from hydrolysis urine by combining acidification with evaporation-crystallization. Environmental Science and Pollution Research, 24, 3872-3881.

Johnston, A. E. \& Richards, I. R. 2003. Effectiveness of different precipitated phosphates as phosphorus sources for plants. Soil Use and Management, 19, 45-49.

Jones, D. L., Cross, P., Withers, P. J., DeLuca, T. H., Robinson, D. A., Quilliam, R. S., Harris, I. M., Chadwick, D. R. \& Edwards-Jones, G. 2013a. Nutrient stripping: the global disparity between food security and soil nutrient stocks. Journal of Applied Ecology, 50, 851-862.

Jones, D. L., Cross, P., Withers, P. J. A., Deluca, T. H., Robinson, D. A., Quilliam, R. S., Harris, I. M., Chadwick, D. R. \& Edwards-Jones, G. 2013b. Nutrient stripping: The global disparity between food security and soil nutrient stocks. Journal of Applied Ecology, 50, 851-862. 
Jones, G. D., Droz, B., Greve, P., Gottschalk, P., Poffet, D., McGrath, S. P., Seneviratne, S. I., Smith, P. \& Winkel, L. H. 2017. Selenium deficiency risk predicted to increase under future climate change. Proceedings of the National Academy of Sciences, 114, 2848-2853.

Jönsson, H., Stenström, T.-A., Svensson, J. \& Sundin, A. 1997. Source separated urine - nutrient and heavy metal content, water saving and faecal contamination. Water Science and Technology, 35, 145-152.

Jönsson, H., Stintzing, A. R., Vinnerås, B. \& Salomon, E. 2004. Guidelines on the use of urine and faeces in crop production, EcoSanRes Programme.

Jönsson, H., Vinnerås, B., Burstrom, A., Höglund, C. \& Stenström, T. A. Source separation of human urine — nitrogen and phosphorus emissions. 8th international conference on the FAO ESCORENA network on recycling of Agricultural, Municipal and Industrial Residues in Agriculture, 1998 Rennes, France. 251-259.

\section{K}

Kadaster, C.-B. 2012. CBS Bestand Bodemgebruik 2012.

Kahiluoto, H., Kuisma, M., Ketoja, E., Salo, T. \& Heikkinen, J. 2015. Phosphorus in manure and sewage sludge more recyclable than in soluble inorganic fertilizer. Environmental Science and Technology, 49, 2115-2122.

Karak, T. \& Bhattacharyya, P. 2011. Human urine as a source of alternative natural fertilizer in agriculture: A flight of fancy or an achievable reality. Resources, Conservation and Recycling, 55, 400-408.

Karak, T., Sonar, I., Nath, J. R., Paul, R. K., Das, S., Boruah, R. K., Dutta, A. K. \& Das, K. 2015. Struvite for composting of agricultural wastes with termite mound: Utilizing the unutilized. Bioresource Technology, 187, 49-59.

Karunanithi, R., Szogi, A. A., Bolan, N., Naidu, R., Loganathan, P., Hunt, P. G., Vanotti, M. B., Saint, C. P., Ok, Y. S. \& Krishnamoorthy, S. 2015. Phosphorus recovery and reuse from waste streams. Advances in Agronomy, 131, 173-250.

Karvelas, M., Katsoyiannis, A. \& Samara, C. 2003. Occurrence and fate of heavy metals in the wastewater treatment process. Chemosphere, 53, 1201-1210.

Katehis, D., Diyamandoglu, V. \& Fillos, J. 1998. Stripping and recovery of ammonia from centrate of anaerobically digested biosolids at elevated temperatures. Water Environment Research, 70, 231-240.

Kathijotes, N., Zlatareva, E., Marinova, S. \& Petrova, V. 2016. Soil fertilization with wastewater biosolids-monitoring changes in the 'soil-fertilizer-plant'system and phosphorus recovery options. Water Science and Technology, 74, 1499-1508.

Katsoyiannis, A. \& Samara, C. 2005. Persistent organic pollutants (POPs) in the conventional activated sludge treatment process: Fate and mass balance. Environmental Research, 97, 245-257.

Kelessidis, A. \& Stasinakis, A. S. 2012. Comparative study of the methods used for treatment and final disposal of sewage sludge in European countries. Waste Management, 32, 1186-1195.

Kelly, P. T. \& He, Z. 2014. Nutrients removal and recovery in bioelectrochemical systems: A review. Bioresource Technology, 153, 351-360.

Kennedy, C., Baker, L., Dhakal, S. \& Ramaswami, A. 2012. Sustainable urban systems. Journal of Industrial Ecology, 16, 775-974.

Kennedy, C., Cuddihy, J. \& Engel-Yan, J. 2007. The changing metabolism of cities. Journal of Industrial Ecology, 11, 43-59.

Keplinger, K. O. \& Hauck, L. M. 2006. The economics of manure utilization: model and application. Journal of Agricultural and Resource Economics, 414-440.

Khai, N. M., Ha, P. Q. \& Öborn, I. 2007. Nutrient flows in small-scale peri-urban vegetable farming systems in Southeast Asia-a case study in Hanoi. Agriculture, Ecosystems \& Environment, 122, 192-202.

King, F. H. 2004. Farmers of forty centuries: Organic farming in China, Korea, and Japan, Courier Corporation.

Kirchmann, H., Börjesson, G., Kätterer, T. \& Cohen, Y. 2016. From agricultural use of sewage sludge to nutrient extraction: A soil science outlook. Ambio, 1-12.

Kirchmann, H., Börjesson, G., Kätterer, T. \& Cohen, Y. 2017. From agricultural use of sewage sludge to nutrient extraction: A soil science outlook. Ambio, 46, 143-154.

Koch, A., Mcbratney, A., Adams, M., Field, D., Hill, R., Crawford, J., Minasny, B., Lal, R., Abbott, L., O'Donnell, A., Angers, D., Baldock, J., Barbier, E., Binkley, D., Parton, W., Wall, D. H., Bird, M., Bouma, J., Chenu, C., Flora, C. B., Goulding, K., Grunwald, S., Hempel, J., Jastrow, J., Lehmann, J., Lorenz, K., Morgan, C. L., Rice, C. W., Whitehead, D., Young, I. \& Zimmermann, M. 2013. Soil Security: Solving the Global Soil Crisis. Global Policy, 4, 434-441.

Kołodyńska, D., Wnetrzak, R., Leahy, J. J., Hayes, M. H. B., Kwapiński, W. \& Hubicki, Z. 2012. Kinetic and adsorptive characterization of biochar in metal ions removal. Chemical Engineering Journal, 197, 295-305. 
Kone, D. 2010. Making urban excreta and wastewater management contribute to cities' economic development: a paradigm shift. Water Policy, 12, 602-610.

Kong, A. Y. Y., Rosenzweig, C. \& Arky, J. 2015. Nitrogen dynamics associated with organic and inorganic inputs to substrate commonly used on rooftop farms. HortScience, 50, 806-813.

Kros, H., Os, J. v., Voogd, J. C., Groenendijk, P., Bruggen, C. v., Molder, R. t. \& Ros, G. 2019. Ruimtelijke allocatie van mesttoediening en ammoniakemissie : beschrijving mestverdelingsmodule INITIATOR versie 5 . Wageningen: Wageningen Environmental Research.

Krüger, O., Fattah, K. P. \& Adam, C. 2016. Phosphorus recovery from the wastewater stream-necessity and possibilities. Desalination and Water Treatment, 57, 15619-15627.

Kruse, A., Koch, F., Stelzl, K., Wüst, D. \& Zeller, M. 2016. Fate of Nitrogen during Hydrothermal Carbonization. Energy and Fuels, 30, 8037-8042.

Kujawa-Roeleveld, K. \& Zeeman, G. 2006. Anaerobic treatment in decentralised and source-separation-based sanitation concepts. Reviews in Environmental Science and Bio/Technology, 5, 115-139.

Kumar, R. \& Pal, P. 2015. Assessing the feasibility of $\mathrm{N}$ and P recovery by struvite precipitation from nutrient-rich wastewater: a review. Environmental Science and Pollution Research, 22, 17453-17464.

Kümmerer, K. 2013. The issue of micropollutants in urban water management. In: LARSEN, T. A., UDERT, K. M. \& LIENERT, J. (eds.) Source separation and decentralization for wastewater management.

Kuntke, P. 2013. Nutrient and energy recovery from urine, Wageningen University.

Kuntke, P., Sleutels, T. H. J. A., Saakes, M. \& Buisman, C. J. N. 2014. Hydrogen production and ammonium recovery from urine by a Microbial Electrolysis Cell. International Journal of Hydrogen Energy, 39, 4771-4778.

Kuntke, P., Śmiech, K. M., Bruning, H., Zeeman, G., Saakes, M., Sleutels, T. H. J. A., Hamelers, H. V. M. \& Buisman, C. J. N. 2012. Ammonium recovery and energy production from urine by a microbial fuel cell. Water Research, 46, 2627-2636.

Kyllingsbæk, A. \& Hansen, J. F. 2007. Development in nutrient balances in Danish agriculture 1980-2004. Nutrient Cycling in Agroecosystems, 79, 267-280.

\section{L}

Lahr, R. H., Goetsch, H. E., Haig, S. J., Noe-Hays, A., Love, N. G., Aga, D. S., Bott, C. B., Foxman, B., Jimenez, J., Luo, T., Nace, K., Ramadugu, K. \& Wigginton, K. R. 2016. Urine Bacterial Community Convergence through Fertilizer Production: Storage, Pasteurization, and Struvite Precipitation. Environmental Science and Technology, 50, 11619-11626.

Lal, R. 2015. Restoring soil quality to mitigate soil degradation. Sustainability (Switzerland), 7, 5875-5895.

Lalander, C., Fidjeland, J., Diener, S., Eriksson, S. \& Vinnerås, B. 2014. High waste-to-biomass conversion and efficient Salmonella spp. reduction using black soldier fly for waste recycling. Agronomy for Sustainable Development, $35,261-271$.

Lalander, C., Hill, G. B. \& Vinnerås, B. 2013. Hygienic quality of faeces treated in urine diverting vermicomposting toilets. Waste Management, 33, 2204-2210.

Larsen, T. \& Lienert, J. 2007. Novaquatis final report. NoMix-a new approach to urban water management. Eawag, Dübendorf.

Larsen, T. A., Alder, A. C., Eggen, R. I., Maurer, M. \& Lienert, J. 2009a. Source separation: will we see a paradigm shift in wastewater handling? : ACS Publications.

Larsen, T. A., Alder, A. C., Eggen, R. I., Maurer, M. \& Lienert, J. 2009b. Source separation: Will we see a paradigm shift in wastewater handling? 1. Environmental science \& technology, 43, 6121-6125.

Larsen, T. A. \& Gujer, W. 1997. The concept of sustainable urban water management. Water Science and Technology, $35,3-10$.

Larsen, T. A., Hoffmann, S., Lüthi, C., Truffer, B. \& Maurer, M. 2016. Emerging solutions to the water challenges of an urbanizing world. Science, 352, 928-933.

Lazarova, Z. \& Spendlingwimmer, R. 2008. Treatment of yellow water by membrane separations and advanced oxidation methods. Water Science and Technology, 58, 419-426.

Le Corre, K. S., Valsami-Jones, E., Hobbs, P. \& Parsons, S. A. 2009. Phosphorus recovery from wastewater by struvite crystallization: A review. Critical Reviews in Environmental Science and Technology.

Leder, C., Rastogi, T. \& Kümmerer, K. 2015. Putting benign by design into practice-novel concepts for green and sustainable pharmacy: Designing green drug derivatives by non-targeted synthesis and screening for biodegradability. Sustainable Chemistry and Pharmacy, 2, 31-36.

Ledezma, P., Kuntke, P., Buisman, C. J. N., Keller, J. \& Freguia, S. 2015. Source-separated urine opens golden opportunities for microbial electrochemical technologies. Trends in Biotechnology, 33, 214-220. 
LeGrand, M., Clark, O. G. \& Chaoui, I. H. I. Rationalizing nutrient flows on an urban planet. American Society of Agricultural and Biological Engineers Annual International Meeting 2014, ASABE 2014, 2014. I: American Society of Agricultural and Biological Engineers, 2536-2542.

Lemming, C., Bruun, S., Jensen, L. S. \& Magid, J. 2017. Plant availability of phosphorus from dewatered sewage sludge, untreated incineration ashes, and other products recovered from a wastewater treatment system. Journal of Plant Nutrition and Soil Science, 180, 779-787.

Lens, P., Zeeman, G. \& Lettinga, G. 2001. Decentralised sanitation and reuse, IWA Publ.

Lettinga, G., Van Lier, J. B., Van Buuren, J. C. L. \& Zeeman, G. 2001. Sustainable development in pollution control and the role of anaerobic treatment. Water Science and Technology, 44, 181-188.

Lettinga, G., van Velsen, A. F. M., Hobma, S. W., de Zeeuw, W. J. \& Klapwijk, A. 1980. Use of Upflox Anaerobic Sludge Blanket (UASB) reactor concept for biological wastewater especially for anaerobic treatment. Biotechnology and Bioengineer, 22, 699-734.

Leusbrock, I., Nanninga, T. A., Lieberg, K., Agudelo-Vera, C. M., Keesman, K. J., Zeeman, G. \& Rijnaarts, H. H. M. 2015. The urban harvest approach as framework and planning tool for improved water and resource cycles. Water Science and Technology, 72, 998-1006.

Li, B., Ju, F., Cai, L. \& Zhang, T. 2015. Profile and Fate of Bacterial Pathogens in Sewage Treatment Plants Revealed by High-Throughput Metagenomic Approach. Environmental Science and Technology, 49, 10492-10502.

Li, H. \& Kwan, M.-P. 2018. Advancing analytical methods for urban metabolism studies. Resources, Conservation and Recycling, 132, 239-245.

Li, R., Teng, W., Li, Y., Wang, W., Cui, R. \& Yang, T. 2017. Potential recovery of phosphorus during the fluidized bed incineration of sewage sludge. Journal of Cleaner Production, 140, 964-970.

Liberti, L., Boari, G., Petruzzelli, D. \& Passino, R. 1981. Nutrient removal and recovery from wastewater by ion exchange. Water Research, 15, 337-342.

Libra, J. A., Ro, K. S., Kammann, C., Funke, A., Berge, N. D., Neubauer, Y., Titirici, M.-M., Fühner, C., Bens, O., Kern, J. \& Emmerich, K.-H. 2011. Hydrothermal carbonization of biomass residuals: a comparative review of the chemistry, processes and applications of wet and dry pyrolysis. Biofuels, 2, 71-106.

Licon Bernal, E. E., Maya, C., Valderrama, C. \& Cortina, J. L. 2016. Valorization of ammonia concentrates from treated urban wastewater using liquid-liquid membrane contactors. Chemical Engineering Journal, 302, 641-649.

Lienert, J., Bürki, T. \& Escher, B. I. 2007. Reducing micropollutants with source control: Substance flow analysis of 212 pharmaceuticals in faeces and urine. Water Science and Technology.

Lind, B. B., Ban, Z. \& Bydén, S. 2000. Nutrient recovery from human urine by struvite crystallization with ammonia adsorption on zeolite and wollastonite. Bioresource Technology, 73, 169-174.

Linder, T. 2019. Making the case for edible microorganisms as an integral part of a more sustainable and resilient food production system. Food Security.

Liu, B., Giannis, A., Zhang, J., Chang, V. W. C. \& Wang, J. Y. 2015. Air stripping process for ammonia recovery from source-separated urine: Modeling and optimization. Journal of Chemical Technology and Biotechnology, 90, 2208-2217.

Liu, D., Xie, B., Dong, C., Liu, G., Hu, D., Qin, Y., Li, H. \& Liu, H. 2018. Effect of fertilizer prepared from human feces and straw on germination, growth and development of wheat. Acta Astronautica, 145, 76-82.

Loganathan, P., Vigneswaran, S., Kandasamy, J. \& Bolan, N. S. 2014. Removal and recovery of phosphate from water using sorption. Critical Reviews in Environmental Science and Technology, 44, 847-907.

Lord, E., Johnson, P. \& Archer, J. 1999. Nitrate Sensitive Areas: a study of large scale control of nitrate loss in England. Soil Use and Management, 15, 201-207.

Lorenz, K. 2015. Organic urban agriculture. Soil Science, 180, 146-153.

Loveland, P. \& Webb, J. 2003. Is there a critical level of organic matter in the agricultural soils of temperate regions: a review. Soil and Tillage Research, 70, 1-18.

Lu, J., Zhang, J., Zhu, Z., Zhang, Y., Zhao, Y., Li, R., Watson, J., Li, B. \& Liu, Z. 2017. Simultaneous production of biocrude oil and recovery of nutrients and metals from human feces via hydrothermal liquefaction. Energy Conversion and Management, 134, 340-346.

Lutchmiah, K., Verliefde, A. R. D., Roest, K., Rietveld, L. C. \& Cornelissen, E. R. 2014. Forward osmosis for application in wastewater treatment: A review. Water Research, 58, 179-197.

Luther, A. K., Desloover, J., Fennell, D. E. \& Rabaey, K. 2015. Electrochemically driven extraction and recovery of ammonia from human urine. Water Research, 87, 367-377. 
M

Maathuis, F. J. M. 2009. Physiological functions of mineral macronutrients. Current Opinion in Plant Biology, 12, 250258.

Magid, J., Eilersen, A. M., Wrisberg, S. \& Henze, M. 2006. Possibilities and barriers for recirculation of nutrients and organic matter from urban to rural areas: A technical theoretical framework applied to the medium-sized town Hillerød, Denmark. Ecological Engineering, 28, 44-54.

Magri, M. E., Fidjeland, J., Jönsson, H., Albihn, A. \& Vinnerås, B. 2015. Inactivation of adenovirus, reovirus and bacteriophages in fecal sludge by $\mathrm{pH}$ and ammonia. Science of the Total Environment, 520, 213-221.

Malila, R., Lehtoranta, S. \& Viskari, E. L. 2019. The role of source separation in nutrient recovery - Comparison of alternative wastewater treatment systems. Journal of Cleaner Production, 219, 350-358.

Maltais-Landry, G., Scow, K., Brennan, E., Torbert, E. \& Vitousek, P. 2016. Higher flexibility in input N: P ratios results in more balanced phosphorus budgets in two long-term experimental agroecosystems. Agriculture, Ecosystems \& Environment, 223, 197-210.

Maltais-Landry, G., Scow, K., Brennan, E. \& Vitousek, P. 2015. Long-Term Effects of Compost and Cover Crops on Soil Phosphorus in Two California Agroecosystems. Soil Science Society of America Journal, 79, 688-697.

Manning, D. A. C. 2015. How will minerals feed the world in 2050? Proceedings of the Geologists' Association, 126, 14-17.

Manning, D. A. C. 2018. Innovation in Resourcing Geological Materials as Crop Nutrients. Natural Resources Research, 27, 217-227.

Manyuchi, M. M., Chiutsi, P., Mbohwa, C., Muzenda, E. \& Mutusva, T. 2018. Bio ethanol from sewage sludge: A bio fuel alternative. South African Journal of Chemical Engineering, 25, 123-127.

Mårald, E. 2006. Our finest gold: Agrarian perspectives on urban technology from the mid-19th century to present-day ecocyclical society. Progress in Industrial Ecology, 3, 393-407.

Marron, N. 2015. Agronomic and environmental effects of land application of residues in short-rotation tree plantations: A literature review. Biomass and Bioenergy, 81, 378-400.

Marschner, H. 2011. Marschner's mineral nutrition of higher plants, Academic press.

Martellozzo, F., Landry, J., Plouffe, D., Seufert, V., Rowhani, P. \& Ramankutty, N. 2014. Urban agriculture: a global analysis of the space constraint to meet urban vegetable demand. Environmental Research Letters, 9, 064025.

Maurer, M., Bufardi, A., Tilley, E., Zurbrügg, C. \& Truffer, B. 2012. A compatibility-based procedure designed to generate potential sanitation system alternatives. Journal of environmental management, 104, 51-61.

Maurer, M., Pronk, W. \& Larsen, T. 2006a. Treatment processes for source-separated urine. Water research, 40, 3151 3166.

Maurer, M., Pronk, W. \& Larsen, T. A. 2006b. Treatment processes for source-separated urine. Water Research.

Maurer, M., Schwegler, P. \& Larsen, T. 2003a. Nutrients in urine: energetic aspects of removal and recovery. Water Science and technology, 48, 37-46.

Maurer, M., Schwegler, P. \& Larsen, T. 2003b. Nutrients in urine: energetic aspects of removal and recovery. Water Science \& Technology, 48, 37-46.

Maurer, M., Schwegler, P. \& Larsen, T. A. 2003c. Nutrients in urine: Energetic aspects of removal and recovery. Water Science and Technology, 48, 37-46.

Maurer, M., Schwegler, P. \& Larsen, T. A. 2003d. Nutrients in urine: energetic aspects of removal and recovery. Water Science and Technology, 48, 37-46.

McBride, M. B. 2003. Toxic metals in sewage sludge-amended soils: Has promotion of beneficial use discounted the risks? Advances in Environmental Research, 8, 5-19.

McClintock, N. 2010. Why farm the city? Theorizing urban agriculture through a lens of metabolic rift. Cambridge journal of regions, Economy and Society, 3, 191-207.

McConville, J., Drangert, J.-O., Tidåker, P., Neset, T.-S., Rauch, S., Strid, I. \& Tonderski, K. 2015. Closing the food loops: guidelines and criteria for improving nutrient management. Sustainability: Science, Practice and Policy, 11, 33-43.

McDonnell, M. J. \& MacGregor-Fors, I. 2016. The ecological future of cities. Science, 352, 936-938.

Mcgarry, M. G., Tongkasame, C. \& Mcgarry, A. G. 1971. Water Reclamation and Algae Harvesting. Source Journal (Water Pollution Control Federation), 43, 824-835.

Mehta, C. M., Khunjar, W. O., Nguyen, V., Tait, S. \& Batstone, D. J. 2015. Technologies to recover nutrients from waste streams: A critical review. Critical Reviews in Environmental Science and Technology, 45, 385-427.

Meinzinger, F. \& Oldenburg, M. 2009. Characteristics of source-separated household wastewater flows: a statistical assessment. Water Science and Technology, 59, 1785-1791. 
Melia, P. M., Cundy, A. B., Sohi, S. P., Hooda, P. S. \& Busquets, R. 2017. Trends in the recovery of phosphorus in bioavailable forms from wastewater. Chemosphere, 186, 381-395.

Meststoffenwet 1986. Uitvoeringsregeling Meststoffenwet. In: MINISTRY OF AGRICULTURE, N. A. F. Q. (ed.).

Metson, G. S. \& Bennett, E. M. 2015. Phosphorus cycling in Montreal's food and urban agriculture systems. PLoS ONE, 10.

Metson, G. S., Cordell, D., Ridoutt, B. \& Mohr, S. 2018. Mapping phosphorus hotspots in Sydney's organic wastes: a spatially explicit inventory to facilitate urban phosphorus recycling. Journal of Urban Ecology, 4, juy009.

Metson, G. S., Hale, R. L., Iwaniec, D. M., Cook, E. M., Corman, J. R., Galletti, C. S. \& Childers, D. L. 2012. Phosphorus in Phoenix: a budget and spatial representation of phosphorus in an urban ecosystem. Ecological Applications, 22, 705-721.

Mihelcic, J. R., Fry, L. M. \& Shaw, R. 2011. Global potential of phosphorus recovery from human urine and feces. Chemosphere, $84,832-839$.

Mikkelsen, R. \& Hartz, T. K. 2008. Nitrogen Sources for Organic Crop Production. Better Crops, 92.

Mininni, G., Blanch, A. R., Lucena, F. \& Berselli, S. 2015. EU policy on sewage sludge utilization and perspectives on new approaches of sludge management. Environmental Science and Pollution Research, 22, 7361-7374.

Ministerie van Landbouw, N. e. V. \& Waterstaat, M. v. I. e. 2017. Zesde Nederlandse actieprogramma betreffende de Nitraatrichtlijn (2018-2021).

Modin, O., Persson, F., Wilén, B. M. \& Hermansson, M. 2016. Nonoxidative removal of organics in the activated sludge process. Critical Reviews in Environmental Science and Technology, 46, 635-672.

Mok, H.-F., Williamson, V. G., Grove, J. R., Burry, K., Barker, S. F. \& Hamilton, A. J. 2014. Strawberry fields forever? Urban agriculture in developed countries: a review. Agronomy for sustainable development, 34, 21-43.

Monfet, E., Aubry, G. \& Ramirez, A. A. 2017. Nutrient removal and recovery from digestate: a review of the technology. Biofuels, 7269, 1-16.

Montanarella, L., Pennock, D. J., McKenzie, N., Badraoui, M., Chude, V., Baptista, I., Mamo, T., Yemefack, M., Aulakh, M. S., Yagi, K., Hong, S. Y., Vijarnsorn, P., Zhang, G. L., Arrouays, D., Black, H., Krasilnikov, P., Sobocká, J., Alegre, J., Henriquez, C. R., Mendonça-Santos, M. d. L., Taboada, M., Espinosa-Victoria, D., AlShankiti, A., AlaviPanah, S. K., Mustafa Elsheikh, E. A. E., Hempel, J., Arbestain, M. C., Nachtergaele, F. \& Vargas, R. 2016. World's soils are under threat. Soil, 2, 79-82.

Montgomery, D. R. 2007. Soil erosion and agricultural sustainability. Proceedings of the National Academy of Sciences of the United States of America, 104, 13268-72.

Morales, N., Boehler, M. A., Buettner, S., Liebi, C. \& Siegrist, H. 2013. Recovery of N and P from urine by struvite precipitation followed by combined stripping with digester sludge liquid at full scale. Water (Switzerland), 5, $1262-1278$

Morgan-Sagastume, F., Valentino, F., Hjort, M., Cirne, D., Karabegovic, L., Gerardin, F., Johansson, P., Karlsson, A., Magnusson, P., Alexandersson, T., Bengtsson, S., Majone, M. \& Werker, A. 2014. Polyhydroxyalkanoate (PHA) production from sludge and municipal wastewater treatment. Water Science and Technology, 69, 177-184.

Mougeot, L. J. Urban agriculture: definition, presence, potentials and risks. In: BAKKER, N., DUBBELING, M., GRUNDEL, S., SABEL KOSCHELLA, U. \& ZEEUW, H. D., eds. Growing cities, growing food: Urban agriculture on the policy agenda. A reader on urban agriculture, 2000 Havana, Cuba. 1-42.

Mougeot, L. J. 2006. Growing better cities: Urban agriculture for sustainable development, IDRC.

Mulbry, W., Westhead, E. K., Pizarro, C. \& Sikora, L. 2005. Recycling of manure nutrients: Use of algal biomass from dairy manure treatment as a slow release fertilizer. Bioresource Technology, 96, 451-458.

Mulier, A., Hofman, G., Baecke, E., Carlier, L., De Brabander, D., De Groote, G., De Wilde, R., Fiems, L., Janssens, G. \& Van Cleemput, O. 2003. A methodology for the calculation of farm level nitrogen and phosphorus balances in Flemish agriculture. European Journal of Agronomy, 20, 45-51.

Muller, A., Ferré, M., Engel, S., Gattinger, A., Holzkämper, A., Huber, R., Müller, M. \& Six, J. 2017. Can soil-less crop production be a sustainable option for soil conservation and future agriculture? Land Use Policy, 69, 102-105.

Muster, T. H., Douglas, G. B., Sherman, N., Seeber, A., Wright, N. \& Güzükara, Y. 2013. Towards effective phosphorus recycling from wastewater: Quantity and quality. Chemosphere, 91, 676-684

\section{$\mathrm{N}$}

Nakhli, S. A. A., Delkash, M., Bakhshayesh, B. E. \& Kazemian, H. 2017. Application of Zeolites for Sustainable Agriculture: a Review on Water and Nutrient Retention. Water, Air, \& Soil Pollution, 228, 464.

Nancharaiah, Y. V., Venkata Mohan, S. \& Lens, P. N. L. 2016. Recent advances in nutrient removal and recovery in biological and bioelectrochemical systems. Bioresource Technology, 215, 173-185. 
Naoum, C., Fatta, D., Haralambous, K. J. \& Loizidou, M. 2001. Removal of heavy metals from sewage sludge by acid treatment. Journal of Environmental Science and Health - Part A Toxic/Hazardous Substances and Environmental Engineering, 36, 873-881.

Neset, T. S. S. \& Cordell, D. 2012. Global phosphorus scarcity: identifying synergies for a sustainable future. Journal of the Science of Food and Agriculture, 92, 2-6.

Nesme, T., Metson, G. S. \& Bennett, E. M. 2018. Global phosphorus flows through agricultural trade. Global Environmental Change, 50, 133-141.

Nicholson, F., Humphries, S., Anthony, S., Smith, S., Chadwick, D. \& Chambers, B. 2012. A software tool for estimating the capacity of agricultural land in England and Wales for recycling organic materials (ALOWANCE). Soil Use and Management, 28, 307-317.

Niewersch, C., Koh, C. N., Wintgens, T., Melin, T., Schaum, C. \& Cornel, P. 2008. Potentials of using nanofiltration to recover phosphorus from sewage sludge. Water Science and Technology, 57, 707-714.

Nigussie, A., Kuyper, T. W., Bruun, S. \& de Neergaard, A. 2016. Vermicomposting as a technology for reducing nitrogen losses and greenhouse gas emissions from small-scale composting. Journal of Cleaner Production, 139, 429-439.

Nowak, B., Wegerer, H., Aschenbrenner, P., Rechberger, H. \& Winter, F. 2012. Sewage sludge ash to phosphate fertilizer by chlorination and thermal treatment: Residence time requirements for heavy metal removal. Environmental Technology (United Kingdom).

Nubé, M. \& Voortman, R. 2011. Human micronutrient deficiencies: linkages with micronutrient deficiencies in soils, crops and animal nutrition. Combating micronutrient deficiencies: Food-based approaches, 7, 289.

O'Neal, J. A. \& Boyer, T. H. 2013. Phosphate recovery using hybrid anion exchange: Applications to source-separated urine and combined wastewater streams. Water Research, 47, 5003-5017.

OAM 2016. Bezoekers (x 1.000) aan musea verenigd in het OAM - Overleg Amsterdamse Musea, 2012-2016. In: OVERLEG AMSTERDAMSE MUSEA (ed.)

Öberg, G. \& Mason-Renton, S. A. 2018. On the limitation of evidence-based policy: Regulatory narratives and land application of biosolids/sewage sludge in BC, Canada and Sweden. Environmental Science and Policy, 84, 88-96.

Öberg, G., Merlinsky, M. G., LaValle, A., Morales, M. \& Tobias, M. 2014. The notion of sewage as waste: a study of infrastructure change and institutional inertia in Buenos Aires, Argentina and Vancouver, Canada.

Öborn, I., Edwards, A., Witter, E., Oenema, O., Ivarsson, K., Withers, P., Nilsson, S. \& Stinzing, A. R. 2003. Element balances as a tool for sustainable nutrient management: a critical appraisal of their merits and limitations within an agronomic and environmental context. European Journal of Agronomy, 20, 211-225.

Oenema, O., Chardon, W., Ehlert, P., van Dijk, K., Schoumans, O. \& Rulkens, W. Proceedings Phosphorus fertilizers from by-products and wastes. International Fertilizer Society, 2012 Cambridge.

Ohura, S., Harada, H., Biswas, B. K., Kondo, M., Ishikawa, S., Kawakita, H., Ohto, K. \& Inoue, K. 2011. Phosphorus recovery from secondary effluent and side-stream liquid in a sewage treatment plant using zirconium-loaded saponified orange waste. Journal of Material Cycles and Waste Management, 13, 293-297.

OIS 2016. Scholen en leerlingen voortgezet onderwijs naar schooladres, schooljaar 2016/2017. In: ONDERZOEK, I. S. (ed.).

Orhon, D. 2015. Evolution of the activated sludge process: the first 50 years. Journal of Chemical Technology \& Biotechnology, 90, 608-640.

Orner, K. D. \& Mihelcic, J. R. 2018. A review of sanitation technologies to achieve multiple sustainable development goals that promote resource recovery. Environmental Science: Water Research \& Technology, 4, 16-32.

Orsini, F., Kahane, R., Nono-Womdim, R. \& Gianquinto, G. 2013. Urban agriculture in the developing world: a review. Agronomy for Sustainable Development, 33, 695-720.

Ostara 2018. Crystal Green.

Ott, C. \& Rechberger, H. 2012. The European phosphorus balance. Resources, Conservation and Recycling, 60, 159-172.

Otterpohl, R., Grottker, M. \& Lange, J. 1997. Sustainable Water and Waste Management in Urban Areas. Water Science and Technology, 35, 121-133.

\section{$\mathrm{P}$}

Parchomenko, A. \& Borsky, S. 2018. Identifying phosphorus hot spots: A spatial analysis of the phosphorus balance as a result of manure application. Journal of Environmental Management, 214, 137-148. 
Parés Viader, R., Jensen, P. E., Ottosen, L. M., Ahrenfeldt, J. \& Hauggaard-Nielsen, H. 2017. Sequential electrodialytic recovery of phosphorus from low-temperature gasification ashes of chemically precipitated sewage sludge. Waste Management, 60, 211-218.

PDOK 2018. Dataset: Basisregistratie Gewaspercelen (BRP). PDOK.

Pearson, L. J., Pearson, L. \& Pearson, C. J. 2010. Sustainable urban agriculture: stocktake and opportunities. International journal of agricultural sustainability, 8, 7-19.

Peccia, J. \& Westerhoff, P. 2015. We Should Expect More out of Our Sewage Sludge. Environmental Science and Technology, 49, 8271-8276.

Perez-Garcia, O., Escalante, F. M. E., de-Bashan, L. E. \& Bashan, Y. 2011. Heterotrophic cultures of microalgae: Metabolism and potential products. Water Research.

Petrik, M. 1954. Utilization of Night-Soil, Sewage, and Sewage Sludge in Agriculture. Bulletin of the World Health Organization, 10, 207-228.

Petzet, S. \& Cornel, P. 2011. Towards a complete recycling of phosphorus in wastewater treatment - options in Germany. Water Science and Technology, 64, 29-35.

Pikaar, I., Matassa, S., Rabaey, K., Bodirsky, B. L., Popp, A., Herrero, M. \& Verstraete, W. 2017. Microbes and the Next Nitrogen Revolution. Environmental Science and Technology, 51, 7297-7303.

Pittmann, T. \& Steinmetz, H. 2017. Polyhydroxyalkanoate production on waste water treatment plants: Process scheme, operating conditions and potential analysis for German and European municipal waste water treatment plants. Bioengineering, 4, 54 .

Poore, J. \& Nemecek, T. 2018. Reducing food's environmental impacts through producers and consumers. 992, 987992.

Pronk, W. \& Koné, D. 2009. Options for urine treatment in developing countries. Desalination, 248, 360-368.

Pronk, W., Palmquist, H., Biebow, M. \& Boller, M. 2006. Nanofiltration for the separation of pharmaceuticals from nutrients in source-separated urine. Water Research, 40, 1405-1412.

Pronk, W., Zuleeg, S., Lienert, J., Escher, B., Koller, M., Berner, A., Koch, G. \& Boller, M. 2007. Pilot experiments with electrodialysis and ozonation for the production of a fertiliser from urine. Water Science and Technology.

Puyol, D., Batstone, D. J., Hülsen, T., Astals, S., Peces, M. \& Krömer, J. O. 2017. Resource recovery from wastewater by biological technologies: opportunities, challenges, and prospects. Frontiers in microbiology, 7, 2106.

\section{$\mathrm{R}$}

Rahman, M. M., Salleh, M. A. M., Rashid, U., Ahsan, A., Hossain, M. M. \& Ra, C. S. 2014. Production of slow release crystal fertilizer from wastewaters through struvite crystallization - A review. Arabian Journal of Chemistry, 7, 139-155.

Rana, S., Biswas, J. K., Rinklebe, J., Meers, E. \& Bolan, N. 2017. Harnessing fertilizer potential of human urine in a mesocosm system : a novel test case for linking the loop between sanitation and aquaculture. Environmental Geochemistry and Health, 39, 1545-1561.

Randall, D. G., Krähenbühl, M., Köpping, I., Larsen, T. A. \& Udert, K. M. 2016. A novel approach for stabilizing fresh urine by calcium hydroxide addition. Water Research, 95, 361-369.

Rasmussen, L. V., Coolsaet, B., Martin, A., Mertz, O., Pascual, U., Corbera, E., Dawson, N., Fisher, J. A., Franks, P. \& Ryan, C. M. 2018. Social-ecological outcomes of agricultural intensification. Nature Sustainability.

Razon, L. F. 2018. Reactive nitrogen: A perspective on its global impact and prospects for its sustainable production. Sustainable Production and Consumption, 15, 35-48.

Rees, W. \& Wackernagel, M. 1996. Urban ecological footprints: why cities cannot be sustainable — and why they are a key to sustainability. Environmental impact assessment review, 16, 223-248.

Renner, R. 2000. Sewage Sludge - Pros \& Cons. Environmental Science and Technology, 34, 431 A-435 A.

Renner, R. 2008. Pipe scales release hazardous metals into drinking water. Environmental Science and Technology, 42, 4241.

Revi, A. \& Rosenzweig, C. 2013. The urban opportunity: Enabling transformative and sustainable development. Background Research Paper for the High-Level Panel of Eminent Persons on the Post-2015 Development Agenda. New York, NY, USA: UN Sustainable Development Solutions Network.

Richardson, A. 2012. A New World Ordure? Thoughts on the use of Humanure in Developed Cities. City, 16, 700-712.

Rijksoverheid. Overzicht aantal uren onderwijstijd [Online]. Available: https://www.rijksoverheid.nl/onderwerpen/ schooltijden-en-onderwijstijd/inhoud/overzicht-aantal-uren-onderwijstijd.

Rijksoverheid. 2014a. Gebruiksruimte en gebruiksnormen [Online]. Available: http://www.drloket.nl/onderwerpen/ mest/dossiers/dossier/gebruiksruimte-en-gebruiksnormen/berekening-van-het-gebruik [Accessed March 18, 2014 2014]. 
Rijksoverheid 2014b. Mestbeleid 2014-2017 Tabellen. In: DIENST REGELINGEN (ed.). Rijksdienst voor Ondernemend Nederland.

Rittmann, B. E., Mayer, B., Westerhoff, P. \& Edwards, M. 2011. Capturing the lost phosphorus. Chemosphere, 84, 846853.

Rockefeller, A. A. 1998. Civilization and sludge: notes on the history of the management of human excreta. Capitalism, Nature, Socialism, 9, 3-18.

Rodríguez Arredondo, M., Kuntke, P., Jeremiasse, A. W., Sleutels, T. H. J. A., Buisman, C. J. N. \& ter Heijne, A. 2015. Bioelectrochemical systems for nitrogen removal and recovery from wastewater. Environ. Sci.: Water Res. Technol., 1, 22-33.

Roefs, I., Meulman, B., Vreeburg, J. H. G. \& Spiller, M. 2017. Centralised, decentralised or hybrid sanitation systems? Economic evaluation under urban development uncertainty and phased expansion. Water Research, 109, 274-286.

Römer, W. 2006. Vergleichende untersuchungen zur pflanzenverfügbarkeit von phosphat aus verschiedenen P-recycling-produkten im keimpflanzenversuch. Journal of Plant Nutrition and Soil Science, 169, 826-832.

Rong, L., Maneerung, T., Ng, J. C., Neoh, K. G., Bay, B. H., Tong, Y. W., Dai, Y. \& Wang, C. H. 2015. Co-gasification of sewage sludge and woody biomass in a fixed-bed downdraft gasifier: Toxicity assessment of solid residues. Waste Management, 36, 241-255.

Ronteltap, M., Maurer, M. \& Gujer, W. 2007. The behaviour of pharmaceuticals and heavy metals during struvite precipitation in urine. Water research, 41, 1859-1868.

Röös, E., Mie, A., Wivstad, M., Salomon, E., Johansson, B., Gunnarsson, S., Wallenbeck, A., Hoffmann, R., Nilsson, U., Sundberg, C. \& Watson, C. A. 2018. Risks and opportunities of increasing yields in organic farming. A review. Agronomy for Sustainable Development, 38.

Rose, C., Parker, A., Jefferson, B. \& Cartmell, E. 2015a. The characterization of feces and urine: a review of the literature to inform advanced treatment technology. Critical reviews in environmental science and technology, 45, 1827-1879.

Rose, C., Parker, A., Jefferson, B. \& Cartmell, E. 2015b. The characterization of feces and urine: A review of the literature to inform advanced treatment technology. Critical Reviews in Environmental Science and Technology, 45, 1827-1879.

Rosemarin, A. \& Ekane, N. 2016. The governance gap surrounding phosphorus. Nutrient Cycling in Agroecosystems, $104,265-279$.

Roy, E. D. 2017. Phosphorus recovery and recycling with ecological engineering: A review. Ecological Engineering, 98, 213-227.

RUAF 2006. Cities Farming for the Future, Urban Agriculture for Green and Productive Cities, Philippines, RUAF Foundation, IDRC, and IIRR.

RVO. Dierlijke mest gebruiksnorm en gebruiksruimte [Online]. Rijksdienst voor Ondernemend Nederland. Available: https://www.rvo.nl/onderwerpen/agrarisch-ondernemen/mestbeleid/mest/gebruiksnormen/dierlijke-mest [Accessed 08/06/2018 2018].

RVO 2014. Tabel 3 Werkingscoëfficiënt 2014-2017. In: 2014-2017, M. (ed.) 01/01/2014 ed.: Rijksdienst voor Ondernemend Nederland.

RVO 2017a. Maximale waarden voor zware metalen. 01Mestbeleid 2017. 01/01/2017 ed.: Rijksdienst voor Ondernemend Nederland.

RVO 2017b. Tabel 1 Stikstofgebruiksnormen 2017. In: 2017, M. (ed.) 01/01/2017 ed.: Rijksdienst voor Ondernemend Nederland.

RVO 2017c. Tabel 4. Maximale waarden voor organische microverontreinigingen in meststoffen per kilogram van het desbetreffende waardegevende bestanddeel. 01Mestbeleid 2017. 01/01/2017 ed.: Rijksdienst voor Ondernemend Nederland.

\section{S}

Safi, Z. 2011. Nutrient cycling and nutrient use efficiency in urban and peri-urban agriculture of Kabul, Afghanistan. Doctor Doctoral dissertation, University of Kassel.

Salutsky, M. L., Dunseth, M., Ries, K. \& Shapiro, J. 1972. Ultimate disposal of phosphate from wastewater by recovery as fertilizer. Effluent Water Treatment Journal, 12.

Sancho, I., Licon, E., Valderrama, C., de Arespacochaga, N., López-Palau, S. \& Cortina, J. L. 2017. Recovery of ammonia from domestic wastewater effluents as liquid fertilizers by integration of natural zeolites and hollow fibre membrane contactors. Science of the Total Environment, 584-585, 244-251.

Sangare, D., Sou/Dakoure, M., Hijikata, N., Lahmar, R., Yacouba, H., Coulibaly, L. \& Funamizu, N. 2015. Toilet compost and human urine used in agriculture: fertilizer value assessment and effect on cultivated soil properties. Environmental technology, 36, 1291-1298. 
Santos, F. M. \& Pires, J. C. M. 2018. Nutrient recovery from wastewaters by microalgae and its potential application as bio-char. Bioresource Technology, 267, 725-731.

Sardans, J. \& Peñuelas, J. 2015. Potassium: A neglected nutrient in global change. Global Ecology and Biogeography, 24, 261-275.

Sartorius, C., von Horn, J. \& Tettenborn, F. 2012. Phosphorus Recovery from Wastewater-Expert Survey on Present Use and Future Potential. Water Environment Research, 84, 313-322.

Schaum, C., Cornel, P. \& Jardin, N. 2007. Phosphorus recovery from sewage sludge ash-a wet chemical approach. Proceeding of the IWA Conference on Biosolids, Moving Forward Wastewater Biosolids Sustainability: Technical, Managerial, and Public Synergy, pp. 24-27.

Scheierling, S. M., Bartone, C. R., Mara, D. D. \& Drechsel, P. 2011. Towards an agenda for improving wastewater use in agriculture. Water International, 36, 420-440.

Scherer, L. \& Pfister, S. 2015. Modelling spatially explicit impacts from phosphorus emissions in agriculture. The International Journal of Life Cycle Assessment, 20, 785-795.

Schock, M. R., Hyland, R. N. \& Welch, M. M. 2008. Occurrence of contaminant accumulation in lead pipe scales from domestic drinking-water distribution systems. Environmental Science and Technology, 42, 4285-4291.

Scholz, R. W., Hellums, D. T. \& Roy, A. A. 2015. Global sustainable phosphorus management: a transdisciplinary venture. Current Science, 1237-1246.

Schön, D. A. 2012. Generative metaphor: A perspective on problem-setting in social policy. Metaphor and Thought.

Schönberg, A., Samiei, K., Kern, H. \& Raupenstrauch, H. 2014. Der RecoPhos-Prozess - Rückgewinnung von Phosphor aus KIärschlammasche. Osterreichische Wasser- und Abfallwirtschaft, 66, 403-407.

Schönning, C., Leeming, R. \& Stenström, T. A. 2002. Faecal contamination of source-separated human urine based on the content of faecal sterols. Water Research, 36, 1965-1972.

Schot, J. \& Geels, F. W. 2008. Strategic niche management and sustainable innovation journeys: theory, findings, research agenda, and policy. Technology analysis \& strategic management, 20, 537-554.

Schroder, J. 2014. The position of mineral nitrogen fertilizer in efficient use of nitrogen and land: a review. Natural resources, 5, 936-948.

Schröder, J. J. \& Neeteson, J. J. 2008. Nutrient management regulations in The Netherlands. Geoderma, 144, 418-425.

Schröder, J. J., Smit, A. L., Cordell, D. \& Rosemarin, A. 2011. Improved phosphorus use efficiency in agriculture: a key requirement for its sustainable use. Chemosphere, $84,822-831$.

Seghezzo, L., Zeeman, G., Van Lier, J. B., Hamelers, H. V. M. \& Lettinga, G. 1998. A review: The anaerobic treatment of sewage in UASB and EGSB reactors. Bioresource Technology, 65, 175-190.

Senecal, J. \& Vinnerås, B. 2017. Urea stabilisation and concentration for urine-diverting dry toilets: Urine dehydration in ash. Science of the Total Environment, 586, 650-657.

Shaheen, S. M., Derbalah, A. S. \& Moghanm, F. S. 2012. Removal of Heavy Metals from Aqueous Solution by Zeolite in Competitive Sorption System. International Journal of Environmental Science and Development, 3, 362-367.

Sharpley, A., Kleinman, P., Jarvie, H. \& Flaten, D. 2016. Distant views and local realities: The limits of global assessments to restore the fragmented phosphorus cycle. Agricultural \& Environmental Letters, 1.

Sheldrick, W. F., Syers, J. K. \& Lingard, J. 2002. A conceptual model for conducting nutrient audits at national, regional, and global scales. Nutr. Cycl. Agroecosys. 62, 61-72.

Shepherd, J. G., Sohi, S. P. \& Heal, K. V. 2016. Optimising the recovery and re-use of phosphorus from wastewater effluent for sustainable fertiliser development. Water Research, 94, 155-165.

Sherwood, S. \& Uphoff, N. 2000. Soil health: Research, practice and policy for a more regenerative agriculture. Applied Soil Ecology, 15, 85-97.

Shilton, A. N., Powell, N. \& Guieysse, B. 2012. Plant based phosphorus recovery from wastewater via algae and macrophytes. Current Opinion in Biotechnology, 23, 884-889.

Shove, E. 2003. Converging Conventions of Comfort, Cleanliness and Convenience. Journal of Consumer Policy, 26, 395-418.

Siegrist, H. 1996. Nitrogen removal from digester supernatant - Comparison of chemical and biological methods. Water Science and Technology.

Simha, P. \& Ganesapillai, M. 2017. Ecological Sanitation and nutrient recovery from human urine: How far have we come? A review. Sustainable Environment Research, 27, 107-116.

Simha, P., Zabaniotou, A. \& Ganesapillai, M. 2016. Continuous urea-nitrogen recycling from human urine: A step towards creating a human excreta based bio-economy. Journal of Cleaner Production, 1-10.

Sinclair, R. 1967. Von Thünen and urban sprawl. Annals of the Association of American Geographers, 57, $72-87$.

Singh, N. K., Singh, R. P. \& Kazmi, A. A. 2017. Environmental impact assessment of a package type IFAS reactor during construction and operational phases: A life cycle approach. Water Science and Technology, 75, 2246-2256.

Smit, A. L., van Middelkoop, J. C., van Dijk, W. \& van Reuler, H. 2015. A substance flow analysis of phosphorus in the 
food production, processing and consumption system of the Netherlands. Nutrient Cycling in Agroecosystems, 103, 1-13.

Smit, J. \& Nasr, J. 1992. Urban agriculture for sustainable cities: using wastes and idle land and water bodies as resources. Environment and urbanization, 4, 141-152.

Smit, J., Nasr, J. \& Ratta, A. 1996. Urban agriculture: food, jobs and sustainable cities. New York, USA, 2, 35-37.

Smit, J., Nasr, J. \& Ratta, A. 2001. Urban Agriculture Food, Jobs and Sustainable Cities, United Nations Development Programme (UNDP).

Sörme, L. \& Lagerkvist, R. 2002. Sources of heavy metals in urban wastewater in Stockholm. Science of the Total Environment, 298, 131-145.

Spångberg, J., Tidåker, P. \& Jönsson, H. 2014. Environmental impact of recycling nutrients in human excreta to agriculture compared with enhanced wastewater treatment. Science of The Total Environment, 493, 209-219.

Specht, K., Siebert, R., Hartmann, I., Freisinger, U. B., Sawicka, M., Werner, A., Thomaier, S., Henckel, D., Walk, H. \& Dierich, A. 2013. Urban agriculture of the future: an overview of sustainability aspects of food production in and on buildings. Agriculture and Human Values, 1-19.

Specht, K., Siebert, R. \& Thomaier, S. 2015. Perception and acceptance of agricultural production in and on urban buildings (ZFarming): a qualitative study from Berlin, Germany. Agriculture and Human Values.

Spiller, M. \& Agudelo, C. Mapping diversity of urban metabolic functions-a planning approach for more resilient cities. Proceedings of the 5th AESOP Young Academics Network Meeting 2011, 15-18 Febr. 2011, Delft, the Netherlands, 2011. 126-139.

Staley, C., Breuillin-Sessoms, F., Wang, P., Kaiser, T., Venterea, R. T. \& Sadowsky, M. J. 2018. Urea amendment decreases microbial diversity and selects for specific nitrifying strains in eight contrasting agricultural soils. Frontiers in Microbiology, 9, 1-13.

Steffen, W., Richardson, K., Rockström, J., Cornell, S. E., Fetzer, I., Bennett, E. M., Biggs, R., Carpenter, S. R., de Vries, W., de Wit, C. A., Folke, C., Gerten, D., Heinke, J., Mace, G. M., Persson, L. M., Ramanathan, V., Reyers, B. \& Sörlin, S. 2015. Planetary boundaries: Guiding human development on a changing planet. Science, 347, 1259855 .

Stendahl, K. \& Jäfverström, S. 2004. Recycling of sludge with the Aqua Reci process. Water Science and Technology, 49, 233-240.

STOWA 2014. Evaluatie Nieuwe Sanitatie Noorderhoek Sneek. Stichting Toegepast Onderzoek Waterbeheer. Available: https://www.stowa.nl/publicaties/evaluatie-nieuwe-sanitatie- noorderhoek-sneek.

STOWA 2018a. Evaluatie nieuwe sanitatie Noorderhoek/ Waterschoon 2. Amersfoort: Stichting Toegepast Onderzoek Waterbeheer.

STOWA. 2018b. Saniwijzer: Nieuwe sanitatie in de praktijk [Online]. Available: https://www.saniwijzer.nl/projecten [Accessed July 10 2019].

Strauss, M. 2000. Human waste (excreta and wastewater) reuse. ETC/SIDA Bibliography on Urban Agriculture.

Strauss, M. 2001. Reuse of wastewater in Urban Agriculture. Annotated Bibliography on Urban Agriculture. Wageningen: ETC-RUAF.

Streiffeler, F. 2001. Potentials of urban and peri-urban agriculture in Africa by the valorization of domestic waste in DESAR. In: LENS, P., ZEEMAN, G. \& LETTINGA, G. (eds.) Decentralised Sanitation and Reuse: Concepts, Systems and Implementation. Cornwall, UK: IWA Publishing.

Struik, P. C. \& Kuyper, T. W. 2017. Sustainable intensification in agriculture: the richer shade of green. A review. Agronomy for Sustainable Development, 37.

Stylianou, M. A., Kollia, D., Haralambous, K.-J., Inglezakis, V. J., Moustakas, K. G. \& Loizidou, M. D. 2007. Effect of acid treatment on the removal of heavy metals from sewage sludge. Desalination, 215, 73-81.

Sukačová, K. \& Červený, J. 2017. Can algal biotechnology bring effective solution for closing the phosphorus cycle? Use of algae for nutrient removal: Review of past trends and future perspectives in the context of nutrient recovery. European Journal of Environmental Sciences, 7, 63-72.

Suttle, N. F. 2010. Mineral nutrition of livestock, Cabi.

Suzuki, Y., Kondo, T., Nakagawa, K., Tsuneda, S., Hirata, A., Shimizu, Y. \& Inamori, Y. 2006. Evaluation of sludge reduction and phosphorus recovery efficiencies in a new advanced wastewater treatment system using denitrifying polyphosphate accumulating organisms. Water Science and Technology, 53, 107-113.

\section{$\mathrm{T}$}

Talboys, P. J., Heppell, J., Roose, T., Healey, J. R., Jones, D. L. \& Withers, P. J. A. 2016. Struvite: a slow-release fertiliser for sustainable phosphorus management? Plant and Soil, 401, 109-123. 
Tarpeh, W. A., Udert, K. M. \& Nelson, K. L. 2017. Comparing ion exchange adsorbents for nitrogen recovery from source-separated urine. Environmental Science and Technology, 51, 2373-2381.

Taylor, J. R. \& Lovell, S. T. 2012. Mapping public and private spaces of urban agriculture in Chicago through the analysis of high-resolution aerial images in Google Earth. Landscape and urban planning, 108, 57-70.

Taylor, J. R. \& Lovell, S. T. 2014. Urban home food gardens in the Global North: research traditions and future directions. Agriculture and Human Values, 31, 285-305.

Tervahauta, T. 2014. Phosphate and organic fertilizer recovery from black water. Wageningen: Wageningen University.

Tervahauta, T., Hoang, T., Hernández, L., Zeeman, G. \& Buisman, C. 2013. Prospects of source-separation-based sanitation concepts: a model-based study. Water, 5, 1006-1035.

Tervahauta, T., Rani, S., Hernández Leal, L., Buisman, C. J. N. \& Zeeman, G. 2014a. Black water sludge reuse in agriculture: Are heavy metals a problem? Journal of Hazardous Materials, 274, 229-236.

Tervahauta, T., Rani, S., Leal, L. H., Buisman, C. J. \& Zeeman, G. 2014b. Black water sludge reuse in agriculture: Are heavy metals a problem? Journal of hazardous materials, 274, 229-236.

Theregowda, R. B., González-Mejía, A. M., Ma, X. \& Garland, J. 2019. Nutrient recovery from municipal wastewater for sustainable food production systems: An alternative to traditional fertilizers. Environmental Engineering Science.

Thomaier, S., Specht, K., Henckel, D., Dierich, A., Siebert, R., Freisinger, U. B. \& Sawicka, M. 2015. Farming in and on urban buildings: Present practice and specific novelties of zero-acreage farming (ZFarming). Renewable Agriculture and Food Systems, 30, 43-54.

Tian, D. \& Niu, S. 2015. A global analysis of soil acidification caused by nitrogen addition. Environmental Research Letters, 10, 024019.

Tidåker, P., Kärrman, E., Baky, A. \& Jönsson, H. 2006. Wastewater management integrated with farming -an environmental systems analysis of a Swedish country town. Resources, Conservation and Recycling, 47, 295-315.

Tidåker, P., Sjöberg, C. \& Jönsson, H. 2007. Local recycling of plant nutrients from small-scale wastewater systems to farmland-A Swedish scenario study. Resources, conservation and recycling, 49, 388-405.

Tilley, E. 2014. Compendium of sanitation systems and technologies, Eawag.

Tilley, E., Atwater, J. \& Mavinic, D. 2008. Effects of storage on phosphorous recovery from urine. Environmental Technology, 29, 807-816.

Toufiq Reza, M., Freitas, A., Yang, X., Hiibel, S., Lin, H. \& Coronella, C. J. 2016. Hydrothermal Carbonization (HTC) of Cow Manure: Carbon and Nitrogen Distributions in HTC Products. Environmental Progress \& Sustainable Energy, 35, 1002-1011.

Trimmer, J. T., Cusick, R. D. \& Guest, J. S. 2017. Amplifying Progress toward Multiple Development Goals through Resource Recovery from Sanitation. Environmental Science and Technology, 51, 10765-10776.

Trimmer, J. T. \& Guest, J. S. 2018. Recirculation of human-derived nutrients from cities to agriculture across six continents. Nature Sustainability, 1, 427-435.

Tun, L. L., Jeong, D., Jeong, S., Cho, K., Lee, S. \& Bae, H. 2016. Dewatering of source-separated human urine for nitrogen recovery by membrane distillation. Journal of Membrane Science, 512, 13-20.

\section{U}

Udert, K., Larsen, T. A. \& Gujer, W. 2006a. Fate of major compounds in source-separated urine. Water Science and Technology, 54, 413-420.

Udert, K. M., Larsen, T. A., Biebow, M. \& Gujer, W. 2003a. Urea hydrolysis and precipitation dynamics in a urine-collecting system. Water Research, 37, 2571-2582.

Udert, K. M., Larsen, T. A. \& Gujer, W. 2003b. Biologically induced precipitation in urine-collecting systems. Water Science and Technology: Water Supply, 3, 71-78.

Udert, K. M., Larsen, T. A. \& Gujer, W. 2006b. Fate of major compounds in source-separated urine. Water Science and Technology, 54, 413-420.

Udo de Haes, H. 2012. Scarcity of Micronutrients in Soil, Feed, Food, and Mineral Reserves-Urgency and Policy Options. Report and Advisory Memorandum for the Dutch Minister of Agriculture and Foreign Trade. Culemborg, the Netherlands: Platform for Agriculture, Innovation, and Society (2012).

Ulén, B. 1997. Leaching of plant nutrients and heavy metals during the composting of household wastes and chemical characterization of the final product. Acta Agriculturae Scandinavica Section B: Soil and Plant Science, 47, 142-148.

Ulrich, A. E. \& Schnug, E. 2013. The Modern Phosphorus Sustainability Movement: A Profiling Experiment. Sustainability, 5, 4523-4545. 
United Nations. 2014. World's population increasingly urban with more than half living in urban areas [Online]. New York. Available: http://www.un.org/en/development/desa/news/population/world-urbanization-prospects.html [Accessed June 12, 2015 2015].

United Nations 2018. The 2018 Revision of World Urbanization Prospects, Online Edition. In: DEPARTMENT OF ECONOMIC AND SOCIAL AFFAIRS, P. D. (ed.).

Usharani, B. \& Vasudevan, N. 2016. Eco-friendly approach for leaching out heavy metals from sewage sludge. Chemistry and Ecology, 32, 507-519.

Uysal, A., Yilmazel, Y. D. \& Demirer, G. N. 2010. The determination of fertilizer quality of the formed struvite from effluent of a sewage sludge anaerobic digester. Journal of Hazardous Materials, 181, 248-254.

\section{$\mathrm{V}$}

van der Bom, F., Nunes, I., Raymond, N. S., Hansen, V., Bonnichsen, L., Magid, J., Nybroe, O. \& Jensen, L. S. 2018 Long-term fertilisation form, level and duration affect the diversity, structure and functioning of soil microbial communities in the field. Soil Biology and Biochemistry.

van der Grinten, E., Spijker, J. \& Lijzen, J. P. A. 2015. Hergebruik van grondstoffen uit afvalwater: Belemmeringen en oplossingsrichtingen aan de hand van de cases fosfaat en cellulose. Rijksinstituut voor Volksgezondheid en Milieu.

van der Hoek, J. P., de Fooij, H. \& Struker, A. 2016. Wastewater as a resource: Strategies to recover resources from Amsterdam's wastewater. Resources, Conservation and Recycling, 113, 53-64.

van Der Schans, J. W. 2010. Urban agriculture in the Netherlands. Urban Agriculture Magazine, 24, 40-42.

van der Schans, J. W. \& Wiskerke, J. S. 2012. Urban agriculture in developed economies. In: VILJOEN, A. \& WISKERKE, J. S. (eds.) Sustainable food planning: evolving theory and practice. Wageningen: Wageningen Academic Publishers.

van Dijk, K. C., Lesschen, J. P. \& Oenema, O. 2016. Phosphorus flows and balances of the European Union Member States. Science of the Total Environment, 542, 1078-1093.

van Drecht, G., Bouwman, A. F., Harrison, J. \& Knoop, J. M. 2009. Global nitrogen and phosphate in urban wastewater for the period 1970 to 2050. Global Biogeochemical Cycles, 23, 1-19.

van Grinsven, H. \& Bleeker, A. 2016. Evaluation of the Manure and fertilisers Act 2016: Synthesis Report. Netherlands Environmental Assessment Agency (PBL).

van Haeff, J. 2012. The Netherlands-Combining efficiently separate collection, anaerobic digestion and composting. In: ATTERO (ed.) ENC Workshop. Rennes, France: Attero.

van Ierssel 2013. Bemestings Advies Uit Je Eigen Stad.

van Loosdrecht, M. C. M. \& Brdjanovic, D. 2014. Anticipating the next century of wastewater treatment. Science.

van Puijenbroek, P., Beusen, A. \& Bouwman, A. 2019. Global nitrogen and phosphorus in urban waste water based on the shared socio-economic pathways. Journal of environmental management, 231, 446-456.

van Thiel, L. 2014. Watergebruik Thuis 2013. Amsterdam: TNS Nipo. Available: https://www.vewin.nl/SiteCollectionDocuments/Publicaties/Cijfers/Watergebruik_Thuis_2013.pdf

van Zon, H. 1986. Een zeer onfrisse geschiedenis. Studies over niet-industriële vervuiling in Nederland, 1850-1920.

Vaneeckhaute, C., Meers, E., Michels, E., Buysse, J. \& Tack, F. M. G. 2013. Ecological and economic benefits of the application of bio-based mineral fertilizers in modern agriculture. Biomass and Bioenergy, 49, 239-248.

Vassilev, S. V., Baxter, D., Andersen, L. K. \& Vassileva, C. G. 2010. An overview of the chemical composition of biomass. Fuel, 89, 913-933.

Vejre, H. 2012. Urban agriculture definitions and common agrarian policy. In: LOHRBERG, F. \& TUIMPE, A. (eds.) COST Action Urban Agriculture Europe: Documentation of 1st Working Group Meeting. Aachen: RWTH Aachen University.

Vernay, A. L., Salcedo Rahola, T. B. \& Ravesteijn, W. Growing food, feeding change: Towards a holistic and dynamic approach of eco-city planning. 3rd International Conference on Next Generation Infrastructure Systems for Eco-Cities, INFRA 2010, 2010 Shenzhen.

Verstraete, W., Van de Caveye, P. \& Diamantis, V. 2009. Maximum use of resources present in domestic "used water". Bioresource Technology, 100, 5537-5545.

Viljoen, A. \& Howe, J. 2012. Continuous productive urban landscapes, Routledge.

Vitousek, P. M., Mooney, H. A., Lubchenco, J. \& Melillo, J. M. 1997. Human Domination of Earth's Ecosystems. Science, 277, 494.

Vitousek, P. M., Naylor, R., Crews, T., David, M. B., Drinkwater, L., Holland, E., Johnes, P., Katzenberger, J., Martinelli, L. \& Matson, P. 2009. Nutrient imbalances in agricultural development. Science, 324, 1519-1520.

Vliegenthart, R. \& van Zoonen, L. 2011. Power to the frame: Bringing sociology back to frame analysis. European Journal of Communication. 
Voortman, R. 2012a. Micronutrients in agriculture and the world food system-future scarcity and implications.

Voortman, R. Micronutrients in agriculture and the world food system - future scarcity and implications. 2012b.

Voskamp, I. M., Spiller, M., Stremke, S., Bregt, A. K., Vreugdenhil, C. \& Rijnaarts, H. H. M. 2018. Space-time information analysis for resource-conscious urban planning and design: A stakeholder based identification of urban metabolism data gaps. Resources, Conservation and Recycling, 128, 516-525.

Voytenko, Y., McCormick, K., Evans, J. \& Schliwa, G. 2016. Urban living labs for sustainability and low carbon cities in Europe: Towards a research agenda. Journal of Cleaner Production, 123, 45-54.

\section{W}

Wadsworth, R., Hallett, S. \& Sakrabani, R. 2018. Phosphate acceptance map: A novel approach to match phosphorus content of biosolids with land and crop requirements. Agricultural Systems, 166, 57-69.

Wall, D. H., Nielsen, U. N. \& Six, J. 2015. Soil biodiversity and human health. Nature, 528, 69-76.

Wang, H.-J., Huang, B., Shi, X.-Z., Darilek, J. L., Yu, D.-S., Sun, W.-X., Zhao, Y.-C., Chang, Q. \& Öborn, I. 2008. Major nutrient balances in small-scale vegetable farming systems in peri-urban areas in China. Nutrient Cycling in Agroecosystems, 81, 203-218.

Wang, M., Ma, L., Strokal, M., Ma, W., Liu, X. \& Kroeze, C. 2018. Hotspots for Nitrogen and Phosphorus Losses from Food Production in China: A County-Scale Analysis. Environmental Science \& Technology, 52, 5782-5791.

Wang, S. \& Peng, Y. 2010. Natural zeolites as effective adsorbents in water and wastewater treatment. Chemical Engineering Journal, 156, 11-24.

Wasserbetriebe, B. 2018. Berliner Pflanze.

Waterschoon. 2011. Het Project [Online]. Available: http://www.waterschoon.nl/project.htm [Accessed 20-05-2014 2014].

Wei, S. P., van Rossum, F., van de Pol, G. J. \& Winkler, M.-K. H. 2018. Recovery of phosphorus and nitrogen from human urine by struvite precipitation, air stripping and acid scrubbing: A pilot study. Chemosphere, 212, 1030-1037.

Wells, M. L., Potin, P., Craigie, J. S., Raven, J. A., Merchant, S. S., Helliwell, K. E., Smith, A. G., Camire, M. E. \& Brawley, S. H. 2017. Algae as nutritional and functional food sources: revisiting our understanding. Journal of Applied Phycology.

Wen, Q., Tutuka, C., Keegan, A. \& Jin, B. 2009. Fate of pathogenic microorganisms and indicators in secondary activated sludge wastewater treatment plants. Journal of Environmental Management, 90, 1442-1447.

Wielemaker, R., Oenema, O., Zeeman, G. \& Weijma, J. 2019. Fertile cities: Nutrient management practices in urban agriculture. Science of The Total Environment.

Wielemaker, R., Stuiver, J., Zeeman, G. \& Weijma, J. in press. Identifying Amsterdam's nutrient hotspots: A new method to map human excreta at building and neighborhood scale.

Wielemaker, R. C., Weijma, J. \& Zeeman, G. 2018a. Harvest to harvest: Recovering nutrients with New Sanitation systems for reuse in Urban Agriculture. Resources, Conservation and Recycling, 128, 426-437.

Wielemaker, R. C., Weijma, J. \& Zeeman, G. 2018b. Harvest to harvest: Recovering nutrients with New Sanitation systems for reuse in Urban Agriculture. Resources, Conservation and Recycling.

Wijst, M. v. d. \& Groot-Marcus, A. P. 1998. Huishoudelijk afvalwater: Berekening van de zuurstofvraag. Wageningen: Landbouwuniversiteit Wageningen. Available: https://www.stowa.nl/publicaties/ huishoudelijk-afvalwater-berekening-van-de-zuurstofvraag

Wilfert, P., Kumar, P. S., Korving, L., Witkamp, G. J. \& Van Loosdrecht, M. C. M. 2015. The Relevance of Phosphorus and Iron Chemistry to the Recovery of Phosphorus from Wastewater: A Review. Environmental Science and Technology, 49, 9400-9414.

Willett, W., Rockström, J., Loken, B., Springmann, M., Lang, T., Vermeulen, S., Garnett, T., Tilman, D., DeClerck, F., Wood, A., Jonell, M., Clark, M., Gordon, L. J., Fanzo, J., Hawkes, C., Zurayk, R., Rivera, J. A., De Vries, W., Majele Sibanda, L., Afshin, A., Chaudhary, A., Herrero, M., Agustina, R., Branca, F., Lartey, A., Fan, S., Crona, B., Fox, E., Bignet, V., Troell, M., Lindahl, T., Singh, S., Cornell, S. E., Srinath Reddy, K., Narain, S., Nishtar, S. \& Murray, C. J. L. 2019. Food in the Anthropocene: the EAT-Lancet Commission on healthy diets from sustainable food systems. The Lancet.

Wilsenach, J., Maurer, M., Larsen, T. \& Van Loosdrecht, M. 2003. From waste treatment to integrated resource management. Water Science and Technology, 48, 1-9.

Wilsenach, J. A., Schuurbiers, C. A. H. \& van Loosdrecht, M. C. M. 2007. Phosphate and potassium recovery from source separated urine through struvite precipitation. Water research, 41, 458-466.

Winker, M., Vinnerås, B., Muskolus, A., Arnold, U. \& Clemens, J. 2009a. Bioresource Technology Fertiliser products from new sanitation systems : Their potential values and risks. Bioresource Technology, 100, 4090-4096. 
Winker, M., Vinnerås, B., Muskolus, A., Arnold, U. \& Clemens, J. 2009b. Fertiliser products from new sanitation systems: Their potential values and risks. Bioresource Technology, 100, 4090-4096.

Withers, P. J., van Dijk, K. C., Neset, T.-S. S., Nesme, T., Oenema, O., Rubæk, G. H., Schoumans, O. F., Smit, B. \& Pellerin, S. 2015. Stewardship to tackle global phosphorus inefficiency: The case of Europe. Ambio, 44, 193-206.

Witzling, L., Wander, M. \& Phillips, E. 2011. Testing and educating on urban soil lead: A case of Chicago community gardens. Journal of Agriculture, Food Systems, and Community Development, 1, 167-185.

Wolfram, M. \& Frantzeskaki, N. 2016. Cities and systemic change for sustainability: prevailing epistemologies and an emerging research agenda. Sustainability, 8, 144.

Wu, X. \& Modin, O. 2013. Ammonium recovery from reject water combined with hydrogen production in a bioelectrochemical reactor. Bioresource Technology, 146, 530-536.

\section{$X$}

Xie, M., Shon, H. K., Gray, S. R. \& Elimelech, M. 2016. Membrane-based processes for wastewater nutrient recovery: Technology, challenges, and future direction. Water Research, 89, 210-221.

Xu, H., He, P., Gu, W., Wang, G. \& Shao, L. 2012. Recovery of phosphorus as struvite from sewage sludge ash. Journal of Environmental Sciences (China), 24, 1533-1538.

Xu, Y., Wang, J., Xu, Z. \& Xu, H. 2001. A study on the PP hollow fiber membrane contactor and its performances for removing ammonia from wastewater or mixed gas: I. Removal and recovery of ammonia from wastewater. Water Science and Technology: Water Supply, 1, 185-194.

\section{Y}

Yang, L., Giannis, A., Chang, V. W. C., Liu, B., Zhang, J. \& Wang, J. Y. 2015. Application of hydroponic systems for the treatment of source-separated human urine. Ecological Engineering, 81, 182-191.

Yao, C., Pan, Y., Lu, H., Wu, P., Meng, Y., Cao, X. \& Xue, S. 2016. Utilization of recovered nitrogen from hydrothermal carbonization process by Arthrospira platensis. Bioresource Technology, 212, 26-34.

Yermán, L., Hadden, R. M., Carrascal, J., Fabris, I., Cormier, D., Torero, J. L., Gerhard, J. I., Krajcovic, M., Pironi, P. \& Cheng, Y. L. 2015. Smouldering combustion as a treatment technology for faeces: Exploring the parameter space. Fuel, 147, 108-116.

Yoshizaki, S. \& Tomida, T. 2000. Principle and process of heavy metal removal from sewage sludge. Environmental Science and Technology, 34, 1572-1575.

You, X., Valderrama, C., Querol, X. \& Cortina, J. L. 2017. Recovery of Ammonium by Powder Synthetic Zeolites from Wastewater Effluents: Optimization of the Regeneration Step. Water, Air, and Soil Pollution, 228.

Yuan, M. H., Chen, Y. H., Tsai, J. Y. \& Chang, C. Y. 2016. Ammonia removal from ammonia-rich wastewater by air stripping using a rotating packed bed. Process Safety and Environmental Protection, 102, 777-785.

\section{Z}

Zasada, I. 2011. Multifunctional peri-urban agriculture-A review of societal demands and the provision of goods and services by farming. Land use policy, 28, 639-648.

Zeeman, G. 2012. New sanitation: bridging cities and agriculture, Wageningen University, Wageningen UR.

Zeeman, G. \& Gerbens, S. 2002. CH4 emissions from animal manure. In: IPCC (ed.) Good Practice Guidance and Uncertainty Management in National Greenhouse Gas Inventories.

Zeeman, G. \& Kujawa-Roeleveld, K. 2011a. Resource recovery from source separated domestic waste (water) streams; full scale results. Water Science and Technology, 64, 1987-1992.

Zeeman, G. \& Kujawa-Roeleveld, K. 2011b. Resource recovery from source separated domestic waste (water) streams; full scale results. Water Science \& Technology, 64.

Zeeman, G., Kujawa, K., De Mes, T., Hernandez, L., De Graaff, M., Abu-Ghunmi, L., Mels, A., Meulman, B., Temmink, H. \& Buisman, C. 2008. Anaerobic treatment as a core technology for energy, nutrients and water recovery from source-separated domestic waste (water). Water Science and Technology, 57, 1207-1212.

Zeraatkar, A. K., Ahmadzadeh, H., Talebi, A. F., Moheimani, N. R. \& McHenry, M. P. 2016. Potential use of algae for heavy metal bioremediation, a critical review. Journal of Environmental Management, 181, 817-831.

Zhang, L. \& Liu, S. 2014. Investigation of organic compounds migration from polymeric pipes into drinking water under long retention times. Procedia Engineering, 70, 1753-1761.

Zhang, X., Davidson, E. A., Mauzerall, D. L., Searchinger, T. D., Dumas, P. \& Shen, Y. 2015. Managing nitrogen for sustainable development. Nature, 528, 51. 
Zhang, Y., Desmidt, E., Van Looveren, A., Pinoy, L., Meesschaert, B. \& Van Der Bruggen, B. 2013. Phosphate separation and recovery from wastewater by novel electrodialysis. Environmental Science and Technology, 47, 5888-5895.

Zhou, X., Li, Y., Li, Z., Xi, Y., Nazim Uddin, S. M. \& Zhang, Y. 2017. Investigation on microbial inactivation and urea decomposition in human urine during thermal storage. Journal of Water Sanitation and Hygiene for Development, 7, 378-386.

Zhou, X., Qu, Y., Kim, B. H., Du, Y., Wang, H., Li, H., Dong, Y., He, W., Liu, J. \& Feng, Y. 2015. Simultaneous current generation and ammonia recovery from real urine using nitrogen-purged bioelectrochemical systems. RSC Adv., 5, 70371-70378.

Zorpas, A. A., Constantinides, T., Vlyssides, A. G., Haralambous, I. \& Loizidou, M. 2000. Heavy metal uptake by natural zeolite and metals partitioning in sewage sludge compost. Bioresource Technology, 72, 113-119. 


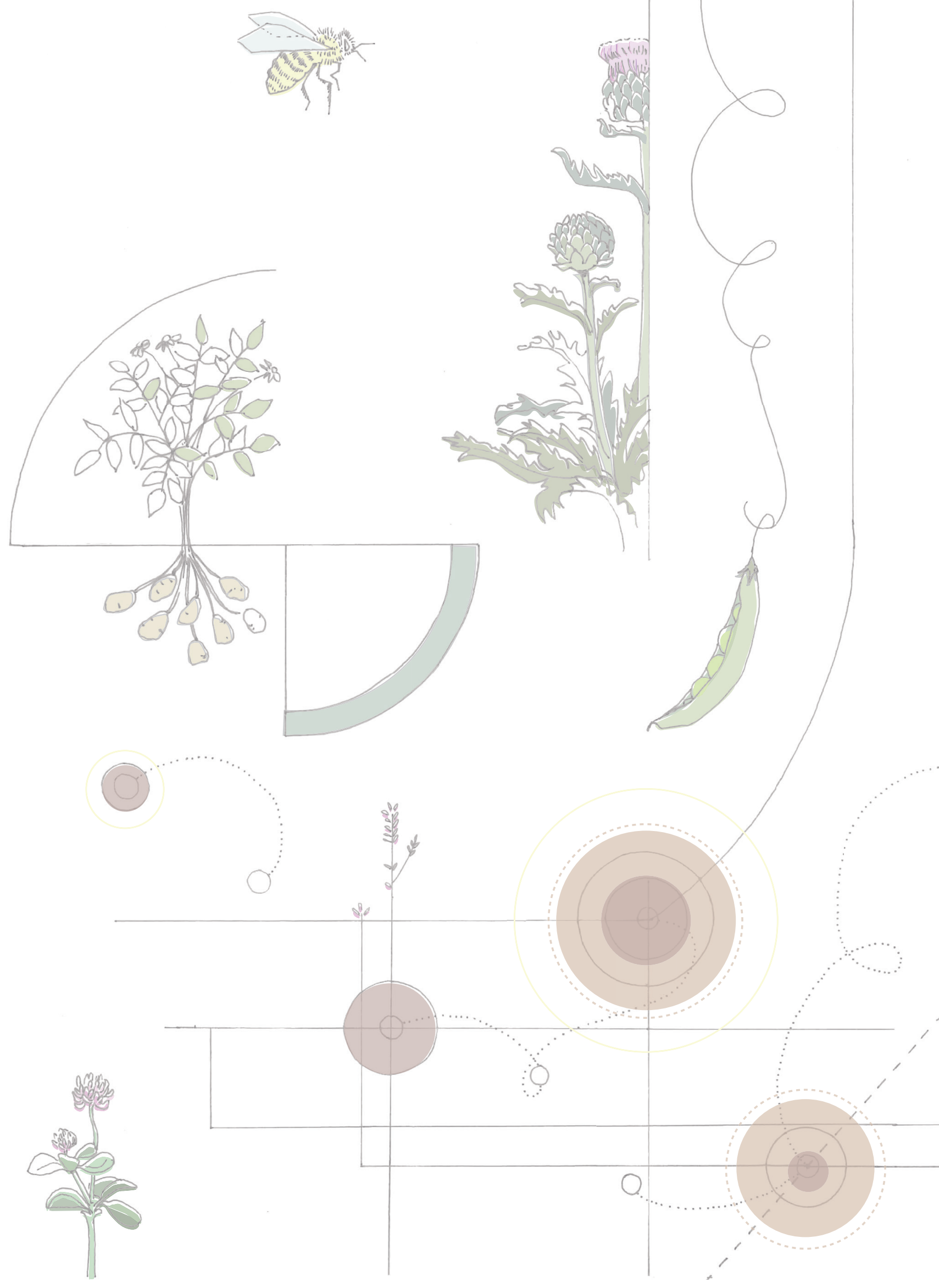




\section{TABLE OF CONTENTS}

\section{Chapter 2}

Harvest to harvest: Recovering nutrients with new sanitation systems for reuse in urban agriculture

Supporting Information I

\section{Chapter 3}

Fertile cities: Nutrient management practices in urban agriculture

Supporting Information II

\section{Chapter 4}

Recycling nutrients contained in human excreta to agriculture: Pathways, processes, and products

Supporting Information III

Supporting Information IV

Supporting Information $\vee$

\section{Chapter 5}

Identifying Amsterdam's nutrient hotspots: A new method to map human excreta at building and neighborhood scale

Supporting Information VI

Chapter 6

Resource Dynamo: A GIS model to match urban nutrient supply with agricultural demand

Supporting Information VII 


\section{SUPPORTING INFORMATION I}

\section{Harvest to harvest: Recovering nutrients with new sanitation systems for reuse in urban agriculture}

\section{Data new sanitation concepts}

This Supporting Information (SI) provides the substance flow analyses for each new sanitation concept (1000 p.e.) and the balance calculations for matching nutrient outputs from new sanitation systems with nutrient demand from urban agriculture, including the calculations for the indicated potential increase in nutrient self-sufficiency per scenario.

This SI contains the following tables:

Table S1.1 Recovery of nitrogen, phosphorus and organic matter for each new sanitation concept for influent of 1000 person equivalents

Table S1.2 Nutrient balances between ground-based urban agriculture and new sanitation concepts

Table S1.3 Nutrient balances between roof-top urban agriculture and new sanitation concepts 
Table S1.1 Recovery of nitrogen, phosphorus and organic matter for each new sanitation concept for influent of 1000 person equivalents

\begin{tabular}{|c|c|c|c|c|c|c|c|c|c|}
\hline \multirow{2}{*}{\begin{tabular}{|l|} 
Treatment \\
sub-stream \\
\end{tabular}} & \multirow{2}{*}{ influent- BW+KW } & \multicolumn{3}{|c|}{ UASB } & \multicolumn{3}{|c|}{ OLAND } & \multicolumn{2}{|c|}{ Struvite } \\
\hline & & Biogas & Sludge & Effluent & Gas & Sludge & Effluent & effluent & precipitate \\
\hline unit & $\mathrm{kg} / 1000 \mathrm{p} / \mathrm{yr}$ & $\mathrm{kg} / \mathrm{yr}$ & $\mathrm{kg} / \mathrm{yr}$ & $\mathrm{kg} / \mathrm{yr}$ & $\mathrm{kg} / \mathrm{yr}$ & $\mathrm{kg} / \mathrm{yr}$ & $\mathrm{kg} / \mathrm{yr}$ & & $\mathrm{kg} / \mathrm{yr}$ \\
\hline COD & 43628.8 & 23559.5 & 8289.5 & 11779.8 & N.R. & N.R. & 5536.5 & 5536.5 & - \\
\hline BOD5 & 21485.5 & 11602.2 & 4082.2 & 5801.1 & N.R. & N.R. & 2726.5 & 2726.5 & - \\
\hline TSS & 52983.0 & 28610.8 & 10066.8 & 14305.4 & N.R. & N.R. & - & - & - \\
\hline TN & 4750.2 & - & 47.5 & 4702.7 & N.R. & N.R. & 1269.7 & 1155.5 & 201.9 \\
\hline TP & 694.3 & - & 229.1 & 465.2 & N.R. & N.R. & - & 18.6 & 446.5 \\
\hline
\end{tabular}

Concept 2: UASB treatment of feces and urine with OLAND, struvite precipitation and sludge disinfection and composting of kitchen waste

\begin{tabular}{|c|c|c|c|c|c|c|c|c|c|}
\hline Treatment & & \multicolumn{3}{|c|}{ UASB } & \multicolumn{3}{|c|}{ OLAND } & \multicolumn{2}{|c|}{ Struvite } \\
\hline sub-stream & Influent-BW & Biogas & Sludge & Effluent & Gas & Sludge & Effluent & effluent & precipitate \\
\hline unit & $\mathrm{kg} / 1000 \mathrm{p} / \mathrm{yr}$ & $\mathrm{kg} / \mathrm{yr}$ & $\mathrm{kg} / \mathrm{yr}$ & $\mathrm{kg} / \mathrm{yr}$ & $\mathrm{kg} / \mathrm{yr}$ & $\mathrm{kg} / \mathrm{yr}$ & $\mathrm{kg} / \mathrm{yr}$ & & \\
\hline COD & 22070.2 & 11917.9 & 4193.3 & 5958.9 & N.R. & N.R. & 2800.7 & 2800.7 & - \\
\hline BOD5 & 9244.6 & 4992.1 & 1756.5 & 2496.0 & N.R. & N.R. & 1173.1 & 1173.1 & - \\
\hline TSS & 24116.4 & 13022.9 & 4582.1 & 6511.4 & N.R. & N.R. & - & - & - \\
\hline TN & 4238.6 & & 42.4 & 4196.3 & N.R. & N.R. & 1133.0 & 1031.0 & 180.6 \\
\hline TP & 621.2 & & 205.0 & 416.2 & N.R. & N.R. & - & 16.6 & 399.5 \\
\hline
\end{tabular}

\begin{tabular}{|c|c|c|c|c|c|c|c|c|c|}
\hline \multirow{2}{*}{\begin{tabular}{|l|} 
Treatment \\
sub-stream
\end{tabular}} & \multirow{2}{*}{ Inffluent Feces+KW } & \multicolumn{3}{|c|}{ UASB } & \multicolumn{3}{|c|}{ OLAND } & \multicolumn{2}{|c|}{ Struvite } \\
\hline & & Biogas & Sludge & Effluent & Gas & Sludge & Effluent & effluent & precipitate \\
\hline \begin{tabular}{|l|l|} 
unit \\
\end{tabular} & $\mathrm{kg} / 1000 \mathrm{p} / \mathrm{yr}$ & $\mathrm{kg} / \mathrm{yr}$ & $\mathrm{kg} / \mathrm{yr}$ & $\mathrm{kg} / \mathrm{yr}$ & $\mathrm{kg} / \mathrm{yr}$ & $\mathrm{kg} / \mathrm{yr}$ & $\mathrm{kg} / \mathrm{yr}$ & $\mathrm{kg} / \mathrm{yr}$ & $\mathrm{kg} / \mathrm{yr}$ \\
\hline COD & 40203.1 & 21709.7 & 7638.6 & 10854.8 & N.R. & N.R. & 5101.8 & 5101.8 & - \\
\hline BOD5 & 20033.1 & 10817.8 & 3806.3 & 5408.9 & N.R. & N.R. & 2542.2 & 2542.2 & - \\
\hline TSS & 42021.0 & 22691.3 & 7984.0 & 11345.7 & N.R. & N.R. & - & - & - \\
\hline TN & 1954.9 & & 19.5 & 1935.3 & N.R. & N.R. & 522.5 & 475.5 & 114.2 \\
\hline TP & 392.8 & & 129.6 & 263.2 & N.R. & N.R. & - & 10.5 & 252.7 \\
\hline
\end{tabular}

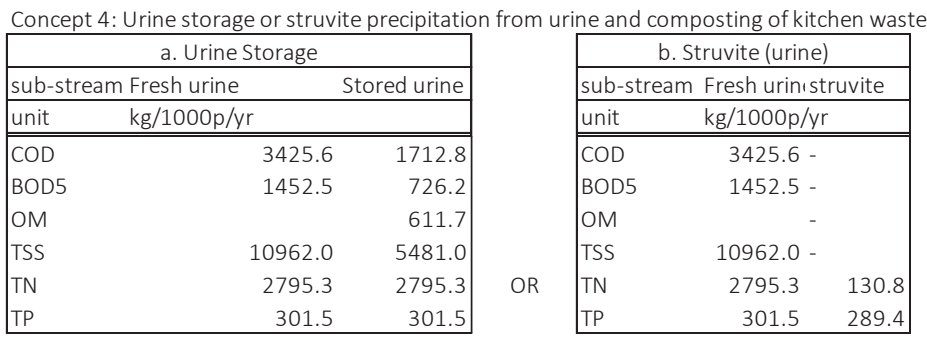




\begin{tabular}{|c|c|c|}
\hline \multicolumn{3}{|c|}{ Sludge Disinfection } \\
\hline $\mathrm{s}$ & ge UASB & Sludge fertilizer \\
\hline \multicolumn{3}{|c|}{$\mathrm{kg} / 1000 \mathrm{p} / \mathrm{yr} \quad \mathrm{kg} / \mathrm{yr}$} \\
\hline COD & 8289.5 & 8289.5 \\
\hline BOD5 & 4082.2 & 4082.2 \\
\hline OM (VS) & & 5921.0 \\
\hline TSS & 10066.8 & 10066.8 \\
\hline TN & 47.5 & 47.5 \\
\hline N Available & & 19.0 \\
\hline TP & 229.1 & 229.1 \\
\hline P Available & & 114.6 \\
\hline
\end{tabular}

\begin{tabular}{|lrr|}
\hline \multicolumn{3}{|c|}{ Compost (kitchen + garden) } \\
\hline \multicolumn{1}{|c|}{ unit } & kgT $/ 1000 \mathrm{p} / \mathrm{yr}$ & compost \\
\hline AND & 123505.2 & 41454.0 \\
Mass & 32743.5 & 8923.5 \\
OM (VS) & 464.0 & 208.8 \\
TN & & 20.9 \\
N Available- & & 54.7 \\
TP & 54.7 & 27.3 \\
\hline P Available- & & \\
\hline
\end{tabular}

\begin{tabular}{|l|cr|}
\hline \multicolumn{3}{|c|}{ Sludge Disinfection } \\
\cline { 2 - 3 } AND & \multicolumn{3}{|c|}{ Sludge UA Sludge fertilizer } \\
\hline \multicolumn{4}{|c}{ AN kg.yr } \\
\hline \hline COD & 4193.3 & 4193.3 \\
BOD5 & 1756.5 & 1756.5 \\
OM (VS) & & 2995.2 \\
TSS & 4582.1 & 4582.1 \\
TN & 42.4 & 42.4 \\
N Available & & 17.0 \\
TP & 205.0 & 205.0 \\
P Available & & 102.5 \\
\hline
\end{tabular}

\begin{tabular}{|lrr|}
\hline \multicolumn{3}{|c|}{ Urine Storage } \\
\cline { 2 - 3 } Anb-strear Fresh urine & Stored urine \\
\hline \multicolumn{1}{|l|}{ unit } & $\mathrm{kg} / 1000 \mathrm{p} / \mathrm{yr}$ \\
\hline \hline COD & 3425.6 & 1712.8 \\
BOD5 & 1452.5 & 726.2 \\
OM & & 611.7 \\
TSS & 10962.0 & 5481.0 \\
TN & 2795.3 & 2795.3 \\
TP & 301.5 & 301.5 \\
\hline
\end{tabular}

\begin{tabular}{|l|cr|}
\hline \multicolumn{3}{|c|}{ Sludge Disinfection } \\
\cline { 2 - 3 } & \multicolumn{3}{|c|}{ Sludge UA Sludge fertilizer } \\
\hline \multicolumn{3}{|c|}{$\mathrm{kg} / 1000 \mathrm{p}, \mathrm{kg} . \mathrm{yr}$} \\
\hline \hline \multirow{5}{*}{ COD } & 7638.6 & 7638.6 \\
BOD5 & 3806.3 & 3806.3 \\
OM (VS) & & 5456.1 \\
TSS & 7984.0 & 7984.0 \\
TN & 19.5 & 19.5 \\
N Available & & 7.8 \\
TP & 129.6 & 129.6 \\
P Available & & 64.8 \\
\hline
\end{tabular}

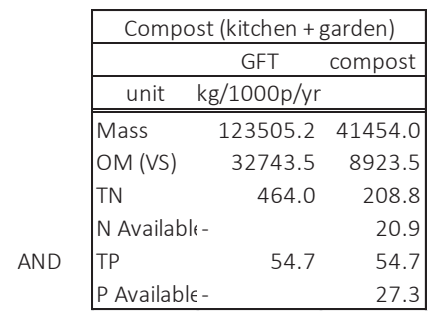


Table S1.2. Nutrient balances between ground-based urban agriculture and new sanitation concepts

\begin{tabular}{|c|c|c|c|c|c|c|c|c|c|}
\hline & 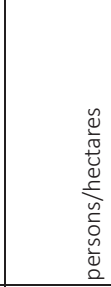 & 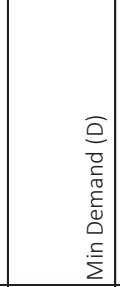 & 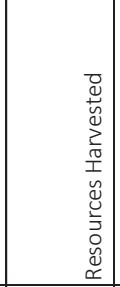 & 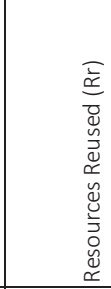 & 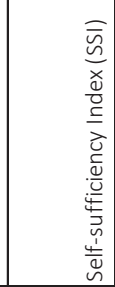 & 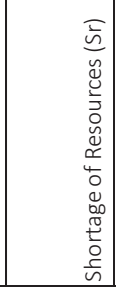 & 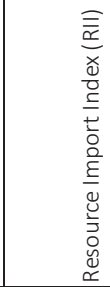 & 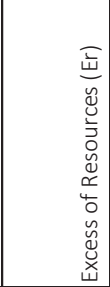 & 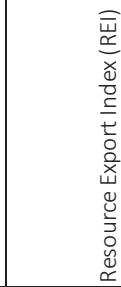 \\
\hline unit & p/ha & $\mathrm{kg} / \mathrm{ha} / \mathrm{yr}$ & $\mathrm{kg} / \mathrm{ha} / \mathrm{yr}$ & $\mathrm{kg} / \mathrm{ha} / \mathrm{yr}$ & $\%$ & $\mathrm{~kg} / \mathrm{ha} / \mathrm{yr}$ & $\%$ & $\mathrm{~kg} / \mathrm{ha} / \mathrm{yr}$ & $\%$ \\
\hline $\begin{array}{l}\text { Ground-based UA + } \\
\text { Concept } 1\end{array}$ & 11.03 & & & & & & & & \\
\hline OM & & 2685.00 & 65.30 & 65.30 & 2.43 & 2619.70 & -97.57 & 0.00 & 0.00 \\
\hline \begin{tabular}{|l|}
$\mathrm{N}$ slow \\
\end{tabular} & & 16.40 & 2.44 & 2.44 & 14.85 & 13.96 & -85.15 & 0.00 & 0.00 \\
\hline N quick & & 92.90 & 0.00 & 0.00 & 0.00 & 92.90 & -100.00 & 0.00 & 0.00 \\
\hline P slow & & 6.19 & 6.19 & 6.19 & 100.00 & 0.00 & 0.00 & 0.00 & 0.00 \\
\hline P quick & & 7.89 & 0.00 & 0.00 & 0.00 & 7.89 & -100.00 & 0.00 & 0.00 \\
\hline $\begin{array}{l}\text { Ground-based UA + } \\
\text { Concept } 2\end{array}$ & 11.69 & & & & & & & & \\
\hline $\mathrm{OM}$ & & 2685.00 & 139.32 & 139.32 & 5.19 & 2545.68 & -94.81 & 0.00 & 0.00 \\
\hline N slow & & 16.40 & 2.55 & 2.55 & 15.57 & 13.85 & -84.43 & 0.00 & 0.00 \\
\hline $\mathrm{N}$ quick & & 92.90 & 0.00 & 0.00 & 0.00 & 92.90 & -100.00 & 36.49 & 1.36 \\
\hline P slow & & 6.19 & 6.19 & 6.19 & 100.00 & 0.00 & 0.00 & 0.82 & 4.98 \\
\hline P quick & & 7.89 & 0.00 & 0.00 & 0.00 & 7.89 & -100.00 & 0.00 & 0.00 \\
\hline $\begin{array}{l}\text { Ground-based UA+ } \\
\text { Concept } 3 \\
\end{array}$ & 26.18 & & & & & & & & \\
\hline OM & & 2685.00 & 142.84 & 106.35 & 3.96 & 2578.65 & -96.04 & 36.49 & 1.36 \\
\hline N slow & & 16.40 & 3.19 & 2.38 & 14.50 & 14.02 & -85.50 & 0.82 & 4.98 \\
\hline N quick & & 92.90 & 73.18 & 73.18 & 78.77 & 19.72 & -21.23 & 0.00 & 0.00 \\
\hline P slow & & 6.19 & 8.31 & 6.19 & 100.00 & 0.00 & 0.00 & 2.12 & 34.31 \\
\hline P quick & & 7.89 & 7.89 & 7.89 & 100.00 & 0.00 & 0.00 & 0.00 & 0.00 \\
\hline \begin{tabular}{|l} 
Ground-based UA+ \\
Concept 4a \\
\end{tabular} & 226.42 & & & & & & & & \\
\hline $\mathrm{OM}$ & & 2685.00 & 2020.42 & 2020.42 & 75.25 & 664.58 & -24.75 & 0.00 & 0.00 \\
\hline $\mathrm{N}$ slow & & 16.40 & 4.73 & 4.73 & 28.83 & 11.67 & -71.17 & 0.00 & 0.00 \\
\hline N quick & & 92.90 & 632.90 & 73.18 & 78.77 & 19.72 & -21.23 & 559.72 & 602.50 \\
\hline P slow & & 6.19 & 6.19 & 6.19 & 100.00 & 0.00 & 0.00 & 0.00 & 0.00 \\
\hline P quick & & 7.89 & 68.25 & 7.89 & 100.00 & 0.00 & 0.00 & 60.36 & 764.85 \\
\hline $\begin{array}{l}\text { Ground-based UA+ } \\
\text { Concept 4b }\end{array}$ & 19.54 & & & & & & & & \\
\hline $\mathrm{OM}$ & & 2685.00 & 174.34 & 174.34 & 6.49 & 2510.66 & -93.51 & 0.00 & 0.00 \\
\hline \begin{tabular}{|l|} 
N slow \\
\end{tabular} & & 16.40 & 2.96 & 2.96 & 18.07 & \begin{tabular}{|r|}
13.44 \\
\end{tabular} & -81.93 & 0.00 & 0.00 \\
\hline N quick & & 92.90 & 0.00 & 0.00 & 0.00 & 92.90 & -100.00 & 0.00 & 0.00 \\
\hline P slow & & 6.19 & 6.19 & 6.19 & 100.00 & 0.00 & 0.00 & 0.00 & 0.00 \\
\hline P quick & & 7.89 & 0.00 & 0.00 & 0.00 & 7.89 & -100.00 & 0.00 & 0.00 \\
\hline
\end{tabular}


Table S1.3. Nutrient balances between roof-top urban agriculture and new sanitation concepts

\begin{tabular}{|c|c|c|c|c|c|c|c|c|}
\hline & 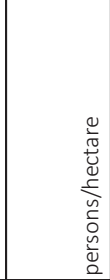 & 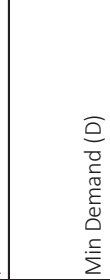 & 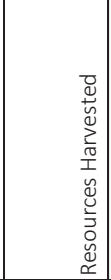 & 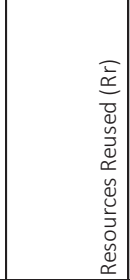 & 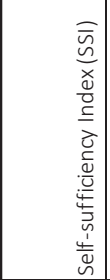 & 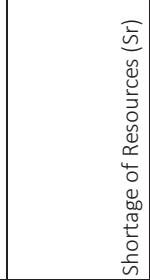 & 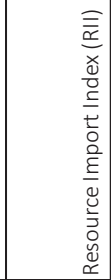 & 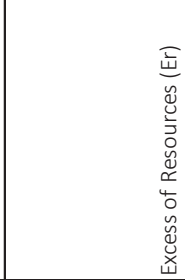 \\
\hline unit & $\mathrm{p} / \mathrm{ha}$ & $\mathrm{kg} / \mathrm{ha} / \mathrm{yr}$ & $\mathrm{kg} / \mathrm{ha} / \mathrm{yr}$ & $\mathrm{kg} / \mathrm{ha} / \mathrm{yr}$ & $\%$ & $\mathrm{~kg} / \mathrm{ha} / \mathrm{yr}$ & $\%$ & $\mathrm{~kg} / \mathrm{ha} / \mathrm{yr}$ \\
\hline Rooftop UA + Concept 1 & 25.09 & & & & & & & \\
\hline OM & & 1742.50 & 148.58 & 148.58 & 8.53 & -1593.92 & 91.47 & 0.00 \\
\hline N slow & & 112.50 & 5.54 & 5.54 & 4.93 & -106.96 & 95.07 & 0.00 \\
\hline $\mathrm{N}$ quick & & NA & 0.00 & 0.00 & 0.00 & 0.00 & 0.00 & 0.00 \\
\hline P slow & & 14.08 & 14.08 & 14.08 & 100.00 & 0.00 & 0.00 & 0.00 \\
\hline P quick & & NA & 0.00 & 0.00 & 0.00 & 0.00 & 0.00 & 0.00 \\
\hline Rooftop UA+Concept 2 & 26.60 & & & & & & & \\
\hline $\mathrm{OM}$ & & 1742.50 & 317.01 & 317.01 & 18.19 & -1425.49 & 81.81 & 0.00 \\
\hline N slow & & 112.50 & 5.81 & 5.81 & 5.16 & -106.69 & 94.84 & 0.00 \\
\hline $\mathrm{N}$ quick & & NA & 0.00 & 0.00 & 0.00 & 0.00 & 0.00 & 0.00 \\
\hline P slow & & 14.08 & 14.08 & 14.08 & 100.00 & 0.00 & 0.00 & 0.00 \\
\hline P quick & & NA & 0.00 & 0.00 & 0.00 & 0.00 & 0.00 & 0.00 \\
\hline Rooftop UA + Concept 3 & 44.35 & & & & & & & \\
\hline $\mathrm{OM}$ & & 1742.50 & 241.99 & 241.99 & 13.89 & -1500.51 & 86.11 & 0.00 \\
\hline N slow & & 112.50 & 5.41 & 5.41 & 4.81 & -107.09 & 95.19 & 0.00 \\
\hline N quick & & NA & 123.98 & 0.00 & 0.00 & 0.00 & 0.00 & 123.98 \\
\hline P slow & & 14.08 & 14.08 & 14.08 & 100.00 & 0.00 & 0.00 & 0.00 \\
\hline P quick & & NA & 13.37 & 0.00 & 0.00 & 0.00 & 0.00 & 13.37 \\
\hline Rooftop UA + Concept 4a & 195.27 & & & & & & & \\
\hline $\mathrm{OM}$ & & 1742.50 & 1742.50 & 1742.50 & 100.00 & 0.00 & 0.00 & 0.00 \\
\hline N slow & & 112.50 & 4.08 & 4.08 & 3.62 & -108.42 & 96.38 & 0.00 \\
\hline N quick & & NA & 25.55 & 25.55 & 0.00 & 0.00 & 0.00 & 25.55 \\
\hline P slow & & 14.08 & 5.34 & 5.34 & 37.90 & -8.74 & 62.10 & 0.00 \\
\hline \begin{tabular}{|l|} 
P quick \\
\end{tabular} & & NA & 56.51 & 56.51 & 0.00 & 0.00 & 0.00 & 56.51 \\
\hline Rooftop UA + Concept 4b & 44.45 & & & & & & & \\
\hline $\mathrm{OM}$ & & 1742.50 & 396.69 & 396.69 & 22.77 & -1345.81 & 77.23 & 0.00 \\
\hline N slow & & 112.50 & 6.74 & 6.74 & 5.99 & -105.76 & 94.01 & 0.00 \\
\hline N quick & & NA & 0.00 & 0.00 & 0.00 & 0.00 & 0.00 & 0.00 \\
\hline P slow & & 14.08 & 14.08 & 14.08 & 100.00 & 0.00 & 0.00 & 0.00 \\
\hline P quick & & NA & 0.00 & 0.00 & 0.00 & 0.00 & 0.00 & 0.00 \\
\hline
\end{tabular}




\section{SUPPORTING INFORMATION II}

\section{Fertile Cities: Nutrient management practices in urban agriculture}

\section{Interview data collection, nutrient budgeting and nutrient ratios}

This Supporting Information (SI) provides the questionnaire used to conduct interviews at urban farms in the Netherlands, as well as data that underpins the calculations made for nutrient budgeting. This includes data on fertilizer composition and equivalencies applied to calculate plant-available nutrient inputs, crop nutrient uptake values as found in literature, as well as the nutrient ratios for the calculated total fertilizer inputs per farm and for crop uptake values. The data collected during the interviews is available upon request via the corresponding author of the published manuscript, although has censored for anonymity.

This SI contains the following tables:

Table S2.1 Questionnaire for interviews at urban farms

Table S2.2 Data sources and assumptions for nutrient budgeting calculations

Table S2.3 Fertilizer composition and equivalencies

Table S2.4 Crop nutrient uptake values from Bosch \& de Jonge, (1989)

Table S2.5 Crop nutrient uptake values from Fink et al., (1999)

Table S2.6 Nutrient ratios for fertilizer inputs at interviewed urban farms

Table S2.7 Nutrient ratios for crop uptake from reference literature: Bosch \& de Jonge (1989) and Fink et al., (1999) 


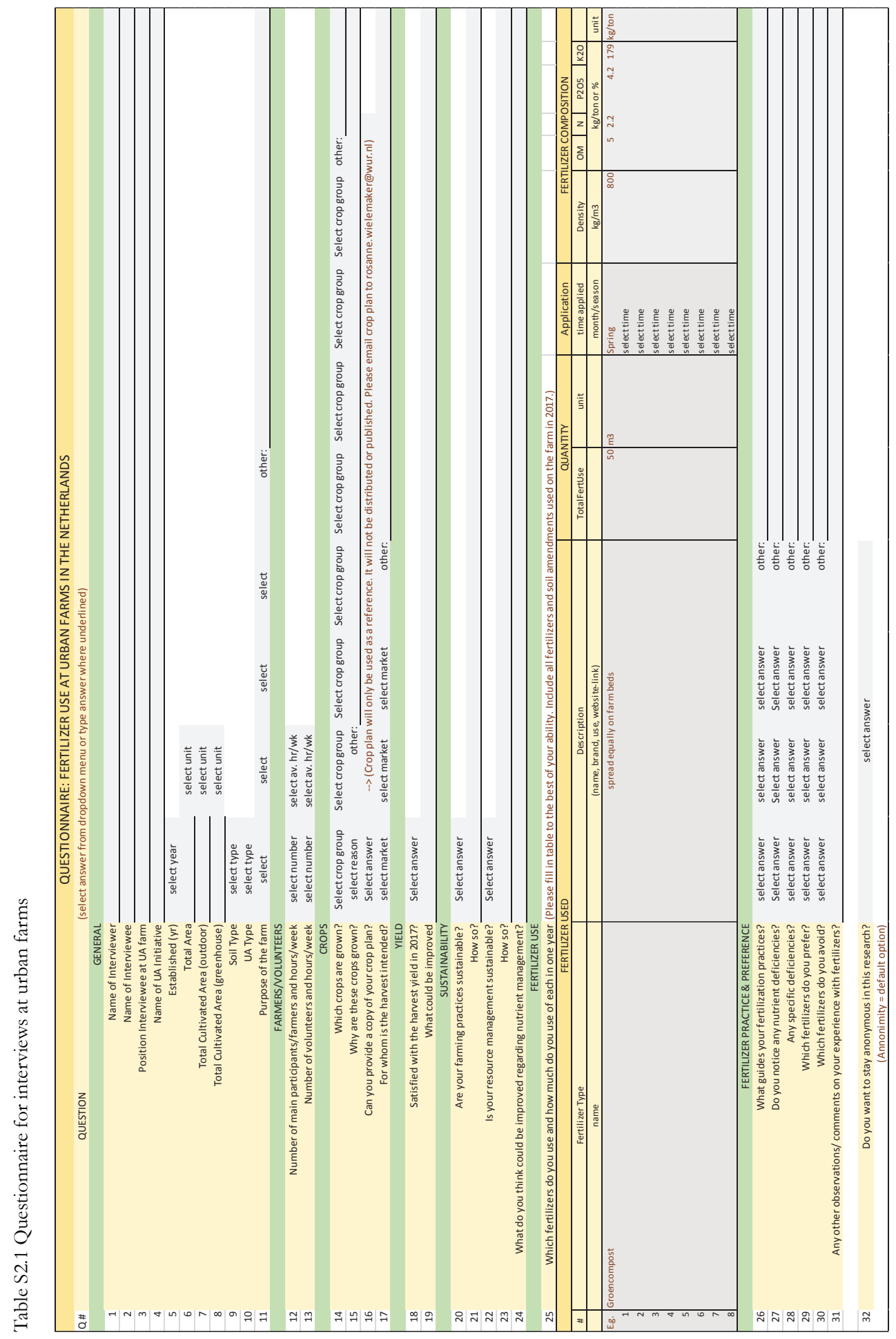


Table S2.2 Data sources and assumptions for nutrient budgeting calculations

\begin{tabular}{|c|c|c|c|c|}
\hline \multirow{2}{*}{\begin{tabular}{|l} 
Data \\
INPUT DATA \\
\end{tabular}} & \multirow{2}{*}{ Description/Equations } & \multirow[t]{2}{*}{ Units } & \multicolumn{2}{|c|}{ Main Data Source } \\
\hline & & & & \\
\hline \multirow{5}{*}{$\begin{array}{l}\text { Animal Manure (i.e., cow, pig, chicken, horse, } \\
\text { sheep, goat, rabbit, and duck) }\end{array}$} & Density & $\mathrm{kg} / \mathrm{m} 3$ & (de Haan \& van Geel, 2013) & Table 8.1 (page 99) \\
\hline & Nutrient composition & $\mathrm{kg} /$ ton & (de Haan \& van Geel, 2013) & Table 8.1 (page 99) \\
\hline & Effective Organic Matter (EOM) & $\mathrm{kg} / \mathrm{ton}$ & (de Haan \& van Geel, 2013) & Table 9.3 (page 111) \\
\hline & & & (Koopmans \& van der Burgt, 2001) & Bijlage 4 Mineraleninhoud van meststoffen \\
\hline & Nitrogen Equivalency (CNtot) & $\%$ & (de Haan \& van Geel, 2013) & Table 8.4 (page 102) \\
\hline \multirow[t]{3}{*}{$\begin{array}{l}\text { Animal-Derived Fertilizers (e.g., bonemeal, chicken } \\
\text { feather powder) }\end{array}$} & Nutrient composition & $\mathrm{kg} / \mathrm{ton}$ & $\begin{array}{l}\text { Fertilizer-specific information per } \\
\text { type/brand from product-specific } \\
\text { websites }\end{array}$ & \\
\hline & EOM & $\mathrm{kg} /$ ton & (de Haan \& van Geel, 2013) & Table 9.3 (page 111) \\
\hline & CNtot & $\%$ & (de Haan \& van Geel, 2013) & Table 8.4 (page 102) \\
\hline \multirow[t]{4}{*}{ Composts (i.e., municipal, green, and mushroom) } & Density & $\mathrm{kg} / \mathrm{m} 3$ & (de Haan \& van Geel, 2013) & Table 8.1 (page 99) \\
\hline & Nutrient composition & $\mathrm{kg} /$ ton & (de Haan \& van Geel, 2013) & Table 8.1 (page 99) \\
\hline & EOM & $\mathrm{kg} /$ ton & (de Haan \& van Geel, 2013) & Table 9.3 (page 111) \\
\hline & CNtot & $\%$ & (de Haan \& van Geel, 2013) & \\
\hline \multirow[t]{3}{*}{$\begin{array}{l}\text { Plant-based Materials (e.g., alfalfa pellets, grape } \\
\text { must) }\end{array}$} & Composition & $\mathrm{kg} / \mathrm{ton}$ & $\begin{array}{l}\begin{array}{l}\text { Fertilizer-specific information per } \\
\text { type/brand from product-specific } \\
\text { websites }\end{array} \\
\text { a }\end{array}$ & \\
\hline & EOM & $\mathrm{kg} / \mathrm{ton}$ & (van Dijk et al., 2005) & Table 3 (page 16) \\
\hline & CNtot & $\%$ & $\begin{array}{l}\text { Rijksdienst voor Ondernemend } \\
\text { Nederland (RVO); Meeting with } \\
\text { Philip Ehlert \& Oene Oenema }\end{array}$ & $\begin{array}{l}\text { Mestbeleid 2014-2017, Tabel } 3 . \\
\text { Werkingscoefficient }\end{array}$ \\
\hline \multirow[t]{3}{*}{ Commercial Fertilizers } & Composition & $\%$ & $\begin{array}{l}\text { fertilizer-specific information per } \\
\text { type/brand }\end{array}$ & \\
\hline & EOM & $\%$ & $?$ & \\
\hline & CNtot & $\%$ & $\begin{array}{l}\text { Rijksdienst voor Ondernemend } \\
\text { Nederland (RVO); Meeting with } \\
\text { Philip Ehlert \& Oene Oenema } \\
\end{array}$ & $\begin{array}{l}\text { Mestbeleid 2014-2017, Tabel } 3 . \\
\text { Werkingscoefficient }\end{array}$ \\
\hline \multicolumn{5}{|l|}{ EQUIVALENTS \& EFFECTIVE OM } \\
\hline \multirow[t]{3}{*}{\begin{tabular}{|l|} 
Nitrogen equivalancy (CNtot) \\
\end{tabular}} & \multirow{3}{*}{$\begin{array}{c}\text { Plant available } \mathrm{N}=\mathrm{CN} \text { tot } * \text { Ntot } \\
{[\mathrm{CN} \text { tot }=(\mathrm{CNm} * \mathrm{Nm}+\mathrm{CNorg} *} \\
\mathrm{Norg} / \mathrm{Ntot} * 100)]\end{array}$} & $\%$ & (de Haan \& van Geel, 2013) & Table 8.4 (page 101) \\
\hline & & & (van Dijk et al., 2005) & Table 1 (page 14) \\
\hline & & & $\begin{array}{l}\text { Rijksdienst voor Ondernemend } \\
\text { Nederland (RVO) }\end{array}$ & $\begin{array}{l}\text { Mestbeleid 2014-2017, Tabel } 3 . \\
\text { Werkingscoefficient }\end{array}$ \\
\hline \multirow[t]{2}{*}{ Humification coefficient } & \multirow[t]{2}{*}{$\begin{array}{l}\text { EOM }=\text { Hummification Coefficient } * \\
\text { OM }\end{array}$} & $\%$ & (de Haan \& van Geel, 2013) & Table 9.3 (page 107) \\
\hline & & & (Koopmans \& van der Burgt, 2001) & Bijlage 4 Mineraleninhoud van meststoffen \\
\hline \multicolumn{5}{|l|}{ DUTCH MANURE \& FERTILIZERS ACT } \\
\hline $\begin{array}{c}\text { Nitrogen application standards } \\
\text { (Stikstofgebruiksnormen) }\end{array}$ & \begin{tabular}{|l|} 
Nitrogen allowances per crop per \\
hectare (see Supplemental Information)
\end{tabular} & $\mathrm{kg} / \mathrm{ha}$ & $\begin{array}{l}\text { Rijksdienst voor Ondernemend } \\
\text { Nederland (RVO) }\end{array}$ & $\begin{array}{l}\text { Mestbeleid 2014-2017, Tabel } 1 . \\
\text { Stikstofgebruiksnormen }\end{array}$ \\
\hline Nitrogen equivalancy (CNtot) & $\begin{array}{l}\text { Coefficients as pertains to Dutch } \\
\text { Manure \& Fertilizers Act to calculate } \\
\text { plant-available nitrogen using } \\
\text { Equation: Plant available } \mathrm{N}=\mathrm{CN} \text { tot * } \\
\text { Ntot ; }\end{array}$ & $\%$ & $\begin{array}{l}\text { Rijksdienst voor Ondernemend } \\
\text { Nederland (RVO) }\end{array}$ & $\begin{array}{l}\text { Mestbeleid 2014-2017, Tabel } 3 \text {. } \\
\text { Werkingscoëfficient dierlijke en andere } \\
\text { organische meststoffen }\end{array}$ \\
\hline Nitrogen from animal manure & $\begin{array}{l}\text { Maximum of } 170 \mathrm{~kg} \mathrm{~N} \text { ha- } 1 \text { yr-1 from } \\
\text { animal manure can be applied }\end{array}$ & $\mathrm{kg} / \mathrm{ha}$ & $\begin{array}{l}\text { Rijksdienst voor Ondernemend } \\
\text { Nederland (RVO) }\end{array}$ & Gebruiksruimte dierlijke mest \\
\hline $\begin{array}{l}\text { Phosphorus application standards } \\
\text { (Fosfaatgebruiksnormen) }\end{array}$ & $\begin{array}{l}\text { Phosphorus allowances per hectare for } \\
\text { three soil P levels (see Supporting } \\
\text { Information) }\end{array}$ & $\mathrm{kg} / \mathrm{ha}$ & $\begin{array}{l}\text { Rijksdienst voor Ondernemend } \\
\text { Nederland (RVO) }\end{array}$ & $\begin{array}{l}\text { Mestbeleid 2014-2017, Tabel } 2 . \\
\text { Fosfaatgebruiksnormen }\end{array}$ \\
\hline Phosphorus Equivalency & $\begin{array}{l}\text { Coefficient of } 50 \% \text { for } \mathrm{P} \text { in composts } \\
\text { calculated using equation: Available P } \\
\text { (compost) }=\mathrm{CP} * \mathrm{P}\end{array}$ & $\%$ & $\begin{array}{l}\text { Rijksdienst voor Ondernemend } \\
\text { Nederland (RVO) }\end{array}$ & \\
\hline Organic matter & $\begin{array}{l}\text { Advice of } 2000 \mathrm{~kg} \text { EOM per hectare per } \\
\text { year }\end{array}$ & $\mathrm{kg} / \mathrm{ha}$ & (de Haan \& van Geel, 2013) & (page 112) \\
\hline \multicolumn{5}{|l|}{ CROP UPTAKE } \\
\hline \multirow[t]{3}{*}{ Vegetable crops $(\mathrm{n}=28)$} & yield & ton/ha & (Bosch \& de Jonge, 1989) & $\begin{array}{l}\text { (page 206) Opname en afvoer van } \\
\text { nutrienten doos vollegrondsgroenten }\end{array}$ \\
\hline & Nutrient composition & $\mathrm{kg} / \mathrm{ha}$ & (Bosch \& de Jonge, 1989) & $\begin{array}{l}\text { (page 206) Opname en afvoer van } \\
\text { nutrienten doos vollegrondsgroenten }\end{array}$ \\
\hline & Nutrient ratios & $\%$ & $\begin{array}{l}\text { Calculted from nutrient composition } \\
\text { (Bosch \& de Jonge, 1989) }\end{array}$ & \\
\hline \multirow[t]{3}{*}{ Vegetable crops $(n=22)$} & Yield & ton/ha & (Fink et al., 1999) & $\begin{array}{l}\text { Table 2. } N-, P-, K \text { - and } M g \text { - contents of } \\
\text { total fresh matter, harvest residues and } \\
\text { marketable yield of vegetable crops. }\end{array}$ \\
\hline & Nutrient composition & $\mathrm{kg} / \mathrm{ha}$ & (Fink et al., 1999) & $\begin{array}{l}\text { Table 2. } N_{-}, P-, K \text { - and } M g \text { - contents of } \\
\text { total fresh matter, harvest residues and } \\
\text { marketable yield of vegetable crops. }\end{array}$ \\
\hline & Nutrient ratios & $\%$ & $\begin{array}{l}\text { Calculated from nutrient } \\
\text { composition (Fink et al., 1999) }\end{array}$ & \\
\hline
\end{tabular}


Table S2.3 Fertilizer composition and equivalencies

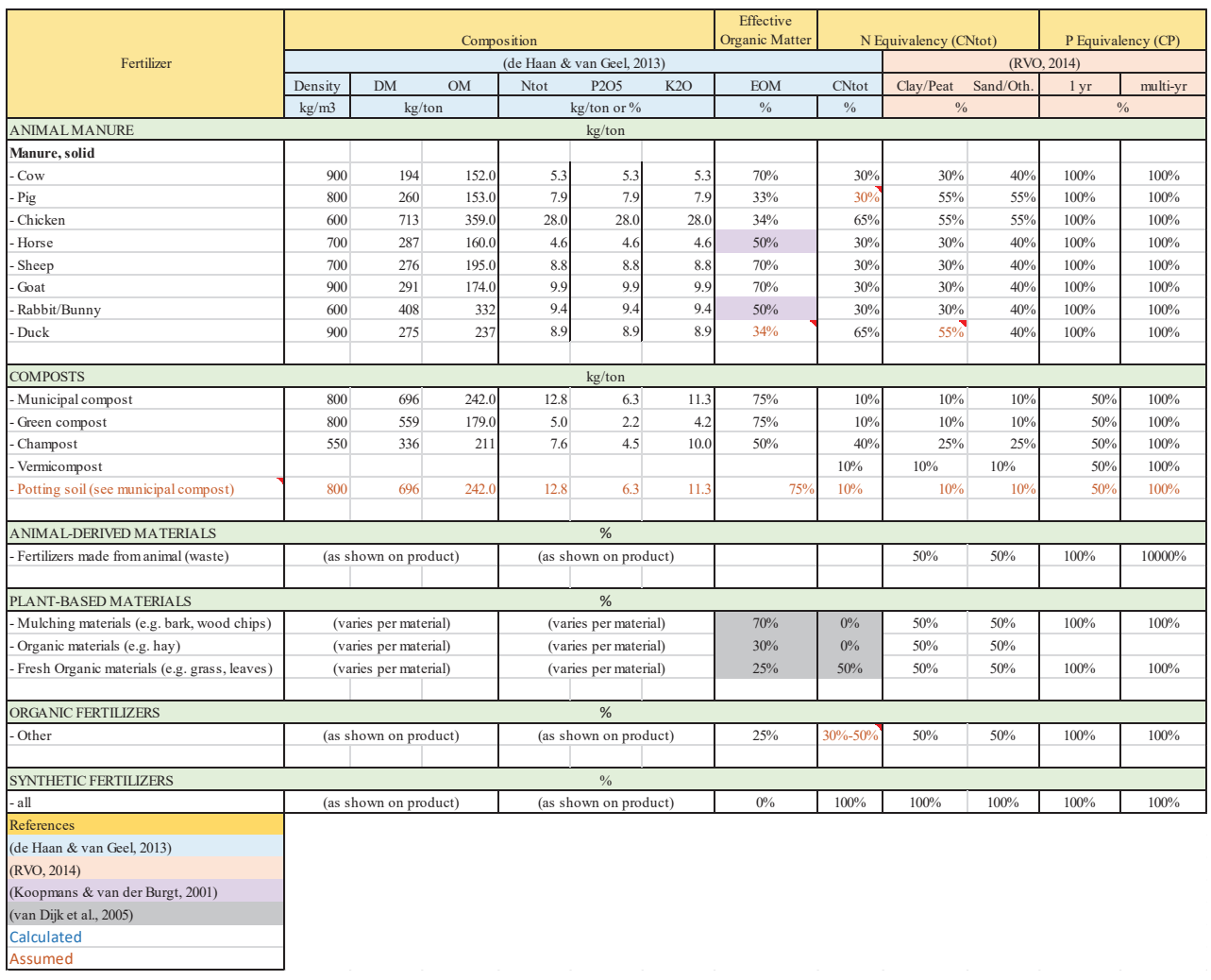


Table S2.4 Crop nutrient uptake from Bosch \& de Jonge (1989)

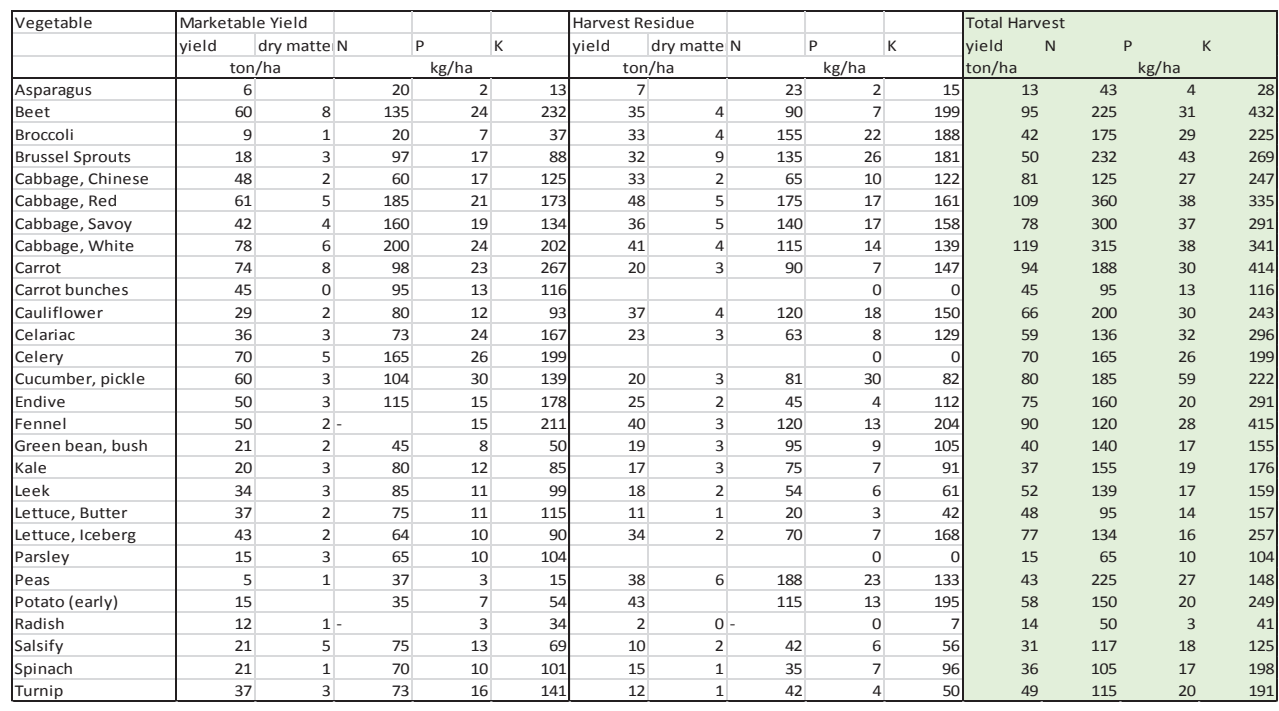

Table S2.5 Crop nutrient uptake from Bosch \& de Jonge (1989)

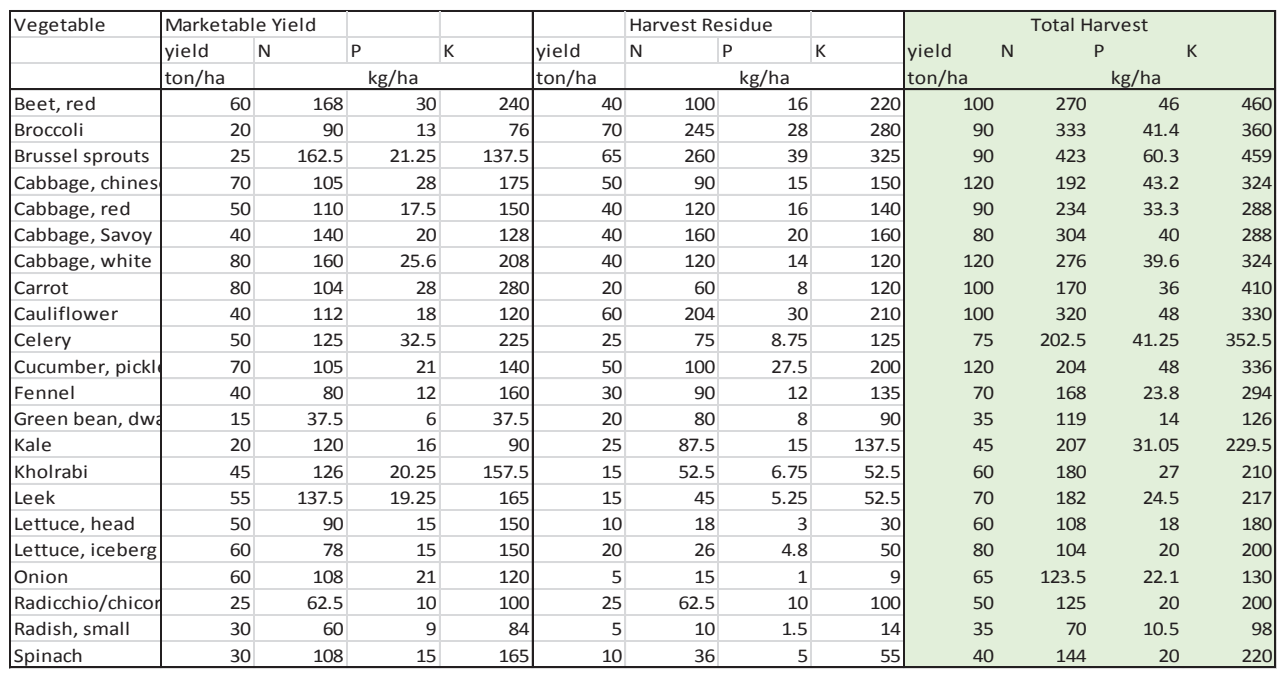


Table S2.6 Nutrient ratios for fertilizer inputs at interviewed urban farms

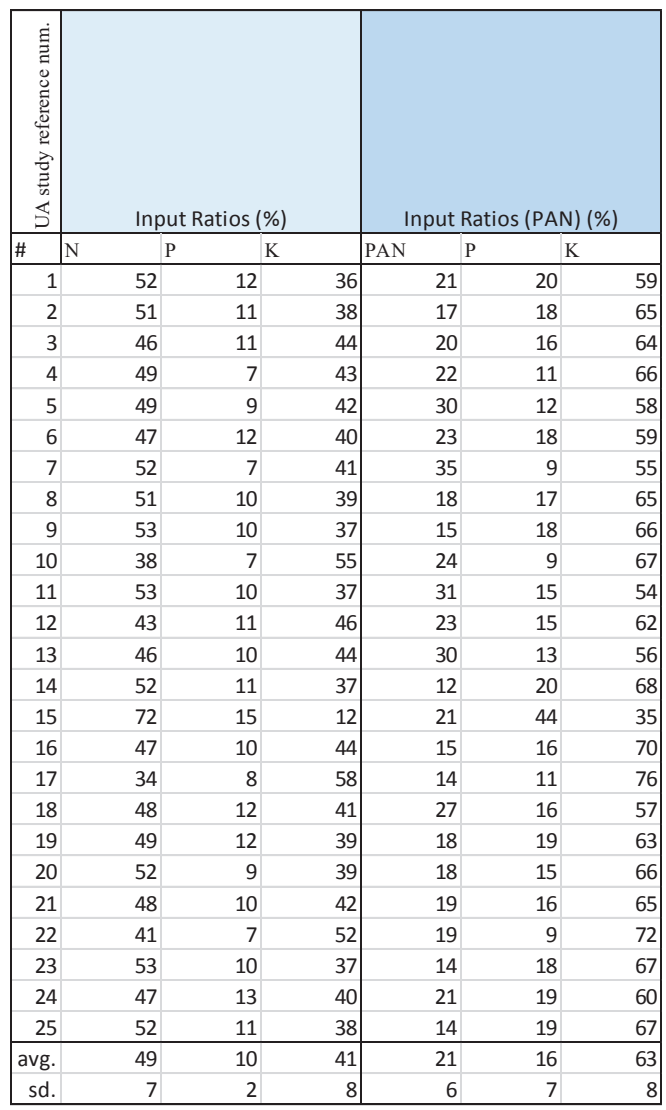

Table S2.7 Nutrient ratios for crop uptake from reference literature: Bosch \& de Jonge (1989) and Fink et al. (1999)

\begin{tabular}{|c|c|c|c|c|c|c|}
\hline & \multicolumn{3}{|c|}{ Nutrient Crop Uptake (kg/ha) } & \multicolumn{3}{|c|}{ Uptake Ratios } \\
\hline & $\mathrm{N}$ & $P$ & K & $\mathrm{N}$ & $\mathrm{P}$ & $\mathrm{K}$ \\
\hline Beet, red & 270 & 46 & 460 & 35 & 6 & 59 \\
\hline Broccoli & 333 & 41.4 & 360 & 45 & 6 & 49 \\
\hline Cabbage, & 234 & 33.3 & 288 & 42 & 6 & 52 \\
\hline Carrot & 170 & 36 & 410 & 28 & 6 & 67 \\
\hline Celery & 202.5 & 41.25 & 352.5 & 34 & 7 & 59 \\
\hline Green be & 119 & 14 & 126 & 46 & 5 & 49 \\
\hline Lettuce, $\mathrm{h}$ & 108 & 18 & 180 & 35 & 6 & 59 \\
\hline Onion & 123.5 & 22.1 & 130 & 45 & 8 & 47 \\
\hline Radish, sn & 70 & 10.5 & 98 & 39 & 6 & 55 \\
\hline Spinach & 144 & 20 & 220 & 38 & 5 & 57 \\
\hline avg. & 203 & 32 & 274 & 40 & 6 & 54 \\
\hline
\end{tabular}




\section{References}

de Haan, J. J. \& van Geel, W. 2013. Adviesbasis voor de bemesting van akkerbouwgewassen - Samenstelling en werking van organische meststoffen [Online]. Available: http://www.kennisakker.nl/kenniscentrum/handleidingen/ adviesbasis-voor-de-bemesting-van-akkerbouwgewassen-samenstelling-en-wer.

Fink, M., Feller, C., Scharpf, H. C., Weier, U., Maync, A., Ziegler, J., Paschold, P. J. \& Strohmeyer, K. 1999. Nitrogen, phosphorus, potassium and magnesium contents of field vegetables-Recent data for fertiliser recommendations and nutrient balances. Journal of Plant Nutrition and Soil Science, 162, 71-73.

Koopmans, C. \& van der Burgt, G.-J. 2001. mineralenbenutting in de biologische landbouw: een integrale benadering. Louis Bolk Instituut.

RVO 2014a. Tabel 2 Fosfaatgebruiksnormen 2014-2017. In: 2014-2017, M. (ed.) 01/01/2014 ed.: Rijksdienst voor Ondernemend Nederland.

RVO 2014b. Tabel 3 Werkingscoëfficiënt 2014-2017. In: 2014-2017, M. (ed.) 01/01/2014 ed.: Rijksdienst voor Ondernemend Nederland.

RVO 2017. Tabel 1 Stikstofgebruiksnormen 2017. In: 2017, M. (ed.) 01/01/2017 ed.: Rijksdienst voor Ondernemend Nederland.

van Dijk, W. \& van Geel, W. 2010. Adviesbasis voor de bemesting van akkerbouw-en vollegrondsgroentegewassen. Lelystad, Praktijkonderzoek Plant \& Omgeving, PPO verslag, 307. 


\section{SUPPORTING INFORMATION III}

\section{Recycling Nutrients Contained in Human Excreta to Agriculture: Pathways, Processes, and Products}

\section{Recovery Pathways}

This Supporting Information (SI) describes the literature search that underpins the analysis and synthesis of recovery pathways presented in the main manuscript. Recovery pathways and treatment processes were identified through a series of search strategies.

A first literature search targeted documents on source-separation and ecological sanitation as well as on the recovery of nutrients from urine, brownwater, blackwater, sewage and sewage sludge (see Table S3.1). From this search a total of 291 documents were selected for further analysis. A database was created containing bibliographic data. Each document was analysed, and categories were iteratively developed for primary inputs, treatment processes, and products rendered by treatment. A second literature search targeted documents more broadly describing recovery of nutrients in human excreta (see Table S3.1). This search returned 493 relevant documents, of which 160 had already been found in the first search. The developed categories were iteratively refined. Additional relevant documents that were found during literature searches designed to inform other parts of our analysis and synthesis (notably the review of treatment processes) were also included and guided the literature search. For instance, when literature on one treatment process (e.g. hydrothermal liquefaction) hinted at other treatment processes not surfaced by the preceding literature searches (e.g. hydrothermal carbonisation), we specifically searched for documents describing recovery pathways including those treatment processes.

We broadly arranged recovery pathways into three groups, each taking as its point of departure one of the three groups of primary inputs described in the main manuscript. Recovery pathways within each group were further arranged into clusters of pathways that feature similar treatment processes and/or render similar products, as illustrated in Figure S3.1. Details on variations within clusters and a list of documents constituting each cluster are provided in Table S3.1. It should be noted that neither Figure S3.1 nor Table S3.1. We stopped our search and analysis once we felt confident that new documents would not add any new processes to the option space presented in the main manuscript. This means that not all documents surfaced by the literature search were analysed in detail and presented in this SI.

This SI contains 2 tables, 1 figure, and 33 pages.

Table S3.1 Search strategies used to inform the development of the option space for nutrient recycling.

Table S3.2 A list of abbreviations used in the preceeding 
Table S3.3 Documents constituting individual clusters as well as overview of variations within individual clusters.

Table S3:4 Description of key mineral precipitates that can be found in fertiliser products rendered by treatment of human excreta and streams containing human excreta.

Table S3.5 Typical sorbents that have been used to extract nutrients from liquid streams during treatment of human excreta and streams containing human excreta.

Figure S3.1 Clusters of recovery pathways. 
Table S3.1 Search strategies used to develop the option space for nutrient recycling.

\begin{tabular}{|c|c|c|}
\hline \multicolumn{3}{|c|}{ SEARCH 1} \\
\hline \multicolumn{2}{|c|}{ Search engine: } & Scopus database \\
\hline \multicolumn{2}{|c|}{ Search date: } & 01 February 2017 \\
\hline \multicolumn{3}{|c|}{ Search terms: } \\
\hline 1.1 & TITLE-ABS-KEY & ("urine separation" OR "urine treatment") \\
\hline 1.2 & TITLE-ABS-KEY & ("black*water" OR “brown*water" OR "yellow*water") AND (sanitation OR “waste*water") \\
\hline 1.3 & TITLE-ABS-KEY & ("ecological sanitation" OR “eco*san" OR "eco sanitation") \\
\hline 1.4 & TITLE & $\begin{array}{l}\text { (*nutrient OR nitrogen OR potassium OR phosphorus) AND (recover* OR recycl*) AND (waste*water OR } \\
\text { sanitation OR sludge) }\end{array}$ \\
\hline 1.5 & TITLE-ABS-KEY & (domestic OR household OR municipal OR human) \\
\hline & AND TITLE & (sanitation OR waste*water) AND (farming OR agriculture* OR horticultur*) \\
\hline \multicolumn{2}{|c|}{ Preliminary result: } & 1422 documents \\
\hline \multicolumn{2}{|c|}{ Manual processing: } & $\begin{array}{l}\text { Scanning based on title and abstract. Documents were considered relevant if they hold information on one } \\
\text { or several recycling pathways. Studies on the recovery from livestock manure or industrial wastewater were } \\
\text { excluded. }\end{array}$ \\
\hline \multicolumn{2}{|c|}{ Final result: } & 291 documents \\
\hline \multicolumn{3}{|c|}{ SEARCH 2} \\
\hline \multicolumn{2}{|c|}{ Search engine: } & Scopus database \\
\hline \multicolumn{2}{|c|}{ Search date: } & 28 November 2017 \\
\hline \multicolumn{3}{|c|}{ Search term: } \\
\hline \multicolumn{2}{|r|}{ TITLE } & 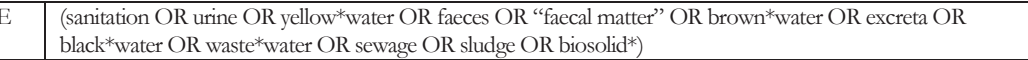 \\
\hline \multicolumn{2}{|c|}{ AND } & (*nutrient* OR phosph* OR nitrogen OR ammoni* OR potas* OR farming OR agricultur* OR horticultur*) \\
\hline \multicolumn{2}{|c|}{ AND } & (recover* OR recycle* OR re*use OR valorisation OR farming OR agricultur* OR horticultur*) \\
\hline \multicolumn{2}{|c|}{ Preliminary results: } & 2171 documents \\
\hline \multicolumn{2}{|c|}{ Automatic exclusion: } & $\begin{array}{l}\text { Studies on the recycling from livestock manure or industrial wastewater were excluded, as well as studies } \\
\text { on contaminants and their fate in the environment. We also aimed to exclude any work to do with any } \\
\text { form of assessment and evaluation of risks, impacts, and perceptions. }\end{array}$ \\
\hline AND & NOT TITLE & \begin{tabular}{l|l} 
(animal OR livestock OR herbivore OR poultry OR swine OR pig* OR bovine OR cow* OR cattle OR dairy \\
OR milk OR meat OR manure)
\end{tabular} \\
\hline AND & TITLE & (hospital OR kidney OR blood OR child* OR women OR men OR metabolite) \\
\hline AND & TITLE & $\begin{array}{l}\text { (oil* OR petroleum OR coal OR mining OR mine OR paper* OR rubber OR pulp* OR *mill OR tannery OR } \\
\text { beamhouse OR brewery OR dredg* OR concrete OR coking OR semiconductor OR electroplating OR } \\
\text { pharmaceutic* OR salin* OR hydroxide OR *ethanol OR furnace OR silo* OR printing OR "disposable } \\
\text { diaper*" OR manufactur* OR sugarbeet OR potato OR "dental metal") }\end{array}$ \\
\hline AND & TITLE & $\begin{array}{l}\text { (risk OR hazard OR prevalence OR disease OR health OR occupation* OR work* OR toxic* OR } \\
\text { schistosomiasis OR virologic* OR bacteriologic* OR hygien* OR epidemiolog* OR infection OR exposure)) }\end{array}$ \\
\hline AND & NOT & $\begin{array}{l}\text { (reservoir OR hydrogeologic OR kinetic* OR speciation OR uptake OR dissipation OR morphology OR fate } \\
\text { OR mobility OR monitoring OR runoff OR bloom*) }\end{array}$ \\
\hline AND & NOT & $\begin{array}{l}\text { (impact* OR planning OR evaluation OR determination OR assess* OR model* OR effect OR policy OR } \\
\text { consequence* OR attitude OR perce* OR preference) }\end{array}$ \\
\hline AND & NOT & (indust*) AND NOT (municipal OR commun* OR domestic OR city OR urban OR campus OR region*) \\
\hline AND & NOT & $\begin{array}{l}\text { (pollutant* OR contamina* OR pah OR las OR "linear alkylbenzene sulfonate" OR phenol* OR pae OR pcb } \\
\text { OR dioxin OR furan OR polybrom* OR * fluor* OR *samine OR pathogen* OR "heavy metal*” OR virus } \\
\text { OR bacteri* OR helminth* OR giardi* OR salmonella OR estrogen OR pcdd OR antibiotic* OR pesticide* } \\
\text { OR aromatic OR hydrocarbon* OR triclo* OR surfactant* OR ibuprofen OR estrone OR estradiol OR } \\
\text { phthalate OR drug* OR glyphosate) AND NOT (remov* OR inactivat* OR destr*) }\end{array}$ \\
\hline AND & NOT TITLE & $\begin{array}{l}\text { (“*ult** waste*” OR “*farming waste*” OR "shrimp waste*” OR "fish waste*” OR "household garbage" OR } \\
\text { "phosph* sludge" OR "phosphoric acid sludge" OR "alum* sludge") }\end{array}$ \\
\hline AND & NOT TITLE & (social OR economic) AND NOT (environmental) \\
\hline Preli & minary results: & 1026 documents \\
\hline Man & ual processing: & $\begin{array}{l}\text { Scanning based on title and abstract. Documents were deemed relevant if they dealt with a description of } \\
\text { recycling pathways other than sewage farming, wastewater irrigation, or land application of sewage sludge. } \\
\text { These traditional pathways are well-established, while our focus was on more novel pathways. }\end{array}$ \\
\hline Final & 1 result: & 493 documents \\
\hline
\end{tabular}


Table S3.2 Documents constituting individual clusters and overview of variations within clusters.

\begin{tabular}{|c|c|}
\hline \multicolumn{2}{|c|}{ ABBREVIATIONS } \\
\hline PI & Primary Input \\
\hline PI-TR & Primary Input - Treatment \\
\hline SI & Secondary Input \\
\hline SUPP & Supplementary Feedstock \\
\hline SEW & Sewage \\
\hline AS & Activated Sludge \\
\hline UASB & Upflow Anaerobic Sludge Blanket \\
\hline FOMBR & Forward Osmosis Membrane Bioreactor \\
\hline SEPT & Septic Tank \\
\hline LSS & Liquid Solid Separation \\
\hline LIQ & Liquid (Effluent or Process Side Stream) \\
\hline EFF & Effluent \\
\hline PSS & Process Side Stream \\
\hline SLU & Sludge (Sludge or Return Activated Sludge) \\
\hline RTS & Return Sludge \\
\hline ASH & Ash \\
\hline STAB & Stabilisation \\
\hline HYGI & Hygienisation \\
\hline B.DEG & Biological Decomposition \\
\hline T.DEC & Thermal Decomposition \\
\hline ACID & Acidification \\
\hline ALKA & Alkalinisation \\
\hline EC & Electrochemical Cell \\
\hline MEC & Microbial Electrochemical Cell \\
\hline MFC & Microbial Fuel Cell \\
\hline FRCO & Freeze Concentration \\
\hline EVAP & Evaporation \\
\hline DIST & Distillation \\
\hline MSMD & Membrane Separation - Membrane Distillation \\
\hline MSRO & Membrane Separation - Reverse Osmosis \\
\hline MSFO & Membrane Separation - Forward Osmosis \\
\hline MSED & Membrane Separation - Electrodialysis \\
\hline ED & Electrodialysis \\
\hline FO & Forward Osmosis \\
\hline PREC & Precipitation \\
\hline SORP & Sorption \\
\hline $\mathrm{AOP}$ & Advanced Oxidation Processes \\
\hline HTL & Hydrothermal Liquefaction \\
\hline INC & Incineration \\
\hline GASI & Gasification \\
\hline PYRO & Pyrolysis \\
\hline SCW & Supercritical Water Process \\
\hline
\end{tabular}


Table S3.3 Documents constituting individual clusters and overview of variations within clusters.

\section{GROUP 1 - URINE OR YELLOWWATER AS PRIMARY INPUT}

\begin{tabular}{|c|c|c|c|c|}
\hline \multicolumn{5}{|c|}{$\begin{array}{l}\text { Prevention of nitrogen losses from urine or yellowwater through prevention of urea hydrolysis in non-hydrolysed (fresh) } \\
\text { urine or prevention of ammonia volatilisation in hydrolysed urine. }\end{array}$} \\
\hline \multicolumn{2}{|c|}{ Urine } & STAB D & \multicolumn{2}{|c|}{ Solution } \\
\hline \multirow{2}{*}{$\begin{array}{l}\text { Target } \\
\text { Nutrient(s) }\end{array}$} & \multicolumn{2}{|l|}{ Stabilisation (STAB) } & \multirow[t]{2}{*}{ Target Product } & \multirow[t]{2}{*}{ Ref } \\
\hline & Process & Variant & & \\
\hline Unspecific & Acidification & Acetic Acid, Sulphuric Acid & Solution & $(1)$ \\
\hline Unspecific & Alkalinisation & $\begin{array}{l}\text { Calcium Hydroxide (Slaked } \\
\text { Lime) }\end{array}$ & Solution, Precipitate & $(2)$ \\
\hline Unspecific & Nitrification & Partial Nitrification & Solution & $(3,4)$ \\
\hline Unspecific & Lactic Acid Fermentation & - & Solution & $(5)$ \\
\hline Unspecific & Electrochemical Potential & ORP of $240 \mathrm{mV}$ or above & Solution & $(6)$ \\
\hline Unspecific & Chemical Oxidation & Ozone, Hydrogen Peroxide & Solution & $(7)$ \\
\hline
\end{tabular}

CLUSTER 1.02 - Hygienisation of Urine or Yellowwater

Pathogen inactivation in urine or yellowwater.

\begin{tabular}{|l|l|l|l|}
\hline \multicolumn{1}{|c|}{ Urine } \\
\hline $\begin{array}{l}\text { Target } \\
\text { Nutrient(s) }\end{array}$ & Hygienisation (HYGI) & Product(s) & Ref \\
\hline Unspecific & Storage & Solution, Precipitate & $(8-11)$ \\
\hline Unspecific & Thermal Storage & Solution & $(12)$ \\
\hline Unspecific & Pasteurisation & Solution & $(9)$ \\
\hline
\end{tabular}

CLUSTER 1.03 - Organic Pollutant Degradation in Urine or Yellowwater

\begin{tabular}{|c|c|c|c|c|}
\hline \multicolumn{5}{|c|}{ Degradation of organic pollutants in urine or yellowwater. } \\
\hline \multicolumn{2}{|c|}{ Urine } & OP.RED & \multicolumn{2}{|l|}{ Solution } \\
\hline \multirow{2}{*}{$\begin{array}{l}\text { Target } \\
\text { Nutrient(s) }\end{array}$} & \multicolumn{2}{|c|}{ Organic Pollutant Reduction (OP.RED) } & \multirow[t]{2}{*}{ Target Product } & \multirow[t]{2}{*}{ Ref } \\
\hline & Process & Variant & & \\
\hline Unspecific & Advanced Oxidation & Ozonation & Solution & $(13)$ \\
\hline Unspecific & Advanced Oxidation & Gamma Radiation & Solution & $(14)$ \\
\hline Unspecific & Advanced Oxidation & Electrochemical Oxidation & Solution & $(14)$ \\
\hline Unspecific & Biological Treatment & $\begin{array}{l}\text { Membrane Bioreactor }+ \\
\text { Effective Microorganisms } \\
(\mathrm{EM})\end{array}$ & Solution & $(15)$ \\
\hline
\end{tabular}


Table S3.3 (continued) Documents constituting individual clusters and overview of variations within clusters.

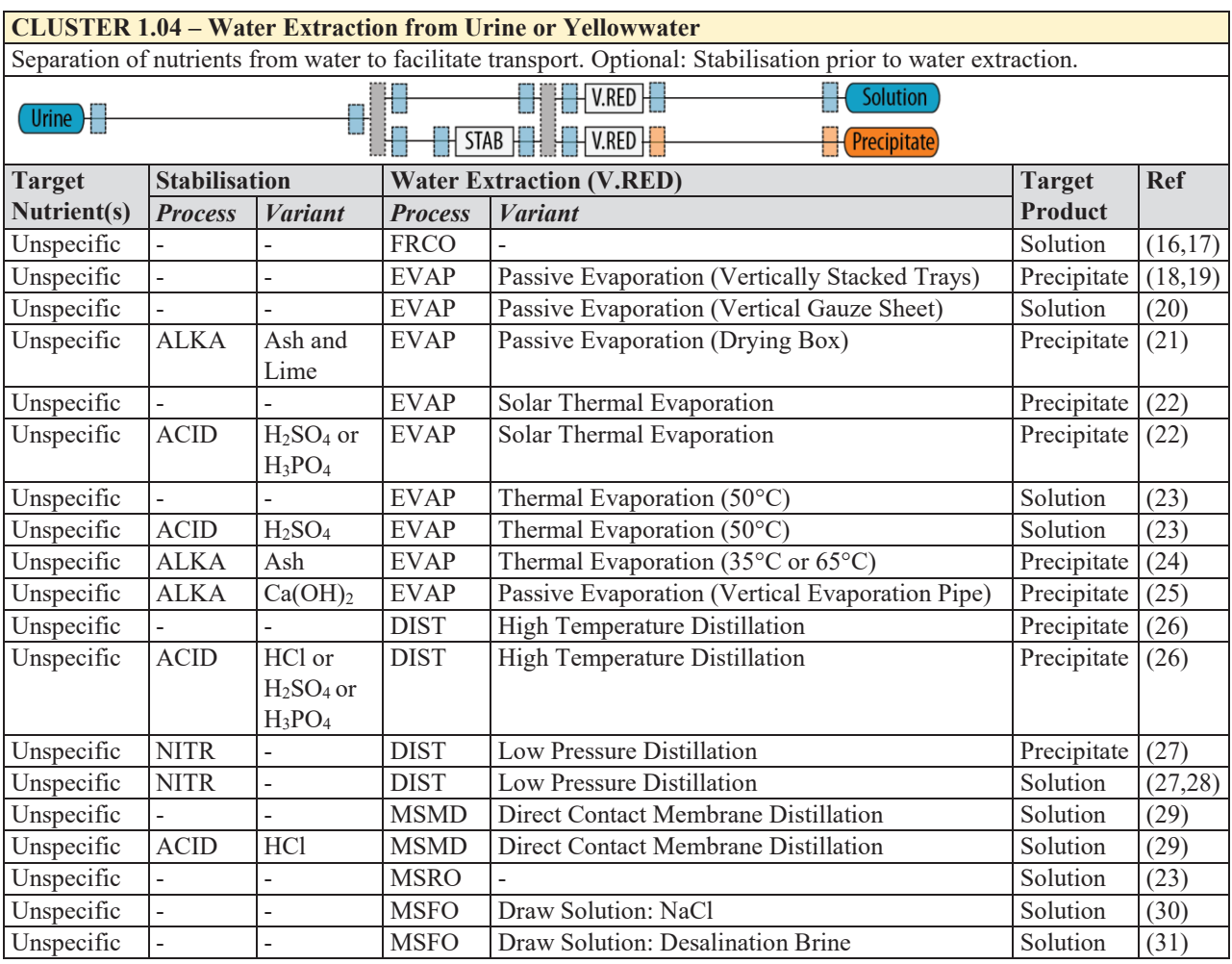

CLUSTER 1.05 - Separation of Nutrients from Organic Pollutants through Membrane Separation Separation of (some of the) nutrients in urine or yellowwater from organic pollutants.

\begin{tabular}{|l|l|l|l|l|}
\hline \multicolumn{3}{|l|}{ Urine } & \multicolumn{3}{|l|}{ Target } & \multirow{2}{*}{ Ref } \\
Target Nutrient(s) & Organic Pollutant Reduction (OP.RED) & Product & \\
\cline { 2 - 6 } & Process & Variant & Solution & $(13,14)$ \\
\hline $\mathrm{N}$ & Nanofiltration & - & Solution & $(32,33)$ \\
\hline Unspecific & Electrodialysis & ED Stack in Abiotic Electrochemical Cell (EC) & Solution & $(34,35)$ \\
\hline Unspecific & Electrodialysis & ED Stack in Microbial Electrochemical Cell (MEC) & &
\end{tabular}

\begin{tabular}{|c|c|c|c|c|}
\hline \multicolumn{5}{|c|}{ CLUSTER 1.06 - Nutrient Extraction through Ammonia Release and Capture } \\
\hline \multicolumn{5}{|c|}{ Nitrogen recovery from urine or yellowwater through the release of ammonia and capture in acid trap. } \\
\hline \multicolumn{5}{|c|}{$\begin{array}{lll}\text { Urine } & \text { GSTR }\end{array}$} \\
\hline \multirow[t]{2}{*}{ Target Nutrient(s) } & \multicolumn{2}{|c|}{ Ammonia Release (STRI) and Capture (SCRU) } & \multirow{2}{*}{$\begin{array}{l}\text { Target } \\
\text { Product }\end{array}$} & \multirow[t]{2}{*}{ Ref } \\
\hline & Release Process & Acid Trap & & \\
\hline NH4-N & Air Stripping & Sulfuric Acid & Solution & $(36,37)$ \\
\hline NH4-N & Air Stripping (Microbial Fuel Cell) & Boric Acid & Solution & $(38)$ \\
\hline NH4-N & Air Stripping (Microbial Fuel Cell) & Sulfuric Acid & Solution & (39) \\
\hline NH4-N & Air Stripping (Microbial Electrolysis Cell) & Boric Acid & Solution & $(40)$ \\
\hline NH4-N & Air Stripping (Microbial Electrolysis Cell) & Sulfuric Acid & Solution & (41) \\
\hline NH4-N & Air Stripping (Electrochemical Cell) & Sulfuric Acid & Solution & $(42)$ \\
\hline NH4-N & Distillation & Sulfuric Acid & Solution & $(23)$ \\
\hline
\end{tabular}


Table S3.3 (continued) Documents constituting individual clusters and overview of variations within clusters.

\begin{tabular}{l}
\hline CLUSTER 1.07 - Nutrient Extraction through Sorption \\
$\begin{array}{l}\text { Nutrient recovery from urine through sorption to sorbent, or sorption to sorbent or resin followed by } \\
\text { desorption/regeneration. Optional: Precipitation in the desorption solution or regenerant. }\end{array}$ \\
\hline Urine \\
\hline
\end{tabular}


Table S3.3 (continued) Documents constituting individual clusters and overview of variations within clusters.

CLUSTER 1.08 - Nutrient Extraction through Precipitation

Nutrient recovery from urine through precipitation induced by the addition of a precipitant and/or pH control, or by electrocoagulation.

\begin{tabular}{|c|c|c|c|c|c|}
\hline Urine & & PREC D & $D$ Precip & tate & \\
\hline $\begin{array}{l}\text { Target } \\
\text { Mineral }\end{array}$ & Variant & Precipitant & $\begin{array}{l}\mathrm{pH} \\
\text { Control }\end{array}$ & Product & Ref \\
\hline MAP & PREC & $\mathrm{MgO}$ & $\mathrm{NaOH}$ & Precipitate & $(36,48)$ \\
\hline MAP & PREC & $\mathrm{MgO}$ & - & Precipitate & $(17,23,44,55,56)$ \\
\hline MAP & PREC (MFC) & $\mathrm{MgCl}_{2}$ & - & Precipitate & $(57)$ \\
\hline MAP & PREC & $\mathrm{MgCl}_{2}$ & - & Precipitate & $(41,55,58-60)$ \\
\hline MAP & PREC & $\mathrm{Mg}(\mathrm{OH})_{2}$ & - & Precipitate & $(55)$ \\
\hline MAP & PREC & $\mathrm{MgCl}_{2}$ & $\mathrm{NaOH}$ & Precipitate & $(48,56,61)$ \\
\hline MAP & PREC & $\mathrm{MgCl}_{2}+\mathrm{Na}_{2} \mathrm{HPO}_{4}$ & $\mathrm{NaOH}$ & Precipitate & $(62-64)$ \\
\hline MAP & PREC & $\mathrm{MgCl}_{2}+\mathrm{Na}_{2} \mathrm{HPO}_{4}$ & - & Precipitate & $(65,66)$ \\
\hline MAP & PREC & $\mathrm{MgSO}_{4}$ & - & Precipitate & $(67)$ \\
\hline MAP & PREC & $\mathrm{MgCO}_{3}$ & - & Precipitate & $(49,67)$ \\
\hline MAP & PREC & Seawater & $\mathrm{NaHCO}_{3}$ & Precipitate & $(68)$ \\
\hline MAP & PREC & Seawater & - & Precipitate & $(69,70)$ \\
\hline MAP & PREC (MFC) & Seawater & - & Precipitate & $(57)$ \\
\hline MAP & PREC & Bittern (Waste Brine) & - & Precipitate & $(60,67)$ \\
\hline MAP & PREC & Reverse Osmosis Brine & - & Precipitate & $(71)$ \\
\hline MAP & PREC & Mg Electrode & - & Precipitate & $(72)$ \\
\hline MAP & PREC & Ash & - & Precipitate & $(73)$ \\
\hline MAP & PREC & - & $\mathrm{NaOH}, \mathrm{H}_{2} \mathrm{SO}_{4}$ & Precipitate & $(74)$ \\
\hline MAP & PREC (MEC) & Bio-Precipitation & - & Precipitate & $(75)$ \\
\hline MPP & PREC & $\mathrm{MgO}$ & - & Precipitate & $(56)$ \\
\hline MPP & PREC & $\mathrm{MgCl}_{2}$ & $\mathrm{NaOH}$ & Precipitate & $(56)$ \\
\hline MPP & PREC & $\mathrm{MgCl}_{2}+\mathrm{Na}_{2} \mathrm{HPO}_{4}$ & $\mathrm{NaOH}$ & Precipitate & $(76-78)$ \\
\hline $\mathrm{CaP}$ & PREC & $\mathrm{Ca}(\mathrm{OH})_{2}$ & - & Precipitate & $(23)$ \\
\hline $\mathrm{FeP}$ & PREC & $\mathrm{Fe}^{3+}\left(\mathrm{FeSO}_{4}\right.$ oxidised by $A$. ferrooxidans $)$ & & Precipitate & (79) \\
\hline
\end{tabular}

\section{CLUSTER 1.09 - Nutrient Extraction through Phototrophic Biomass Growth}

Nutrient recovery from urine through growth of microalgae, cyanobacteria, or zoooplankton in tanks or photobioreactors. Optional: Stabilisation prior to phototrophic biomass growth.

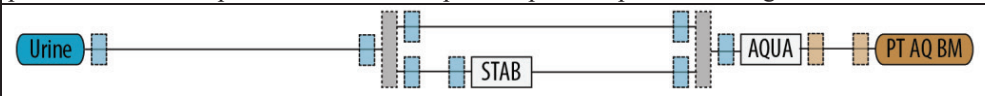

\begin{tabular}{|l|l|l|l|l|l|}
\hline $\begin{array}{l}\text { Target } \\
\text { Nutrient(s) }\end{array}$ & Stabilisation & Reactor Type & Organism & \multicolumn{2}{l|}{ Ref } \\
\hline Unspecific & - & Photobioreactor & Microalgae & Chlorella sorokiniana & $(80-82)$ \\
\hline Unspecific & - & Photobioreactor & Microalgae & Desmodesmus abundans & $(83)$ \\
\hline Unspecific & - & Tank & Microalgae & Scenedesmus acuminatus & $(84)$ \\
\hline Unspecific & - & Photobioreactor & Microalgae & Spirulina platensis & $(85)$ \\
\hline Unspecific & Nitrification & Photobioreactor & Microalgae & Spirulina platensis & Feng et al. (2008) \\
\hline Unspecific & Nitrification & Photobioreactor & Cyanobacteria & Arthrospira platensis & $(86)$ \\
\hline Unspecific & - & Tank & Zooplankton & Daphnia magna & $(84)$ \\
\hline
\end{tabular}


Table S3.3 (continued) Documents constituting individual clusters and overview of variations within clusters.

GROUP 2 - FAECES, EXCRETA, BROWNWATER, OR BLACKWATER AS PRIMARY INPUT

\begin{tabular}{|c|c|c|c|c|c|c|}
\hline \multirow{2}{*}{\multicolumn{7}{|c|}{\begin{tabular}{|l|l|l} 
CLUSTER 2.01 - Granulation in Wet Organic Fraction \\
Phosphate precinitation in sludge-line of faecal-derived matter
\end{tabular}}} \\
\hline & & & faecal-derived & & & \\
\hline \multicolumn{7}{|c|}{ Excreta] DPI-TR } \\
\hline \multicolumn{3}{|c|}{ SLU] } & \multicolumn{2}{|l|}{ DPREC D } & & \\
\hline $\begin{array}{l}\text { Target } \\
\text { Mineral(s) }\end{array}$ & PI & PI-TR & Variant & Precipitant & $\mathrm{pH}$ Control & Ref \\
\hline $\mathrm{CaP}$ & Blackwater & UASB & Granulation & - & - & (87) \\
\hline
\end{tabular}

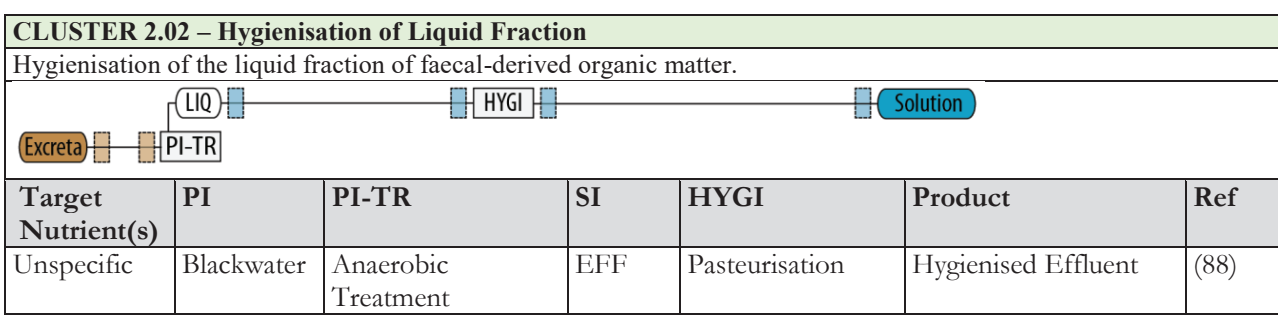

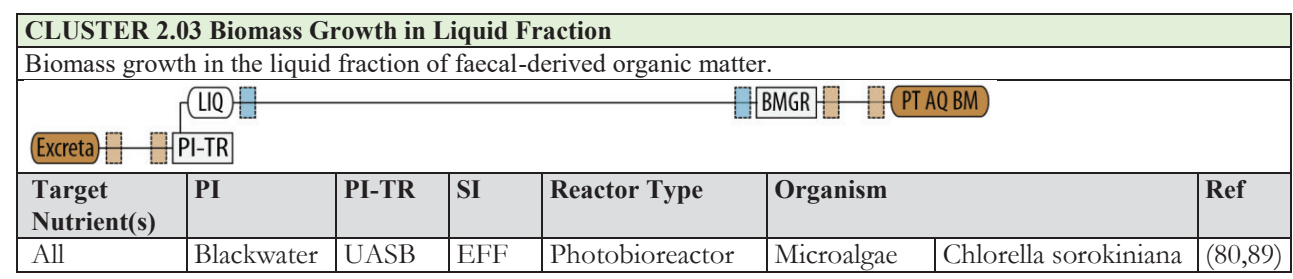

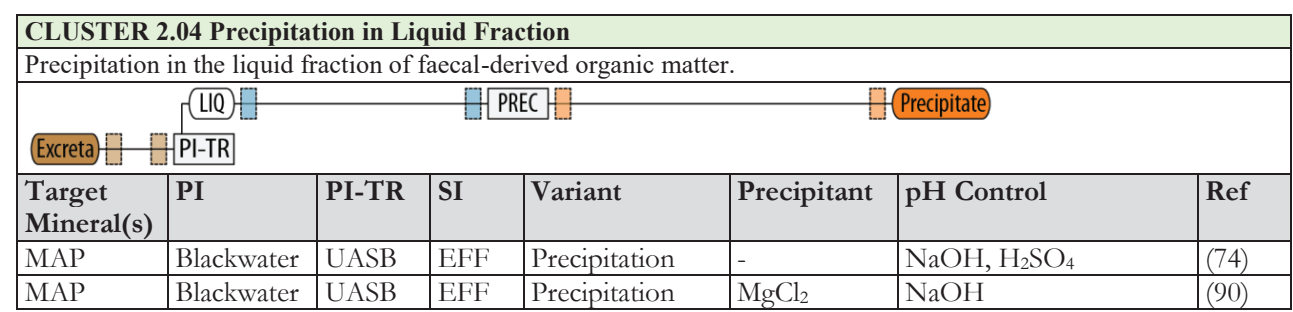

\begin{tabular}{|c|c|c|c|c|c|}
\hline \multicolumn{6}{|c|}{ CLUSTER 2.05 Hygienisation of Feedstock } \\
\hline \multicolumn{6}{|c|}{ Hygienisation of (the organic fraction of) faecal-derived organic matter. } \\
\hline \multicolumn{6}{|c|}{ Faeces $]$} \\
\hline \multicolumn{3}{|c|}{ Excreta) DPI-TR } & \multirow{2}{*}{ HYGI } & \multicolumn{2}{|l|}{ FDOM } \\
\hline & SLU) & $t$ & & & \\
\hline PI & PI-TR & SI & HYGI & Product & Ref \\
\hline Faeces & - & - & Desiccation & Dehydrated Faeces & $(91)$ \\
\hline Faecal Sludge & - & - & Lactic Acid Fermentation & Hygienised Faecal Sludge & $(92)$ \\
\hline Faecal Sludge & - & - & Lime Treatment & Hygienised Faecal Sludge & $(92)$ \\
\hline
\end{tabular}


Table S3.3 (continued) Documents constituting individual clusters and overview of variations within clusters.

\begin{tabular}{|l|l|l|l|l|l|l|l|l|}
\hline \multicolumn{2}{|l|}{ CLUSTER 2.06 Biological decomposition of Feedstock or Solid Fraction } \\
\hline Biological decomposition of (the organic fraction of) faecal-derived organic matter. \\
\hline Faeces
\end{tabular}

\begin{tabular}{|l|l|l|l|}
\hline \multicolumn{6}{|l|}{ CLUSTER 2.07 Thermal Decomposition of Feedstock } \\
\hline Thermal decomposition of (the organic fraction of) faecal-derived organic matter. \\
\hline Faeces
\end{tabular}


Table S3.3 (continued) Documents constituting individual clusters and overview of variations within clusters.

GROUP 3 - SEWAGE AS PRIMARY INPUT

\begin{tabular}{|c|c|c|c|c|c|}
\hline \multirow{2}{*}{\multicolumn{6}{|c|}{$\begin{array}{l}\text { CLUSTER 3.01 Hygienisation of Liquid Fraction } \\
\text { Hygienisation of sewage-derived liquid streams. }\end{array}$}} \\
\hline & & & & & \\
\hline \multicolumn{6}{|c|}{ 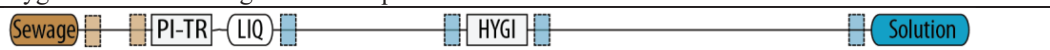 } \\
\hline $\begin{array}{l}\text { Target } \\
\text { Nutrient(s } \\
\text { ) }\end{array}$ & PI-TR & SI & HYGI & Product & Ref \\
\hline Unspecific & AS & EFF & AOP & Hygienised Effluent (Solution) & \\
\hline
\end{tabular}

\begin{tabular}{|l|l|l|l|l|l|l|l|l|}
\hline \multicolumn{6}{|l|}{ CLUSTER 3.02 Water Extraction from Liquid Fraction } \\
\hline \multicolumn{7}{|l|}{ Water extraction from sewage-derived liquid streams. Optional: stabilisation prior to water extraction. } \\
\hline $\begin{array}{l}\text { Tewage) } \\
\text { Nutrient(s) }\end{array}$ & PI-TR & SI & \multicolumn{2}{|l|}{ Stabilisation (STAB) } & Volume Reduction (V.RED) & $\begin{array}{l}\text { Target } \\
\text { Product }\end{array}$ & Ref \\
\hline Unspecific & AS & PSS & - & - & EVAP & $\begin{array}{l}\text { Thermal Evaporation } \\
\left(50^{\circ} \mathrm{C}\right)\end{array}$ & Solution & $(23)$ \\
\hline Unspecific & AS & PSS & $\begin{array}{l}\text { ACI } \\
\text { D }\end{array}$ & $\mathrm{H}_{2} \mathrm{SO}_{4}$ & EVAP & $\begin{array}{l}\text { Thermal Evaporation } \\
\left(50^{\circ} \mathrm{C}\right)\end{array}$ & Solution & $(23)$ \\
\hline Unspecific & AS & PSS & - & - & MSRO & - & Solution & $(23)$ \\
\hline Unspecific & AS & PSS & $\begin{array}{l}\text { ACI } \\
\text { D }\end{array}$ & $\mathrm{H}_{2} \mathrm{SO}_{4}$ & MSRO & - & Solution & $(23)$ \\
\hline Unspecific & AS & EFF & - & - & MSFO & - & Solution & $(114)$ \\
\hline
\end{tabular}

\begin{tabular}{|c|c|c|c|c|c|c|c|}
\hline \multicolumn{8}{|c|}{ Nutrient extraction from sewage-derived liquid stream: membrane separation. } \\
\hline \multicolumn{8}{|c|}{ (Sewage) [PI-TR- (LIQ)] DN.EXT] } \\
\hline $\begin{array}{l}\text { Target } \\
\text { Nutrient(s) }\end{array}$ & PI-TR & SI & Stabilisation & Volume & ion & Target Product & Ref \\
\hline Unspecific & AS & PSS & - & MSED & ED Stack in MEC & Solution & $(115$ \\
\hline
\end{tabular}

\begin{tabular}{|c|c|c|c|c|c|c|}
\hline \multicolumn{7}{|c|}{ CLUSTER 3.04 Nutrient Extraction from Liquid Fraction (Ammonia Release and Capture) } \\
\hline \multicolumn{7}{|c|}{ Nutrient extraction from sewage-derived liquid streams: ammonia release and capture. } \\
\hline \multicolumn{5}{|c|}{ (Sewage) BPI-TR-(PSS) $\quad$ GSTRI BSCRU } & \multicolumn{2}{|l|}{ Solution } \\
\hline $\begin{array}{l}\text { Target } \\
\text { Nutrient }\end{array}$ & $\begin{array}{l}\text { PI- } \\
\text { TR }\end{array}$ & SI & Ammonia Release & Scrubbing & Target Product & Ref \\
\hline NH4-N & AS & PSS & Air Stripping & $\mathrm{H} 2 \mathrm{SO} 4$ & Ammonium Sulfate & $(116-118)$ \\
\hline NH4-N & AS & PSS & Microbial Electrolysis Cell & $\mathrm{HCl}$ & Ammonium Chloride & $(119)$ \\
\hline NH4-N & AS & PSS & Electrochemical Cell & $\mathrm{H} 2 \mathrm{SO} 4$ & Ammonium Sulfate & $(120)$ \\
\hline
\end{tabular}


Table S3.3 (continued) Documents constituting individual clusters and overview of variations within clusters.

\begin{tabular}{|c|c|c|c|c|c|c|c|c|}
\hline \multicolumn{9}{|c|}{ CLUSTER 3.05 Nutrient Extraction from Liquid Fraction (Sorption) } \\
\hline \multicolumn{9}{|c|}{$\begin{array}{l}\text { Nutrient recovery from sewage-derived liquid streams through sorption. Sorption to sorbent, or sorption to } \\
\text { sorbent/resin followed by desorption/regeneration. Optional: precipitation from the regenerant. }\end{array}$} \\
\hline \multicolumn{4}{|l|}{ Sewage } & \multicolumn{3}{|c|}{ 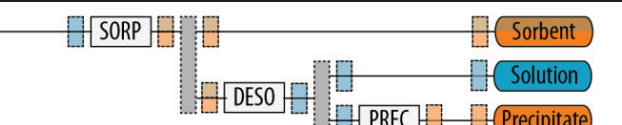 } & & \\
\hline $\begin{array}{l}\text { Target } \\
\text { Nutrient(s) }\end{array}$ & PI & TR & SI & Sorbent / Resin & \begin{tabular}{l|l} 
Deso./ \\
Regen.
\end{tabular} & Recov. & Product & Ref \\
\hline NH4-N & SEW & UASB & EFF & Zeolite & - & - & Sorbent & $(121)$ \\
\hline NH4-N & SEW & AS & EFF & Zeolite & $\mathrm{NaOH}$ & STRI & Solution & $(122)$ \\
\hline NH4-N & SEW & AS & EFF & Zeolite (Synthetic Zeolite) & $\begin{array}{l}\mathrm{NaOH} \\
\mathrm{KOH}\end{array}$ & - & Solution & $(123)$ \\
\hline $\begin{array}{l}\text { NH4- } \\
\text { N,P,K }\end{array}$ & SEW & AS & PSS & Zeolite (Metal Modified) & - & - & Sorbent & $(124)$ \\
\hline NH4-N,P,K & SEW & AS & EFF & Anion Exchange Resin + Zeolite & $\mathrm{NaCl}$ & PREC & Precipitate & $(125-129)$ \\
\hline NH4-N,P & SEW & AS & $\mathrm{EFF}$ & $\begin{array}{l}\text { Zeolite (Clinoptilolite) (Metal Oxide } \\
\text { Mod.) }\end{array}$ & - & - & Sorbent & $(130)$ \\
\hline NH4-N,P & SEW & AS & EFF & Palygorskite (Metal Oxide Modified) & - & - & Sorbent & $(131)$ \\
\hline NH4-N,P & - & - & - & Amphoteric Straw Cellulose [Wheat] & - & - & Sorbent & $(132)$ \\
\hline NH4-N,P & SEW & AS & EFF & Hydrotalcite + Zeolite & $\mathrm{NaCl}$ & PREC & Precipitate & $(133)$ \\
\hline $\mathrm{P}$ & SEW & AS & EFF & Biochar [Raw Corn] & - & - & Sorbent & $(134)$ \\
\hline $\mathrm{P}$ & SEW & AS & EFF & Biochar [Sewage Sludge] & - & - & Sorbent & $(135)$ \\
\hline $\mathrm{P}$ & SEW & AS & EFF & Biochar [Sewage Sludge + Ochre] & - & - & Sorbent & $(135)$ \\
\hline $\mathrm{P}$ & SEW & AS & $\mathrm{EFF}$ & Biochar (Iron Modified) & - & - & Sorbent & $(136,137)$ \\
\hline $\mathrm{P}$ & - & - & - & Calcinated Waste Egg Shells & - & - & Sorbent & $(138)$ \\
\hline $\mathrm{P}$ & SEW & AS & PSS & Anion Exchange Resin (Metal Modif.) & $\mathrm{NaOH}$ & - & Solution & $(54)$ \\
\hline $\mathrm{P}$ & SEW & AS & EFF & Anion Exchange Resin (Metal Modif.) & $\mathrm{NaOH}$ & - & Solution & $(54)$ \\
\hline $\mathrm{P}$ & SEW & AS & EFF & Anion Exchange Resin (Metal Modif.) & \begin{tabular}{l|l|l}
$\mathrm{NaOH}$ \\
$+\mathrm{NaCl}$ \\
\end{tabular} & PREC & Precipitate & $(139)$ \\
\hline $\mathrm{P}$ & SEW & AS & EFF & Anion Exchange Resin & $\mathrm{NaOH}$ & PREC & Precipitate & $(140,141)$ \\
\hline $\mathrm{P}$ & SEW & AS & PSS & Anion Exchange Resin (Iron Oxide) & $\mathrm{NaOH}$ & PREC & Precipitate & $(142)$ \\
\hline $\mathrm{P}$ & SEW & AS & EFF & Iron Oxy-Hydroxide & $\mathrm{NaOH}$ & PREC & Precipitate & $(140)$ \\
\hline $\mathrm{P}$ & SEW & AS & EFF & Iron-Manganese Oxy-Hydroxide & $\mathrm{NaOH}$ & PREC & Precipitate & $(140)$ \\
\hline $\mathrm{P}$ & SEW & AS & EFF & Zirconium Ferrite & $\mathrm{NaOH}$ & - & Solution & $(143-145)$ \\
\hline $\mathrm{P}$ & SEW & AS & EFF & Zirconium Sulphate & - & - & Sorbent & $(146)$ \\
\hline $\mathrm{P}$ & SEW & AS & EFF & Magnetite & $\mathrm{NaOH}$ & PREC & Precipitate & $(147)$ \\
\hline $\mathrm{P}$ & SEW & AS & EFF & $\begin{array}{l}\text { Waste Carpet (Wool Rich) (Metal } \\
\text { Oxide Modified) }\end{array}$ & $\mathrm{NaCl}$ & - & Solution & $(148)$ \\
\hline $\mathrm{P}$ & SEW & AS & EFF & $\begin{array}{l}\text { Saponified Orange Waste (Zirconium } \\
\text { Loaded) }\end{array}$ & $\mathrm{NaOH}$ & - & Solution & $(144,149)$ \\
\hline $\mathrm{P}$ & SEW & AS & PSS & $\begin{array}{l}\text { Saponified Orange Waste (Zirconium } \\
\text { Loaded) }\end{array}$ & $\mathrm{NaOH}$ & - & Solution & $(149)$ \\
\hline $\mathrm{P}$ & SEW & AS & EFF & Clay Minerals & $\mathrm{NaOH}$ & PREC & Precipitate & $(150-153)$ \\
\hline $\mathrm{P}$ & SEW & AS & EFF & Activated Alumina & $\mathrm{NaOH}$ & PREC & Precipitate & $(150-153)$ \\
\hline $\mathrm{P}$ & SEW & AS & EFF & Nanocomposites (Zirconium Based) & $\mathrm{NaOH}$ & PREC & Precipitate & $(154,155)$ \\
\hline $\mathrm{P}$ & SEW & AS & EFF & Nanocomposites (Lanthanum Based) & $\mathrm{NaOH}$ & - & Solution & $(156)$ \\
\hline $\mathrm{P}$ & - & - & - & Okara (Zirconium Loaded) & $\begin{array}{l}\mathrm{NaOH} \\
+\mathrm{HCl}\end{array}$ & - & Solution & $(157)$ \\
\hline $\mathrm{P}$ & - & - & - & $\begin{array}{l}\text { Metal-Organic Frameworks } \\
\text { (Lanthanide Based) }\end{array}$ & $\mathrm{NaOH}$ & - & Solution & $(158)$ \\
\hline $\mathrm{P}$ & - & - & - & Coated Carboxylated Surface & $\mathrm{NaOH}$ & PREC & Precipitate & (159) \\
\hline
\end{tabular}


Table S3.3 (continued) Documents constituting individual clusters and overview of variations within clusters.

\begin{tabular}{|c|c|c|c|c|c|c|}
\hline \multicolumn{7}{|c|}{ CLUSTER 3.06 Nutrient Extraction from Liquid Fraction (Precipitation) } \\
\hline \multicolumn{7}{|c|}{ Precipitation in sewage-derived liquid stream. } \\
\hline \multicolumn{7}{|c|}{$\begin{array}{ll}\text { (Sewage) } & \text { DREC } \\
\text { (SI-TR } & \end{array}$} \\
\hline $\begin{array}{l}\text { Target } \\
\text { Mineral(s) }\end{array}$ & PI & PI-TR & SI & Precipitant & pH Control & Ref \\
\hline MAP & SEW & AS & PSS & $\mathrm{MgO}$ & - & $(23,118)$ \\
\hline MAP & SEW & AS & PSS & $\mathrm{Mg}(\mathrm{OH})_{2}$ & $\mathrm{NaOH}$ & $(160,161)$ \\
\hline MAP & SEW & AS & PSS & $\mathrm{MgCl}_{2}$ & $\mathrm{NaOH}$ & $(161-168)$ \\
\hline MAP & SEW & AS & PSS & $\mathrm{MgCl}_{2}$ & - & $(116,169)$ \\
\hline MAP & SEW & $\mathrm{AS}+\mathrm{FO}$ & PSS & $\mathrm{MgCl}_{2}$ & - & $(170)$ \\
\hline MAP & SEW & AS & PSS & Magnesium Source (Unspecified) & - & $(171)$ \\
\hline MAP & SEW & AS & PSS & Bacteria Induced & & $(172)$ \\
\hline $\mathrm{MAP}, \mathrm{CaP}$ & SEW & FOMBR & EFF & - & $\mathrm{CO}_{2}, \mathrm{NaHCO}_{3}$ & $(173-176)$ \\
\hline $\mathrm{CaP}$ & SEW & $\mathrm{AS}$ & PSS & $\mathrm{CaCl}_{2}$ & - & $(116,177)$ \\
\hline $\mathrm{CaP}$ & SEW & $\mathrm{AS}$ & PSS & $\mathrm{CaCl}_{2}$ & Air Stripping & $(178)$ \\
\hline $\mathrm{CaP}$ & SEW & $\mathrm{AS}$ & PSS & $\mathrm{Ca}(\mathrm{OH})_{2}$ & - & $(23)$ \\
\hline $\mathrm{CaP}$ & SEW & AS & PSS & $\mathrm{Ca}(\mathrm{OH})_{2}($ Waste Lime $)$ & - & $(169)$ \\
\hline $\mathrm{CaP}$ & SEW & AS & EFF & $\mathrm{MgO}($ Seed $)$ & - & (179) \\
\hline $\mathrm{CaP}$ & SEW & AS & EFF & CSH (Seed) & - & $(180)$ \\
\hline $\mathrm{CaP}$ & SEW & AS & PSS & CSH (Seed) & - & $(180-185)$ \\
\hline $\mathrm{CaP}$ & SEW & AS & PSS & Sand (Seed) & - & $(186)$ \\
\hline $\mathrm{CaP}$ & SEW & AS & EFF & Converter Slag (Seed) & $\mathrm{NaOH}$ & $(187)$ \\
\hline $\mathrm{CaP}$ & SEW & AS & EFF & Concrete Sludge Derived Sorbent (Seed) & - & $(188)$ \\
\hline AlP & SEW & AS & PSS & $\mathrm{AlCl}_{3}$ & - & (116) \\
\hline FeP & SEW & AS & PSS & $\mathrm{FeCl}_{3}$ & - & (189) \\
\hline MPP & SEW & AS & EFF & $\mathrm{MgSO}_{4}$ & $\mathrm{NaOH}$ & (190) \\
\hline $\mathrm{MgP}+\mathrm{NaP}$ & SEW & FOMBR & EFF & $\mathrm{MgCl}_{2}$ & $\mathrm{NaOH}$ & (191) \\
\hline
\end{tabular}

\begin{tabular}{|c|c|c|c|c|c|c|c|c|c|}
\hline \multicolumn{10}{|c|}{$\begin{array}{l}\text { CLUSTER 3.07 Nutrient Extraction from Liquid Fraction (Phototrophic Biomass Growth) } \\
\text { Biomass growth in sewage-derived liquid streams. Growth of microalgae, cyanobacteria and aquatic plants. } \\
\text { Optional: mobilisation of nutrients from sludge. Optional: sorption and desorption prior to biomass growth. }\end{array}$} \\
\hline \multicolumn{7}{|c|}{ 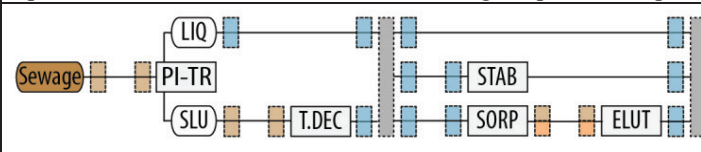 } & \multicolumn{2}{|c|}{ DAQUA PPTAQBM } & \\
\hline PI & PI-TR & SI & TDEC & $\begin{array}{l}\text { STAB / } \\
\text { SORP }\end{array}$ & ELUT & Reactor & \multicolumn{2}{|l|}{ Organism } & Ref \\
\hline SEW & UASB & EFF & - & - & - & Pond & \begin{tabular}{|l|} 
Aquatic \\
Plants
\end{tabular} & Duckweed & $(192,193)$ \\
\hline SEW & AS & EFF & - & - & & Pond & Algae & & $(194,195)$ \\
\hline SEW & AS & EFF & - & - & & PBR & Microalgae & Scenedesmus obliquus & (196) \\
\hline SEW & AS & PSS & - & - & - & PBR & Microalgae & Chlorella sp. & (197) \\
\hline SEW & AS & SLU & HTL & - & - & PBR & Microalgae & Aurantiochytrium & (198) \\
\hline SEW & AS & SLU & HTL & - & - & PBR & Microalgae & Euglena gracilis & (198) \\
\hline SEW & - & - & - & - & - & Tank & Microalgae & Chlorella sp. & (199) \\
\hline SEW & AS & EFF & - & - & - & PBR & Microalgae & Chlorella sp. & $(200-202)$ \\
\hline- & - & - & - & $\begin{array}{l}\text { Silica } \\
\text { (Amine } \\
\text { Grafted) }\end{array}$ & $\mathrm{H}_{2} \mathrm{O}(\mathrm{DI})$ & PBR & Cyanobacteria & & $(203)$ \\
\hline- & - & - & - & Zeolite & $\begin{array}{l}\mathrm{H}_{2} \mathrm{O}(\mathrm{DI}) \\
\text { Cultivation } \\
\text { Media }\end{array}$ & PBR & Microalgae & Chlorella vulgaris & (204) \\
\hline
\end{tabular}


Table S3.3 (continued) Documents constituting individual clusters and overview of variations within clusters.

\begin{tabular}{|l|l|l|l|l|l|}
\hline \multicolumn{2}{|l|}{ CLUSTER 3.08 Hygienisation } \\
\hline \multicolumn{2}{|l|}{ Hygienisation of sewage-derived streams. } \\
\hline Sewage & PI-TR-SLU & & \\
\hline $\begin{array}{l}\text { Target } \\
\text { Nutrient(s) }\end{array}$ & PI-TR & SI & HYGI & Product & Ref \\
\hline Unspecific & Septic Tank & SLU & Ammonia Sanitisation & Hygienised Sludge & $(10)$ \\
\hline Unspecific & AS & SLU & Ammonia Sanitisation & Hygienised Sludge & $(205)$ \\
\hline
\end{tabular}

\begin{tabular}{|c|c|c|c|c|c|c|c|c|c|}
\hline \multicolumn{10}{|c|}{ CLUSTER 3.09 Biological Decomposition } \\
\hline \multicolumn{10}{|c|}{ Biological decomposition of sewage-derived streams. } \\
\hline (Sewage) : & PI-TR & SLU & 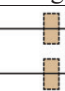 & $\overline{\square S U}$ & ${ }_{\mathrm{BP}}$ & $\begin{array}{l}D \\
B \text { B.DEG }\end{array}$ & FD OM & & \\
\hline $\begin{array}{l}\text { Target } \\
\text { Nutrient(s) }\end{array}$ & PI & PI-TR & SI & SUPP & $\begin{array}{l}\text { B.DEG } \\
\text { (Anaerobic) }\end{array}$ & B.DEG (Aer & obic) & Product & Ref \\
\hline Unspecific & SEW & AS & SLU & & & Composting & & Compost & $(206)$ \\
\hline Unspecific & SEW & LSS & SLU & & & & Vermicomp. & Vermicomp. & $(207,208)$ \\
\hline Unspecific & SEW & AS & SLU & & & & Vermicomp. & Vermicomp. & $(209,210)$ \\
\hline Unspecific & SEW & SEPT & SLU & Charcoal & $\begin{array}{l}\text { Lactic Acid } \\
\text { Fermentation }\end{array}$ & Composting & & Terra Preta & $(105)$ \\
\hline Unspecific & SEW & AS & SLU & Charcoal & $\begin{array}{l}\text { Lactic Acid } \\
\text { Fermentation }\end{array}$ & Composting & & Terra Preta & $(105)$ \\
\hline
\end{tabular}

\begin{tabular}{|l|l|l|l|l|l|}
\hline CLUSTER 3.10 Thermal Decomposition & \\
\hline \multicolumn{5}{|l|}{ Thermal decomposition of sewage-derived streams. } \\
\hline
\end{tabular}

\begin{tabular}{|l|}
\hline CLUSTER 3.11 Nutrient Extraction in Sludge-Line (Precipitation) \\
\hline Phosphate precipitation in sludge-line of sewage-derived matter. \\
\hline Sewage) Precipitate \\
\hline
\end{tabular}

\begin{tabular}{|l|l|l|l|l|l|l|}
\hline $\begin{array}{l}\text { Target } \\
\text { Mineral(s) }\end{array}$ & PI & PI-TR & SI & $\begin{array}{l}\text { Induction of } \\
\text { Precipitation }\end{array}$ & pH Control & Ref \\
\hline MAP & SEW & AS & SLU & $\mathrm{MgCl}_{2}$ & $\mathrm{CO}_{2}$ & $(213,214)$ \\
\hline MAP & SEW & AS & SLU & $\mathrm{MgCl}_{2}+\mathrm{KH}_{2} \mathrm{PO}_{4}$ & $\mathrm{NaOH}$ or $\mathrm{HCl}$ \\
\hline MAP & SEW & AS & SLU & $\mathrm{Mg}(\mathrm{OH})_{2}$ & $\mathrm{CO}_{2}$ & $(215)$ \\
\hline MAP & SEW & AS & SLU & $\mathrm{Mg}(\mathrm{OH})_{2}$ & $\mathrm{H}_{2} \mathrm{SO}_{4}$ & $(213)$ \\
\hline CaP & SEW & AS & SLU & $\mathrm{CaCl}$ & $(216)$ \\
\hline CaP & SEW & AS & SLU & $\mathrm{Ca}(\mathrm{OH})_{2}$ & $\mathrm{CO}_{2}$ & $(213)$ \\
\hline CaP & SEW & AS & SLU & CSH (Seed) & - & $(213)$ \\
\hline FeP & SEW & AS & SLU & Waste Iron Scrap & $\mathrm{NaOH}$ or $\mathrm{HCl}$ & $(217)$ \\
\hline
\end{tabular}


Table S3.3 (continued) Documents constituting individual clusters and overview of variations within clusters.

\begin{tabular}{|l}
\hline CLUSTER 3.12 Nutrient Extraction (Ammonia Release and Capture) \\
\hline Nitrogen recovery from excreta-derived streams through ammonia release and capture in acid trap. \\
\hline Sewage) \\
\hline $\begin{array}{l}\text { Target } \\
\text { Nutrient }\end{array}$
\end{tabular}

\begin{tabular}{|c|c|c|c|c|c|c|c|c|c|}
\hline \multicolumn{10}{|c|}{ CLUSTER 3.13 Nutrient Extraction (Release from Sewage Sludge) } \\
\hline \multicolumn{10}{|c|}{ Release of $\mathrm{P}$ from sewage sludge. } \\
\hline \multicolumn{10}{|c|}{ 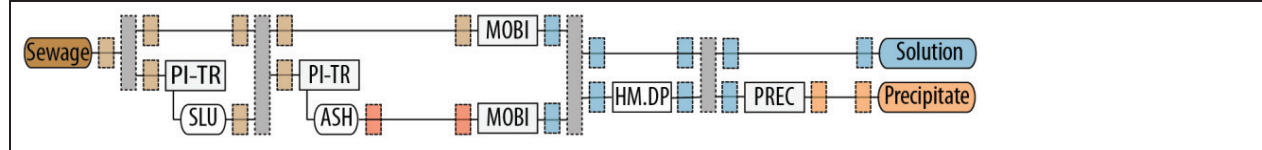 } \\
\hline $\begin{array}{l}\text { Target } \\
\text { Nutrient }\end{array}$ & PI & PI-TR & SI & MOBI & HM.DEP & PREC & \begin{tabular}{|l|} 
Target \\
Product
\end{tabular} & \begin{tabular}{|l} 
Process \\
Name
\end{tabular} & Ref \\
\hline $\mathrm{P}$ & SEW & AS & RTS & ANAER & MSED & - & Solution & & $(220)$ \\
\hline $\mathrm{P}$ & SEW & AS & RTS & ANAER & & PREC & Precipitate & & $(221-230)$ \\
\hline $\mathrm{P}$ & SEW & $\mathrm{AS}$ & SLU & Sulfate & & - & Solution & & $(231)$ \\
\hline $\mathrm{P}$ & SEW & $\mathrm{AS}$ & SLU & Sulfate & HM.PREC & PREC & Precipitate & & $(231)$ \\
\hline $\mathrm{P}$ & SEW & AS & SLU & Acidification & & PREC & Precipitate & Aqua Reci & $(232)$ \\
\hline $\mathrm{P}$ & SEW & AS & RTS & Acidification & & PREC & Precipitate & & (233) \\
\hline $\mathrm{P}$ & SEW & AS & SLU & Acidification & MSNF & - & Solution & & $(234,235)$ \\
\hline $\mathrm{P}$ & SEW & AS & SLU & Acidification & MSED & PREC & Precipitate & & $(236)$ \\
\hline $\mathrm{P}$ & SEW & AS & SLU & Acidification & HM.PREC & PREC & Precipitate & Gifhorn & $(237)$ \\
\hline $\mathrm{P}$ & SEW & $\mathrm{AS}$ & SLU & Acidification & CMPLX & PREC & Precipitate & Stuttgart & $(238)$ \\
\hline $\mathrm{P}$ & SEW & $\mathrm{AS}$ & RTS & Alkalinisation & MSED & - & \begin{tabular}{|l|} 
Solution \\
\end{tabular} & & $(239)$ \\
\hline$P$ & SEW & AS & RTS & $\begin{array}{l}\text { Alkalinisation- } \\
\text { Microwaving }\end{array}$ & & PREC & Precipitate & & $(240,241)$ \\
\hline $\mathrm{P}$ & SEW & AS & RTS & $\begin{array}{l}\text { Alkalisation- } \\
\text { Hydrolysis }\end{array}$ & & PREC & Precipitate & & $(242,243)$ \\
\hline $\mathrm{P}$ & SEW & AS & RTS & Fermentation & & PREC & Precipitate & & $(244)$ \\
\hline $\mathrm{P}$ & SEW & AS & RTS & $\begin{array}{l}\text { Hydrolysis- } \\
\text { Fermentation } \\
\end{array}$ & MSED & PREC & Precipitate & & (245) \\
\hline $\mathrm{P}$ & SEW & AS & RTS & BES & & PREC & Precipitate & & $(246,247)$ \\
\hline $\mathrm{P}$ & SEW & AS & RTS & \begin{tabular}{|l|} 
Ozonation \\
\end{tabular} & & PREC & Precipitate & & $(248,249)$ \\
\hline $\mathrm{P}$ & SEW & AS & SLU & $\mathrm{CO} 2$ & & PREC & Precipitate & Budenheim & \\
\hline
\end{tabular}


Table S3.3 (continued) Documents constituting individual clusters and overview of variations within clusters.

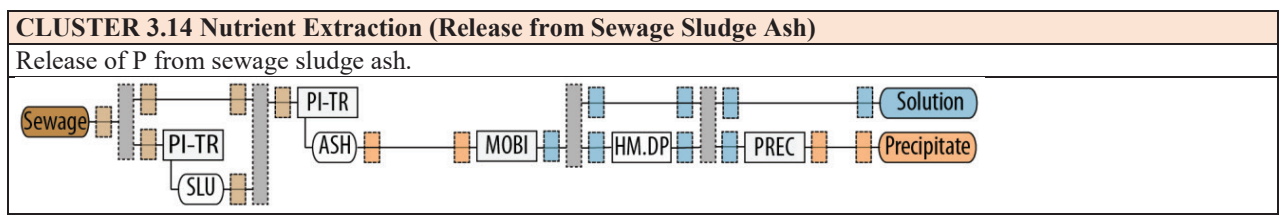

\begin{tabular}{|c|c|c|c|c|c|c|c|c|c|c|}
\hline \multirow{2}{*}{$\begin{array}{l}\text { Target } \\
\text { Nutrient } \\
\mathrm{P}\end{array}$} & \multirow{2}{*}{\begin{tabular}{|l|} 
PI \\
SEW \\
\end{tabular}} & \multicolumn{2}{|c|}{ PI-TR } & \multirow{2}{*}{$\begin{array}{l}\text { SI } \\
\mathrm{ASH}\end{array}$} & \multirow{2}{*}{\begin{tabular}{|l|} 
MOBI \\
ACID \\
\end{tabular}} & \multirow{2}{*}{$\begin{array}{l}\text { HM.DEP } \\
-\end{array}$} & \multirow{2}{*}{$\begin{array}{l}\text { PREC } \\
- \\
\end{array}$} & \multirow{2}{*}{\begin{tabular}{|l|} 
Target \\
Product
\end{tabular}} & \multirow[t]{2}{*}{ Process Name } & \multirow{2}{*}{\begin{tabular}{|l|} 
Ref \\
$(250-254,254)$ \\
\end{tabular}} \\
\hline & & AS & INC & & & & & & & \\
\hline $\mathrm{P}$ & SEW & AS & INC & $\mathrm{ASH}$ & ACID & SEQ.IEX & - & \begin{tabular}{|l} 
Solution \\
\end{tabular} & & $(237)$ \\
\hline $\mathrm{P}$ & SEW & AS & INC & $\mathrm{ASH}$ & ACID & SOLV.EX & - & Solution & & $(255)$ \\
\hline $\mathrm{P}$ & SEW & AS & INC & $\mathrm{ASH}$ & ACID & SOLV.EX & PREC & Precipitate & PASCH & \\
\hline $\mathrm{P}$ & SEW & AS & INC & $\mathrm{ASH}$ & ACID & EKIN & - & Solution & & $(256)$ \\
\hline $\mathrm{P}$ & SEW & AS & INC & ASH & ACID & MSNF & - & Solution & & $(234,257)$ \\
\hline $\mathrm{P}$ & SEW & AS & INC & $\mathrm{ASH}$ & ACID & MSED & - & Solution & & $(258,259)$ \\
\hline $\mathrm{P}$ & SEW & AS & GASI & $\mathrm{ASH}$ & ACID & MSED & - & Solution & & $(259,260)$ \\
\hline $\mathrm{P}$ & SEW & AS & INC & $\mathrm{ASH}$ & ACID & MSED & PREC & Precipitate & & $(236)$ \\
\hline $\mathrm{P}$ & SEW & AS & INC & $\mathrm{ASH}$ & ACID & ALKA & PREC & Precipitate & SESAL-Phos & $(261-263)$ \\
\hline $\mathrm{P}$ & SEW & AS & PYRO & $\mathrm{ASH}$ & ACID & - & - & Solution & & $(252)$ \\
\hline $\mathrm{P}$ & SEW & AS & SCW & ASH & ACID & - & & Solution & & $(264)$ \\
\hline $\mathrm{P}$ & SEW & AS & GASI & $\mathrm{ASH}$ & ACID & - & - & \begin{tabular}{|l|} 
Solution \\
\end{tabular} & & $(265)$ \\
\hline $\mathrm{P}$ & SEW & AS & INC & $\mathrm{ASH}$ & ACID & - & PREC & Precipitate & CleanMAP & \\
\hline $\mathrm{P}$ & SEW & AS & INC & $\overline{\mathrm{ASH}}$ & ACID & - & PREC & Precipitate & LOTUS & \\
\hline $\mathrm{P}$ & SEW & AS & INC & $\mathrm{ASH}$ & ACID & - & PREC & Precipitate & Leachphos & \\
\hline $\mathrm{P}$ & SEW & AS & INC & $\mathrm{ASH}$ & ACID & - & PREC & Precipitate & & $(266,267)$ \\
\hline $\mathrm{P}$ & SEW & AS & INC & $\mathrm{ASH}$ & ACID & HM.PREC & PREC & Precipitate & SEPHOS & $(257)$ \\
\hline $\mathrm{P}$ & SEW & AS & INC & $\mathrm{ASH}$ & ACID & HM.SORP & PREC & Precipitate & & $(268)$ \\
\hline $\mathrm{P}$ & SEW & AS & SCW & ASH & ACID & SORP & PREC & Precipitate & & (269) \\
\hline
\end{tabular}

\begin{tabular}{|c|c|c|c|c|c|c|c|c|}
\hline \multicolumn{9}{|c|}{ CLUSTER 3.15 Ash Treatment } \\
\hline \multicolumn{9}{|c|}{ Ash treatment. } \\
\hline $\begin{array}{l}\text { Target } \\
\text { Nutrient }\end{array}$ & PI & PI-T & & SI & MOBI & \begin{tabular}{|l|} 
Target \\
Product
\end{tabular} & \begin{tabular}{|l|} 
Process \\
Name
\end{tabular} & Ref \\
\hline $\mathrm{P}$ & SEW & $\mathrm{AS}$ & INC & $\mathrm{ASH}$ & $\begin{array}{l}\text { Thermo-Chemical } \\
\text { Treatment }\end{array}$ & Ash & & $(270,271)$ \\
\hline $\mathrm{P}$ & SEW & AS & INC & $\mathrm{ASH}$ & $\begin{array}{l}\text { Thermo-Chemical } \\
\text { Treatment }\end{array}$ & Ash & & $(272,273)$ \\
\hline $\mathrm{P}$ & SEW & AS & INC & ASH & $\begin{array}{l}\text { Thermo-Reductive } \\
\text { Treatment }\end{array}$ & Solution & & $(274)$ \\
\hline $\mathrm{P}$ & SEW & $\mathrm{AS}$ & - & SLU & Smelt Gasification & Slag & Mephrec & \\
\hline $\mathrm{P}$ & SEW & $\mathrm{AS}$ & INC & ASH & Smelt Gasification & Slag & ATZ & $(275)$ \\
\hline
\end{tabular}


Table S3.4 Description of key mineral precipitates that can be found in fertiliser products rendered by treatment of human excreta and streams containing human excreta.

\begin{tabular}{|c|c|}
\hline Precipitate & Description \\
\hline MAP & $\begin{array}{l}\text { Struvite is a phosphate mineral with the formula } \mathrm{MgNH}_{4} \mathrm{PO}_{4} \cdot 6 \mathrm{H}_{2} \mathrm{O} \text { and consists of } \\
\text { magnesium }\left(\mathrm{Mg}^{2+}\right) \text {, ammonium }\left(\mathrm{NH}_{4}^{+}\right) \text {, and orthophosphate }\left(\mathrm{PO}_{4}{ }^{3-}\right) \text { in equal molar } \\
\text { concentrations. Struvite is also commonly referred to as magnesium ammonium phosphate } \\
\text { hexahydrate, or simply magnesium ammonium phosphate (MAP). }\end{array}$ \\
\hline MPP & $\begin{array}{l}\text { Several struvite analogues or struvite-type compounds exist, which together with struvite } \\
\text { form the struvite group (Mathew and Schroeder, 1979; Graeser et al., 2008; Weil, 2008). } \\
\text { The general formula for minerals of the struvite group is }[A][M]\left[X \mathrm{O}_{4}\right] \cdot n \mathrm{H}_{2} \mathrm{O} \text {, where: } A= \\
\mathrm{NH}_{4}, \mathrm{~K}, \mathrm{Na}, \mathrm{Tl}, \mathrm{Rb} ; M=\mathrm{Mg}, \mathrm{Co}, \mathrm{Ni} ; X=\mathrm{P}, \mathrm{As} \text {; and } n=6,7 \text {. The struvite analogue with } \\
\text { the formula } \mathrm{MgKPO}_{4} \cdot 6 \mathrm{H}_{2} \mathrm{O} \text { is of most interest in the context of nutrient recovery from } \\
\text { human excreta }(\mathrm{Xu} \text { et al., } 2012,2015 \text { ). It is commonly referred to as magnesium potassium } \\
\text { phosphate hexahydrate, or simply magnesium potassium phosphate (MPP). }\end{array}$ \\
\hline Ca-P & $\begin{array}{l}\text { Calcium phosphates are a family of mineral salts containing calcium cations }\left(\mathrm{Ca}^{2+}\right) \text { and } \\
\text { phosphate anions }\left(\mathrm{PO}_{4}^{3}\right) \text { (Dorozhkin and Epple, 2002). Common calcium phosphate } \\
\text { precipitates include hydroxylapatite }\left(\mathrm{Ca}_{10}\left(\mathrm{PO}_{4}\right)_{6}(\mathrm{OH})_{2}\right) \text {, brushite }\left(\mathrm{CaHPO}_{4} \cdot \mathrm{H}_{2} \mathrm{O}\right) \text {, } \\
\text { carbonated hydroxylapatite as well as amorphous calcium phosphate }\left(\mathrm{Ca}_{\mathrm{x}}\left(\mathrm{PO}_{4}\right)_{\mathrm{y}} \cdot \mathrm{nH}_{2} \mathrm{O}\right) \text {. A } \\
\text { carbonated hydroxylapatite is a hydroxylapatite where carbonate groups }\left(\mathrm{CO}_{3}{ }^{2-}\right) \text { are inserted } \\
\text { into the apatite structure to replace phosphate and/or hydroxyl }\left(\mathrm{OH}^{-}\right) \text {groups }\left(\mathrm{Pham} \mathrm{Minh} \mathrm{et}^{\text {et }}\right. \\
\text { al., 2014). }\end{array}$ \\
\hline Al-P & $\begin{array}{l}\text { Aluminium phosphates are a family of mineral salts containing aluminium cations }\left(\mathrm{Al}^{3+}\right) \\
\text { and phosphate anions }\left(\mathrm{PO}_{4}^{3}\right) . \mathrm{AlPO}_{4} \text { is the most common aluminium phosphate in the } \\
\text { context of the recovery of nutrients in human excreta. }\end{array}$ \\
\hline $\mathrm{Fe}(\mathrm{III})-\mathrm{P}$ & $\begin{array}{l}\text { Ferric phosphates are a family of mineral salts containing iron(III) cations }\left(\mathrm{Fe}^{3+}\right) \text { and } \\
\text { phosphate anions }\left(\mathrm{PO}_{4}{ }^{3}\right) . \mathrm{FePO}_{4} \text { is the most common ferric phosphate in the context of the } \\
\text { recovery of nutrients in human excreta. }\end{array}$ \\
\hline Mg-P & $\begin{array}{l}\text { Magnesium phosphates are salts of magnesium }\left(\mathrm{Mg}^{2+}\right) \text { and phosphate }\left(\mathrm{PO}_{4}{ }^{3-}\right) \text { appearing } \\
\text { as magnesium phosphate monobasic }\left(\mathrm{Mg}\left(\mathrm{H}_{2} \mathrm{PO}_{4}\right)_{2}\right) \text {, dibasic }\left(\mathrm{MgHPO}_{4}\right) \text {, or tribasic } \\
\left(\mathrm{Mg}_{3}\left(\mathrm{PO}_{4}\right)_{2}\right) \text { as well as amorphous magnesium phosphate. Anhydrous forms include } \\
\text { newberyite }\left(\mathrm{MgHPO}_{4} \cdot 3 \mathrm{H}_{2} \mathrm{O}\right) \text { and bobierrite }\left(\mathrm{Mg}_{3}\left(\mathrm{PO}_{4}\right)_{2} \cdot 8 \mathrm{H}_{2} \mathrm{O}\right) \text {. }\end{array}$ \\
\hline K-P & $\begin{array}{l}\text { Potassium phosphates are salts of potassium }\left(\mathrm{K}^{+}\right) \text {and phosphate }\left(\mathrm{PO}_{4}{ }^{3-}\right) \text { appearing as } \\
\text { potassium phosphate monobasic }\left(\mathrm{KH}_{2} \mathrm{PO}_{4}\right) \text {, dibasic }\left(\mathrm{K}_{2} \mathrm{HPO}_{4}\right) \text {, or tribasic }\left(\mathrm{K}_{3} \mathrm{PO}_{4}\right) \text {. }\end{array}$ \\
\hline Other & $\begin{array}{l}\text { Other minerals that can be precipitated during nutrient recovery from human excreta } \\
\text { include ammonium dihydrogenphosphate/nitrophosammite }\left(\mathrm{NH}_{4} \mathrm{H}_{2} \mathrm{PO}_{4}\right) \text {, potassium } \\
\text { chloride (sylvite) }(\mathrm{KCl}) \text {, natrium chlorice (halite) }(\mathrm{NaCl}) \text {, potassium bicarbonate } \\
\left.\left(\mathrm{KHCO}_{3}\right) \text {, gypsum }(\mathrm{CaSO})_{4}\right) \text {, potassium sulfate }\left(\mathrm{K}_{2} \mathrm{SO}_{4}\right) \text {, calcite }\left(\mathrm{CaCO}_{3}\right) \text {, dolomite } \\
\left.\left(\mathrm{CaMg} \mathrm{CO}_{3}\right)_{2}\right) \text { and chemically complex phosphates such as montgomeryite } \\
\left(\mathrm{Ca}_{4} \mathrm{MgAl}_{4}\left(\mathrm{PO}_{4}\right)_{6}(\mathrm{OH})_{4} \cdot 12 \mathrm{H}_{2} \mathrm{O}\right) \text {. }\end{array}$ \\
\hline
\end{tabular}

Table 4: Typical sorbents that have been used to extract nutrients from liquid streams during treatment of huma excreta and streams containing human excreta.

\begin{tabular}{|l|l|}
\hline Charcoal & $\begin{array}{l}\text { Activated carbon or biochar. Can be obtained from a variety of feedstocks, including faecal- } \\
\text { derived organic matter and phototrophic biomass. }\end{array}$ \\
\hline Calcined struvite & $\begin{array}{l}\text { Struvite from which ammonium has been removed through thermal treatment in the absence } \\
\left.\text { of or under limited supply of air or oxygen (e.g. at } 200^{\circ} \mathrm{C} \text { for } 3 \mathrm{~h}\right) \text { (Latifian } \text { et al., 2014) }\end{array}$ \\
\hline Aluminosilicates & $\begin{array}{l}\text { Minerals composed of aluminium, silicon, oxygen, and coutercations. Zeolites (e.g. } \\
\text { clinoptilolite) and palygorskite are the most common ones used as sorbents. }\end{array}$ \\
\hline Calcium silicates & $\begin{array}{l}\text { Minerals composed of calcium, silicon, oxygen, and coutercations. Wollastonite is the most } \\
\text { common one used as sorbent. }\end{array}$ \\
\hline Calcium oxide & $\begin{array}{l}\text { CaO. For example prepared from egg shells through calcination egg shells (Köse and } \\
\text { Kivanç, 2011). }\end{array}$ \\
\hline Other & $\begin{array}{l}\text { Other sorbent material include polymeric resins as well as metal modified charcoal, mineral } \\
\text { sorbents, or polymeric resins. }\end{array}$ \\
\hline
\end{tabular}




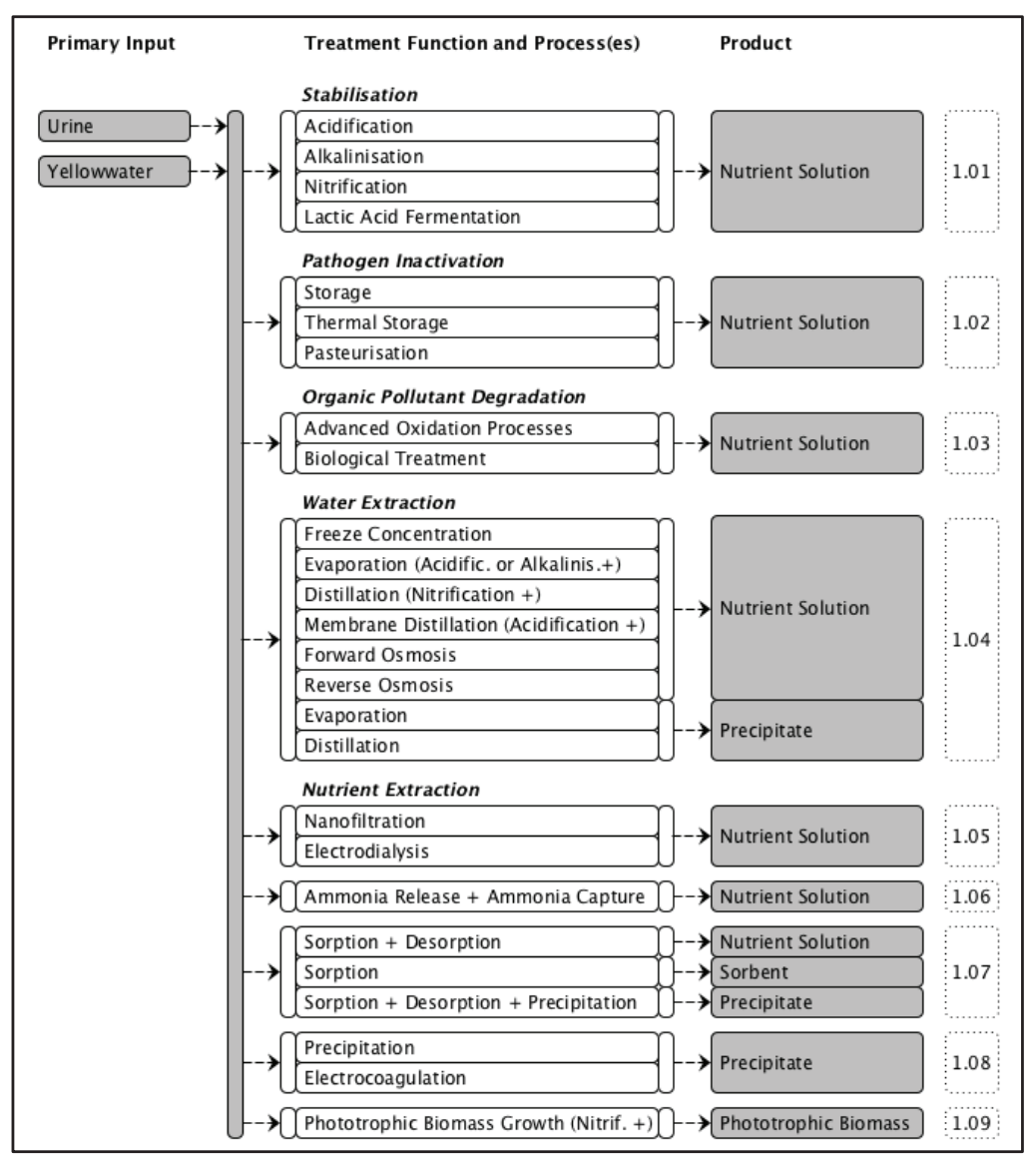

Figure S3.1 Clusters of recovery pathways. (a) Recovery pathways starting from source-separated urine or yellowwater. 


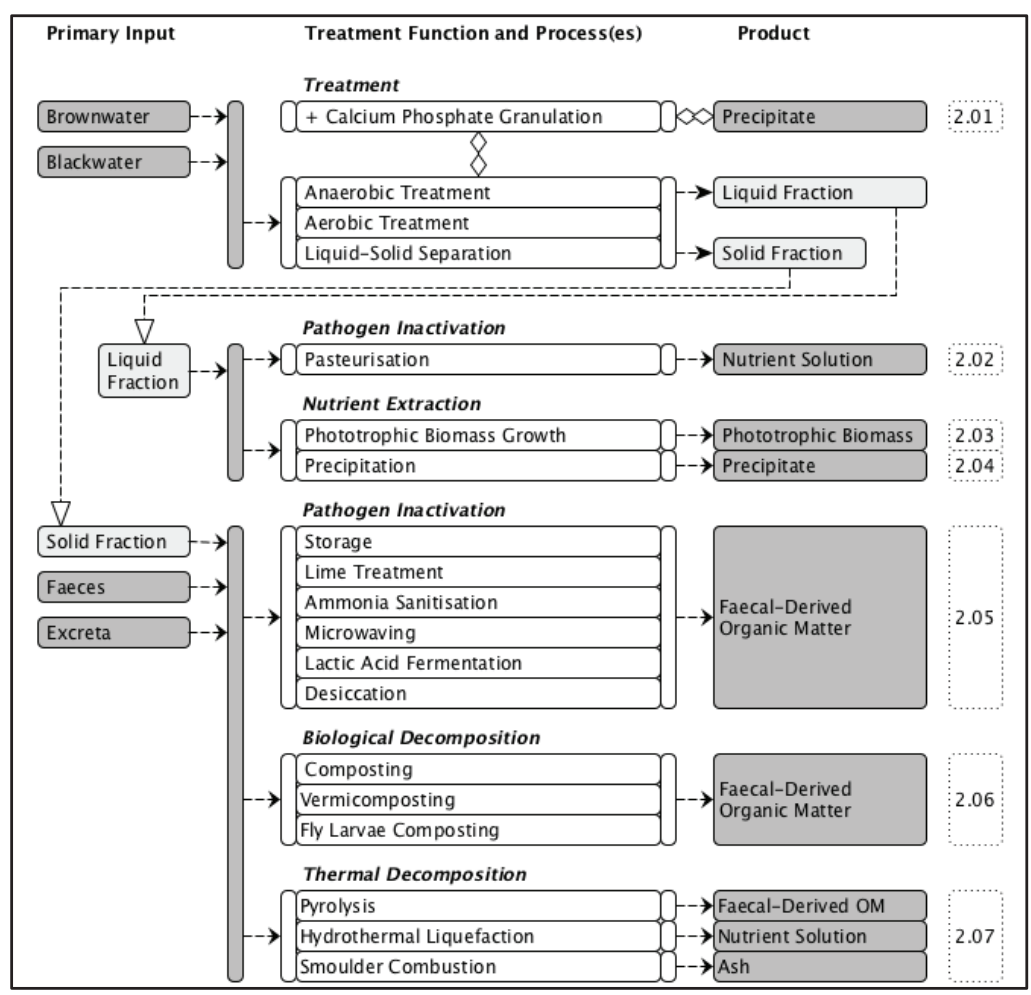

Figure S3.1 Clusters of recovery pathways. (b) Recovery pathways starting from source-separated faeces, brownwater, excreta, or blackwater. 


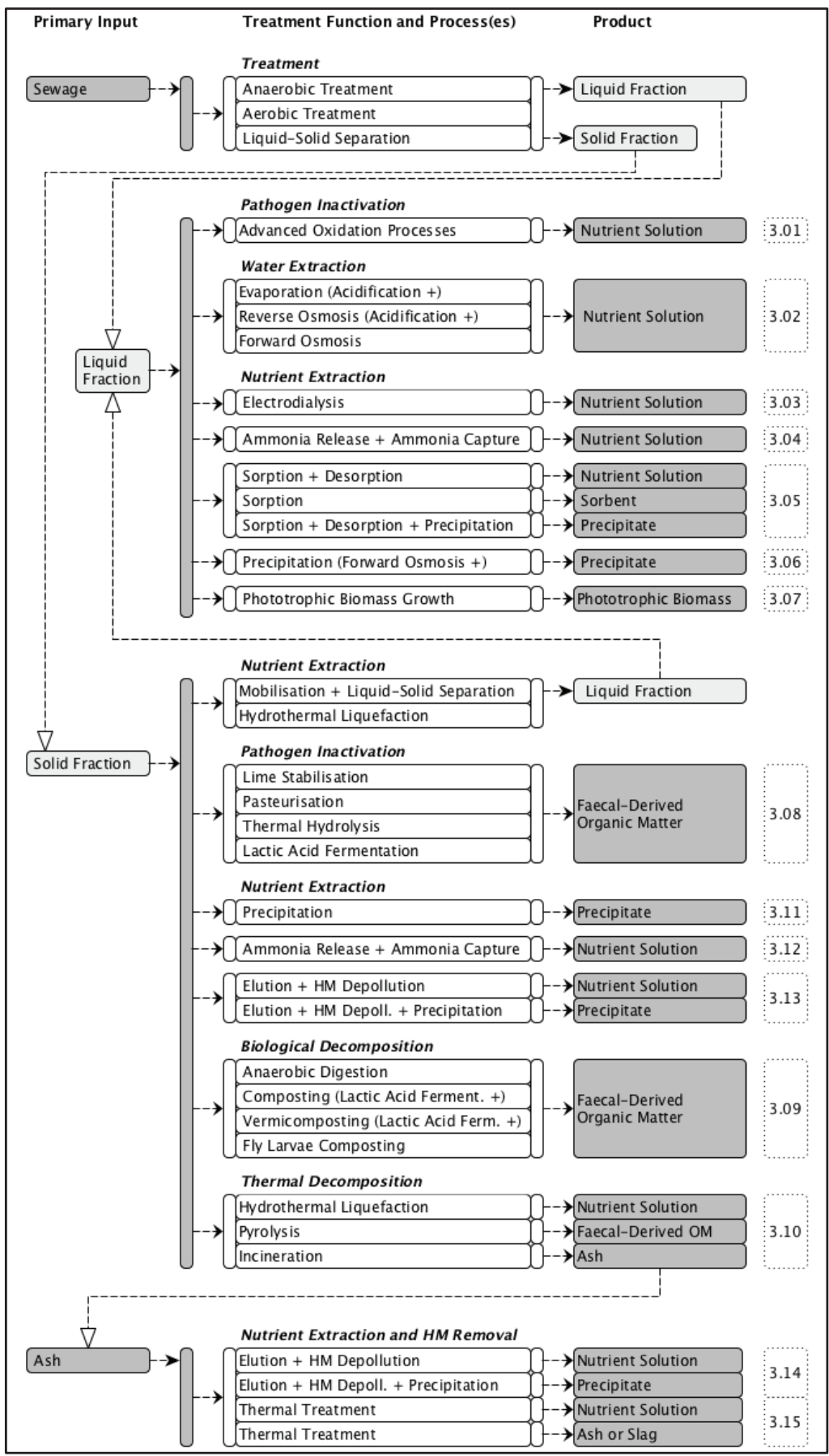

Figure S3.1 Clusters of recovery pathways. (c) Recovery pathways starting from sewage. 


\section{References}

1. Hellström, D., Johansson, E. and Grennberg, K. (1999) 'Storage of human urine: Acidification as a method to inhibit decomposition of urea', Ecological Engineering, 12(3-4), pp. 253-269. doi: 10.1016/S0925-8574(98)000743.

2. Randall, D. G. et al. (2016) 'A novel approach for stabilizing fresh urine by calcium hydroxide addition', Water Research, 95, pp. 361-369. doi: 10.1016/j.watres.2016.03.007.

3. Sun, F. Y. et al. (2012) 'Stabilization of source-separated urine by biological nitrification process: Treatment performance and nitrite accumulation', Water Science and Technology, 66(7), pp. 1491-1497. doi: 10.2166/ wst.2012.337.

4. Udert, K. M. et al. (2003) 'Nitrification and autotrophic denitrification of source-separated urine', Water Science and Technology, 48(1), pp. 119-130. doi: doi: 10.1021/es048422m.

5. Andreev, N., Ronteltap, M., Boincean, B. and Lens, P. N. L. (2017) 'Treatment of Source-Separated Human Feces via Lactic Acid Fermentation Combined with Thermophilic Composting', Compost Science \& Utilization, 25(4), pp. 220-230. doi: 10.1080/1065657X.2016.1277809.

6. Ikematsu, M. et al. (2007) 'Electrochemical treatment of human urine for its storage and reuse as flush water', Science of the Total Environment, 382(1), pp. 159-164. doi: 10.1016/j.scitotenv.2007.03.028.

7. Zhang, Y. et al. (2013) 'Stabilization of source-separated human urine by chemical oxidation', Water Science and Technology, 67(9), pp. 1901-1907. doi: 10.2166/wst.2013.055.

8. Andersson, E. (2015) 'Turning waste into value: Using human urine to enrich soils for sustainable food production in Uganda', Journal of Cleaner Production. Elsevier Ltd, 96, pp. 290-298. doi: 10.1016/j.jclepro.2014.01.070.

9. Lahr, R. H. et al. (2016) 'Urine Bacterial Community Convergence through Fertilizer Production: Storage, Pasteurization, and Struvite Precipitation', Environmental Science and Technology, 50(21), pp. 11619-11626. doi: 10.1021/acs.est.6b02094.

10. Tidåker, P., Sjöberg, C. and Jönsson, H. (2007) 'Local recycling of plant nutrients from small-scale wastewater systems to farmland-A Swedish scenario study', Resources, Conservation and Recycling, 49(4), pp. 388-405. doi: 10.1016/j.resconrec.2006.05.004.

11. Tilley, E., Atwater, J. and Mavinic, D. (2008) 'Effects of storage on phosphorous recovery from urine', Environmental Technology, 29(7), pp. 807-816.

12. Zhou, X. et al. (2015) 'Simultaneous current generation and ammonia recovery from real urine using nitrogen-purged bioelectrochemical systems', RSC Adv. Royal Society of Chemistry, 5(86), pp. 70371-70378. doi: 10.1039/ C5RA11556F.

13. Pronk, W. et al. (2006) 'Nanofiltration for the separation of pharmaceuticals from nutrients in source-separated urine', Water Research, 40(7), pp. 1405-1412. doi: 10.1016/j.watres.2006.01.038.

14. Lazarova, Z. and Spendlingwimmer, R. (2008) 'Treatment of yellow water by membrane separations and advanced oxidation methods', Water Science and Technology, 58(2), pp. 419-426. doi: 10.2166/wst.2008.667.

15. Abdel-Shafy, H. I. and Mansour, M. S. M. (2016) 'Integration of effective microorganisms and membrane bioreactor for the elimination of pharmaceutical active compounds from urine for safe reuse', Journal of Water Reuse and Desalination, 6(4), pp. 495-504. doi: 10.2166/wrd.2016.141.

16. Gulyas, H. et al. (2004) 'Freeze concentration for enrichment of nutrients in yellow water from no-mix toilets', Water Science and Technology, 50(6), pp. 61-68.

17. Lind, B. B., Ban, Z. and Bydén, S. (2000) 'Nutrient recovery from human urine by struvite crystallization with ammonia adsorption on zeolite and wollastonite', Bioresource Technology, 73(2), pp. 169-174. doi: 10.1016/ S0960-8524(99)90157-8.

18. Bethune, D. N., Chu, A. and Ryan, M. C. (2014) 'Passive evaporation of source-separated urine from dry toilets: a lab study', Journal of Water, Sanitation and Hygiene for Development, 4(4), p. 654. doi: 10.2166/washdev.2014.058.

19. Bethune, D. N., Chu, A. and Ryan, M. C. (2016) 'Passive evaporation of source-separated urine from dry toilets: UES optimization and dry product accumulation over time', Journal of Water, Sanitation and Hygiene for Development, 6(1), pp. 96-103. doi: 10.2166/washdev.2016.103.

20. Pahore, M. M., Ito, R. and Funamizu, N. (2010) 'Rational design of an on-site volume reduction system for source-separated urine', Environmental Technology, 31(4), pp. 399-408. doi: 10.1080/09593330903505654.

21. Dutta, S. and Vinnerås, B. (2016) 'Fertilizer from dried human urine added to ash and lime -A potential product from eco-sanitation system', Water Science and Technology, 74(6), pp. 1436-1445. doi: 10.2166/wst.2016.324.

22. Antonini, S. et al. (2012) 'Solar thermal evaporation of human urine for nitrogen and phosphorus recovery in Vietnam', Science of the Total Environment. Elsevier B.V., 414, pp. 592-599. doi: 10.1016/j.scitotenv.2011.11.055.

23. Ek, M. et al. (2006) 'Concentration of nutrients from urine and reject water from anaerobically digested sludge', Water Science and Technology, 54(11-12), pp. 437-444. doi: 10.2166/wst.2006.924. 
24. Senecal, J. and Vinnerås, B. (2017) 'Urea stabilisation and concentration for urine-diverting dry toilets: Urine dehydration in ash', Science of the Total Environment. The Authors, 586, pp. 650-657. doi: 10.1016/j.scitotenv.2017.02.038.

25. Eawag (no date) Blue Diversion AUTARKY Wastewater Treatment off the Grid.

26. Jiang, S. et al. (2017) 'Effect of initial $\mathrm{pH}$ and $\mathrm{pH}$-adjusted acid on nutrient recovery from hydrolysis urine by combining acidification with evaporation-crystallization', Environmental Science and Pollution Research. Environmental Science and Pollution Research, 24(4), pp. 3872-3881. doi: 10.1007/s11356-016-8052-8.

27. Udert, K. M. and Wächter, M. (2012) 'Complete nutrient recovery from source-separated urine by nitrification and distillation', Water Research, 46(2), pp. 453-464. doi: 10.1016/j.watres.2011.11.020.

28. Fumasoli, A. et al. (2016) 'Operating a pilot-scale nitrification/distillation plant for complete nutrient recovery from urine', Water Science and Technology, 73(1), pp. 215-222. doi: 10.2166/wst.2015.485.

29. Tun, L. L. et al. (2016) 'Dewatering of source-separated human urine for nitrogen recovery by membrane distillation', Journal of Membrane Science. Elsevier, 512, pp. 13-20. doi: 10.1016/j.memsci.2016.04.004.

30. Liu, Q. et al. (2016) 'Integrated forward osmosis-membrane distillation process for human urine treatment', Water Research. Elsevier Ltd, 91, pp. 45-54. doi: 10.1016/j.watres.2015.12.045.

31. Zhang, J. et al. (2014) 'Mining nutrients (N, P, K) from urban source seprated urine by forward osmosis', Environmental Science and Technology, 48(6), pp. 3386-3394.

32. Pronk, W., Biebow, M. and Boller, M. (2006) 'Electrodialysis for recovering salts from a urine solution containing micropollutants', Environmental Science and Technology, 40(7), pp. 2414-2420. doi: 10.1021/es051921i.

33. Pronk, W. et al. (2007) 'Pilot experiments with electrodialysis and ozonation for the production of a fertiliser from urine', in Water Science and Technology, pp. 219-227. doi: 10.2166/wst.2007.575.

34. Chen, X. et al. (2017) 'The microbial electrochemical current accelerates urea hydrolysis for recovery of nutrients from source-separated urine', Environmental Science and Technology Letters, 4(7), pp. 305-310. doi: $10.1021 /$ acs.estlett.7b00168.

35. Tice, R. C. and Kim, Y. (2014) 'Energy efficient reconcentration of diluted human urine using ion exchange membranes in bioelectrochemical systems', Water Research. Elsevier Ltd, 64, pp. 61-72. doi: 10.1016/j. watres.2014.06.037.

36. Antonini, S. et al. (2011) 'Nitrogen and Phosphorus Recovery from Human Urine by Struvite Precipitation and Air Stripping in Vietnam', Clean - Soil, Air, Water, 39(12), pp. 1099-1104. doi: 10.1002/clen.201100036.

37. Liu, B. et al. (2015) 'Air stripping process for ammonia recovery from source-separated urine: Modeling and optimization', Journal of Chemical Technology and Biotechnology, 90(12), pp. 2208-2217. doi: 10.1002/jctb.4535.

38. Kuntke, P. et al. (2012) 'Ammonium recovery and energy production from urine by a microbial fuel cell', Water Research, 46(8), pp. 2627-2636. doi: 10.1016/j.watres.2012.02.025.

39. Zhou, X. et al. (2017) 'Investigation on microbial inactivation and urea decomposition in human urine during thermal storage', Journal of Water Sanitation and Hygiene for Development, 7(3), pp. 378-386. doi: 10.2166/ washdev.2017.142.

40. Kuntke, P. et al. (2014) 'Hydrogen production and ammonium recovery from urine by a Microbial Electrolysis Cell', International Journal of Hydrogen Energy. Elsevier Ltd, 39(10), pp. 4771-4778. doi: 10.1016/j. ijhydene.2013.10.089.

41. Zamora, P. et al. (2017) 'Long-term operation of a pilot-scale reactor for phosphorus recovery as struvite from source-separated urine', Journal of Chemical Technology and Biotechnology, 92(5), pp. 1035-1045. doi: 10.1002/jctb.5079.

42. Luther, A. K. et al. (2015) 'Electrochemically driven extraction and recovery of ammonia from human urine', Water Research. Elsevier Ltd, 87, pp. 367-377. doi: 10.1016/j.watres.2015.09.041.

43. Ganesapillai, M., Simha, P. and Zabaniotou, A. (2015) 'Closed-loop fertility cycle: Realizing sustainability in sanitation and agricultural production through the design and implementation of nutrient recovery systems for human urine', Sustainable Production and Consumption. Elsevier B.V., 4(August), pp. 36-46. doi: 10.1016/j. spc.2015.08.004.

44. Ganesapillai, M. et al. (2016) 'Nutrient Recovery and Recycling from Human Urine: A Circular Perspective on Sanitation and Food Security', Procedia Engineering. Elsevier B.V., 148, pp. 346-353. doi: 10.1016/j.proeng.2016.06.461.

45. Simha, P., Zabaniotou, A. and Ganesapillai, M. (2016) 'Continuous urea-nitrogen recycling from human urine: A step towards creating a human excreta based bio-economy', Journal of Cleaner Production. Elsevier Ltd, pp. 1-10. doi: 10.1016/j.jclepro.2017.01.062.

46. Bai, X. et al. (2017) 'Recovery of Ammonium in Urine by Biochar Derived from Faecal Sludge and its Application as Soil Conditioner', Waste and Biomass Valorization. Springer Netherlands, 0(0), pp. 1-10. doi: 10.1007/ s12649-017-9906-0. 
47. Tarpeh, W. A., Udert, K. M. and Nelson, K. L. (2017) 'Comparing ion exchange adsorbents for nitrogen recovery from source-separated urine', Environmental Science and Technology, 51(4), pp. 2373-2381. doi: 10.1021/ acs.est.6b05816.

48. Latifian, M., Holst, O. and Liu, J. (2014) 'Nitrogen and Phosphorus Removal from Urine by Sequential Struvite Formation and Recycling Process', Clean - Soil, Air, Water, 42(8), pp. 1157-1161. doi: 10.1002/clen.201300070.

49. Xu, S. et al. (2015) 'Nitrogen and phosphate recovery from source-separated urine by dosing with magnesite and zeolite', Polish Journal of Environmental Studies, 24(5), pp. 2269-2275. doi: 10.15244/pjoes/43611.

50. Casadellà, A. et al. (2016) 'Clinoptilolite-based mixed matrix membranes for the selective recovery of potassium and ammonium’, Water Research, 90, pp. 62-70. doi: 10.1016/j.watres.2015.12.017.

51. Kocatürk, N. P. and Baykal, B. B. (2012) 'Recovery of Plant Nutrients from Dilute Solutions of Human Urine and Preliminary Investigations on Pot Trials', Clean - Soil, Air, Water, 40(5), pp. 538-544. doi: 10.1002/ clen.201100193.

52. Allar, A. D. and Beler-Baykal, B. (2015) 'Stagewise processing of yellow water using clinoptilolite for nitrogen and phosphorus recovery and higher residual quality', Water Science and Technology, 71(12), pp. 1869-1874. doi: $10.2166 /$ wst.2015.176.

53. Xu, K., Zhang, Chuke, et al. (2017) 'Optimizing the modification of wood waste biochar via metal oxides to remove and recover phosphate from human urine', Environmental Geochemistry and Health. Springer Netherlands, pp. 1-10. doi: 10.1007/s10653-017-9986-6.

54. O’Neal, J. A. and Boyer, T. H. (2013) 'Phosphate recovery using hybrid anion exchange: Applications to source-separated urine and combined wastewater streams', Water Research. Elsevier Ltd, 47(14), pp. 5003-5017. doi: 10.1016/j.watres.2013.05.037.

55. Barbosa, S. G. et al. (2016) 'A design of experiments to assess phosphorous removal and crystal properties in struvite precipitation of source separated urine using different Mg sources', Chemical Engineering Journal, 298, pp. 146-153. doi: 10.1016/j.cej.2016.03.148.

56. Wilsenach, J. A., Schuurbiers, C. A. H. and van Loosdrecht, M. C. M. (2007) 'Phosphate and potassium recovery from source separated urine through struvite precipitation', Water Research, 41(2), pp. 458-466. doi: 10.1016/j. watres.2006.10.014.

57. Merino-Jimenez, I. et al. (2017) 'Enhanced MFC power production and struvite recovery by the addition of sea salts to urine', Water Research. Elsevier Ltd, 109, pp. 46-53. doi: 10.1016/j.watres.2016.11.017.

58. Başakçılardan-Kabakc1, S., İpekoğlu, A. N. and Talınlı, İ. (2007) 'Precipitation of Urinary Phosphate', Environmental Engineering Science, 24(10), pp. 1399-1408. doi: 10.1089/ees.2006.0136.

59. Kabdaşli, I. et al. (2006) 'Nitrogen recovery by struvite precipitation from anthropogenic nutrient solution', in Fresenius Environmental Bulletin, pp. 1146-1149. doi: 10.2166/wst.2006.433.

60. Liu, X. et al. (2014) 'Fate of phosphorus in diluted urine with tap water', Chemosphere. Elsevier Ltd, 113, pp. 146-150. doi: 10.1016/j.chemosphere.2014.04.093.

61. Ronteltap, M. et al. (2010) 'Struvite precipitation from urine - Influencing factors on particle size', Water Research. Elsevier Ltd, 44(6), pp. 2038-2046. doi: 10.1016/j.watres.2009.12.015.

62. Liu, Z. et al. (2008) 'Urea hydrolysis and recovery of nitrogen and phosphorous as MAP from stale human urine', Journal of Environmental Sciences, 20(8), pp. 1018-1024. doi: 10.1016/S1001-0742(08)62202-0.

63. Liu, Q. et al. (2010) 'Pilot Study on Removal and Recovery of Nitrogen and Phosphorus in Human Urine by Crystallization of Magnesium Ammonium Phosphate', Advanced Materials Research, 113-116, pp. 2310-2313. doi: 10.4028/www.scientific.net/AMR.113-116.2310.

64. Tünay, O., Kabdasli, I. and Tatli, M. B. (2009) 'Nitrogen removal and recovery from human urine by struvite precipitation', International Journal of Environment and Waste Management, 3, pp. 382-392.

65. Zang, G.-L. et al. (2012) 'Nutrient removal and energy production in a urine treatment process using magnesium ammonium phosphate precipitation and a microbial fuel cell technique', Physical Chemistry Chemical Physics, 14(6), p. 1978. doi: 10.1039/c2cp23402e.

66. Jin, Z. et al. (2014) 'Microbial and metabolic characterization of a denitrifying phosphorus-uptake/side stream phosphorus removal system for treating domestic sewage', Biodegradation, 25(6), pp. 777-786. doi: 10.1007/ s10532-014-9698-x.

67. Etter, B. et al. (2011) 'Low-cost struvite production using source-separated urine in Nepal', Water Research. Elsevier Ltd, 45(2), pp. 852-862. doi: 10.1016/j.watres.2010.10.007.

68. Mackey, H. R. et al. (2014) 'Combined seawater toilet flushing and urine separation for economic phosphorus recovery and nitrogen removal: A laboratory-scale trial', Water Science and Technology, 70(6), pp. 1065-1073. doi: $10.2166 /$ wst.2014.335.

69. Dai, J. et al. (2014) 'An exploratory study on seawater-catalysed urine phosphorus recovery (SUPR)', Water Research. Elsevier Ltd, 66, pp. 75-84. doi: 10.1016/j.watres.2014.08.008. 
70. Tang, W. T. et al. (2015) 'Microbial ureolysis in the seawater-catalysed urine phosphorus recovery system: Kinetic study and reactor verification', Water Research. Elsevier Ltd, 87, pp. 10-19. doi: 10.1016/j.watres.2015.09.004.

71. Yao, S. et al. (2017) 'On-site nutrient recovery and removal from source-separated urine by phosphorus precipitation and short-cut nitrification-denitrification', Chemosphere. Elsevier Ltd, 175, pp. 210-218. doi: 10.1016/j. chemosphere.2017.02.062.

72. Hug, A. and Udert, K. M. (2013) 'Struvite precipitation from urine with electrochemical magnesium dosage', Water Research. Elsevier Ltd, 47(1), pp. 289-299. doi: 10.1016/j.watres.2012.09.036.

73. Sakthivel, S. R., Tilley, E. and Udert, K. M. (2012) 'Wood ash as a magnesium source for phosphorus recovery from source-separated urine', Science of the Total Environment. Elsevier B.V., 419, pp. 68-75. doi: 10.1016/j. scitotenv.2011.12.065.

74. Kemacheevakul, P., Polprasert, C. and Shimizu, Y. (2011) 'Phosphorus recovery from human urine and anaerobically treated wastewater through $\mathrm{pH}$ adjustment and chemical precipitation', Environmental Technology, 32(7), pp. 693-698. doi: 10.1080/09593330.2010.510537.

75. Ledezma, P. et al. (2017) 'Recovering Nitrogen as a Solid without Chemical Dosing: Bio-Electroconcentration for Recovery of Nutrients from Urine', Environmental Science and Technology Letters, 4(3), pp. 119-124. doi: 10.1021 /acs.estlett.7b00024.

76. Xu, K. et al. (2012) 'Laboratory experiments on simultaneous removal of $\mathrm{K}$ and $\mathrm{P}$ from synthetic and real urine for nutrient recycle by crystallization of magnesium-potassium-phosphate-hexahydrate in a draft tube and baffle reactor', Chemosphere. Elsevier Ltd, 88(2), pp. 219-223. doi: 10.1016/j.chemosphere.2012.02.061.

77. Xu, K. et al. (2015) 'The precipitation of magnesium potassium phosphate hexahydrate for $\mathrm{P}$ and $\mathrm{K}$ recovery from synthetic urine', Water Research. Elsevier Ltd, 80, pp. 71-79. doi: 10.1016/j.watres.2015.05.026.

78. Xu, K., Zhang, Chi, et al. (2017) 'Removal and recovery of N, P and K from urine via ammonia stripping and precipitations of struvite and struvite-K', Water Science and Technology, 75(1), pp. 155-164. doi: 10.2166/ wst.2016.494.

79. Jadhav, U. and Hocheng, H. (2016) 'Recovery of phosphorus from source separated urine by Acidithiobacillus ferrooxidans culture supernatant', Ecological Engineering. Elsevier B.V., 92, pp. 90-96. doi: 10.1016/j.ecoleng.2016.03.048.

80. de Wilt, A. et al. (2016) 'Micropollutant removal in an algal treatment system fed with source separated wastewater streams', Journal of Hazardous Materials. Elsevier B.V., 304, pp. 84-92. doi: 10.1016/j.jhazmat.2015.10.033.

81. Tuantet, K. et al. (2014) 'Nutrient removal and microalgal biomass production on urine in a short light-path photobioreactor', Water Research, 55, pp. 162-174. doi: 10.1016/j.watres.2014.02.027.

82. Zhang, S. et al. (2014) 'Urban nutrient recovery from fresh human urine through cultivation of Chlorella sorokiniana', Journal of Environmental Management. Elsevier Ltd, 145, pp. 129-136. doi: 10.1016/j.jenvman.2014.06.013.

83. Piltz, B. and Melkonian, M. (2017) 'Immobilized microalgae for nutrient recovery from source-separated human urine', Journal of Applied Phycology. Journal of Applied Phycology, pp. 1-9. doi: 10.1007/s10811-017$1266-4$.

84. Adamsson, M. (2000) 'Potential use of human urine by greenhouse culturing of microalgae (Scenedesmus acuminatus), zooplankton (Daphnia magna) and tomatoes (Lycopersicon)', Ecological Engineering, 16(2), pp. 243-254. doi: 10.1016/S0925-8574(00)00064-1.

85. Chang, Y. et al. (2013) 'Cultivation of Spirulina platensis for biomass production and nutrient removal from synthetic human urine’, Applied Energy. Elsevier Ltd, 102, pp. 427-431. doi: 10.1016/j.apenergy.2012.07.024.

86. Coppens, J. et al. (2016) 'Nitrification and microalgae cultivation for two-stage biological nutrient valorization from source separated urine', Bioresource Technology. Elsevier Ltd, 211, pp. 41-50. doi: 10.1016/j. biortech.2016.03.001.

87. Tervahauta, T. et al. (2014) 'Calcium phosphate granulation in anaerobic treatment of black water: A new approach to phosphorus recovery’, Water Research, 48(1), pp. 632-642. doi: 10.1016/j.watres.2013.10.012.

88. Forbis-Stokes, A. A. et al. (2016) 'On-Site Fecal Sludge Treatment with the Anaerobic Digestion Pasteurization Latrine’, Environmental Engineering Science, 33(11), pp. 898-906. doi: 10.1089/ees.2016.0148.

89. Vasconcelos Fernandes, T. et al. (2015) 'Closing Domestic Nutrient Cycles Using Microalgae’, Environmental Science and Technology, 49(20), pp. 12450-12456. doi: 10.1021/acs.est.5b02858.

90. De Graaff, M. S. et al. (2011) 'Energy and phosphorus recovery from black water', Water Science and Technology, 63(11), pp. 2759-2765. doi: 10.2166/wst.2011.558.

91. Udert, K. M., Etter, B. and Gounden, T. (2016) 'Promoting sanitation in South Africa through nutrient recovery from urine', Gaia, 25(3), pp. 194-196. doi: 10.14512/gaia.25.3.12.

92. Anderson, C. et al. (2015) 'Lactic acid fermentation, urea and lime addition: Promising faecal sludge sanitizing methods for emergency sanitation', International Journal of Environmental Research and Public Health, 12(11), pp. 13871-13885. doi: 10.3390/ijerph121113871. 
93. Fidjeland, J. et al. (2013) 'The potential for self-sanitisation of faecal sludge by intrinsic ammonia', Water Research, 47(16), pp. 6014-6023. doi: 10.1016/j.watres.2013.07.024.

94. Magri, M. E. et al. (2015) 'Inactivation of adenovirus, reovirus and bacteriophages in fecal sludge by $\mathrm{pH}$ and ammonia', Science of the Total Environment. Elsevier B.V., 520, pp. 213-221. doi: 10.1016/j.scitotenv.2015.03.035.

95. Ogunyoku, T. A., Habebo, F. and Nelson, K. L. (2016) 'In-toilet disinfection of fresh fecal sludge with ammonia naturally present in excreta', Journal of Water, Sanitation and Hygiene for Development, 6(1), pp. 104-114. doi: $10.2166 /$ washdev.2015.233.

96. Fidjeland, J., Svensson, S. E. and Vinnerås, B. (2015) 'Ammonia sanitization of blackwater for safe use as fertilizer', Water Science and Technology, 71(5), pp. 795-800. doi: 10.2166/wst.2015.033.

97. Nordin, A. C. and Vinnerås, B. (2015) 'Sanitising black water by auto-thermal aerobic digestion (ATAD) combined with ammonia treatment', Water Science and Technology, 72(12), pp. 2112-2121. doi: 10.2166/wst.2015.432.

98. Hill, G. B., Baldwin, S. A. and Vinnerås, B. (2013) 'Composting toilets a misnomer: Excessive ammonia from urine inhibits microbial activity yet is insufficient in sanitizing the end-product', Journal of Environmental Management. Elsevier Ltd, 119, pp. 29-35. doi: 10.1016/j.jenvman.2012.12.046.

99. Park, J., Chon, K. and Cho, J. (2015) 'Science Walden: new horizons of combined ecological sanitation with separated urine/feces and treatment wetlands', Desalination and Water Treatment. Taylor \& Francis, 54(4-5), pp. 1353-1360. doi: 10.1080/19443994.2014.903528.

100. Hill, G. B. and Baldwin, S. A. (2012) 'Vermicomposting toilets, an alternative to latrine style microbial composting toilets, prove far superior in mass reduction, pathogen destruction, compost quality, and operational cost', Waste Management. Elsevier Ltd, 32(10), pp. 1811-1820. doi: 10.1016/j.wasman.2012.04.023.

101. Lalander, C., Hill, G. B. and Vinnerås, B. (2013) 'Hygienic quality of faeces treated in urine diverting vermicomposting toilets', Waste Management. Elsevier Ltd, 33(11), pp. 2204-2210. doi: 10.1016/j.wasman.2013.07.007.

102. Yadav, K. D., Tare, V. and Ahammed, M. M. (2010) 'Vermicomposting of source-separated human faeces for nutrient recycling', Waste Management. Elsevier Ltd, 30(1), pp. 50-56. doi: 10.1016/j.wasman.2009.09.034.

103. Andreev, N., Ronteltap, M., Boincean, B., Wernli, M., et al. (2017) 'Lactic acid fermentation of human urine to improve its fertilizing value and reduce odour emissions', Journal of Environmental Management, 198, pp. 63-69. doi: 10.1016/j.jenvman.2017.04.059.

104. Factura, H. et al. (2010) 'Terra Preta sanitation: Re-discovered from an ancient Amazonian civilisation - Integrating sanitation, bio-waste management and agriculture', Water Science and Technology, 61(10), pp. 2673-2679. doi: $10.2166 /$ wst.2010.201.

105. De Gisi, S., Petta, L. and Wendland, C. (2014) 'History and technology of Terra Preta sanitation', Sustainability (Switzerland), 6(3), pp. 1328-1345. doi: 10.3390/su6031328.

106. Vinnerås, B. and Jönsson, H. (2002) 'The performance and potential of faecal separation and urine diversion to recycle plant nutrients in household wastewater', Bioresource Technology, 84(3), pp. 275-282. doi: 10.1016/ S0960-8524(02)00054-8.

107. Cofie, O. O. et al. (2006) 'Solid-liquid separation of faecal sludge using drying beds in Ghana: Implications for nutrient recycling in urban agriculture', Water Research, 40(1), pp. 75-82. doi: 10.1016/j.watres.2005.10.023.

108. Yadav, K. D., Tare, V. and Ahammed, M. M. (2012) 'Integrated composting-vermicomposting process for stabilization of human faecal slurry', Ecological Engineering. Elsevier B.V., 47, pp. 24-29. doi: 10.1016/j.ecoleng.2012.06.039.

109. Butkovskyi, A. et al. (2016) 'Mitigation of micropollutants for black water application in agriculture via composting of anaerobic sludge', Journal of Hazardous Materials, 303, pp. 41-47. doi: 10.1016/j.jhazmat.2015.10.016.

110. Oarga Mulec, A. et al. (2016) 'Composting of the solid fraction of blackwater from a separation system with vacuum toilets - Effects on the process and quality', Journal of Cleaner Production, 112, pp. 4683-4690. doi: 10.1016/j.jclepro.2015.07.080.

111. Lu, J. et al. (2017) 'Simultaneous production of biocrude oil and recovery of nutrients and metals from human feces via hydrothermal liquefaction', Energy Conversion and Management. Elsevier Ltd, 134, pp. 340-346. doi: $10.1016 /$ j.enconman.2016.12.052.

112. Fabris, I. et al. (2017) 'Continuous, self-sustaining smouldering destruction of simulated faeces', Fuel. The Authors, 190, pp. 58-66. doi: 10.1016/j.fuel.2016.11.014.

113. Yermán, L. et al. (2015) 'Smouldering combustion as a treatment technology for faeces: Exploring the parameter space', Fuel, 147, pp. 108-116. doi: 10.1016/j.fuel.2015.01.055.

114. Xue, W. et al. (2015) 'Seawater-driven forward osmosis for enriching nitrogen and phosphorous in treated municipal wastewater: Effect of membrane properties and feed solution chemistry', Water Research. Elsevier Ltd, 69, pp. 120-130. doi: 10.1016/j.watres.2014.11.007.

115. Zhang, F., Li, J. and He, Z. (2014) 'A new method for nutrients removal and recovery from wastewater using a bioelectrochemical system', Bioresource Technology. Elsevier Ltd, 166, pp. 630-634. doi: 10.1016/j. biortech.2014.05.105. 
116. Huang, H., Liu, J. and Ding, L. (2015) 'Recovery of phosphate and ammonia nitrogen from the anaerobic digestion supernatant of activated sludge by chemical precipitation', Journal of Cleaner Production. Elsevier Ltd, 102, pp. 437-446. doi: 10.1016/j.jclepro.2015.04.117.

117. Katehis, D., Diyamandoglu, V. and Fillos, J. (1998) 'Stripping and recovery of ammonia from centrate of anaerobically digested biosolids at elevated temperatures', Water Environment Research, 70(2), pp. 231-240. doi: 10.2175/106143098X127080.

118. Morales, N. et al. (2013) 'Recovery of N and P from urine by struvite precipitation followed by combined stripping with digester sludge liquid at full scale', Water (Switzerland), 5(3), pp. 1262-1278. doi: 10.3390/w5031262.

119. Wu, X. and Modin, O. (2013) 'Ammonium recovery from reject water combined with hydrogen production in a bioelectrochemical reactor', Bioresource Technology. Elsevier Ltd, 146, pp. 530-536. doi: 10.1016/j. biortech.2013.07.130.

120. Desloover, J. et al. (2012) 'Electrochemical resource recovery from digestate to prevent ammonia toxicity during anaerobic digestion', Environmental Science and Technology, 46(21), pp. 12209-12216. doi: 10.1021/ es3028154.

121. Smith, D. P. and Smith, N. T. (2016) 'Local-scale recovery of wastewater nitrogen for edible plant growth', Water Science and Technology, 73(6), pp. 1287-1292. doi: 10.2166/wst.2015.598.

122. Sancho, I. et al. (2017) 'Recovery of ammonia from domestic wastewater effluents as liquid fertilizers by integration of natural zeolites and hollow fibre membrane contactors', Science of the Total Environment, 584-585, pp. 244-251. doi: 10.1016/j.scitotenv.2017.01.123.

123. You, X. et al. (2017) 'Recovery of Ammonium by Powder Synthetic Zeolites from Wastewater Effluents: Optimization of the Regeneration Step', Water, Air, and Soil Pollution. Water, Air, \& Soil Pollution, 228(10). doi: $10.1007 / \mathrm{s} 11270-017-3577-0$.

124. Hermassi, M. et al. (2017) 'Recovery of nutrients (N-P-K) from potassium-rich sludge anaerobic digestion sidestreams by integration of a hybrid sorption-membrane ultrafiltration process: Use of powder reactive sorbents as nutrient carriers', Science of the Total Environment. Elsevier B.V., 599-600, pp. 422-430. doi: 10.1016/j.scitotenv.2017.04.140.

125. Liberti, L. et al. (1981) 'Nutrient removal and recovery from wastewater by ion exchange', Water Research, 15(3), pp. 337-342. doi: 10.1016/0043-1354(81)90038-5.

126. Liberti, L. et al. (1988) 'Field demonstrations of the rim-nut process for nutrients recovery from municipal wastewater', Nuclear and Chemical Waste Management, 8(1), pp. 83-86. doi: 10.1016/0191-815X(88)90011-3.

127. Liberti, L., Petruzzelli, D. and De Florio, L. (2001) 'Rem nut ion exchange plus struvite precipitation process', Environmental Technology (United Kingdom), 22(11), pp. 1313-1324. doi: 10.1080/09593330409355443.

128. Petruzzelli, D. et al. (2003) 'A new phosphate-selective sorbent for the Rem Nut ${ }^{\circledR}$ process. Laboratory investigation and field experience at a medium a wastewater treatment plant', Water Science and Technology, 48(1), pp. 179-184.

129. Petruzzelli, D. et al. (2004) 'A phosphate-selective sorbent for the REM NUT® process: field experience at Massafra Wastewater Treatment Plant', Reactive and Functional Polymers, 60, pp. 195-202. doi: 10.1016/j.reactfunctpolym.2004.02.023.

130. Guaya, D. et al. (2016) 'Recovery of ammonium and phosphate from treated urban wastewater by using potassium clinoptilolite impregnated hydrated metal oxides as N-P-K fertilizer', Journal of Environmental Chemical Engineering. Elsevier B.V., 4(3), pp. 3519-3526. doi: 10.1016/j.jece.2016.07.031.

131. Wang, H. et al. (2017) 'Eco-friendly synthesis of self-existed magnesium oxide supported nanorod-like palygorskite for enhanced and simultaneous recovery of nutrients from simulated wastewater through adsorption and in-situ struvite formation', Applied Clay Science. Elsevier B.V., 135, pp. 418-426. doi: 10.1016/j. clay.2016.10.027.

132. Wang, X. et al. (2016) 'Recovery of Ammonium and Phosphate from Wastewater by Wheat Straw-based Amphoteric Adsorbent and Reusing as a Multifunctional Slow-Release Compound Fertilizer', ACS Sustainable Chemistry and Engineering, 4(4), pp. 2068-2079. doi: 10.1021/acssuschemeng.5b01494.

133. Mishima, Y. and Araki, H. (2010) 'Phosphorus recovery from wastewater by hydrotalcite and zeolite', Lowland Technology International, 12(2), pp. 17-22.

134. Zhang, T. et al. (2013) 'Application of Biochar for Phosphate Adsorption and Recovery from Wastewater', Periodical Advanced Materials Research (Volumes 750 - 752), 750-752(August 2013), pp. 1-5. doi: 10.4028/www. scientific.net/AMR.750-752.1389.

135. Shepherd, J. G., Sohi, S. P. and Heal, K. V. (2016) 'Optimising the recovery and re-use of phosphorus from wastewater effluent for sustainable fertiliser development', Water Research. Elsevier Ltd, 94, pp. 155-165. doi: 10.1016/j.watres.2016.02.038. 
136. Dunkel, C. E., Shrestha, D. S. and Moller, G. (2016) 'Environmental assessment of phosphorus recovery from municipal waste water using N-E-W techTM system', 2016 ASABE Annual International Meeting, Orlando, Florida, USA, 17-20 July, 2016, p. 162460532. doi: 10.13031/aim.20162460532.

137. Moller, G. et al. (2016) 'N-E-W TECHTM: RESOURCE RECOVERY IN A NOVEL FUNCTIONALIZED BIOCHAR, CATALYTIC OXIDATION, REACTIVE FILTRATION WATER TREATMENT FOR N/P REMOVAL/RECOVERY AND PATHOGEN/PRIORITY SUBSTANCE DESTRUCTION', in 10th European Waste Water Management Conference.

138. Köse, T. E. and Kivanç, B. (2011) 'Adsorption of phosphate from aqueous solutions using calcined waste eggshell', Chemical Engineering Journal, 178, pp. 34-39. doi: 10.1016/j.cej.2011.09.129.

139. Sengupta, S. and Pandit, A. (2011) 'Selective removal of phosphorus from wastewater combined with its recovery as a solid-phase fertilizer', Water Research. Elsevier Ltd, 45(11), pp. 3318-3330. doi: 10.1016/j. watres.2011.03.044.

140. Kalaitzidou, K. et al. (2016) 'Pilot-Scale Phosphate Recovery from Secondary Wastewater Effluents', Environmental Processes. Environmental Processes, 3, pp. 5-22. doi: 10.1007/s40710-016-0139-1.

141. Midorikawa, I. et al. (2008) 'Recovery of high purity phosphorus from municipal wastewater secondary effluent by a high-speed adsorbent’, Water Science and Technology, 58(8), pp. 1601-1607. doi: 10.2166/wst.2008.537.

142. Bottini, A. and Rizzo, L. (2012) 'Phosphorus Recovery from Urban Wastewater Treatment Plant Sludge Liquor by Ion Exchange', Separation Science and Technology, 47(4), pp. 613-620. doi: 10.1080/01496395.2011.627904.

143. Suzuki, Y. et al. (2006) 'Evaluation of sludge reduction and phosphorus recovery efficiencies in a new advanced wastewater treatment system using denitrifying polyphosphate accumulating organisms', Water Science and Technology, 53(6), pp. 107-113. doi: 10.2166/wst.2006.179.

144. Biswas, B. K. et al. (2008) 'Removal and recovery of phosphorus from water by means of adsorption onto orange waste gel loaded with zirconium', Bioresource Technology, 99(18), pp. 8685-8690. doi: 10.1016/j. biortech.2008.04.015.

145. Ishiwata, T. et al. (2010) 'Removal and recovery of phosphorus in wastewater by superconducting High Gradient Magnetic Separation with ferromagnetic adsorbent', Physica C: Superconductivity and its Applications. Elsevier B.V., 470(20), pp. 1818-1821. doi: 10.1016/j.physc.2010.05.214.

146. Lee, S. H. et al. (2007) 'Phosphorus recovery by mesoporous structure material from wastewater', Water Science and Technology, 55(1-2), pp. 169-176. doi: 10.2166/wst.2007.052.

147. Xiao, X. et al. (2017) 'Phosphorus removal and recovery from secondary effluent in sewage treatment plant by magnetite mineral microparticles', Powder Technology. Elsevier B.V., 306, pp. 68-73. doi: 10.1016/j.powtec.2016.10.066.

148. Collinson, S. and Duplá García, O. (2013) 'Modification of Waste Carpet with Hydrated Ferric Oxide for Recycling as an Adsorbent Material to Recover Phosphate from Wastewater', Science of Advanced Materials, 5(10), pp. $1427-1435$

149. Ohura, S. et al. (2011) 'Phosphorus recovery from secondary effluent and side-stream liquid in a sewage treatment plant using zirconium-loaded saponified orange waste', Journal of Material Cycles and Waste Management, 13(4), pp. 293-297. doi: 10.1007/s10163-011-0029-6.

150. Urano, K. and Tachikawa, H. (1991b) 'Process Development for Removal and Recovery of Phosphorus from Wastewater by a New Adsorbent. 2. Adsorption Rates and Breakthrough Curves', Industrial and Engineering Chemistry Research, 30(8), pp. 1897-1899. doi: 10.1021/ie00056a033.

151. Urano, K. and Tachikawa, H. (1991a) 'Process Development for Removal and Recovery of Phosphorus from Wastewater by a New Adsorbent. 1. Preparation Method and Adsorption Capability of a New Adsorbent', Industrial and Engineering Chemistry Research, 30(8), pp. 1893-1896. doi: 10.1021/ie00056a032.

152. Urano, K. and Tachikawa, H. (1992) 'Process Development for Removal and Recovery of Phosphorus from Wastewater by a New Adsorbent. 3. Desorption of Phosphate and Regeneration of Adsorbent', Industrial and Engineering Chemistry Research, 31(6), pp. 1510-1513. doi: 10.1021/ie00006a012.

153. Urano, K., Tachikawa, H. and Kitajima, M. (1992) 'Process Development for Removal and Recovery of Phosphorus from Wastewater by a New Adsorbent. 4. Recovery of Phosphate and Aluminum from Desorbing Solution’, Industrial and Engineering Chemistry Research, 31(6), pp. 1513-1515. doi: 10.1021/ie00006a013.

154. Drenkova-Tuhtan, A. et al. (2017) 'Pilot-scale removal and recovery of dissolved phosphate from secondary wastewater effluents with reusable $\mathrm{ZnFeZr}$ adsorbent @ Fe3O4/SiO2particles with magnetic harvesting, Water Research. Elsevier Ltd, 109, pp. 77-87. doi: 10.1016/j.watres.2016.11.039.

155. Fang, L., Wu, B. and Lo, I. M. C. (2017) 'Fabrication of silica-free superparamagnetic ZrO2@Fe3O4 with enhanced phosphate recovery from sewage: Performance and adsorption mechanism', Chemical Engineering Journal Elsevier B.V., 319, pp. 258-267. doi: 10.1016/j.cej.2017.03.012. 
156. Wu, B. et al. (2017) 'Highly efficient and selective phosphate removal from wastewater by magnetically recoverable $\mathrm{La}(\mathrm{OH}) 3 / \mathrm{Fe} 3 \mathrm{O} 4$ nanocomposites', Water Research. Elsevier Ltd, 126, pp. 179-188. doi: 10.1016/j. watres.2017.09.034.

157. Nguyen, T. A. H. et al. (2015) 'Adsorption of phosphate from aqueous solutions and sewage using zirconium loaded okara (ZLO): Fixed-bed column study', Science of the Total Environment, 523, pp. 40-49. doi: 10.1016/j. scitotenv.2015.03.126.

158. Liu, H. et al. (2016) 'Effective adsorption of phosphate from aqueous solution by La-based metal-organic frameworks', RSC Advances. Royal Society of Chemistry, 6(107), pp. 105282-105287. doi: 10.1039/C6RA24568D.

159. Saha, B., Chakraborty, S. and Das, G. (2009) 'A mechanistic insight into enhanced and selective phosphate adsorption on a coated carboxylated surface', Journal of Colloid and Interface Science. Elsevier Inc., 331(1), pp. 21-26. doi: 10.1016/j.jcis.2008.11.007.

160. von Munch, E. and Barr, K. (2001) 'Controlled struvite crystallization for removing phosphorus from anaerobic digester side stream', Water Research, 35(1), pp. 151-159.

161. Xavier, L. D. et al. (2014) 'Study of the recovery of phosphorus from struvite precipitation in supernatant line from anaerobic digesters of sludge', Water Science and Technology: Water Supply, 14(5), pp. 751-757. doi: 10.2166/ws.2014.033.

162. Adnan, A. et al. (2004) 'Preliminary investigation into factors affecting controlled struvite crystallization at the bench scale', Journal of Environmental Engineering and Science, 3(3), pp. 195-202. doi: 10.1139/s03-082.

163. Bhuiyan, M. I. H., Mavinic, D. S. and Koch, F. A. (2008) 'Phosphorus recovery from wastewater through struvite formation in fluidized bed reactors: A sustainable approach', Water Science and Technology, 57(2), pp. 175-181. doi: 10.2166/wst.2008.002.

164. Cullen, N., Baur, R. and Schauer, P. (2013) 'Three years of operation of North America's first nutrient recovery facility', Water Science and Technology, 68(4), pp. 763-768. doi: 10.2166/wst.2013.260.

165. Fattah, K. P. et al. (2008) 'Determining the feasibility of phosphorus recovery as struvite from filter press centrate in a secondary wastewater treatment plant', Journal of Environmental Science and Health - Part A Toxic/Hazardous Substances and Environmental Engineering, 43(7), pp. 756-764. doi: 10.1080/10934520801960052.

166. Fattah, K. P. et al. (2010) 'Use of carbon dioxide stripping for struvite crystallization to save caustic dosage: performance at pilotscale operationPaper submitted to the Journal of Environmental Engineering and Science.', Canadian Journal of Civil Engineering, 37(9), pp. 1271-1275. doi: 10.1139/L10-055.

167. Fattah, K. P. and Mavinic, D. S. (2011) 'Modeling and optimization of struvite precipitation process for phosphorus recovery from wastewater', 2011 4th International Conference on Modeling, Simulation and Applied Optimization, ICMSAO 2011, pp. 2-7. doi: 10.1109/ICMSAO.2011.5775497.

168. Garcia-Belinchón, C. et al. (2013) 'Struvite recovery: Pilot-scale results and economic assessment of different scenarios', Water Practice and Technology, 8(1), pp. 119-130. doi: 10.2166/wpt.2013.013.

169. Ahn, Y. H. and Speece, R. E. (2006) 'A novel process for organic acids and nutrient recovery from municipal wastewater sludge', Water Science and Technology, 53(12), pp. 101-109. doi: 10.2166/wst.2006.411.

170. Xie, M. et al. (2014) 'Toward Resource Recovery from Wastewater: Phosphorus Extraction from Digested Sludge using Hybrid Forward Osmosis--Membrane Distillation Process', Environmental Science \& Technology Letters, 1, pp. 191-195. doi: 10.1021/ez400189z.

171. Pastor, L. et al. (2008) 'Sewage sludge management for phosphorus recovery as struvite in EBPR wastewater treatment plants', Bioresource Technology, 99(11), pp. 4817-4824. doi: 10.1016/j.biortech.2007.09.054.

172. Soares, A. et al. (2014) 'Bio-Struvite: A new route to recover phosphorus from wastewater', Clean - Soil, Air, Water, 42(7), pp. 994-997. doi: 10.1002/clen.201300287.

173. Qiu, G. and Ting, Y. P. (2014) 'Direct phosphorus recovery from municipal wastewater via osmotic membrane bioreactor (OMBR) for wastewater treatment’, Bioresource Technology. Elsevier Ltd, 170, pp. 221-229. doi: 10.1016/j.biortech.2014.07.103.

174. Qiu, G. et al. (2015) 'Direct and complete phosphorus recovery from municipal wastewater using a hybrid microfiltration-forward osmosis membrane bioreactor process with seawater brine as draw solution', Environmental Science and Technology, 49(10), pp. 6156-6163. doi: 10.1021/es504554f.

175. Qiu, G. et al. (2016a) 'The potential of hybrid forward osmosis membrane bioreactor (FOMBR) processes in achieving high throughput treatment of municipal wastewater with enhanced phosphorus recovery', Water Research. Elsevier Ltd, 105, pp. 370-382. doi: 10.1016/j.watres.2016.09.017.

176. Qiu, G. et al. (2016b) 'Towards high through-put biological treatment of municipal wastewater and enhanced phosphorus recovery using a hybrid microfiltration-forward osmosis membrane bioreactor with hydraulic retention time in sub-hour level', Bioresource Technology. Elsevier Ltd, 219, pp. 298-310. doi: 10.1016/j. biortech.2016.07.126. 
177. Takiguchi, N. et al. (2004) 'A laboratory-scale test of anaerobic digestion and methane production after phosphorus recovery from waste activated sludge', Journal of Bioscience and Bioengineering, 97(6), pp. 365-368. doi: 10.1016/S1389-1723(04)70219-4.

178. Dai, H. et al. (2016) 'An efficient approach for phosphorus recovery from wastewater using series-coupled air-agitated crystallization reactors', Chemosphere. Elsevier Ltd, 165, pp. 211-220. doi: 10.1016/j.chemosphere.2016.09.001.

179. Angel, R. (1999) 'Removal of Phosphate from Sewage as Amorphous Calcium Phosphate', Environmental Technology, 20(7), pp. 709-720. doi: 10.1080/09593332008616865.

180. Berg, U. et al. (2006) 'Phosphorus removal and recovery from wastewater by tobermorite-seeded crystallisation of calcium phosphate', Water Science and Technology, 53(3), pp. 131-138. doi: 10.2166/wst.2006.084.

181. Berg, U. et al. (2007) 'P-RoC-Phosphorus recovery from wastewater by crystallisation of calcium phosphate compounds', Journal of Residuals Science \& Technology, 4(3), pp. 121-126.

182. Ehbrecht, A. et al. (2011) 'P-Recovery fromsewage by seeded crystallisation in a pilot plant in batch mode technology', Water Science and Technology, 63(2), pp. 339-344. doi: 10.2166/wst.2011.061.

183. Okano, K. et al. (2013) 'Novel technique for phosphorus recovery from aqueous solutions using amorphous calcium silicate hydrates (A-CSHs)', Water Research. Elsevier Ltd, 47(7), pp. 2251-2259. doi: 10.1016/j. watres.2013.01.052.

184. Okano, K., Yamamoto, Y., et al. (2016) 'A simple technology for phosphorus recovery using acid-treated concrete sludge', Separation and Purification Technology. Elsevier B.V., 165, pp. 173-178. doi: 10.1016/j.seppur.2016.03.054.

185. Okano, K., Miyamaru, S., et al. (2016) 'A mobile pilot-scale plant for in situ demonstration of phosphorus recovery from wastewater using amorphous calcium silicate hydrates', Separation and Purification Technology. Elsevier B.V., 170, pp. 116-121. doi: 10.1016/j.seppur.2016.06.040.

186. Rensink, J. H., Eggers, E. and Donker, H. J. G. W. (1991) 'High Biological Nutrient Removal From Domestic Waste-Water inCombination With Phosphorus Recycling', Water. Science and. Technology., 23(4-6), pp. 651-657.

187. Kim, E. H. et al. (2006) 'Recovery of phosphates from wastewater using converter slag: Kinetics analysis of a completely mixed phosphorus crystallization process', Chemosphere, 63(2), pp. 192-201. doi: 10.1016/j. chemosphere.2005.08.029.

188. Iizuka, A. et al. (2012) 'Phosphorus adsorbent derived from concrete sludge (PAdeCS) and its phosphorus recovery performance', Industrial and Engineering Chemistry Research, 51(34), pp. 11266-11273. doi: 10.1021/ ie 301225 g.

189. Lin, L. et al. (2017) 'Recovery of organic carbon and phosphorus from wastewater by Fe-enhanced primary sedimentation and sludge fermentation', Process Biochemistry. Elsevier Ltd, 54, pp. 135-139. doi: 10.1016/j. procbio.2016.12.016.

190. Nakao, S., Nishio, T. and Kanjo, Y. (2017) 'Simultaneous recovery of phosphorus and potassium as magnesium potassium phosphate from synthetic sewage sludge effluent', Environmental Technology (United Kingdom). Taylor \& Francis, 38(19), pp. 2416-2426. doi: 10.1080/09593330.2016.1264485.

191. Huang, L., Lee, D.-J. and Lai, J.-Y. (2015) 'Forward osmosis membrane bioreactor for wastewater treatment with phosphorus recovery', Bioresource Technology. Elsevier Ltd, 198, pp. 418-423. doi: 10.1016/j. biortech.2015.09.045.

192. El-Shafai, S. A. et al. (2007) 'Nitrogen recovery in an integrated system for wastewater treatment and tilapia production', Environmentalist, 27(2), pp. 287-302. doi: 10.1007/s10669-007-9005-z.

193. Nasr, F. A., Doma, H. S. and Nassar, H. F. (2009) 'Treatment of domestic wastewater using an anaerobic baffled reactor followed by a duckweed pond for agricultural purposes', Environmentalist, 29(3), pp. 270-279. doi: 10.1007/s10669-008-9188-y.

194. Craggs, R. et al. (2014) 'High rate algal pond systems for low-energy wastewater treatment, nutrient recovery and energy production', New Zealand Journal of Botany. Taylor \& Francis, 52(1), pp. 60-73. doi: 10.1080/0028825X.2013.861855.

195. Shelef, G., Azov, Y. and Moraine, R. (1982) 'Nutrients Remov Al and Recovery in a Two-Stage High Rate Algal W Astew Ater Treatment System'.

196. Voltolina, D., Gómez-Villa, H. and Correa, G. (2005) 'Nitrogen removal and recycling by Scenedesmus obliquus in semicontinuous cultures using artificial wastewater and a simulated light and temperature cycle', Bioresource Technology, 96(3), pp. 359-362. doi: 10.1016/j.biortech.2004.04.004.

197. Rusten, B. and Sahu, A. K. (2011) 'Microalgae growth for nutrient recovery from sludge liquor and production of renewable bioenergy', Water Science and Technology, 64(6), pp. 1195-1201. doi: 10.2166/wst.2011.722.

198. Aida, T. M. et al. (2016) 'Nutrient recovery from municipal sludge for microalgae cultivation with two-step hydrothermal liquefaction', Algal Research. Elsevier B.V., 18, pp. 61-68. doi: 10.1016/j.algal.2016.06.009. 
199. Wang, H. et al. (2016) 'A novel PSB-EDI system for high ammonia wastewater treatment, biomass production and nitrogen resource recovery: PSB system', Water Science and Technology, 74(3), pp. 616-624. doi: 10.2166/ wst.2016.254.

200. Valverde-Pérez, B., Ramin, E., et al. (2015) 'EBP2R - An innovative enhanced biological nutrient recovery activated sludge system to produce growth medium for green microalgae cultivation', Water Research, 68, pp. 821-830. doi: 10.1016/j.watres.2014.09.027.

201. Valverde-Pérez, B., Fuentes-Martínez, J. M., et al. (2015) Control Structure Design of an Innovative Enhanced Biological Nutrient Recovery Activated Sludge System Coupled with a Photobioreactor, 12th International Symposium on Process Systems Engineering and 25th European Symposium on Computer Aided Process Engineering. Elsevier. doi: http://dx.doi.org/10.1016/B978-0-444-63576-1.50120-5.

202. Valverde-Pérez, B. et al. (2016) 'Control structure design for resource recovery using the enhanced biological phosphorus removal and recovery (EBP2R) activated sludge process', Chemical Engineering Journal, 296, pp. 447-457. doi: 10.1016/j.cej.2016.03.021.

203. Kim, J. et al. (2016) 'Efficient recovery of nitrate and phosphate from wastewater by an amine-grafted adsorbent for cyanobacterial biomass production', Bioresource Technology. Elsevier Ltd, 205, pp. 269-273. doi: 10.1016/j. biortech.2016.01.055.

204. Markou, G. et al. (2015) 'Cultivation of Chlorella vulgaris and Arthrospira platensis with recovered phosphorus from wastewater by means of zeolite sorption', International Journal of Molecular Sciences, 16(2), pp. 4250-4264. doi: 10.3390/ijms16024250.

205. Nordin, A. C., Olsson, J. and Vinnerås, B. (2015) 'Urea for Sanitization of Anaerobically Digested Dewatered Sewage Sludge’, Environmental Engineering Science, 32(2), pp. 86-94. doi: 10.1089/ees.2013.0230.

206. Randall, C. W. (2003) 'Potential societal and economic impacts of wastewater nutrient removal and recycling', Water Science and Technology, 48(1), pp. 11-17.

207. Gajurel, D. et al. (2007) 'Potential of filter-vermicomposter for household wastewater pre-treatment and sludge sanitisation on site', in Water Science and Technology, pp. 65-70. doi: 10.2166/wst.2007.128.

208. Liu, J. et al. (2009) 'Ceramsite-vermifilter for domestic wastewater treatment and reuse: An option for rural agriculture', in 2009 International Conference on Energy and Environment Technology, ICEET 2009, pp. 472-475. doi: 10.1109/ICEET.2009.352.

209. He, X. et al. (2016) 'Effect of vermicomposting on concentration and speciation of heavy metals in sewage sludge with additive materials', Bioresource Technology. Elsevier Ltd, 218, pp. 867-873. doi: 10.1016/j. biortech.2016.07.045.

210. Yang, J. et al. (2014) 'Insight into the roles of earthworm in vermicomposting of sewage sludge by determining the water-extracts through chemical and spectroscopic methods', Bioresource Technology. Elsevier Ltd, 154, pp. 94-100. doi: 10.1016/j.biortech.2013.12.023.

211. Bridle, T. and Pritchard, D. (2004) 'Energy and nutrient recovery from sewage sludge via pyrolysis', Water Science Technololgy, 50(February), pp. 169-175.

212. Li, R. et al. (2017) 'Potential recovery of phosphorus during the fluidized bed incineration of sewage sludge', Journal of Cleaner Production. Elsevier Ltd, 140, pp. 964-970. doi: 10.1016/j.jclepro.2016.06.177.

213. Heinzmann, B. and Engel, G. (2007) 'Induced Magnesium Ammonia Phosphate Precipitation to Prevent Incrustations and Measures for Phosphorus Recovery', Moving Forward. Wastewater Biosolids Sustainability: Technical, Managerial, and Public Synergy, pp. 511-518. doi: 10.2166/WPT.2006051.

214. Marchi, A. et al. (2015) 'Full-scale phosphorus recovery from digested waste water sludge in Belgium - Part I: Technical achievements and challenges', Water Science and Technology, 71(4), pp. 487-494. doi: 10.2166/ wst.2015.023.

215. Zhang, C. and Chen, Y. (2009) 'Simultaneous nitrogen and phosphorus recovery from sludge-fermentation liquid mixture and application of the fermentation liquid to enhance municipal wastewater biological nutrient removal', Environmental Science and Technology, 43(16), pp. 6164-6170. doi: 10.1021/es9005948.

216. Shimamura, K. et al. (2008) 'Development of a process for the recovery of phosphorus resource from digested sludge by crystallization technology', Water Science and Technology, 57(3), pp. 451-456. doi: 10.2166/ wst.2008.065.

217. Petzet, S. and Cornel, P. (2012) 'Prevention of Struvite Scaling in Digesters Combined With Phosphorus Removal and Recovery-The FIX-Phos Process', Water Environment Research, 84(3), pp. 220-226. doi: Doi 10.2175/ $106143012 \times 13347678384125$.

218. Zheng, W. et al. (2013) 'Remove and recover phosphorus during anaerobic digestion of excess sludge by adding waste iron scrap', Journal of the Serbian Chemical Society, 78(2), pp. 303-312. doi: 10.2298/JSC120205057Z.

219. Horttanainen, M., Deviatkin, I. and Havukainen, J. (2017) 'Nitrogen release from mechanically dewatered sewage sludge during thermal drying and potential for recovery', Journal of Cleaner Production. Elsevier Ltd, 142, pp. 1819-1826. doi: 10.1016/j.jclepro.2016.11.102. 
220. Wang, X. et al. (2013) 'Phosphate recovery from excess sludge by conventional electrodialysis (CED) and electrodialysis with bipolar membranes (EDBM)', Industrial and Engineering Chemistry Research, 52(45), pp. 15896-15904. doi: 10.1021/ie4014088.

221. Dai, H. et al. (2017) 'Effects of supersaturation control strategies on hydroxyapatite (HAP) crystallization for phosphorus recovery from wastewater', Environmental Science and Pollution Research. Environmental Science and Pollution Research, 24(6), pp. 5791-5799. doi: 10.1007/s11356-016-8236-2.

222. Saktaywin, W. et al. (2006) 'Operation of a new sewage treatment process with technologies of excess sludge reduction and phosphorus recovery', in Water Science and Technology, pp. 217-227. doi: 10.2166/wst.2006.424.

223. Xia, C. W. et al. (2014) 'A novel approach for phosphorus recovery and No wasted sludge in enhanced biological phosphorus removal process with external COD addition', Applied Biochemistry and Biotechnology, 172(2), pp. 820-828. doi: 10.1007/s12010-013-0575-6.

224. Yan, P. et al. (2013b) 'Pilot-scale test of an advanced, integrated wastewater treatment process with sludge reduction, inorganic solids separation, phosphorus recovery, and enhanced nutrient removal (SIPER)', Bioresource Technology. Elsevier Ltd, 142, pp. 483-489. doi: 10.1016/j.biortech.2013.05.071.

225. Yan, P. et al. (2013a) 'Evaluation of sludge reduction and carbon source recovery from excess sludge by the advanced Sludge reduction, Inorganic solids separation, Phosphorus recovery, and Enhanced nutrient Removal (SIPER) wastewater treatment process', Bioresource Technology. Elsevier Ltd, 150, pp. 344-351. doi: 10.1016/j.biortech.2013.10.038.

226. Yan, P., Ji, F. Y., et al. (2015) 'Investigation into cyclic utilization of carbon source in an advanced sludge reduction, inorganic solids separation, phosphorus recovery, and enhanced nutrient removal (SIPER) wastewater treatment process', Water Science and Technology, 72(9), pp. 1628-1634. doi: 10.2166/wst.2015.382.

227. Yan, P., Guo, J. S., et al. (2015) 'Enhanced nitrogen and phosphorus removal by an advanced simultaneous sludge reduction, inorganic solids separation, phosphorus recovery, and enhanced nutrient removal wastewater treatment process', Bioresource Technology. Elsevier Ltd, 183, pp. 181-187. doi: 10.1016/j.biortech.2015.02.070.

228. Zou, H. and Lu, X. (2016) 'An innovative continuous flow BNR-IC process for nutrients removal and phosphorus recovery from synthetic and real domestic wastewater', Journal of Environmental Engineering and Landscape Management, 24(2), pp. 116-123. doi: 10.3846/16486897.2015.1113180.

229. Zou, H. and Wang, Y. (2016) 'Phosphorus removal and recovery from domestic wastewater in a novel process of enhanced biological phosphorus removal coupled with crystallization', Bioresource Technology. Elsevier Ltd, 211, pp. 87-92. doi: 10.1016/j.biortech.2016.03.073.

230. Zou, H., Lu, X. and Li, T. (2014) 'A novel process for nutrients removal and phosphorus recovery from domestic wastewater by combining BNR with induced crystallization', Journal of Environmental Engineering and Landscape Management, 22(4), pp. 274-283. doi: 10.3846/16486897.2014.931284.

231. Mejia Likosova, E. et al. (2013) 'Understanding colloidal FeSx formation from iron phosphate precipitation sludge for optimal phosphorus recovery', Journal of Colloid and Interface Science. Elsevier Inc., 403, pp. 16-21. doi: 10.1016/j.jcis.2013.04.001.

232. Stendahl, K. and Jäfverström, S. (2004) 'Recycling of sludge with the Aqua Reci process', Water Science and Technology, 49(1), pp. 233-240.

233. Guilayn, F. et al. (2017) 'Sequencing biological acidification of waste-activated sludge aiming to optimize phosphorus dissolution and recovery', Environmental Technology (United Kingdom), 38(11), pp. 1399-1407. doi: 10.1080/09593330.2016.1230653.

234. Niewersch, C. et al. (2008) 'Potentials of using nanofiltration to recover phosphorus from sewage sludge', Water Science and Technology, 57(5), pp. 707-714. doi: 10.2166/wst.2008.119.

235. Thong, Z. et al. (2016) 'Molecular Design of Nanofiltration Membranes for the Recovery of Phosphorus from Sewage Sludge', ACS Sustainable Chemistry and Engineering, 4(10), pp. 5570-5577. doi: 10.1021/acssuschemeng.6b01299.

236. Güney, K., Weidelener, A. and Krampe, J. (2008) 'Phosphorus recovery from digested sewage sludge as MAP by the help of metal ion separation', Water Research, 42(18), pp. 4692-4698. doi: 10.1016/j.watres.2008.08.016.

237. Levlin, E. et al. (2002) 'Effects of phosphorus recovery requirements on Swedish sludge management', Water Science and Technology, 46(4-5), pp. 435-440.

238. Antakyali, D. et al. (2013) 'Large-scale application of nutrient recovery from digested sludge as struvite', Water Practice and Technology, 8(2), pp. 256-262. doi: 10.2166/wpt.2013.027.

239. Guedes, P. et al. (2016) 'Electrodialytic treatment of sewage sludge: Current intensity influence on phosphorus recovery and organic contaminants removal', Chemical Engineering Journal. Elsevier B.V., 306, pp. 1058-1066. doi: $10.1016 /$ j.cej.2016.08.040.

240. Wang, Y. et al. (2016) 'Effect of organic matter on phosphorus recovery from sewage sludge subjected to microwave hybrid pretreatment', Journal of Environmental Sciences (China). Elsevier B.V., 39, pp. 29-36. doi: 10.1016/j.jes.2015.10.008. 
241. Wang, Y. et al. (2017) 'Microwave-assisted digestion and $\mathrm{NaOH}$ treatment of waste-activated sludge to recover phosphorus by crystallizing struvite', Environmental Technology (United Kingdom), 38(10), pp. 1211-1222. doi: 10.1080/09593330.2016.1220630.

242. Bi, W., Li, Y. and Hu, Y. (2014) 'Recovery of phosphorus and nitrogen from alkaline hydrolysis supernatant of excess sludge by magnesium ammonium phosphate’, Bioresource Technology. Elsevier Ltd, 166, pp. 1-8. doi: 10.1016/j.biortech.2014.04.092.

243. Wang, Y. et al. (2014) 'Nutrient release, recovery and removal from waste sludge of a biological nutrient removal system', Environmental Technology (United Kingdom), 35(21), pp. 2734-2742. doi: 10.1080/09593330.2014.920048.

244. Chen, Y. et al. (2013) 'Efficient recovery of carbon, nitrogen, and phosphorus from waste activated sludge', Water Science \& Technology, 68(4), p. 916. doi: 10.2166/wst.2013.331.

245. Tao, B. et al. (2016) 'Recovery and concentration of thermally hydrolysed waste activated sludge derived volatile fatty acids and nutrients by microfiltration, electrodialysis and struvite precipitation for polyhydroxyalkanoates production', Chemical Engineering Journal. Elsevier B.V., 295, pp. 11-19. doi: 10.1016/j.cej.2016.03.036.

246. Fischer, F. et al. (2011) 'Microbial fuel cell enables phosphate recovery from digested sewage sludge as struvite', Bioresource Technology. Elsevier Ltd, 102(10), pp. 5824-5830. doi: 10.1016/j.biortech.2011.02.089.

247. Happe, M. et al. (2016) 'Scale-up of phosphate remobilization from sewage sludge in a microbial fuel cell', Bioresource Technology. Elsevier Ltd, 200, pp. 435-443. doi: 10.1016/j.biortech.2015.10.057.

248. Qiang, Z. et al. (2015) 'Operation performance of an A/A/O process coupled with excess sludge ozonation and phosphorus recovery: A pilot-scale study’, Chemical Engineering Journal. Elsevier B.V., 268, pp. 162-169. doi: 10.1016/j.cej.2015.01.054.

249. Saktaywin, W. et al. (2005) 'Advanced sewage treatment process with excess sludge reduction and phosphorus recovery', Water Research, 39(5), pp. 902-910. doi: 10.1016/j.watres.2004.11.035.

250. Donatello, S., Tong, D. and Cheeseman, C. R. (2010) 'Production of technical grade phosphoric acid from incinerator sewage sludge ash (ISSA)', Waste Management. Elsevier Ltd, 30(8-9), pp. 1634-1642. doi: 10.1016/j. wasman.2010.04.009.

251. Gorazda, K. et al. (2017) 'Fertilisers production from ashes after sewage sludge combustion - A strategy towards sustainable development', Environmental Research. Elsevier, 154(July 2016), pp. 171-180. doi: 10.1016/j. envres.2017.01.002.

252. Kleemann, R. et al. (2017) 'Comparison of phosphorus recovery from incinerated sewage sludge ash (ISSA) and pyrolysed sewage sludge char (PSSC)', Waste Management. Elsevier Ltd, 60, pp. 201-210. doi: 10.1016/j. wasman.2016.10.055.

253. Lee, M. and Kim, D. J. (2017) 'Identification of phosphorus forms in sewage sludge ash during acid pre-treatment for phosphorus recovery by chemical fractionation and spectroscopy', Journal of Industrial and Engineering Chemistry. The Korean Society of Industrial and Engineering Chemistry, 51, pp. 64-70. doi: 10.1016/j. jiec.2017.02.013.

254. Pettersson, A., Åmand, L. E. and Steenari, B. M. (2008) 'Leaching of ashes from co-combustion of sewage sludge and wood-Part II: The mobility of metals during phosphorus extraction', Biomass and Bioenergy, 32(3), pp. 236-244. doi: 10.1016/j.biombioe.2007.09.006.

255. Hong, K. J. et al. (2005) 'Study on the recovery of phosphorus from waste-activated sludge incinerator ash', Journal of Environmental Science and Health - Part A Toxic/Hazardous Substances and Environmental Engineering, 40(3), pp. 617-631. doi: 10.1081/ESE-200046614.

256. Sturm, G. et al. (2010) 'Electrokinetic phosphorus recovery from packed beds of sewage sludge ash: Yield and energy demand', in Journal of Applied Electrochemistry, pp. 1069-1078. doi: 10.1007/s10800-009-0061-6.

257. Schaum, C., Cornel, P. and Jardin, N. (2007) 'Phosphorus recovery from sewage sludge ash-a wet chemical approach', Proceeding of the IWA Conference on ..., pp. 583-590.

258. Guedes, P. et al. (2014) 'Phosphorus recovery from sewage sludge ash through an electrodialytic process', Waste Management. Elsevier Ltd, 34(5), pp. 886-892. doi: 10.1016/j.wasman.2014.02.021.

259. Parés Viader, R., Jensen, P. E., Ottosen, L. M., Thomsen, T. P., et al. (2017) 'Comparison of phosphorus recovery from incineration and gasification sewage sludge ash', Water Science and Technology, 75(5), pp. 1251-1260. doi: 10.2166/wst.2016.620.

260. Parés Viader, R., Jensen, P. E., Ottosen, L. M., Ahrenfeldt, J., et al. (2017) 'Sequential electrodialytic recovery of phosphorus from low-temperature gasification ashes of chemically precipitated sewage sludge', Waste Management, 60, pp. 211-218. doi: 10.1016/j.wasman.2016.11.030.

261. Lim, B. H. and Kim, D. J. (2017) 'Selective acidic elution of Ca from sewage sludge ash for phosphorus recovery under $\mathrm{pH}$ control', Journal of Industrial and Engineering Chemistry. The Korean Society of Industrial and Engineering Chemistry, 46, pp. 62-67. doi: 10.1016/j.jiec.2016.10.016.

262. Petzet, S. et al. (2011) 'Recovery of phosphorus and aluminium from sewage sludge ash by a new wet chemical elution process (SESAL-Phos-recovery process)', Water Science and Technology, 64(3), pp. 693-699. doi: 
10.2166/wst.2011.682.

263. Petzet, S., Peplinski, B. and Cornel, P. (2012) 'On wet chemical phosphorus recovery from sewage sludge ash by acidic or alkaline leaching and an optimized combination of both', Water Research. Elsevier Ltd, 46(12), pp. 3769-3780. doi: 10.1016/j.watres.2012.03.068.

264. Acelas, N. Y. et al. (2014) 'Supercritical water gasification of sewage sludge: Gas production and phosphorus recovery', Bioresource Technology. Elsevier Ltd, 174, pp. 167-175. doi: 10.1016/j.biortech.2014.10.003.

265. Atienza-Martínez, M. et al. (2014) 'Phosphorus recovery from sewage sludge char ash', Biomass and Bioenergy, 65, pp. 42-50. doi: 10.1016/j.biombioe.2014.03.058.

266. Liu, Y. and Qu, H. (2016) 'Design and optimization of a reactive crystallization process for high purity phosphorus recovery from sewage sludge ash', Journal of Environmental Chemical Engineering. Elsevier B.V., 4(2), pp. 2155-2162. doi: 10.1016/j.jece.2016.03.042.

267. Liu, Y. and Qu, H. (2015) PAT for Reactive Crystallization Process Optimization for Phosphorus Recovery from Sewage Sludge, Computer Aided Chemical Engineering. Elsevier. doi: 10.1016/B978-0-444-63577-8.50107-8

268. Xu, H. et al. (2012) 'Recovery of phosphorus as struvite from sewage sludge ash', Journal of Environmental Sciences (China). The Research Centre for Eco-Environmental Sciences, Chinese Academy of Sciences, 24(8), pp. 1533-1538. doi: 10.1016/S1001-0742(11)60969-8.

269. Zhai, Y. et al. (2014) 'Recovery of phosphorus from sewage sludge in combination with the supercritical water process', Water Science and Technology, 70(6), pp. 1108-1114. doi: 10.2166/wst.2014.344.

270. Adam, C. et al. (2008) 'Phosphorrückgewinnung aus Klärschlammaschen - Ergebnisse aus dem EU-Projekt SUSAN', Osterreichische Wasser- und Abfallwirtschaft, 60(3-4), pp. 55-64. doi: 10.1007/s00506-008-0152-3.

271. Adam, C. et al. (2009) 'Thermochemical treatment of sewage sludge ashes for phosphorus recovery', Waste Management, 29(3), pp. 1122-1128. doi: 10.1016/j.wasman.2008.09.011.

272. Cordova Udaeta, M. et al. (2017) 'Recovery of phosphorus from Sewage Sludge Ash (SSA) by heat treatment followed by high gradient magnetic separation and flotation', Advanced Powder Technology, 28(3), pp. 755-762. doi: 10.1016/j.apt.2016.11.023.

273. Herzel, H. et al. (2016) 'Sewage sludge ash - A promising secondary phosphorus source for fertilizer production', Science of the Total Environment. Elsevier B.V., 542, pp. 1136-1143. doi: 10.1016/j.scitotenv.2015.08.059.

274. Schönberg, A. et al. (2014) 'Der RecoPhos-Prozess - Rückgewinnung von Phosphor aus KIärschlammasche', Osterreichische Wasser- und Abfallwirtschaft, 66(11-12), pp. 403-407. doi: 10.1007/s00506-014-0193-8.

275. Mocker, M., Löh, I. and Stenzel, F. (2010) 'Wertstoffgewinnung aus Verbrennungsrückständen', Chemie-Ingenieur-Technik, 82(11), pp. 1977-1983. doi: 10.1002/cite.201000130. 


\section{SUPPORTING INFORMATION IV}

\section{Recycling Nutrients Contained in Human Excreta to Agriculture: Pathways, Processes, and Products.}

\section{Developing the Option Space for Nutrient Recovery}

This Supporting Information (SI) describes the step-wise development of the option space for nutrient recovery.

This SI contains 2 tables, 2 figures, and 5 pages.

Table S4.1 Description of key mineral precipitates that can be found in fertiliser products rendered by treatment of human excreta and streams containing human excreta.

Table S4.2 Typical sorbents that have been used to extract nutrients from liquid streams during treatment of human excreta and streams containing human excreta.

Figure S4.1 Development of the option space.

Figure S4.2 Pathways from primary input to product (simplified representation). 


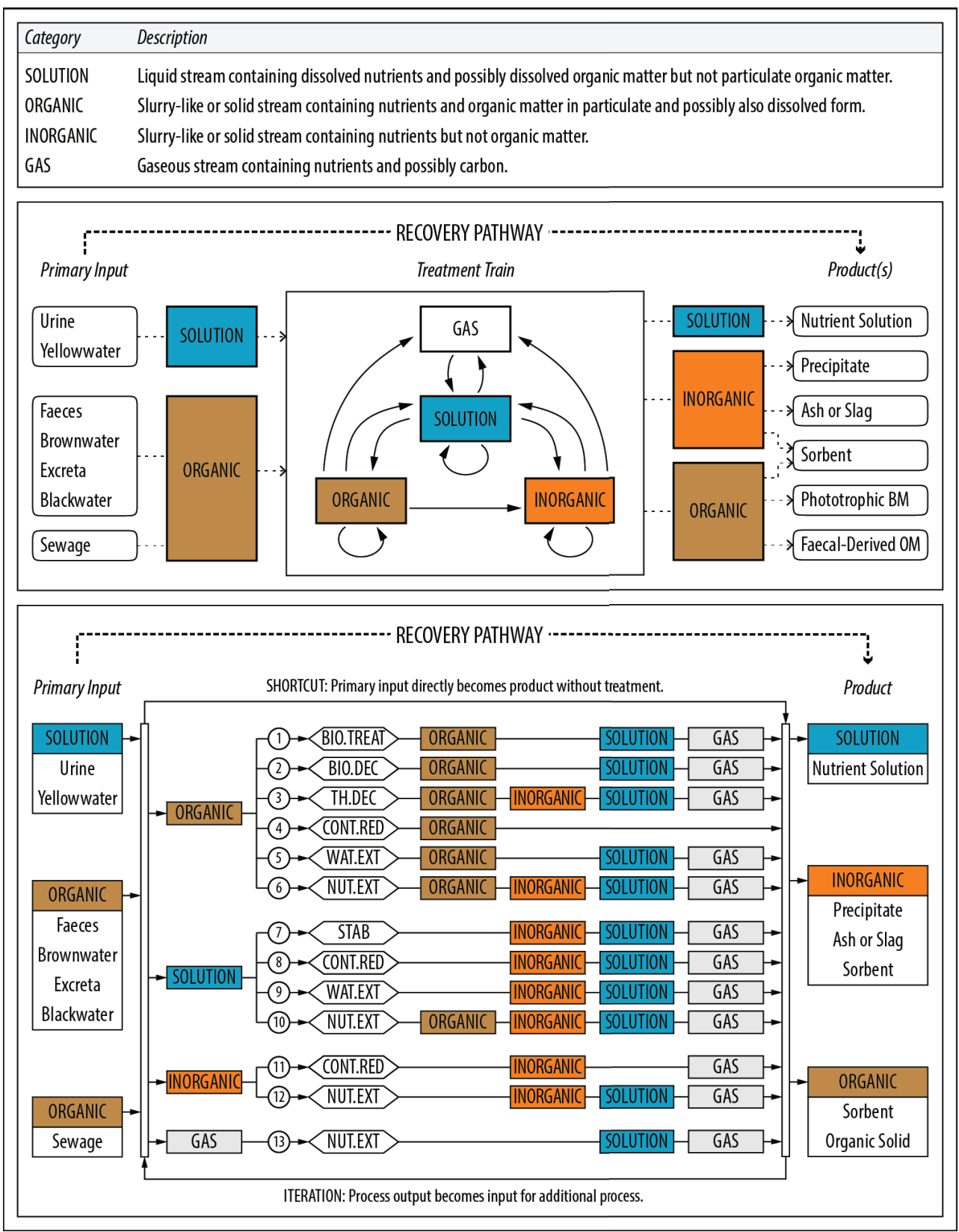

Figure S4.1 Development of the option space. (a) Categories of similar process inputs and outputs. (b) How treatment processes can convent an input belonging to one category to an output belonging to the same or a different category. (c) Refined input-output model that forms the backbone of the option space for recovery pathways shown in Figure 4 in the main manuscript. 

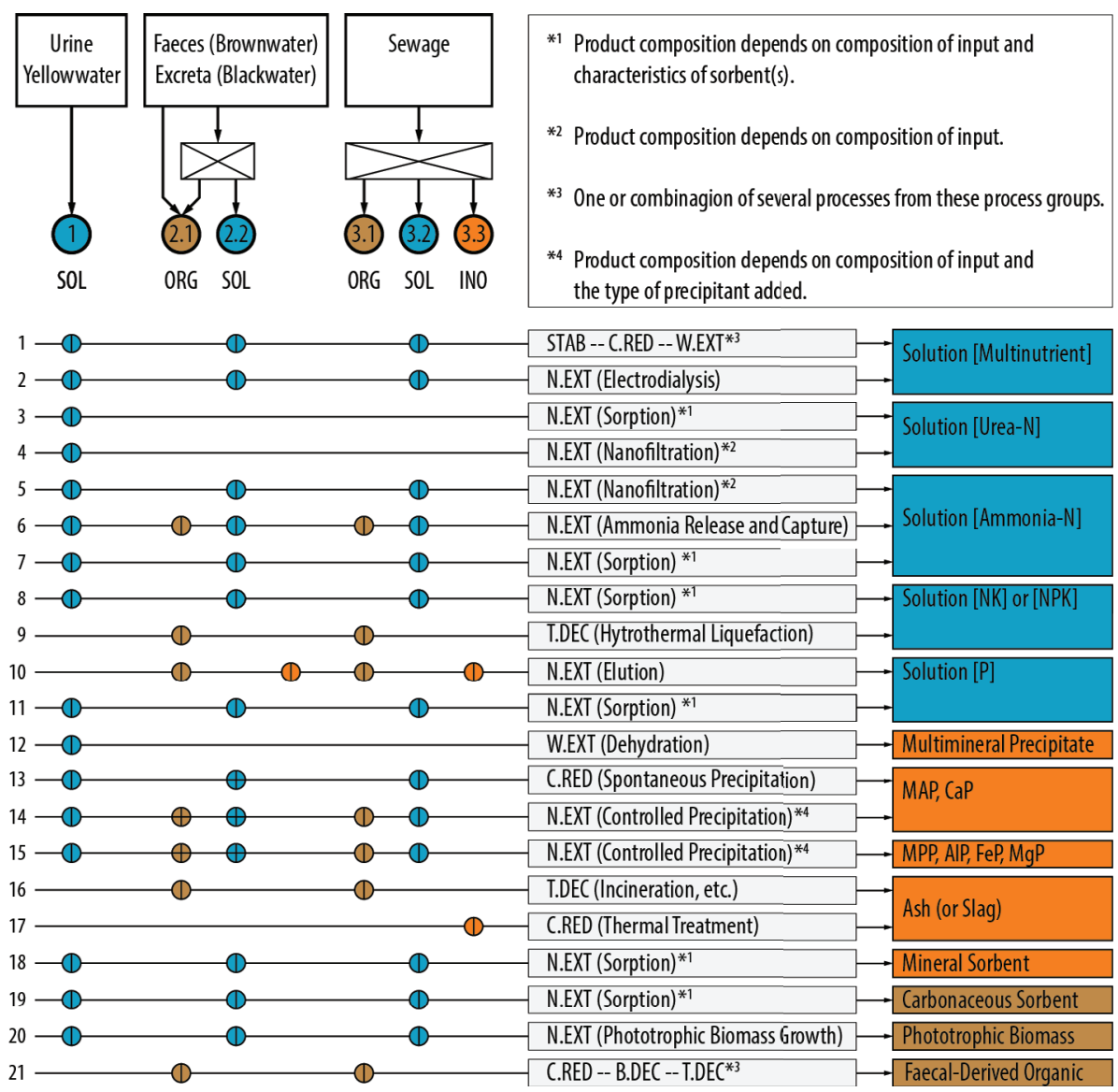

Figure S4.2 Pathways from primary input to product (simplified representation). 
Table S4.1 Description of key mineral precipitates that can be found in fertiliser products rendered by treatment of human excreta and streams containing human excreta.

\begin{tabular}{|c|c|}
\hline Precipitate & Description \\
\hline MAP & $\begin{array}{l}\text { Struvite is a phosphate mineral with the formula } \mathrm{MgNH}_{4} \mathrm{PO}_{4} \cdot 6 \mathrm{H}_{2} \mathrm{O} \text { and consists of } \\
\text { magnesium }\left(\mathrm{Mg}^{2+}\right) \text {, ammonium }\left(\mathrm{NH}_{4}^{+}\right) \text {, and orthophosphate }\left(\mathrm{PO}_{4}^{3-}\right) \text { in equal molar } \\
\text { concentrations. Struvite is also commonly referred to as magnesium ammonium phosphate } \\
\text { hexahydrate, or simply magnesium ammonium phosphate (MAP). }\end{array}$ \\
\hline MPP & $\begin{array}{l}\text { Several struvite analogues or struvite-type compounds exist, which together with struvite } \\
\text { form the struvite group (1-3). The general formula for minerals of the struvite group is } \\
{[A][M]\left[\mathrm{XO}_{4}\right] \cdot n \mathrm{H}_{2} \mathrm{O} \text {, where: } A=\mathrm{NH}_{4}, \mathrm{~K}, \mathrm{Na}, \mathrm{Tl}, \mathrm{Rb} ; M=\mathrm{Mg}, \mathrm{Co}, \mathrm{Ni} ; \mathrm{X}=\mathrm{P}, \text { As; and } n=} \\
6,7 \text {. The struvite analogue with the formula } \mathrm{MgKPO}_{4} \cdot 6 \mathrm{H}_{2} \mathrm{O} \text { is of most interest in the } \\
\text { context of nutrient recovery from human excreta }(4,5) \text {. It is commonly referred to as } \\
\text { magnesium potassium phosphate hexahydrate, or simply magnesium potassium phosphate } \\
(\mathrm{MPP}) \text {. }\end{array}$ \\
\hline Ca-P & $\begin{array}{l}\text { Calcium phosphates are a family of mineral salts containing calcium cations }\left(\mathrm{Ca}^{2+}\right) \text { and } \\
\text { phosphate anions }\left(\mathrm{PO}_{4}^{3}\right) \quad(6) \text {. Common calcium phosphate precipitates include } \\
\text { hydroxylapatite }\left(\mathrm{Ca} 10\left(\mathrm{PO}_{4}\right)_{6}(\mathrm{OH})_{2}\right) \text {, brushite }\left(\mathrm{CaHPO}_{4} \cdot \mathrm{H}_{2} \mathrm{O}\right) \text {, carbonated hydroxylapatite as } \\
\text { well as amorphous calcium phosphate }\left(\mathrm{Ca}_{\mathrm{x}}\left(\mathrm{PO}_{4}\right)_{\mathrm{y}} \cdot \mathrm{nH}_{2} \mathrm{O}\right) \text {. A carbonated hydroxylapatite is a } \\
\text { hydroxylapatite where carbonate groups }\left(\mathrm{CO}_{3}^{2-}\right) \text { are inserted into the apatite structure to } \\
\text { replace phosphate and/or hydroxyl }(\mathrm{OH}) \text { groups }(7) \text {. }\end{array}$ \\
\hline Al-P & $\begin{array}{l}\text { Aluminium phosphates are a family of mineral salts containing aluminium cations }\left(\mathrm{Al}^{3+}\right) \\
\text { and phosphate anions }\left(\mathrm{PO}_{4}^{3}\right) \text {. AlPO } \mathrm{PO}_{4} \text { is the most common aluminium phosphate in the } \\
\text { context of the recovery of nutrients in human excreta. }\end{array}$ \\
\hline $\mathrm{Fe}(\mathrm{III})-\mathrm{P}$ & $\begin{array}{l}\text { Ferric phosphates are a family of mineral salts containing iron(III) cations }\left(\mathrm{Fe}^{3+}\right) \text { and } \\
\text { phosphate anions }\left(\mathrm{PO}_{4}^{3}\right) . \mathrm{FePO}_{4} \text { is the most common ferric phosphate in the context of } \\
\text { the recovery of nutrients in human excreta. }\end{array}$ \\
\hline Mg-P & $\begin{array}{l}\text { Magnesium phosphates are salts of magnesium }\left(\mathrm{Mg}^{2+}\right) \text { and phosphate }\left(\mathrm{PO}_{4}{ }^{3-}\right) \text { appearing as } \\
\text { magnesium phosphate monobasic }\left(\mathrm{Mg}\left(\mathrm{H}_{2} \mathrm{PO}_{4}\right)_{2}\right) \text {, dibasic }\left(\mathrm{MgHPO}_{4}\right) \text {, or tribasic } \\
\left(\mathrm{Mg}_{3}\left(\mathrm{PO}_{4}\right)_{2}\right) \text { as well as amorphous magnesium phosphate. Anhydrous forms include } \\
\text { newberyite }\left(\mathrm{MgHPO}_{4} \cdot 3 \mathrm{H}_{2} \mathrm{O}\right) \text { and bobierrite }\left(\mathrm{Mg}_{3}\left(\mathrm{PO}_{4}\right)_{2} \cdot 8 \mathrm{H}_{2} \mathrm{O}\right) \text {. }\end{array}$ \\
\hline K-P & $\begin{array}{l}\text { Potassium phosphates are salts of potassium }\left(\mathrm{K}^{+}\right) \text {and phosphate }\left(\mathrm{PO}_{4}^{3-}\right) \text { appearing as } \\
\text { potassium phosphate monobasic }\left(\mathrm{KH}_{2} \mathrm{PO}_{4}\right) \text {, dibasic }\left(\mathrm{K}_{2} \mathrm{HPO}_{4}\right) \text {, or tribasic }\left(\mathrm{K}_{3} \mathrm{PO}_{4}\right) \text {. }\end{array}$ \\
\hline Other & $\begin{array}{l}\text { Other minerals that can be precipitated during nutrient recovery from human excreta } \\
\text { include ammonium dihydrogenphosphate/nitrophosammite }\left(\mathrm{NH}_{4} \mathrm{H}_{2} \mathrm{PO}_{4}\right) \text {, potassium } \\
\text { chloride (sylvite) }(\mathrm{KCl}) \text {, natrium chlorice (halite) }(\mathrm{NaCl}) \text {, potassium bicarbonate }\left(\mathrm{KHCO}_{3}\right) \text {, } \\
\text { gypsum }\left(\mathrm{CaSO}_{4}\right) \text {, potassium sulfate }\left(\mathrm{K}_{2} \mathrm{SO}_{4}\right) \text {, calcite }(\mathrm{CaCO}) \text {, dolomite }\left(\mathrm{CaMg}\left(\mathrm{CO}_{3}\right)_{2}\right) \text {, and } \\
\text { chemically complex phosphates such as montgomeryite }\left(\mathrm{Ca}_{4} \mathrm{MgAl}_{4}\left(\mathrm{PO}_{4}\right)_{6}(\mathrm{OH})_{4} \cdot 12 \mathrm{H}_{2} \mathrm{O}\right) \text {. }\end{array}$ \\
\hline
\end{tabular}

Table S4.2 Typical sorbents that have been used to extract nutrients from liquid streams during treatment of human excreta and streams containing human excreta.

\begin{tabular}{|l|l|}
\hline Charcoal & $\begin{array}{l}\text { Activated carbon or biochar. Can be obtained from a variety of feedstocks, including faecal- } \\
\text { derived organic matter and phototrophic biomass. }\end{array}$ \\
\hline Calcined struvite & $\begin{array}{l}\text { Struvite from which ammonium has been removed through thermal treatment in the } \\
\left.\text { absence of or under limited supply of air or oxygen (e.g. at } 200^{\circ} \mathrm{C} \text { for } 3 \mathrm{~h}\right)(8) \text {. }\end{array}$ \\
\hline Aluminosilicates & $\begin{array}{l}\text { Minerals composed of aluminium, silicon, oxygen, and coutercations. Zeolites (e.g. } \\
\text { clinoptilolite) and palygorskite are the most common ones used as sorbents. }\end{array}$ \\
\hline Calcium silicates & $\begin{array}{l}\text { Minerals composed of calcium, silicon, oxygen, and coutercations. Wollastonite is the most } \\
\text { common one used as sorbent. }\end{array}$ \\
\hline Calcium oxide & CaO. For example prepared from egg shells through calcination egg shells (9). \\
\hline Other & $\begin{array}{l}\text { Other sorbent material include polymeric resins as well as metal modified charcoal, mineral } \\
\text { sorbents, or polymeric resins. }\end{array}$ \\
\hline
\end{tabular}




\section{References}

1. Mathew, M. and Schroeder, L. W. (1979) 'Crystal structure of a struvite analogue, MgKPO4.6H2O', Acta Crystallographica Section B Structural Crystallography and Crystal Chemistry, 35(1), pp. 11-13. doi: 10.1107/ S0567740879002429.

2. Graeser, S. et al. (2008) 'Struvite- ( K ), $\mathrm{KMgPO} 4 \cdot 6 \mathrm{H} 2 \mathrm{O}$, the potassium equivalent of struvite - a new mineral', European journal of Mineralogy, 20(May), pp. 629-633. doi: 10.1127/0935-1221/2008/0020-1810.

3. Weil, M. (2008) 'The struvite-type compounds M $\mathrm{Mg}(\mathrm{H} 2 \mathrm{O}) 6]$ ( X O 4), where M = Rb, Tl and X = P, As', Crystal Research and Technology, 43(12), pp. 1286-1291. doi: 10.1002/crat.200800403.

4. Xu, K. et al. (2012) 'Laboratory experiments on simultaneous removal of $\mathrm{K}$ and $\mathrm{P}$ from synthetic and real urine for nutrient recycle by crystallization of magnesium-potassium-phosphate-hexahydrate in a draft tube and baffle reactor', Chemosphere. Elsevier Ltd, 88(2), pp. 219-223. doi: 10.1016/j.chemosphere.2012.02.061.

5. Xu, K. et al. (2015) 'The precipitation of magnesium potassium phosphate hexahydrate for $\mathrm{P}$ and $\mathrm{K}$ recovery from synthetic urine', Water Research. Elsevier Ltd, 80, pp. 71-79. doi: 10.1016/j.watres.2015.05.026.

6. Dorozhkin, S. V and Epple, M. (2002) 'Biological and medical significance of calcilum phosphates', Angewandte Chemie International Edition, 41(17), pp. 3130-3146. doi: 10.1002/1521-3773(20020902)41.

7. Pham Minh, D., Nzihou, A. and Sharrock, P. (2014) 'Carbonated hydroxyapatite starting from calcite and different orthophosphates under moderate hydrothermal conditions: Synthesis and surface reactivity in simulated body fluid', Materials Research Bulletin. Elsevier Ltd, 60, pp. e292-e299. doi: 10.1016/j.materresbull.2014.08.052.

8. Latifian, M., Holst, O. and Liu, J. (2014) 'Nitrogen and Phosphorus Removal from Urine by Sequential Struvite Formation and Recycling Process', Clean - Soil, Air, Water, 42(8), pp. 1157-1161. doi: 10.1002/clen.201300070.

9. Köse, T. E. and Kivanç, B. (2011) 'Adsorption of phosphate from aqueous solutions using calcined waste eggshell', Chemical Engineering Journal, 178, pp. 34 -39. doi: 10.1016/j.cej.2011.09.129. 


\section{SUPPORTING INFORMATION V}

\section{Recycling Nutrients Contained in Human Excreta to Agriculture: Pathways, Processes, and Products.}

\section{Treatment Processes}

This Supporting Information (SI) describes treatment processes that aim to facilitate recycling of nutrients (and organic matter) contained in human excreta to agriculture. The focus is on briefly summarising each treatment process as well as on outlining the fate of nutrients (nitrogen, phosphorus, and potassium), organic matter (carbon), and contaminants (pathogens, organic pollutants, heavy metals) during treatment. To this end, we specifically searched for literature to complement the documents describing recovery pathways as presented in SI1.

This SI contains 35 pages.

Table S5.1 Summary table of treatment processes, their repective process outputs and nutrient and pollutant partitioning across process outputs

Figure S5.1 Summary figure of treatment processes 


\section{TABLE OF CONTENTS}

Biological treatment

Aerobic and Anaerobic Treatment

Bioelectrochemical Systems

Biological decomposition

Anaerobic Processes: Fermentation, Anaerobic Digestion

Aerobic Processes: Composting, Vermicomposting, Fly Larvae Composting

Hydrothermal Processes: Thermal Hydrolysis, HTC, HTL, HTG, HTO

Thermal Processes

Hygienisation

Storage, Pasteurisation, Ammonia Sanitisation, Desiccation, Lime Stabilisation

Separation

Thermal Drying

Mobilisation-Separation

Stabilisation

Chemical Processes: Alkalinisation, Acidification

Biological Processes: Nitrification, Lactic Acid Fermentation

Hygienisation and depollution

Storage, Thermal Storage, Pasteurisation

Advanced Oxidation, Biological Treatment

Separation

Freezing and Melting

Vaporisation: Evaporation, Distillation, Membrane Distillation

Membrane Separation: Forward Osmosis, Reverse Osmosis, Nanofiltration, Electrodialysis

Ammonia Release and Capture

Phototrophic Biomass Growth

Controlled Precipitation

Sorption

Separation

Elution, Thermal Treatment

\begin{tabular}{|l|l|l|}
\hline LEGEND Carbon \\
T.: Some of the respective constituents may go to this fraction. \\
The majority of the respective constituents go to this fraction.
\end{tabular}




\begin{tabular}{|c|c|c|}
\hline \multicolumn{3}{|c|}{$\begin{array}{l}\text { BIOLOGICAL TREATMENT - Aerobic and Anaerobic Treatment } \\
\text { Summary }\end{array}$} \\
\hline \multirow{2}{*}{\multicolumn{3}{|c|}{$\begin{array}{l}\text { The activated sludge process is an aerobic treatment process that was discovered roughly a century ago and has since } \\
\text { been a central tenet of sewage treatment at municipal sewage treatment plants (STPs) (1). The process renders a purified } \\
\text { effluent devoid of suspended and dissolved organic matter, an organic residual commonly referred to as sewage sludge } \\
\text { (in European regulations) or biosolids (in North American regulations), as well as gaseous emissions. Over the years, } \\
\text { biological nitrogen (N) and biological or chemical phosphorus (P) removal processes have been incorporated into overall } \\
\text { process design to meet ever stricter effluent standards for N and P (2). More recently, nonoxidative removal of organics } \\
\text { has received increased attention, for example through the formation and intracellular storage of polyhydroxyalkanoates } \\
\text { (PHA) or other precursors for high-value products (3). } \\
\text { Anaerobic treatment processes are an alternative way to treat municipal sewage that requires much less energy (4). } \\
\text { Anaerobic treatment renders a purified effluent, an organic residual, and biogas. }\end{array}$}} \\
\hline & & \\
\hline \multicolumn{2}{|c|}{ Activated Sludge Process } & Organic $\rightarrow$ ACT.SLU $\rightarrow$ Solution Effluent \\
\hline \multicolumn{2}{|c|}{ Anaerobic Treatment } & 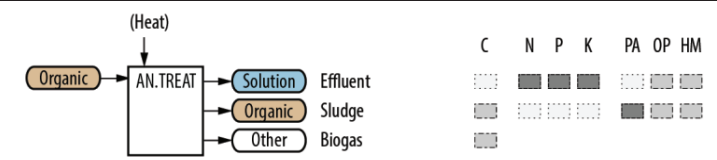 \\
\hline \multicolumn{3}{|c|}{ Fate of Constituents } \\
\hline \multirow{2}{*}{\multicolumn{3}{|c|}{$\begin{array}{l}\text { The fate of nutrients in STPs based on the activated sludge process depends on process design. Without the incorporation } \\
\text { of dedicated nutrient removal processes, most of the soluble } \mathrm{N}, \mathrm{P} \text { and } \mathrm{K} \text { remains in the effluent, with some soluble } \mathrm{N} \\
\text { released to the atmosphere in the form of ammonia }\left(\mathrm{NH}_{3}\right) \text { or nitrous oxide }\left(\mathrm{N}_{2} \mathrm{O}\right) \text { (if nitrification takes place). Process } \\
\text { designs that include nutrient removal processes aim to incorporate soluble } \mathrm{P} \text { into the sludge and enhance } \mathrm{N} \text { removal } \\
\text { through the release of dinitrogen gas }\left(\mathrm{N}_{2}\right) \text { to the atmosphere following nitrification and denitrification. Nitrogen release } \\
\text { in the form of nitrous oxide }\left(\mathrm{N}_{2} \mathrm{O}\right) \text { can still occur as a result of incomplete denitrification. Nitrous oxide is a potent } \\
\text { climate gas. Pathogens tend to accumulate in the sludge, and heavy metals partition fairly equally between effluent and } \\
\text { sludge. The partitioning behaviour of organic pollutants depends on the compound, with a tendency towards sorbing to } \\
\text { the sludge. } \\
\text { Anaerobic treatment focuses on the conversation of organic matter to biogas and the nutrients } \mathrm{N}, \mathrm{P} \text {, and } \mathrm{K} \text { mostly remain } \\
\text { in the effluent. Some organic pollutants can be degraded during anaerobic treatment. }\end{array}$}} \\
\hline & & \\
\hline Reference & \multicolumn{2}{|c|}{ Information Extracted } \\
\hline$(5)$ & \multicolumn{2}{|c|}{$\begin{array}{l}\text { AS. } \mathrm{N}_{2} \mathrm{O} \text { emissions are generally below } 1 \% \text { of influent } \mathrm{N} \text { but STPs nevertheless are significant } \mathrm{N}_{2} \mathrm{O} \\
\text { emitters. }\end{array}$} \\
\hline (6) & \multicolumn{2}{|c|}{$\begin{array}{l}\text { AS. Up to } 15 \% \text { of influent } \mathrm{N} \text { is emitted as } \mathrm{N}_{2} \mathrm{O} \text {. About } 1 \% \text { of influent } \mathrm{COD} \text { is in the form of methane, } \\
\text { which can be emitted to the atmosphere during the activated sludge process. }\end{array}$} \\
\hline (7) & \multicolumn{2}{|c|}{ AS. Effective removal of most bacterial pathogens from the treated effluent. } \\
\hline (8) & \multicolumn{2}{|c|}{$\begin{array}{l}\text { AS. Good potential to remove pathogens from the treated effluent. Sorption to the sludge is an important } \\
\text { removal pathway. }\end{array}$} \\
\hline (9) & \multicolumn{2}{|c|}{$\begin{array}{l}\text { AS. Relative distribution between treated effluent and waste sludge varied largely among } 7 \text { PCBs and } 19 \\
\text { organochlorine pesticides. }\end{array}$} \\
\hline$(10)$ & \multicolumn{2}{|c|}{ AT. About $61 \%$ of $\mathrm{P}$ was found to be released with the digester effluent. } \\
\hline$(11)$ & \multicolumn{2}{|c|}{$\begin{array}{l}\text { AT. Removal of organic pollutants during anaerobic treatment (UASB + OLAND) of blackwater mixed } \\
\text { with kitchen waste. Observed removal efficiencies of well beyond } 50 \% \text { for the selected personal care } \\
\text { products (fragrances, biocides, preservatives, UV-filters) and their biotransformation products. }\end{array}$} \\
\hline$(12)$ & \multicolumn{2}{|c|}{ AT. Only some organic pollutants have been found to be decomposed during anaerobic treatment. } \\
\hline$(13)$ & \multicolumn{2}{|c|}{ AT. Estrogen degradation only partial. } \\
\hline
\end{tabular}




\section{BIOLOGICAL DECOMPOSITION - Bioelectrochemical Systems} Summary

Microbial fuel cells (14-16) and microbial electrolysis cells are emerging bioelectrochemical system (BES) aiming at converting organic matter into electricity or hydrogen, respectively.

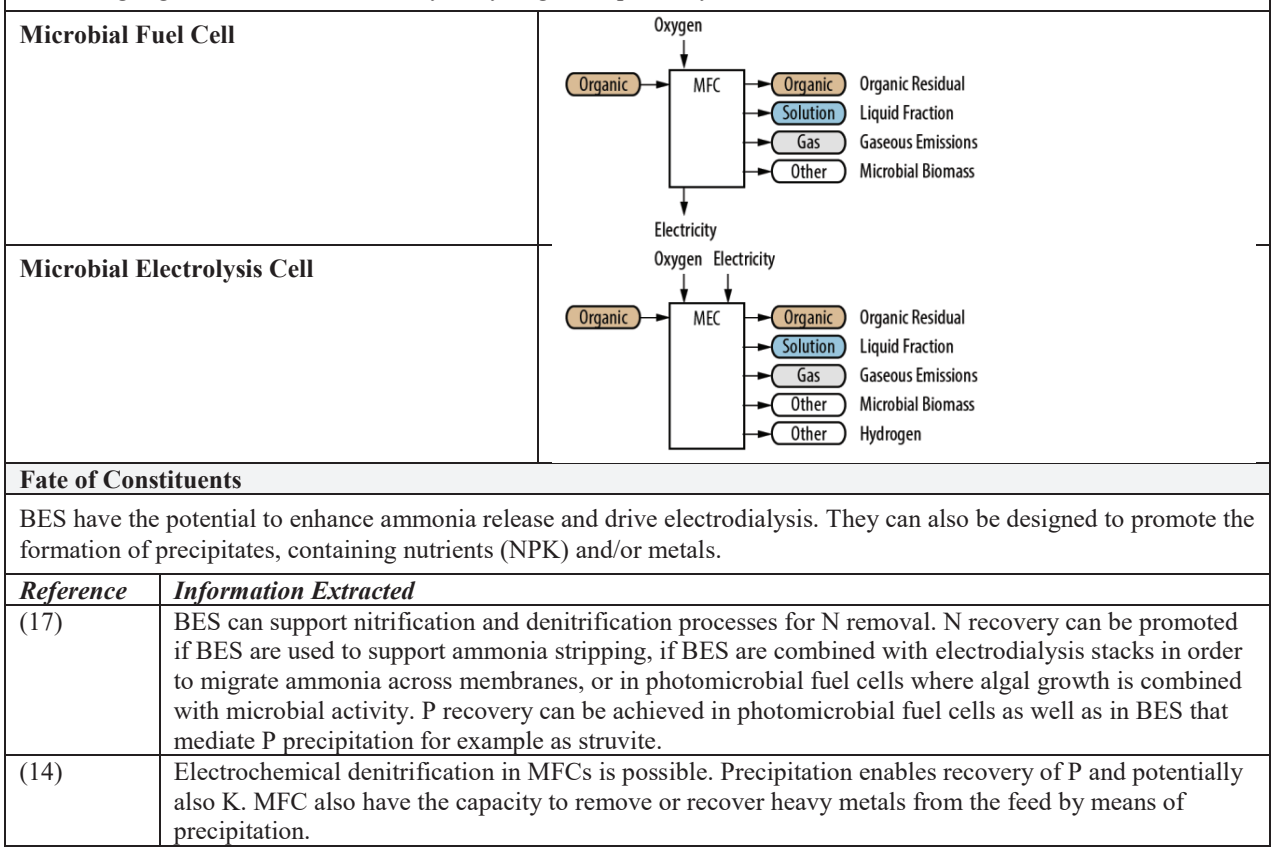




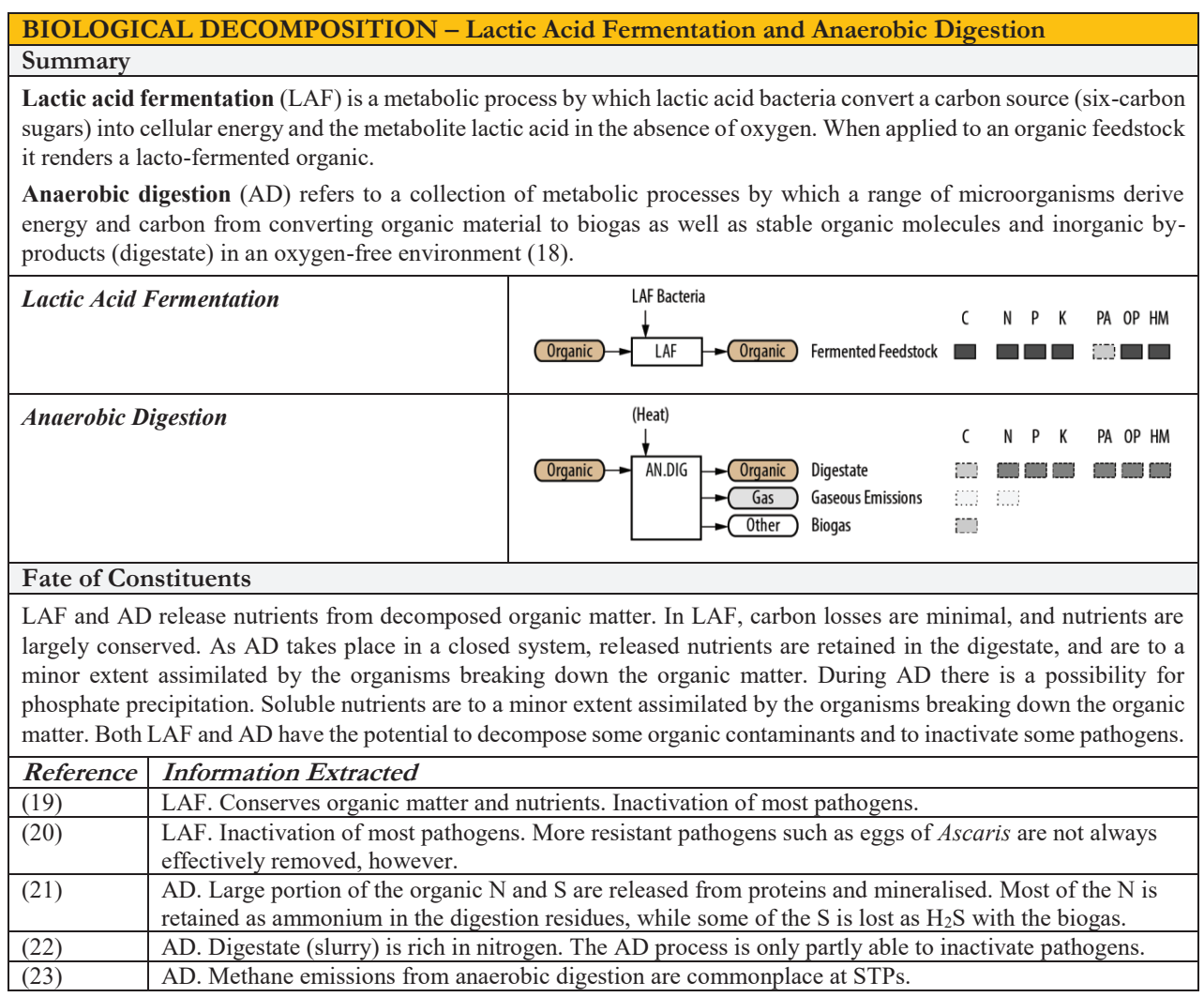




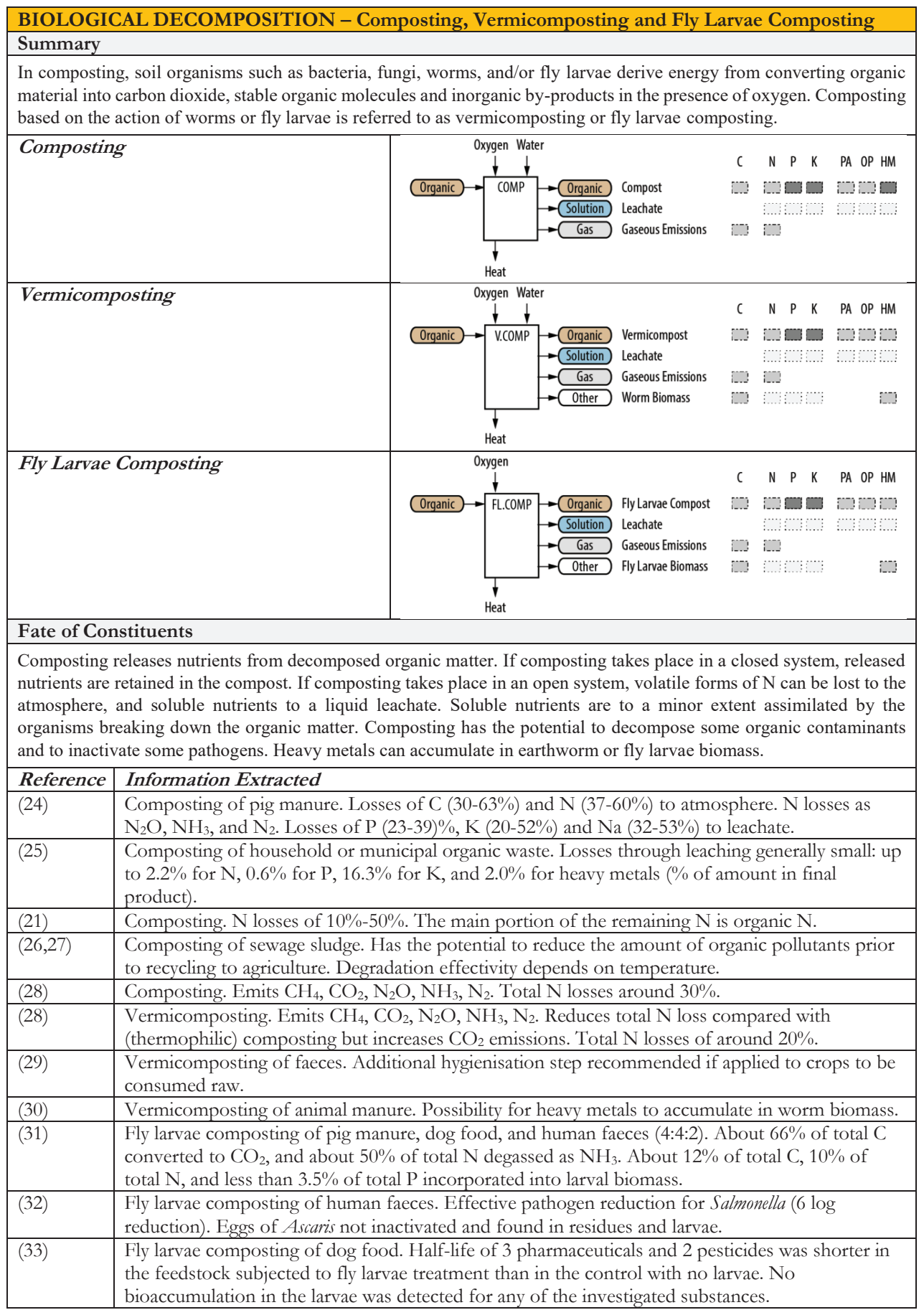




\section{THERMAL DECOMPOSITION - Hydrothermal Processes}

\section{Summary}

Hydrothermal processes include thermal hydrolysis, hydrothermal carbonisation (HTC), hydrothermal liquefaction (HTL), hydrothermal gasification (HTG), and hydrothermal oxidation (HTO). These processes have in common that wet organic matter is the process input, which is then converted into gaseous, liquid, and solid outputs.

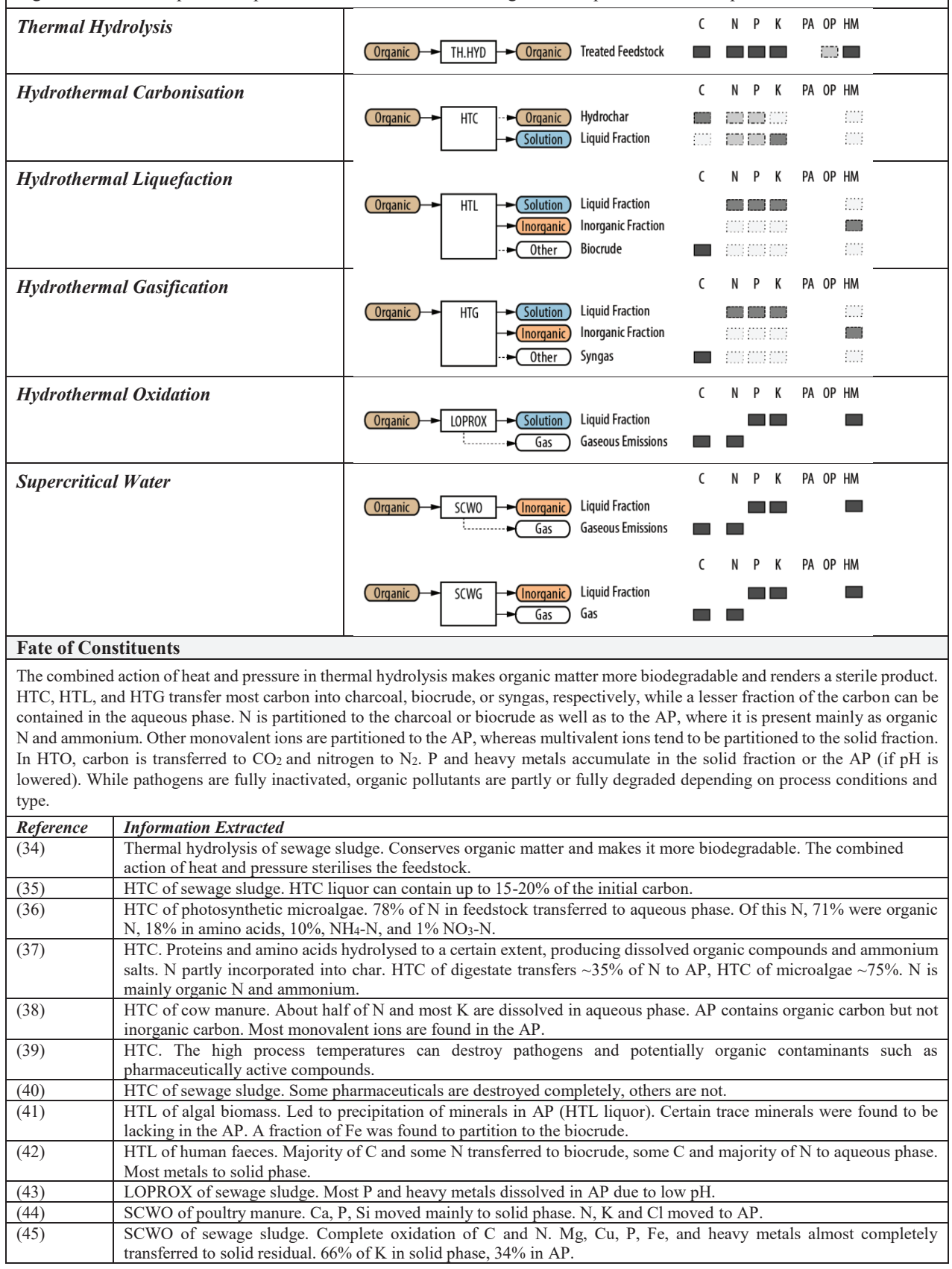




\section{THERMAL DECOMPOSITION - Thermal Processes} Summary

Thermal processes include pyrolysis, gasification, and combustion. These processes have in common that dry organic matter is the process input, which is then converted into gaseous, liquid, and/or solid outputs.

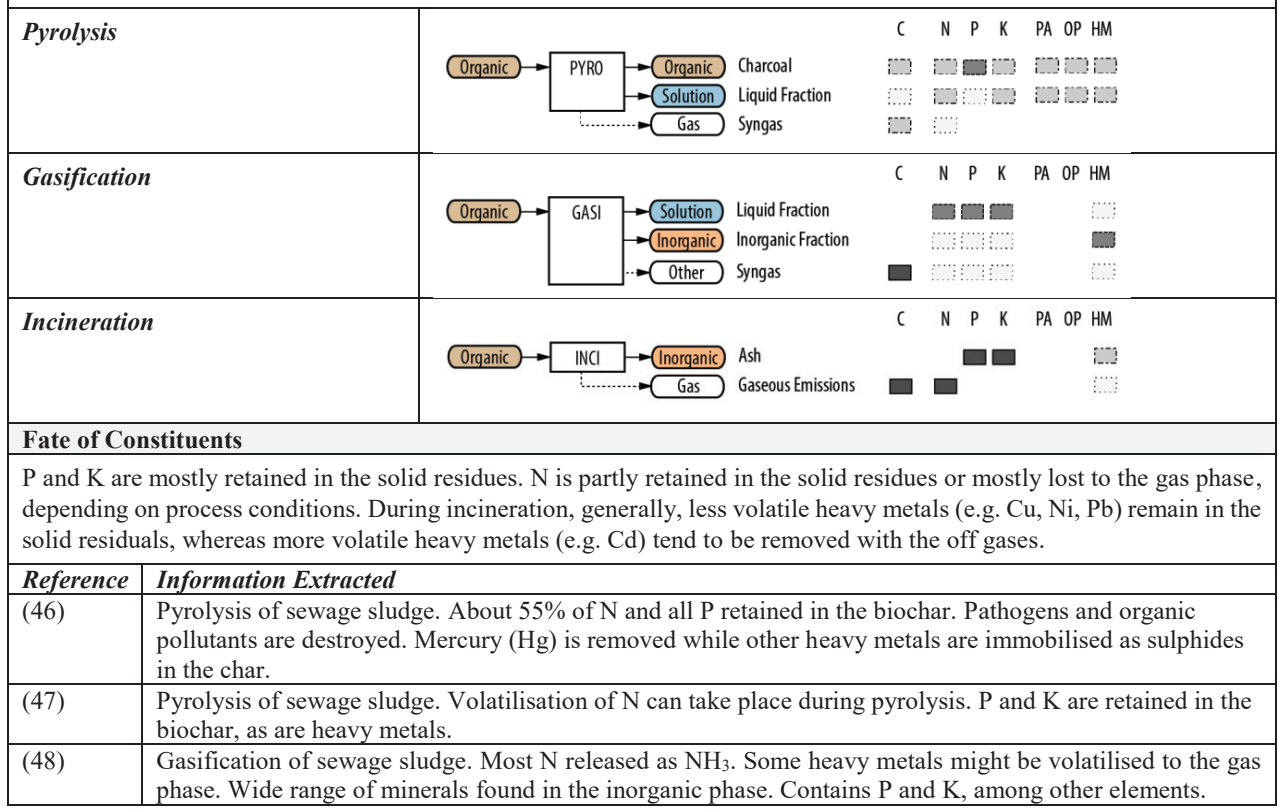




\section{HYGIENISATION - Storage, Pasteurisation, Ammonia Sanitisation, Desiccation}

\section{Summary}

Pathogen inactivation during storage makes use of natural die-off of pathogens while outside of a host. Pasteurisation achieves pathogen inactivation by exposure to high temperatures during a short period of time. Ammonia sanitisation makes use of the toxicity of ammonia to microbes and can be achieved by the addition of ammonia or urea, as urea is quickly degraded to ammonium by ubiquitous urea hydrolysing enzymes (21). Desiccation consists of adding a dry substance such as plant ash, lime, dried soil, or sawdust to the feedstock. Lime treatment consists of the addition of lime in order to raise $\mathrm{pH}$.

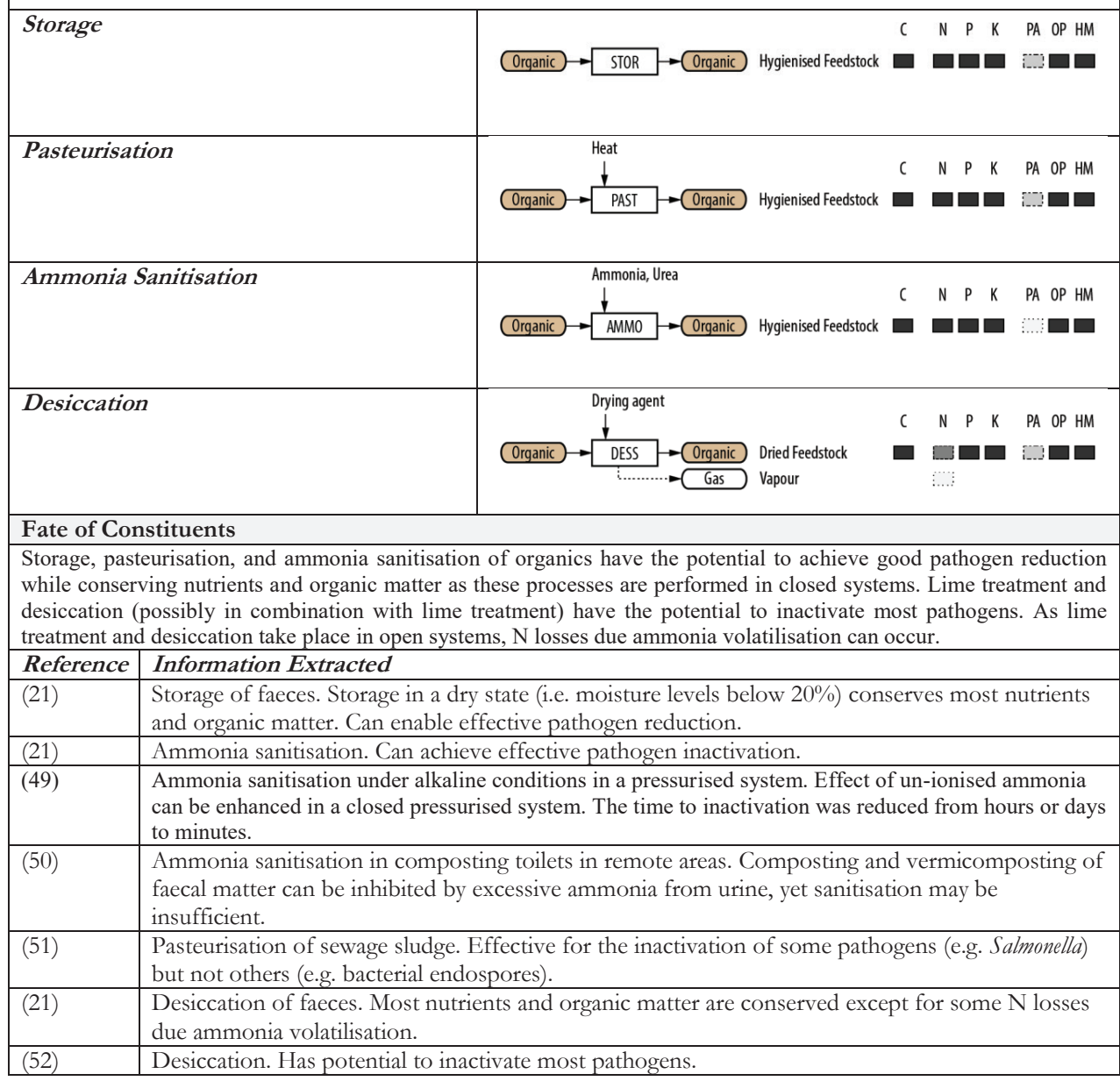




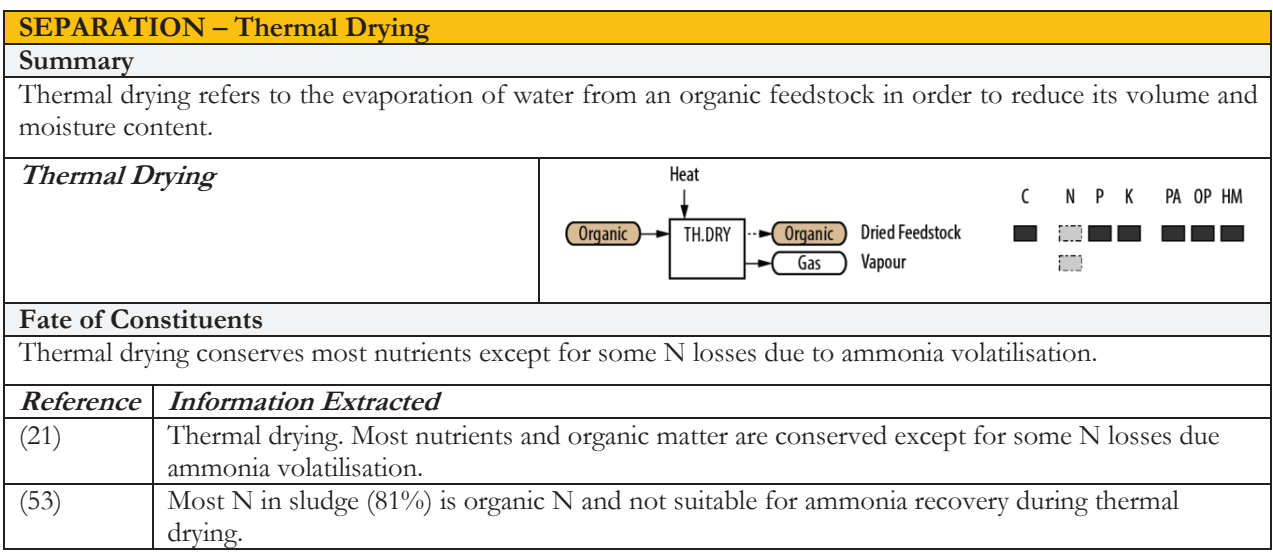

\section{SEPARATION - Mobilisation-Separation}

\section{Summary}

P extraction from the solid fraction of an organic such as sludge to a nutrient solution requires the extraction of $\mathrm{P}$ from the solid to the liquid phase followed by liquid-solid separation. A range of processes has been investigated to achive mobilisation of $\mathrm{P}$ from sludge, including ozonation (54), additional anaerobic tanks or zones in EBPR (enhanced biological phosphorus removal) schemes (55), bioelectrochemical systems (56,57), and acid elution (58-60). The former two are applicable for sewage sludge rendered by biological P removal, whereas the latter two are more adequate for sewage sludge rendered by chemical P removal.

Mobilisation - Separation
Fate of Constituents
The main purpose of mobilisation-separation processes is to transfer $\mathrm{P}$ from the solid fraction of an organic to a
liquid.




\section{STABILISATION - Chemical Processes}

\section{Summary}

Alkalinisation and acidification are two common processes applied to aqueous solutions such as urine or treated effluent to prevent ammonia volatilisation. Alkalinisation is best applied to urea-rich solutions (notably fresh urine), as high $\mathrm{pH}$ prevents urea hydrolysis but shifts the ammonia-ammonium equilibrium towards volatile ammonium. Acidification is applicable to both urea-rich solutions (notably fresh urine) and ammonia-rich solutions (notably the effluent or liquid process-side streams in the treatment of sewage or other streams containing human faeces), as low $\mathrm{pH}$ prevents urea hydrolysis and shifts the ammonia-ammonium equilibrium towards non-volatile ammonium. Acidification is less applicable to hydrolysed urine, however, given the its high buffer capacity.

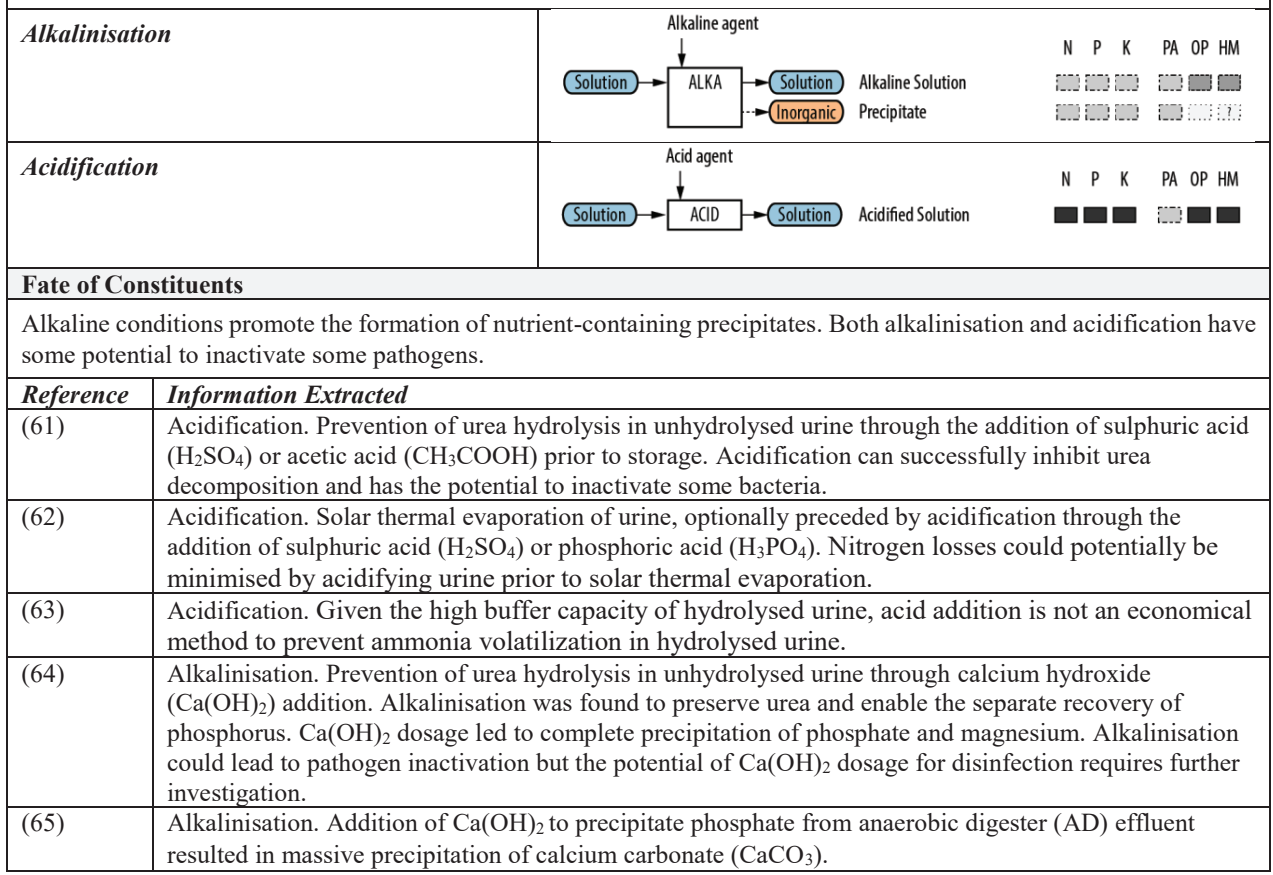




\section{STABILISATION - Biological Processes}

\section{Summary}

Nitrification refers to the biological oxidation of ammonia and ammonium to nitrite $\left(\mathrm{NO}_{2}{ }^{-}\right)$and nitrate $\left(\mathrm{NO}_{3}{ }^{-}\right)$and is best applied to solutions rich in ammonia nitrogen such as hydrolysed urine. LAF is best applied to urea-rich solutions such as fresh urine as the decrease in $\mathrm{pH}$ is enough to inhibit urea hydrolysis. As a result of the high buffer capacity of hydrolysed urine, LAF is not sufficient to sufficiently lower the $\mathrm{pH}$ to prevent ammonia volatilisation.

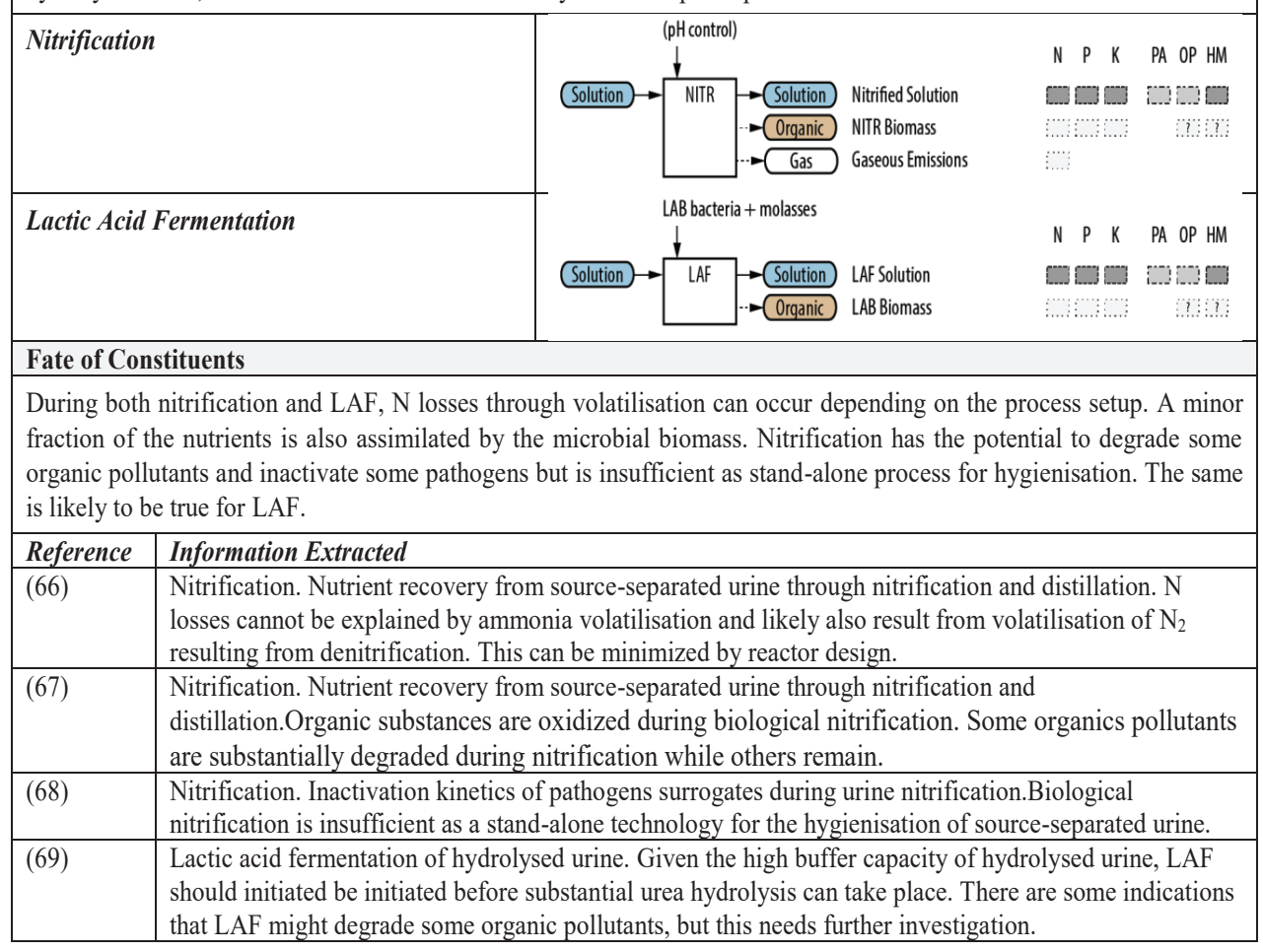




\section{HYGIENISATION AND DEPOLLUTION - Storage, Thermal Storage, Pasteurisation}

\section{Summary}

During storage at ambient temperatures under alkaline conditions and in a closed container, pathogen inactivation mainly results from the combined effect of alkaline $\mathrm{pH}$ and un-ionised ammonia $(49,70)$. The World Health Organization recommends storage at $20^{\circ} \mathrm{C}$ or higher for at least six months in order to assure safe application of human urine (WHO, 2006). During thermal storage (e.g. at $70^{\circ} \mathrm{C}$ for 1 week) and pasteurisation (e.g. at $80^{\circ} \mathrm{C}$ for 30 minutes), pathogen inactivation is greatly enhanced by the high temperatures and hygienisation can be achieved after minutes or days rather than months $(70,71)$.

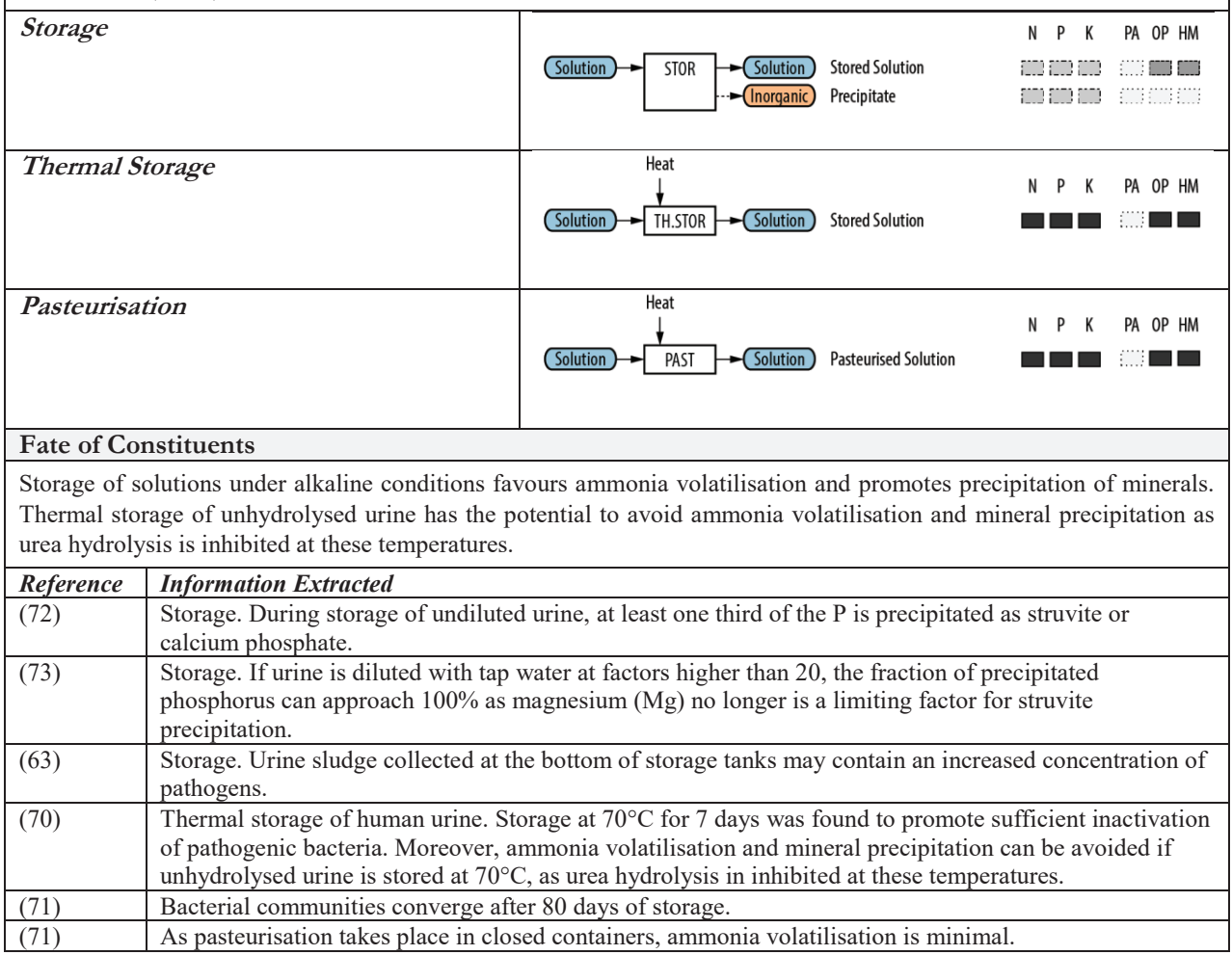




\section{HYGIENISATION AND DEPOLLUTION - Advanced Oxidation, Biological Treatment}

\section{Summary}

Advanced oxidation processes (AOPs) are processes that generate hydroxyl radicals or other oxidative radical species in sufficient quantities to degrade organic contaminants (74), or less commonly, to inactivate pathogens $(74,75)$. Oxidation can also be achieved through microbial processes in biological treatment.

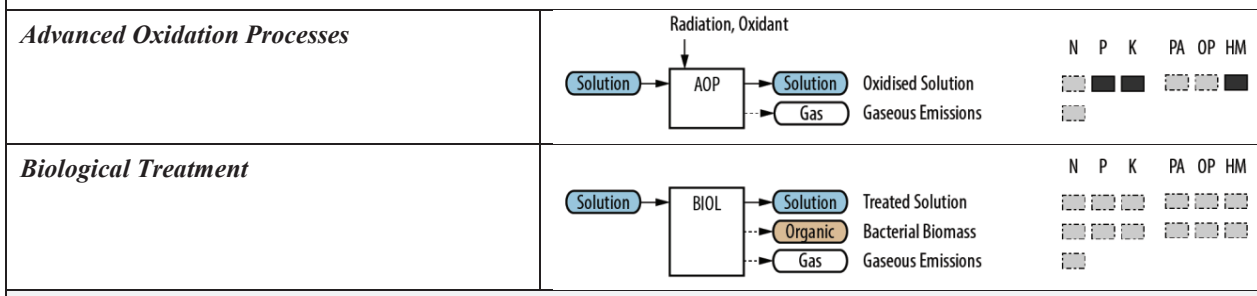

Fate of Constituents

In advanced oxidation, $\mathrm{N}$ losses can occur if ammonia nitrogen is oxidised to $\mathrm{N}_{2}$. In biological oxidation, significant $\mathrm{N}$ losses can occur due to biological nitrification and denitrification, and nutrients are assimilated to some extent by the microorganisms. Both chemical and biological oxidation have the potential to degrade organic pollutants such as pharmaceutically active compounds. Degradation efficiencies rely heavily upon the properties of the contaminants and the selected process and operating conditions. There are also indications that biological treatment might be useful to remove heavy metals from solutions.

\begin{tabular}{|l|l|}
\hline Reference & Information Extracted \\
\hline $\mathbf{7 4 )}$ & $\begin{array}{l}\text { AOPs in wastewater treatment. AOPs based on hydroxyl radicals hardly react with ammonia nitrogen. } \\
\text { Sulfate radicals have a similar strong oxidative capacity and short lifespan but different reaction patterns } \\
\text { from hydroxyl radicals and can readily oxidise ammonia nitrogen. Treatment efficiency as for organic } \\
\text { pollutant degradation relies primarily on the type of AOP, physical/chemical properties of target } \\
\text { pollutants, and operational conditions. }\end{array}$ \\
\hline $\mathbf{( 7 5 )}$ & $\begin{array}{l}\text { Light-assisted AOPs for urine treatment. UV-based AOPs are effective for OP removal and pathogen } \\
\text { inactivation. }\end{array}$ \\
\hline $\mathbf{7 6 )}$ & $\begin{array}{l}\text { Biological treatment. Elimination of pharmaceutical active compounds in urine by biological oxidation } \\
\text { in membrane bioreactor (MBR). Up to 85\% N removal through nitrification and denitrification. More } \\
\text { than } 65 \% \text { of P contained in effluent. }\end{array}$ \\
\hline $\begin{array}{l}\text { Biological treatment. Microbial bioremediation for heavy metal removal from treated effluent. Microbial } \\
\text { bioremediation is a potential method for the removal of heavy metal pollution in sewage effluents before } \\
\text { discharged to the environment. }\end{array}$ \\
\hline
\end{tabular}




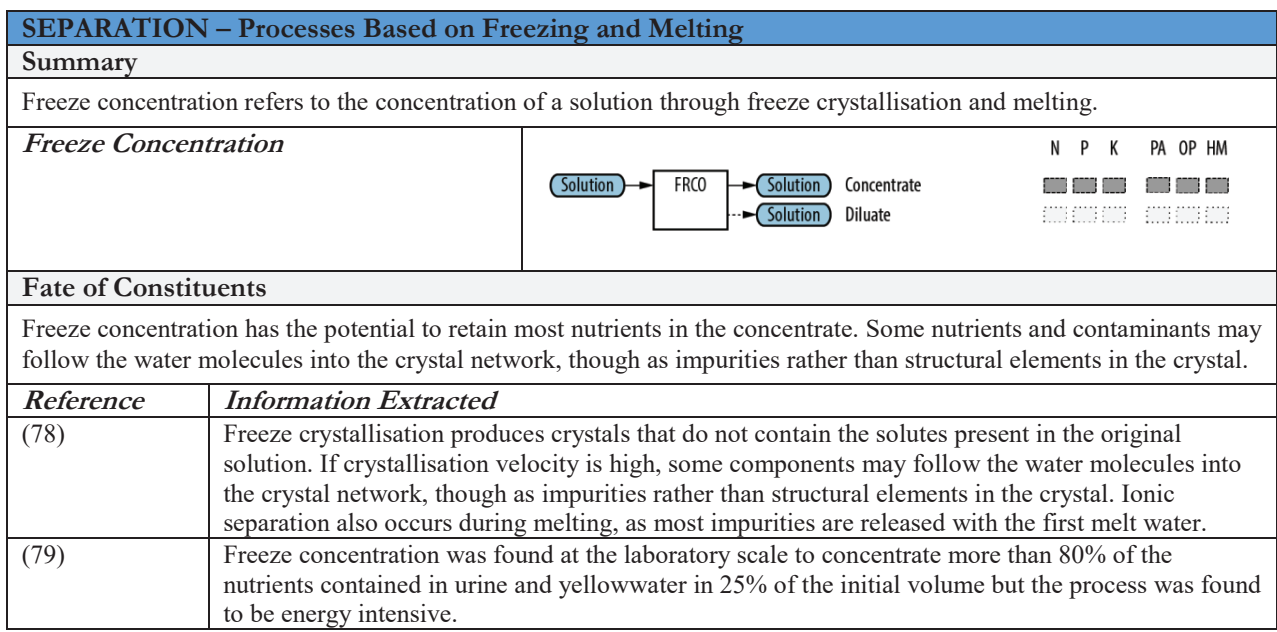




\section{SEPARATION - Processes Based on Vaporisation}

Summary

Evaporation refers to the phase transition from liquid to gas phase through vaporisation from the surface of a liquid to a gas not saturated with the evaporating substance. Distillation refers to vaporisation from the bulk liquid when the boiling point of the liquid is reached. Membrane distillation (MD) is a membrane separation process driven by a vapour pressure difference induced by a temperature gradient across a microporous hydrophobic membrane.

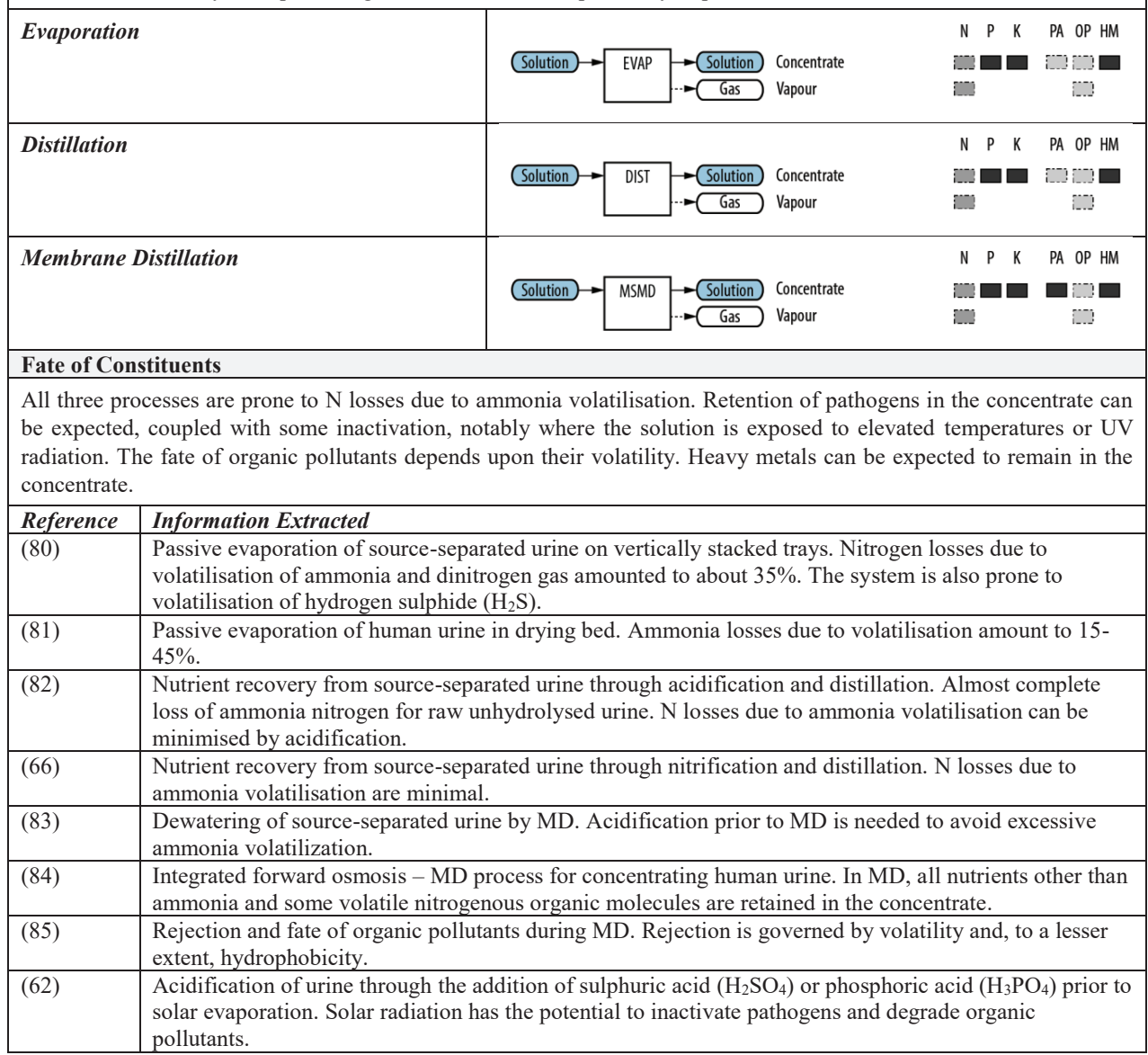




\section{SEPARATION - Processes Based on Membrane Separation}

\section{Summary}

Forward osmosis (FO) is a membrane separation process driven by an osmotic pressure difference induced by a concentration gradient across a semi-permeable membrane. In FO, a draw solution of high concentration (relative to the feed solution) induces a net flow of water from the feed solution (e.g. urine or treated effluent) through the membrane to the draw solution, leading to a separation of water in the feed solution from its solutes. The draw solution needs to be either renewed or regenerated, where regeneration involves separating the water from the draw solution. Reverse osmosis (RO) is a membrane separation process driven by a hydraulic pressure difference across a semi-permeable membrane In RO, the solvent passes through the membrane, while some dissolved and suspended species are retained by the membrane. Nanofiltration (NF) is similar to RO but NF membranes have larger pores than RO membranes.

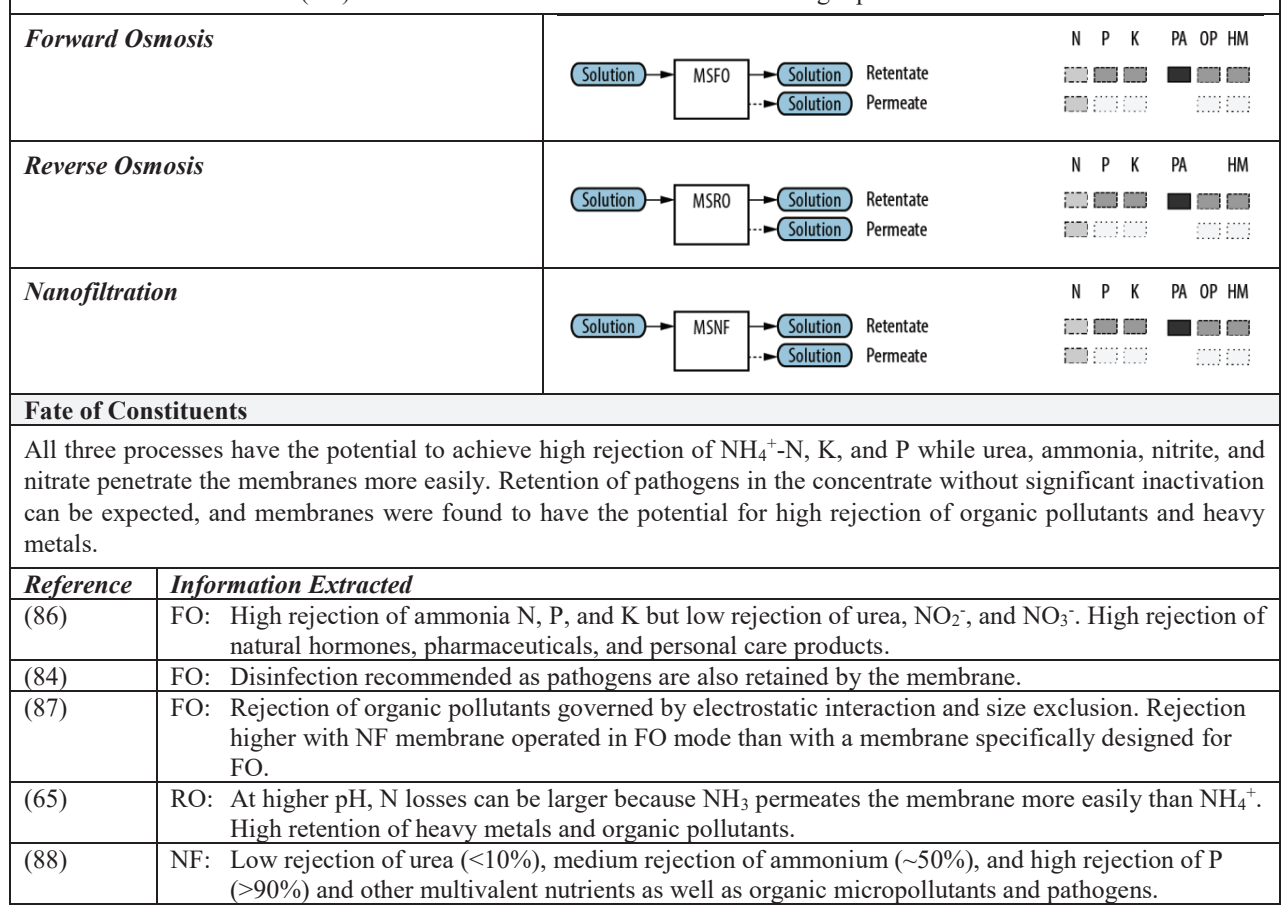


SEPARATION - Processes Based on Membrane Separation Summary

Electrodialysis (ED) is a membrane separation process driven by an electric potential gradient across ion exchange membranes and is useful to transport ions from one solution to another. One or several pairs of anion and cation exchange membranes are placed between two (biotic or abiotic) electrodes, forming an ED stack with one or several ED cells, each delimited by an anion and a cation exchange membrane. Anions and cations can permeate through the membrane of the opposite charge but are rejected by the membrane of the same charge. The alternating charge of the ion exchange membranes induces a transport of ions from the feed stream to the concentrate or electrode stream. ED differs from other membrane separation processes in that the dissolved species are removed from the feed solution rather than the solvent, although water is transported through the membrane to some extent.

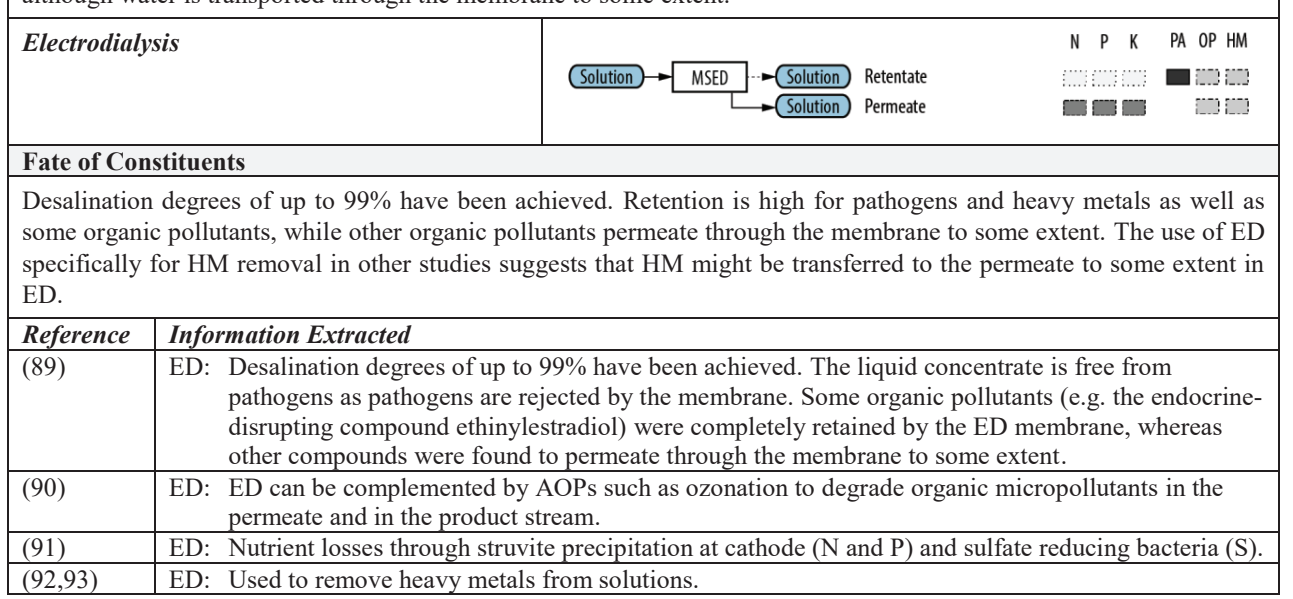




\section{SEPARATION - Processes Based on Ammonia Release and Capture}

\section{Summary}

Stripping is a physical separation process where one or several components are removed from a liquid stream by a gas stream. In sewage and urine treatment, ammonia stripping is a common process for nitrogen removal. Ammonia stripping releases ammonia from a solution to the gas phase. The stripping gas can be passed through an acid trap where ammonia is captured and concentrated as a high purity ammonium product.

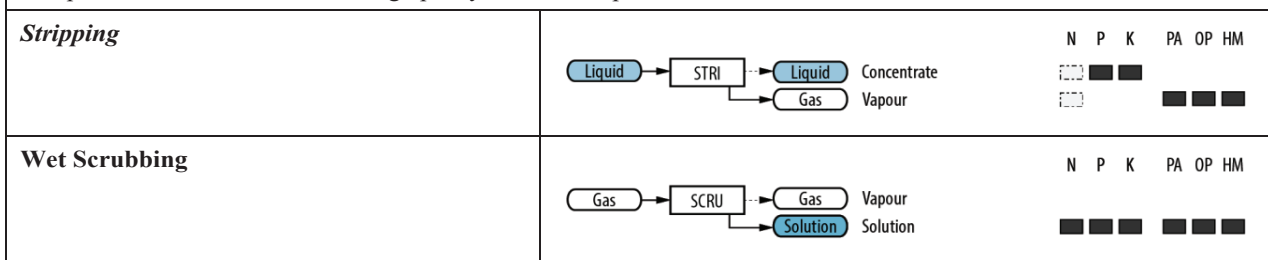

Wet scrubbing is a common process to clean gases and has also found application to capture ammonia from a gas stream and concentrate it in a high purity liquid ammonium product. Acid traps that have been used to this end include sulphuric acid $\left(\mathrm{H}_{2} \mathrm{SO}_{4}\right)(94-97)$, boric acid $\left(\mathrm{H}_{3} \mathrm{BO}_{3}\right)$, hydrochloric acid $(\mathrm{HCl})$ (e.g. 98), nitric acid $\left(\mathrm{HNO}_{3}\right)$ (e.g. 53) and phosphoric acid $\left(\mathrm{H}_{3} \mathrm{PO}_{4}\right)$ (e.g. 99).

Pathogens, organic pollutants, and heavy metals can be expected to largely remain in the residual solution from which ammonia has been stripped.

\section{SEPARATION - Processes based on Phototrophic Biomass Growth}

\section{Summary}

Through phototrophic biomass growth in aquatic or terrestrial systems, nutrients can be extracted from solutions and incorporated into phototrophic biomass. Phototrophic biomass growth refers to biomass growth where photosynthesis is the energy source for cell metabolic processes and the carbon source is carbon dioxide (photoautotrophs) or other organic matter (photoheterotrophs). Technologies that render phototrophic biomass include cultivation in for instance open ponds, closed photobioreactors, or hydroponic systems. Cultivated organisms range from cyanobacteria and microalgae to aquatic and terrestrial plants. Algal treatment systems have received particular attention. Other studies have investigated how municipal STPs based on the activated sludge process could be optimised to provide growth medium for microalgae cultivation (100-102), or how nutrients can be concentrated through sorption to and desorption from mineral sorbents to enhance phototrophic biomass growth $(103,104)$.

\begin{tabular}{|c|c|c|c|c|}
\hline \multicolumn{2}{|c|}{ Phototrophic Biomass Growth } & 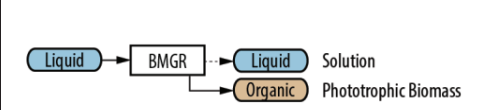 & 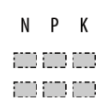 & 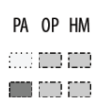 \\
\hline \multicolumn{5}{|c|}{ Fate of Constituents } \\
\hline \multicolumn{5}{|c|}{$\begin{array}{l}\text { Algal systems have been found to have the potential to simultaneously extract } \mathrm{N} \text { and } \mathrm{P} \text { but algal growth can be limited } \\
\text { by deficiencies of micronutrients such as magnesium. Algal systems have also been found to have the potential to extract } \\
\text { organic pollutants and heavy metals through sorption. }\end{array}$} \\
\hline Reference & \multicolumn{4}{|c|}{ Information Extracted } \\
\hline (105) & \multicolumn{4}{|c|}{ Algal systems have been found to have the potential to extract organic pollutants through sorption. } \\
\hline$(106,107)$ & \multicolumn{4}{|c|}{ Algal systems have been found to have the potential to extract heavy metals through sorption. } \\
\hline
\end{tabular}




\section{SEPARATION - Processes based on Precipitation through the Addition of a Precipitant}

\section{Summary}

Precipitation is the process of transferring a solute from a liquid to a solid in amorphous or crystalline form and can be induced in a solution or a wet organic matrix. The precipitation of ferric and aluminium phosphates has long been applied at municipal STPs in order to reduce phosphorus (P) concentrations in the effluent (108). More recently, the precipitation of struvite (also known as magnesium ammonium phosphate, MAP) and calcium phosphates in urine, yellowwater, or aqueous streams at municipal STPs have received particular attention $(109,110)$ and is generally achieved through the addition of precipitants that increase the availability and concentration of magnesium and calcium ions. Common magnesium sources include magnesium oxide $(\mathrm{MgO})$, magnesium chloride $\left(\mathrm{MgCl}_{2}\right)$, magnesium sulphate $\left(\mathrm{MgSO}_{4}\right)$, magnesium carbonate $\left(\mathrm{MgCO}_{3}\right)$, magnesium plates (electrochemical dosage), and seawater. Common calcium sources include calcium hydroxide $\left(\mathrm{Ca}(\mathrm{OH})_{2}\right)$ and calcium silicate hydrate $(\mathrm{CSH})$. Precipitation in the Mg-Ca-NH3-PO4 system has been explored thoroughly (110-117). In the absence of ammonium, it is possible to precipitate the struvite analogue magnesium potassium phosphate (MPP) (118-121). Other studies have targeted aluminium phosphate (Huang, Lee, and Lai 2015), ferric phosphate (123), or magnesium and sodium phosphates (Huang, Lee, and Lai 2015).

\begin{tabular}{|l|l|}
\hline Precipitation & \\
\hline Fate of Constituents \\
\hline $\begin{array}{l}\text { Pathogens may accumulate in precipitate if in the form of a slurry or powder. Pharmaceuticals have been found to attach } \\
\text { to the surface of precipitates rather than being incorporated in the crystal structure and can be removed by washing. It } \\
\text { can be expected that the findings regarding pharmaceuticals can be extrapolated to organic pollutants in general. }\end{array}$ \\
\hline Reference & Information Extracted \\
\hline$(63)$ & Pathogens may accumulate in precipitate if in the form of a slurry. \\
\hline$(71,124)$ & Pathogens may accumulate in precipitate if in the form of a powder. \\
\hline$(125,126)$ & $\begin{array}{l}\text { Pharmaceuticals have been found to attach to the surface of precipitates rather than being } \\
\text { incorporated in the crystal structure. }\end{array}$ \\
\hline$(127)$ & Pharmaceuticals can be removed from precipitates by washing. \\
\hline
\end{tabular}


Sorption is an umbrella term for process where one or several substances become attached to another substance referred to as sorbent. Sorption includes absorption, adsorption, and ion exchange (IEX). Absorption refers to the uptake by the bulk of the sorbent, and adsorption to the adherence onto the surface of the sorbent. In IEX, the uptake of ions of one kind by the ion exchanger is balanced by a release of ions of a different kind from the ion exchanger. IEX resins are either cation resins that exchange positively charged ions or anion resins that exchange negatively charged ions. A strict differentiation between absorption, adsorption, and IEX is often difficult if not impossible, as these processes often take place in parallel and with similar materials acting as sorbent or ion exchanger. The release of substances from a sorbent is referred to as desorption, the release of ions from an IEX resin is referred to as regeneration. Common desorption/regeneration media include ultrapure or de-ionised water (e.g. 128) as well as several kinds of acids including citric and hydrochloric acid.

When a nutrient-loaded sorbent is the target product of sorption, sorbents commonly used to this end include various forms of charcoal, aluminosilicate and calcium silicate minerals, and calcium oxides. When sorption is followed by desorption and regeneration, also synthetic sorbents/resins such as clinoptilolite-based mixed matrix membranes (e.g. Casadella et al., 2016), zirconium loaded okara (e.g. Nguyen et al., 2015), lanthanide-based metal-organic frameworks (e.g. Liu et al., 2016), or zirconium ferrite (e.g. Ishiwata et al., 2010) have been investigated.

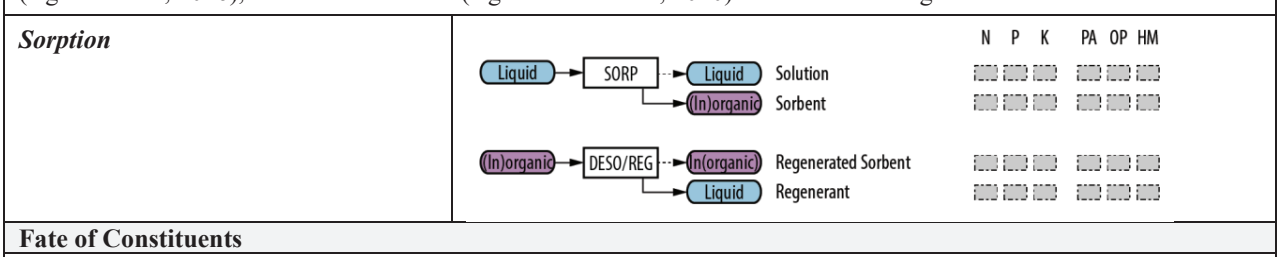

Charcoal has been shown to have the potential to adsorb urea, $\mathrm{NH}_{4}{ }^{+}$, and $\mathrm{PO}_{4}^{-}$. Mineral sorbents generally have good cation exchange properties and good affinity for $\mathrm{NH}_{4}{ }^{+}$and $\mathrm{K}^{+}$; because of differences in the selectivity towards certain cations, it is possible to transfer $\mathrm{NH}_{4}{ }^{+}$and $\mathrm{K}^{+}$but not $\mathrm{Na}^{+}$. Mineral sorbents have also been shown to act as precipitation nuclei for the surface precipitation of phosphates, for instance as calcium phosphate, notably if $\mathrm{Ca}^{2+}$ is released in exchange for $\mathrm{NH}_{4}{ }^{+}$or $\mathrm{K}^{+}$. Charcoal and mineral sorbents do not only remove nutrients from aqueous solutions. Charcoal has the potential to remove some waterborne pathogens, organic pollutants, and heavy metals from solutions. Mineral sorbents have the potential to remove organic pollutants and heavy metals from solutions.

\begin{tabular}{|l|l|}
\hline Reference & Information Extracted \\
\hline$(129)$ & Charcoal. Urea removal through physical adsorption \\
\hline$(130,131)$ & $\begin{array}{l}\text { Charcoal. Ammonium removal through chemical sorption in the form of reactions of ammonium with } \\
\text { oxygen function groups at biochar surface. }\end{array}$ \\
\hline$(132)$ & Charcoal. Phosphorus removal through through anion-exchange. \\
\hline$(131)$ & Charcoal. Phosphorus removal through chemical sorption. \\
\hline$(133)$ & $\begin{array}{l}\text { Zeolites. Good affinity for } \mathrm{NH}_{4}{ }^{+} \text {and } \mathrm{K}^{+} \text {through ion exchange. Differences in selectivity means that } \\
\mathrm{NH}_{4}{ }^{+} \text {and } \mathrm{K}^{+} \text {is sorbed while } \mathrm{Na}^{+} \text {is not. }\end{array}$ \\
\hline$(134)$ & $\mathrm{Wollastonite}$ Removes $\mathrm{NH}_{4}{ }^{+}$through cation exchange. $\mathrm{PO}_{4}{ }^{3-}$ are not sorbed as negatively charged. \\
\hline$(135-137)$ & Zeolites. Precipitation nuclei for P. \\
\hline$(134,138)$ & Wollastonite. Precipitation nuclei for P. \\
\hline$(139)$ & Calcinated egg shells. Precipitation nuclei for P. \\
\hline$(140)$ & Charcoal. Some waterborne pathogens transferred to activated carbon. \\
\hline$(141)$ & Charcoal. Common in water treatment for removing organic pollutants. \\
\hline$(142)$ & Charcoal. Has the potential to remove certain organic pollutants. \\
\hline$(143)$ & Charcoal. Has the potential to remove heavy metal ions. \\
\hline$(144,145)$ & Charcoal. Can reduce heavy metal plant availability in soils. \\
\hline$(146)$ & Zeolites. Used as selective adsorbents for removing certain organic pollutants from aqueous solutions. \\
\hline$(147)$ & Calcinated egg shells. Useful for the removal of certain organic micropollutants from aqueous solutions. \\
\hline$(148)$ & Calcinated egg shells. Have been shown useful for the removal of certain organic micropollutants. \\
\hline$(149,150)$ & Zeolites. Have the potential to remove heavy metals from aqueous solutions and slurries. \\
\hline$(151)$ & Wollastonite. Has the potential to sorb heavy metals. \\
\hline$(152)$ & Calcinated egg shells. Useful for the removal of heavy metals. \\
\hline
\end{tabular}




\begin{tabular}{|c|c|c|c|}
\hline \multicolumn{4}{|c|}{ SEPARATION - Elution, Thermal Treatment } \\
\hline \multicolumn{4}{|c|}{$\begin{array}{l}\text { Thermo-chemical treatment of ashes refers to ash treatment at temperatures of around } 1000^{\circ} \mathrm{C} \text { with the addition of } \\
\text { chloride additives that facilitate the formation of heavy metal compounds with low evaporation temperatures. Thermo- } \\
\text { reductive treatment of ashes refers to ash treatment at temperatures of around } 1500{ }^{\circ} \mathrm{C} \text { and at very low partial oxygen } \\
\text { pressure. Once transferred to the gaseous phase, } \mathrm{P} \text { can be condensated to } \mathrm{P}_{4} \text { or } \mathrm{H}_{3} \mathrm{PO}_{4} \text {. Elution or leaching is the process } \\
\text { of extracting substances from a solid by dissolving them in a liquid. Elution is useful for example to extract } \mathrm{P} \text { from ashes. }\end{array}$} \\
\hline \multicolumn{2}{|c|}{ Thermal Treatment } & $\begin{array}{l}\text { Heat, chloride additives } \\
\text { Inorganic } \longrightarrow \text { TH.CHEM } \longrightarrow \text { Garganic Depolluted Ash } \\
\end{array}$ & 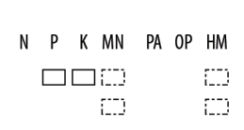 \\
\hline \multicolumn{2}{|l|}{ Elution } & Inorganic $\longrightarrow$ (Inorganic Leached Ash & 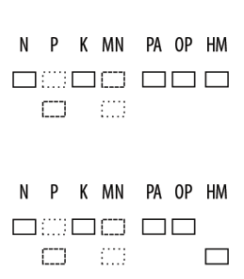 \\
\hline \multicolumn{4}{|c|}{ Fate of Constituents } \\
\hline \multicolumn{4}{|c|}{$\begin{array}{l}\text { At temperatures around } 1500^{\circ} \mathrm{C} \text {, many common phosphate compounds are reduced and vaporised. Removal efficiencies } \\
\text { during thermal treatment depend on the heavy metal and the process design and range from almost no to nearly complete } \\
\text { removal. Virtually no P is being lost in the process. Near complete separation from heavy metals can ideally be achieved. } \\
\text { Alkaline elution dissolves phosphorus and aluminium to some extent, but not iron and heavy metals; acidic leaching } \\
\text { dissolves phosphorus as well as metals. }\end{array}$} \\
\hline Reference & Informc & & \\
\hline \multicolumn{4}{|l|}{$(153)$} \\
\hline \multicolumn{4}{|l|}{$(154)$} \\
\hline \multicolumn{4}{|l|}{$(45)$} \\
\hline$(48)$ & Leach & & \\
\hline
\end{tabular}


Figure S5.1 Summary figure of treatment processes (Process groups associated with each number label are described in Table S5.1)

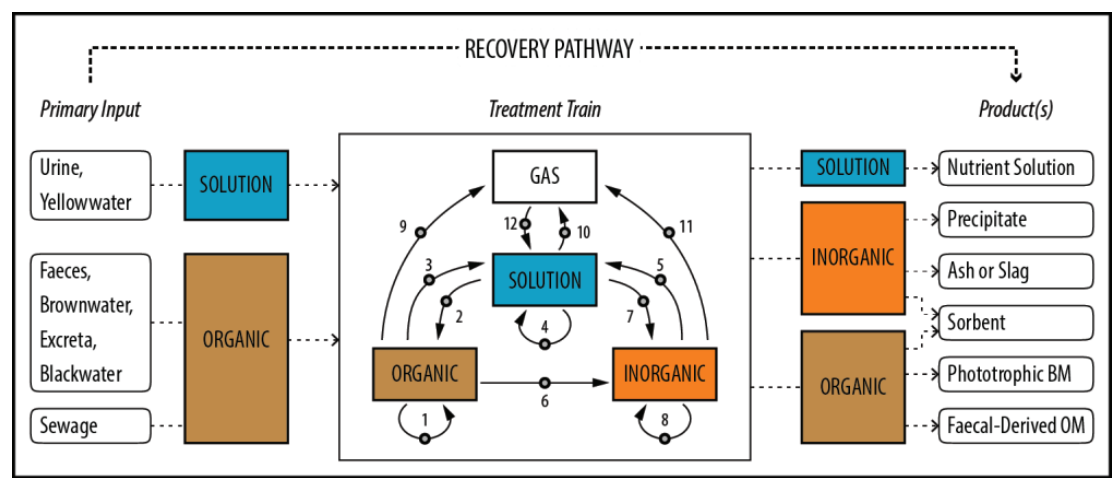


Table S5.1 Summary table of treatment processes, their repective process outputs and nutrient and pollutant partitioning across process outputs (numbers in first column refer to numbers in Figure S5.1)

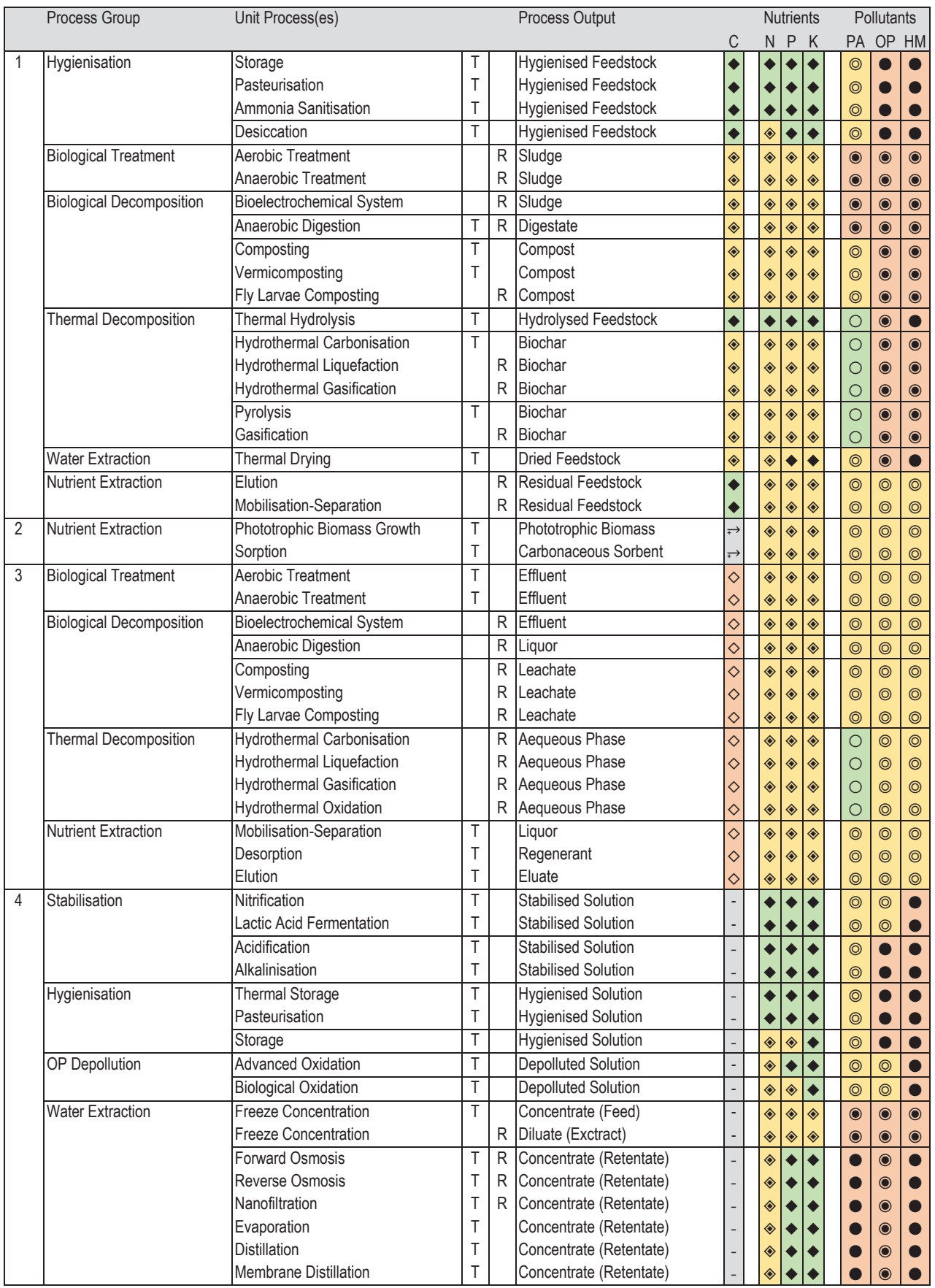


Table S5.1 (continued) Summary table of treatment processes, their repective process outputs and nutrient and pollutant partitioning across process outputs (numbers in first column refer to numbers in Figure S5.1)

\begin{tabular}{|c|c|c|c|c|c|c|c|c|c|c|c|c|}
\hline & Process Group & Unit Process(es) & & & Process Output & & & rient & & & Illutar & \\
\hline & & & & & & C & $\mathrm{N}$ & $\mathrm{Pr}$ & $\mathrm{K}$ & PA & $\mathrm{OP}$ & $\mathrm{HM}$ \\
\hline 4 & Separation & Electrodialysis & $T$ & & Concentrate (Extract) & - & $\bullet$ & $\bullet$ & $\bullet$ & 0 & (0) & (0) \\
\hline & & Electrodialysis & & $\mathrm{R}$ & Diluate (Feed) & - & $\diamond$ & $\Delta<<$ & $\diamond$ & 0 & (2) & (2) \\
\hline & & Forward Osmosis & $T$ & $\mathrm{R}$ & Diluate (Draw Solution) & - & $\Delta$ & $\Delta<$ & $\diamond$ & 0 & 0 & 0 \\
\hline & & Reverse Osmosis & $\mathrm{T}$ & $\mathrm{R}$ & Diluate (Permeate) & - & $\Delta$ & $\diamond$ & $\diamond$ & 0 & 0 & 0 \\
\hline & & Nanofiltration & $\mathrm{T}$ & $\mathrm{R}$ & Diluate (Permeate) & - & $\diamond$ & $\diamond<$ & $\diamond$ & 0 & 0 & 0 \\
\hline & & Phototrophic Biomass Growth & & $\mathrm{R}$ & Residual (Feed) & - & $\diamond$ & $\diamond \mid<$ & $\diamond$ & (0) & () & (0) \\
\hline & & Sorption & & $\mathrm{R}$ & Residual (Feed) & - & $\diamond$ & $\diamond \mid$ & $\diamond$ & (0) & (0) & (2) \\
\hline & & Precipitation & & $\mathrm{R}$ & Residual (Feed) & - & $\diamond$ & $\Delta$ & $\diamond$ & (2) & () & (0) \\
\hline & & Stripping & & $\mathrm{R}$ & Residual (Feed) & - & $\diamond$ & $\bullet$ & $\bullet$ & & 0 & 0 \\
\hline 5 & Nutrient Extraction & Desorption & $T$ & & Regenerant & - & $\bullet$ & $\bullet$ & $\bullet$ & (0) & (2) & (2) \\
\hline & & Elution & $\mathrm{T}$ & & Regenerant & - & $\bullet$ & 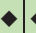 & $\bullet$ & () & () & () \\
\hline 6 & Nutrient Extraction & Controlled Precipitation & $T$ & & Precipitate & $\diamond$ & $\diamond$ & $\bullet$ & $\diamond$ & 0 & 0 & 0 \\
\hline & Thermal Decomposition & Hydrothermal Carbonisation & & $\mathrm{R}$ & Ash & $\diamond$ & $\diamond$ & $\bullet$ & $\bullet$ & 0 & 0 & 0 \\
\hline & & Hydrothermal Liquefaction & & $\mathrm{R}$ & Ash & $\diamond$ & $\diamond$ & $\bullet$ & $\bullet$ & 0 & 0 & ○ \\
\hline & & Hydrothermal Gasification & & $\mathrm{R}$ & Ash & $\diamond$ & $\diamond$ & - & $\bullet$ & 0 & 0 & 0 \\
\hline & & Hydrothermal Oxidation & & $\mathrm{R}$ & Ash & $\diamond$ & $\diamond$ & $\bullet$ & $\bullet$ & 0 & 0 & ○ \\
\hline & & Pyrolysis & & $\mathrm{R}$ & Ash & $\diamond$ & $\diamond$ & $\bullet$ & $\bullet$ & 0 & 0 & 0 \\
\hline & & Combustion & & $\mathrm{R}$ & Ash & $\diamond$ & $\diamond$ & - & - & 0 & 0 & o \\
\hline 7 & Nutrient Extraction & Controlled Precipitation & $T$ & & Precipitate & $\diamond$ & $\diamond$ & $\Delta$ & $\Delta$ & () & (0) & () \\
\hline & Nutrient Extraction & Sorption & $T$ & & Mineral Sorbent & $\diamond$ & $\diamond$ & $\diamond$ & $\diamond$ & (2) & (2) & (2) \\
\hline 8 & Depollution & Thermal Treatment & $T$ & & Depolluted Ash & $\bullet$ & $\bullet$ & $\Delta$ & $\bullet$ & 0 & 0 & (?) \\
\hline 9 & Biological Treatment & Aerobic Treatment & & $\mathrm{R}$ & Gaseous Emissions & $\diamond$ & $\diamond$ & $\Delta<$ & $\diamond$ & 0 & 0 & 0 \\
\hline & Biological Treatment & Anaerobic Treatment & & $\mathrm{R}$ & Gaseous Emissions & $\diamond$ & $\diamond$ & $\diamond \mid<$ & $\diamond$ & 0 & 0 & 0 \\
\hline & Biological Decomposition & Anaerobic Digestion & & $\mathrm{R}$ & Gaseous Emissions & $\diamond$ & $\diamond$ & $\diamond \mid<$ & $\diamond$ & O & 0 & O \\
\hline & & Composting & & $\mathrm{R}$ & Gaseous Emissions & $\diamond$ & $\diamond$ & $\diamond \mid<$ & $\diamond$ & 0 & 0 & 0 \\
\hline & & Vermicomposting & & $\mathrm{R}$ & Gaseous Emissions & $\diamond$ & $\diamond$ & $\diamond \mid$ & $\diamond$ & 0 & ○ & 0 \\
\hline & & Fly Larvae Composting & & $\mathrm{R}$ & Gaseous Emissions & $\diamond$ & $\diamond$ & $\diamond$ & $\diamond$ & 0 & 0 & 0 \\
\hline & Thermal Decomposition & Hydrothermal Carbonisation & & $\mathrm{R}$ & Gaseous Emissions & $\diamond$ & $\diamond$ & $\Delta$ & $\diamond$ & 0 & 0 & 0 \\
\hline & & Hydrothermal Liquefaction & & $\mathrm{R}$ & Gaseous Emissions & $\diamond$ & $\diamond$ & $\Delta<$ & $\diamond$ & 0 & 0 & 0 \\
\hline & & Hydrothermal Gasification & & $\mathrm{R}$ & Gaseous Emissions & $\diamond$ & $\diamond$ & $\diamond$ & $\diamond$ & 0 & 0 & 0 \\
\hline & & Hydrothermal Oxidation & & $\mathrm{R}$ & Gaseous Emissions & $\diamond$ & $\diamond$ & $\diamond \mid$ & $\diamond$ & 0 & 0 & O \\
\hline & & Pyrolysis & & $\mathrm{R}$ & Gaseous Emissions & $\diamond$ & $\diamond$ & $\diamond$ & $\diamond$ & 0 & 0 & 0 \\
\hline & & Gasification & & $\mathrm{R}$ & Gaseous Emissions & $\diamond$ & $\diamond$ & $\diamond$ & $\diamond$ & 0 & 0 & 0 \\
\hline & & Combustion & & $\mathrm{R}$ & Gaseous Emissions & $\diamond$ & $\diamond$ & $\diamond \mid<$ & $\diamond$ & 0 & 0 & 0 \\
\hline & Hygienisation & Desiccation & & $\mathrm{R}$ & Gaseous Emissions & $\diamond$ & $\Delta$ & $\Delta<$ & $\diamond$ & 0 & 0 & 0 \\
\hline & & Lime Treatment & & $\mathrm{R}$ & Gaseous Emissions & $\diamond$ & $\Delta$ & $\diamond$ & $\diamond$ & 0 & 0 & 0 \\
\hline & Water Extraction & Thermal Drying & & $\mathrm{R}$ & Gaseous Emissions & $\diamond$ & $\diamond$ & $\Delta<$ & $\diamond$ & 0 & 0 & 0 \\
\hline 10 & Nutrient Extraction & Stripping & $\mathrm{T}$ & & Gaseous Emissions & $\diamond$ & $\diamond$ & $\diamond<$ & $\diamond$ & 0 & 0 & 0 \\
\hline & Water Extraction & Evaporation & & $R$ & Gaseous Emissions & $\diamond$ & $\diamond$ & $\diamond<$ & $\diamond$ & 0 & 0 & 0 \\
\hline & Water Extraction & Distillation & & $\mathrm{R}$ & Gaseous Emissions & $\diamond$ & $\diamond$ & $\diamond$ & $\diamond$ & 0 & 0 & 0 \\
\hline & Water Extraction & Membrane Distillation & & $\mathrm{R}$ & Gaseous Emissions & $\diamond$ & $\diamond$ & $\diamond<$ & $\diamond$ & 0 & 0 & 0 \\
\hline 11 & Nutrient Extraction & Thermal Treatment & & $\mathrm{R}$ & Gaseous Emissions & - & - & $\bullet$ & $\diamond$ & 0 & 0 & (2) \\
\hline & HM Depollution & Thermal Treatment & & $\mathrm{R}$ & Gaseous Emissions & - & - & $\diamond<$ & $\diamond$ & 0 & 0 & 0 \\
\hline 12 & Nutrient Extraction & Condensation & $T$ & & Condensate & - & $\bullet$ & 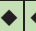 & • & - & - & - \\
\hline & Nutrient Extraction & Wet Scrubbing & $T$ & & Scrubbing Liquid & - & $\bullet$ & - & - & (2) & (2) & (?) \\
\hline$\bullet$ & Present in input, mostly car & ured in this output. & & & & & & & & & & \\
\hline$\diamond$ & Present in input, partially ce & tured in this output. & & & & & & & & & & \\
\hline$\diamond$ & Present in input, not captur & in this output. & & & & & & & & & & \\
\hline- & Not present in input. & & & & & & & & & & & \\
\hline$\Rightarrow$ & Not present in input, added & om other stream. & & & & & & & & & & \\
\hline 0 & Fully inactivated/degraded/ & moved. & & & & & & & & & & \\
\hline () & Fully or partially inactivated & egraded/removed. & & & & & & & & & & \\
\hline O & Partially inactivated/degrad & d/removed. & & & & & & & & & & \\
\hline - & Hardly inactivated/degrade & removed. & & & & & & & & & & \\
\hline
\end{tabular}




\section{References}

1. Orhon, D. (2015) 'Evolution of the activated sludge process: the first 50 years', Journal of Chemical Technology \& Biotechnology, 90(4), pp. 608-640. doi: 10.1002/jctb.4565.

2. Cooper, P. F. (2001) 'Historical aspects of wastewater treatment', in Lens, P. N. L., Zeeman, G., and Lettinga, G. (eds) Decentralised sanitation and reuse: concepts, systems and implementation. IWA, pp. 11-38.

3. Modin, O. et al. (2016) 'Nonoxidative removal of organics in the activated sludge process', Critical Reviews in Environmental Science and Technology, 46(7), pp. 635-672. doi: 10.1080/10643389.2016.1149903.

4. McCarty, P. L. and Smith, D. P. (1986) 'Anaerobic wastewater treatment', Environmental Science and Technology, 20(12), pp. 1200-1206. doi: 10.1021/es00154a002.

5. Law, Y. et al. (2012) 'Nitrous oxide emissions from wastewater treatment processes', Philosophical Transactions of the Royal Society B: Biological Sciences, 367(1593), pp. 1265-1277. doi: 10.1098/rstb.2011.0317.

6. Campos, J. L. et al. (2016) 'Greenhouse Gases Emissions from Wastewater Treatment Plants: Minimization, Treatment, and Prevention', Journal of Chemistry, 2016. doi: 10.1155/2016/3796352.

7. Li, B. et al. (2015) 'Profile and Fate of Bacterial Pathogens in Sewage Treatment Plants Revealed by High-Throughput Metagenomic Approach', Environmental Science and Technology, 49(17), pp. 10492-10502. doi: 10.1021/ acs.est.5b02345.

8. Wen, Q. et al. (2009) 'Fate of pathogenic microorganisms and indicators in secondary activated sludge wastewater treatment plants', Journal of Environmental Management. Elsevier Ltd, 90(3), pp. 1442-1447. doi: 10.1016/j. jenvman.2008.09.002.

9. Katsoyiannis, A. and Samara, C. (2005) 'Persistent organic pollutants (POPs) in the conventional activated sludge treatment process: Fate and mass balance', Environmental Research, 97(3), pp. 245-257. doi: 10.1016/j. envres.2004.09.001.

10. de Graaff, M. S. et al. (2011) 'Fate of hormones and pharmaceuticals during combined anaerobic treatment and nitrogen removal by partial nitritation-anammox in vacuum collected black water', Water Research. Elsevier Ltd, 45(1), pp. 375-383. doi: 10.1016/j.watres.2010.08.023.

11. Butkovskyi, A. et al. (2016) 'Fate of personal care and household products in source separated sanitation', Journal of Hazardous Materials. Elsevier B.V., 320, pp. 427-434. doi: 10.1016/j.jhazmat.2016.08.059.

12. Zeeman, G. and Kujawa-Roeleveld, K. (2013) Anaerobic treatment of source- separated domestic wastewater, Source separation and decentralization for wastewater management.

13. des Mes, T. Z. D. et al. (2008) 'Anaerobic biodegradation of estrogens - Hard to digest', Water Science and Technology, 57(8), pp. 1177-1182. doi: 10.2166/wst.2008.102.

14. Gude, V. G. (2016) 'Wastewater treatment in microbial fuel cells - An overview', Journal of Cleaner Production. Elsevier Ltd, 122, pp. 287-307. doi: 10.1016/j.jclepro.2016.02.022.

15. He, L. et al. (2017) 'Advances in microbial fuel cells for wastewater treatment', Renewable and Sustainable Energy Reviews, 71(May 2016), pp. 388-403. doi: 10.1016/j.rser.2016.12.069.

16. Santoro, C. et al. (2017) 'Microbial fuel cells: From fundamentals to applications. A review', Journal of Power Sources. Elsevier B.V, 356, pp. 225-244. doi: 10.1016/j.jpowsour.2017.03.109.

17. Kelly, P. T. and He, Z. (2014) 'Nutrients removal and recovery in bioelectrochemical systems: A review', Bioresource Technology. Elsevier Ltd, 153, pp. 351-360. doi: 10.1016/j.biortech.2013.12.046.

18. Demirbas, A., Taylan, O. and Kaya, D. (2016) 'Biogas production from municipal sewage sludge (MSS)', Energy Sources, Part A: Recovery, Utilization and Environmental Effects. Taylor \& Francis, 38(20), pp. 3027-3033. doi: 10.1080/15567036.2015.1124944.

19. Scheinemann, H. A. et al. (2015) 'Hygienisation and Nutrient Conservation of Sewage Sludge or Cattle Manure by Lactic Acid Fermentation'. doi: 10.1371/journal.pone.0118230.

20. Andreev, N. et al. (2018) 'Lactic acid fermentation of human excreta for agricultural application', Journal of Environmental Management. Elsevier Ltd, 206, pp. 890-900. doi: 10.1016/j.jenvman.2017.11.072.

21. Jonsson, H. et al. (2004) 'Guidelines on the Use of Urine and Faeces in Crop Production', EcoSanRes Publication Series, 2, pp. 1-35.

22. Lohri, C. R. et al. (2017) 'Treatment technologies for urban solid biowaste to create value products: a review with focus on low- and middle-income settings', Reviews in Environmental Science and Biotechnology, 16(1), pp. 81-130. doi: 10.1007/s11157-017-9422-5.

23. Daelman, M. R. J. et al. (2012) 'Methane emission during municipal wastewater treatment', Water Research. Elsevier Ltd, 46(11), pp. 3657-3670. doi: 10.1016/j.watres.2012.04.024.

24. Tiquia, S. M., Richard, T. L. and Honeyman, M. S. (2002) 'Carbon, nutrient, and mass loss during composting.pdf', Nutrient Cycling in Agroecosystems, 62(1), pp. 15-24. 
25. Ulén, B. (1997) 'Leaching of plant nutrients and heavy metals during the composting of household wastes and chemical characterization of the final product', Acta Agriculturae Scandinavica Section B: Soil and Plant Science, 47(3), pp. 142-148. doi: 10.1080/09064719709362454.

26. Sadef, Y., Poulsen, T. G. and Bester, K. (2014) 'Modeling organic micro pollutant degradation kinetics during sewage sludge composting', Waste Management. Elsevier Ltd, 34(11), pp. 2007-2013. doi: 10.1016/j.wasman.2014.07.001.

27. Sadef, Y. et al. (2016) 'Uncertainty in degradation rates for organic micro-pollutants during full-scale sewage sludge composting', Waste Management. Elsevier Ltd, 56, pp. 396-402. doi: 10.1016/j.wasman.2016.06.018.

28. Nigussie, A. et al. (2016) 'Vermicomposting as a technology for reducing nitrogen losses and greenhouse gas emissions from small-scale composting', Journal of Cleaner Production. Elsevier Ltd, 139(x), pp. 429-439. doi: 10.1016/j.jclepro.2016.08.058.

29. Lalander, C., Hill, G. B. and Vinnerås, B. (2013) 'Hygienic quality of faeces treated in urine diverting vermicomposting toilets', Waste Management. Elsevier Ltd, 33(11), pp. 2204-2210. doi: 10.1016/j.wasman.2013.07.007.

30. Song, X. et al. (2014) 'Heavy metal and nutrient changes during vermicomposting animal manure spiked with mushroom residues', Waste Management, 34(11), pp. 1977-1983. doi: 10.1016/j.wasman.2014.07.013.

31. Lalander, C. et al. (2014) 'High waste-to-biomass conversion and efficient Salmonella spp. reduction using black soldier fly for waste recycling', Agronomy for Sustainable Development, 35(1), pp. 261-271. doi: 10.1007/ s13593-014-0235-4.

32. Lalander, C. et al. (2013) 'Faecal sludge management with the larvae of the black soldier fly (Hermetia illucens) - From a hygiene aspect', Science of the Total Environment. Elsevier B.V., 458-460, pp. 312-318. doi: 10.1016/j.scitotenv.2013.04.033.

33. Lalander, C. et al. (2016) 'Fate of pharmaceuticals and pesticides in fly larvae composting', Science of the Total Environment. The Authors, 565, pp. 279-286. doi: 10.1016/j.scitotenv.2016.04.147.

34. Barber, W. P. F. (2016) 'Thermal hydrolysis for sewage treatment: A critical review', Water Research. Elsevier Ltd, 104, pp. 53-71. doi: 10.1016/j.watres.2016.07.069.

35. Villamil, J. A. et al. (2018) 'Valorisation of the liquid fraction from hydrothermal carbonisation of sewage sludge by anaerobic digestion', Journal of Chemical Technology and Biotechnology, 93(2), pp. 450-456. doi: 10.1002/ jctb. 5375 .

36. Yao, C. et al. (2016) 'Utilization of recovered nitrogen from hydrothermal carbonization process by Arthrospira platensis', Bioresource Technology. Elsevier Ltd, 212, pp. 26-34. doi: 10.1016/j.biortech.2016.03.166.

37. Kruse, A. et al. (2016) 'Fate of Nitrogen during Hydrothermal Carbonization', Energy and Fuels, 30(10), pp. 80378042. doi: 10.1021 /acs.energyfuels.6b01312.

38. Toufiq Reza, M. et al. (2016) 'Hydrothermal Carbonization (HTC) of Cow Manure: Carbon and Nitrogen Distributions in HTC Products', Environmental Progress \& Sustainable Energy, 35(4), pp. 1002-1011. doi: 10.1002/ ep.

39. Libra, J. A. et al. (2011) 'Hydrothermal carbonization of biomass residuals: a comparative review of the chemistry, processes and applications of wet and dry pyrolysis', Biofuels, 2(1), pp. 71-106. doi: 10.4155/bfs.10.81.

40. vom Eyser, C. et al. (2015) 'Pharmaceutical load in sewage sludge and biochar produced by hydrothermal carbonization', Science of the Total Environment. Elsevier B.V., 537, pp. 180-186. doi: 10.1016/j.scitotenv.2015.08.021.

41. Edmundson, S. et al. (2017) 'Phosphorus and nitrogen recycle following algal bio-crude production via continuous hydrothermal liquefaction', Algal Research. Elsevier, 26(June), pp. 415-421. doi: 10.1016/j.algal.2017.07.016.

42. Lu, J. et al. (2017) 'Simultaneous production of biocrude oil and recovery of nutrients and metals from human feces via hydrothermal liquefaction', Energy Conversion and Management. Elsevier Ltd, 134, pp. 340-346. doi: 10.1016/j.enconman.2016.12.052.

43. Blöcher, C., Niewersch, C. and Melin, T. (2012) 'Phosphorus recovery from sewage sludge with a hybrid process of low pressure wet oxidation and nanofiltration', Water Research, 46(6), pp. 2009-2019. doi: 10.1016/j. watres.2012.01.022.

44. Yanagida, T. et al. (2009) 'Recovery of activated carbon catalyst, calcium, nitrogen and phosphate from effluent following supercritical water gasification of poultry manure', Bioresource Technology. Elsevier Ltd, 100(20), pp. 4884-4886. doi: 10.1016/j.biortech.2009.05.042.

45. Stendahl, K. and Jäfverström, S. (2004) 'Recycling of sludge with the Aqua Reci process', Water Science and Technology, 49(1), pp. 233-240.

46. Bridle, T. and Pritchard, D. (2004) 'Energy and nutrient recovery from sewage sludge via pyrolysis', Water Science Technololgy, 50(February), pp. 169-175.

47. Hossain, M. K. et al. (2011) 'Influence of pyrolysis temperature on production and nutrient properties of wastewater sludge biochar', Journal of Environmental Management. Elsevier Ltd, 92(1), pp. 223-228. doi: 10.1016/j. jenvman.2010.09.008. 
48. Gorazda, K. et al. (2017) 'Sewage sludge as a fuel and raw material for phosphorus recovery: Combined process of gasification and P extraction', Waste Management. Elsevier Ltd, 73, pp. 404-415. doi: 10.1016/j.wasman.2017.10.032.

49. Fitzmorris, K. B. et al. (2007) 'Decrease of Time for Pathogen Inactivation in Alkaline Disinfection Systems Using Pressure', Water Environment Research, 79(4), pp. 388-395. doi: 10.2175/106143006X111862.

50. Hill, G. B., Baldwin, S. A. and Vinnerås, B. (2013) 'Composting toilets a misnomer: Excessive ammonia from urine inhibits microbial activity yet is insufficient in sanitizing the end-product', Journal of Environmental Management. Elsevier Ltd, 119, pp. 29-35. doi: 10.1016/j.jenvman.2012.12.046.

51. Arthurson, V. (2008) 'Proper sanitization of sewage sludge: A critical issue for a sustainable society', Applied and Environmental Microbiology, 74(17), pp. 5267-5275. doi: 10.1128/AEM.00438-08.

52. Udert, K. M., Etter, B. and Gounden, T. (2016) 'Promoting sanitation in South Africa through nutrient recovery from urine', Gaia, 25(3), pp. 194-196. doi: 10.14512/gaia.25.3.12.

53. Horttanainen, M., Deviatkin, I. and Havukainen, J. (2017) 'Nitrogen release from mechanically dewatered sewage sludge during thermal drying and potential for recovery', Journal of Cleaner Production. Elsevier Ltd, 142, pp. 1819-1826. doi: 10.1016/j.jclepro.2016.11.102.

54. Suzuki, Y. et al. (2006) 'Evaluation of sludge reduction and phosphorus recovery efficiencies in a new advanced wastewater treatment system using denitrifying polyphosphate accumulating organisms', Water Science and Technology, 53(6), pp. 107-113. doi: 10.2166/wst.2006.179.

55. Heinzmann, B. (2005) 'Phosphorus recycling in sewage treatment plants with biological phosphorus removal.', Water science and technology : a journal of the International Association on Water Pollution Research, 52(10-11), pp. 543-8.

56. Happe, M. et al. (2016) 'Scale-up of phosphate remobilization from sewage sludge in a microbial fuel cell', Bioresource Technology. Elsevier Ltd, 200, pp. 435-443. doi: 10.1016/j.biortech.2015.10.057.

57. Fischer, F. et al. (2011) 'Microbial fuel cell enables phosphate recovery from digested sewage sludge as struvite', Bioresource Technology. Elsevier Ltd, 102(10), pp. 5824-5830. doi: 10.1016/j.biortech.2011.02.089.

58. Antakyali, D. et al. (2013) 'Large-scale application of nutrient recovery from digested sludge as struvite', Water Practice and Technology, 8(2), pp. 256-262. doi: 10.2166/wpt.2013.027.

59. Güney, K., Weidelener, A. and Krampe, J. (2008) 'Phosphorus recovery from digested sewage sludge as MAP by the help of metal ion separation', Water Research, 42(18), pp. 4692-4698. doi: 10.1016/j.watres.2008.08.016.

60. Niewersch, C. et al. (2008) 'Potentials of using nanofiltration to recover phosphorus from sewage sludge', Water Science and Technology, 57(5), pp. 707-714. doi: 10.2166/wst.2008.119.

61. Hellström, D., Johansson, E. and Grennberg, K. (1999) 'Storage of human urine: Acidification as a method to inhibit decomposition of urea', Ecological Engineering, 12(3-4), pp. 253-269. doi: 10.1016/S0925-8574(98)000743.

62. Antonini, S. et al. (2012) 'Solar thermal evaporation of human urine for nitrogen and phosphorus recovery in Vietnam', Science of the Total Environment. Elsevier B.V., 414, pp. 592-599. doi: 10.1016/j.scitotenv.2011.11.055.

63. Udert, K. M., Larsen, T. A. and Gujer, W. (2006) 'Fate of major compounds in source-separated urine', Water Science and Technology, 54(11-12), pp. 413-420. doi: 10.2166/wst.2006.921.

64. Randall, D. G. et al. (2016) 'A novel approach for stabilizing fresh urine by calcium hydroxide addition', Water Research, 95, pp. 361-369. doi: 10.1016/j.watres.2016.03.007.

65. Ek, M. et al. (2006) 'Concentration of nutrients from urine and reject water from anaerobically digested sludge', Water Science and Technology, 54(11-12), pp. 437-444. doi: 10.2166/wst.2006.924.

66. Udert, K. M. and Wächter, M. (2012) 'Complete nutrient recovery from source-separated urine by nitrification and distillation’, Water Research, 46(2), pp. 453-464. doi: 10.1016/j.watres.2011.11.020.

67. Fumasoli, A. et al. (2016) 'Operating a pilot-scale nitrification/distillation plant for complete nutrient recovery from urine’, Water Science and Technology, 73(1), pp. 215-222. doi: 10.2166/wst.2015.485.

68. Bischel, H. N. et al. (2015) 'Inactivation kinetics and mechanisms of viral and bacterial pathogen surrogates during urine nitrification', Environ. Sci.: Water Res. Technol. Royal Society of Chemistry, 1(1), pp. 65-76. doi: 10.1039/C4EW00065J.

69. Andreev, N. et al. (2017) 'Lactic acid fermentation of human urine to improve its fertilizing value and reduce odour emissions', Journal of Environmental Management, 198, pp. 63-69. doi: 10.1016/j.jenvman.2017.04.059.

70. Zhou, X. et al. (2017) 'Investigation on microbial inactivation and urea decomposition in human urine during thermal storage', Journal of Water Sanitation and Hygiene for Development, 7(3), pp. 378-386. doi: 10.2166/ washdev.2017.142.

71. Lahr, R. H. et al. (2016) 'Urine Bacterial Community Convergence through Fertilizer Production: Storage, Pasteurization, and Struvite Precipitation', Environmental Science and Technology, 50(21), pp. 11619-11626. doi: 10.1021/acs.est.6b02094. 
72. Tilley, E., Atwater, J. and Mavinic, D. (2008) 'Effects of storage on phosphorous recovery from urine', Environmental Technology, 29(7), pp. 807-816.

73. Udert, K. M., Larsen, T. A. and Gujer, W. (2003) 'Biologically induced precipitation in urine-collecting systems', Water Science and Technology: Water Supply, 3(3), pp. 71-78. doi: 10.1016/S0043-1354(03)00071-X.

74. Deng, Y. and Zhao, R. (2015) 'Advanced Oxidation Processes (AOPs) in Wastewater Treatment', Current Pollution Reports, 1(3), pp. 167-176. doi: 10.1007/s40726-015-0015-z.

75. Giannakis, S., Rtimi, S. and Pulgarin, C. (2017) 'Light-assisted advanced oxidation processes for the elimination of chemical and microbiological pollution of wastewaters in developed and developing countries', Molecules, 22(7). doi: $10.3390 /$ molecules22071070.

76. Abdel-Shafy, H. I. and Mansour, M. S. M. (2016) 'Integration of effective microorganisms and membrane bioreactor for the elimination of pharmaceutical active compounds from urine for safe reuse', Journal of Water Reuse and Desalination, 6(4), pp. 495-504. doi: 10.2166/wrd.2016.141.

77. Al-Musharafi, S. K. (2016) 'Heavy Metals in Sewage Treated Effluents: Pollution and Microbial Bioremediation from Arid Regions', The Open Biotechnology Journal, 10(Suppl-2, M8), pp. 352-362. doi: 10.2174/1874070701610010352.

78. Lind, B. B., Ban, Z. and Bydén, S. (2001) 'Volume reduction and concentration of nutrients in human urine', Ecological Engineering, 16(4), pp. 561-566. doi: 10.1016/S0925-8574(00)00107-5.

79. Gulyas, H. et al. (2004) 'Freeze concentration for enrichment of nutrients in yellow water from no-mix toilets', Water Science and Technology, 50(6), pp. 61-68.

80. Bethune, D. N., Chu, A. and Ryan, M. C. (2016) 'Passive evaporation of source-separated urine from dry toilets: UES optimization and dry product accumulation over time', Journal of Water, Sanitation and Hygiene for Development, 6(1), pp. 96-103. doi: 10.2166/washdev.2016.103.

81. Dutta, S. and Vinnerås, B. (2016) 'Fertilizer from dried human urine added to ash and lime -A potential product from eco-sanitation system', Water Science and Technology, 74(6), pp. 1436-1445. doi: 10.2166/wst.2016.324.

82. Jiang, S. et al. (2017) 'Effect of initial $\mathrm{pH}$ and $\mathrm{pH}$-adjusted acid on nutrient recovery from hydrolysis urine by combining acidification with evaporation-crystallization', Environmental Science and Pollution Research. Environmental Science and Pollution Research, 24(4), pp. 3872-3881. doi: 10.1007/s11356-016-8052-8.

83. Tun, L. L. et al. (2016) 'Dewatering of source-separated human urine for nitrogen recovery by membrane distillation', Journal of Membrane Science. Elsevier, 512, pp. 13-20. doi: 10.1016/j.memsci.2016.04.004.

84. Liu, Q. et al. (2016) 'Integrated forward osmosis-membrane distillation process for human urine treatment', Water Research. Elsevier Ltd, 91, pp. 45-54. doi: 10.1016/j.watres.2015.12.045.

85. Wijekoon, K. C. et al. (2014) 'Rejection and fate of trace organic compounds (TrOCs) during membrane distillation', Journal of Membrane Science. Elsevier, 453, pp. 636-642. doi: 10.1016/j.memsci.2013.12.002.

86. Zhang, J. et al. (2014) 'Mining nutrients (N, P, K) from urban source seprated urine by forward osmosis', Environmental Science and Technology, 48(6), pp. 3386-3394.

87. Alturki, A. A. et al. (2013) 'Removal of trace organic contaminants by the forward osmosis process', Separation and Purification Technology. Elsevier B.V., 103, pp. 258-266. doi: 10.1016/j.seppur.2012.10.036.

88. Pronk, W. et al. (2006) 'Nanofiltration for the separation of pharmaceuticals from nutrients in source-separated urine', Water Research, 40(7), pp. 1405-1412. doi: 10.1016/j.watres.2006.01.038.

89. Pronk, W., Biebow, M. and Boller, M. (2006) 'Electrodialysis for recovering salts from a urine solution containing micropollutants', Environmental Science and Technology, 40(7), pp. 2414-2420. doi: 10.1021/es051921i.

90. Pronk, W. et al. (2007) 'Pilot experiments with electrodialysis and ozonation for the production of a fertiliser from urine', in Water Science and Technology, pp. 219-227. doi: 10.2166/wst.2007.575.

91. Tice, R. C. and Kim, Y. (2014) 'Energy efficient reconcentration of diluted human urine using ion exchange membranes in bioelectrochemical systems', Water Research. Elsevier Ltd, 64, pp. 61-72. doi: 10.1016/j. watres.2014.06.037.

92. Smara, A. et al. (2007) 'Removal of heavy metals from diluted mixtures by a hybrid ion-exchange/electrodialysis process', Separation and Purification Technology, 57(1), pp. 103-110. doi: 10.1016/j.seppur.2007.03.012.

93. Delimi, R., Boutemine, N. and Benredjem, Z. (2013) 'Removal of heavy metals from Water Rinsing of Plating Baths by Electrodialysis', E3S Web of Conferences, 1, p. 25011. doi: 10.1051/e3sconf/20130125011.

94. Antonini, S. et al. (2011) 'Nitrogen and Phosphorus Recovery from Human Urine by Struvite Precipitation and Air Stripping in Vietnam', Clean - Soil, Air, Water, 39(12), pp. 1099-1104. doi: 10.1002/clen.201100036.

95. Desloover, J. et al. (2012) 'Electrochemical resource recovery from digestate to prevent ammonia toxicity during anaerobic digestion', Environmental Science and Technology, 46(21), pp. 12209-12216. doi: 10.1021/ es3028154.

96. Latifian, M., Holst, O. and Liu, J. (2014) 'Nitrogen and Phosphorus Removal from Urine by Sequential Struvite Formation and Recycling Process', Clean - Soil, Air, Water, 42(8), pp. 1157-1161. doi: 10.1002/clen.201300070. 
97. Huang, H., Liu, J. and Ding, L. (2015) 'Recovery of phosphate and ammonia nitrogen from the anaerobic digestion supernatant of activated sludge by chemical precipitation', Journal of Cleaner Production. Elsevier Ltd, 102, pp. 437-446. doi: 10.1016/j.jclepro.2015.04.117.

98. Wu, X. and Modin, O. (2013) 'Ammonium recovery from reject water combined with hydrogen production in a bioelectrochemical reactor', Bioresource Technology. Elsevier Ltd, 146, pp. 530-536. doi: 10.1016/j. biortech.2013.07.130.

99. Licon Bernal, E. E. et al. (2016) 'Valorization of ammonia concentrates from treated urban wastewater using liquid-liquid membrane contactors', Chemical Engineering Journal. Elsevier B.V., 302, pp. 641-649. doi: 10.1016/j.cej.2016.05.094.

100. Valverde-Pérez, B., Fuentes-Martínez, J. M., et al. (2015) Control Structure Design of an Innovative Enhanced Biological Nutrient Recovery Activated Sludge System Coupled with a Photobioreactor, 12th International Symposium on Process Systems Engineering and 25th European Symposium on Computer Aided Process Engineering. Elsevier. doi: http://dx.doi.org/10.1016/B978-0-444-63576-1.50120-5.

101. Valverde-Pérez, B., Ramin, E., et al. (2015) 'EBP2R - An innovative enhanced biological nutrient recovery activated sludge system to produce growth medium for green microalgae cultivation', Water Research, 68, pp. 821-830. doi: 10.1016/j.watres.2014.09.027.

102. Valverde-Pérez, B. et al. (2016) 'Control structure design for resource recovery using the enhanced biological phosphorus removal and recovery (EBP2R) activated sludge process', Chemical Engineering Journal, 296, pp. 447-457. doi: 10.1016/j.cej.2016.03.021.

103. Markou, G. et al. (2015) 'Cultivation of Chlorella vulgaris and Arthrospira platensis with recovered phosphorus from wastewater by means of zeolite sorption', International Journal of Molecular Sciences, 16(2), pp. 4250-4264. doi: 10.3390/ijms16024250.

104. Kim, J. et al. (2016) 'Efficient recovery of nitrate and phosphate from wastewater by an amine-grafted adsorbent for cyanobacterial biomass production', Bioresource Technology. Elsevier Ltd, 205, pp. 269-273. doi: 10.1016/j. biortech.2016.01.055.

105. de Wilt, A. et al. (2016) 'Micropollutant removal in an algal treatment system fed with source separated wastewater streams', Journal of Hazardous Materials. Elsevier B.V., 304, pp. 84-92. doi: 10.1016/j.jhazmat.2015.10.033.

106. Zeraatkar, A. K. et al. (2016) 'Potential use of algae for heavy metal bioremediation, a critical review', Journal of Environmental Management. Elsevier Ltd, 181, pp. 817-831. doi: 10.1016/j.jenvman.2016.06.059.

107. Demey, H., Vincent, T. and Guibal, E. (2018) 'A novel algal-based sorbent for heavy metal removal', Chemical Engineering Journal. Elsevier, 332(September 2017), pp. 582-595. doi: 10.1016/j.cej.2017.09.083.

108. De-Bashan, L. E. and Bashan, Y. (2004) 'Recent advances in removing phosphorus from wastewater and its future use as fertilizer (1997-2003)’, Water Research, 38(19), pp. 4222-4246. doi: 10.1016/j.watres.2004.07.014.

109. Melia, P. M. et al. (2017) 'Trends in the recovery of phosphorus in bioavailable forms from wastewater', Chemosphere. Elsevier Ltd, 186, pp. 381-395. doi: 10.1016/j.chemosphere.2017.07.089.

110. Vasenko, L. and Qu, H. (2017) 'Effect of NH4-N/P and Ca/P molar ratios on the reactive crystallization of calcium phosphates for phosphorus recovery from wastewater', Journal of Crystal Growth. Elsevier, 459(October 2016), pp. 61-66. doi: 10.1016/j.jcrysgro.2016.11.076.

111. Ronteltap, M. et al. (2010) 'Struvite precipitation from urine - Influencing factors on particle size', Water Research. Elsevier Ltd, 44(6), pp. 2038-2046. doi: 10.1016/j.watres.2009.12.015.

112. Triger, A., Pic, J. S. and Cabassud, C. (2012) 'Determination of struvite crystallization mechanisms in urine using turbidity measurement', Water Research, 46(18), pp. 6084-6094. doi: 10.1016/j.watres.2012.08.030.

113. Liu, X. et al. (2013) 'Influence of process parameters on phosphorus recovery by struvite formation from urine', Water Science and Technology, 68(11), pp. 2434-2440. doi: 10.2166/wst.2013.514.

114. Ronteltap, M., Maurer, M. and Gujer, W. (2007a) 'Struvite precipitation thermodynamics in source-separated urine', Water Research, 41(5), pp. 977-984. doi: 10.1016/j.watres.2006.11.046.

115. Barbosa, S. G. et al. (2016) 'A design of experiments to assess phosphorous removal and crystal properties in struvite precipitation of source separated urine using different Mg sources', Chemical Engineering Journal, 298, pp. 146-153. doi: 10.1016/j.cej.2016.03.148.

116. Valls, A. et al. (2010) 'Preference assessment for the management of sewage sludge application on agricultural soils', International Journal of Multicriteria Decision Making, 1(1), p. 4. doi: 10.1504/IJMCDM.2010.033684.

117. Muster, T. H. et al. (2013) 'Towards effective phosphorus recycling from wastewater: Quantity and quality', Chemosphere. Elsevier Ltd, 91(5), pp. 676-684. doi: 10.1016/j.chemosphere.2013.01.057.

118. Wilsenach, J. A., Schuurbiers, C. A. H. and van Loosdrecht, M. C. M. (2007) 'Phosphate and potassium recovery from source separated urine through struvite precipitation', Water Research, 41(2), pp. 458-466. doi: 10.1016/j.watres.2006.10.014.

119. Xu, K. et al. (2015) 'The precipitation of magnesium potassium phosphate hexahydrate for $\mathrm{P}$ and $\mathrm{K}$ recovery from synthetic urine', Water Research. Elsevier Ltd, 80, pp. 71-79. doi: 10.1016/j.watres.2015.05.026. 
120. Xu, K. et al. (2012) 'Laboratory experiments on simultaneous removal of $\mathrm{K}$ and $\mathrm{P}$ from synthetic and real urine for nutrient recycle by crystallization of magnesium-potassium-phosphate-hexahydrate in a draft tube and baffle reactor', Chemosphere. Elsevier Ltd, 88(2), pp. 219-223. doi: 10.1016/j.chemosphere.2012.02.061.

121. Nakao, S., Nishio, T. and Kanjo, Y. (2017) 'Simultaneous recovery of phosphorus and potassium as magnesium potassium phosphate from synthetic sewage sludge effluent', Environmental Technology (United Kingdom). Taylor \& Francis, 38(19), pp. 2416-2426. doi: 10.1080/09593330.2016.1264485.

122. Huang, L., Lee, D.-J. and Lai, J.-Y. (2015) 'Forward osmosis membrane bioreactor for wastewater treatment with phosphorus recovery', Bioresource Technology. Elsevier Ltd, 198, pp. 418-423. doi: 10.1016/j. biortech.2015.09.045.

123. Lin, L. et al. (2017) 'Recovery of organic carbon and phosphorus from wastewater by Fe-enhanced primary sedimentation and sludge fermentation', Process Biochemistry. Elsevier Ltd, 54, pp. 135-139. doi: 10.1016/j. procbio.2016.12.016.

124. Decrey, L. et al. (2011) 'Fate of the pathogen indicators phage $\Phi X 174$ and Ascaris suum eggs during the production of struvite fertilizer from source-separated urine', Water Research, 45(16), pp. 4960-4972. doi: 10.1016/j. watres.2011.06.042.

125. Escher, B. I. et al. (2006) 'Monitoring the removal efficiency of pharmaceuticals and hormones in different treatment processes of source-separated urine with bioassays', Environmental Science and Technology, 40(16), pp. 5095-5101. doi: 10.1021/es060598w.

126. Ronteltap, M., Maurer, M. and Gujer, W. (2007b) 'The behaviour of pharmaceuticals and heavy metals during struvite precipitation in urine', Water Research, 41(9), pp. 1859-1868. doi: 10.1016/j.watres.2007.01.026.

127. Schürmann, B. et al. (2012) 'Fate of pharmaceuticals and bacteria in stored urine during precipitation and drying of struvite', Water Science and Technology, 65(10), pp. 1774-1780. doi: 10.2166/wst.2012.041.

128. Simha, P. and Ganesapillai, M. (2017) 'Ecological Sanitation and nutrient recovery from human urine: How far have we come? A review', Sustainable Environment Research. Elsevier Ltd, 27(3), pp. 107-116. doi: 10.1016/j. serj.2016.12.001.

129. Kameda, T., Ito, S. and Yoshioka, T. (2017) 'Kinetic and equilibrium studies of urea adsorption onto activated carbon: Adsorption mechanism', Journal of Dispersion Science and Technology. Taylor \& Francis, 38(7), pp. 1063-1066. doi: 10.1080/01932691.2016.1219953.

130. Cai, Y. et al. (2016) 'Sorption/Desorption Behavior and Mechanism of NH4+ by Biochar as a Nitrogen Fertilizer Sustained-Release Material', Journal of Agricultural and Food Chemistry, 64(24), pp. 4958-4964. doi: 10.1021 /acs.jafc.6b00109.

131. Takaya, C. A. et al. (2016) 'Phosphate and ammonium sorption capacity of biochar and hydrochar from different wastes', Chemosphere. Elsevier Ltd, 145, pp. 518-527. doi: 10.1016/j.chemosphere.2015.11.052.

132. Trazzi, P. A. et al. (2016) 'Adsorption and desorption of phosphate on biochars', Journal of Environmental Chemical Engineering. Elsevier B.V., 4(1), pp. 37-46. doi: 10.1016/j.jece.2015.11.005.

133. Jaskūnas, A. (2015) 'Adsorption of potassium ions on natural zeolite : kinetic and equilibrium studies', 26(2), pp. 69-78.

134. Hedström, A. (2006) 'Wollastonite as reactive filter medium for sorption of wastewater ammonium and phosphorus', Environmental Technology, 27(7), pp. 801-809. doi: 10.1080/09593332708618693.

135. Karapinar, N. (2009) 'Application of natural zeolite for phosphorus and ammonium removal from aqueous solutions', Journal of Hazardous Materials, 170(2-3), pp. 1186-1191. doi: 10.1016/j.jhazmat.2009.05.094.

136. Guaya, D. et al. (2016) 'Recovery of ammonium and phosphate from treated urban wastewater by using potassium clinoptilolite impregnated hydrated metal oxides as N-P-K fertilizer', Journal of Environmental Chemical Engineering. Elsevier B.V., 4(3), pp. 3519-3526. doi: 10.1016/j.jece.2016.07.031.

137. Wan, C. et al. (2017) 'Simultaneous recovery of nitrogen and phosphorus from sludge fermentation liquid by zeolite adsorption: Mechanism and application', Separation and Purification Technology. Elsevier B.V., 180, pp. 1-12. doi: 10.1016/j.seppur.2017.02.031.

138. Gustafsson, J. P. et al. (2008) 'Phosphate removal by mineral-based sorbents used in filters for small-scale wastewater treatment', Water Research, 42(1-2), pp. 189-197. doi: 10.1016/j.watres.2007.06.058.

139. Köse, T. E. and Kivanç, B. (2011) 'Adsorption of phosphate from aqueous solutions using calcined waste eggshell', Chemical Engineering Journal, 178, pp. 34 -39. doi: 10.1016/j.cej.2011.09.129.

140. Busscher, H. J. et al. (2006) 'Removal of two waterborne pathogenic bacterial strains by activated carbon particles prior to and after charge modification', Environmental Science and Technology, 40(21), pp. 6799-6804. doi: 10.1021/es061282r.

141. Nam, S.-W. et al. (2014) 'Adsorption characteristics of selected hydrophilic and hydrophobic micropollutants in water using activated carbon', Journal of Hazardous Materials. Elsevier B.V., 270, pp. 144-152. doi: 10.1016/j. jhazmat.2014.01.037. 
142. Tong, Y., Mayer, B. K. and McNamara, P. J. (2016) 'Triclosan adsorption using wastewater biosolids-derived biochar', Environ. Sci.: Water Res. Technol. Royal Society of Chemistry, 2(4), pp. 761-768. doi: 10.1039/C6EW00127K.

143. Kołodyńska, D. et al. (2012) 'Kinetic and adsorptive characterization of biochar in metal ions removal', Chemical Engineering Journal, 197, pp. 295-305. doi: 10.1016/j.cej.2012.05.025.

144. Al-Wabel, M. I. et al. (2015) 'Conocarpus biochar as a soil amendment for reducing heavy metal availability and uptake by maize plants', Saudi Journal of Biological Sciences. King Saud University, 22(4), pp. 503-511. doi: 10.1016/j.sjbs.2014.12.003.

145. Kim, H. S. et al. (2015) 'Effect of biochar on heavy metal immobilization and uptake by lettuce (Lactuca sativa L.) in agricultural soil', Environmental Earth Sciences. Springer Berlin Heidelberg, 74(2), pp. 1249-1259. doi: 10.1007/s12665-015-4116-1.

146. De Ridder, D. J. et al. (2012) 'Zeolites for nitrosamine and pharmaceutical removal from demineralised and surface water: Mechanisms and efficacy', Separation and Purification Technology. Elsevier B.V., 89, pp. 71-77. doi: 10.1016/j.seppur.2012.01.025.

147. Chraibi, S. et al. (2016) 'Utilization of Calcined Eggshell Waste as an Adsorbent for the Removal of Phenol from Aqueous Solution', Journal of Encapsulation and Adsorption Sciences, 06(04), pp. 132-146. doi: 10.4236/ jeas.2016.64010.

148. Tsai, W. T. et al. (2008) 'Utilization of ground eggshell waste as an adsorbent for the removal of dyes from aqueous solution’, Bioresource Technology, 99(6), pp. 1623-1629. doi: 10.1016/j.biortech.2007.04.010.

149. Zorpas, A. A. et al. (2000) 'Heavy metal uptake by natural zeolite and metals partitioning in sewage sludge compost', Bioresource Technology, 72(2), pp. 113-119. doi: 10.1016/S0960-8524(99)00110-8.

150. Shaheen, S. M., Derbalah, A. S. and Moghanm, F. S. (2012) 'Removal of Heavy Metals from Aqueous Solution by Zeolite in Competitive Sorption System', International Journal of Environmental Science and Development, 3(4), pp. 362-367. doi: 10.7763/IJESD.2012.V3.248.

151. Babel, S. (2003) 'Low-cost adsorbents for heavy metals uptake from contaminated water: a review', Journal of Hazardous Materials, 97(1-3), pp. 219-243. doi: 10.1016/S0304-3894(02)00263-7.

152. Choi, H. J. and Lee, S. M. (2015) 'Heavy metal removal from acid mine drainage by calcined eggshell and microalgae hybrid system', Environmental Science and Pollution Research, 22(17), pp. 13404-13411. doi: 10.1007/ s11356-015-4623-3.

153. Egle, L., Rechberger, H. and Zessner, M. (2015) 'Overview and description of technologies for recovering phosphorus from municipal wastewater', Resources, Conservation and Recycling. Elsevier B.V., 105, pp. 325-346. doi: 10.1016/j.resconrec.2015.09.016.

154. Schönberg, A. et al. (2014) 'Der RecoPhos-Prozess - Rückgewinnung von Phosphor aus KIärschlammasche’, Osterreichische Wasser- und Abfallwirtschaft, 66(11-12), pp. 403-407. doi: 10.1007/s00506-014-0193-8. 


\title{
Supporting Information VI
}

\section{Identifying Amsterdam's nutrient hotspots: A new method to map human excreta at building and neighborhood scale}

\author{
Input Data
}

This Supporting Information (SI) includes tables with collected input data used in for calculations and the GIS analysis. Table S1 provides an overview of the types of data collected and the respective references. Table S2 shows the weekly time use data distributed over locations: home, work, school and other. This Table S3 is an example table of the data that underpins the building hotspots figures. Please contact the corresponding author to receive the excel spreadsheet with building and neighborhood data.

Table S6.1 Overview of all collected input data and references

Table S6.2 Time use distribution

Table S6.3 Phosphorus loads per building (example table)

\section{Nitrogen and Potassium Hotspots}

This Supporting Information (SI) includes two figures: one of the nitrogen hotspots at building and neighborhood scale, and one for the potassium hotspots at building and neighborhood scale. As with phosphorus, shown in the manuscript, the depiction of both building and neighborhood load profiles shows the relevance of mapping nutrients at varying scales. Buildings with high loads don't necessarily fall in neighborhoods with high loads and vice versa neighborhoods with high loads don't necessarily contain buildings with high loads.

Figure S6.1. Plotted neighborhood phosphorus loads

Figure S6.2 Building and neighborhood nitrogen loads

Figure S6.3 Building and neighborhood potassium loads 
Table S6.1 Overview of all collected input data and references

\begin{tabular}{|c|c|c|c|c|}
\hline & \multicolumn{2}{|l|}{ Input data } & \multicolumn{2}{|l|}{ Main Data Source } \\
\hline & Description & Variable & Organization/Reference & Year \\
\hline \multirow[t]{3}{*}{ Geographic boundary } & City & & $\begin{array}{l}\text { Basisregistratie Adressen en Gebouwen (BAG) via } \\
\text { ESRI, Nederland }\end{array}$ & 2017 \\
\hline & Neighborhood & $x$ & Onderzoek, Informatie en Statistiek (OIS) & 2016 \\
\hline & Building & $x$ & $\begin{array}{l}\text { Basisregistratie Adressen en Gebouwen (BAG) via } \\
\text { ESRI, Nederland }\end{array}$ & 2017 \\
\hline \multirow[t]{2}{*}{ Residential } & Number of individuals per boundary & $\mathrm{I}_{\mathrm{x}}$ & Provided by the Municipality of Amsterdam & 2015 \\
\hline & $\begin{array}{l}\text { Number of hours individuals spend } \\
\text { within boundary }\end{array}$ & $\mathrm{T}_{\mathrm{x}}$ & (CBS, 2013); (Cloin et al., 2013) & 2011 \\
\hline \multirow[t]{2}{*}{ Commercial } & Number of individuals per building & $\mathrm{I}_{\mathrm{x}}$ & Municipality of Amsterdam & 2015 \\
\hline & $\begin{array}{l}\text { Number of hours individuals spend } \\
\text { within boundary }\end{array}$ & $\mathrm{T}_{\mathrm{x}}$ & (CBS, 2013); (Cloin et al., 2013) & 2011 \\
\hline \multirow[t]{2}{*}{ Schools } & Number of individuals per building & $\mathrm{I}_{\mathrm{x}}$ & Onderzoek, Informatie en Statistiek (OIS) & $2016 / 2017$ \\
\hline & $\begin{array}{l}\text { Number of hours individuals spend } \\
\text { within boundary }\end{array}$ & $T_{\mathrm{x}}$ & $\begin{array}{l}\text { Centraal Bureau voor de Statistiek (CBS) (ages 12- } \\
\text { 18); Rijksoverheid (ages < 12) }\end{array}$ & 2014 \\
\hline \multirow[t]{2}{*}{$\begin{array}{l}\text { Public Institutions } \\
\text { (Museums, theater, } \\
\text { concert halls) }\end{array}$} & Number of individuals per building & $\mathrm{I}_{\mathrm{x}}$ & $\begin{array}{l}\text { Overleg Amsterdamse Musea (OAM) (museums } \\
\text { 2012-2016); Centraal Bureau voor de Statistiek } \\
\text { (CBS) (theater/concert halls 2014-2016) }\end{array}$ & 2016 \\
\hline & $\begin{array}{l}\text { Number of hours individuals spend } \\
\text { within boundary }\end{array}$ & $\mathrm{T}_{\mathrm{x}}$ & Google (https://www.google.com/business/) & 2018 \\
\hline \multirow{4}{*}{$\begin{array}{l}\text { Characterization of } \\
\text { urine and feces, and } \\
\text { frequency of excretion }\end{array}$} & Nutrient content in urine & $\mathrm{N}_{U}$ & (Meinzinger \& Oldenburg, 2009) & \\
\hline & Nutrient content in feces & $\mathrm{N}_{\mathrm{F}}$ & (Meinzinger \& Oldenburg, 2009) & \\
\hline & Frequency of urination & $f_{U}$ & (Rose et al., 2015) & \\
\hline & Frequency of defecation & $f_{F}$ & (Rose et al., 2015) & \\
\hline \multirow{2}{*}{$\begin{array}{l}\text { Number of excretion } \\
\text { events per time frame }\end{array}$} & Number of urination events & $\mathrm{V}_{U}$ & (Rose et al., 2015) & \\
\hline & Number of defecation events & $V_{F}$ & (Rose et al., 2015) & \\
\hline
\end{tabular}

Figure S6.1 Phosphorus loads for neighborhoods in Group I-V. The slope increases after 2000 kg P yr-1 neighborhood-1. The mean value is $812 \mathrm{~kg}$ P yr-1 neighborhood-1.

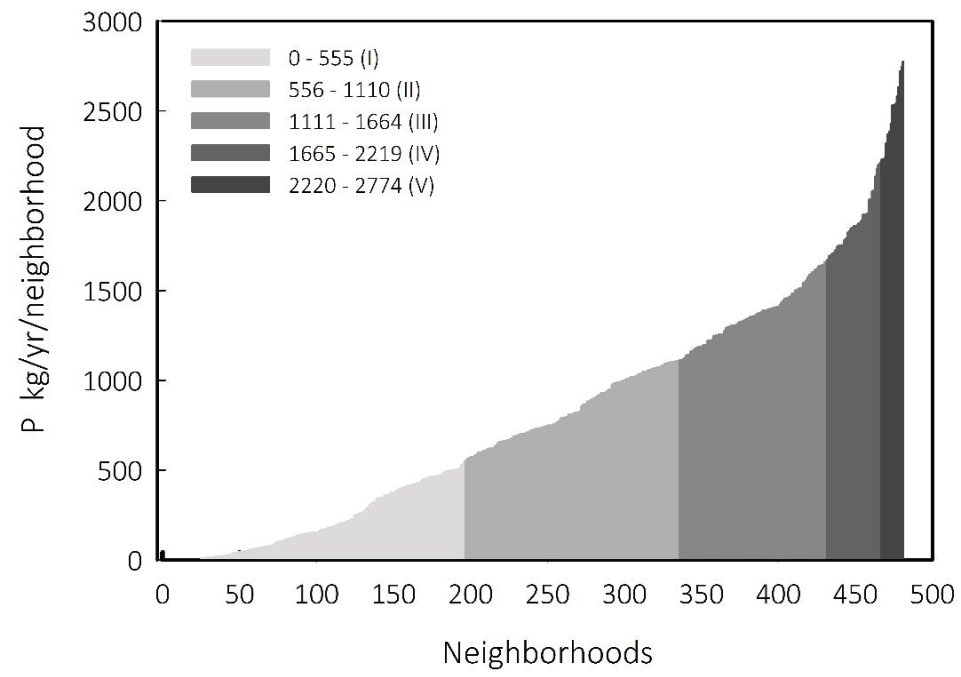


Table S6.2 Time use distribution

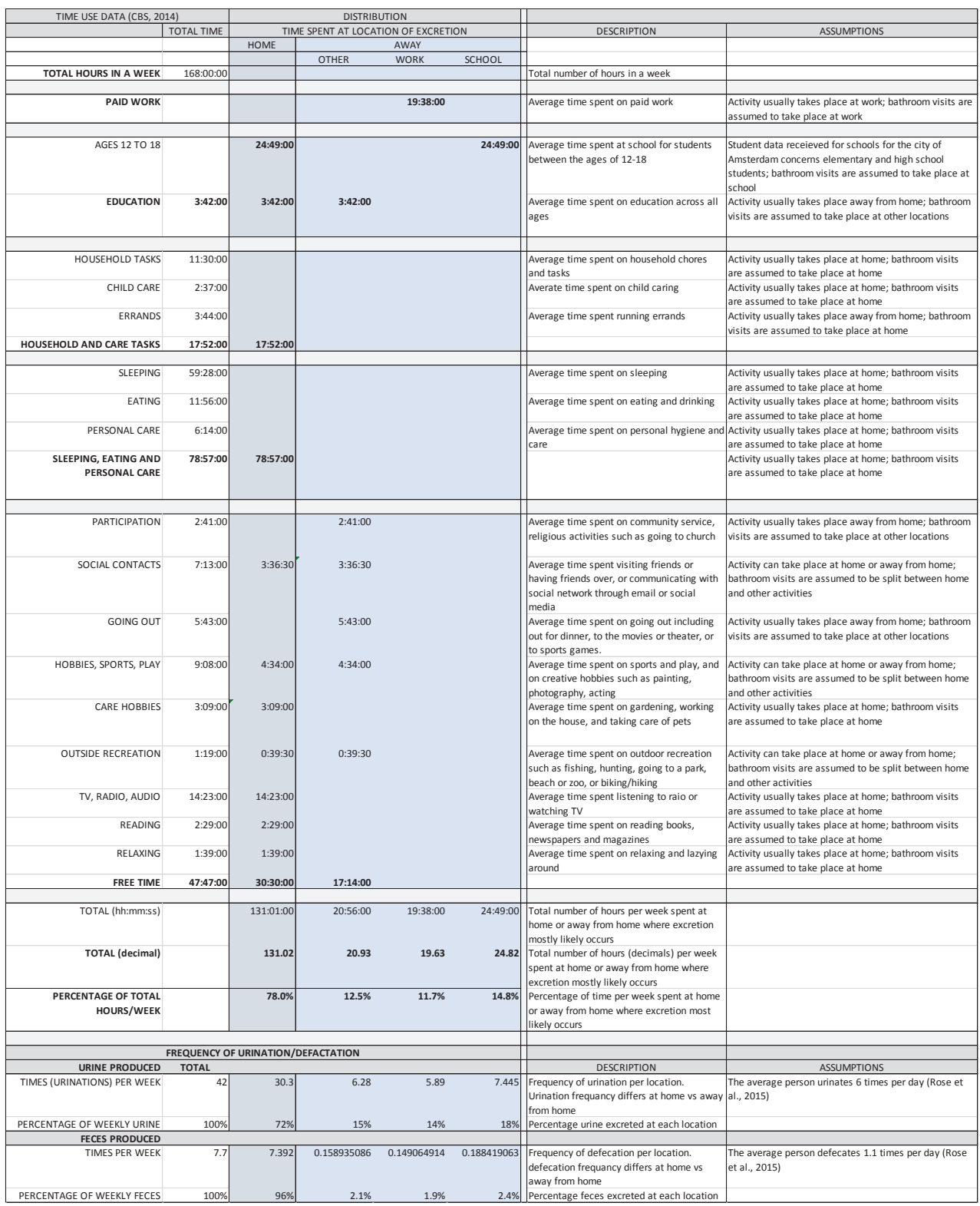




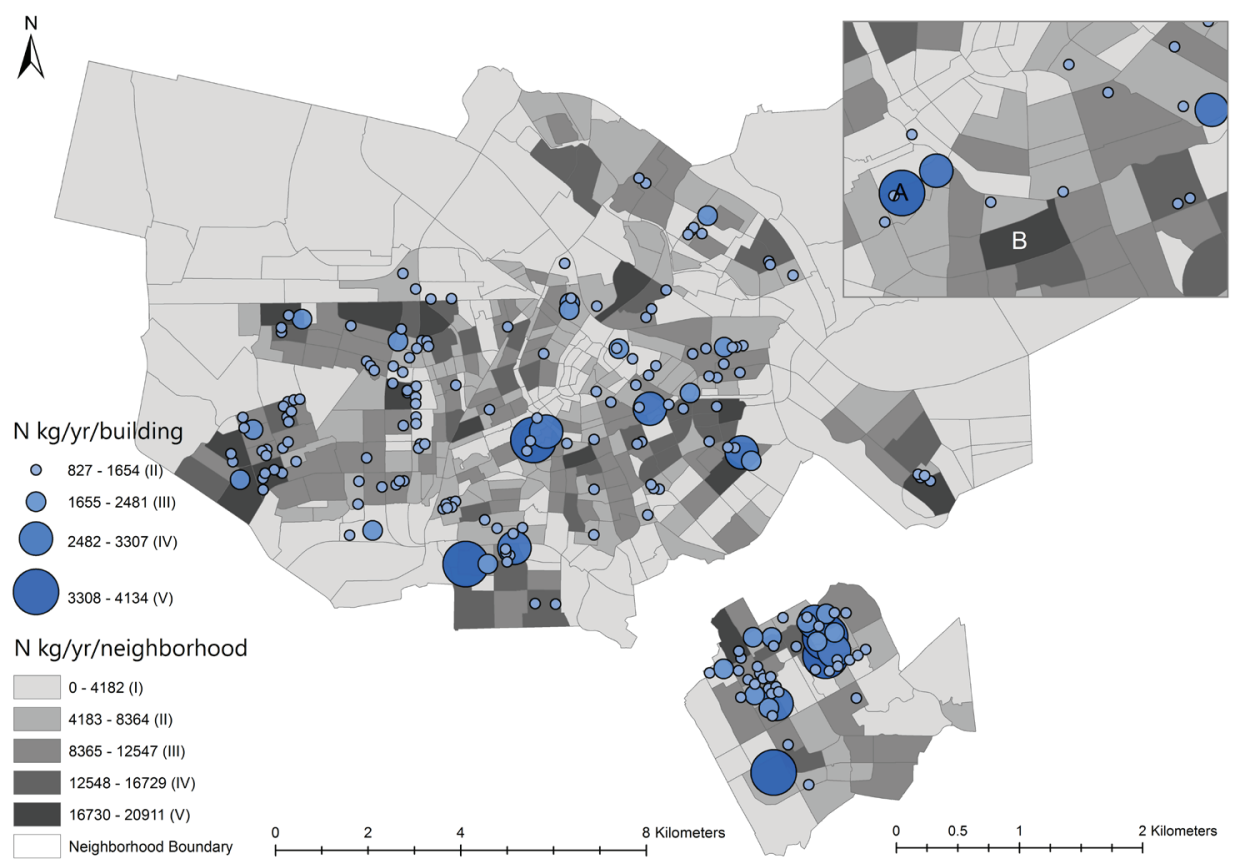

Figure S6.2 Building and neighborhood nitrogen loads

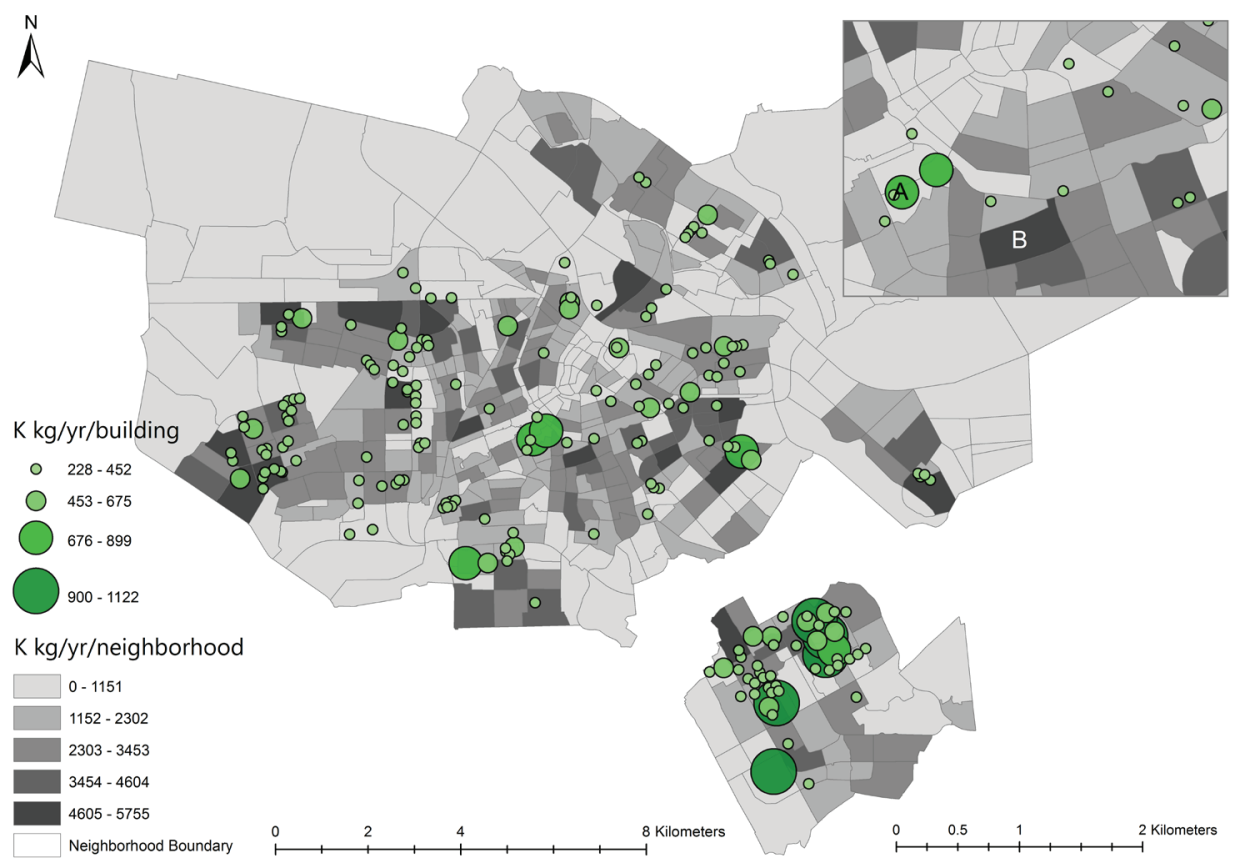

Figure S6.3 Building and neighborhood potassium loads 
Table S6.3 Phosphorus loads per building (example table)

\begin{tabular}{|c|c|c|c|c|c|c|}
\hline Object & Identificatie \# & $\begin{array}{l}\mathrm{P} \mathrm{kg} / \mathrm{yr} / \text { building } \\
\text { (value range) }\end{array}$ & $\begin{array}{l}\text { Load } \\
\text { class }\end{array}$ & Shape Length & Shape Area & Building Function \\
\hline 34 & 0363100012081902 & $109-218$ & II & 256.6227936 & 1411.541505 & RESIDENTIAL \\
\hline 48 & 0363100012095344 & $109-218$ & $\|$ & 227.294863 & 1226.804606 & RESIDENTIAL \\
\hline 50 & 0363100012095578 & $109-218$ & II & 274.5287247 & 1993.054041 & RESIDENTIAL \\
\hline 76 & 0363100012109685 & $109-218$ & II & 235.3081715 & 1271.942439 & RESIDENTIAL \\
\hline 152 & 0363100012166152 & $109-218$ & II & 294.2611486 & 2650.729527 & VISITORS \\
\hline 163 & 0363100012186093 & $109-218$ & II & 856.0096054 & 6553.385114 & RESIDENTIAL \\
\hline 84 & 0363100012112179 & $109-218$ & II & 711.9574424 & 17646.21556 & RESIDENTIAL \\
\hline 169 & 0363100012237532 & $109-218$ & II & 242.8229095 & 2182.230279 & RESIDENTIAL \\
\hline 18 & 0363100012074496 & $109-218$ & II & 386.7731605 & 1954.948936 & RESIDENTIAL \\
\hline 61 & 0363100012099895 & $109-218$ & II & 672.1273285 & 8818.62579 & STUDY \\
\hline 185 & 0363100012239988 & $109-218$ & II & 306.6068155 & 5228.985927 & RESIDENTIAL \\
\hline 188 & 0363100012242034 & $109-218$ & II & 253.6177777 & 2312.858126 & RESIDENTIAL \\
\hline 4 & 0363100012063809 & $109-218$ & II & 288.2374242 & 2016.10979 & RESIDENTIAL \\
\hline 45 & 0363100012093461 & $109-218$ & II & 235.3458887 & 1814.794467 & RESIDENTIAL \\
\hline 150 & 0363100012164981 & $109-218$ & II & 671.2438609 & 3802.733471 & RESIDENTIAL \\
\hline 154 & 0363100012169740 & $109-218$ & II & 200.0793664 & 1314.743959 & RESIDENTIAL \\
\hline 178 & 0363100012238846 & $109-218$ & II & 329.2595203 & 3929.182731 & RESIDENTIAL \\
\hline 68 & 0363100012104501 & $109-218$ & II & 434.6694637 & 2536.474799 & RESIDENTIAL \\
\hline 70 & 0363100012105724 & $109-218$ & II & 269.5869416 & 4125.910424 & RESIDENTIAL \\
\hline 156 & 0363100012173447 & $109-218$ & II & 278.2514703 & 2782.003277 & VISITORS \\
\hline 46 & 0363100012095184 & $109-218$ & II & 368.4809845 & 1908.705659 & RESIDENTIAL \\
\hline 100 & 0363100012119700 & $109-218$ & II & 645.9290981 & 3301.189834 & RESIDENTIAL \\
\hline 172 & 0363100012237838 & $109-218$ & II & 333.875539 & 4927.86298 & VISITORS \\
\hline 192 & 0363100012249646 & $109-218$ & II & 329.8626646 & 3719.798142 & RESIDENTIAL \\
\hline 79 & 0363100012110795 & $109-218$ & II & 492.4868328 & 2770.428342 & RESIDENTIAL \\
\hline 139 & 0363100012149391 & $109-218$ & II & 213.1985651 & 1971.220246 & RESIDENTIAL \\
\hline 128 & 0363100012144956 & $109-218$ & II & 483.1488815 & 3149.528011 & RESIDENTIAL \\
\hline 19 & 0363100012074757 & $109-218$ & II & 1361.475925 & 15498.52195 & WORK \\
\hline 69 & 0363100012104977 & $109-218$ & $\|$ & 241.6582048 & 3619.702426 & RESIDENTIAL \\
\hline 95 & 0363100012116511 & $109-218$ & II & 115.3955844 & 713.1913538 & WORK \\
\hline 165 & 0363100012219321 & $109-218$ & II & 539.1797758 & 4602.542566 & RESIDENTIAL \\
\hline
\end{tabular}




\section{References}

BAG 2017. Basisregistratie Adressen en Gebouwen (BAG).

CBS 2013. Bijlage B TBO 2011/2012 Onderzoeksdocumentatie. Centraal Bureau voor de Statistiek.

CBS 2014. Tijdsbesteding in uren per week; persoonskenmerken. 29-07-2014 ed. Den Haag: Centraal Bureau voor de Statistiek.

CBS 2017. Kerncijfers wijken en buurten 2015. 01-03-2017 ed. Den Haag: Centraal Bureau voor de Statistiek.

Cloïn, M., van den Broek, A., van den Dool, R., de Haan, J., de Hart, J., van Houwelingen, P., Tiessen-Raaphorst, A., Sonck, N. \& Spit, J. 2013. Met oog op de tijd: Een blik op de tijdsbesteding van Nederlanders. In: CLOÏN, M. (ed.). Den Haag: Sociaal en Cultureel Planbureau,.

Meinzinger, F. \& Oldenburg, M. 2009. Characteristics of source-separated household wastewater flows: a statistical assessment. Water Science and Technology, 59, 1785-1791.

OAM 2016. Bezoekers (x 1.000) aan musea verenigd in het OAM - Overleg Amsterdamse Musea, 2012-2016. In: OVERLEG AMSTERDAMSE MUSEA (ed.).

OIS 2016. Scholen en leerlingen voortgezet onderwijs naar schooladres, schooljaar 2016/2017. In: ONDERZOEK, I. S. (ed.).

Rose, C., Parker, A., Jefferson, B. \& Cartmell, E. 2015. The characterization of feces and urine: a review of the literature to inform advanced treatment technology. Critical reviews in environmental science and technology, 45, 1827-1879. 


\title{
Supporting Information VII
}

\section{Resource Dynamo: A GIS model to match urban nutrient supply with agricultural demand}

\author{
Input Data
}

This Supporting Information (SI) includes a description of the Resource Dynamo Model including input data and data sources used to quantify and match phosphorus supply and demand in the municipality of Amsterdam, as well as assumptions and specifications used for the model.

Table S7.1 Valuation of the soil phosphate concentration for cropland per phosphate class (Pwvalue) and respective phosphate and phosphorus allowances starting in 2020.

Table S7.2 Valuation of the soil phosphate concentration for grassland per phosphate class (Pwvalue) and respective phosphate and phosphorus allowances starting in 2020.

Table S7.3 Assumptions and input data used to quantify and match phosphorus supply and demand for the municipality of Amsterdam 


\section{Phosphate application rates}

Overview of phosphate application allowances based on the 'Zesde Nederlandse actieprogramma betreffende de Nitraatrichtlijn (2018 - 2021)' (Ministerie van Landbouw and Waterstaat, 2017)

Table S7.1 Valuation of the soil phosphate concentration for cropland per phosphate class (Pw-value) and respective phosphate and phosphorus allowances starting in 2020.

\begin{tabular}{|c|c|c|c|}
\hline $\begin{array}{l}\text { Valuation of the soil } \\
\text { phosphate concentration }\end{array}$ & $\begin{array}{c}\text { Phosphate classes } \\
\text { Pw-value, mg }\end{array}$ & $\begin{array}{l}\text { Phosphate allowances, } \\
\mathrm{kg} \mathrm{P}_{2} \mathrm{O}_{5} / \mathrm{ha}\end{array}$ & $\begin{array}{l}\text { Phosphorus allowances, } \\
\text { kg P/ha }\end{array}$ \\
\hline & $\mathrm{P}_{2} \mathrm{O}_{5} / \mathrm{L}$ & \multicolumn{2}{|c|}{ From 2020 onwards } \\
\hline Poor (P-fixing) & $<25$ & 120 & 52.37 \\
\hline Low & $25-35$ & 80 & 34.91 \\
\hline Neutral & $36-45$ & 70 & 30.55 \\
\hline Ample & $46-55$ & 60 & 26.18 \\
\hline High & $>55$ & 40 & 17.46 \\
\hline
\end{tabular}

Table S7.2 Valuation of the soil phosphate concentration for grassland per phosphate class (P-Al-value) and respective phosphate and phosphorus allowances starting in 2020.

\begin{tabular}{|c|c|c|c|}
\hline $\begin{array}{l}\text { Valuation of the soil } \\
\text { phosphate concentration }\end{array}$ & $\begin{array}{c}\text { Phosphate classes } \\
\text { P-Al-value, mg }\end{array}$ & $\begin{array}{l}\text { Phosphate allowances, } \\
\qquad \mathrm{kg} \mathrm{P}_{2} \mathrm{O}_{5} / \text { ha }\end{array}$ & $\begin{array}{l}\text { Phosphorus allowances, } \\
\text { kg P/ha }\end{array}$ \\
\hline & $\mathrm{P}_{2} \mathrm{U}_{5} / \mathrm{L}$ & \multicolumn{2}{|c|}{ From 2020 onwards } \\
\hline Poor (P-fixing) & $<16$ & 120 & 52.37 \\
\hline Low & $16-26$ & 105 & 45.82 \\
\hline Neutral & $27-40$ & 95 & 41.46 \\
\hline Ample & $41-50$ & 90 & 39.28 \\
\hline High & $>50$ & 75 & 32.73 \\
\hline
\end{tabular}




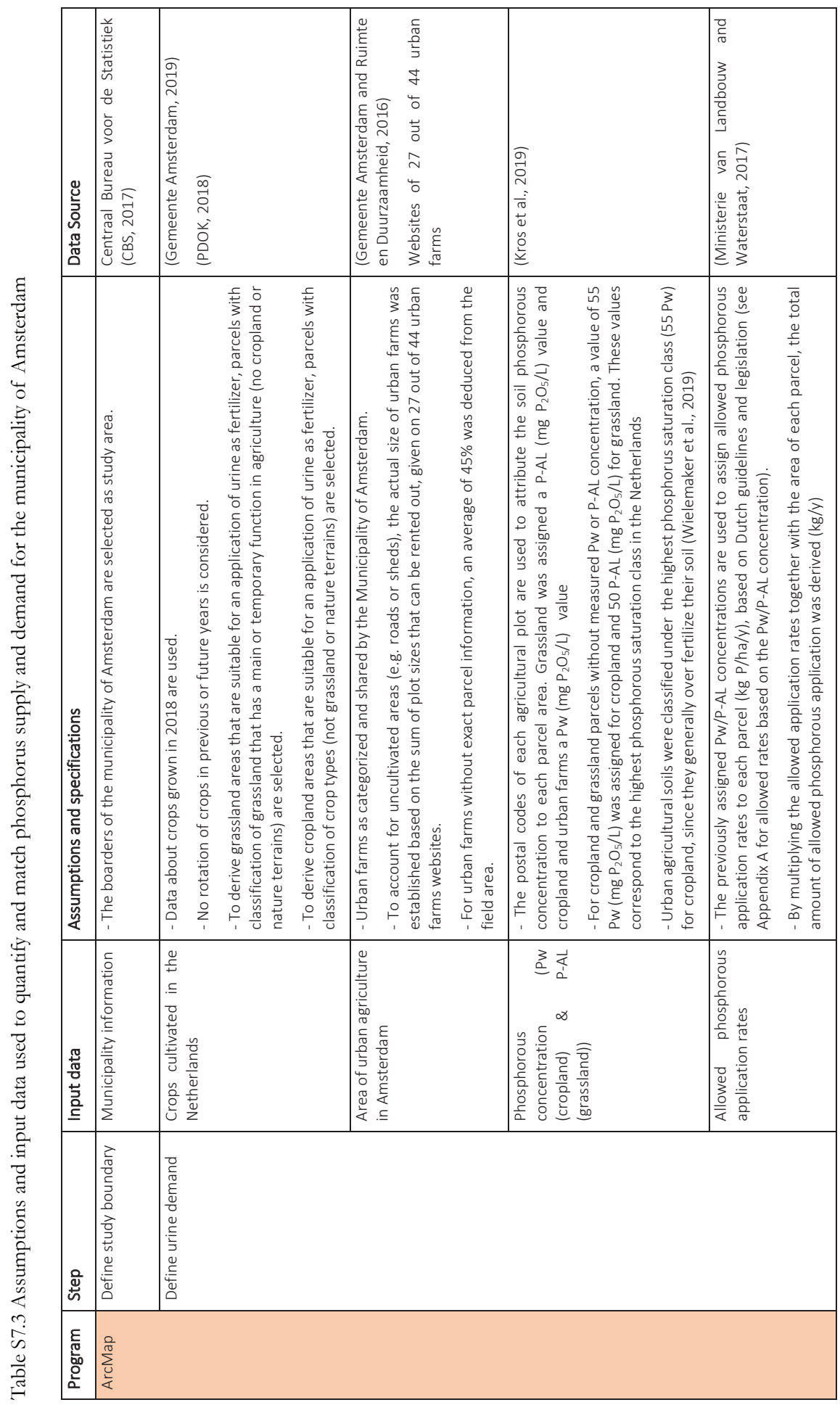




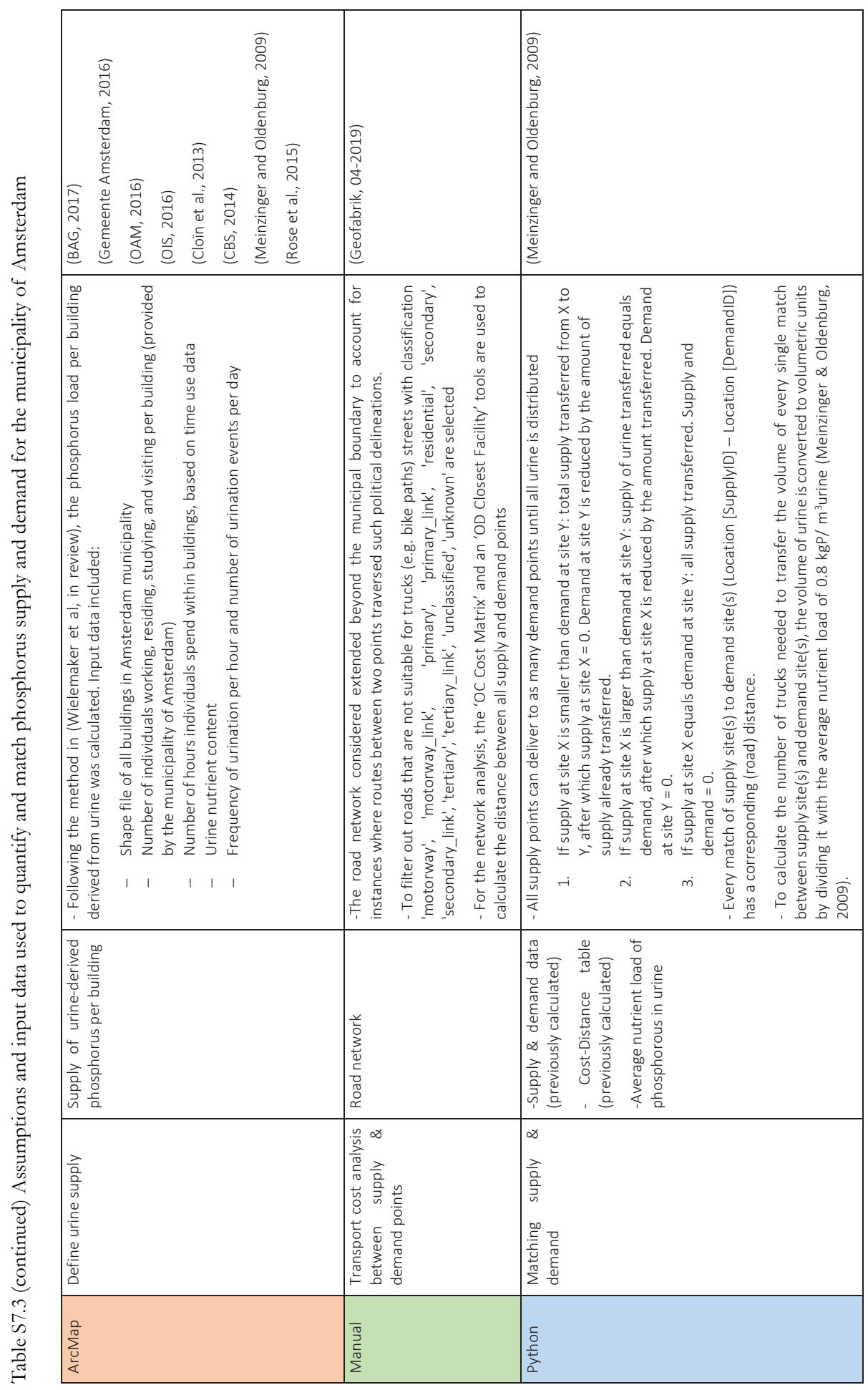




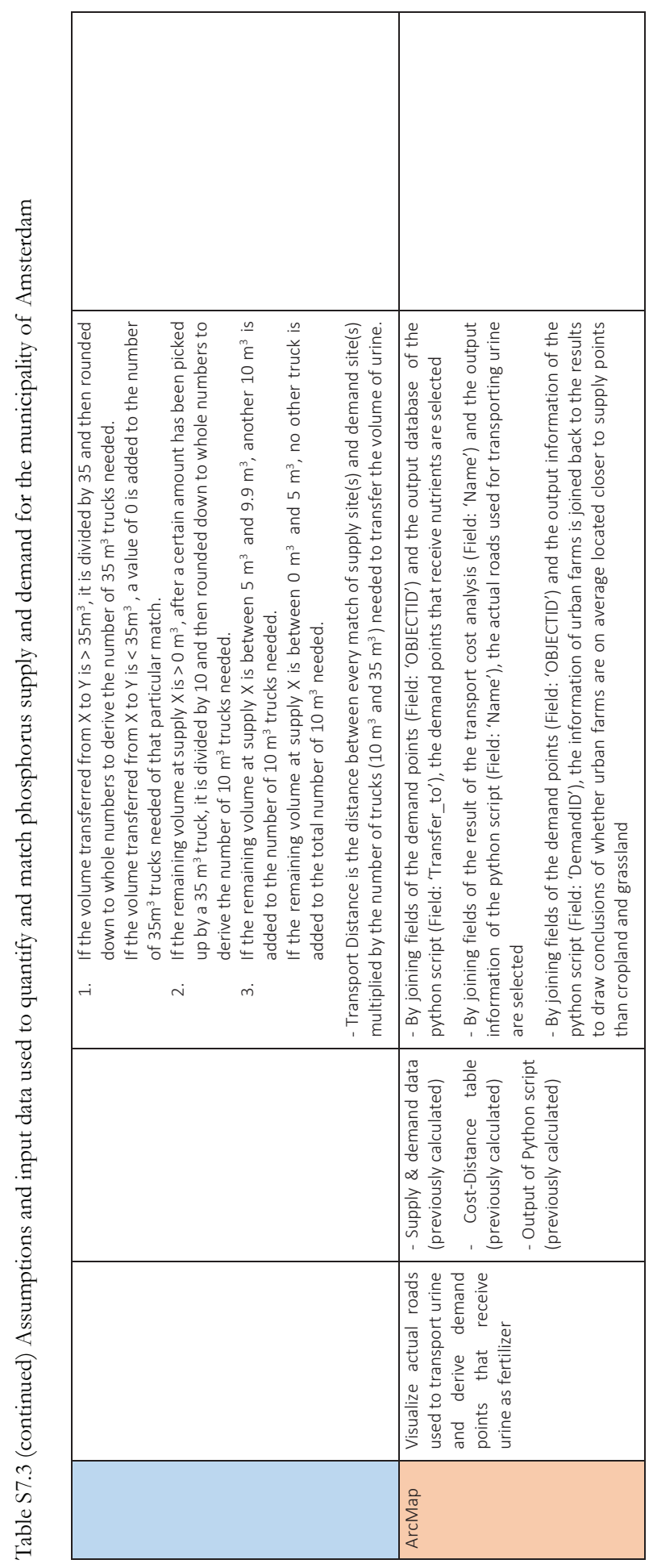




\section{References}

BAG 2017. Basisregistratie Adressen en Gebouwen (BAG).

CBS 2014. Tijdsbesteding in uren per week; persoonskenmerken. 29-07-2014 ed. Den Haag: Centraal Bureau voor de Statistiek.

Cloïn, M., van den Broek, A., van den Dool, R., de Haan, J., de Hart, J., van Houwelingen, P., Tiessen-Raaphorst, A., Sonck, N. \& Spit, J. 2013. Met oog op de tijd: Een blik op de tijdsbesteding van Nederlanders. In: CLOÏN, M. (ed.). Den Haag: Sociaal en Cultureel Planbureau,.

Gemeente Amsterdam 2016. AMSInwoners.

Gemeente Amsterdam \& Ruimte en Duurzaamheid 2016. Stadslandbouw. 07-12-2016 ed.

Geofabrik 04-2019. Open StreetMap-Netherlands-latest.osm.pbf.

Kros, H., Os, J. v., Voogd, J. C., Groenendijk, P., Bruggen, C. v., Molder, R. t. \& Ros, G. 2019. Ruimtelijke allocatie van mesttoediening en ammoniakemissie : beschrijving mestverdelingsmodule INITIATOR versie 5 . Wageningen: Wageningen Environmental Research.

Meinzinger, F. \& Oldenburg, M. 2009. Characteristics of source-separated household wastewater flows: a statistical assessment. Water Science and Technology, 59, 1785-1791.

Ministerie van Landbouw, N. e. V. \& Waterstaat, M. v. I. e. 2017. Zesde Nederlandse actieprogramma betreffende de Nitraatrichtlijn (2018-2021).

OAM 2016. Bezoekers (x 1.000) aan musea verenigd in het OAM - Overleg Amsterdamse Musea, 2012-2016. In: OVERLEG AMSTERDAMSE MUSEA (ed.).

OIS 2016. Scholen en leerlingen voortgezet onderwijs naar schooladres, schooljaar 2016/2017. In: ONDERZOEK, I. S. (ed.).

PDOK 2018. Dataset: Basisregistratie Gewaspercelen (BRP). PDOK.

Rose, C., Parker, A., Jefferson, B. \& Cartmell, E. 2015. The characterization of feces and urine: a review of the literature to inform advanced treatment technology. Critical reviews in environmental science and technology, 45 , 1827-1879.

Wielemaker, R., Oenema, O., Zeeman, G. \& Weijma, J. 2019. Fertile cities: Nutrient management practices in urban agriculture. Science of The Total Environment. 


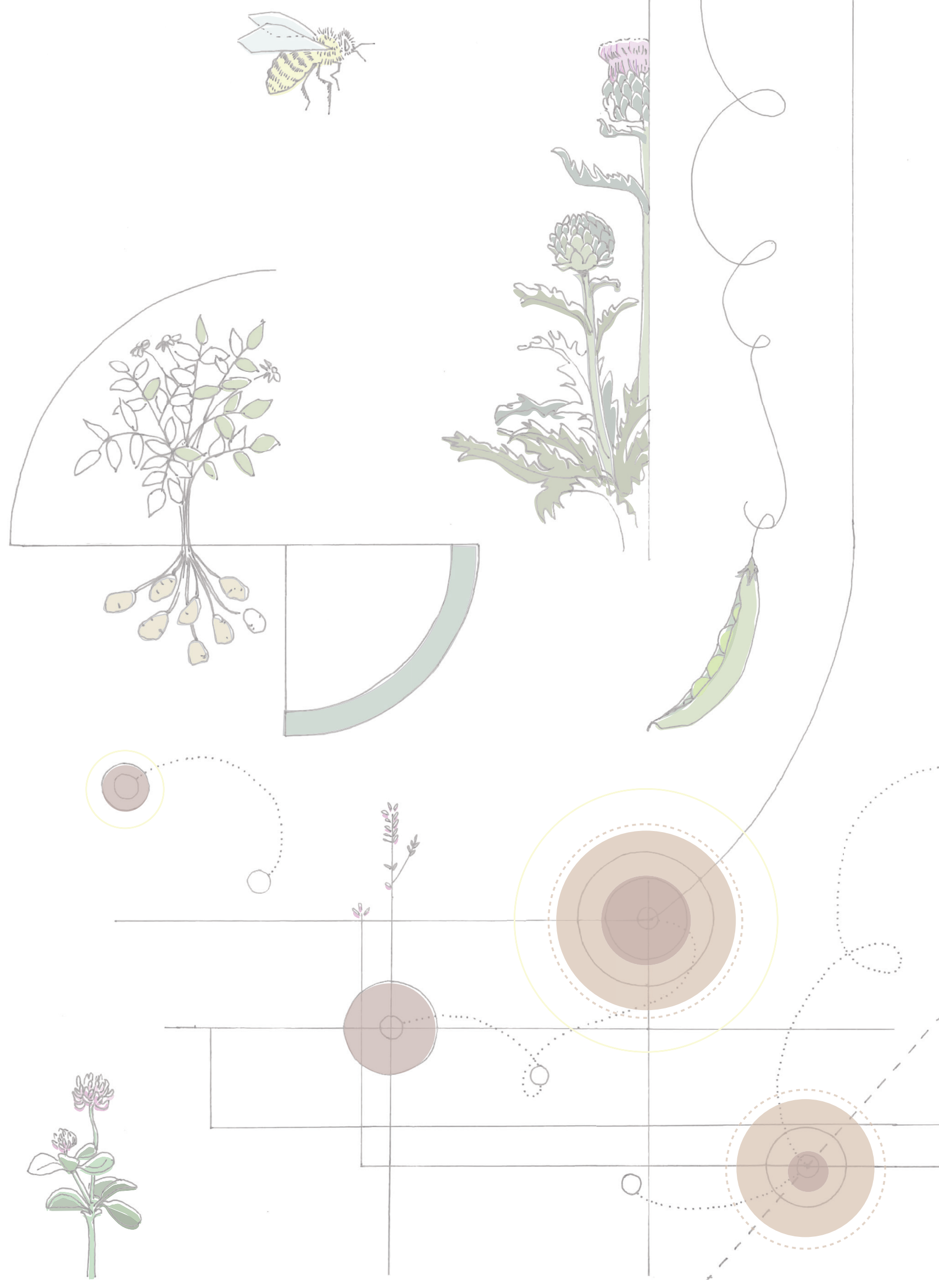




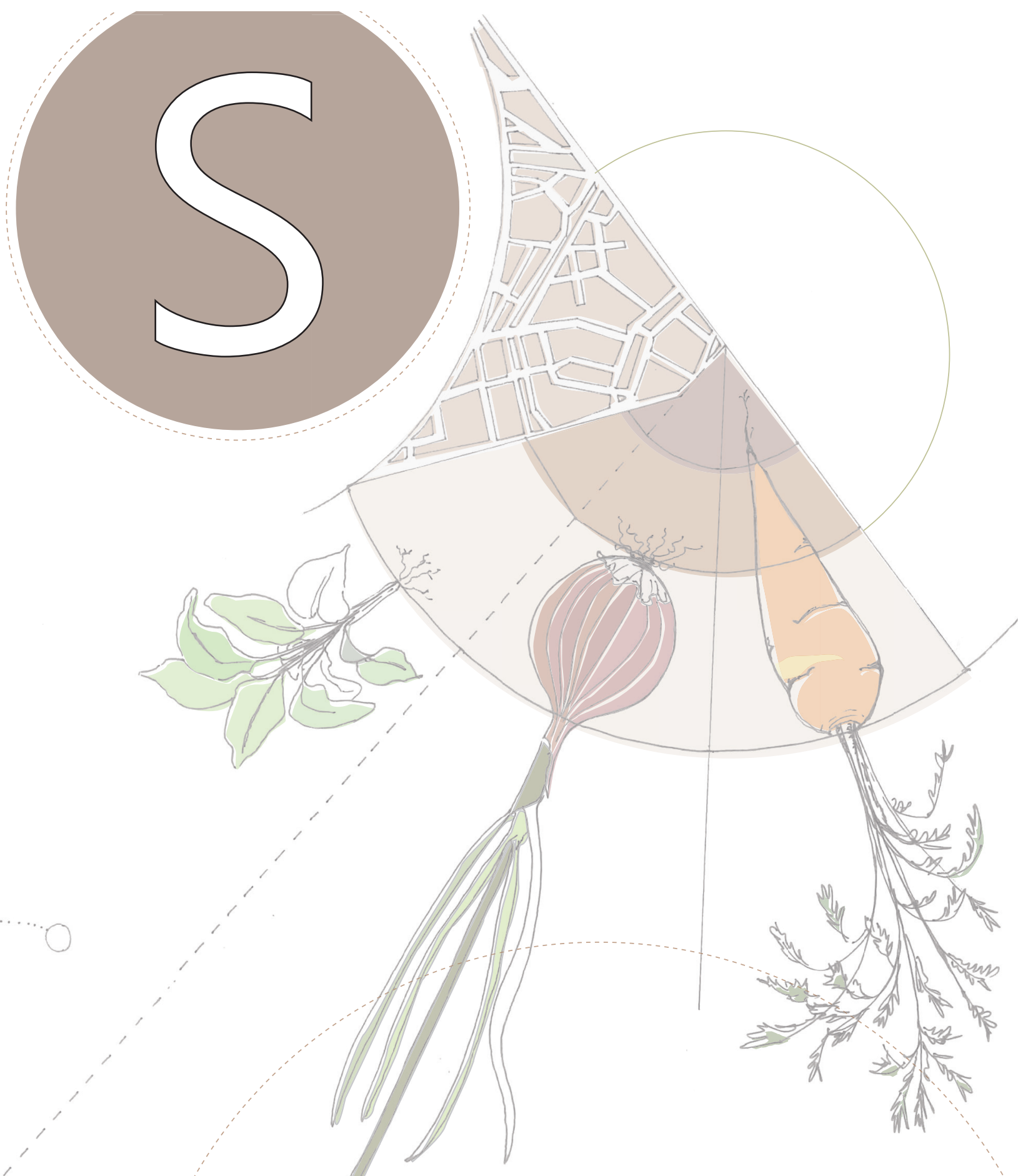

SUMMARY

SAMENVATTING

RESUMEN 


\section{Summary}

Nutrient cycling occurs in ecosystems as a result of various natural driving sources (e.g., solar energy, tectonic energy, gravity), and interacts with the larger biogeochemical cycles through a system of inputs and outputs, which vary in space and time. In agroecosystems, nutrient cycling and management refers to the replacement of nutrients, withdrawn during crop harvesting, through biological processes such as nitrogen fixation or through the addition of organic material and/or mineral fertilizers to agricultural fields. Until the 19th century human excreta ('nightsoil') and organic waste were recycled to agriculture to replenish farm land with nutrients and organic matter in (peri-)urban areas.

With the onset of cheap chemical fertilizer production and the implementation of extensive waterborne sanitation infrastructures, however, the use of human excreta was largely abandoned. Despite the increased global agricultural productivity due to the use of synthetic fertilizers and the improved human health conditions due to sanitization, both developments critically altered nutrient flows, with corresponding consequences. To start, the production of synthetic fertilizers exhausts fossil and mineral resources. The production of nitrogen is energy intensive, currently sourcing energy from fossil fuels, while the sourcing of phosphorus and potassium, as well as several micronutrients, is dependent on finite, and spatially-concentrated, ore reserves. Second, nutrient balances in agriculture vary globally and include the distinct depletion of nutrients from agricultural soils in some places and the accumulation of nutrients in soils and water systems in other places. Finally, nutrients contained in consumed food, which are subsequently excreted in the form of urine and feces, together contribute the largest fraction of nutrients to domestic wastewater. Current management strategies of human excreta contribute to irretrievable losses of nutrients in particular to water bodies and in landfills. Cognizant of the limitations of industrial fertilizer production and use, and of current sanitation infrastructures, it has become increasingly evident that present patterns of nutrient flows are unsustainable in the long term.

Given the abundance and concentration of wastewater production in cities, cities play a key role in new approaches for recycling nutrients contained in human excreta to agriculture. Developments in urban agriculture and new sanitation systems bring about new narratives to the status quo of both food production and human excreta management, and reintroduce the opportunity to partially close nutrient cycles at the urban scale. Urban agriculture is the production of food in and around (peri-urban) a city and manifestations include both low-tech and high-tech production systems, such as, community gardens, ground-based farms, rooftop farms, rooftop greenhouses, and (multi-story) indoor farms. New sanitation systems collect, transport and treat streams containing human excreta and aim to recover valuable resources from those streams. Based on the premise that nutrient recovery is most cost-effective from streams with high nutrient and low contaminant concentrations, new sanitation systems uphold source- separation of streams. 
Developments in both urban agriculture and new sanitation have occurred in parallel, yet autonomously. The recognition of the mutual benefit for nutrient exchange between urban agriculture and new sanitation has increased. In this regard, urban agriculture has a demand for nutrients and new sanitation a supply of nutrients, which if matched, can facilitate nutrient recycling and thereby minimize nutrient losses. Nevertheless, numerous challenges remain to match nutrient flows between urban agriculture and new sanitation. Not only do the quantities and qualities of nutrient demand and supply need to be matched - taking into account parameters for plant requirements, as well as human hygiene and environmental safety (e.g. pathogens, heavy metals) - but also spatial and temporal dynamics of demand and supply (e.g. when and where fertilizers are needed and when and where nutrients are excreted) need to be optimized for coupling of nutrient flows.

The objective of this research is to contribute to uncovering the potential of integrating urban agriculture and new sanitation so as to establish nutrient recirculation between the two. Specific objectives include (i) an analysis of nutrient demand and supply, (ii) an evaluation of spatial and temporal aspects of supply and demand matching, and (iii) a reflection on trade-offs for improved nutrient recycling within the urban environment. This thesis primarily focuses on the three macronutrients, nitrogen $(\mathrm{N})$, phosphorus $(\mathrm{P})$ and potassium $(\mathrm{K})$, as well as organic matter $(\mathrm{OM})$, although, other macro- and micronutrients are tangentially discussed. The central question of this thesis research is: 'what is the potential to recycle nutrients present in human excreta as fertilizer to agriculture within the urban and peri-urban environment?' To address this question, four sub-questions are defined:

1. What is the demand for nutrients by urban agriculture?

2. What quantity and quality of recovered nutrient-containing products can new sanitation systems render?

3. How do spatial and temporal conditions influence the potential to match nutrient demand by urban agriculture with nutrient supply by recovered products?

4. What trade-offs need to be considered when matching nutrient flows between urban agriculture and new sanitation systems?

After the introduction in Chapter 1, Chapter 2 presents a first exploration to couple urban agriculture and new sanitation using the Urban Harvest Approach (UHA). The UHA is an approach to improve urban resource management towards self-sufficiency by applying the following management strategies: demand minimization, output minimization and multi-sourcing. Novel to this research is adapting the UHA, until now extensively applied to the urban water cycle, to nitrogen, phosphorus and organic matter loads for two urban agriculture typologies, groundbased and rooftop, and four new sanitation concepts. Results show an achieved self-sufficiency of $100 \%$ for phosphorus and partial self-sufficiency for nitrogen and organic matter. The study also indicated that nutrient management in urban agriculture is largely undocumented and 
unregulated, identifying the need for more comprehensive data gathering of nutrient demand.

To further examine nutrient management in urban agriculture, Chapter 3 presents data collected from a total of 25 ground-based urban agriculture initiatives in the Netherlands on i) preferences for types of fertilizers, and ii) quantity and quality of fertilizers used, including nutrient composition and organic matter content. Results show over-fertilization of fields compared to nutrient demand based on crop nutrient uptake (450\% for total nitrogen, $600 \%$ for phosphorus and $250 \%$ for potassium) and compared to legal application limits for $\mathrm{N}$ and $\mathrm{P}$ in conventional agriculture in the Netherlands. In conclusion, in future assessments, nutrient demand should reflect crop uptake values rather than current nutrient management practices at urban farms.

Chapter 4 presents an overview of conceivable recovery pathways for the recovery of nutrients from streams containing human excreta. Recovery pathways are outlined starting from urine and yellow water, feces and excreta and starting from brown water, black water and sewage, as well as a summary of recovered products rendered per pathway and their application potential in agriculture. The review allows for the identification of broader trends and patterns regarding efforts that facilitate recycling of nutrients contained in human excreta to agriculture. The review suggests that there is scope to explore how to maximize nutrient recovery by combining pathways and products, and including a broader range of nutrients. To this end, the review provides a template for designing and combining nutrient recovery pathways.

In addition to laying bare nutrient flows in urban agriculture and new sanitation, there is scope to ask questions that go beyond nutrient quantities and qualities of demand and supply. Nutrient recirculation is also challenged by a spatial disconnect where food is consumed and human excreta produced, and where food is produced. Chapter 5 presents results of the developed geographic information systems (GIS)-based methodology that provides a spatially explicit inventory of promising locations for nutrient 'harvesting' from human excreta. The results quantify the nutrient recovery potential per building and neighborhood for nitrogen, phosphorus and potassium, identifying locations with relatively high nutrient excretion (termed nutrient hotspots). In Amsterdam, 193 buildings identified as phosphorus hotspots together produce 32.5 tons of phosphorus annually, $10 \%$ of the city's annual load of 330.5 tons. The methodology is new in the field of nutrient mapping, especially at the smallest geographical scale: per building, though the presentation of results at two spatial scales attest to the value of spatial resolution for the generation and interpretation of results, and in their usefulness in decision-making.

Chapter 6 builds on the previous study to further extend the usefulness of the model in informing decision-making practitioners strategies for recycling nutrients to agricultural fields. The GIS model matches discrete locations (in this case building nutrient hotspots) with areas of agricultural demand (urban agriculture, cropland and grassland sites). The model further optimizes for minimum transport distance between supply and demand points, and calculates and maps the transport routes according to existing road networks. To disclose the potential of the model, phosphorus supply in human-derived urine is matched with phosphorus demands 
by agricultural fields within the municipality of Amsterdam on a temporal scale of 1 year. The value of such geospatial models is in their potential contribution to planning capacity of needed interventions, for example, in the transition to a circular economy.

Chapter 7 offers a perspective on the current framing of resource recovery as part of waste management systems, and calls for a needed reframing human excreta management as part of food and farming systems. Such reframing brings to the fore six aspects of critical importance which are currently underrated. Increased consideration of these aspects has the potential to better guide human excreta management towards global food, soil, and nutrient security without compromising other priorities related to human and environmental health.

The thesis ends with a concluding chapter in which a synthesis of the results on the potential to redirect nutrients contained in human excreta to urban agriculture is presented. Chapter 8 places the results of this thesis within a broader perspective on (urban) nutrient management. The synthesis contemplates aligning future spatial arrangements of agricultural systems and the reuse of recovered products at various proximities from the urban center. As such, the embeddedness of urban agriculture in the city lends itself for establishing local nutrient cycles, especially by using nutrients in forms too costly (i.e. bulky) to export back to rural and global agricultural hinterlands. Nonetheless, there is a need for increased understanding of nutrient flows in terms of quantity and quality, especially considering the diversity of (future) urban agriculture and new sanitation typologies, as well as their point sources, direction and connection to other spatial characteristics. While the contribution of integrated urban agriculture and new sanitation systems towards a circular nutrient economy will remain partial, the necessity for systems changes in both food provisioning and resource management strategies should welcome further exploration of their integration. 


\section{Samenvatting}

Nutriëntencycli komen voor in ecosystemen als gevolg van verschillende natuurlijke energiebronnen (bijv. zonne-energie, tektonische energie, zwaartekracht) en ze zijn verbonden met de grotere biogeochemische cycli via een systeem van in- en uitgaande stromen. In de landbouw wordt de nutriëntencyclus actief beïnvloed door nutriënten in de bodem, die tijdens het oogsten van gewassen worden onttrokken, aan te vullen door middel van biologische processen zoals stikstofbinding of door toevoeging van organisch materiaal en / of minerale meststoffen aan landbouwgronden. Tot de 19e eeuw werd hiervoor in en rond stedelijke gebieden menselijke ontlasting en organisch afval gebruikt, waardoor er sprake was van een lokale nutriëntencyclus.

Met de grootschalige productie van kunstmest en de wijdverbreide implementatie van riolering kwam er echter een einde aan het gebruik van menselijke ontlasting als meststof. Ondanks de toegenomen mondiale landbouwproductiviteit als gevolg van de toepassing van synthetische meststoffen en de verbeterde gezondheidstoestand van de mens als gevolg van afvalwaterzuivering, kennen beide ontwikkelingen ook negatieve gevolgen. Allereerst put de productie van synthetische meststoffen fossiele en minerale bronnen uit. De productie van stikstof is namelijk energie-intensief en is momenteel afhankelijk van fossiele brandstoffen, terwijl de winning van fosfor en kalium, evenals die van verscheidene micronutriënten, afhankelijk is van eindige minerale reserves die zich op een beperkt aantal plekken in de wereld bevinden. Ten tweede variëren nutriëntenbalansen in de landbouw wereldwijd, waarbij op sommige plekken sprake is van een duidelijke uitputting van nutriënten in landbouwgronden en op andere plekken voedingsstoffen zich ophopen in de bodem en in watersystemen. Tot slot vormen nutriënten die worden geconsumeerd als voedsel en vervolgens worden uitgescheiden als urine en ontlasting, de grootste fractie van nutriënten in huishoudelijk afvalwater. Deze nutriënten gaan grotendeels onherstelbaar verloren in oppervlakte- en grondwater en op stortplaatsen, ten gevolge van de huidige manier waarop afvalwaterzuiveringsprocessen zijn ingericht. Deze gevolgen van de productie en het gebruik van synthetische meststoffen en van de huidige sanitaire infrastructuur, hebben in toenemende mate zichtbaar gemaakt dat het huidige patroon aan nutriëntenstromen op de lange termijn niet duurzaam is.

Doordat in steden veel afvalwater wordt geproduceerd, spelen steden een sleutelrol voor nieuwe benaderingen om nutriënten uit menselijke ontlasting toe te passen in de landbouw. Ontwikkelingen in de stadslandbouw en nieuwe sanitatiesystemen bieden alternatieven voor zowel de huidige manier van voedselproductie als voor de manier waarop afvalwater wordt behandeld. Deze ontwikkelingen geven de mogelijkheid om de nutriëntencyclus op stedelijke schaal opnieuw gedeeltelijk te sluiten. Stadslandbouw is de productie van voedsel in en rondom de stad. Dit type landbouw omvat zowel low-tech als high-tech productiesystemen, waaronder gemeenschapstuinen, boerderijen, daktuinen, dakkassen, en 'vertical farming', waarbij het telen plaatsvindt in gebouwen, vaak in meerdere etages. Nieuwe sanitatiesystemen verzamelen, transporteren en behandelen afvalwater en hebben tot doel waardevolle grondstoffen (inclusief 
nutriënten) uit die stromen te winnen. Op basis van het uitgangspunt dat nutriëntterugwinning het meest kosteneffectief is uit geconcentreerde stromen met hoge nutriënten- en lage verontreinigingconcentraties, scheiden nieuwe sanitatiesystemen stromen aan de bron (zoals het gescheiden inzamelen van toilet water en grijswater).

Ontwikkelingen in zowel stadslandbouw als nieuwe sanitatie hebben parallel, maar autonoom plaats gevonden. In toenemende mate wordt het wederzijdse voordeel voor de uitwisseling van nutriënten tussen stadslandbouw en nieuwe sanitatie erkend. Stadslandbouw heeft namelijk een vraag naar nutriënten en nieuwe sanitatie een aanbod van nutriënten, die, indien op elkaar afgestemd, het hergebruik van nutriënten mogelijk kunnen maken en het verlies aan nutriënten kunnen minimaliseren. Desalniettemin zijn er nog tal van uitdagingen om de nutriëntenstromen tussen stadslandbouw en nieuwe sanitatie op elkaar af te stemmen. Zo moet de kwantiteit en de kwaliteit van de vraag naar en het aanbod aan nutriënten op elkaar worden afgestemd - rekening houdend met plantvereisten, evenals menselijke hygiëne en milieuveiligheid (bijvoorbeeld ziekteverwekkers, zware metalen). Ook ruimtelijke en temporele kwesties van de vraag en het aanbod (bijvoorbeeld wanneer en waar meststoffen nodig zijn en wanneer en waar nutriënten worden uitgescheiden) moeten worden geoptimaliseerd voor de koppeling van nutriënten stromen.

Het doel van dit onderzoek is bij te dragen aan het begrijpen van het potentieel om de recirculatie van nutriënten tussen nieuwe sanitatie en stadslandbouw tot stand te brengen. Specifieke doelstellingen zijn (i) een analyse van de vraag naar en het aanbod van nutriënten, (ii) een evaluatie van ruimtelijke en temporele aspecten van de koppeling tussen vraag en aanbod, en (iii) een reflectie op de mogelijke afwegingen van het hergebruik van nutriënten in de stedelijke omgeving. Dit proefschrift richt zich voornamelijk op de drie macronutriënten stikstof $(\mathrm{N})$, fosfor $(\mathrm{P})$ en kalium $(\mathrm{K})$, evenals organische stof $(\mathrm{OM})$; andere macro- en micronutriënten worden in beperkte mate besproken. De centrale vraag van dit proefschrift is: 'wat is het potentieel om nutriënten die aanwezig zijn in menselijke ontlasting als meststof her te gebruiken in de stadslandbouw'. Om deze vraag te beantwoorden, zijn vier deelvragen gedefinieerd:

1. Wat is de nutriëntenvraag vanuit de stadslandbouw?

2. Welke kwantiteit en kwaliteit van teruggewonnen nutriënten-bevattende producten kunnen nieuwe sanitatiesystemen opleveren?

3. Hoe beïnvloeden ruimtelijke en temporele omstandigheden het potentieel om de nutriëntenvraag door stadslandbouw te koppelen met het aanbod van nutriënten in de teruggewonnen producten?

4. Met welke afwegingen moet rekening worden gehouden bij het afstemmen van nutriëntenstromen tussen stadslandbouw en nieuwe sanitatiesystemen?

$\mathrm{Na}$ de inleiding in hoofdstuk 1, presenteert hoofdstuk. 2 een eerste verkenning om stadslandbouw en nieuwe sanitatiesystemen te combineren met behulp van de Urban Harvest Approach (UHA). 
De UHA is een benadering om het beheer van stedelijke grondstoffen voor zelfvoorziening te verbeteren door de volgende managementstrategieën toe te passen: vraagminimalisatie, reductie van uitgaande afval stromen door het herwinnen en recyclen van grondstoffen, en de resterende vraag voorzien via hernieuwbare en lokale bronnen. Nieuw in dit onderzoek is het aanpassen van het UHA, tot nu toe uitgebreid toegepast op de stedelijke watercyclus, op stikstof, fosfor en organische stof voor twee stadslandbouwtypen, in volle grond en op het dak, en vier nieuwe sanitatiesystemen. De resultaten tonen een mogelijke zelfvoorziening van $100 \%$ voor fosfor en gedeeltelijke zelfvoorziening voor stikstof en organische stof. De studie geeft ook aan dat het beheer van nutriënten in de stadslandbouw grotendeels ongedocumenteerd en niet gereguleerd is, en identificeert de behoefte aan uitgebreidere gegevensverzameling over de vraag naar nutriënten vanuit de stadslandbouw.

Om het beheer van nutriënten in de stadslandbouw verder te onderzoeken, presenteert hoofdstuk 3 gegevens van in totaal vijfentwintig grondgebonden stadslandbouw initiatieven in Nederland met betrekking tot i) voorkeur voor soorten meststoffen, en ii) gebruikte kwantiteit en kwaliteit van meststoffen, inclusief de samenstelling van de meststoffen wat nutriënten en organische stof gehalte betreft. Resultaten tonen overbemesting van de standslandbouw gronden aan in vergelijking met de vraag naar nutriënten, gebaseerd op de opname van nutriënten door gewassen (450\% voor totale stikstof, $600 \%$ voor fosfor en $250 \%$ voor kalium) en vergeleken met wettelijke toepassingslimieten voor $\mathrm{N}$ en $\mathrm{P}$ in de gangbare landbouw in Nederland. Concluderend, zou in toekomstige beoordelingen met betrekking tot bemesting in de stadlandbouw de vraag naar nutriënten een weerspiegeling moeten zijn van de waarden voor opname door gewassen in plaats van de huidige praktijken van toediening van meststoffen.

Hoofdstuk 4 presenteert een overzicht van denkbare sanitatie 'routes' voor het terugwinnen van nutriënten uit afvalwaterstromen die menselijke uitwerpselen bevatten. Deze routes worden geschetst, voor de stromen urine en geel water, fecaliën en bruin water, zwart water en rioolwater, evenals een samenvatting van teruggewonnen producten die per route worden weergegeven en hun toepassingspotentieel in de landbouw. Deze review maakt het mogelijk om bredere trends en patronen te identificeren die het hergebruik van nutriënten in menselijke ontlasting in de landbouw vergemakkelijken. De beoordeling geeft aan dat er ruimte is om te onderzoeken hoe het terugwinnen van nutriënten kan worden geoptimaliseerd door het combineren van routes en producten, en met een breder scala aan nutriënten. Hierbij kan het gepresenteerde overzicht een raamwerk vormen voor het ontwerpen en combineren van routes voor het terugwinnen van nutriënten.

Naast het rapporteren over nutriëntenstromen in de stadslandbouw en nieuwe sanitatie, is er ruimte om vragen te stellen die verder gaan dan het in kaart brengen van de kwantiteiten en kwaliteiten van vraag en aanbod. Een uitdaging voor de recirculatie van nutriënten is de ruimtelijke ontkoppeling tussen daar waar voedsel wordt geconsumeerd en menselijke uitwerpselen worden geproduceerd en daar waar voedsel wordt geproduceerd. Hoofdstuk 5 presenteert de resultaten 
van de ontwikkelde methode, op basis van geografische informatiesystemen (GIS), die het mogelijk maakt een ruimtelijke inventarisatie te maken van veelbelovende locaties voor het 'oogsten' van nutriënten uit menselijke uitwerpselen. De resultaten kwantificeren het potentieel voor het terugwinnen van nutriënten per gebouw en wijk voor stikstof, fosfor en kalium, waarbij locaties worden geïdentificeerd met een relatief hoge uitscheiding van nutriënten ('hotspots' genoemd). In Amsterdam zijn 193 gebouwen geïdentificeerd als fosfor hotspots en jaarlijks produceren ze samen 32,5 ton fosfor, 10\% van de jaarlijkse vracht van de stad van 330,5 ton. De methode is nieuw op het gebied van het in kaart brengen van nutriëntenstromen, vooral op de kleinste geografische schaal: per gebouw. De presentatie van resultaten op twee ruimtelijke schalen (gebouw en wijk) toont de waarde van ruimtelijke resolutie voor het genereren en interpreteren van resultaten aan, en hun bruikbaarheid in besluitvorming.

Hoofdstuk 6 bouwt voort op de vorige studie om het nut van het model bij het informeren van besluitvormingsstrategieën voor het recyclen van nutriënten naar landbouwgebieden verder uit te breiden. Het GIS-model koppelt concrete locaties (in dit geval de hotspots op gebouw schaal) aan gebieden met agrarische vraag (stadslandbouw, akker- en grasland). Het model optimaliseert verder voor de minimale transportafstand tussen vraag- en aanbodpunten door transportroutes te berekenen en deze in kaart te brengen volgens bestaande wegennetwerken. Om het potentieel van het model te onthullen, wordt het aanbod van fosfor in van mensen afkomstige urine gekoppeld aan de vraag naar fosfor door landbouwvelden binnen de gemeente Amsterdam op een temporele schaal van 1 jaar. De waarde van dergelijke ruimtelijke modellen ligt in hun potentiële bijdrage aan het plannen van benodigde interventies, bijvoorbeeld in de overgang naar een circulaire economie.

Hoofdstuk 7 biedt een perspectief op het huidige kijk op het terugwinnen van nutriënten als onderdeel van afvalbeheersystemen, en roept op tot een noodzakelijk herformulering van het beheer van menselijke ontlasting als onderdeel van voedsel- en landbouwsystemen. Zo'n herformulering brengt zes aspecten van cruciaal belang naar voren die momenteel worden onderschat. Meer aandacht voor deze aspecten biedt kansen om het beheer van menselijke ontlasting beter te sturen naar wereldwijde nutriëntenzekerheid, bodemvruchtbaarheid en voedselzekerheid, zonder afbreuk te doen aan andere prioriteiten met betrekking tot de gezondheid van mens en milieu.

Het proefschrift eindigt met een synthese betreffende het potentieel om nutriënten in menselijke ontlasting toe te passen in de stadslandbouw. Dit hoofdstuk 8 plaatst de resultaten van dit proefschrift in een breder perspectief van (stedelijk) nutriëntenbeheer. In deze synthese wordt uiteengezet hoe toekomstige ruimtelijke ordeningen van landbouwsystemen en het hergebruik van teruggewonnen nutriënten-bevattende producten op verschillende afstanden van het stedelijke centrum met elkaar in lijn kunnen worden gebracht. Als zodanig leent de indeling van stadslandbouw zich voor het tot stand brengen van lokale nutriëntenkringlopen, vooral voor de toepassing van nutriëntenproducten die te duur zijn (d.w.z. volumineus en/of zwaar) om 
terug te exporteren naar landelijke en internationale landbouwgronden. Desalniettemin is er behoefte aan een beter begrip van de kwantiteit en kwaliteit van nutriëntenstromen, vooral met het oog op de diversiteit van (toekomstige) stadslandbouw en nieuwe sanitatietypen, evenals hun puntbronnen, richting en verband met andere ruimtelijke kenmerken. Ondanks dat de integratie van stadslandbouw en nieuwe sanitatiesystemen slechts voor een deel zal bijdragen aan een circulaire nutriënteneconomie, is het van belang om bij de noodzakelijke systeemwijzigingen van zowel voedselvoorziening als strategieën voor grondstoffenbeheer hun integratie verder te verkennen. 


\section{Resumen}

El ciclo de nutrientes ocurre en los ecosistemas como resultado de varias fuentes naturales de conducción (por ejemplo: energía solar, energía tectónica, gravedad). También, interactúa con los ciclos biogeoquímicos más grandes a través de un sistema de flujos de entrada y salida, que varían en el espacio y el tiempo. En los agroecosistemas, el ciclo y manejo de nutrientes se refiere al reemplazo de nutrientes, extraídos durante la cosecha del cultivo, a través de procesos biológicos como la fijación de nitrógeno o mediante la adición de material orgánico y/o fertilizantes minerales a los campos agrícolas. Hasta el siglo XIX, la excreta humana y los desechos orgánicos se reciclaban en la agricultura para reponer las tierras agrícolas con nutrientes y materia orgánica en las áreas (peri) urbanas.

Con el inicio de la producción de fertilizantes químicos y la implementación de extensas infraestructuras de saneamiento a base de agua, se abandonó en gran medida el uso de excretas humanas. A pesar del aumento de la productividad agrícola mundial debido al uso de fertilizantes sintéticos y la mejora de las condiciones de salud humana debido al saneamiento, ambos desarrollos alteraron críticamente los flujos de nutrientes, con varias consecuencias. En primer lugar, la producción de fertilizantes sintéticos agota los recursos fósiles y minerales. La producción de nitrógeno, es intensiva en consumo de energía, actualmente obtenida través de combustibles fósiles. La provisión de fósforo y potasio, así como varios micronutrientes, depende de reservas de mineral finitas y concentradas espacialmente en pocos países. En segundo lugar, los equilibrios de nutrientes en la agricultura difieren a nivel mundial. En algunos lugares, los nutrientes de los suelos agrícolas se están agotando mientras que en otros existe acumulación de nutrientes en los suelos y en el agua. En tercer lugar, el contenido de nutrientes en los alimentos se excretan en forma de orina y heces. Juntos aportan la mayor fracción de nutrientes en aguas residuales domésticas. Las estrategias actuales de gestión de la excreta humana contribuyen a pérdidas irrecuperables de nutrientes, en particular para los cuerpos de agua y los vertederos. Las limitaciones de la producción y el uso de fertilizantes industriales, y de las infraestructuras de saneamiento actuales muestran que los flujos actuales de nutrientes son insostenibles a largo plazo.

Debido a la abundancia y concentración de la producción de aguas residuales en las ciudades, las ciudades juegan un papel clave en los nuevos enfoques para reciclar los nutrientes de las excretas humanas. Los avances en la agricultura urbana y los nuevos sistemas de saneamiento generan nuevas narrativas sobre el status quo de la producción de alimentos y el manejo de las excretas humanas. Estos avances (re)introducen la oportunidad de cerrar parcialmente los ciclos de nutrientes a escala urbana. La agricultura urbana se define como la producción de alimentos en una ciudad y sus alrededores (periurbana). La agricultura urbana incluye sistemas de producción de baja y alta tecnología, como huertos comunitarios, granjas comerciales, huertos en la azotea, invernaderos en la azotea y vertical farms (multi-pisos). Los nuevos sistemas de saneamiento recolectan, transportan y tratan flujos que contienen excretas humanas y tienen como objetivo 
recuperar recursos valiosos de esas flujos. Los nuevos sistemas de saneamiento mantienen la separación de fuentes de los flujos, ya que la recuperación de nutrientes es más rentable en flujos con altas concentraciones de nutrientes y bajas concentraciones de contaminantes.

Tanto la agricultura urbana como los sistemas de nuevo saneamiento se han desarrollado en paralelo, pero de manera autónoma. A pesar de ello, el reconocimiento del beneficio mutuo por el intercambio de nutrientes entre la agricultura urbana y el nuevo saneamiento va en aumento. La demanda de nutrientes de la agricultura urbana podría en efecto ser cubierta por la oferta de nutrientes del nuevo saneamiento al combinar los dos sistemas, facilitando el reciclaje de nutrientes y, por lo tanto, minimizando las pérdidas. Sin embargo, quedan numerosos desafíos para equilibrar los flujos de nutrientes entre la agricultura urbana y el nuevo saneamiento. No solo deben coincidir las cantidades, sino también la calidad de la demanda y oferta, teniendo en cuenta los parámetros para los requisitos de la planta, así como la higiene humana y la seguridad ambiental (por ejemplo: patógenos, metales pesados). Además, es importante considerar la dinámica espacial y temporal de la demanda y oferta (por ejemplo, cuándo y dónde se necesitan fertilizantes y cuándo y dónde se excretan los nutrientes) para optimizar los flujos de nutrientes.

El objetivo de esta investigación es contribuir al descubrimiento del potencial de integrar la agricultura urbana y el nuevo saneamiento para establecer la recirculación de nutrientes entre los dos. Los objetivos específicos incluyen (i) un análisis de la demanda y la oferta de nutrientes, (ii) una evaluación de los aspectos espaciales y temporales de la oferta y la demanda, y (iii) una reflexión sobre las compensaciones para mejorar el reciclaje de nutrientes en el entorno urbano. Esta tesis se centra principalmente en los tres macronutrientes: nitrógeno $(\mathrm{N})$, fósforo $(\mathrm{P})$ y potasio $(\mathrm{K})$, así como en la materia orgánica $(\mathrm{OM})$.Otros macro y micronutrientes se discuten de forma tangencial. La pregunta central de esta investigación de tesis es: '¿Cuál es el potencial de reciclar los nutrientes presentes en la excreta humana como fertilizante para la agricultura dentro del entorno urbano y periurbano?' Para abordar esta pregunta, se definen cuatro subpreguntas:

1. ¿Cuál es la demanda de nutrientes por parte de la agricultura urbana?

2. ¿Qué cantidad y calidad de nutrientes pueden ser recuperados a partir de los nuevos sistemas de saneamiento?

3. ¿Cómo influyen las condiciones espaciales y temporales en el potencial de balancear la demanda de nutrientes de la agricultura urbana con la oferta de nutrientes de los productos recuperados?

4. ¿Qué compensaciones deben considerarse al hacer coincidir los flujos de nutrientes entre la agricultura urbana y los nuevos sistemas de saneamiento?

Despues de la introduccion, en el Capitulo 1, el Capitulo 2 presenta una primera exploración para unir la agricultura urbana y el nuevo saneamiento utilizando el Urban Harvest Approach (UHA). El UHA es un procedimiento para mejorar la gestión de los recursos urbanos hacia la autosuficiencia mediante la aplicación de las siguientes estrategias: minimización de la demanda, minimización de gastos y residuos, y provisión desde múltiples fuentes. La novedad de esta 
investigación es la adaptación del UHA que hasta ahora ha sido ampliamente aplicado solamente al ciclo urbano del agua. En esta tesis investigamos las cargas de nitrógeno, fósforo y materia orgánica para dos tipologías de agricultura urbana, y cuatro conceptos de nuevo saneamiento. Los resultados muestran una autosuficiencia lograda del 100\% para el fósforo y una autosuficiencia parcial para el nitrógeno y la materia orgánica. El estudio también indica que el manejo de nutrientes en la agricultura urbana en gran parte no es documentado ni regulado. Concluimos que una recopilación de datos más completa de la demanda de nutrientes es necesaria.

Para examinar más a fondo el manejo de nutrientes en la agricultura urbana, el Capitulo 3 presenta datos recopilados de un total de 25 huertos de agricultura urbana en los Países Bajos. Los datos incluyen: i)preferencias para los tipos de fertilizantes, y ii)cantidad y calidad de fertilizantes utilizados, incluyendo la composición de nutrientes y contenido de materia orgánica. Los resultados muestran una fertilización excesiva de los campos en comparación con la demanda de nutrientes, basada en la utilización de nutrientes por medio de los cultivos (450\% para el nitrógeno total, $600 \%$ para el fósforo y $250 \%$ para el potasio). La fertilización también excede los límites legales de aplicación de N y P en la agricultura convencional en los Países Bajos. En conclusión, en evaluaciones futuras, la demanda de nutrientes debería reflejar los valores de absorción de los cultivos en lugar de las prácticas actuales de manejo de nutrientes en los huertos urbanas.

El Capitulo 4 presenta una visión general de posibles opciones para la recuperación de nutrientes contenidos en la excreta humana. Las vías de recuperación se describen a partir de orina y agua amarilla, heces y excreta y a partir de aguas negras y aguas residuales. También se presenta un resumen de los productos recuperados por vía y su potencial de aplicación en la agricultura. La revisión de literatura permite la identificación de tendencias y patrones más amplios con respecto a los esfuerzos que facilitan el reciclaje de nutrientes contenidos en las excretas humanas para la agricultura. La revisión sugiere que hay margen para explorar cómo maximizar la recuperación de nutrientes combinando vías y productos, e incluyendo una gama más amplia de nutrientes. Con este fin, la revisión proporciona un registro para diseñar y combinar vías de recuperación de nutrientes.

Al examinar los flujos de nutrientes en la agricultura urbana y el nuevo saneamiento, hay aspectos que van más allá de las cantidades y calidades de nutrientes y las de la demanda y la oferta. La recirculación de nutrientes también se ve desafiada por una desconexión espacial: donde se consumen alimentos y se producen excretas humanas, y donde se producen alimentos. El Capitulo 5 presenta los resultados de la metodología desarrollada basada en los sistemas de información geográfica (SIG) que proporciona un inventario explícito de ubicaciones prometedoras para la 'recolección' de nutrientes de la excreta humana. Los resultados cuantifican el potencial de recuperación de nutrientes por edificio y vecindario para nitrógeno, fósforo y potasio, identificando ubicaciones con excreción de nutrientes relativamente alta (denominados 'focos' de nutrientes). En Ámsterdam, 193 edificios fueron identificados como focos de fósforo y juntos 
producen 32.5 toneladas de fósforo anualmente, el 10\% de la carga anual de la ciudad de 330.5 toneladas. La metodología es nueva en el entendimiento de flujos de nutrientes, especialmente en la escala geográfica más pequeña: por edificio. Además, la presentación de resultados en dos escalas espaciales atestigua el valor de la resolución espacial para la generación e interpretación de resultados, y su utilidad en toma de decisiones.

El Capitulo 6 se basa en el estudio anterior para ampliar aún más la utilidad del modelo para informar las estrategias de los profesionales que toman decisiones para reciclar nutrientes hacia los campos agrícolas. El modelo SIG hace coincidir ubicaciones discretas (en este caso, los focos) con áreas de demanda agrícola (agricultura urbana, granjas de cultivo y pastizales). El modelo optimiza aún más la distancia mínima de transporte entre los puntos de oferta y demanda, y calcula y mapea las rutas de transporte de acuerdo con las redes de carreteras existentes. Para revelar el potencial del modelo, el suministro de fósforo en la orina derivada del ser humano se correlaciona con las demandas de fósforo de los campos agrícolas dentro del municipio de Ámsterdam en una escala temporal de 1 año. El valor de tales modelos geoespaciales tiene una potencial contribución a la capacidad de planificación de las intervenciones necesarias, por ejemplo, en la transición a una economía circular.

El Capitulo 7 ofrece una perspectiva sobre el marco actual de la recuperación de recursos como parte de los sistemas de gestión de residuos, y exige un nuevo marco necesario para la gestión de las excretas humanas como parte de los sistemas alimentarios y agrícolas. Tal reformulación pone de relieve seis aspectos de importancia crítica que actualmente están subestimados. Una mayor consideración de estos aspectos tiene el potencial de guiar mejor el manejo de las excretas humanas hacia la seguridad global de los alimentos, el suelo y los nutrientes sin comprometer otras prioridades relacionadas con la salud humana y ambiental.

La tesis finaliza con un capítulo en el que se presenta una síntesis de los resultados sobre el potencial de redirigir los nutrientes contenidos en las excretas humanas a la agricultura (urbana). El Capitulo 8 coloca los resultados de esta tesis dentro de una perspectiva más amplia sobre el manejo de nutrientes. La síntesis contempla la alineación de futuros arreglos espaciales de los sistemas agrícolas y la reutilización de productos recuperados en varias proximidades del centro urbano. Como tal, la integración de la agricultura urbana en la ciudad se presta para establecer ciclos de nutrientes locales, especialmente mediante el uso de nutrientes en formas demasiado costosas (es decir, voluminosas) para exportar de regreso a las zonas rurales y globales agrícolas. Además, existe la necesidad de una mayor comprensión de los flujos de nutrientes en términos de cantidad y calidad, especialmente teniendo en cuenta la diversidad de la (futura) agricultura urbana y las tipologías de nuevo saneamiento. Aunque la contribución de la agricultura urbana y los nuevos sistemas de saneamiento hacia una economía circular de nutrientes seguirá siendo parcial, la necesidad de cambios en los sistemas tanto en el suministro de alimentos como en las estrategias de gestión de recursos debería ser el punto de inicio para su integración. 


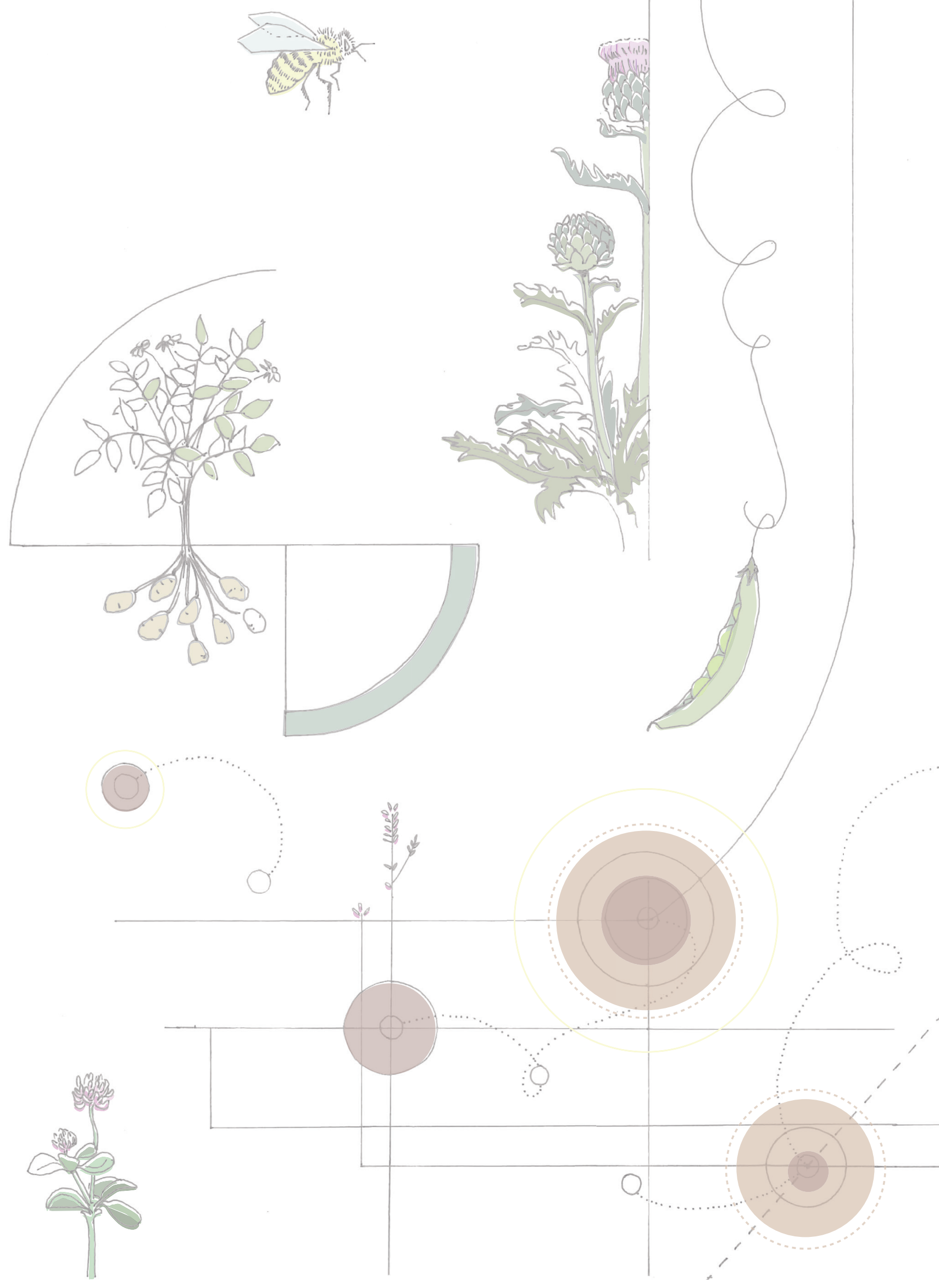




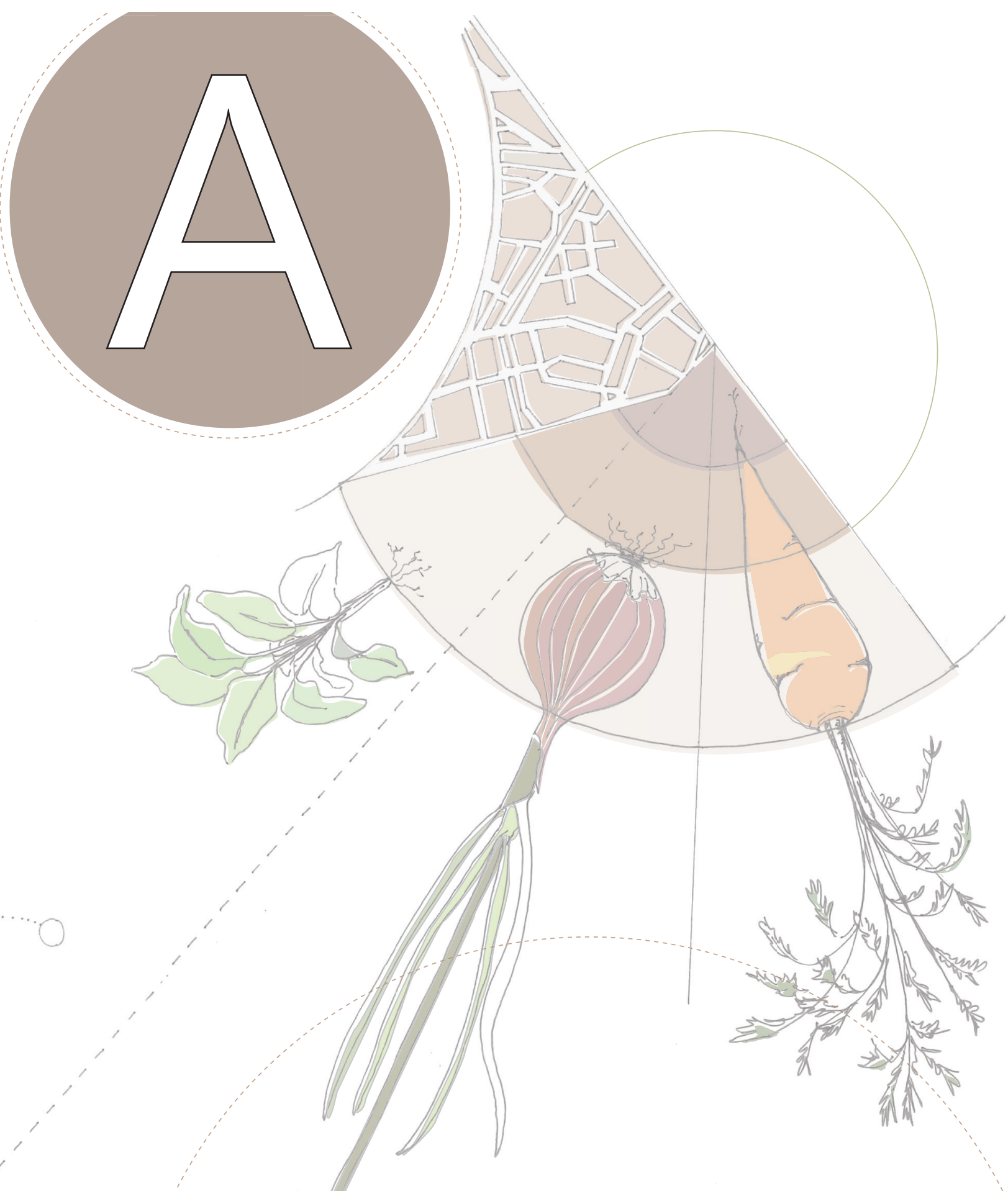

\section{ACKNOWLEDGEMENTS}




\section{Acknowledgements}

I would like to extend my appreciation to those who supported me throughout this $\mathrm{PhD}$ odyssey, both professionally and personally.

To start, I would like to thank the Department of Environmental Technology for fostering an inspiring, open, intellectual and engaged environment. The combination of three research groups and thus the diversity of research focuses leads to interesting conversations and opportunities for cross pollination of ideas and methods. Thanks to Huub and Cees, the staff and all of my colleagues for creating an unbeatable standard of 'togetherness'.

I thank my supervision team for their lose reigns yet sharp feedback. I was given the freedom to pursue research and activities that interested me yet, given structure when taken by the details. Jan Weijma: thank you for your daily guidance and supervision, and your constructive and detailed feedback on manuscripts. I very much enjoyed working together, and learned for your calm and critical reasoning, and practical approach to the tasks at hand. Thank you for always having an open door to discuss work, as well as personal struggles or successes. I am especially appreciative of the flexibility you granted me in planning my work, but also in balancing my work and free time interests. Thank you for allowing me to travel often and for long periods of time during the last four years, without any reproach. Grietje Zeeman, thank you for the discussions, for your support, for checking-up on my progress, planning and state of mind, and for the confidence boosts. Oene Oenema, thank you for your supervision on all things related to nutrients: in soil, plants and fertilizers. I am particularly grateful for your quick replies and to-the-point feedback on manuscripts. I would also like to thank Ingo Leusbrock for his supervision at the beginning of my $\mathrm{PhD}$ trajectory.

To my other co-authors: Robin Harder, with you I can get serious about this field, turn it inside out and upside down. Even fleshing out basic terms, such as 'reuse', 'recycling' and 'recovery', spawns long discussions. I've learned immensely from our collaborations, for which I am so grateful. Thank you for all the 'shit' talking, the hard work and for the friendship. John Stuiver, thank you for helping me smooth out the glitches in my GIS model with much patience. Gunilla Öberg and Sverker Molander, thank you for the helpful input on manuscripts, time and time again. Carolin Wilken and Wei-Shan Chen (Momo), the energy and engagement during our meetings with Jan were top-notch! Thank you so much! I would also like to thank MSc students Stefan Vasilev, Ines Weingarten, Tong Shen, Mathieu Chaix-Bar, Dieuwertje Roelofsen for their hard work and dedication to their research topics. It was a pleasure to supervise each of you.

To the dynamic and international community at ETE. To those who listened, those who served as my anchor when doubt and confusion prevailed; those that cheered me on; those that made me smile and laugh during and beyond working hours: THANK YOU! A shout out to those who have graduated, moved up or moved on: Yvonne, Justine, Arnoud, Sanne, Leire, Sam, Lucia, Dandan, Koen, Momo, Nora, Yujie. To those still biting the bullet, or rather, enjoying the ride: Laura, 
Ilse, Joeri, Thomas, Carlos, Dainis, Sanne, Farzaneh, Adrian, Delaram, Silvi, Jess, Tania, Vincent, Emilius, Kasper, Koen, Annemerel, Merijn, Dilan, Margo, Selin, Yue, Shiyang, Ivonne, Pim, Yu, Bingnan, Rieks, Elackiya, Shokouh, Suzanne, Bernou, Kamonashish, Pradip, Sha, Jiyao, Indra.

Many thanks to Liesbeth, Gea, Marjolein, Wies and the others at the secretariat for their organizational support. Liesbeth, especially, thank you for all the answers. Thanks to Kasia for your support and guidance, to Darja for your positive personality and interest, to Miriam for your advice and humor, to Huub for checking in from time to time, to Alette for the sound advice to 'know when to stop'. Many thanks to Arnoud for the routine sport sessions, to Andrea por ser detallista, por su cariño y su humor, to Carlos por la calma que compartes, to Adrian por las clases de salsa, to Silvi por su energia, to Farzaneh for the care and travels in Chile, to Delaram for checking in, to Thomas for the recipe exchange, to Pim for the travel tips and tops. To my Stitch $\&$ Bitch clan: thanks for the dinners, the chocolate and tea, the gossip and the stitching. It has been so fun to share this hobby with you all and it's good to know alumni are still welcome.

A special thanks to my officemates, Laura, Leire, Emilius, Azie, Dandan, and Vincent, at T'T.1.091 for the friendly and supportive office environment. We got serious and quiet when we needed to be focused and productive, but we also got chatty when we wanted to relax and share. Most remarkable was the cultural diversity in our room (Spain, Indonesia, Brazil, Malaysia, China, the Netherlands and Costa Rica), which became most evident when we started naming 'exotic' fruit. Azie: thanks for the genuine care, for teaching us about your country and religion, and for bringing us souvenirs from your travels. Emilius: thanks for being so relaxed and calm, and for being a great team player. Dandan: thank you for your positive outlook and great laugh! We're still waiting for that morning Tai Chi lesson! Vincent: thanks for filling the room with colorful origami! Laura, thank you for owning the spot in the middle of the room. You became a solid center point that I could turn to for advice, for a good laugh or a hug. Thank you for always caring, your friendship and for smiling with your eyes. Leire: it's not only thanks to the volume of hair on your head that you're able to fill a room. You're warmth and energy are impressive and infectious; you make every day feel like a party! Thank you for our long conversations in the office and a good dance here and there!

To my paranymphs: Joeri, thanks for your openness, for being dependable, for saying it as it is, for questioning habits, and for the best conversation starters. Apart from my family, you're the only other person with whom I'll switch between English, Spanish and Dutch within conversations, or even within one sentence. Thanks for the friendship and the many funxtion trainings. Ilse: we have intertwined our roles as colleagues and as friends seamlessly. What serendipitous timing that we embarked on our PhD odysseys in parallel, and what luck to be paired in interests, drive, values, creativity, and even perfectionism. At work I especially enjoyed our brainstorm sessions over cups of coffee at Impulse, and the endless feedback rounds on our scientific figures. 'Why did you make that line green and not blue?' Who knows what writing a paper together would have been like! Ilse, ik kan je niet genoeg bedanken voor je steun, je tijd, je advies, de reizen, de knuffels, en het lachen. 
It's good to have friends who are always in for sharing a bottle of cava in celebration of achievements (however small): Ilse, Tim, Thijs en Merel. Etentjes, aan de rijn, bruiloften, yoga, dansen, goeie gesprekken en vele flessen cava. Ik waardeer jullie en onze vriendschappen enorm. Tim: bedankt voor je oprechte interesse en vragen, Thijs: voor je rustige en positieve energie, Merel: je manier van aanpakken, dat je voor alles open en klaar staat, en de plantjes verzorgen. Hanne: Als er iemand is bij wie ik altijd terecht kan, dan ben jij dat. Ik heb zo veel te danken an jou en je mateloze steun, enthousiasme, troost, en liefde. Gewoon mogen zijn wie we zijn. Jeroen: bedankt voor de vriendschap en de fijne gesprekken sinds dag één in Wageningen. Melle: snel de tijd om nog even een koffietje te drinken voor dat één van ons weer op reis vertrok. Bedankt voor je praktische blik, je openheid, en vooral voor de vele constructieve sessies afgelopen maanden. Ivar, Mireille, Teun, Eli, Tim: altijd feest met jullie, rond een kampvuur in de groene Kempen of op de Veluwe, en lange avonden gezelschapsspelletjes doen. Bedankt voor de top momenten! Iris: bedankt voor alle uitwisselingen van creatieve ideeën en je vriendschap. Elisa: bedankt voor de fijne gesprekken, de wandelingen en je steun. Didi: je vriendschap en zorg is voor mij onmisbaar geweest. Bedankt voor alle leuke uitjes samen, het sporten, koken en verven, en de lange wandelingen langs de Rijn. To 'las chicas': thank you for the decades of friendship, the endless love, and the best reunions. I will gladly be known to you as your friend who studies 'how poop will save the world'. To my fellow Pioneers: thank you for the support from afar and the strong friendships! The Mechelen crew: bedankt om mij te omarmen en voor de fijne momenten in België en in Nederland. Fatima, gracias por su apoyo constante, su cariño y la amistad.

I am endlessly grateful for the continuous support I have received from my family during the $\mathrm{PhD}$ trajectory. Metje: bedankt voor je interesse in mijn werk, je advies en je steun. Joke en Anton, mijn 'ouders' in Nederland, bedankt voor jullie open deuren. Vera: je bent voor mij een dierbare vriendin. Bedankt voor het luisteren, het delen, je vertrouwen en betrouwbaarheid. Opa en Oma, Opa en Piety: bedankt voor jullie interesse in alles wat wij als kleinkinderen deden, de bewaarde krantenartikelen, de verhalen en fijne gesprekken en jullie bodemloze liefde. Geertje, Daniël, Sarah, Sebastiaan, Mikkel, Oda, August, Adriaan en Johanna: bedankt voor de fijne momenten samen, jullie interesse en liefde, en de gastvrijheid in Mechelen, in Noorwegen en in Duitsland. Het voelt alsof we altijd samen op wereldreis zijn.

Thomas: thanks for your listening ear and for your sound advice, the kind that only older brothers can give. I continue to learn from you and am impressed by your integrity, knowledge and conviction. Thanks for the couch crashes, the recipes, the new tunes, the phone calls, and the shared inside jokes. Pappa en Mamma, Frans en Margreet: bedankt voor de onvoorwaardelijke steun en grenzeloze vertrouwen. Jullie hebben mij de mogelijkheid gegeven om mijn ambities na te streven en mijn vleugels uit te strekken. Zonder jullie had ik hier niet gestaan. Simon: bedankt voor de tijd en ruimte die je mij hebt gegeven om deze mijlpaal te kunnen halen. De vele rondjes langs de dijk waren voor mij onmisbaar: met een theetje voor mij en een biertje voor jou, onze dromen en perspectieven delen. Je geeft mij houvast, en ik weet dat ik altijd op jou kan rekenen. Ik kijk uit naar onze volgende avonturen. Sjoeke, ik zie u graag. 


\section{About the author}

Rosanne Cornélie Wielemaker was born in San José, Costa Rica on February 15 th $^{\text {t }} 1989$ and grew up just outside of the city in Escazú. After completing secondary school at the American international Country Day School, she moved to Portland, OR in the United States of America to pursue her Bachelor of Arts degree in Environmental Studies (magna cum lande) at Lewis \& Clark College. It was there that Rosanne's more focused interest in urban food systems germinated. She conducted independent research on the role of urban agriculture in Havana, Cuba, and her thesis, which received departmental honors, focused on the utopian visions of urban

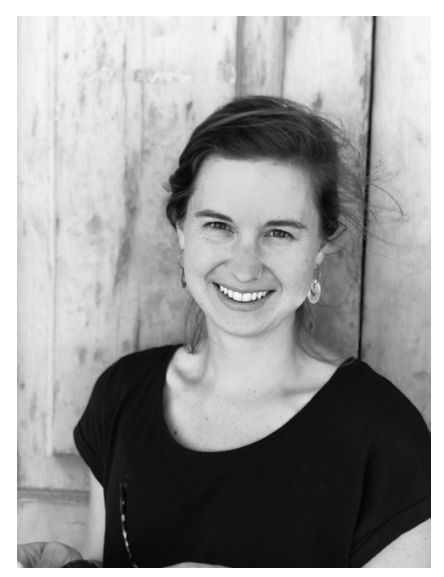
agriculture in cities. In 2011, she moved south to the sprawled city of Phoenix, AZ to put her own convictions of urban farming to the test and worked as General Farm Assistant at Farmyard LLC.

Rosanne moved to Wageningen, the Netherlands in 2012 to obtain a Master of Science degree in Urban Environmental Management (cum laude) from Wageningen University. Growing up in a country of septic tanks, the introduction to large centralized wastewater treatment plants that remove, yet fail to recover, valuable resources caught her attention during lectures. Her master's thesis focused on the recovery of nutrients from domestic wastewater with new sanitation systems for reuse in urban agriculture, and received the 2015 Rachel Carson Thesis Award from the Dutch Association of Environmental Professionals (VVM). During the last year of her master's Rosanne was accepted to the SENSE Honors Program of the Research School for Socio-Economic and Natural Sciences of the Environment (SENSE) to write a PhD research proposal. Her proposal was awarded a second place prize by the Wageningen Institute for Environment and Climate Research (WIMEK). In 2014, Rosanne once again crossed the Atlantic Ocean to Washington D.C. in the United States of America to undertake an internship position at the Department of Agriculture at the Embassy of the Netherlands. Here she investigated the market and knowledgetransfer opportunities in the urban agriculture sector in North America.

Rosanne started as a PhD Researcher in 2015 at the Department of Environmental Technology in the Urban Systems Engineering group under the supervision of Dr Jan Weijma, Prof. Dr Grietje Zeeman, and Prof. Dr Oene Oenema. Her researched aimed to achieve a systematic understanding of urban nutrient flows, specifically focused on the recovery of nutrients from human excreta for reuse as fertilizers in agricultural systems. Her research dealt with matching both quantity and quality of nutrient supply and demand, meanwhile considering relevant spatial and temporal dynamics. The focus of her work on localized, new sanitation systems and urban agriculture is rooted in the idea that resource cycles should be closed at the smallest scale first, before moving outwards to regional or even global scales. 


\section{SENSE}

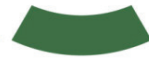

Netherlands Research School for the

Socio-Economic and Natural Sciences of the Environment

\section{I P L O M A}

for specialised PhD training

The Netherlands research school for the Socio-Economic and Natural Sciences of the Environment (SENSE) declares that

\section{Rosanne Cornélie Wielemaker}

born on 15 February 1989 in San José, Costa Rica

has successfully fulfilled all requirements of the educational PhD programme of SENSE.

Wageningen, 19 December 2020

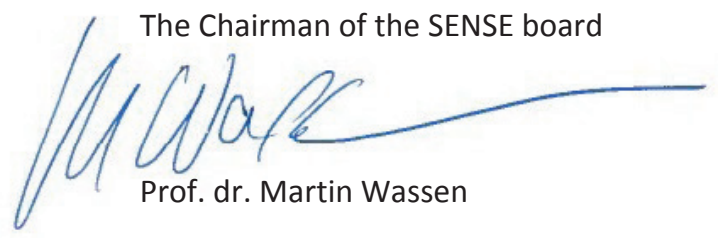

the SENSE Director of Education

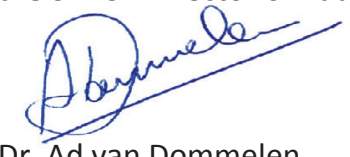

Dr. Ad van Dommelen

The SENSE Research School has been accredited by the Royal Netherlands Academy of Arts and Sciences (KNAW)

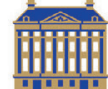

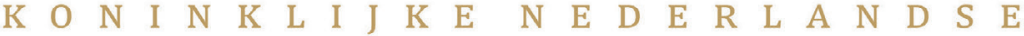
$\begin{array}{llllllllllllllllllllllll}\text { A } & \text { K } & \text { A } & \text { D } & \text { E } & \text { M } & \text { I } & \text { E } & \text { V } & \text { A } & \text { N } & \text { W } & \text { E } & \text { T } & \text { E } & \text { N } & \text { S } & \text { C } & \text { H } & \text { A } & \text { P } & \text { P } & \text { E } & \text { N }\end{array}$ 


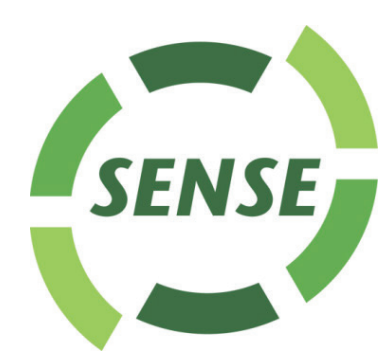

The SENSE Research School declares that Rosanne Cornélie Wielemaker has successfully fulfilled all requirements of the educational PhD programme of SENSE with a work load of $49.3 \mathrm{EC}$, including the following activities:

\section{$\underline{\text { SENSE PhD Courses }}$}

- Environmental research in context (2015)

- Research in context activity: Creating content and video-presenting of accessible MOOC lecture on 'Faeces to Fertilizer' as part of 'Co-creating Sustainable Cities', and developing best practices for successful video-presentations (2017)

\section{Other PhD and Advanced MSc Courses}

- Nutrient Management, Wageningen University (2018)

- Techniques for Writing and Presenting a Scientific Paper, Wageningen Graduate Schools (2015)

- GIS in Practice, PE\&RC and SENSE (2016)

- Project and Time Management, Wageningen Graduate Schools (2015)

- Infographics and Iconography, Wageningen Graduate Schools (2018)

- Scientific Artwork, Wageningen Graduate Schools (2019)

- Adobe InDesign Essential Training, Wageningen Graduate Schools (2019)

\section{Selection of Management and Didactic Skills Training}

- Lecturer in the MSc courses 'Energy, Water and Nutrient Cycles in the Built Environment', 'Foodscapes, Urban Lifestyles and Transition' and 'Circular Systems Engineering' (2015-2019)

- Supervisor of four MSc students (2016-2019)

- Supervisor of one BSc student (2017)

- Member of Department Trip Committee to Chile to promote knowledge-exchange between Dutch and Chilean parties on water treatment and management (2018)

\section{Selection of Oral Presentations}

- Harvest to Harvest: Recovering nutrients with New Sanitation systems for reuse in Urban Agriculture, 7th International AESOP Conference, 7 - 9 October 2015, Torino, Italy

- Identifying and mapping urban nutrient hotspots, 7th Specialized Conference on Resources Oriented Sanitation, 15-18 October 2018, Haifa, Israel

- Integration of urban agriculture and new sanitation: opportunities for nutrient cycling, Novel approaches for future (waste)water treatment and resource recovery, 6 March 2019, Santiago, Chile

o Mapping Amsterdam's nutrient hotspots, EcoCity, 7-11 October 2019, Vancouver, Canada

SENSE coordinator PhD education

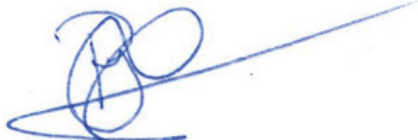

Dr. ir. Peter Vermeulen 
The research described in this thesis was financially supported by the Netherlands Organisation for Scientific Research/Dutch Research Council (NWO) under grant agreement 869.15.016.

Financial support from Wageningen University and NWO for printing this thesis is gratefully acknowledged.

Cover design and artwork by Rosanne Wielemaker

Printed by Proefschriftmaken on FSC-certified paper 

